\title{
Die Konstituierung von Cultural Property
}

Forschungsperspektiven

Regina F. Bendix, Kilian Bizer and Stefan Groth (dir.)

Publisher: Göttingen University Press

Year of publication: 2010

Published on OpenEdition Books: 12 avril 2017

Series: Göttingen Studies in Cultural Property

Electronic EAN: 9782821875425

\section{Qboooks}

http://books.openedition.org

\section{Printed version}

EAN (Print version): 9783941875616

Number of pages: 333

Electronic reference

BENDIX, Regina F. (Hrsg.) ; BIZER, Kilian (Hrsg.) ; und GROTH, Stefan (Hrsg.). Die Konstituierung von Cultural Property: Forschungsperspektiven. Neuauflage [Online]. Göttingen: Göttingen University Press, 2010 (Erstellungsdatum: 21 septembre 2021). Online verfügbar: <http://books.openedition.org/gup/ 488>. ISBN: 9782821875425

(c) Göttingen University Press, 2010

Nutzungsbedingungen

http://www.openedition.org/6540 


\section{Die Konstituierung von Cultural Property}

Forschungsperspektiven

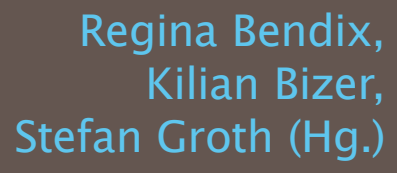

Göttinger Studien zu Cultural Property, Band 1 

Regina Bendix, Kilian Bizer, Stefan Groth (Hg.)

Die Konstituierung von Cultural Property: Forschungsperspektiven

This work is licensed under the Creative Commons License 3.0 "by-nd", allowing you to download, distribute and print the document in a few copies for private or educational use, given that the document stays unchanged and the creator is mentioned. You are not allowed to sell copies of the free version.

$$
\text { (c) }
$$

SORMERIGHIS RESERVED 
erschienen als Band 1 in der Reihe „Göttinger Studien zu Cultural Property“ im Universitätsverlag Göttingen 2010 
Regina Bendix, Kilian Bizer, Stefan Groth (Hg.)

Die Konstituierung von Cultural Property: Forschungsperspektiven

Göttinger Studien zu

Cultural Property, Band 1

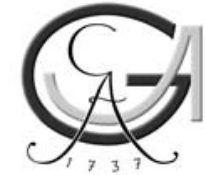

Universitätsverlag Göttingen 2010 


\section{Bibliographische Information der Deutschen Nationalbibliothek}

Die Deutsche Nationalbibliothek verzeichnet diese Publikation in der Deutschen Nationalbibliographie; detaillierte bibliographische Daten sind im Internet über $<$ http://dnb.ddb.de $>$ abrufbar.

Gedruckt mit Unterstützung der Deutschen Forschungsgemeinschaft (DFG)

Dieses Buch ist auch als freie Onlineversion über die Homepage des Verlags sowie über den OPAC der Niedersächsischen Staats- und Universitätsbibliothek (http://www.sub.uni-goettingen.de) erreichbar und darf gelesen, heruntergeladen sowie als Privatkopie ausgedruckt werden. Es gelten die Lizenzbestimmungen der Onlineversion. Es ist nicht gestattet, Kopien oder gedruckte Fassungen der freien Onlineversion zu veräußern.

Satz und Layout: Stefan Groth

Umschlaggestaltung: Margo Bargheer, Stefan Groth Titelabbildung: Stefan Groth

(C) 2010 Universitätsverlag Göttingen http:/ / univerlag.uni-goettingen.de ISBN: 978-3-941875-61-6

ISSN: $2190-8672$ 
„Göttinger Studien zu Cultural Property“ / „Göttingen Studies in Cultural Property“

\section{Reihenherausgeber}

Regina Bendix

Kilian Bizer

Brigitta Hauser-Schäublin

Gerald Spindler

Peter-Tobias Stoll

\section{Editorial Board}

Andreas Busch, Göttingen

Rosemary Coombe, Toronto

Ejan Mackaay, Montreal

Dorothy Noyes, Columbus

Achim Spiller, Göttingen

Bernhard Tschofen, Tübingen

\section{Homepage}

http://gscp.cultural-property.org 



\section{Inhaltsverzeichnis}

Vorwort der Herausgeber

Cultural Property als interdisziplinäre Forschungsaufgabe:

Eine Einleitung. 1

Regina Bendix und Kilian Bizer

\section{Zwischen Heritage und Cultural Property}

Flexibilisierte Kultur zwischen „Tradition, Modernität und Markt“: Akteursbezogene Verwendungs- und Bedeutungsvielfalt von „,kulturellem Erbe“ 25

Brigitta Hauser-Schäublin und Karin Klenke

Sbek Thom nach seiner UNESCO Zertifizierung: Eine Untersuchung der Dynamik des kambodschanischen Schattentheaters nach seiner Ernennung zum immateriellen Kulturerbe .45

Aditya Eggert

Cultural Property und das Heritage-Regime der UNESCO:

Parallelen in ideellen und wirtschaftlichen Inwertsetzungsprozessen von kulturellen Elementen

Arnika Peselmann und Philipp Socha

\section{Parameter des Schutzes von Cultural Property}

Die UNESCO-Konvention zum Schutz des immateriellen (Kultur-)

Erbes der Menschheit von 2003: Öffnung des Welterbekonzepts

oder Stärkung der kulturellen Hoheit des Staates?

Sven Mißling 
Ausdrucksformen der Folklore:

Freie und abhängige Schöpfungen

Philipp Zimbehl

Der Schutz kultureller Güter:

Die Ökonomie der Identität.

Marianna Bicskei, Kilian Bizer und Zulia Gubaydullina

Ethnographische Filmarbeit und Copyright:

Überlegungen zur Situation in Indonesien

Beate Engelbrecht

\section{Muster und Motivationen im Verhandeln von Cultural Property}

Perspektiven der Differenzierung:

Multiple Ausdeutungen von traditionellem Wissen

indigener Gemeinschaften in WIPO Verhandlungen

Stefan Groth

Die verborgene Effektivität minimaler Resultate in internationalen Verhandlungen: Der Fall der WIPO 197

Matthias Lankau, Kilian Bizer und Zulia Gubaydullina

\section{Forschen über Cultural Property}

Die technische Dimension der Konstitution/Konstruktion von Cultural Property 221

Johannes Müske

Der zunehmende Geltungsbereich kultureller Besitztümer und ihrer Politik 235

Rosemary Coombe

\section{Ausblick}

On Cultural Property and Its Protection:

A Law and Economics Comment 261 Ejan MacNay 


\section{Vorwort}

Die interdisziplinäre Forschergruppe zur Konstituierung von Cultural Property nahm ihre Arbeit am 1. Juni 2008 auf. Interdisziplinarität ist ein wissenschaftliches Abenteuer, während dessen sich nicht nur scheinbar unüberwindbare epistemologische Grenzziehungen zeigen, sondern die Forschenden auch Durchbrüche oder zumindest Durchblicke durch gerade diese Grenzen erleben. Neben Forschungsergebnissen, die die jeweilige disziplinäre Forschung fördern, ist es uns ein Anliegen, in diesem ersten Band unserer Publikationsreihe auch einige Ergebnisse vorzustellen, die das Zusammenwirken von Kultur- und Sozialwissenschaften mit juristischen und wirtschaftswissenschaftlichen Disziplinen exemplarisch zeigen. Dies ist unserem Forschungsthema geschuldet, denn die Konstituierung von Cultural Property resultiert auch aus Ideen und Konzepten, die aus dem Wissenstransfer in die Öffentlichkeit aus den beteiligten Fächern in Vergangenheit und Gegenwart erwachsen.

Der Band versammelt Ergebnisse aus disziplinären Teilprojekten mit Beiträgen, die sich aus interdisziplinärer Zusammenarbeit ergaben. Sie werden ergänzt von einem Beitrag und einem Kommentare von Fellows und Mitgliedern unseres Expertennetzwerks. Gerade diesen möchten wir danken für die vielfältigen Impulse und die konstruktive Arbeit mit uns in Workshops und vielen Einzelgesprächen während kürzerer und längerer Aufenthalte: Rosemary Coombe (Toronto) und Ejan Mackaay (Montreal) verbrachten mehrmonatige Fellow Aufenthalte in Göttingen und haben unser Denken und Argumentieren durch viele Einzel- und Gruppengesprächen, bei denen sie sich für die Interessen und Fragen der Nachwuchskräfte unserer Forschergruppe interessiert haben, vorangebracht. Bruno S. Frey (Zürich) verbrachte eine kurze aber produktive Fellow-Strecke mit uns in Göttingen und Valdimar Hafstein (Reykjavík), Jason Baird Jackson (Bloomington), Dorothy Noyes (Columbus) sowie Silke von Lewinsky (München) haben 
während ihrer Kurzbesuche in Vorträgen und Gesprächen unser Forschungsthema reflektiert. Keebet von Benda-Beckmann (Halle), Michael Hahn (Lausanne), Sabine Maasen (Basel) sowie Nele Matz-Lück (Heidelberg) vermittelten eine Außenperspektive in unsern Workshops, und Christoph Brumann (Halle), Tatjana Flessas (London) und William Logan (Burwood Victoria) nahmen als äußerst aktive Diskutanten an der ersten Klausurtagung teil. Dank geht aber auch an die studentischen und wissenschaftlichen Hilfskräfte, die sowohl in der Koordination wie auch in den Einzelprojekten tatkräftige Unterstützung leisten. Den Koordinatorinnen der Forschergruppe, Arnika Peselmann und Marianna Bicskei, sei an dieser Stelle namentlich gedankt für ihren Einsatz, der den reibungslosen Ablauf vieler paralleler Unternehmungen gewährleistet. Dank gilt auch den MTV-Kräften der beteiligten Institute und Lehrstühle, die sich in bewundernswerter Weise mit den sich stets verändernden Modalitäten von Mittelabruf, Abrechnung, Werk- und Honorarverträgen und Reisekostengesetzen befassen.

Unsere Forschergruppe wird durch die Deutsche Forscbungsgemeinschaft gefördert, die Antragstellung ebenso wie infrastrukturelle Aspekte der laufenden Arbeit wurden und werden durch die Georg-August-Universität Göttingen mitgetragen. Beiden Förderinstitutionen sei hiermit gedankt.

Regina Bendix, Kilian Bizer, Stefan Groth

Göttingen, im Juni 2010 


\title{
Cultural Property als interdisziplinäre Forschungs- aufgabe: Eine Einleitung
}

\author{
Regina Bendix und Kilian Bizer
}

Mit „Triumph der Medizinmänner“ betitelte die Frankfurter Allgemeine Zeitung einen Bericht über die juristische Auseinandersetzung einer südafrikanischen Gemeinschaft gegen das deutsche Pharmaunternehmen Schwabe. Mit Wörtern wie „Medizinmänner“ im Kampf gegen „Biopiraterie“ ruft der Artikel den kolonialen Hintergrund hervor, aus welchem sich diese Auseinandersetzungen um eine GeranienWurzel und das traditionelle Wissen um ihre hustenheilende Kraft speist. Doch spricht er auch von einer „Gemeinschaft" (und nicht etwa einem „Stamm") und berichtet über die Unterstützung durch die Nichtregierungsorganisation „African Center for Biosafety": Damit sind Nomenklaturen und Akteure der postkolonialen Gegenwart genannt, die für die zunehmende öffentliche und internationale Auseinandersetzung um Eigentum an Kultur charakteristisch sind. ${ }^{1}$ Indigenes Wissen um Pflanzen ist nur einer von vielen Bereichen immaterieller Kultur, deren potentieller Wert unter dem Gesichtspunkt von Cultural Property ins Rampenlicht gerückt ist. Im Juni 2010 berichtete der Daily Telegraph über eine Regierungsinitiative Indiens, in Vorbereitung auf Patentierung Yoga Positionen filmisch festzuhalten. „Es ist wie mit Fußball und Großbritannien“, wird ein Guru im Gespräch mit einem britischen Journalisten zitiert, „Ihr habt der Welt Fußball gegeben, das war wun-

${ }^{1}$ Frankfurter Allgemeine Zeitung, 01.02.2010, S. 15. 
derbar und generös. Aber stellt Euch vor, es gäbe Leute, die zu sagen beginnen, sie hätten den Sport erfunden. Das wäre doch ärgerlich. “2

Dies sind nur zwei aus einer Vielzahl von Beispielen, die die theoretische Frage, ob Eigentum an Kultur sinnvoll sein kann, in konkrete Verhandlungsräume getragen haben. Eigentum an materiellen Kulturgütern erscheint zumindest regelbar, wenn auch das gesetzliche Arbeiten mit natürlichen und juristischen Personen als Eigentümern nicht universell geteilt wird. Das zunehmende und weltweite wirtschaftliche Interesse an immateriellen Kulturgütern wie traditionellem Wissen und kulturellen Ausdrucksformen erweitert und problematisiert den Spielraum. Nach westlichem Selbstverständnis befinden sich die immateriellen Dimensionen von Kultur in der Public Domain, jeder kann sie für sich nutzen. Ansprüche indigener Gruppen, Aborigines, ethnischer Interessensgemeinschaften und kultureller Gemeinschaften, Rechte an ihrer Kultur selbst zu verwalten, greift die Grundfesten dieser Annahme an.

Gibt es gute Gründe dafür, kulturelles Eigentum an immateriellen Gütern zu schaffen? Und gibt es gute Gründe dafür, auch für materielles Kulturgut einen internationalen Prozess der Inwertsetzung zu betreiben und damit faktische Verfügungsrechte über Renditen zu schaffen? Die Positionen in diesen Fragen fallen durchaus unterschiedlich aus. Während die einen hervorheben, dass Kultur in der Public Domain einen lebendigen Umgang mit ihr ermöglicht, stellen die anderen heraus, dass erst durch Eigentum für Gruppen oder Privatpersonen sich der Schutz derselben sowie ihre Pflege und Entwicklung auch wirtschaftlich lohnt. Gleichzeitig betonen andere, dass die Zweckentfremdung oder Misappropriation von Kultur durch kommerzielle Anbieter die Identität bestimmter Gruppen beeinträchtigt und deshalb Inwertsetzungsprozesse rund um Kultur auch unter Gesichtspunkten der Menschenrechte zu betrachten sind.

Unabhängig davon, wie man im Einzelfall diese Fragen selbst beantworten möchte, befinden sich die Vereinten Nationen in verschiedenen Foren in einem Abstimmungsprozess darüber, bestimmte materielle Objekte ebenso wie immaterielle Praxen über einen speziellen Status in Wert zu setzen (zum Beispiel bei der UNESCO), bei immateriellen Kulturgütern zu ermitteln, auf welche Weise diese denn, analog zu geistigem Eigentum, mit Verfügungsrechten versehen werden können (zum Beispiel bei der WIPO) und inwiefern solche Verfügungsrechte auch unter dem Gesichtspunkt des Schutzes gefährdeter menschlicher Populationen betrachtet werden soll (zum Beispiel bei der CBD).

Seit Juni 2008 arbeitet an der Universität Göttingen eine interdisziplinäre Forschergruppe an solchen Fragen unter dem Gesichtspunkt der Konstituierung von Cultural Property. Diese Einleitung stellt die Thematik, ihre Begrifflichkeiten und unsere Forschungsschwerpunkte vor, um so die hier versammelten Ausschnitte der in den ersten achtzehn Monaten erzielten Teilergebnisse zu rahmen. Die Erkennt-

2 "India moves to patent hundreds of yoga postures", http://www.telegraph.co.uk/news/worldnews/asia/india/7809883/India-moves-to-patent-hundreds-of-yoga-postures.html (Zugriff am 21.06. 2010, Übersetzung der Autoren). 
nisinteressen der beteiligten kultur- und sozialwissenschaftlichen Disziplinen, der Volkswirtschaftslehre sowie der Rechtswissenschaften im Bereich des Zivil- und des Völkerrechts unterscheiden sich erheblich durch ihr jeweiliges wissenschaftliches ebenso wie gesellschaftliches Selbstverständnis. Das, so die Forschungsgrundlage unserer Unternehmung, nur über seine Konstituierungsprozesse nachvollziehbare Konzept des Cultural Property, liegt jedoch bereits in seiner Benennung zwischen den Fächern; sowohl das Verstehen wie das Erklären des Phänomens bedürfen deshalb der interdisziplinären Zusammenarbeit.

Der Begriff Cultural Property fügt sich zusammen aus „Eigentum“ - einer üblicherweise rechtlich definierten und verankerten und wirtschaftlich zentralen Kategorie sowie dem Adjektiv „kulturell“, wodurch gleich mehrere Schleusen geöffnet werden: Es präsentieren sich die Fragen nach der Zurechenbarkeit des Eigentums, der Veräußerbarkeit von Kultur sowie nach globalen Maßstäben dessen, welche Rechte und Pflichten mit dem Eigentum einhergehen können, worin wiederum die Definitionsproblematik von „Kultur“ einerseits sowie kulturell divergierende Konzepte von Eigentum andererseits zu der komplexen Forschungsmatrix beitragen.

Hieraus ergibt sich eine Spannbreite von Forschungsfragen, die selbstredend in Etappen und Ausschnitten zu behandeln sind. Es eröffnet sich ein Themenkomplex, zu dem die unterschiedlichen Fachperspektiven sowohl an Grundlagenwissen für die jeweils eigene Disziplin wie auch an interdisziplinär generierten Erkenntnissen für die global-gesellschaftliche Reflexion der Umsetzbarkeit des Cultural Property-Konzeptes in gelebte juristische und wirtschaftliche Praxis beitragen können. Die Zusammensetzung unseres Forschungsteams privilegiert spezifische Fokussierungen sowohl von übergeordneten Fragen wie auch von Fallstudien, die in unserem ersten Forschungskonzept Niederschlag fanden. Eine Konzentration auf Akteure, Diskurse, und Kontexte innerhalb der Fallstudien dient dazu, bereits bestehende und sich herausbildende Regeln im Bereich von Cultural Property differenziert betrachten und vergleichen zu können. Zu Grunde liegende Konzepte, die Wahl der Fallstudien sowie die Erfahrungen mit dem interdisziplinären Zusammenarbeiten sollen im Folgenden skizziert werden.

\section{Zur Konstituierung eines Begriffes}

Kulturelles Eigentum wird erstmalig im ausgehenden 19. Jahrhundert als international relevanter Typus von Eigentum umschrieben. Manilio Frigo hält dies wie folgt fest (2004:367):

According to an established rule of customary international law, the destruction, pillage, looting or confiscation of works of art and other items of public or private cultural property in the course of armed conflicts must be considered unlawful. The illicit character of the above practices may be asserted at least since the codification of that rule in the Hague Convention respecting the Laws and Customs of War on Land, adopted and revised re- 
spectively by the First and Second Peace Conferences of 1899 and 1907, and in the 1907 Hague Convention concerning Bombardment by Naval Forces in Time of War.

Im internationalen juristischen Kontext genannt wurde der Begriff allerdings erst in der Haager Konvention von 1954 zum Schutz von Cultural Property im Falle bewaffneten Konflikts (ebd.). Gemeint waren hiermit insbesondere „kulturelle Güter" der - im westlichen Sinne - hochkulturellen Art: die Erbeutung von Gemälden und Skulpturen, wertvollen Bibliotheksbeständen und dergleichen mehr. Die Rückführung von Kriegsbeute an staatliche und private Institutionen nach dem zweiten Weltkrieg bedurfte der Legitimation auf völkerrechtlicher Ebene und der Entwicklung von international geltenden Rechtsinstrumenten.

Verschiedene Nachkriegsdynamiken auf globaler Ebene führten zu einer sukzessiven Erweiterung der Begriffsbedeutung von Cultural Property. Die Befreiung und Entlassung vieler kolonialer Territorien in staatliche Souveränität förderte den Anspruch auf die Rückführung von kulturellen Gütern aus kolonialem Bestand weit über das hinaus, was unter dem westlichen Verständnis von hochkulturellen Gütern gefasst worden war. Die Erweiterung des Verständnisses von „kulturrelevantem" Eigentum lässt sich vor allem über zwei Bewegungen verfolgen, nämlich die Bemühungen um den Schutz von Kulturgütern vor Zerstörung und Vergessen sowie die Versuche, kulturelles Eigentum mit Nutzungsrechten zu versehen.

Die verschiedenen Heritage-Konventionen der United Nations Educational, Scientific and Cultural Organization (UNESCO) lassen nachvollziehen, wie die Bemühung um den Schutz von als wertvoll erachteten archäologischen und architektonischen Beständen zu der noch breiter gefassten UNESCO-Konvention von 1972 zum Schutze des Kultur- und Naturerbes der Welt führte. ${ }^{3}$ Hierin zeigt sich einerseits eine Annäherung der Begrifflichkeit von Cultural Property und Cultural Heritaget, wobei die Betonung deutlich auf ideellen Werten liegt. Die weitere Entwicklung der Schutzprogramme innerhalb der UNESCO reflektiert sodann auch das beständig differenziertere Bemühen, Typen von schützenswerter Kultur zu identifizieren: So wurden verschiedene Kategorien von Kulturlandschaften als Teil des Weltkulturerbes heraus differenziert; unter dem Begriff des Memory of the World wurde ein UNESCO-Register des Dokumentenerbes eröffnet; dem Kulturerbe unter Wasser wurde eine weitere UNESCO-Konvention gewidmet und 2003 gelang schließlich nach langem Ringen auch die UNESCO-Konvention zum Schutze des mündlichen und immateriellen Kulturerbes (Hafstein 2004, 2007). Das Heritage-Denken hat also, wie die über dreißigjährige Zeitspanne zeigt, nach und nach von einer eher statischen und materiellen Auffassung des Schutzwürdigen zu einer zunehmend prozessualen Konzeption von kulturellem Erbe gefunden, die sich in der 2003er Konvention wie folgt ausdrückt (UNESCO 2003:3):

\footnotetext{
${ }^{3}$ Vgl. zu der Entwicklung innerhalb der UNESCO seit 1954 Weigelt (2008).

${ }^{4}$ Vgl. Frigo 2004, O’Keefe 1992.
} 
This intangible culture heritage, transmitted from generation to generation, is constantly recreated by communities and groups in response to their environment, their interaction with nature and their history, and provides them with a sense of identity and continuity, thus promoting respect for cultural diversity and human creativity.

Bemerkenswert ist zudem die Verschiebung vom Eigentums- zum Erbebegriff innerhalb der UNESCO. Der euro-amerikanische Eigentumsbegriff, der auch in der Haager Konvention von 1954 unhinterfragt figuriert, und der im juristischen Gebrauch eine umfassende Verfügungsgewalt beinhaltet, wurde durch den sehr viel weniger scharf konturierten Begriff eines globalen Heritage ersetzt (Weigelt 2008, vgl. Bendix 2000).

Parallel zur Ausdifferenzierung eines globalen Heritage-Nachkriegsregimes, das sich von früheren, regional und national agierenden Denk- und Heimatschutzbestrebungen und -gesetzen ableitet, sich aber von diesen in Ausmaß und Anspruch unterscheidet (Tschofen 2007), erwuchs auch das Bestreben, insbesondere immaterielle kulturelle Güter - Traditionen, kulturelle Wissensbestände, etc. - wirtschaftlich zu nutzen. Mit anderen Worten: Der reale Eigentumsanspruch verschaffte sich neben ideellen, identitätsstiftenden Ansprüchen zunehmend Geltung. Wie die Völkerrechtlerin Silke von Lewinski zusammenfasst, reicht das westlich geprägte Verlangen, verloren geglaubtes, kulturelles Wissens in vermeintlich „unberührten“ Gruppen wiederzufinden, weit zurück. Diese Gruppen - heute oft unter dem Begriff ,,indigene Kulturen“ gefasst ${ }^{5}$ - wehren sich zunehmend gegen die Nutzung ihrer kulturellen Ressourcen (Lewinski 2003:1-3). ${ }^{6}$ Zum einen wird damit eine quasi-kolonialistische Haltung seitens großer Konzerne und Wirtschaftsmächte geahndet. Zum anderen reflektieren diese Bestrebungen um Eigenverantwortung und Kontrolle nicht nur politische, sondern durchaus auch wirtschaftliche Interessen: Für viele indigene Gruppierungen ist ihre Kultur eine der wenigen Ressourcen, die sie im internationalen Markt nutzen können. Entsprechend brisant ist das Bemühen, Regulierungen von Cultural Property auf internationaler Ebene herbeizuführen. Diese Entwicklung hat 2000 innerhalb einer weiteren Institution der United Nations, der World Intellectual Property Organization (WIPO), zur

\footnotetext{
5 Auf eine Diskussion des Begriffs der Indigenität wird hier verzichtet, nicht ohne darauf zu verweisen, dass ethnische Gruppen und andere kulturelle ebenso wie religiöse Minoritäten innerhalb von Nationalstaaten, nomadische Gruppen etc. oft ihre eigenen Gruppenbezeichnungen bevorzugen und der Begriff des Indigenen ebenso wie die genannten anderen Begriffe jeweils aus spezifischen Kontexten erwuchsen und eine Verallgemeinerung nur mit caveat genutzt werden kann. „Kulturelle Gruppen" ist vielleicht - um der Vergleichbarkeit willen - am ehesten brauchbar, communities ist ein weiterer Begriff, der in manchen internationalen Gremien sowie auch seitens Repräsentanten von manchen Gruppen genutzt wird, da dieses Konzept keine ethnische/genetische Komponente beinhalten muss (wenn es dies beim Gebrauch mancher Akteure dennoch oft tut).

${ }^{6}$ Lau (2000) hat die zumindest partiell auf die Fusion von New Age Interessen und kapitalistische Prinzipien zurückzuführende Wertschöpfung aus fremd-kulturellem Wissen durch westliche Industrien an verschiedenen Fallbeispielen nachvollzogen. Comaroff und Comaroff nutzen große Teile ihres kritischen Essays Ethnicity Inc. (2009), um die Selbstvermarktung seitens ethnischer Gruppen zu problematisieren und zu verstehen.
} 
Regina Bendix und Kilian Bizer

Gründung eines internationalen Komitees geführt (IGC), das sich damit befasst, inwiefern Cultural Property mit den juristischen Kategorien des geistigen Eigentum Urheber und Patentrecht, Trademark - gehandhabt werden kann.

Die skizzierten Bereiche des innerhalb der UNESCO diskutierten Cultural Heritage und der nicht nur innerhalb der WIPO diskutierten Cultural Property zeigen in ihrer institutionellen Verortung und unterschiedlichen Benennung den Versuch, zwischen ideellen und wirtschaftlichen Werten von Kulturgütern zu unterscheiden. Doch ist es mehr als deutlich, dass die unweigerlich miteinander verbundenen ideellen Werte von Kulturelementen - archäologisch, historisch, ästhetisch, lebenspraktisch - einer potentiellen wirtschaftlichen Inwertsetzung nicht zwangsläufig entgegenstehen müssen. Die Entscheidung über den Wert eines Kulturgutes unterliegt äußerst komplexen Faktoren (Groth 2009). Die Entscheidung über den Besitzanspruch eines kulturellen Gutes, sei dies materieller oder immaterieller Art, lässt sich ebenfalls nur schwer festlegen, wie Dorothy Noyes unter Rückgriff auf Salomons Urteil feststellte (2006).

Natürlich gibt es verschiedene andere Bereiche, in welchen die Wertschöpfung aus kulturellen Ressourcen gang und gäbe ist - am deutlichsten sicher im Feld des Tourismus beispielsweise in Verbindung mit Stätten des Weltkulturerbes. Die diskursive Zuspitzung zu Cultural Property ergab sich jedoch in den zwei geschilderten Arenen von UNESCO und WIPO, weshalb die in der ersten Phase des Forschungsunternehmens initiierten Göttinger Forschungsvorhaben den HeritageKomplex einerseits und Verhandlungsbühnen und -prozesse in diesen Organisationen andererseits fokussiert haben.

\section{Zur Anlage des interdisziplinären Forschungsvorhabens}

Wem gehört Kultur? Wer hat Anspruch auf kulturelles Eigentum? Wie und von wem können rechtsgültige Entscheidungen hierzu gefällt werden? Seit einigen Jahren werden diese Fragen zunehmend in breiterer Öffentlichkeit und mit wachsender Brisanz diskutiert. Je nachdem, wem diese Frage gestellt wird, folgen eine Reihe weiterer Fragen gesellschaftspolitischer, wirtschaftlicher oder wissenschaftlicher Natur. Je globaler die Basis der beteiligten Akteure, desto komplexer präsentieren sich die Fragen. Die Initiatorin der Göttinger Forschergruppe begegnete Cultural Property erstmals als Vorsitzende einer internationalen Fachvereinigung, welche ab 2004 als beobachtende Organisation bei den Sitzungen des Intergovernmental Committees zu Cultural Property der WIPO partizipieren konnte. Vertraut mit Diskussionen rund um die Inwertsetzung von Kultur, die seit den 1960er Jahren in der Kulturanthropologie/Europäischen Ethnologie dokumentiert und teilweise virulent diskutiert worden sind und werden ${ }^{7}$, erschien dieses WIPO-Forum als eine

\footnotetext{
7 Zum Beispiel zu Folklorismus, Tourismus und ethnischem Marketing Bausinger 1988, EvansPritchard 1987, Graburn 1979, vgl. zusammenfassend Bendix 1997:188-218, sowie jüngere und jüngste Arbeiten wie Hemme, Tauschek und Bendix 2007, Hemme 2009, Zimmermann 2009.
} 
neue Ebene, die zu verstehen auch für Kulturwissenschaftler/innen wesentlich war. Die Veräußerung von Kultur in ein Wirtschaftsgut stellt - nach der bereits seit der Romantik verfolgbaren politischen Instrumentalisierung von Kultur im Rahmen der Nationalstaatsbildung - eine weitere Handlungsebene dar, innerhalb der sich neue kulturelle Praxen und damit neue lebensweltliche Perspektiven herausbilden. Hinzu kam, dass an verschiedenen internationalen Fachtagungen sowohl der Jurist Wend Wendland, Deputy Director der Global IP Division der WIPO wie auch der Abteilungsleiter der UNESCO-Sektion für immaterielles Kulturerbe, der Sprachwissenschaftler Rieks Smeets, auftraten und dabei einerseits ihre institutionellen Anliegen vorstellten und andererseits um kulturwissenschaftliches Interesse und Begleitung warben. Die Nähe zwischen Fragen des kulturellen Eigentums und des Kulturerbes wurde damit gleichsam inszeniert.

Diese Foren und ihre Auswirkungen auf die kulturelle Praxis erregen kulturwissenschaftliches Interesse insbesondere auch deshalb, weil die Akteure innerhalb der WIPO und des IGC - und partiell trifft dies auch für UNESCO $\mathrm{zu}^{8}$ - kaum mehr direkten Bezug zu den verhandelten Kulturgütern haben, sondern diese vielmehr in unterschiedlicher Weise evaluieren, zertifizieren und verwalten. Es sind Diplomat/innen und Regierungsvertreter/innen, meist geschult in Jura und Wirtschaftswissenschaften, die sich mit den internationalen Handelsparametern von Kulturgütern beschäftigen. Völkerrechtliche und vor allem staatliche Handelsgesetze sowie Prinzipien einer profitablen Volkswirtschaft stehen im Vordergrund, während der direkte Bezug zu den kulturellen Praxen und Artefakten fehlt. Im Tourismusgewerbe bieten Akteure eines Ortes oder einer Region Aspekte ihrer Kultur von Kunsthandwerk bis zu Festivitäten und historischen Monumenten Besuchern zu Kauf und Besichtigung an und profitieren davon mehr oder weniger direkt. Hierzu gibt es seit den 1970er Jahren eine reichhaltige und wachsende Sekundärliteratur inklusive verschiedener interdisziplinärer Fachzeitschriften. ${ }^{9}$ Die Dynamiken in diesem und anderen mit Kulturgütern befassten Wirtschaftsbereichen weisen jedoch global, grob verallgemeinernd betrachtet, ein starkes Nord-Süd Gefälle auf. Manche Akteure erkennen und nutzen den Wert kultureller Ressourcen von Gruppen, denen sie selbst nicht angehören. Diese Aneignungen haben die Problematisierung von Cultural Property auf internationaler Ebene in Gang gebracht und auch zur Herausbildung des WIPO IGCs geführt.

\footnotetext{
8 Die Gremien der UNESCO für Kulturerbe werden jedoch explizit durch verschiedene Expertengremien unterstützt, allen voran der International Council on Museums and Sites: "ICOMOS is named in the 1972 UNESCO World Heritage Convention as one of the three formal advisory bodies to the World Heritage Committee, along with the World Conservation Union - IUCN, based at Gland (Switzerland), and the International Centre for the Study of the Preservation and Restoration of Cultural Property (ICCROM), based in Rome (Italy)." "“Icomos and the World Heritage Convention", http://www.international.icomos.org/icomos/world_heritage/icomoswh_eng.htm, Zugriff am 04.04.2010).

${ }^{9}$ Genannt seien hier aus den rund 70 Fachzeitschriften, die spezifisch der Tourismusforschung gewidmet sind, nur die Zeitschriften Annals of Tourism Research (seit 1972), die auch als die erste für dieses interdisziplinäre Feld gelten kann, sowie das Journal for Tourism and Cultural Change (seit 2003), das sich thematisch vermehrt auch Fragen stellt, wie sie in unserer Forschergruppe behandelt werden.
} 
Diese Sachverhalte können zwar durchaus kulturwissenschaftlich dokumentiert werden, doch bedarf es, so die Göttinger Einsicht, eines interdisziplinären Zugriffes, um Forschungsresultate zu erzielen, die das handelnde Selbstverständnis der beteiligten Akteure nicht nur aus der - vielleicht verständlicherweise - oft kritischen Perspektive der Kulturwissenschaften wahrnehmen ${ }^{10}$, sondern die Praxen und Handlungshorizonte aller beteiligten Akteursgruppen mit einbeziehen. Manche Kulturwissenschaftler/innen verstehen sich nachwievor als Fürsprecher/innen von marginalen communities in einer postkolonialen Welt, doch selbst der jüngste, fast zynisch verfasste Essay des sozialanthropologischen Paares John L. und Jean Comaroff zu den Typen ethnischer Vermarktung (2009) deutet letztendlich auf eine Verunsicherung, inwieweit eine solche Fürsprecherrolle immer angezeigt oder gewünscht ist. ${ }^{11}$

Um die interdisziplinäre Arbeit aufgreifen zu können, galt es, einerseits die Definitionsproblematik des Kulturbegriffs zu konfrontieren und andererseits die Existenz kulturell divergierender Konzepte von Eigentum hervorzuheben. Eine wissenschaftliche Beschäftigung mit Cultural Property lässt rasch die Problematik erkennen, dass aus den vielfältigen Manifestationen von Kultur - verstanden im Sinne von Clifford Geertz als ein Gewebe von Bedeutungen, das der Mensch selbst hervorbringt und in dem er sich fortwährend bewegt - nur einige wenige kulturelle Elemente ausgewählt werden, die dann als eigentumswürdig erklärt und genutzt werden. Durch ein wie auch immer begründetes Herausheben ausgewählter, kultureller Äußerungen aus der Selbstverständlichkeit von Kultur erhalten diese Elemente einen Sonderstatus. Um sie ranken sich die Diskussionen über Cultural Property, die den Sonderstatus in verschiedener Weise markieren, reglementieren und dadurch auch fixieren. Kultur im Sinne ethnologisch-kulturanthropologischer Konzeption ist beständig wandelbar; kulturelle Praxen im Sinne von Pierre Bourdieu werden in einem unreflektierten Habitus ausgeführt. Werden kulturelle Objekte und Praxen aus dieser selbstverständlichen Einbettung herausgehoben, so bedarf es der Reflexion und Reglementierung. „Kultur“ ist jedoch nicht nur ein wissenschaftliches Konzept, sondern auch ein Alltagsbegriff, der je nach Kontext und Akteuren sowie deren Motivationen und Zielen unterschiedlich verwendet

\footnotetext{
${ }^{10}$ So argumentierte etwa Martin Scharfe im Eröffnungsvortrag der bisher größten deutschsprachigen Fachtagung zu Kulturerbe: „Man kann inzwischen wissen, dass die Kulturwissenschaftlerin, der Kulturwissenschaftler dem Begriff des Kulturellen Erbes misstrauen muss, weil er - ein erster Punkt! - ein Begriff der politischen Praxis ist, das heißt nicht so sehr ein Hilfsmittel fürs Nachdenken als vielmehr ein Vehikel des Machens und der politischen Gestaltung. Das heißt aber auch: Audruck konkurrierender Mächte“ (2009:15-6). Unter den insgesamt 42 Beiträgen dieses Tagungsbandes sind dennoch auch ein Gutteil an Fallstudien, die Kulturerbe Praxen empirisch untersuchen, mit anderen Wertschöpfungssystemen in Verbindung bringen und damit dazu beitragen, Polarisierungen zu überwinden.

11 Welche Rolle Kultur- und Sozialanthropologie in diesen Prozessen einnehmen, wie sie sich unterschiedlich politisch verorten je nach impliziter oder expliziter politisch-ideologischer Position und je nach Fachverständnis wäre eine eigene Studie wert . Immerhin lässt sich sagen, dass diese Fächer sich insbesondre seit dem zweiten Weltkrieg konsequent mit der Ethik ihres Forschens und Publizierens auseinandersetzen - was zum Beispiel an verschiedenen, in der American Antbropological Association diskutierten Fällen, deutlich wird.
} 
wird. Eine klare Grenzziehung zwischen dem ethnologisch-kulturanthropologischen Kulturbegriff und dem in seiner Definitionsbreite schillernden Alltagsverständnis von Kultur ist oft nur schwer möglich.

Diese begriffliche Heterogenität zeigt sich auch in den beteiligten Wissenschaften unserer Forschergruppe. Der Kulturbegriff, der in den verschiedenen Teilprojekten verwendet wird, steht deshalb - notwendigerweise - im Spannungsfeld zweier Pole der geistes- und gesellschaftswissenschaftlichen Beschäftigung mit Kultur. Sowohl für das juristische wie das wirtschaftswissenschaftliche Arbeiten ist es wichtig, eine Bestimmung dessen vorzunehmen, was unter „Kultur“ zu verstehen ist, damit normative Perspektiven zur Nutzung und/oder zum Schutz von Cultural Property überhaupt erarbeitet werden können. Ein diese Handlungszusammenhänge organisierender Kulturbegriff, der letztlich auf den Tylor'schen Kulturbegriff (1871) zurück geht, wurde von der UNESCO anlässlich der Weltkulturkonferenz in Mexiko (1982) folgendermaßen definiert - eine Definition auf welche sich diese Gesellschaftswissenschaften in unserer Forschergruppe anfänglich stützten:

[...] in its widest sense, culture may now be said to be the whole complex of distinctive spiritual, material, intellectual and emotional features that characterize a society or social group. It includes not only the arts and letters, but also modes of life, the fundamental rights of the human being, value systems, traditions and beliefs (UNESCO 1982: Präambel).

Die kulturanthropologisch-ethnologischen Projekte arbeiten dagegen mit einem offenen, konstruktivistischen Kulturbegriff, da diese Projekte darauf ausgerichtet sind $\mathrm{zu}$ untersuchen, was unterschiedliche Akteursgruppen in ihren jeweiligen Kontexten unter Kultur verstehen und mit welchem Ziel. Kultur in diesem Sinn wird als aushandelbarer Prozess von mit unterschiedlichen Motivationen und Zielen ausgestatteten Akteursgruppen begriffen. Diese Projekte verzichten deshalb auf eine a priori Bestimmung von Kultur, die sich retrospektiv auch für die Rechts- und Wirtschaftswissenschaften nicht wirklich ausgezahlt hat, weil die schillernden Facetten des Kulturbegriffs lediglich für die Annäherung an den Forschungsgegenstand hilfreich, sich aber keineswegs als für die Forschungsarbeit konstitutiv erwies.

Ziel der interdisziplinären Zusammenarbeit insgesamt ist es, durch die konkrete Analyse von Fallstudien die Diskurse, Prozesse und Motivationen von Akteursgruppen aufzuzeigen, die zur Bestimmung eines bevorzugten Spektrums kultureller Manifestationen im Sinne von Cultural Property führen, hinter welchem sich auch eine Normierung von eben verschiedenen Auslegungen des Kulturbegriffes verbergen. Die Teilprojekte haben deswegen Kultur als das verstanden, was die lokalen, nationalen oder internationalen Akteure darunter verstehen und haben sich auf dieser Basis dann mit den Akteuren und ihren Motivationen sowie den Prozessen beschäftigt, die zu einem wie auch immer gearteten Schutz vor Aneignung durch Dritte führen. 
Auch der Eigentumsbegriff musste interdisziplinär durchdacht werden, um zu einer gegenseitig akzeptablen Vorstellung eines sozial konstituierten Eigentums zu gelangen - wobei hier die Vorstellungen der Fächer näher beieinander lagen. In vielen wissenschaftlichen Disziplinen basieren Konzepte von Eigentum auf europäischen, historisch gewachsenen Eigentumsbegriffen. Eigentumsrechte erwuchsen hier einerseits durch die zum Beispiel im frühneuzeitlichen England einsetzenden Auseinandersetzungen um Eigentum sowie um die Nutzungsrechte und pflichten der Allmende. Die Befürwortung privaten Eigentums geht allerdings bereits auf Aristoteles Formulierungen zurück, die unter dem Stichwort „The Tragedy of the Commons “ in jüngerer Zeit erweiternd diskutiert wurden. ${ }^{12}$ Landeigentum bleibt auch bei der Konstituierung von Cultural Property eine latent wichtige Komponente, vor allem für die sogenannten indigenen Völker, bei der Argumente um die intrinsische Verbindung von territorialem und kulturellem Eigentum in komplexe institutionelle Aushandlungsprozesse überführt werden.

Ein Problemspektrum in der globalen Konstituierung von Cultural Property ergibt sich aus dieser bis vor kurzem unreflektierten Dominanz des westlich geprägten Eigentumsbegriffes, denn Definitionen von „Eigentum“ sowie das Spektrum von Haltungen bezüglich Eigentumsarten unterscheiden sich je nach kulturellem Umfeld und historischer Epoche (Siegrist und Sugermann 1999). Bereits in der westlichen Denk- und Handlungstradition ist der Begriff des Eigentums komplex. Die industrielle Revolution trug dazu bei, dass der Eigentumsbegriff sich von Landbesitz auf weitere Ressourcen ausdehnte, die als handelbares Gut auf Märkten gekauft und verkauft werden. Eine sich differenzierende Marktwirtschaft führte dazu, dass nicht nur materielle sondern auch geistige Ressourcen - das sogenannte intellectual property (IP) - zwecks Wertschöpfung herangezogen wurden (wobei der Schutz geistigen Eigentums bereits im 15. Jahrhundert belegbar ist). Die Entwicklung des Urhebergedankens und damit des Urheber- und Patentrechtes unter anderem als Anreizsysteme für die Schaffung neuer Werke und Produkte, brachte in der westlichen Praxis zunehmend einen an das Individuum gebunden Eigentumsgedanken hervor (Goldstein 1994, 2001, Patterson 1968, Vaidhyanathan 2001), der in der modernen Volkswirtschaftslehre vielleicht seinen Höhepunkt gefunden hat und die Zuteilung möglichst aller Verfügungsrechte am privaten Eigentum fordert.

Für Güter, die in der Konstituierung von Cultural Property aus der kulturellen Praxis herausgegriffen werden, gestaltet sich diese Eigentumsperspektive auch in westlichen, postindustriellen Gesellschaften problematisch. Denn Cultural Property weist oft die Charakteristika eines „Gruppengutes“ auf - eines Gutes also, wofür das englische “commons” oder die deutsche „Allmende“ eher zutreffen. Die Versuche, ein Cultural Property als privates Gut so zu operationalisieren, dass es westlichen wirtschaftlichen und juristischen Parametern entspricht, verlangt einerseits nach einem stabilen Kulturbegriff und andererseits nach einer Festlegung der Be-

\footnotetext{
12 Hardins (1968) Artikel hierzu stimulierte die Verwendung der „Allmende-Tragödie“ in Diskursfeldern von Umweltschutz bis biologischer Evolution.
} 
sitzverhältnisse für ein kollektives Gut, wie dies Kulturgüter repräsentieren. ${ }^{13}$ Inwiefern Kollektive aus Cultural Property Nutzen ziehen können, aber auch verpflichtet werden können, ,ihr“ Cultural Property nur so einzusetzen, wie dies das Kollektiv entscheidet, birgt weitere Problematiken, die mit dynamischen Konzeptionen von kultureller Entwicklung und Veränderung sowie der Kreativität des Individuums schwer zu vereinbaren sind.

Erweitert sich die Handlungs- und damit Forschungsbasis von westlichen auf nicht-westliche, postkoloniale Gesellschaften und indigene Völker, muss das kulturell divergente Verständnis von „Eigentum“ zusätzlich in Betracht gezogen werden. Die westliche Begriffspraxis ist in ihrer begrenzten Verwendung von Gruppengüterkonzepten und ihrem Fokus auf privaten Verfügungsrechten zu limitiert (vgl. Hann 1998, Kasten 2004). Deshalb kombiniert die Göttinger Forschergruppe Teilprojekte auf europäischer, südostasiatischer sowie internationaler Ebene, die dieses divergente Begriffsverständnis aufzeigen. Sie befassen sich zudem mit Cultural Property Bereichen von Heritage-Praxen bis zur Archivierung von Kultur, die mit einem historisch jeweils anderen Verständnis von Eigentum umgeben sind.

Um diese Vielfalt als Ausgangspunkt für das gemeinsame Forschungsanliegen auf einen Nenner zu bringen, haben sich die beteiligten Wissenschaftler/innen auf eine Definition geeinigt, die Eigentum als Resultat sozialer Beziehungen betrachtet (vgl. Hann 1998). Netzwerke sozialer Beziehungen (bzw. deren Akteure) bringen Regelungen hervor, welche Gebrauch und Zugang zu materiellen und immateriellen Ressourcen festlegen. Solche Netzwerke müssen im Zusammenhang mit Produktionsweisen, politisch-ideologischen Werten und Machtverhältnissen (mit entsprechenden Akteuren) betrachtet werden. Um die Konstituierung von Cultural Property zu verstehen, gilt es, das Verhältnis von Ressource, Identität und Wert(en) zu erkennen, welches in einem dynamischen Prozess zur Überführung oder Transformation von kulturellen Gütern und Praxen in Cultural Property mündet. Durch die interdisziplinäre Zusammenarbeit setzt sich die Forschergruppe das Ziel, eine Systematik der Grundstrukturen dieses Transformationsprozesses in seiner sozialen, politischen und organisatorischen Vernetzung herauszuarbeiten.

Die Wissenschaftler/innen des Forschungsteams bringen spezifische fachwissenschaftliche und methodische Kompetenzen mit sowie die Einsicht, dass Cultural Property, angesiedelt zwischen Kultur, Wirtschaft und Recht, durch interdisziplinäre Zusammenarbeit angegangen werden muss. Aus den Kulturwissenschaften Kulturanthropologie/Europäische Ethnologie sowie der Ethnologie beteiligen sich Fachkräfte, die in vorherigen Arbeiten mit Teilaspekten des Gesamtforschungsvorhabens konfrontiert worden sind und dabei Fragen der ideologischen und ökonomischen Instrumentalisierung von Kultur verschiedentlich aufgegriffen haben; zusätzliche Expertise besteht auch zur Rolle von Technik (Fixierung kultureller Praxen auf Ton und Bild) in der Verwandlung von Kultur in Cultural Property. Die

13 Aus der wachsenden Literatur zur Frage der Besitzverhältnisse von Kulturgütern seien Brown 2003, Coombe 1998, Hafstein 2004, Kasten 2004 und Noyes 2006 genannt. 
juristische Expertise wird ebenfalls in mehrfacher Hinsicht gefordert, gilt es doch einerseits die tatsächlichen und unterschiedlichen rechtlichen Rahmenbedingungen, in welchen sich die Propertization abspielen soll, vergleichend zu eruieren und andererseits bedarf es auch der völkerrechtliche Dimension, die für viele Cultural Property Konstituierungsversuche wesentlich ist, die sich aber oft zwischen nationalstaatliche Rechtsbestimmungen lagert. Schließlich sind wirtschaftliche Muster und volkswirtschaftspolitische Entscheidungen ein integraler Teil der Motivationen mit welchen die zu untersuchenden Akteure ausgestattet sind.

In gemeinsamer Diskussion und unter Berücksichtigung der theoretischen, methodischen und geographischen Erfahrungen und Kompetenzen, wurden Erkenntnisziele für die Forschergruppe herausgearbeitet. Die Untersuchung der Konstituierung von Cultural Property soll die komplexe Globalisierung und Vernetzung wirtschaftlicher und juristischer Regimes in einer postkolonialen Welt aufzeigen. Dabei liegt uns einerseits daran, den Ist-Zustand dieser Konstituierung von Regelbildung in vierfacher Hinsicht nachvollziehbar zu machen. Erstens unterscheiden sich die Konstituierungsprozesse in westlichen, spätmodernen und postindustriellen lokal-globalen Strängen im Vergleich zu postkolonialen lokal-globalen Strängen. Zweitens unterscheiden sich die Prozesse in politisch-wirtschaftlichen Identifikationsprozessen (das heisst in der Verbindung zu Heritage-Praxen) von den Entscheidungsfindungen zu Rechten an Cultural Property in Medien (Dokumentarfilm, Schallarchivierung und -nutzung). Drittens bestehen wesentliche Unterschiede im rechtlichen Status bzw. Anspruch verschiedener Nutzergruppen. Und viertens streben wir den Diskurs über reflektierte Vorschläge zu potentiellen SollZuständen im Bereich Cultural Property in Form von Empfehlungen und Empfehlungsbeschränkungen an.

\section{Forschungsanliegen und Forschungspraxis: Erste Stationen}

Dieser Band legt erste Ergebnisse vor, die sich, vor dem Hintergrund der skizzierten Erkenntnisziele in den ersten achtzehn Monaten der Forschung herauskristallisiert haben. Die sechs geförderten Teilprojekte waren so konzeptioniert, dass die Teilresultate für eine beständige Verdichtung und Verlinkung sorgen. Die ethnographischen Fallstudien konzentrieren sich auf Südostasien (Kambodscha und Indonesien), das deutschsprachige Europa sowie auf das Feld der internationalen Organisationen und Gremien, mit einem Schwerpunkt auf der WIPO. Aus diesen Studien fließen Resultate in die institutionenökonomische und -juristische sowie die völkerrechtliche Arbeit, wobei insbesondere im Bereich der internationalen Organisationen auch gemeinsame Feldforschung unternommen wurde. Wie in empirisch angelegten Arbeiten immer zu gewärtigen haben sich im Lauf der ersten Forschungsstrecken die Fokussierungen verändert beziehungsweise dem Feld entsprechend justiert. 
Der Band gruppiert die Ergebnisse in vier Teilen. Während sich Teil 1 mit dem Konnex von Heritage und Cultural Property beschäftigt, fokussiert Teil 2 auf Schutzmechanismen für Cultural Property. Teil 3 fasst die Ergebnisse aus der Feldforschung bei der WIPO zusammen, und Teil 4 vermittelt einen Einblick in die Technikaspekte der Eigentumsbildung sowie einen Überblick über Cultural Property Forschung aus internationaler Perspektive. Teil 5 entwickelt einen ersten Ausblick auf das weitere Vorgehen durch den Kommentar von einem unserer auswärtigen Experten.

Im ersten Teil werden Forschungsergebnisse vorgelegt, die die Verzahnung von Heritage-Entscheidungen und Praxen des Cultural Property illustrieren. Die Ethnologinnen Brigitta Hauser-Schäublin und Karin Klenke nutzen Forschungsergebnisse aus ihrem Forschungsaufenthalt in Indonesien, um die Verwendungs- und Bedeutungsvielfalt von kulturellem Erbe seitens verschiedener Akteursgruppen herauszuarbeiten und zu systematisieren. Anhand des noch laufenden UNESCONominierungsprozesses in Sulawesi für ein "Tana Torajan Traditional Settlement" wird gezeigt, wie verschiedene involvierte Akteursgruppen auf lokaler, regionaler und nationaler Ebene diese Aufgabe auch mit unterschiedlichen Bedeutungssystemen und Zielvorstellungen angehen. Gleichsam auf globaler Ebene betrachten die Autorinnen sodann die Auswirkungen von Zertifizierungsprozessen kultureller Güter einerseits durch die UNESCO, etwa bei der alle regionalen Varianten umfassende Nominierung von indonesischer Batik, die den Weg dieses Kunsthandwerks auf dem Weltmarkt mitbestimmen wird. Andererseits werden im Rahmen von Verhandlungen in der WIPO angetroffene Fälle indonesischen, kulturell geprägten Handwerkswissens vom Silberschmied bis zur Gewürzzusammenstellung betrachtet, bei denen sich indonesische Akteure plötzlich ausländischen Patentnehmern ,ihrer“ kulturellen Praxen gegenüber sahen. Mit dem Begriff der „flexibilisierten Kultur" wollen die Autorinnen die Spannbreite von ideellen und wirtschaftlichen Nutzungen aufzeigen und verdeutlichen, wie unterschiedliches Selbstverständnis von kulturellen Eigentums- und Nutzungsrechten in internationalen Zertifizierungsprozessen aufeinandertrifft. Die Komplexität des anstehenden Bedarfs für Regelsetzungen wird hierdurch verdeutlicht.

Die Ethnologin Aditya Eggert liefert eine erste Bilanz aus der Erforschung der UNESCO-Nominierung des kambodschanischen Schattentheaters Sbek Thom als immaterielles Weltkulturerbe. Sie schildert die notwendigen Schritte bis zur Nominierung - eine neue kulturelle Praxis, die auch in jedem Staat divergierend durchgeführt wird. Auf der Basis ihrer Feldforschung mit Sbek Thom Ensembles im Kontext kambodschanischer Kulturpolitik kann Eggert belegen, dass die ideelle Ehrung vornehmlich wirtschaftliche Folgen hat. So räumt sie ein, dass die UNESCOZertifizierung zwar ein Verschwinden des Sbek Thom verhindert hat. Gleichzeitig stellt sie aber „die Tendenz zur kommerziellen Nutzung dieses kulturellen Phänomens“ fest, das ein „höheres Maß an Kontrolle seiner Praxis durch Regierungsakteure und insbesondere die Steigerung des Wettbewerbsklimas in Zusammenhang mit seinem Gebrauch“ zur Folge hat. Zu untersuchen bleibt die Rolle von juristi- 
schen und ökonomischen Faktoren in diesen sich herausbildenden kambodschanischen Regeln im Umgang mit einer Heritage-Nominierung.

Ein Vergleich der wirtschaftlichen, ideellen und rechtlichen Diskurse um eine potentiell entstehende und eine vor kurzem aberkannte Heritage-Nominierung in Sachsen wird im nächsten Beitrag angestellt. Die Kulturanthropologin Arnika Peselmann und der Völkerrechtler Philipp Socha stellen die Fallbeispiele „Montanregion Erzgebirge“ und „Dresdner Elbtal“" im Rahmen eines Vergleichs der diskursiven Wirkungsmacht von Cultural Heritage und Cultural Property Regimes vor. Die beiden Fälle zeigen, dass der Status Weltkulturerbe durch die Inwertsetzung von identitätsstiftender „Wirkung Parallelen zu den Debatten um Cultural Property aufweist, die sich ebenso mit den Implikationen der Verrechtlichung von Kultur auf die kollektive Identität Kulturschaffender und den damit einhergehenden ökonomischen Effekten befasst und diese kritisch hinterfragt". Ähnlich wie bei den Forschungsergebnissen aus Südostsien wird hier verdeutlicht, dass ein Erfassen und Verstehen von Weltkulturerbe bedingt, dass es „unter Beachtung derselben Problemkomplexe, die bei Cultural Property untersucht werden, betrachtet" wird.

Im zweiten Teil verschiebt sich der Fokus auf die Untersuchung der Parameter, unter denen kulturelle Elemente Schutz erfahren und wie dieser Schutz sich gestaltet. Der Völkerrechtler Sven Mißling unterzieht die UNESCO-Konvention zum immateriellen Kulturerbe von 2003 einer sorgfältigen Analyse. Er arbeitet dabei heraus, dass ein Effekt dieser für die UNESCO zwar in mancher Hinsicht progressiven Neubestimmung von Kultur als einem dynamischen Prozess - im Unterschied zu der mit statischem Kulturkonzept arbeitenden Konvention von 1972 - die Staaten, die die Konvention ratifiziert haben, mit Rechten über kulturelle „Güter“ der immateriellen Art versieht, die sie vorher nicht inne hatten. Zudem wird ein in verschiedenen Forschungsverläufen unserer Projektarbeit notierter Aspekt hierdurch besonders verdeutlicht: Rechte und Anerkennung von „Kultur“, wie sie im Rahmen von UNESCO und anderen Organisationen der United Nations verhandelt werden, haben keine universelle Gültigkeit, sondern werden, wie alle völkerrechtlichen Richtlinien, in jeweils staatlichen Regelwerken ratifiziert. Von UNESCO geleitete Nominierungsprozesse sorgen zwar für globales Renommee, die Umsetzung unterliegt jedoch, wie auch die bereits zusammengefassten Fallstudien verdeutlichen, nicht nur der kulturellen Spezifik eines jeweils lokal-regionalen Habitus, sondern auch den lokal geltenden juristischen Regelwerken. Wie diese Regelwerke im Rahmen einer jeweils staatlich geprägten Kulturpolitik implementiert und genutzt werden, bedarf weiterer vergleichender Forschung.

Der Zivilrechtler Philipp Zimbehl fokussiert sodann auf die Optionen, die für den potentiellen Schutz von Cultural Property bereits vorliegen, so wie dies auch im Rahmen des IGCs in der WIPO angedacht ist. Er nutzt das Beispiel von Folklore, im Kontext von WIPO eher traditional cultural expression genannt. Herausgearbeitet werden hier einerseits die bereits bestehenden rechtlichen Grundlagen des Urheberrechts: Zimbehl eruiert, inwiefern dies für Folklore geltend gemacht werden kann. In einem weiteren Teil wendet sich Zimbehl sodann den bereits existieren- 
den sui generis Optionen zu und überprüft, was deren Auswirkungen auf Folklore beziehungsweise auf Akteure, die sich um solchen Rechtsschutz bemühen, sein könnten. Zimbehls ausblickende Schlussfolgerungen ergeben, dass die Rechtswissenschaft bezüglich mancher kulturellbestimmter Aspekte zu dem zu beurteilenden Material kulturwissenschaftliche Expertise benötigen, während die Kulturwissenschaft juristischen Beistand braucht, um die Frage, „wie eine Rechtsregel in ein bestehendes System zu integrieren ist" adäquat aufgreifen zu können.

Die Wirtschaftswissenschaftler/innen Marianna Bicskei, Kilian Bizer und Zulia Gubaydullina nutzen den Zusammenhang zwischen Kulturgütern und Identität für eine ökonomische Analyse, in der Identität in die Nutzenfunktion der Individuen integriert ist: Sie gehen davon aus, dass zwischen direkten und indirekten Effekten der Nutzung und der Produktion kultureller Güter zu unterscheiden ist und trennen die drei Akteursgruppen der Kulturträger, der Kulturproduzenten und der Kulturkonsumenten. Von diesen steht die Gruppe der Kulturträger im Mittelpunkt, für die die jeweiligen kulturellen Güter identitätsstiftend wirken. Zu den direkten Effekten, die dann auftreten können, wenn andere das Kulturgut produzieren oder konsumieren, zählen sie marktliche Erlöse, während indirekte Effekte die Wirkungen auf die Identität sind. Auf diese Weise verdeutlichen sie, dass die Vielfalt kultureller Güter sich nach den jeweiligen Wirkungen unterscheiden lässt und nur relativ wenige davon unmittelbare negative Effekte auf die Identität von Kulturträgern haben. Für diese Fälle, so greifen sie die Anregung von Zimbehl auf, wären dann besondere Schutzkategorien wie sui generis Optionen denkbar, um den Schutz zu verbessern. In den meisten anderen Fällen kann es einer klassischen Abwägung von Nutzen und Kosten überlassen bleiben, ob Schutzkategorien zum Einsatz kommen oder nicht.

Die visuelle Anthropologin und Ethnologin Beate Engelbrecht stellt sodann praxisnah die Frage nach der Rechtslage im Bereich eines ethnographischen, transkulturellen Filmes, der je nach Verständnis der beteiligten Akteure, sowohl ein kulturelles Eigentum abbilden wie auch ein urheberrechtlich geschütztes Autorenwerk darstellen kann. Die Erörterung anhand eines konkreten, sich entfaltenden kulturellen Events - bearbeitet im Zusammenhang mit unserem im indonesischen Sulawesi angesiedelten Teilprojekt - mit Protagonist/innen und Filmenden aus unterschiedlichen Welten und daher auch divergierendem Verständnis von Eigentumsfragen, verdeutlicht, wie viele Kontexte berücksichtigt werden müssen, um sich selbst als Autorin eine - immer noch Lücken offen lassende - Absicherung zu schaffen, die gleichzeitig auch den Schutzes der kulturellen Eigentumsrechte der gefilmten community berücksichtigt. Das Beiziehen vorhandener juristischer Regelwerke, das Befolgen von ethischen Praxen westlicher filmender Feldforschung und die parallele Dokumentation des Umgangs mit einheimischer filmischer Begleitung eines wichtigen Familienrituals zeigen die Vielschichtigkeit auf, in welcher sich Cultural Property konstituieren kann.

Der dritte Teil des Bandes fokussiert auf Ergebnisse aus der interdisziplinären Feldforschung beim Intergovernment Committee (IGC) in Genf. Stefan Groth nutzt 
das Analyseraster der linguistischen Anthropologie, um die Verhandlungsmuster und Motivationen, wie sie im Projektverlauf im IGC der WIPO kommunikationsethnographisch dokumentiert worden sind, zu durchleuchten. Im Fokus liegen unterschiedliche Perspektiven auf das traditionelle Wissen indigener Gemeinschaften in Verhandlungen um Cultural Property Rights. Das Durchleuchten von verschiedener Terminologieverwendung im Verhandlungsgeschehen legt frei, dass verschiedene Akteursgruppen das Konzept „traditionelles Wissen“ und damit verknüpfte Sozialstrukturen und Wissensbestände unterschiedlich interpretieren - auf Grund der ideologischen sowie strategischen Modelle und Vorstellungen, die ihr Verhalten und Verhandeln im Rahmen des Geschehens in der WIPO auch mitbestimmt. Die fünf hier freigelegten Deutungen davon, was unter traditionellem Wissen verstanden wird, bieten auch eine Erklärung dafür, warum Verhandlungsteilnehmer/innen stets zu Grundsatzfragen zurückkehren müssen und nur minimale Fortschritte im Verhandlungsverlauf zu verzeichnen sind. Ohne auf die Reflektionsebene der spezifischen Terminologieverwendung zugreifen zu können (oder zu wollen), bleiben vielen Akteuren diese Unterschiede im sprachlichen Selbstverständnis verborgen, und es kommt - was unsere Forschung insgesamt beim WIPO IGC belegt hat - zu vielen Wiederholungen, vorhersehbaren Kommunikationsmustern und wenigen Resultaten. ${ }^{14}$

Denselben Verhandlungsprozess des Intergovernmental Committee on Intellectual Property and Genetic Resources, Traditional Knowledge and Folklore der WIPO untersuchen parallel Kilian Bizer, Zulia Gubaydullina und Matthias Lankau aus ökonomischer Perspektive. Seit das Komitee 2001 ins Leben gerufen wurde, haben die Verhandlungen nicht zu substantiellen Ergebnissen, sondern nur zu minimalen Fortschritten geführt. Der Beitrag sucht - parallel zu dem beschriebenen Ansatz von Stefan Groth - einen ökonomischen Erklärungsansatz dafür, dass Länder an diesen kostspieligen und scheinbar ineffektiven Verhandlungen teilnehmen, da klassische ökonomische Theorien hier zu versagen scheinen. Sie wählen ein einfaches Verhandlungsmodell, in dem sich „Blockadeländer“ mit Kosten konfrontiert sehen, wenn ein Mandat auf Grund fehlender Verhandlungsergebnisse ausläuft. Länder, die auf Fortschritt drängen, sehen sich keinen solchen Mandatsverlustkosten ausgesetzt, weswegen ihnen dies die Möglichkeit eröffnet, Zugeständnisse von den Blockadeländern zu fordern. Der Verhandlungsprozess führt zu minimal results, die auf den ersten Blick ineffektiv erscheinen. Allerdings gibt es dennoch gute Gründe für diese Verhandlungen. Obwohl verborgen für einen Außenstehenden, kann das Land, das auf Verhandlungsfortschritt abzielt, zunächst eine kleine, längerfristig sogar eine größere Verbesserung seines Status Quo erwarten, wohingegen sich für das Blockadeland die aus den Zugeständnissen resultierenden erwarteten Kosten

\footnotetext{
14 Vgl. hierzu auch das unpublizierte Vortragsmanuskript von Regina Bendix und Stefan Groth, "Stalling and Speeding: Ways of Speaking at WIPO's Intergovernmental Committee on Cultural Property", gehalten im Workshop W019 "The Anthropology of the United Nations" des EASA Kongresses in Ljubljana, 26-29 August, 2008.
} 
kleiner sind als seine Mandatsverlustkosten. Innerhalb dieses Modells ist es also für beide Spieler sinnvoll, an den Verhandlungen teilzunehmen. Die scheinbare Immobilität der Verhandlungen in der WIPO gewinnt vor dem Hintergrund dieser minimal results an Rationalität und es ist ökonomisch erklärbar, warum internationale Verhandlungen sich oft über viele Dekaden hinziehen, ohne abgebrochen zu werden.

Der vierte Teil des Bandes beginnt mit Johannes Müskes Versuch, die Forschung zu Cultural Property in den breiteren Rahmen der kulturwissenschaftlichen Technikforschung zu stellen. Er arbeitet heraus, dass jede Dimension der Konstituierung von Cultural Property sowie auch der Verhandlungen über diesen Komplex letztendlich von technischen Selbstverständlichkeiten und Neuerungen abhängen. Die in diesem Teilprojekt erforschten Grenzen der Propertisierung kulturellen Ausdrucks wurden am Beispiel von archivierten Tönen und Klängen untersucht und werden hier unter dem Gesichtspunkt von Speichermedien, Archiven und deren Rolle in der Konstituierung Cultural Property betrachtet.

Einen äußerst hilfreichen Überblick über den gegenwärtigen Forschungsstand zu Fragen von Cultural Property bietet sodann die Ethnologin und Juristin Rosemary Coombe. Den hier in Übersetzung vorliegenden Aufsatz verfasste Coombe während ihres dreimonatigen Fellow Aufenthaltes im Sommer 2009 und publizierte ihn im Annual Review of Law and Society.

Im fünften und letzten Teil findet sich sodann ein Kommentar von einem Kollegen, der uns als Fellows begleitet hat: Der kanadische Jurist und Experte im Bereich von Gesetz und Wirtschaft, Ejan Makaay verbrachte drei Monate im Herbst 2009 mit dem Forschungsteam und verfasste einen evaluierenden Ausblick nach unserer ersten Klausurtagung.

\section{Erfahrungen aus dem Experiment der interdisziplinä- ren Öffnung}

Wie jedes interdisziplinäre Vorhaben so birgt auch dieses einige Gefahren: Die größte Gefahr ist stets, dass man angesichts der vielfältigen Fragen zwischen den Disziplinen in den Hintergrund treten lässt, welche Fragen sich disziplinär stellen. Dieser Gefahr begegnet man wirkungsvoll, indem die Teilgruppen der disziplinär eng verwandten Forschung groß genug bleiben, um innerhalb der Disziplinen auf die eigene Methodik und den Stand der Forschung reflexiv arbeiten zu können. Doch kaum ist diese Gefahr gebannt, zeigt sich die zweite: Bleibt vor lauter disziplinären Fragestellungen noch Raum für die trans- oder interdisziplinäre Arbeit?

Transdisziplinär waren in unserer Göttinger Forschergruppe zunächst eine Reihe von Begriffsklärungen vorzunehmen, um zum Beispiel den Beitrag jeder Disziplin zum Kultur- und zum Eigentumsbegriff zu verdeutlichen. Cultural Property ist, wie der Begriff bereits aussagt, in verschiedenen wissenschaftlichen Feldern zu verorten. Eigentum wird vielleicht vornehmlich in Wirtschaft und Recht ange- 
siedelt, doch unterliegt ein Verständnis davon, was Eigentum sein kann und wie Eigentumsverhältnisse zustande kommen auch einem kulturellen Selbstverständnis. Chris Hann formuliert, aus kulturwissenschaftlicher Sicht, dass Eigentumsverhältnisse sich nicht aus einer Mensch-Ding Beziehung ergeben sondern aus der Beziehung, dem kulturell geprägten Verhandeln zwischen Menschen (1998:4). Diese sicher auch interdisziplinär vertretbare Blickrichtung muss dennoch auch eingestehen, dass die Ausgestaltung dieser Beziehung, sowohl hinsichtlich der wirtschaftlichen Interessen wie auch deren Normierung in juristischen und damit gesellschaftlich akzeptierten Parametern ebenfalls von Wissenschaften mit langen Fachtraditionen untersucht und mitgestaltet werden. Kulturwissenschaften leben zwar mit einem Selbstbild, dass sie das Verstehen dem Planen und Normieren vorziehen, aber auch sie haben, in verschiedenen Formen des Wissenstransfers Einfluss auf gesellschaftliche Realitäten. Gerade Cultural Property ist ein Paradebeispiel hierfür, da kulturwissenschaftliche Studien und Konzepte im Lauf von mehr als hundert Jahren auch ihren Teil dazu beigetragen haben, dass Akteure sich Kultur als in Teilen oder als Ganzes veräußerbar vorstellen konnten.

Gerade dieser Dialog zwischen den eindeutig normativ orientierten Rechtswissenschaften und den zumindest (auch) normativ arbeitenden Wirtschaftswissenschaften auf der einen Seite und den positiv-analytisch oder deskriptiv arbeitenden Kulturwissenschaften auf der anderen Seite trug dazu bei, vorsichtig mit den Ansprüchen an die jeweils andere Disziplin heranzutreten. In diesen Klärungsprozessen etwa zwischen Ethnologie und Völkerrecht oder zwischen Kulturanthropologie und Wirtschaftswissenschaft haben sich Schnittstellen für interdisziplinäre Fragestellungen ergeben, die wir entweder in gemeinsamen Beiträgen oder in Parallelbeiträgen bearbeitet haben. Diese gehen insofern über die transdisziplinäre Perspektive hinaus als sie nicht nur Ergebnisse der einen Disziplin an die andere weitergeben, sondern sich gegenseitig in der Wahrnehmung und Präzisierung der Fragestellung befruchten und damit die Perspektive der eigenen Disziplin erweitern. Allerdings sehen wir, dass diese Ansätze den sprichwörtlichen zarten Pflänzchen gleichen, die noch der intensiven Pflege bedürfen, um weitere Früchte tragen zu können.

Die interdisziplinäre Konfiguration der Göttinger Forschergruppe zu Cultural Property ist in dieser Zusammensetzung von Wissenschaften bisher kaum erprobt: Die Kulturwissenschaften Kulturanthropologie/Europäische Ethnologie sowie die Ethnologie, die trotz verwirrend ähnlicher Namen benachbarte aber dennoch differente Fachhistorien und damit auch leicht divergierende Erkenntnisinteressen aufweisen ${ }^{15}$, sind beide gleichermaßen konfrontiert in ihren empirischen For-

\footnotetext{
15 In Göttingen tragen die ehemalige Volkskunde und Völkerkunde heute die Namen Kulturanthropologie/Europäische Ethnologie (angesiedelt in der philosophischen Fakultät) und Ethnologie (sozialwissenschaftliche Fakultät). Während diese Fächer von Außenstehenden, ja selbst von Mitgliedern unseres Forschungsteams bisweilen als sehr ähnlich betrachtet werden, unterscheiden sich ihre Fachgeschichten und somit auch ihre Fachidentitäten erheblich. Im Aufgreifen unserer Forschungsthematik hat sich eine Zusammenarbeit als ersprießlich und wichtig erwiesen, gerade weil die Fachperspektiven komplementär aber nicht identisch sind. Die Umbenennung dieser Fächer im deutschsprachi-
} 
schungsfeldern mit Phänomenen, die im weitesten Sinne als Cultural Property bezeichnet werden müssen. Aber auch innerhalb der Rechtswissenschaft ist die Perspektive des Völkerrechts, die stärker auf die internationale Rechtsentwicklung fokussiert, also das international law making, hinlänglich verschieden von der zivilrechtlichen Perspektive des Wirtschaftsrechts, das rechtsvergleichend eher die gewachsenen Strukturen der nationalen Gesetzgebung in den Mittelpunkt rückt. Beide juristischen Disziplinen verfügen ihrerseits über eine gewisse Nähe zur Wirtschaftswissenschaft, auch wenn letztere ausgehend vom Paradigma des rational choice manchmal sehr eng erscheinende Erklärungsmuster verfolgt. Auch wenn also innerhalb der beiden Gruppenteile Kulturwissenschaft und Rechts- und Wirtschaftswissenschaften Unterscheide bestehen, die es für die interdisziplinäre Forschung zu überwinden gilt, so liegen die eigentlichen Herausforderungen im Brückenbau zwischen diesen Gruppenteilen.

Nach unserer Erfahrung überwindet man die Hürden trans- und interdisziplinärer Zusammenarbeit durch gegenseitige intellektuelle wie interkulturelle Einladungen, sich mit den unterschiedlichen Erkenntnisinteressen und entsprechend auch verschiedenen Fachkulturen auseinanderzusetzen. Diese Einladungen haben wir nach dreijähriger Diskussionsphase im Vorfeld der Forschergruppe an unsere Mitarbeiter/innen weitergegeben, indem wir anhand von Lektürekursen jeweilige Eckpfeiler der eigenen Disziplin in Nachmittagsseminaren benannt und diskutiert haben. Ziel dieser Seminare war es, die Perspektive der anderen zumindest ansatzweise zu verstehen, um die von dort kommenden Fragen einordnen zu können und sich an die andersartigen Analyseraster und die damit in Verbindung stehenden Sprach-, Schreib-, Lese-, Forschungs- und Veröffentlichungsmuster zu gewöhnen. Letztere zeigten sich selbst in der Zusammenstellung der ersten Ergebnisse für diesen Band: Schreibstil und Verweispraxen unterscheiden sich, Kompromisse müssen gefunden werden - und dass dies auch zu schaffen ist, verweist auf die Fähigkeit, manche kaum mehr reflektierte Fachgewohnheit auch zumindest zwischenzeitlich ablegen zu können, ohne sich um Einbußen im eigenfachlichen Renommé zu sorgen.

Doch die Kompromisse reichen weiter: Sie zeigen sich vor allem beim gemeinsamen Schreiben von Beiträgen, weil das disziplinäre Denken auch Textstrukturen verlangt, die Autor/innen für einen gemeinsamen Beitrag aktiv auflösen und neu aufbauen müssen. Eine große Hilfe dabei sind gemeinsame Workshops wie der in diesem Tagungsband zusammengefasste im November 2009, weil sie schon im Vorfeld zur Verschriftlichung des Gedachten zwingen, die in aller Regel mit einer analytischen Schärfung einhergeht. Zusätzlich sorgt aber die intensive, um Verstehen ringende Diskussion für ein Erkennen von parallelen Fragestellungen und

gen Raum als Konsequenz der wissenschaftshistorischen Vergangenheitsbewältigung seit den 1960er Jahren hat zu einer Namensvielfalt geführt, die sich auch nicht unbedingt verbessert, wenn die Englischen Bezeichnungen genutzt werden, da auch diese sich in der englischsprachigen Welt von Großbritannien über die USA und Kanada bis Indien oder Australien wiederum Unterschiede aufzeigen. Wir haben es in diesem Band den Autor/innen überlassen, wie sie ihr jeweils eigenes Fach nennen. 
Antworten. Dass es gerade zu diesem Verständnis kam, verdanken wir auch unseren auswärtigen Experten und Fellows, deren kritischer Blick von außen uns ein ums andere Mal Anregungen gab, uns noch stärker aufeinander einzulassen. Auch aus diesem Grund betrachten wir die hier vorgelegten Ergebnisse als einen ersten Zwischenstand, den wir in Vorbereitung auf den nächsten Workshop an einigen Stellen erweitern und verbessern können. 




\section{Teil 1}

\section{Zwischen Heritage und Cultural Property}





\title{
Flexibilisierte Kultur zwischen „Tradition, Moder- nität und Markt": Akteursbezogene Verwendungs- und Bedeutungsvielfalt von „kulturellem Erbe“
}

\author{
Brigitta Hauser-Schäublin und Karin Klenke
}

\section{Einleitung}

Wenn „Kultur“ im Sinn von Cultural Property zu „Eigentum“ wird und dadurch (neue) „Eigentümer“ erhält, ist dies das Resultat einer Verdinglichung von materieller oder auch immaterieller Kultur sowie von Auswahl- und Identifizierungsprozessen durch bestimmte Akteure. „Kulturelles Eigentum“ beschränkt sich deshalb auf ausgewählte, aus dem selbstverständlichen und alltäglichen Zusammenhang einer sozialen Gruppe herausgelöste Elemente (Brown 2004)ํ․ Jenseits des ursprünglichen kulturellen und sozialen Kontextes erlangen diese Elemente ein Eigenleben, das durch das Handeln von Akteursgruppen, deren Motive und Ziele sowie deren Beziehungsnetze bestimmt wird (vgl. dazu Benda-Beckmann et al. 2006b). Die Handlungen, durch welche die kulturellen Elemente je nach Situation strategisch geformt werden, führen zu einem vom ursprünglichen „Dasein“ verschiedenen und vielfältigen sozialen Leben dieser Dinge, Praxen und Ideen (vgl. Appadurai 1986). Diese Verdinglichung von Kultur wird - wenn sie ,geschützt“ oder zertifiziert werden soll - durch eine kategoriale Transformation kaschiert: „Eigentum“ und „Kultur“ werden in einer überhöhenden Weise zu „kulturellem

\footnotetext{
${ }^{1}$ Kirshenblatt-Gimblett spricht von „meta-kulturellen Handlungen“, die eine kulturelle Ausdrucksform oder Hervorbringung einer bestimmten Gesellschaft zu einem "global cultural commons" und "global public spheres" transformieren (2006).
} 
Erbe“ stilisiert. Inzwischen bedienen sich weltweit die verschiedensten Akteursgruppen dieses Begriffs, um auf den besonderen Wert (jenseits des nackten ökonomischen Wertes) und die Authentizität des Gegenstandes, den er bezeichnet, hinzuweisen². Welcher Wert und welche Authentizität damit gemeint sind, bleibt zumeist unscharf und lässt deshalb ein breites Spektrum von Deutungs- und Handlungszusammenhängen zu. Dieser Einsatz- und Bedeutungsvielfalt von als „Erbe“ deklarierten kulturellen Elementen, die Staaten internationalen Organisationen zur Zertifizierung vorschlagen, sind wir auf unseren Forschungen über Angkor und Immaterielles Kulturgut (intangible heritage) in Kambodscha und über den Prozess der Nominierung der Tana Toraja Traditional Settlements (in Sulawesi, Indonesien) als "Cultural Landscape" immer wieder aufs Neue begegnet.

In den offiziellen Diskursen bilden „kulturelles Erbe“ und „Identität“ einen gemeinsamen Nenner. Sie tauchen in allen Nominierungsanträgen an die UNES$\mathrm{CO}$ und in den Konventionen und Handbüchern, beispielsweise der World Intellectual Property Organisation (WIPO) ständig auf (vgl. Anico und Peralta 2009)3. So heißt es etwa in der Einleitung zum Abschnitt "Intellectual property and traditional cultural expressions" der WIPO (WIPO 2010:56): "The cultural heritage of a community or nation lies at the heart of its identity [...]." Diese Formulierung zeigt, wie „kulturelles Erbe“ und „Identität" als symbolische Brücke dienen, um eine lokale Gruppe (community) und eine Nation sozusagen auf Augenhöhe miteinander zu verbinden. Hier entsteht der Eindruck, als würden die lokale Gruppe und die Nation darunter jeweils das Gleiche verstehen, gleiche Ziele haben und als gleichberechtigte Partner den Zu- und Umgang mit diesem Erbe bestimmen. In Wirklichkeit laufen diese Zuschreibungen auf den unterschiedlichen Ebenen parallel, wobei diese Ebenen jedoch mit unterschiedlicher Macht und Handlungsfähigkeit im nationalen und internationalen Kontext ausgestattet sind.

„Identität“ ist, wie auch „Erbe“, ein vielschichtiger Begriff. „Identität“ erlangt nur in Interaktionen zwischen Eigenem und Fremden eine Bedeutung, denn die Rückberufung darauf dient der Selbstdefinition und Abgrenzung gegenüber Anderen. Er ist ein Instrument zur Schaffung von Gemeinsamkeit (sowie Zugehörigkeit) und Differenz. Identität ist prozessual und kontextabhängig und kann deshalb, als strategische Ressource, dazu verwendet werden, sich in politischen und gesellschaftlichen Zusammenhängen immer wieder neu zu positionieren.

In international politics [...] identity seems to have become a symbolic public good, the defence of which asserts a legitimacy that is beyond criticism or opposition. Reified into a sacred and holy apotheosis 'identity' is

2 Zum heritage-Begriff vgl. Hemme, Tauschek und Bendix 2007a.

${ }^{3}$ In der UNESCO-Konvention von 1972 (Convention Concerning the Protection of the World Cultural and Natural Heritage) taucht der Begriff identity noch nicht auf. Die inflationäre Verwendung dieses Begriffs, zusammen mit heritage, begann in den 1990er Jahren. Der Begriff heritage, so schreiben Anico und Peralta - und das gleiche gilt für identity -, "has become so epidemic: everyone has to have one" (2009:2). 
something to which everyone has a right. It allows the pursuit of narrowly sectional interests to pass - covered by at least a fig leaf of sincerity - as a defence of the ineffable. It is a difficult card to trump [...] Jenkins 2004:9-10).

Der Rückbezug auf Identität schafft, so kann man aus diesem Zitat schließen, allen Akteuren, ob indigenen Gruppen, Regionen oder Nationalstaaten Handlungsspielräume, die andere nicht in Frage zu stellen oder gar zu beschränken wagen - es sei denn, sie führen ebenfalls ihre „Identität“ ins Feld.

Die Verbindung von beiden Begriffen, „Erbe“ und „Identität“, bringt ein doppelt symbolisches "good" hervor, das nahezu mit einer sakralen Aura versehen ist. Hinter dieser überhöhenden Bezeichnung verbergen sich jedoch, je nach Akteursgruppe, die sich dieser Begriffe bedient, verschiedene Vorstellungen, Strategien und Hoffnungen. ${ }^{4}$

Die Untersuchungen in Kambodscha zeigten beispielsweise, dass jede Akteursgruppe auf den verschiedenen, hierarchisch geordneten Ebenen unterschiedliche Motive und Ziele besitzt und dementsprechend auch gegenüber der nächst tieferen Ebene dominante Bedeutungszuschreibungen an kulturellen Elementen vornimmt. So kommt es gelegentlich zu Synergien und Harmonisierungen, öfter jedoch zu Polyphonien und Widersprüchen (Miura 2005, 2009, Neth 2009, Eggert in diesem Band). Ähnliche Prozesse zeichnen sich bei den Toraja in Sulawesi (Indonesien) $\mathrm{ab}$, auf die wir in diesem Beitrag genauer eingehen werden. ${ }^{5}$

In unseren Forschungen in Indonesien und Kambodscha haben wir versucht, die Transformation von Kultur, die als kulturelles Erbe ausgewählt wurde, von der lokalen bis zur internationalen Ebene zu verfolgen. Wir möchten in diesem Beitrag aufzeigen, wie Auswahl, Begründungen für die Auswahlkriterien und Interpretation derselben je nach Akteursgruppen variieren. Diese Vielfalt von Handlungsstrategien, Zielen und Bedeutungen verdeutlicht, wie kulturelles Erbe durch einen Verdinglichungsprozess zu einem Gut wird, das verschiedenste Akteure, oft losgelöst voneinander, immer wieder als Instrument neu formen und darüber verfügen: Kultur wird flexibilisiert. Diese unterschiedlichen, untereinander manchmal nur lose verbundenen Ebenen können folgendermaßen charakterisiert werden:

(1) Die regionale/lokale Ebene, auf der die Gruppe angesiedelt ist, aus deren Lebenswelt das kulturelle Element stammt. Diese lokale Ebene ist nicht nur der „Ursprung“, sondern auch das „Ende“ des Verdinglichungsprozesses. Denn vor Ort werden nach erfolgreich verlaufener Nominierung beispielsweise als Weltkulturerbe der UNESCO durch den Nationalstaat (oder von ihm beauftragte Dritte) Maßnahmen durchgeführt, die zum konkreten Erhalt des kulturellen Erbe dienen.

\footnotetext{
${ }^{4}$ Wir werden im Folgenden aus Gründen der Lesbarkeit den Begriff „kulturelles Erbe“ ohne Anführungszeichen schreiben, übernehmen ihn jedoch nicht als wissenschaftliches Konzept, sondern als Wertbegriff im skizzierten Sinn.

${ }^{5}$ Die Empirie zu dieser Darstellung stammt aus der noch laufenden Feldforschung von Karin Klenke und muss daher als vorläufige Interpretation verstanden werden.
} 
Brigitta Hauser-Schäublin und Karin Klenke

Diese Maßnahmen basieren auf Richtlinien der Regierung und der zur Managementberatung herangezogenen internationalen Experten. Lokale Bedürfnisse, Wünsche, Ziele und selbst überlieferte Rechte müssen oft hinter den hierarchisch übergeordneten Prioritäten zurücktreten. ${ }^{6}$

(2) Die nationale Ebene. Diese ist geprägt von den Behörden (meist Ministerien), in deren Obhut das international anzuerkennende kulturelle Erbe gestellt ist, sowie deren strategische und rechtliche Vorgaben. Diese orientieren sich an innenund außenpolitischen Strategien und Zielen der Regierung. Der nationale Umgang mit kulturellem Erbe ist auch bestimmt durch das Verhältnis von Mehrheit und Minderheiten und deren Berücksichtigung. Und schließlich sind es die Akteure in den verschiedenen Gremien und Behörden, welche den Handlungsspielraum individuell gestalten.

(3) Die höchste Ebene, nämlich die Ebene der internationalen Organisationen in Interaktion mit den Vertretern der Mitgliedstaaten. ${ }^{7}$

Im Folgenden können wir uns nur mit Ausschnitten aus diesen komplexen Verhältnissen beschäftigen. Im ersten Teil befassen wir uns mit dem noch nicht abgeschlossenen Nominierungsprozess von Tana Toraja Traditional Settlements zur Cultural Landscape und zeigen, wie verschiedene Akteursgruppen gemäß ihrer jeweiligen Vorstellungen und Ziele den nominierten Orten ${ }^{8}$ unterschiedliche Bewertungen und Bedeutungen zuschreiben. Im zweiten Teil analysieren wir kurz die Gemeinsamkeiten von UNESCO und WIPO bezüglich des Schutzes von kulturellen Ausdrucksformen und ihrer Instrumente. Am Beispiel Indonesiens verfolgen wir, welche Erfahrungen, Werte und strategischen Überlegungen die Nutzung dieser internationalen Instrumente und damit die Aushandlungen zwischen nationalen und internationalen Akteuren prägen.

\section{Fallstudie Toraja}

\subsection{Die Region Toraja}

Die Toraja leben überwiegend als Reisbauern in der Region Toraja im Hochland der Provinz Süd-Sulawesi. ${ }^{9}$ In vorkolonialer Zeit siedelte die Bevölkerung im Nor-

\footnotetext{
${ }^{6}$ Vgl. dazu die Untersuchungen von Miura zu Angkor (2004, 2005, 2009).

7 Brigitta Hauser-Schäublin nahm an Sitzungen des Intergovernmental Committee on Intellectual Property and Genetic Resources, Traditional Knowledge and Folklore der World Intellectual Property Organization (WIPO) in Genf (Sommer 2009) und des Intergovernmental Committee for the Safeguarding of the Intangible Cultural Heritage der UNESCO in Abu Dhabi (Herbst 2009) teil.

8 Um den Begriff „Stätten“ zu vermeiden, der Assoziationen von unbelebten, musealen Monumenten hervorruft, wählen wir den Begriff „Orte“. Dies bedeutet jedoch nicht, dass alle nominierten Orte bewohnte kleine Dörfer darstellen.

${ }^{9}$ Der Landkreis Tana Toraja teilte sich im Jahr 2008 in die Landkreise Tana Toraja und Toraja Utara. Wenn wir im geographischen Sinne von „Toraja“ sprechen, meinen wir diese beiden Landkreise. 2004 lebten in den beiden Landkreisen Tana Toraja und Toraja Utara ca. 450.000 Menschen, wobei
} 
den des heutigen Toraja in autonomen, teilweise verfeindeten Dörfern mit jeweils eigenen politischen Führern, zwischen denen ausgeprägte Rivalitätsbeziehungen herrschten, die bis heute virulent sind (Nooy-Palm 1979). Im Süden existierten dagegen drei stärker hierarchisierte größere politische Einheiten, die von „Königen" angeführt wurden und wiederum in Rivalitätsbeziehungen untereinander sowie zu den jeweiligen politischen Führern im Norden standen (Crystal 1970, 1974). Eine kontinuierliche zentrale politische Organisation existierte nicht.

Die Gesellschaft war hierarchisch in Adlige, Freie und Sklaven gegliedert, wobei die Adligen etwa 10 Prozent, die Freien etwa 20 Prozent und die Sklaven entsprechend 70 Prozent der Bevölkerung stellten. Obwohl diese Stratifizierung heute keine rechtliche Grundlage mehr hat, ist ihre Bedeutung im Alltag sowie ihre Konsequenzen für politische Partizipation, ökonomischen Erfolg, rituelle Praxis etc. bis heute ungebrochen. ${ }^{10}$ Totenfeste werden gemäß dem sozialen Status des bzw. der Verstorbenen durchgeführt und stellen damit eine zentrale soziale Arena zur Austragung von Statuswettkämpfen sowohl der Adligen untereinander als auch zwischen Adligen und ökonomisch potenten, aufstrebenden Freien und Sklaven dar (Adams 1984, Nooy-Palm 1986, Volkman 1985).

Die EinwohnerInnen der Hochebene sind heute zu knapp 88 Prozent christlichen Glaubens, ca. 7,5 Prozent sind Muslime und 2,8 Prozent sind AnhängerInnen der vorchristlichen Religion. Die Bildungseinrichtungen der niederländischen Mission haben wie in anderen christlichen Regionen Indonesiens zu einem erheblichen Bildungserfolg der (überwiegend adligen) Toraja beigetragen, die heute auf allen Ebenen der regionalen, lokalen und nationalen Behörden und Institutionen, in Universitäten sowie in der Wirtschaft erfolgreich sind.

Eine zentrale Rolle in der sozialen Organisation spielen die adligen Ahnenhäuser (tongkonan). Je älter ein Ahnenhaus ist und je mehr weitere Ahnenhäuser davon abstammen, desto bedeutungsvoller ist seine Stellung und desto höher das Prestige des Familienverbandes. Ahnenhäuser müssen regelmäßig zu Ehren des Gründerpaares von ihren Deszendenzgruppen renoviert werden, was wiederum die soziale und ökonomische Potenz der Besitzer unter Beweis stellt und deren Prestige erhöht. Entsprechend bemisst sich das Alter eines Ahnenhauses nicht aus dem Alter des Baumaterials, also seiner physischen Existenz, sondern aus seiner sozialen Existenz (im Sinne von Lévi-Strauss „société à maison“, vgl. Carstens und HughJones 1995), die sich durch die Jahrhunderte immer wieder - ganz oder teilweise neu materialisieren kann (vgl. dazu auch Nooy-Palm 2001, Waterson 1990, 2003). Die Ahnenhäuser werden ergänzt durch die ihnen gegenüberstehenden Reisspeicher, deren Anzahl je nach Reichtum der Besitzer variiert. Nach lokalem Ver-

geschätzt mindestens ebenso viele Toraja in der Migration innerhalb Indonesien oder im Ausland leben.

${ }^{10}$ Wir sprechen also nicht von ehemaligen Adligen, Freien und Sklaven, sondern der lokalen Praxis folgend von Adligen, Freien und Sklaven, wenngleich es keine staatlich-rechtliche Grundlage für diese Kategorien gibt. 
Brigitta Hauser-Schäublin und Karin Klenke

ständnis sind die Ahnenhäuser, die Zeremonialplätze (rante) mit den früher anlässlich adliger Totenfeste gesetzten Menhiren (simbuang) und die Grabstätten mit Felsgräbern (liang) zwar häufig räumlich voneinander getrennt, jedoch existentiell miteinander verbunden, da sich in diesen materiellen Strukturen der Lebenszyklus des Menschen spiegelt: „Es gibt einen Ort, wo wir geboren werden und leben [die Häuser], einen Ort, wo wir als Tote gefeiert werden [die Zeremonialplätze] und einen Ort, wo wir begraben werden [die Grabstätten]“.

Toraja ist von der Zentralregierung in den späten 1970er Jahren zu einem touristischen Ziel ernannt und ausgebaut worden. Von 1986 bis 1994, dem Höhepunkt der BesucherInnenzahlen, kletterte die Gesamtzahl der Touristen pro Jahr von 133.316 auf 261.552 (Adams 2006:16). Nach dem Sturz Suhartos und den Attentaten von Bali brachen die bereits rückläufigen BesucherInnenzahlen dramatisch ein und sanken auf 32.000 im Jahr 2005 (BPSTT 2006:211). Im Mittelpunkt des touristischen Interesses stehen bis heute die Totenfeste sowie die Grabstätten und die Ahnenhäuser.

\subsection{Der Prozess der Nominierung}

Im Jahr 2000 wurde zunächst Ke’te’ Kesu' als einzelnes Toraja-Dorf zur Zertifizierung als World Heritage auf die vorläufige Liste (tentative list) der UNESCO gesetzt. Diese Nominierung erfolgte nach beharrlichen Bemühungen der Bevölkerung von Ke'te' Kesu', genauer: der adeligen Deszendenzgruppen des Ahnenhauses Kesu', das eines der drei ältesten und prestigeträchtigsten Ahnenhäuser von Toraja darstellt und dessen Abstammungsgruppen nicht nur über beachtliche ökonomische Ressourcen verfügen, sondern auch politisch und administrativ bis auf höchste nationale Ebenen sehr gut vernetzt sind. Im April 2001 wurde anlässlich einer Ortsbegehung während des subregionalen Treffens "Global Strategy and Periodic Reporting for World Heritage Cultural Properties in Southeast Asia" in Tana Toraja dringend eine Ausweitung und Neuformulierung der Nominierung als Cultural Landscape angeregt. Schon Anfang Mai 2001 zog die indonesische Regierung die Nominierung von Ke'te' Kesu' zurück und begann an der Nominierung als Cultural Landscape zu arbeiten. Das entsprechende Nominierungsdokument inklusive des Managementplans existiert bereits seit 2005 in schriftlicher Form. Hier wurden elf Orte nominiert, von denen acht im Norden und drei im Süden von Toraja liegen. Bis auf einen waren alle bereits als „touristisches Objekt“ registriert. Die Nominierung wurde jedoch zugunsten des Antrags von Bali zurückgestellt. Im Oktober 2009 wurde der überarbeitete Antrag als Tana Toraja Traditional Settlement schließlich mit diesen elf Orten als Cultural Landscape auf die tentative list der UNESCO gesetzt. Solche „traditionellen Siedlungen“ repräsentieren in erster Linie die soziale Schicht der Adeligen und umfassen idealerweise Ahnenhäuser, Reisspeicher, einen Zeremonialplatz mit Menhiren, eine Beerdigungsstätte mit Felsgräbern, einen Bambuswald, Reisfelder sowie Weideplätze für die Wasserbüffel. Die auf der tentative list aufgeführten elf Orte weisen diese als zentral angesehenen 
Kriterien in je unterschiedlichen Kombinationen auf. Für 2010 ist eine weitere Überarbeitung durch das für die Nominierung zuständige Ministerium für Kultur und Tourismus geplant, bei dem Anzahl und Verteilung der Orte neu gestaltet werden sollen.

Auf lokaler Ebene ist weder bei den damals federführend beteiligten BeamtInnen noch in den zuständigen Behörden oder in den nominierten Ort selbst bekannt, welche Orte letztendlich in die Nominierung von 2005 aufgenommen wurden. Unbekannt ist auf lokal-regionaler Ebene auch, weshalb noch keine Zertifizierung erfolgt ist und dass eine Neubearbeitung aussteht. Gerade an diesem Beispiel wird deutlich, wie lückenhaft der Informationsaustausch zwischen lokaler, regionaler und nationaler Ebene ist und wie mangelhaft die Entscheidungsprozesse koordiniert sind - wenigstens aus lokaler Sicht. Hinzu kommt, dass das Gros der nichtadeligen und ökonomisch nicht erfolgreichen Bevölkerung weder formal noch inhaltlich in den Nominierungsprozess involviert ist.

Im Folgenden werden wir die Vervielfältigung von Bedeutungen schlaglichtartig an den Beispielen der konkurrierenden Begründungen für die Auswahl der Dörfer sowie an verschiedenen Auffassungen von Authentizität illustrieren.

\subsection{Konkurrierende Begründungen für die Auswahl der nominierten Orte}

Wie bereits erwähnt werden den einzelnen Orten auf lokaler Ebene Bedeutungen verliehen, über die jedoch keinesfalls Einigkeit besteht. In der auf Prestige und Konkurrenz beruhenden Gesellschaft der Toraja wird der Nominierungsprozess als innergesellschaftliche Strategie des Prestige-Erwerbs genutzt und gedeutet. Vor dem Hintergrund der Rivalitätsbeziehungen ist man sich der Bedeutung des Prädikats „Weltkulturerbe“ sehr bewusst, das den herausragenden Wert des eigenen Ortes - und das für die gesamte Menschheit! - international bestätigt.

Wie nun sehen diese konkurrierenden Begründungen aus? Dass acht der elf nominierten Orte im Norden liegen, wird gerade von Adligen aus dem Süden häufig darauf zurückgeführt, dass zwar die Landräte von Tana Toraja immer Vertreter des Südens gewesen seien, die für die Nominierung zuständigen LeiterInnen des Amtes für Tourismus und Kultur jedoch stets aus dem Norden gekommen seien und den Nominierungsprozess zu Gunsten ihrer Region beeinflusst hätten. Die Kriterien für die Auswahl gründeten sich nach dieser Lesart auf historischen Rivalitätsbeziehungen. Fünf der im Norden liegenden Orte und zwei der drei aus dem Süden haben zudem engste historische Verbindungen zu Ke'te' Kesu', während beispielsweise das ebenfalls zu den drei ältesten Ahnenhäusern von Toraja gehörende Ahnenhaus Kaero im Königtum Sangalla in der Nominierung leer ausgegangen ist. ${ }^{11}$ Die Angehörigen des Ahnenhauses Kesu' haben es nach lokalem Ver-

\footnotetext{
11 Adams (2009) hat die Strategien der adligen Familie von Ke'te' Kesu' im Kontext von Tourismus und heritage sowie deren Wettstreit mit der vom Ahnenhaus Kaero in Sangalla abstammenden adligen Familie, der auf dem touristischen Feld sowie - eine frühe Form der „Heritage-isierung“ - unter
} 
ständnis geschafft, im lokalen Statuswettkampf einen mächtigen Verbündeten zu gewinnen: die UNESCO, die, so lautet die Annahme, demnächst die Bedeutung von Ke'te' Kesu' für die Menschheit bestätigen wird.

Von anderen Akteuren wird der historische Prozess des politischen Zusammenschlusses als wichtigstes Kriterium angesehen. Ke'te' Kesu' aus dem Norden sowie Lemo und Londa aus dem Süden, die geographisch nahe beieinander liegen, so meinte ein Vertreter des Ortes Lemo, seien zu Recht als (vermeintlich einzige) Orte nominiert worden: Immerhin habe hier um 1683 angesichts der drohenden Invasion der Bugis die erste politische Vereinigung als Toraja stattgefunden. Zudem, so führte er weiter aus, hätten die niederländischen Kolonialherren danach den König von Makale und das Oberhaupt von Ke’te' Kesu' als Verwalter von Toraja eingesetzt, was die Bedeutung dieser Region als politischem Zentrum nur unterstreichen würde. Die UNESCO-Nominierung, so lässt sich diese Interpretationsweise der (vermeintlich nur drei) ausgewählten Orte zusammenfassen, würde diesen - retrospektiv harmonisierten - historischen Tatsachen Rechnung tragen, denn diese Orte symbolisierten die gemeinsame politische Identität der Toraja.

Die Verwaltung des Landkreises hatte wiederum andere Kriterien im Sinn, als sie der UNESCO ausgewählte Orte zur Nominierung vorschlug: Entscheidend war nach Aussage einer damaligen Leiterin des Amtes für Tourismus und Kultur, dass die Orte bereits als touristische Objekte erschlossen waren, über eine attraktive natürliche Umgebung verfügten, dass adat (überlieferte Traditionen) und die vorchristliche Religion aluk to dolo noch lebendig waren, dass der Distrikt, in dem sie liegen, über ein ausreichend hohes Bruttosozialprodukt verfügte, die EinwohnerInnen der Idee der Entwicklung gegenüber aufgeschlossen waren sowie eine gute infrastrukturelle Anbindung gegeben war. Außerdem hätte aus Gründen der Gerechtigkeit ein Ort aus dem Norden, einer aus dem Süden und einer aus der Mitte nominiert werden sollen. Bei einer gewissen Kompromissbereitschaft gegenüber den Kriterien der UNESCO wird in dieser Sichtweise den vorgeschlagenen Orten die Bedeutung eines Motors der lokalen ökonomischen Entwicklung zugewiesen.

Das Kriterium der Vollständigkeit (integrity), von der UNESCO definiert als "a measure of the wholeness and intactness of the natural and/or cultural heritage and its attributes" (UNESCO 2005b:\$88) ist durch das Wechselspiel zwischen den verschiedenen Ebenen zu einem lokalen Argument geworden. Grundsätzlich gehört, wie bereits erwähnt, zu jedem Ahnenhaus ein Zeremonialplatz sowie eine Grabstätte, wobei meistens mehrere verstreut liegende Ahnenhäuser gemeinsam eine Grabstätte und ein Zeremonialfeld besitzen, die jedoch geographisch weit auseinander liegen können. Die UNESCO fordert, dass die für den Schutz als notwendig erachteten Kernzonen (core zones) alle materiellen Strukturen, die zu dem outstanding universal value beitragen, umfassen müssen (UNESCO 2005b:\$100), wor-

anderem durch den Bau von Museen und einer Bibliothek ausgetragen wurde, differenziert geschildert. 
an man sich bei der Nominierung von Toraja Traditional Settlements jedoch nicht gehalten hat. So entsprechen dem UNESCO-Kriterium der Vollständigkeit nur drei der nominierten Orte, während die core zones bei den Orten, die weniger kompakt sind, so gelegt wurden, dass nur die Grabstätte, nur der Zeremonialplatz oder nur die Ahnenhäuser erfasst wurden. Das UNESCO-Kriterium der Vollständigkeit ist nun auch zu einem Argument auf der lokalen Ebene geworden, mit dem bestimmten nominierten Orten die Eignung zum Weltkulturerbe abgesprochen wird, da sie ja - dies aber erst in den neuen Grenzen der core zones - nicht „vollständig“" seien. ${ }^{12}$

Nach diesem Überblick über konkurrierende Bedeutungen auf lokaler Ebene nehmen wir die konkurrierenden Bedeutungen zwischen den verschiedenen Ebenen in den Blick.

\subsection{Authentizität}

Ein komplexes Wechselspiel zwischen den verschiedenen Ebenen lässt sich bei den Begründungen der von der UNESCO geforderten „Authentizität“ beobachten. ${ }^{13}$ Die regelmäßige Renovierung von Ahnenhäusern auch mit neuen Materialien, der Wiederaufbau von physisch nicht mehr existenten Ahnenhäusern, bauliche Veränderungen wie etwa der Umbau des offenen Bereiches unter der Wohnebene zu einem geschlossenen Erdgeschoss, werden auf lokaler Ebene nicht als Abweichung von materieller Authentizität verstanden. Vielmehr wird dies kulturell folgerichtig als legitime Realisierung des Gebots der beständigen Bewahrung, Renovierung und Verbesserung des eigenen Ahnenhauses als Reverenz an das Gründerpaar angesehen. Die Belassung eines Ahnenhauses in seinem ursprünglichen Zustand kann umgekehrt als ein Zeichen dafür interpretiert werden, dass es von seiner Deszendenzgruppe vernachlässigt wird oder diese nicht genug Geld bzw. den gemeinsamen Willen hat, es adäquat zu erhalten. Während "spirit" und "feeling" dieser mit neuen Materialien gestalteten Ahnenhäuser und Reisspeicher also völlig ,authentisch“ sind, mangelt es ihnen an der „Echtheit" von "materials” and "substances", die jedoch von den meisten Toraja als eher unwesentlich empfunden wird. ${ }^{14}$

\footnotetext{
${ }^{12}$ Es ist zu vermuten, dass hierfür pragmatische Aspekte ausschlaggebend waren, da eine den maximalen Schutz bietende core zone, die sich über mehrere Quadratkilometer ökonomisch höchst divers genutzten Landes erstrecken würde, als nicht praktikabel angesehen wurde. Abgesehen davon hat man bei den meisten nominierten Orten offensichtlich einfach die Grenzen, die sie als touristisches Objekt definierten, übernommen.

${ }_{13}$ Bezieht sich in den Operational Guidelines Authentizität auf "material", "design", "workmanship" sowie "setting", wurde sie bei der Nara Conference 1994 jedoch neu formuliert als "form and design, materials and substance, use and function, traditions and techniques, location and setting, and spirit and feeling." Wir beschränken uns auf den Aspekt von "materials and substance" sowie "spirit and feeling".

${ }^{14}$ Die Idee des Ahnenhauses kann auch in ganz anderer Form fortgeführt werden. So gibt es Häuser im Stil der Bugis, die völlig mit Toraja-Schnitzereien bedeckt sind, moderne Steinhäuser, auf dessen Dach ein Miniatur-Ahnenhaus steht oder mächtige, frisch renovierte Ahnenhäuser, neben denen eine
} 
Andere Toraja argumentieren jedoch, dass auch die Authentizität des Materials einen relevanten Aspekt der ehrenvollen Erinnerung des Gründerpaares darstelle. Eine zentrale Rolle in dieser Diskussion hat hierbei das Dach von Reisspeichern und Ahnenhäusern eingenommen, das üblicherweise mit Bambus gedeckt, in den letzten Jahrzehnten aber aus Kostengründen vielfach durch Wellblech ersetzt wurde. Die nominierten Dörfer, deren Ahnenhäuser und Reisspeicher Wellblechdächer aufweisen, erfüllen in den Augen der Verfechter der materiellen Authentizität somit die Anforderungen für eine Nominierung nicht und beweisen zudem, dass diese Dörfer die Bedeutung des „Schutzes unseres kulturellen Erbes“ nicht verstanden hätten.

Hier spielt auch der Einfluss des Tourismus eine Rolle, da manche Dörfer die Erfahrung gemacht haben, dass Häuser mit Wellblechdächern für TouristInnen, die das „Ursprüngliche“ und „Natürliche“ suchen, nicht attraktiv sind. Materielle Echtheit ist deshalb ein neues Kriterium, das erst im Kontext von Tourismus und des Diskurses vom „kulturellen Erbe“ entstanden ist.

Auf regionaler und nationaler Ebene, also bei der Landesbehörde für Denkmalschutz in Makassar, sowie in der Abteilung Archäologie des Ministeriums für Kultur und Tourismus in Jakarta, die für die Nominierung zuständig ist, steht die Authentizität des Materials im Vordergrund. Mehrere Dörfer haben bereits Gelder beim Landesamt für Denkmalschutz beantragt und bewilligt bekommen, um ihre Ahnenhäuser wieder mit Bambus zu decken. Authentizität, so sagte man im Ministerium in Jakarta, kann wieder neu hergestellt werden. Allerdings bevorzugt wiederum das Amt für öffentliche Ordnung auf lokaler und regionaler Ebene in Übereinstimmung mit dem nationalen Ethos der Entwicklung und des Fortschritts ein zeitgemäß-akkurates Erscheinungsbild der Dörfer. Ein anderer nominierter Ort mit besten familiären Beziehungen zu eben diesem Amt hat daher - sehr zum Verdruss des Amtes für Denkmalschutz - bereits neue Wellblechdächer, gepflasterte Wege für die Besucher, niedrige Mäuerchen zur Einfriedung der Ahnenhäuser, sowie kleine Regenwassergräben um die Reisspeicher herum erhalten. Zudem säumen die neu gepflasterten Wege kleine, aus Beton gegossene und an Windlichter erinnernde Miniaturen von Ahnenhäusern, die mit ihrem dekorativen Effekt an Bali erinnern sollen. Diese und andere aus Sicht mancher Toraja angemessenen Verbesserungen der ausgewählten Orte werden allerdings in dem Nominierungsdokument als zu beseitigende Eingriffe in die Authentizität beklagt.

In ihren Bemühungen um „kulturelle Echtheit“ gehen die zuständigen Denkmalschutzbehörden auf regionaler und nationaler Ebene jedoch noch einen Schritt weiter: Sie bedauern, dass die gesamte Lebensweise der Toraja nicht mehr „authentisch“ sei, da vor allem das Christentum die „echte“ Kultur bereits weitgehend

moderne zweistöckige Villa mit Säulen, Balkonen, schmiedeeisernen Geländern, Tiffany-Fenstern etc. steht. Selbstverständlich - und vielleicht bedauerlicherweise - würden sich diese Orte niemals als Weltkulturerbe qualifizieren, obwohl sie "spirit" und "feeling" bestens repräsentieren. Zum tongkonan als "ethnic marker" und die lokale ebenfalls umstrittenen Bedeutungen der Schnitzmotive siehe Adams 1998, 2006. 
zerstört habe. ${ }^{15}$ Als Beispiel wird Kande Api angeführt, ein Ort, der auf seinen Zeremonialplatz eine Kirche gebaut hat. Auf lokaler Ebene dagegen wird das Christentum als unproblematischer, selbstverständlicher und zentraler Bestandteil der kulturellen Identität angesehen, der in keinerlei Widerspruch zur kulturellen Authentizität steht. Der Bau einer Kirche auf dem Zeremonialplatz stellt aus lokaler Perspektive eine positive Entwicklung dar, sozusagen das, was die UNESCO in der Definition einer organically evolved landscape "significant material evidence of its historic evolution" nennt.

Es ist jedoch gerade diese „echte“ Kultur, auf die regionale und nationale Behörden so viel Wert legen, die den Toraja in der indonesischen Hierarchie kulturell-gesellschaftlicher Entwicklung den recht unvorteilhaften Platz einer in frühen Zeiten stehen gebliebenen Gesellschaft zuweist. So erwähnt das Nominierungsdokument mehrfach, dass "Tana Toraja traditional settlement and culture still retain the characteristics of early Austronesian culture [...] In this regard, the heritage has an indispensable scientific value as a source of analogy to study the past' (S. 25, Hervorhebung im Original ${ }^{16}$ : Die Toraja, so scheint es, sollen in einem Land, das sich sonst dem Fortschritt und der Entwicklung verschrieben hat, die Rolle des archaischen Anderen ausfüllen.

Konstituiert sich aus Perspektive der UNESCO die Einzigartigkeit der Toraja aus ihrer Differenz zu anderen Kulturen Indonesiens, wird Differenz also vertikal - in der historischen Entwicklung - verstanden, so fächert sich für die Toraja auf lokaler Ebene ein horizontales Feld von Differenzen und konkurrierenden Bedeutungen und Werten auf. Hier repräsentieren die nominierten Orte eben nicht alle gleichermaßen „die Lebensweise der Toraja“. In einer von tradierten Konkurrenzen und Statuswettkämpfen - sowohl zwischen dem Süden und dem Norden, als auch innerhalb des Nordens - gezeichneten Gesellschaft erfahren die nominierten Orte vor dem Hintergrund der Auseinandersetzungen mit den benachbarten Bugis oder den niederländischen Kolonialherren ganz spezifische Zuweisungen von Bedeutungen und Werten.

Verdinglichte Kultur, so hat sich gezeigt, gewinnt ein Eigenleben. Dabei nutzen lokale Akteure die Diskurse von heritage und Identität durchaus zur Förderung eigener, lokal bedeutsamer Ziele. Wie wir im Folgenden sehen werden, verbinden sich auf der Ebene nationaler/internationaler Interaktionen mit den kulturellen Elementen noch weitere, ganz neue Bedeutungen und Ziele, die meistens vorwiegend ökonomisch bestimmt und nur noch beschränkt (wenn überhaupt) an Werte und Sinngebung der Ursprungsgesellschaft anknüpfen.

\footnotetext{
15 Es ist nicht ohne gewisse Ironie, dass nach Jahrzehnten des staatlichen Drucks auf die StaatsbürgerInnen, als Zeichen der Modernität und Entwicklung einer der Weltreligionen beizutreten, genau diese Religionszugehörigkeit nun den RepräsentantInnen eines anderen Bereichs des Staates die gesamte Lebensweise als unecht erscheinen lässt.

${ }^{16}$ Die Beziehung zwischen Toraja und Bugis - so wurde mir mehrfach mit gesenkter Stimme von den buginesischen und javanischen Mitarbeitern der Denkmalbehörde mitgeteilt - sei ja auch dadurch charakterisiert, dass die Bugis früher Toraja als Sklaven gehalten haben. Das Thema aber sei ein Tabu.
} 


\section{3 „Kulturelles Erbe“ in internationalen Gremien}

\subsection{UNESCO und WIPO als alternative Instrumente}

UNESCO und WIPO unterscheiden sich grundsätzlich voneinander, auch wenn es, wie gezeigt werden soll, bezüglich kulturellem Erbe Überschneidung/Ergänzung gibt. Die UNESCO bemüht sich um die Förderung und Bewahrung von kulturellen Äußerungen, die dadurch eine besondere ästhetische Wertzuschreibung erfahren und als einzigartig anerkannt werden. Bei der WIPO stehen der ökonomische Wert von Ideen und Produkten sowie deren Schutz im Vordergrund.

Die UNESCO mit ihren Committees for the Safeguarding of the Intangible Cultural Heritage und World Heritage sind die wichtigsten internationalen Gremien, die kulturelles Erbe durch Eintragung auf ihrer Liste zu einer besonderen Anerkennung verhelfen. An den Sitzungen der UNESCO schlagen Mitgliedstaaten dem Gremium vor, einzelne Kulturgüter, die sie nach einem langen Procedere der Auswahl und Begründung in ihrem Land nominierungsfähig gemacht haben, in die Liste herausragender kultureller Hervorbringungen aufzunehmen.

Diese internationalen Gremien diskutieren auf unterschiedliche Art und Weise und mit ebenso verschiedenen Zielen, wie kulturelle Hervorbringungen definiert, kategorisiert und geschützt werden können. Bei der UNESCO geschieht dies aufgrund der Konventionen von 1972 und 2003; eine Eintragung des von ihnen vorgeschlagenen Erbes auf der Liste von Immateriellem Kulturgut bzw. der Welterbe ist das Ziel all jener Länder, die sich um die Nominierung einzelner Kulturgüter bemühen. ${ }^{17}$

Wie sich herausstellte, werden diese internationalen Organisationen von verschiedenen Staaten als Möglichkeit wahrgenommnen, alternativ oder chronologisch gestaffelt einzelne Kulturgüter anerkennen bzw. schützen zu lassen. Im Vordergrund stehen - wie das Beispiel Batik Indonesia verdeutlichen wird - ökonomische und strategische Überlegungen zur Selbstbehauptung auf einem globalisierten Markt.

Im Unterschied zur UNESCO und ihren Konventionen ringen die Delegierten der Mitgliedstaaten der WIPO noch immer um die Präliminarien einer verbindlichen Konvention. ${ }^{18}$ Bei der WIPO stehen geistiges Eigentum (intellectual property) und, mehr oder weniger abgeleitet davon, genetische Ressourcen, traditionelles

\footnotetext{
17 An der Sitzung in Abu Dhabi hatten China 22, Japan 13 und Korea 5 Vorschläge zur Nominierung als intangible heritage eingereicht. Dieser zweifellos inflationären Entwicklung wurde im Hinblick auf die Zukunft durch eine ergänzende Regelung der Richtlinien, die in der gleichen Sitzung verabschiedet wurde, Einhalt geboten.

18 Stefan Groth hat sich in seinen Forschungen auf die WIPO-Verhandlungen konzentriert; vgl. seinen Beitrag in diesem Band ebenso wie denjenigen von Marianna Bicskei und Sven Mißling.
} 
kulturelles Wissen und Folklore im Vordergrund. ${ }^{19}$ Die Organisation hat zum Ziel, ein Intellectual Property-System zu entwickeln, welches Schutz und Zugang zu kulturellen Ressourcen in dem Sinne regelt, dass Kreativität und Innovationen begünstigt, ökonomische Entwicklungen gefördert werden und das öffentliche Interesse gewahrt bleibt. Die Palette von geistigem und kulturellem Eigentum, das individuell und gemeinschaftliche Hervorbringungen und Schöpfungen umfasst, ist äußerst breit. Die vorgesehenen Schutzinstrumente umfassen patents, trademarks, industrial design, collective markers und geographic indicators (als Teile von collective markers) sowie copyright (WIPO 2010:69, 123). Während sich die meisten dieser Schutzinstrumente vor allem an gesellschaftlicher Individualisierung und damit zusammenhängend mit individuellen Kreationen - im Sinne von Autorenschaft - und deren ökonomischen Inwertsetzung orientieren, befasst sich der Schutz von traditional cultural expressions mit dem kulturellen Erbe (cultural heritage) von "indigenous, local and other cultural communities" 20 und deren Künstlern (56). Leitend bei der Schaffung dieses Teilbereichs der WIPO war die UNESCO-Konvention von 2005, das „Übereinkommen zum Schutz und zur Förderung der Vielfalt kultureller Ausdrucksformen“ (UNESCO 2005a); dieses wird als „Magna Charta der internationalen Kulturpolitik“ bezeichnet, weil es „das Menschenrecht auf kulturelle Selbstbestimmung im Völkerrecht" verankerte (Deutsche UNESCO Kommission 2006). Bei der WIPO geht es vor allem um jene Bereiche von kulturellem Erbe, die im Schnittpunkt zwischen Tradition, Modernität und Markt ("tradition, modernity and the marketplace”, WIPO 2010:56) stehen, also über den „Selbstzweck“ von Kultur bzw. deren Alläglichkeit hinausgehen und im Fokus von Begehrlichkeit auch von externen Akteuren stehen, vor allem von transnationalen Unternehmen und Industrienationen. ${ }^{21}$ Hier versucht die WIPO, das Wissen von "traditional knowledge holders" (62) vor nicht-autorisierter Aneignung, Weitergabe und Vermarktung zu schützen und eine Nutzung, die aus der Sicht der Herkunftsgesellschaft als unangemessen, verfälschend oder gar beleidigend betrachtet wird, zu verhindern. Umgekehrt sollen dadurch indigene Gruppen ermächtigt werden, innovativ mit ihren Traditionen umzugehen, damit sie diese auch ökonomisch nutzen können.

\footnotetext{
19 Dieser letztgenannte Teilbereich wurde vor allem durch Artikel 8 der "Convention on Biological Diversity" in Rio 1991 initiiert (Clift 2007:196). Genetische Ressourcen und biologische Diversität über beides verfügen die Länder des Südens bekanntlich sehr viel mehr als die Industrienationen bildet denjenigen Bereich, an dem sich die Positionen der Länder des Nordens von denen des Südens diametral gegenüber stehen.

20 An anderer Stelle als "indigenous and traditional communities" bezeichnet (WIPO 2010:63).

21 Anlässlich der Sitzung des Intergovernmental Committee der WIPO im Sommer 2009 wurde deutlich, wie sehr dieser Bereich ein Zankapfel ökonomischer Interessen zwischen den Ländern des Nordens und des Südens ist. Besonders eindrücklich fand ich die Tatsache, dass sich die Delegationen der meisten Länder des Südens in der Formulierung einer Richtlinie einig waren; die zahlenmäßig weit unterlegenen (aber umso machtvolleren) Länder des Nordens wehrten sich (mit einzelnen Ausnahmen) vehement gegen eine verbindliche Formulierung. Da es keine Abstimmungen gibt, sondern nur „Konsensus“ hatten die Länder des Südens das Nachsehen.
} 


\subsection{Batik zwischen kulturellem Erbe und geschütztem Markenzeichen}

Anlässlich der Sitzung des Intergovernmental Committee for the Safeguarding of Intangible Heritage in Abu Dhabi (2009) wurde „Indonesian Batik“ - eine Kategorie, welche alle Batikarten unterschiedlich regional-kultureller Herkunft umfasst in die UNESCO-Liste aufgenommen. Wie ein Vertreter der indonesischen Delegation (wozu auch Vertreter der Handelskammer zählten) erklärte, soll diese Zertifizierung dazu dienen, die ,echten“ indonesischen Produkte von den billigen Batikimporten (Stoffe mit Batikmustern, die im Druckverfahren hergestellt sind) abzugrenzen. Man habe, so ein Vertreter der Handelskammer, der Regierung bereits vorgeschlagen, eine Regelung zu erlassen, nach der Batikdrucke - ob importiert oder im eigenen Land hergestellt - mit „Batikmotiv“ gekennzeichnet werden müssten - und zwar in den Stoff eingewebt. Dem Vertreter der Handelskammer war es klar, dass diese Auszeichnungspflicht die Flut der billigen Batikdrucke, welche Touristen und auch der lokalen Bevölkerung als „echt“ verkauft werden, nicht verhindern, jedoch eindämmen kann. Die ,echten“ mit Batikmustern verzierten Textilien sind von Hand gefertigt (Reserveverfahren mit Wachs) und der Preis beträgt deshalb ein Vielfaches von gedruckten Batiken (vgl. UNESCO 2009). Aus diesem Grund wird gerade ein Großteil der Indonesier weiterhin gedruckte Batikstoffe - sie dienen als traditionelles Bekleidungsstück (sarong) - kaufen.

Den Eintrag von Batik auf der UNESCO-Liste hatte zuvor ein anderer indonesischer Vertreter an der WIPO-Konferenz als Vorstufe zur Erlangung einer international anerkannten und geschützten Handelsmarke (als collective mark mit geografischem Indikator) benannt. Da es jedoch noch keine verabschiedete Konvention gebe, habe sich Indonesien in einem ersten Schritt für die Nominierung durch die UNESCO entschieden. Indonesien betrachte die UNESCONominierung als präventive Maßnahme, die indirekt verhindern könne, dass eine Firma in einem anderen Land die indonesische Batiktechnik und ihre Motive patentieren lassen könne. ${ }^{22}$ Auf jeden Fall werde die Aufmerksamkeit der Weltöffentlichkeit auf den indonesischen Batik gelenkt, der durch die Nominierung in seiner Authentizität bestätigt worden sei.

Auf meine Frage, ob - nachdem bereits Malaysia dagegen protestiert hatte, dass Indonesien die Batiktechnik für sich beanspruche, obwohl diese in Malaysia ebenfalls praktiziert werde - dieser nationale Anspruch auf eine Technik nicht andere Länder übervorteile, meinte er: Die geografische Herkunft „Indonesien“ lasse anderen Ländern die Möglichkeit, beispielsweise ihre eigenen Batikstoffe ebenfalls als Intangible Heritage der UNESCO vorzuschlagen. ${ }^{23}$ Aus diesem Grund habe man nicht einzelne Muster, sondern „Indonesian Batik“ auf die Liste

\footnotetext{
22 Auch Jaszi et al. haben festgestellt, dass Indonesien befürchtet, andere Staaten bzw. NichtIndonesier könnten handwerkliche Erzeugnisse aus Indonesien patentieren lassen bzw. copyright für sich beantragen (2009:17).

${ }^{23}$ Was nur möglich ist, wenn diese Stoffe auf eigenen Traditionen aufbauen und sich deshalb in der Art unterscheiden.
} 
setzen lassen. Und er fügte hinzu, ,wir alle wissen, dass Kultur nicht an Landesgrenzen Halt macht."

Bereits 2007 führte Indonesien eine eigene, landesintern gültige Zertifizierung von Batik ein. So vergibt Indonesien seither das Markenzeichen (trademark) in einem Verfahren, das aus zwei Schritten besteht: Unternehmen müssen ihre Produkte zuerst daraufhin überprüfen lassen, ob sie dem „Nationalen Indonesischen Standard“ (Standar Nasional Indonesia) entsprechen. Bestehen die Textilien diese Tests, so kann das Unternehmen im Batikzentrum in Yogyakarta die eigentliche Zertifizierung beantragen. Dieses Batikzentrum (Balai Besar Kerajinan dan Batik) unterzieht die Textilien weiteren Untersuchungen (vor allem bezüglich der Reserve- und Färbetechnik, der Muster sowie der Qualität der Stoffe). Nach bestandenen Tests wird das Markenzeichen „Batik Indonesia“ vergeben, auf das Copyright im indonesischen Copyright Büro eingetragen ist. Jede dieser Textilien dieses Unternehmens wird danach mit diesem Markenzeichen ausgezeichnet. Diese Auszeichnung ist drei Jahre gültig und muss oder kann danach erneuert werden. ${ }^{24}$ Gerade diese landesinterne Zertifizierung zeigt, dass nicht eine lokale Gruppe oder gar individuelle Batikkünstler im Vordergrund stehen, sondern Unternehmen, die, so kann man annehmen, ihre eigenen Ziele und Strategien besitzen.

\subsection{Lokale Schöpfungen, Rechte und internationale Regeln}

Das Beispiel des Batik hat gezeigt, wie aus einem ursprünglich lokalen Handwerk, das in kleinsten Manufakturen ausgeübt wurde, inzwischen ein von national anerkannten Unternehmen hergestelltes Handelsprodukt geworden ist, das nationalen Ausleseverfahren und Standardisierungen unterzogen wird. Diese Batiktextilien zwar ausgezeichnet durch die UNESCO als einzigartiges immaterielles kulturelles Erbe Indonesiens - befinden sich als geschütztes Handelsprodukt auf dem Weg zum Weltmarkt.

Der internationale Markt mit seinen eigenen Spielregeln stellt indigene Künstler und deren Werke vor völlig neue Situationen. Ein Beamter des indonesischen Ministeriums für Kultur und Tourismus schilderte anlässlich der Sitzung des Intergovernmental Committee der WIPO im Sommer 2009 den Fall eines indonesischen Silberschmieds, der plötzlich mit ihm völlig unvertrauten - internationalen Vorstellungen und Regeln über künstlerischer Schöpfung im Sinne von „Originalität“", „Original“, „Kopie“ und damit zusammen hängenden Rechten konfrontiert wurde, was schließlich dazu führte, dass ihm der Zugang zum internationalen Markt verwehrt blieb:

Ein indonesischer Silberschmied wollte seine Arbeiten, die auf traditionellen Mustern aufbauen, in einer Ausstellung in den USA zeigen (und ver-

24 Jaszi nennt diese Art der Zertifizierung "a hybrid of a collective mark and a certification mark" (2009:102-103). 
kaufen) und stellte einen entsprechenden Antrag auf Import. Die Einfuhr seiner kunsthandwerklichen Arbeiten wurde ihm nicht erlaubt. Als Begründung wurde angegeben, dass das Muster, das die meisten seiner Arbeiten aufwiesen, bereits in den U.S.A. patentiert sei. ${ }^{25}$

Dieses Beispiel illustriert, neben der Relevanz von Schutzmaßnahmen für kulturelle Hervorbringungen „traditioneller Gemeinschaften“, auch das unterschiedliche Verständnis von Nutzung und Rechten an Mustern. Während offensichtlich eine Einzelperson bzw. eine Firma eine Patentierung für ein (ursprünglich aus Indonesien stammendes) Muster in den USA als geistigen Eigentum beantragt und erhalten hatte, ging der indonesische Silberschmied von einem Repertoire von Mustern aus seiner eigenen Kultur aus, aus dem jedermann im Sinne des „Open Access" frei schöpfen könne. Sein kreativer Umgang mit traditionellen Mustern bedeutete deshalb nicht, dass er diese als Person (bzw. als Künstler, senian) im Sinne von Urheberschaft für sich beanspruchte. Er vertrat eine mit internationalen Regeln unvereinbare Anschauung und Praxis von Rechten. Der indonesische Delegierte formulierte es so:

Unser Leben war immer ein gemeinschaftliches Leben (communal life). Als [das Konzept] „Geistiges Eigentum“ (Intellectual Property) in Indonesien eingeführt wurde, gab es Verwirrung: Wie ist es möglich, dass es individuelle Rechte gibt? [...] Die Menschen in ländlichen Gebieten sind darüber verwirrt. Denn wenn jemand beispielsweise versucht, eine Skulptur nachzuschnitzen und eine große Ähnlichkeit mit dem Vorbild erreicht, dann freuen sich die Leute darüber und bewerten dies als eine gute Leistung.

Diese Beschreibung zeigt, dass in „traditionellen Gemeinschaften“ Indonesiens die Kategorie des künstlerisch-schöpferischen Individuums als autonomer Erschaffer oder gar Erfinder nicht existierte, sondern die schaffende Person und ihre Hervorbringung als Teil und Resultat der Gemeinschaft galt. Sie stand (und steht) in ständiger Interaktion mit anderen Mitgliedern, wobei jedes ständig aus dem gesamten kulturellen „Reservoir“" schöpft - und Neues hinzufügt. In diesem Verständnis von sozialer Verwobenheit gibt es keine exklusiv zu bewertende individuelle Leistung. Aus der indonesischen Perspektive wird damit die Besonderheit der westlichen Konzeption des Künstlers als unabhängiger und individueller geistiger Schöpfer erkennbar.

Nachahmen wurde und wird in Indonesien nicht als (partielles) Wegnehmen des „Originals“ verstanden, als Plagiat oder gar als versuchte Fälschung. Die „Kopie“ tut dem „Original“ - sofern diese Gegenüberstellung überhaupt zutreffend ist

\footnotetext{
25 Vgl. dazu die Website www.budaya-indonesia.org, auf der 27 kulturelle Hervorbringungen aus verschiedenen Teilen Indonesiens aufgelistet sind, die alle von Unternehmen mit Sitz im Ausland angeeignet und kommerzialisiert wurden. Das Beispiel des Silberschmieds (aus Bali) ist erwähnt, aber auch bestimmte indonesische Gewürzmischungen, die heute von Konzernen außerhalb Indonesiens hergestellt und in großem Stil vermarktet werden. Auch der Kaffee der Toraja ist ein solches Beispiel.
} 
- keinen Abbruch und vermindert dessen (ideellen) Wert auch nicht (vgl. dazu auch Aragon und Leach 2008), sie kann vielmehr als Bewunderung und Respekt vor der Leistung eines anderen verstanden werden.

Wie der Delegierte weiter darlegte, orientiert sich der indonesische Staat heute an westlichen, inzwischen international verbindlich gewordenen Konzepten von Geistigem Eigentum und Autorenschaft, um selbst aktiv werden zu können und Übervorteilungen zu verhindern. Er unternimmt auch entsprechende Anstrengungen, diese Konzepte einer breiten Bevölkerung zu vermitteln. Das Ziel Indonesiens sei es, für alles, was das Land und seine Bewohner hervorgebracht haben, international die höchstrangigen Rechte zu erlangen. Die Staatsvertreter an den Sitzungen waren in der Regel Juristen, welche im Wirtschaftsministerium, im Ministerium für Kultur und Tourismus (eine Kombination zweier Bereiche, die für sich spricht) und des Außenministeriums tätig sind.

Sie wählen, wie kurz erwähnt, die verschiedenen internationalen Organisationen und deren Instrumente danach aus, welche Organisation auf welche Weise zum besten Schutz ihrer kulturellen Produkte beitragen kann und sehen diese auch als Alternativen, teilweise eine als Vorstufe der anderen. Beim Batik, so meinte ein Vertreter, wäre die Erreichung einer collective mark wirkungsvoller (und ertragreicher) als die Eintragung auf der Intangible Heritage-Liste der UNESCO. Der Vertreter Indonesiens, mit dem wir anlässlich der WIPO-Konferenz gesprochen hatten, nimmt jeweils an den Sitzungen der WTO (TRIPS), WIPO und der UNESCO teil, um die Möglichkeiten für den Schutz kultureller Errungenschaften Indonesiens auszuloten und wenn möglich zu ergreifen. Nach den Prioritäten befragt, welche er aus der Sicht Indonesiens diesen Organisationen beimisse, antwortete er: WIPO, dann WTO und dann UNESCO; diese Reihung habe nichts mit einer Geringschätzung von Kultur zu tun, aber letztlich gehe es um ökonomische Interessen und da sei es wichtig, dass Indonesien vorne mit dabei sei. Das seien Fragen, die heute in allen Ländern heiß diskutiert würden.

\section{Schluss}

Die Beispiele des Silberschmieds und der Toraja haben gezeigt, wie auf den verschiedenen Schauplätzen - lokal, national, international - unterschiedliche Werte und Regelungen bezüglich kultureller Hervorbringungen im Kontext von Autorenschaft sowie von Authentizität aufeinander prallen: Der Silberschmied, der von einem ganz anderen Selbstverständnis vom Umgang mit Schöpfungen im Rahmen seiner Kultur ausgeht, muss plötzlich zur Kenntnis nehmen, dass ein Angehöriger eines anderen Staates dieselben Muster bereits als geistiges Eigentum hat patentieren lassen - und er selbst diese nicht mehr offiziell verwenden darf. Die Toraja sehen überwiegend in der kontinuierlichen sozialen Existenz eines Ahnenhauses die Authentizität gewahrt, während die materielle Authentizität zweitrangig ist, die 
jedoch auf nationaler und internationaler Ebene größte Bedeutung hat. Dazwischen steht der indonesische Staat, der versucht, kulturelle Errungenschaften so zu schützen, dass diese auf dem internationalen Markt als indonesische Handelsprodukte bestehen können. Diese Beispiele verdeutlichten auch, dass die Aneignungsund Aushandlungsprozesse hierarchisch geordnet sind. Ganz ausgeblendet wird in diesem letztlich ökonomisch bestimmten Wettlauf in internationalen Organisationen um die Zertifizierung und Patentierung von Kultur die Frage nach Transformation der ideellen Bedeutungen, welche die Ursprungsgesellschaft diesen Mustern (Silberschmiedarbeiten und Batik) und den ausgewählten Toraja-Orten beimessen, und die sukzessive Sinnveränderung oder gar -entleerung, die damit einher geht.

In allen erwähnten internationalen Organisationen sind, wie bereits erwähnt, die Nationalstaaten die Mitglieder und Verhandlungspartner und - verständlicherweise - nicht die von der WIPO besonders hervorgehobenen lokalen Gruppen. ${ }^{26}$ Lokale Gruppen werden damit nicht als eigenständige Akteure anerkannt, sondern als solche, über die der Staat die Autorität besitzt und die durch die internationalen Regelungen in der Regel verstärkt und legitimiert wird.

Die Problematik im Umgang mit verdinglichter Kultur liegt nicht nur darin, dass die Ebene der national/internationalen Beziehungen nur sehr schwach mit der lokalen und regionalen Ebene verbunden ist.

Auch das Verhältnis der Gruppen innerhalb eines Staates ist nicht frei von Komplikationen. Ob sich etwa alle lokalen Gruppen in Indonesien mit Batik als nationalem heritage zufrieden geben, bleibt abzuwarten. So wurde in Toraja bereits Unzufriedenheit artikuliert, dass „die Javaner“ angeblich klassische Toraja-Muster bei Batiken verwenden und man sich deshalb um das bak cipta (Urheberrecht) bemühen müsse. Andererseits haben Holzschnitzer der Toraja begonnen, für den lukrativen touristischen Markt die traditionellen flächigen geometrischen und floralen Schnitzmuster durch balinesische Motive und Techniken, also etwa dreidimensionale realistische Landschaftsszenen, zu ergänzen und sehen das wiederum als Ausdruck einer legitimen künstlerischen Inspiration an (Adams 2006). Da „Kultur““ erst durch soziale Interaktion entsteht - durch Kommunikation und Austausch stellt sich tatsächlich die Frage, wohin eine zunehmende Verrechtlichung künstlerischer Ausdrucksformen innerhalb (und außerhalb) Indonesiens führen soll und wird.

Die Frage, die WIPO sich selber stellt, ist angesichts der Problematik der Beziehungen zwischen einzelnen Akteursgruppen und dem Staat, in dem sie leben und der sie nach außen vertritt, mehr als berechtigt: "To whom, if anyone, does a nation's cultural heritage "belong" and "which intellectual property policies best serve cultural diversity and cultural pluralism" (WIPO 2010:57)?

\footnotetext{
26 In allen diesen Organisationen sind NGOs zugelassen (ohne Stimmrecht). Die tatsächlichen Zugangsmöglichkeiten zu diesen Gremien sind jedoch durch eine Vielzahl von Faktoren begrenzt: Sprachkenntnisse, Beziehungsnetze, finanzielle Möglichkeiten, nationale Unterstützung, Zugang zu Informationen und die Fähigkeit, diesen zum Teil komplizierten, ritualisierten Verhandlungsformen folgen zu können und dabei erst noch eine eigene Handlungsfähigkeit zu entwickeln.
} 




\title{
Sbek Thom nach seiner UNESCO Zertifizierung: Eine Untersuchung der Dynamik des kambodschanischen Schattentheaters nach seiner Ernennung zum immateriellen Kulturerbe
}

\author{
Aditya Eggert
}

\section{Einleitung}

Eines der ethnologischen Projekte innerhalb der Interdisziplinären Forschergruppe zu Cultural Property an der Georg-August-Universität Göttingen untersucht die Prozesse der Konstituierung eines „Weltkulturerbes“ und deren Bedeutung am Beispiel Kambodscha. Im Rahmen dieses Projektes führte ich von April bis September 2009 eine Feldforschung zu den aktuellen Strukturen, Prozessen und Diskursen durch, die in der Konstituierung von immateriellem Kulturerbe in Kambodscha beteiligt sind. Ausgangspunkt der Untersuchung waren die Institutionen und Akteursgruppen, die auf den verschiedenen Stufen der Konstituierung von immateriellem Kulturerbe in Kambodscha eine Rolle spielen - einschließlich ihrer widersprüchlichen Interessen, Motivationen und Ziele. Diese Stufen sind im Einzelnen: (1) Auswahl immaterieller Kulturelemente ${ }^{1}$ für die Nominierung; (2) Konzeption des Nominierungsantrags und Einreichung bei der UNESCO; (3) Prüfung der Anfrage und Entscheidung über die Aufnahme in die Repräsentative Liste oder in

\footnotetext{
1 „Kulturelement“ oder „kulturelles Element“ wird in diesem Beitrag definiert als ein ursprünglich in eine kulturelle Gesamtheit eingebettetes kulturelles Phänomen, welches durch bestimmte Institutionen und Akteure aus diesem Zusammenhang herausgenommen wurde, um es der UNESCO als immaterielles Kulturerbe vorzuschlagen.
} 
die Liste des gefährdeten immateriellen Kulturerbes; sowie (4) Nutzung und Gestaltung des neuen kulturellen Artefakts.

Stufen eins bis drei umfassen den formalen Prozess der Nominierung und Ernennung eines immateriellen Kulturerbes, während Stufe vier sich unmittelbar an diesen Prozess anschließt. Gegenstand dieses Beitrags ist die vierte Stufe der Konstituierung von immateriellem Kulturerbe, welche die Nutzung und Gestaltung des neuen kulturellen Artefakts betrifft. Integraler Bestandteil einer ethnologischen Forschung über die Konstituierung eines immateriellen Kulturerbes ist es, die möglichen Auswirkungen einer solchen Zertifizierung ${ }^{2}$ auf die weitere Entwicklung des entsprechenden kulturellen Elements zu überprüfen. Ziel ist es hier, die Dynamik der verschiedenen Gruppen zu verfolgen, die das immaterielle Kulturerbe nach seiner UNESCO Zertifizierung nutzen und gestalten. Inwiefern wird das neue kulturelle Artefakt von diesen Gruppen in Wert gesetzt? Hat eine Revitalisierung des immateriellen Kulturelements stattgefunden, wie es in Artikel 2.3 der UNESCO 2003 Konvention als Ziel der Listung formuliert ist (UNESCO 2003)? Innerhalb der Forschung zu Cultural Property ist es besonders interessant zu untersuchen, ob bestimmte Gruppen Rechte in Bezug auf dieses immaterielle Kulturerbe beanspruchen oder ob Wettbewerbstendenzen bzw. Rivalitäten um das neue kulturelle Artefakt beobachtet werden können. Gleichermaßen ist es von Bedeutung zu überprüfen, wer überhaupt die Möglichkeit besitzt, das immaterielle Kulturerbe zu nutzen und $\mathrm{zu}$ formen und welche Konsequenzen sich aus diesen Konstellationen letztendlich für das immaterielle Kulturerbe selbst ergeben. Diese Aspekte werden im Hinblick auf die weitere Gestaltung des Forschungsprojektes diskutiert.

Nach Erläuterung des Forschungsgegenstandes und der Herangehensweise innerhalb des Forschungsprojektes im zweiten Abschnitt, werde ich im dritten Abschnitt einen kurzen Überblick über den aktuellen Forschungsstand geben. Der vierte und fünfte Abschnitt befasst sich anschließend mit der Dynamik des kambodschanischen Schattentheaters nach seiner Zertifizierung durch die UNESCO. In der Schlussfolgerung im sechsten Abschnitt fasse ich die entwickelten Theorien zusammen und erörtere ihre Bedeutung im Rahmen der Cultural Property Diskurse.

\footnotetext{
${ }^{2}$ Die Ernennung einer lebendigen kulturellen Ausdrucksform zum immateriellen Kulturerbe ist eine Auszeichnung der UNESCO, die nach gewissen Selektionskriterien durchgeführt wird und mit gewissem Status und Begünstigungen verbunden ist (Zugang zum "Intangible Cultural Heritage Fund", internationale Unterstützung, Entwicklung zu einem touristischen Anziehungspunkt, etc.), um die international konkurriert wird. Da gerade diese Wettbewerbssituation und die sich daraus ergebenden Rivalitäten zwischen einzelnen Akteuren in diesem Beitrag diskutiert werden, ziehe ich hier den Begriff „Zertifizierung“ dem neutraleren Begriff „Listung“ vor.
} 


\section{Forschungsgegenstand und Herangehensweise}

\subsection{Forschungsgegenstand}

Die vorliegende Untersuchung wird anhand des $\mathrm{Khmer}^{3}$ Schattentheaters Sbek Thom durchgeführt, das im Jahr 2005, nach dem Königlichen Ballett in 2003, von der UNESCO zum „Meisterwerk des mündlichen und immateriellen Kulturerbes der Menschheit" erklärt worden ist. ${ }^{4}$ Beide kulturellen Elemente wurden 2008 in die „Repräsentative Liste des immateriellen Kulturerbes der Menschheit" aufgenommen (ebd.). Sbek Thom eignet sich insofern für diese Untersuchung, als dass es die letzte der in Kambodscha erfolgten Auszeichnungen zum immateriellen Kulturerbe erhielt und bislang noch keine umfassende Studie über seine weitere Entwicklung existiert. ${ }^{5}$

In der Vorstellung der Khmer ist das Khmer Schattentheater auf die Angkor Zeit zwischen dem neunten und 15. Jahrhundert zurückzuführen (Pech 1995, UNESCO und MoCFA 2004). Es wird weitgehend davon ausgegangen, dass es sich in den vergangenen nahezu tausend Jahren nicht verändert hat. Ein Großteil der existierenden Literatur zu Sbek Thom bezieht sich daher auf die Angkor-Zeit. Dennoch ist es wichtig sich bewusst zu machen, dass diese Kulturform aufgrund von politischen und religiösen Veränderungen seit der Angkor-Zeit einem kontinuierlichem Wandel unterworfen war. Die Zeit der Roten Khmer stellt in diesem Zusammenhang nur einen der „Umbrüche“ dar, die über einen langen Zeitraum hinweg in Kambodscha stattgefunden haben. Sie macht deutlich, wie das Schattentheater (und auch andere Kunstformen) im Laufe der Geschichte beeinflusst wurden. Unter dem Regime der Roten Khmer kam ein Großteil der gestalterischen und darstellenden Künste innerhalb des Landes nahezu vollständig zum Erliegen. ${ }^{6}$ Der Prozess der Neubelebung des immateriellen Kulturerbes in Kambodscha begann erst im Jahr 1979, als die überlebenden Meister und Kulturschaffenden unter

\footnotetext{
${ }^{3}$ Mit dem Begriff „Khmer“ beziehe ich mich in diesem Beitrag sowohl auf die in Kambodscha mehrheitlich vertretene ethnische Gruppe der Khmer als auch auf das für ihre Kultur verwendete Adjektiv. „Kambodschanisch“ wird hier als Adjektiv für die Zugehörigkeit zu dem Staat Kambodscha verwendet, in dem außer der ethnischen Mehrheit der Khmer auch noch weitere Minderheiten und indigene Gruppen leben, die alle „Kambodschaner“ sind.

4 Vgl. http://www.unesco.org/culture/ich/index.php?pg=00011 (Zugriff am 15.04.2010).

${ }^{5}$ Ich werde mich in dieser Studie auf das so genannte ,große“ Schattentheater Sbek Thom beschränken. In Kambodscha existieren darüber hinaus noch zwei andere Schattentheaterformen: das farbige Schattentheater Sbek Por und das „kleine“ Schattentheater Sbek. Thoch. Um die Auswirkungen der UNESCO Zertifizierung des großen Schattentheaters auch auf die Dynamik dieser beiden Schattentheaterformen zu untersuchen, ist allerdings eine zusätzliche Studie erforderlich.

${ }^{6}$ Unter dem Versuch der Roten Khmer, in Kambodscha einen kommunistischen Agrarstaat zu schaffen, kamen in der Zeit von 1975 bis 1979 circa 90 Prozent der kambodschanischen Künstler, Tänzer, Musiker, Schauspieler, Dramaturgen und Dichter ums Leben. Ein Großteil der kulturellen Zeugnisse wurde in dieser Zeit zerstört, so dass viele der kulturellen Ausdrucksformen für immer verloren gingen (Cambodian Living Arts 2007, Pech 1995).
} 
der Initiative des ehemaligen Kulturministers (1981-1989) Chheng Phon zusammenkamen, um das wiederzubeleben, was noch übrig war (Heywood 2008).

Da eine ausführliche wissenschaftliche Beschreibung der Sbek Thom Gruppen zur Zeit seiner Nominierung fehlt, dienen mir die Angaben im UNESCO Nominierungsantrag, sowie Literatur aus der Zeit vor der UNESCO Nominierung als Referenzrahmen für die Untersuchung möglicher Veränderungen. ${ }^{7}$ Jedoch können Verzerrungen in der zeitlichen Wahrnehmung nicht vermieden werden, so dass sich Veränderungen in Verbindung mit Sbek Thom, die von meinen Gesprächspartnern im Feld beschrieben wurden, gegebenenfalls auf Transformationen beziehen, die bereits in der Zeit vor der Nominierung stattgefunden haben. Darüber hinaus ist es schwierig zu beurteilen, ob die Veränderungen im Schattentheater tatsächlich auf die UNESCO Zertifizierung zurückzuführen sind oder ob noch andere Einflüsse eine Rolle spielen. Um ein noch genaueres Bild von den möglichen Wandlungen des Schattentheaters sowie von ihrer Verknüpfung mit der UNESCO Zertifizierung zu bekommen, müssen noch mehr Gesprächspartner in Betracht gezogen und weitere Beobachtungen durchgeführt werden.

\subsection{Herangehensweise}

Folgende Kriterien dienen als Grundlage für die Analyse der weiteren Entwicklung des Sbek Thom nach seiner Zertifizierung als immaterielles Kulturerbe: (1) Anzahl der Schattentheatergruppen; (2) Grad der Aktivität des Schattentheaters in diesen Gruppen; (3) Struktur und Organisation der Gruppen; (4) Kooperation und Wettbewerb zwischen den Gruppen; (5) Zusammenarbeit der Schattentheatergruppen mit der Regierung. Da die verschiedenen Kriterien stark miteinander verwoben sind, erfolgt ihre Abhandlung zum Teil über mehrere Abschnitte hinweg. Die für die Analyse verwendeten Daten wurden mittels teilnehmender Beobachtung sowie mit Hilfe von narrativen und semi-strukturierten Interviews auf der Basis eines Fragebogens gewonnen. Diese Daten wurden durch Material aus relevanter Literatur im "Centre for Khmer Studies" in Siem Reap, in der "Royal University of Fine Arts" und im UNESCO Feldbüro in Phnom Penh ergänzt. In der vorliegenden Studie wird in erster Linie die Situation der Gruppen in Siem Reap und in Phnom Penh betrachtet, nicht aber der Gruppe in Banteay Meanchey, da die Untersuchung dieser Gruppe noch aussteht.

Die Interviews mit den Schattentheatergruppen in Siem Reap und in Phnom Penh wurden hauptsächlich mit den Leitern, Direktoren und Koordinatoren dieser Gruppen geführt. Um jedoch einen Einblick in die Perspektive, in die Stellung und

\footnotetext{
7 Allerdings ist zu beachten, dass der Nominierungsantrag nach gewissen von der UNESCO festgelegten Vorschriften zu verfassen ist. Daher ist es möglich, dass es in der Konzeption dieses Antrags zu gewissen Selektions- und Konstruktionsprozessen hinsichtlich des verwendeten Materials gekommen ist. Aus diesem Grund habe ich die aus dem Nominierungsantrag gewonnenen Informationen mit Daten aus meinen eigenen Interviews, sowie mit zusätzlicher Literatur belegt (insoweit diese vorhanden beziehungsweise verfügbar war).
} 
in die Probleme der gesamten Gruppe zu erhalten, ist es notwendig, in einem nächsten Feldaufenthalt Interviews mit allen Gruppenmitgliedern und auch Gruppendiskussionen durchzuführen. In der Lektüre des vorliegenden Beitrags ist zu beachten, dass das Forschungsprojekt noch nicht abgeschlossen ist. Offene Fragen und potentielle Lücken werden während meines nächsten Feldforschungsaufenthaltes sowie in den folgenden Forschungsphasen berücksichtigt.

\section{3 Überblick über den aktuellen Forschungsstand}

Nur wenig Literatur existiert zu Sbek Thom aus der Zeit vor den Roten Khmer (1975-1979), in der ein Großteil der kulturellen Zeugnisse Kambodschas zerstört worden ist. Jedoch sind Manuskripte von einigen Sbek Thom Meistern wie Nab Chum und Ti Chean erhalten geblieben, die sich bei den Sbek Thom Gruppen in Siem Reap in Verwahrung befinden. Englische oder französische Beschreibungen zu Sbek Thom finden sich auch in Werken von Pelliot (1902), Bois (1906), Groslier (1913, 1929), Thierry (1963) und Thiounn (1956). Bizot (1973), Saveros (1977) und Schriften des "Buddhist Institute" (1937) in Phnom Penh behandeln die Erzählung des Khmer Schattentheaters, das Reamkers. Brunet (1969) gibt einen Einblick in die Geschichte, in die Ausrüstung, in die Darbietung und in die Musik des Sbek Thom.

Nach der Zeit der Roten Khmer verfasste Pech im Jahre 1994 das bislang umfassendste Werk zu Sbek Thom in englischer Sprache. Zur gleichen Zeit führte Miura (1994) eine kurze Studie zu dem Thema "Information on Cambodian Shadow Puppets" für die UNESCO durch, in der sie die zu dieser Zeit in Siem Reap aktiven Gruppen beschreibt. Im Jahre 2004 wurde Sbek. Thom schließlich in das "Inventory of the Intangible Cultural Heritage in Cambodia" (UNESCO und MoCFA 2004) aufgenommen, was seinen Weg in die Nominierung für das immaterielle Kulturerbe ebnete 9 . Da das Gutachten zur Nominierung derzeit nicht für die Öffentlichkeit zugänglich ist (E-Mail von Proschan, 07.10.2009) können die Kriterien, die für die Zertifizierung von Sbek Thom ausschlaggebend waren an dieser Stelle nicht aufgeführt werden. Diese Informationen sind in einem nächsten Feldaufenthalt gegebenenfalls direkt bei den Verantwortlichen des Kulturministeriums in Phnom Penh zu ermitteln (ebd.).

\footnotetext{
8 Als Epik, die vom Kampf zwischen dem Guten und dem Bösen handelt, findet das Reamker seinen Ursprung im indischen Ramayana. In Kambodscha galt es zunächst als bedeutendste Erzählung innerhalb der Brahmanischen Religion und wurde später zu einem Teil der buddhistischen Lehre (Pech 1995).

9 Nach den Richtlinien der UNESCO kann ein kulturelles Element nur für das immaterielle Kulturerbe nominiert werden, wenn es in die nationale Bestandsliste des immateriellen Kulturerbes aufgenommen worden ist. Diese Liste wird von der UNESCO auch als "tentative list" bezeichnet (UNESCO 2008, 2010).
} 
Darüber hinaus werden aktuelle Studien zu Sbek Thom von Fukutomi10 am "Department of Social and Cultural Anthropology" der "University of the Sacred Heart" in Tokio, Japan, betrieben. An Veröffentlichungen ist daraus bisher in der Zeitschrift "Asian Literature" die japanische Übersetzung der Epik Reamker in der Version des Meisters Ti Chean erschienen (Fukutomi 1999, 2001). Es bleibt abzuwarten welche Erkenntnisse in Zukunft noch aus diesen Studien für die Entwicklung des Schattentheaters nach seiner Zertifizierung als immaterielles Kulturerbe gezogen werden können.

\section{Schattentheatergruppen und ihre Aktivität}

Die Nominierung des Khmer Schattentheaters Sbek Thom für die Zertifizierung als immaterielles Kulturerbe durch die UNESCO wurde von einem eigens dafür zusammengestellten "Intangible Cultural Heritage Committee” (ICHC) innerhalb des "Ministry of Culture and Fine Arts" (MoCFA) in Phnom Penh vorgenommen. Im Zuge dieser Nominierung wurde eine Auswahl an Sbek Thom Gruppen getroffen, die der UNESCO als "recognized practitioners of the tradition" (MoCFA 2004a) vorgeschlagen wurden. Im Folgenden werde ich einen Einblick sowohl in die offiziell anerkannten als auch in die unabhängigen Sbek Thom Gruppen geben.

\subsection{Offiziell anerkannte Gruppen}

Laut UNESCO Nominierungsantrag zählten im Jahre 2004 insgesamt vier Gruppen des Khmer Schattentheaters zu den "recognized practitioners of the tradition" (MoCFA 2004a) ${ }^{11}$ : (1) Wat Svay Dangkum, Siem Reap; (2) Wat Reach Bo, Siem Reap; (3) Sbek Thom, Banteay Meanchey; (4) Sbek Thom, Department of Performing Arts (MoCFA). Dabei bleibt bislang jedoch unklar, welche Kriterien dem MoCFA zur Auszeichnung dieser Gruppen als "recognized practitioners" dienten und welche weiteren Sbek Thom Gruppen zu dieser Zeit existierten, die diesen Status nicht erhalten haben. Während meiner Forschung konnten zwei der oben genannten Gruppen als aktiv ${ }^{12}$ identifiziert werden: die Gruppe Wat Svay Dangkum (Sala Kanseing) ${ }^{13}$ und die Gruppe Wat Reach Bo. Die Gruppe Sala Kanseing

\footnotetext{
${ }^{10}$ Fukutomi Tomoko ist seit 1997 Praktizierende des Khmer Schattentheaters und zugleich Trägerin der Gruppe Sala Kanseing in Siem Reap (siehe unten, Interview mit Fukutomi, 15.04.2009).

11 Ich werde in diesem Beitrag die Veränderungen innerhalb und zwischen den Schattentheatergruppen in den Mittelpunkt stellen. Nähere Informationen zu den einzelnen Gruppen sind im UNESCO Nominierungsantrag zu finden (MoCFA 2004a, 2004b).

12 Den Begriff ,aktiv“ verstehe ich hier als das regelmäßige Stattfinden von Proben und Auftritten des Khmer Schattentheaters.

13 Der Leiter der Sbek Thom Gruppe Sala Kanseing, zugleich Enkel des Meisters Ti Chean, betonte, dass sich diese Gruppe im "Sala Kanseing Village" innerhalb der "Svay Dangkum Commune" befinde und aus diesem Grund „Sala Kanseing Gruppe“ genannt werden sollte. In diesem Beitrag wird
} 
probte zum Zeitpunkt der Forschung samstags und sonntags jeweils für zwei Stunden und bereitete sich auf eine Tournee im November 2009 mit dem "Modern Puppet Centre" in Japan vor (E-Mail von Fukutomi, 10.10.2009, Interview mit Ti Cheans Enkel, 13.06.2009). Darüber hinaus waren keine Auftritte in Kambodscha geplant. Die Gruppe Wat Reach Bo trainierte jeden Freitag, Samstag und Sonntag ebenfalls für zwei Stunden (laut "Class Schedule" der Gruppe) und trat sowohl regelmäßig für Touristen im Amansara Hotel in Siem Reap auf, als auch gelegentlich für Reisegruppen (Interview mit Om, 09.04.2009). Vertreter aus beiden Gruppen erklärten, dass sie mit der Ausübung des Schattentheaters das Ziel verfolgen, Sbek Thom so zu bewahren, wie es durch die ,alten Lehrer“14 überliefert worden war (Interview mit Ti Cheans Enkel und Ly Rida, 17.04.2009). Nab Rin, Erzähler der Gruppe in Sala Kanseing, betonte im Nominierungsantrag:

I learned Sbek Thom from my ancestors and follow the same styles as they have taught me, I never change or create a new style of performing acts, even the way I narrate the scripts I never change - I preserve the original texts from ancient times. If people don't wish to watch our show, I don't know how to deal with this; how to please the spectators. I dare not to reform what my ancestors left me (MoCFA 2004b, bestätigt im Interview mit Nab Rin, 13.06.2009).

Verschiedene Quellen zu Sbek Thom verweisen darauf, dass mit Sbek Thom nur die Epik Reamker aufgeführt wird. Diese Epik ist stark mit den religiösen Überzeugungen der Khmer verbunden (Brunet 1969, MoCFA 2004a, Pech 1995, UNESCO und MoCFA 2004). Da das Khmer Schattentheater einen Akt der Verehrung ${ }^{15}$ beinhaltet, wird es als heilig angesehen (Brunet 1969, MoCFA 2004a, Pech 1995). Wenn der Respekt gegenüber den alten Lehrern und Meistern des Sbek Thom sowie seine Heiligkeit die Ausübenden daran hindert, ihre Kulturform weiterzuentwickeln und sie an das zeitgenössische Leben anzupassen, so lässt das darauf schließen, dass Sbek Thom ein in sich starres immaterielles Kulturelement ist. Allerdings sind ,immateriell“" und „starr“ zwei sich einander widersprechende Begriffe, denn „immateriell“ bedeutet „lebendig“ und lebendiges Kulturerbe ist

deshalb für die Gruppe Wat Svay Dangkum der Ausdruck „Sala Kanseing“ verwendet (Interview mit Ti Cheans Enkel, 17.04.2009).

${ }_{14}$ Dem Nominierungsantrag zufolge wird die Gruppe Sala Kanseing gemeinhin als die älteste der bestehenden Sbek Thom Gruppen betrachtet. Sie wurde in den 1950er und 1960er Jahren zunächst von Meister Ta Mao geleitet, gefolgt von Ta Nab Bun, Khong Det, Nab Chum, Ti Chean, Nab Rin, and Nab Keng (MoCFA 2004b). Aus der Perspektive der heutigen Gruppe geht ihr Wissen allerdings auf den Meister Ti Chean zurück, der im Jahre 2000 verstarb (Interview mit Ti Cheans Enkel und der Beraterin Gruppe Sala Kanseing, 17.04.2009). Das Wissen der Gruppe Wat Reach Bo hingegen geht laut Nominierungsantrag auf den Meister Khong Det zurück (MoCFA 2004b, Interview mit Ly, 17.04.2009).

15 Der Akt der Verehrung, der sowohl während der Eingangszeremonie als auch während der Abschlusszeremonie stattfindet, dient insbesondere den Göttern Shiva und Vishnu, dem in der Handlung vertretenen Asketen, sowie den alten Lehrern des Schattentheaters (Brunet 1969, Pech 1995). 
aufgrund der fortlaufenden Interaktion seiner Träger mit der Umwelt einem kontinuierlichen Wandel unterworfen. Und wenn verhindert wird, dass sich ein immaterielles Kulturelement weiter entwickelt, ist es dann nicht seiner Vitalität beraubt und erreicht somit einen Zustand der Erstarrung? Es sind weitere Untersuchungen erforderlich um herauszufinden, in welchem Maße Sbek Thom und seine Erzählung doch anpassungsfähig sind und demnach dazu geeignet, mit der Gesellschaft zu wachsen, oder ob noch andere Faktoren beteiligt sind, die ihre Entwicklung hemmen. Es ist anzunehmen, dass ein kulturelles Element von der Bevölkerung eher aufrechterhalten wird, wenn es in seiner Anpassung an das zeitgenössische Leben flexibel ist, als wenn es rigide und Veränderungen gegenüber weitgehend unaufgeschlossen ist.

Was die Gruppe in Banteay Meanchey anbetrifft, so wiesen zwei meiner Gesprächspartner darauf hin, dass diese Schattentheatergruppe nicht mehr aktiv ist (Interview mit Thach, 30.07.2009, Interview mit Mao, 08.08.2009). Interessant ist dabei zu erwähnen, dass diese Gruppe im UNESCO Nominierungsantrag die einzige ist, bei der keine detaillierte Aufstellung seiner Mitglieder erfolgt ist. Es ist also fraglich, ob diese Informationen nicht verfügbar waren oder ob sich die Gruppe zu diesem Zeitpunkt bereits im Prozess der Auflösung befand. Dieser Sachverhalt ist bei einem nächsten Feldforschungsaufenthalt genauer zu untersuchen.

Auch im Interview mit dem stellvertretenden Direktor des "Department of Performing Arts", Pok Saran (08.08.2009), stellte sich heraus, dass die Gruppe innerhalb seines Departments ${ }^{16}$ das Schattentheater zurzeit nicht mehr ausübt. Laut Pok sind Darbietungen des Sbek Thom äußerst selten und zum Zeitpunkt des Interviews fanden überhaupt keine Aufführungen statt. Das große Khmer Schattentheater, so Pok, gehört zwar zum Repertoire der Gruppe, wird aber nur trainiert, wenn eine Aufführung geplant ist. Dabei wird Sbek Thom entweder im Ausland aufgeführt oder für Touristen, die eine Darbietung wünschen. Die letzte Veranstaltung fand allerdings im Juni 2008 für das Bayon TV in Phnom Penh statt. Da momentan eine größere Nachfrage von Gästen für den Volkstanz besteht, konzentriert sich die Gruppe auf diese Kulturform.

Um genaue Rückschlüsse auf die Vitalität des Sbek Thom nach seiner UNESCO Zertifizierung ziehen zu können, ist es von Bedeutung zu wissen, warum die beiden Gruppen ihre Ausübung des Schattentheaters eingestellt haben. Ein Grund dafür könnte sein, dass keine kompetenten Künstler oder Musiker mehr zur Verfügung standen, um das Stück in seiner vollständigen Besetzung ${ }^{17}$ durchführen zu

\footnotetext{
16 Die Sbek Thom Gruppe innerhalb des "Department of Performing Arts" im MoCFA setzt sich aus "senior performers" zusammen, die ihren akademischen Grad in den 1960er und 1970er Jahren an der "Royal University of Fine Arts" (RUFA) in Phnom Penh erlangt haben. Zu dieser Zeit wurden die Meister Ti Chean und Ta Dub aus Siem Reap nach Phnom Penh eingeladen, um die Studenten an der RUFA zu unterrichten (Interview mit Pok, 08.08.2009). Pok zufolge gehört nicht nur das Schattentheater Sbek Thom zu dem Repertoire der Gruppe, sondern auch der klassische Tanz, der Volkstanz, das männliche Maskentheater Lakhaon Khol und das kleine Schattentheater Sbek Thoch (Ayang).

17 Das große Schattentheater setzt sich aus insgesamt 150 verschiedenen Schattentheaterfiguren zusammen, die von ungefähr zehn Darstellern manipuliert werden. Eine Aufführung aller Episoden
} 
können. Da es sich beim Sbek Thom um eine Kunstform handelt, die nicht nur technisch sehr anspruchsvoll ist, sondern auch eine besondere spirituelle Gabe erfordert, gestaltet es sich schwierig, geeignete Künstler zu finden, so zumindest die Einschätzung des ehemaligen Kulturministers Chheng Phon im Interview (02.07.2009). Und aus den Erläuterungen von Brunet (1969) scheint es, als könne Sbek Thom nur seine besondere und im Ritual erforderliche Wirkung entfalten, wenn es in seiner vollständigen Besetzung aufgeführt wird. Möglicherweise standen aber auch nicht genügend finanzielle Mittel für die Entlohnung der Künstler oder für die Beschaffung bzw. für die Reparatur der benötigten Schattentheaterpuppen, Instrumente und anderen Ausrüstungsgegenstände zur Verfügung. Der Mangel an Schattentheaterpuppen, die in ihrer Fabrikation und Beschaffung äuBerst aufwendig sind, stellt heutzutage ein Haupthindernis für die Ausübung dieser Kunst dar. Schließlich könnte auch das Fehlen der Nachfrage und somit des Publikums für die Inaktivität der Gruppen verantwortlich sein. Wenn in der Bevölkerung nur mangelndes Interesse an einer Sbek Thom Aufführung besteht, wird den Gruppen nicht nur die Existenzgrundlage, sondern auch die Moral entzogen und die Mitglieder müssen sich anderweitig orientieren.

Im Fall der Gruppe im "Department of Performing Arts" scheint es, als seien nicht genügend Anfragen von „Gästen“ vorhanden. Allerdings stellt sich dabei die Frage, wer diese Gäste sind - Einheimische oder Touristen, Staatsbürger oder Ausländer? Ebenfalls ist fraglich, warum derzeit eine größere Nachfrage nach Darbietungen im Volkstanz als nach Aufführungen des Sbek Thom besteht. Nimmt man die Weltkulturerbestätten der UNESCO als Beispiel, so hat sich gezeigt, dass aufgrund der durch die UNESCO unterstützten Öffentlichkeitsarbeit ein besonderes touristisches Interesse an dem Besuch dieser Stätten besteht. Es wäre zu erwarten, dass sich diese Entwicklungen auch im Bereich des immateriellen Kulturerbes zeigen. Dieser Effekt ist zwar bei dem im Jahr 2003 zertifizierten Königlichen Ballett zu beobachten, bei dem ein erhebliches Maß an Bewusstseinsbildung durch die Prinzessin und ehemalige Kulturministerin Norodom Bopha Devi (1999_ 2004) betrieben wurde (Heywood 2008), nicht jedoch bei dem Schattentheater Sbek Thom. Ob der Grund darin liegt, dass die Zertifizierung des Sbek Thom noch nicht lange genug zurück liegt, dass im Fall von Sbek Thom die treibenden Kräfte fehlen oder ob andere Aspekte eine Rolle spielen, kann zu diesem Zeitpunkt noch nicht eindeutig beurteilt werden.

Ausschlaggebend ist in jedem Fall, wer darüber entscheidet, was den Gästen präsentiert wird und ob sie überhaupt von der Zertifizierung des Sbek Thom zum immateriellen Kulturerbe wissen. Möglicherweise kann fehlende Bewusstseinsbildung als Grund für die ausbleibende Nachfrage des Sbek Thom identifiziert wer-

des Reamker dauert sieben Nächte in Folge (Brunet 1969). Das Erlernen der Handlungsabläufe und der äußerst komplexen Manipulation der Figuren erfordert einen langwierigen Trainingsprozess, der meist bereits in der Kindheit beginnt (ebd.). Das Sbek Thom wird durch ein musikalisches Ensemble, dem Phleng Pin Peat, begleitet, welches in seiner großen Besetzung aus neun und in seiner kleinen Besetzung aus fünf verschiedenen Instrumenten besteht (Pech 1995). 
den. Hierbei stellt sich die Frage, warum das MoCFA Sbek Thom, als kürzlich zertifiziertes immaterielles Kulturerbe, nicht zum elementaren Bestandteil seines kulturellen Programms macht. Die soeben beschriebenen Bedingungen lassen nicht darauf schließen, dass auf der Seite der kambodschanischen Regierung die in der UNESCO 2003 Konvention (UNESCO 2003) geforderten Bemühungen bestehen, die zertifizierten immateriellen Kulturformen aktiv in der Bevölkerung zu verbreiten und zu fördern. Das verleitet zu zwei Annahmen: das Khmer Schattentheater ist äußerst lebendig, so dass es keiner zusätzlichen Unterstützung bedarf; oder es ist keinesfalls lebendig und die UNESCO Zertifizierung ist als Maßnahme zu verstehen, es vor dem endgültigen Verschwinden zu bewahren oder um andere Interessen zu verfolgen. In Anbetracht der oben beschriebenen Situation des Khmer Schattentheaters erscheinen die beiden letzten Erklärungen eher plausibel. Allerdings sind noch zusätzliche Interviews mit der örtlichen Bevölkerung sowie extensive Beobachtungen der aktuellen Verhältnisse des Schattentheaters notwendig um festzustellen, in welchem Kontext Sbek Thom heute tatsächlich noch aufgeführt wird und ob Sbek Thom auch in der einheimischen Bevölkerung noch ein gewisses $\mathrm{Ma} ß$ an Bedeutung besitzt. Wie die Untersuchungen der Gruppen in Siem Reap und im "Department of Performing Arts" in Phnom Penh gezeigt haben, wird Sbek Thom dort vor allem für Touristen und für das Fernsehen aufgeführt. Das lässt vermuten, dass kommerzielle Interessen mit seiner Darbietung verbunden sind. Um zu überprüfen, welche Interessen das MoCFA außerdem noch mit der UNESCO Zertifizierung verfolgt haben könnte, werde ich im Folgenden ein Blick auf die unabhängigen Sbek Thom Gruppen werfen.

\subsection{Unabhängige Gruppen}

Neben den im UNESCO Nominierungsantrag aufgeführten Sbek. Thom Gruppen wurden von mir während meiner Feldforschung noch zwei weitere aktive Schattentheatergruppen identifiziert: (1) Kok Tlok Association of Artists, Phnom Penh; (2) Sovanna Phum, Cambodia Art Association, Phnom Penh.

Die "Kok Tlok Association of Artists" (Kok Tlok) wurde 2006 von einem französisch-kambodschanischen Linguisten gegründet und verfolgt das Ziel, klassische Theaterformen wie Sbek Thom, Lakbaon Bassac und Yike neu aufleben zu lassen und einer breiten Öffentlichkeit in Kambodscha zugänglich zu machen sowohl in der Stadt als auch auf dem Land. ${ }^{18}$ Gleichzeitig möchte dieser Verein neue Strömungen im Bereich der darstellenden Künste schaffen und diese in das zeitgenössische Leben der Khmer integrieren. Kok Tlok hat mit den Proben des Schattentheaters im Frühjahr 2009 begonnen und seit August 2009 regelmäßig öffentliche (im Chenla Theatre und im MoCFA) und private Veranstaltungen organisiert. Die Gruppe plant derzeit die Beteiligung am „Festival Mondial des

\footnotetext{
${ }^{18}$ Vgl. http:/ /www.kokthloktheatre.org (Zugriff am 15.04.2010).
} 
Théâtres de Marionnettes“19 im September 2011 in Frankreich, um Sbek Thom auch weltweit bekannt zu machen (Interview mit Thach, 25.01.2010). Zum Zeitpunkt der Forschung stand die Gruppe mit dem MoCFA im Hinblick auf eine mögliche Zusammenarbeit in Verhandlung. Für die Umsetzung seiner Projekte ist Kok Tlok allerdings vollständig auf die Kooperation mit amerikanischen NGOs wie der "Friends of Khmer Culture" und "AMRITA Performing Arts" angewiesen und wird von dem Verein „Association des Amis de Kok Tlok“ in Frankreich unterstützt (ebd., Kok Tlok 2009).

Sovanna Phum wurde 1994 von einer französischen Künstlerin als unabhängiger Verein der Khmer Kunst gegründet ${ }^{20}$ (Sovanna Phum 2010). Ziel dieses Vereins ist die Neubelebung, Bewahrung und Förderung der Khmer Kultur für ein lokales und internationales Publikum. Umgesetzt wird dieses Ziel mit Hilfe eines Zentrums in Phnom Penh, das dem Training, dem Austausch und der Entwicklung der darstellenden Khmer Künste dient. In seinen regulären Darbietungen, die jeden Samstag und Sonntag auf der eigenen Bühne stattfinden, verbindet Sovanna Phum Elemente des Sbek Thom, des klassischen Tanzes, des klassischen Theaters und des Zirkus mit modernen Kunst- und Kulturformen, um die Öffentlichkeit für aktuelle soziale Probleme zu sensibilisieren. In Zusammenhang mit Sbek Thom verfolgt der Verein einen eher beweglichen Ansatz:

Shadow puppetry [...] is powerful and mysterious in its immateriality, capturing the imagination of people for thousands of years. Even within the structured storylines every performance leaves room for improvisation. This allows the art form to remain a relevant, living part of the culture of the time, able to respond to the contemporary needs of the population (Sovanna Phum 2010).

Sovanna Phum bezieht seine finanziellen Mittel aus dem Kartenverkauf seiner regulären Vorstellungen, aus dem Verkauf kultureller Produkte wie z.B. Musikinstrumente, Masken und Schattentheaterfiguren sowie aus der Unterstützung durch internationale Geber.

Auffällig ist, dass beide unabhängigen Gruppen eine Praxis in Bezug auf Sbek Thom vertreten, die einem Wandel gegenüber eher aufgeschlossen ist und mehr mit dem zeitgenössischen Leben der Khmer verbunden ist. Das widerspricht der zuvor gemachten Annahme, dass Sbek Thom ein in sich starres und weitgehend unveränderliches Kulturelement ist ${ }^{21}$. Außerdem ist $\mathrm{zu}$ beachten, dass Sovanna

\footnotetext{
${ }^{19}$ Nähere Informationen zum Festival finden sich auf der folgenden Webseite: http://www.festivalmarionnette.com/

${ }^{20}$ Vgl. http://shadow-puppets.org/ (Zugriff am 15.04.2010).

${ }^{21} \mathrm{Um}$ ein noch tiefgehenderes Verständnis von den Veränderungen des Sbek Thom nach seiner UNESCO Zertifizierung zu ermöglichen, muss eine Studie zu dem Khmer Konzept von Wandel durchgeführt werden. Das würde helfen zu verstehen, was Sbek Thom Künstler selbst unter Wandel bzw. unter Veränderungen begreifen und damit zum Verständnis ihrer Aussagen erheblich beitragen.
} 
Phum bereits vor der UNESCO Nominierung des Khmer Schattentheaters gegründet wurde, im Nominierungsantrag aber nicht als "recognized practitioner" erwähnt wurde. Was ist der Grund dafür? Erfüllte Sovanna Phum nicht die erforderlichen Kriterien des MoCFA für einen Status als anerkannte Gruppe oder war dieser Verein nicht an einer Zusammenarbeit mit dem MoCFA interessiert? Dafür wäre es aufschlussreich, die Bedingungen zu kennen, die mit einem Status als "recognized practitioner" verbunden sind. Müssen sich diese Gruppen im MoCFA oder in einer anderen Institution der Regierung registrieren? Und ist diese Registrierung mit gewissen finanziellen oder praktischen Vorteilen für die Schattentheatergruppen verknüpft oder sind diese Gruppen ganz im Gegenteil besonderen Einschränkungen unterworfen?

Eine dieser Einschränkungen könnte sich beispielsweise auf die künstlerische Freiheit in der Ausübung des Sbek Thom beziehen. Ich möchte an dieser Stelle hervorheben, dass für das Königliche Ballett, das im Jahre 2003 von der UNESCO zum „Meisterwerk des mündlichen und immateriellen Kulturerbes der Menschheit" erklärt worden ist (UNESCO 2008), mittlerweile ein Kontrollsystem eingerichtet wurde, welches die künstlerische Leistung der Tanzgruppen in Siem Reap beurteilt und eine Genehmigung für Auftritte in Restaurants und Hotels einfordert (Interview mit Oun und Sun, 21.04.2009; Interview mit Tim 14.04.2009)22. Im Fall der "Khmer Arts Academy", einer Schule für klassischen Khmer Tanz in Phnom Penh, hinderte das MoCFA eine Tanzgruppe im Jahre 2007 an der Ausreise nach Österreich, weil sie dort eine Khmer Version des Stückes „Romeo und Julia“ aufführen wollte. Ein hoher Beamter des MoCFA lieferte dazu folgende Erklärung:

Those people [of the Khmer Arts Academy] don't understand the tradition of Cambodian dance, and they are breaking this UNESCO spirit, because UNESCO, this is already proclaimed as world heritage, you cannot change the performance of world heritage (Interview mit UNESCO Vertreter, 14.08.2009).

Die Aussage des Regierungsbeamten macht deutlich, dass dieser Akteur die Zertifizierung als immaterielles Kulturerbe als einen Prozess begreift, der eine bestimmte kulturelle Praxis definiert, festschreibt und sie in dieser Form für verbindlich erklärt. Der Status des immateriellen Kulturerbes wird damit als Instrument genutzt, um bestimmte Gruppen hinsichtlich ihrer künstlerischen Freiheit zu kontrollieren. Auf diese Weise wird der natürliche Vorgang der Erneuerung und Ge-

Eine solche Studie würde allerdings den Rahmen dieses Beitrags sprengen und muss an anderer Stelle durchgeführt werden.

22 Weitere Untersuchungen sind erforderlich um herauszufinden, wie dieses Kontrollsystem in der Praxis aussieht. Es ist insbesondere von Interesse, ob tatsächlich eine technische Überprüfung der Tanzaufführungen stattfindet - und wenn ja durch wen - oder ob dieses System praktisch darauf begrenzt ist, eine Gebühr für die Auftrittsgenehmigung in Hotels und Restaurants zu verlangen. In diesem Fall wäre das Kontrollsystem nicht mehr als eine zusätzliche Einnahmequelle der Regierung und würde nicht primär dazu dienen, die Qualität der Darbietung zu gewährleisten. 
staltung des immateriellen Kulturelements unterbunden und die Dynamik seiner weiteren Entwicklung durch die kulturelle Politik eines Regierungsakteurs gesteuert.

Es wird entscheidend sein zu verfolgen, ob ein solches Kontrollsystem auch für Sbek Thom eingerichtet wird. Der Einfluss der Regierung könnte sich als Grund für die Rigidität des Sbek Thom bei den anerkannten Gruppen in Siem Reap erweisen, die hauptsächlich für Touristen aufführen und dabei eine künstlich konservierte kulturelle Praxis zur Schau stellen. Interessant ist in dieser Hinsicht, wie sich die Zusammenarbeit zwischen Kok Tlok und dem MoCFA weiterhin gestalten wird. Wird diese Gruppe den Status einer offiziell anerkannten Gruppe erhalten? Und was bedeutet eine Zusammenarbeit mit dem MoCFA für Kok Tlok? Im Hinblick auf das "Festival Mondial des Théâtres de Marionnettes" 2011 in Frankreich ist fraglich, ob die Beteiligung der Gruppe von der Regierung zugelassen wird oder ob andere Sbek Thom Gruppen das vorrangige Recht auf eine Teilnahme beanspruchen werden.

Nach Vertretern der UNESCO und des MoCFA in Phnom Penh (Interview mit Hong 13.07.2009, Interview mit Mao 08.08.2009) ist außerdem ein Projekt zur Förderung von Sbek Thom geplant, das durch den "Japan Funds in Trust" finanziert werden soll. Hierbei stellt sich die Frage, welche der Schattentheatergruppen zur Teilnahme an diesem Projekt zugelassen sein werden und unter welchen Kriterien. Welche Ziele sind mit diesem Projekt verbunden und welche Konsequenzen ergeben sich daraus letztendlich für Sbek Thom? Welches sind die Interessen aller beteiligten Akteure? Die Antwort auf diese Fragen wird entscheidend sein für die weitere Entwicklung des Khmer Schattentheaters - Erstarrung oder Flexibilität sowie auch für die Dynamik zwischen den aktiven Schattentheatergruppen. Im folgenden Abschnitt werde ich einen Blick auf die strukturellen Veränderungen werfen, die in den Schattentheatergruppen seit der UNESCO Zertifizierung bereits stattgefunden haben.

\section{Struktur und Organisation der Schattentheatergruppen}

\subsection{Die Gruppe Sala Kanseing}

Hinsichtlich der Gruppe Sala Kanseing in Siem Reap haben seit der UNESCO Zertifizierung von Sbek Thom strukturelle Veränderungen stattgefunden. Nach dem Tod von Meister Ti Chean im Jahr 2000 wurde die Leitung dieser Gruppe nach den Aussagen seines Enkels von diesem übernommen. Die Koordination erfolgte durch Ti Cheans ehemalige japanische Schülerin Fukutomi (Interview mit Fukutomi, 15.04.2009, Interview mit Ti Cheans Enkel, 17.04.2009). ${ }^{23}$ In einem

\footnotetext{
23 Diese Konstellation wird auch im "Cambodia Arts Directory" beschrieben, einer Zusammenstellung von Institutionen, Organisationen, Vereinigungen und Akteuren, die sich in Kambodscha im Kulturbereich engagieren (Visiting Arts 2001).
} 
Interview mit einem weiteren Mitglied der Gruppe, das im UNESCO Nominierungsantrag zu Sbek Thom als Leiter der Gruppe angegeben ist (MoCFA 2004b), wies dieser jedoch darauf hin, dass er die Gruppe als Leiter und Lehrer übernahm und "only the name of Ti Chean, [...] the sign of Ti Chean" beibehielt (Interview mit Gruppenmitglied Sala Kanseing, 21.04.2009). Er erklärte außerdem, dass er die Gruppe Sala Kanseing im Jahre 2005 aufgrund von Korruption gemeinsam mit dem Erzähler verlassen hatte (ebd.). Offensichtlich kam ihm aus seiner Sicht nicht die erforderliche Macht, Anerkennung und Entlohnung für seine Dienste als Leiter, Lehrer und Darsteller zu. Er gründete infolgedessen 2007 seine eigene Gruppe in Kok Krasang ${ }^{24}$. Ti Cheans Enkel betonte, dass der Gründer der Schattentheatergruppe in Kok Krasang weiterhin den Namen von Ti Chean verwendete, obwohl er nicht die gleichen Rechte wie Ti Cheans Enkel besaß, der - im Gegensatz zum Gründer der Gruppe in Kok Krasang - ein direkter Nachfahre von Ti Chean ist:

Yes, he [the creator of the Kok Krasang group] uses the name of Ti Chean [...]. There is only one teacher of Sbek Thom: Ti Chean, but [the creator of the Kok Krasang group] wants to use Ti Chean's name to make himself popular. But he is not the son of Ti Chean, so he doesn't have the same rights like his son. [The Kok Krasang group] doesn't have all the material for performance, but some people invite it to play. But most people want to find out about the Ti Chean group, not about [the Kok Krasang group]. The population and the tourists who want to see Sbek Thom, they come to Ti Chean's group not to [the Kok Krasang group] (Interview mit Ti Cheans Enkel, 17.04.2009).

Scheinbar haben sich innerhalb der Gruppe Sala Kanseing seit der UNESCO Zertifizierung von Sbek Thom Unstimmigkeiten, Machtkämpfe und Rechtsansprüche unter den Gruppenmitgliedern entwickelt. Diese Ansprüche beziehen sich nicht nur auf die Teilnahme in und auf die Kontrolle über die Gruppe, sondern auch auf den Namen von Meister Ti Chean einschließlich der Kompetenzen, die mit diesem Namen assoziiert werden. Die Vergütung der innerhalb der Gruppe geleisteten Dienste spielte eine zusätzliche Rolle. Diese Annahme wird durch die Tatsache verstärkt, dass sich die Gruppe auf Einladung des "Foundation Modern Puppet Centre" zu einer Tournee in Japan wieder vereinigte, um die eingeforderte Qualität der Darbietung zu gewährleisten, die nur in der ursprünglichen Besetzung möglich war (Interview mit Miura, 03.06.2009). Eine Analyse des Wettbewerbs zwischen unterschiedlichen Sbek Thom Gruppen in Siem Reap wäre unvollständig, würde man nicht auch die Entwicklungen in der Gruppe Wat Reach Bo betrachten.

\footnotetext{
${ }^{24}$ Zum Zeitpunkt meines Besuches in Siem Reap im April 2009 hatte die Gruppe in Kok Krasang ihre Sbek Thom Proben aus Mangel an Nachfrage eingestellt und wurde hier aus diesem Grund nicht als aktive Gruppe erwähnt (Interview mit Gruppenmitglied Sala Kanseing, 21.04.2009).
} 


\subsection{Die Gruppe Wat Reach Bo}

Die Gruppe Wat Reach Bo wurde laut UNESCO Nominierungsantrag im Jahre 1995 durch den Abt Pin Sem gegründet und wird seitdem finanziell von Kambodschanern "now living overseas" (MoCFA 2004a) unterstützt25. Die Landesbeauftragte von "Cambodian Living Arts" wies darauf hin, dass diese Gruppe seit Jahren die gleichen Stücke aufführt und keine Möglichkeit hat sich weiter zu entwickeln, da ihr ein Sbek Thom Meister fehlt (Interview mit Thum, 01.07.2009). Die Beraterin der Gruppe Sala Kanseing erwähnte zudem in einem Interview (17.04.2009), dass Pin Sem seine Schattentheatergruppe auf der Basis von Aufnahmen gegründet hatte, die von einem seiner Gesandten in der Gruppe Sala Kanseing gemacht worden sind. Für die Preisgabe ihres Wissens über Sbek Thom, hatte die Gruppe Sala Kanseing einen finanziellen Ausgleich erhalten. Die Beraterin betonte allerdings auch, dass das Kopieren der Sbek Thom Darbietung unweigerlich Veränderungen sowohl in ihrem Inhalt, als auch in ihrer Form mit sich bringen würde, die letztendlich zu Qualitätsverlust führen könnten. Hinzu kommt ihrer Meinung nach, dass die kambodschanische Regierung nicht genügend Kontrolle ausübt, um das Kopieren und Modifizieren von Wissen in Bezug auf immaterielles Kulturerbe zu verhindern:

I am very sorry about the guy who copied from here to create another group. Because they don't copy all, if you study $100 \%$ then you only copy $60 \%$ or $70 \%$. [...] For me, it is very difficult to control everything, as I am very old. And it is very hard for the law in Cambodia to control everything and to prevent copying. If someone wants to copy, it is very easy for him (Interview mit Beraterin der Gruppe Sala Kanseing, 17.04.0209).

Diese Aussagen machen erneut die Besorgnis der Gruppe Sala Kanseing über ihre Rechte in Bezug auf das Sbek Thom Wissen deutlich, das auf ihren alten Meister Ti Chean zurückgeht. Ti Chean ist zu einem Label für Sbek Thom geworden, um das sich verschiedene Gruppen mit divergierenden Interessen streiten. Auf der einen Seite steht die Sorge um die Reinheit und um die Qualität der auf dem Wissen von Ti Chean beruhenden Sbek Thom Aufführung in der Gruppe Sala Kanseing, die sich als Hüter von Ti Cheans Wissen versteht. Auf der anderen Seite mag für die neu gegründete Gruppe in Kok Krasang der Nutzen dieses Labels zu Marketingzwecken und zur Erhöhung des Prestiges eine wichtige Rolle spielen. In welcher Beziehung die Gruppe Wat Reach Bo zu diesem Label steht, bleibt in Zukunft noch zu überprüfen.

\footnotetext{
${ }^{25} \mathrm{Da}$ sich Pin Sem während meines gesamten Feldforschungsaufenthaltes in den USA aufhielt, war es nicht möglich, ein persönliches Gespräch mit ihm zu führen. Die Gruppe wird nun durch seinen ehemaligen Schüler, Vann Sopheavuth, geleitet. Finanzielle Unterstützung erhält diese Gruppe außerdem von der amerikanisch-kambodschanischen NGO "Cambodian Living Arts" (CLA), die auch touristische Darbietungen für die Gruppe organisiert (Interview mit Om, 09.04.2009).
} 


\subsection{Ein internationales Netzwerk um Sbek Thom}

In der Beschreibung der verschiedenen Schattentheatergruppen fällt auf, dass auch diverse ausländische Institutionen und Akteure an der Konstituierung von Sbek Thom als immaterielles Kulturerbe in Kambodscha beteiligt sind: die Gruppe Sala Kanseing wurde durch eine Japanerin koordiniert, die Gruppe Wat Reach Bo wird von Gebern in den USA unterstützt, Kok Tlok wird von einem französischen Verein gesponsert, Sovanna Phum wird von einer französischen Künstlerin geleitet und das geplante Projekt zur Förderung von Sbek Thom soll durch den "Japan Funds in Trust" finanziert werden. Ein komplexes internationales Netzwerk an Institutionen und Akteuren hat sich um Sbek Thom entwickelt, wobei alle ihre eigenen und teilweise widersprüchlichen Interessen und Motive mit der Beteiligung an Sbek Thom verfolgen. Die Tatsache, dass Initiativen und finanzielle Ressourcen zur Förderung des Khmer Schattentheaters hauptsächlich aus dem Ausland stammen, lässt vermuten, dass die Strukturen und Programme der Regierung nicht ausreichen, um die verschiedenen Sbek Thom Gruppen zu unterhalten. Eines der Absichten innerhalb des Forschungsprojektes ist es, ein detailliertes Bild dieser transnationalen Querverbindungen einschließlich ihrer Verknüpfung mit Regierungsstrukturen und der Zertifizierung von Sbek Thom als immaterielles Kulturerbe zu zeichnen. Ziel ist es letztendlich, die Auswirkungen dieser Strukturen auf die weitere Entwicklung des Khmer Schattentheaters zu untersuchen.

\section{$6 \quad$ Fazit}

In diesem Beitrag habe ich die Dynamik des Khmer Schattentheaters sowie der Gruppen behandelt, die dieses kulturelle Phänomen nach seiner UNESCO Zertifizierung als immaterielles Kulturerbe nutzen und gestalten. Im Hinblick auf die Auswirkungen der UNESCO Zertifizierung auf Sbek Thom wurden vier Entwicklungen diskutiert:

Offiziell anerkannte und unterstützte Gruppen wie die Gruppen Sala Kanseing und Wat Reach Bo in Siem Reap verfolgen einen eher traditionellen und rigiden Sbek Thom Ansatz. Sie führen ihre Kunst hauptsächlich für Touristen auf. Aufgrund der individuellen Auslegung von UNESCO Regeln und Vorschriften durch einen Regierungsakteur in einer einflussreichen Position, wird Sbek Thom mit der Ernennung zum immateriellen Kulturerbe als kulturelles Konstrukt definiert, festgeschrieben und in dieser Form für verbindlich erklärt. Die UNESCO Zertifizierung wird durch diesen Regierungsakteur als Instrument genutzt, um Kontrolle auf die Sbek Thom Gruppen hinsichtlich ihrer künstlerischen Freiheit auszuüben. Die natürliche Entwicklung des Sbek Thom durch Neuerung und Kreativität auf Seiten der Künstler wird unterdrückt und das lebendige Kulturerbe verkümmert zu einem starren Museumsstück, das zu kommerziellen Zwecken genutzt wird. Ferner dient es der Regierung dazu, einen internationalen Ruf auf der Basis der UNESCO Zertifizierung und einer konstruierten Khmer Identiät zu schaffen. Die traditionellen 
Sbek Thom Gruppen ringen um ihr Überleben, da ihre Existenz von touristischen Anfragen abhängt, während das Interesse der lokalen Bevölkerung an ihren traditionellen Sbek Thom Aufführungen begrenzt ist.

Die Initiative unabhängiger Sbek Thom Gruppen wie der Kok Tlok Association of Artists und Sovanna Phum, die nach Freiraum in ihrem künstlerischen Ausdruck streben und einen eher zeitgenössischen Sbek Thom Ansatz verfolgen, zeigt, dass die weitere Entwicklung von Sbek Thom dennoch möglich ist, aber von der Sichtweise der entsprechenden Akteursgruppen auf das kulturelle Phänomen abhängt. Ihr Status als unabhängige Gruppen lässt darauf schließen, dass Gruppen mit einem flexiblen und eher innovativen Sbek Thom Ansatz von aktuellen Akteuren der Regierung nicht unterstützt werden, da sie ihrem Konzept von Sbek Thom als festgeschriebenes und verbindliches kulturelles Artefakt nicht entsprechen. Da diese Gruppen nicht durch die Regierung gefördert werden, suchen sie finanzielle Unterstützung bei nationalen und internationalen Gebern.

Durch die Auswahl von "recognized practitioners of the tradition" im UNESCO Nominierungsantrag wurden einige Sbek Thom Gruppen bevorzugt, während andere unberücksichtigt blieben. Dadurch wurde ein Wettbewerbsklima geschaffen, dass Spannungen zwischen den bestehenden Sbek Thom Gruppen begünstigt. Diese Spannungen haben sich bereits bei den Sbek Thom Gruppen in Siem Reap manifestiert. Es ist anzunehmen, dass die Festschreibung einer Gruppenhierarchie für die Gruppe Sala Kanseing innerhalb des UNESCO Nominierungsantrags durch die Zuordnung von ausgewählten Personen zu bestimmten Positionen, zu den Spannungen und Machtkämpfen in der Gruppe Sala Kanseing beigetragen hat. Die UNESCO Zertifizierung ist zu einem Auslöser für Rechtsansprüche auf das Label Ti Chean geworden, welches das Wissen und die Expertise des im Nominierungsantrag erwähnten Meisters Ti Chean repräsentiert. Verschiedene Parteien innerhalb der Gruppe Sala Kanseing wollen dieses Label einerseits zu Marketingzwecken und andererseits zur Gewährleistung der Originalität der Sbek Thom Aufführung sowie seiner Abstammung von Meister Ti Chean nutzen.

Da von der kambodschanischen Regierung offenbar nur sehr begrenzte Ressourcen für die Förderung von Sbek Thom zur Verfügung gestellt werden und da die unabhängigen Gruppen in ihrer Existenz auf externe Geber angewiesen sind, hat sich der Wettbewerb um Sbek Thom auf Institutionen und Akteure in der internationalen Gemeinschaft ausgeweitet, welche die Förderung von Sbek Thom finanziell unterstützen. Diese Umstände lassen darauf schließen, dass ein von der UNESCO als immaterielles Kulturerbe zertifiziertes Element wie Sbek. Thom von Akteuren und Institutionen auf der lokalen, nationalen und internationalen Ebene als eine attraktive Ressource für die Umsetzung ihrer Ziele und Interessen angesehen wird.

Vier Jahre nach der Zertifizierung von Sbek Thom können die in diesem Beitrag beschriebenen Entwicklungen vorerst als Zwischenbilanz betrachtet werden. Sie legen nahe, dass die UNESCO Zertifizierung zwar ein Verschwinden des Sbek Thom verhindert hat, sie betonen jedoch auch die Tendenz zur kommerziellen 
Nutzung dieses kulturellen Phänomens, das höhere Maß an Kontrolle seiner Praxis durch Regierungsakteure und insbesondere die Steigerung des Wettbewerbsklimas in Zusammenhang mit seinem Gebrauch. Um eine langfristige Stellungnahme zu dem Einfluss der UNESCO Zertifizierung als immaterielles Kulturerbe auf das kulturelle Phänomen und auf seine Nutzung durch gewisse lokale, nationale und internationale Akteure abgeben zu können, muss die Entwicklung des Sbek Thom über einen längeren Zeitraum hinweg beobachtet werden. Allerdings kann angenommen werden, dass die in diesem Beitrag beschriebenen Entwicklungen in den kommenden Jahren ungehindert ihren Lauf nehmen werden, da die Politik der derzeitigen Regierungsakteure nicht alle existierenden Sbek Thom Gruppen berücksichtigt und letztendlich alle um ihre Existenz kämpfen müssen. Das geplante japanische Förderprojekt zu Sbek Thom sowie die Auswahl an Institutionen und Akteuren, die daran beteiligt sein werden, könnten mehr Aufschluss über die weitere Entwicklung dieses kulturellen Artefaktes geben. 




\title{
Cultural Property und das Heritage-Regime der UNESCO: Parallelen und Interaktionen bei ideellen und wirtschaftlichen Inwertsetzungsprozessen von kulturellen Elementen
}

\author{
Arnika Peselmann und Philipp Socha
}

\section{Einleitung}

Die Inwertsetzung und Nutzbarmachung von kollektiv generierten Wissensbeständen, die sich in Erzeugnissen oder Praxen realisieren, wird in internationalen politischen Foren, aber auch in wissenschaftlichen Kontexten über alle disziplinären Grenzen hinweg diskutiert und begrifflich mit dem Terminus Cultural Property gefasst. Cultural Property wird jedoch kontextabhängig unterschiedliche Bedeutung zugeschrieben: So im juristischen Verständnis als Cultural Property Rights sui generis und in Diskussionen der Critical Legal Studies um die kulturelle Dimension von verschiedenen Rechtsinstrumenten. Aber auch als eine diskursive Strategie und kulturelle Praxis, bei der Akteure Zugehörigkeit und Eigentum an materiellen oder immateriellen kulturellen Elementen ausdrücken. Die Konstituierung eines Cultural Property kann umschrieben werden als Prozess, bei dem ein kulturelles Element auf der Basis komplexer Selektionsprozesse aus seinem Kontext gelöst wird und eine Wertzuschreibung erfährt, die ökonomisch und/oder ideell sein kann, sich aus dem Wertesystem des betreffenden Kollektivs ableitet und/oder von außen projiziert wird. Der Prozess, innerhalb dessen sich ein Cultural Property formiert, kann von unterschiedlichsten Interessenlagen und Beeinflussung diver- 
genter und teilweise auch konkurrierender Akteursgruppen, Organisationen und Institutionen geprägt sein.

Die hier nur abstrakt umschriebene Konstituierung von Cultural Property kann auch beim Zertifizierungsprozess von UNESCO-Weltkulturerbe-Stätten beobachtet werden: Die Ernennung lokaler kultureller Elemente als Erbe der Menschheit ist Resultat lokaler, nationaler und internationaler Selektierungs- und Stilisierungsmuster von Kultur und eröffnet Möglichkeiten sowohl der ideellen als auch der ökonomischen Nutzbarmachung, die die Formierung eines Cultural Property (noch weiter) befördern können. Die Wechselwirkung dieser Prozesse im Kontext von Weltkulturerbe (WKE) wird als Teil der wissenschaftlichen Auseinandersetzung um Cultural Property diskutiert. Der hier gewählte interdisziplinäre Ansatz setzt einen auf den Welterbekontext fokussierten Überblick der gegenwärtig geführten Debatte um Cultural Property in Bezug zu den Narrativen und Argumentationen, die im Kontext zweier Feldstudien sichtbar werden. Es handelt sich dabei zum einen um den verlorenen Welterbestatus des Dresdner Elbtals und zum anderen um die Nominierungsvorbereitung einer deutsch-tschechischen UNESCO-Industriekulturlandschaft Montanregion Erzgebirge. In den zwei konkreten Settings wird die Relevanz der Inwertsetzung und ökonomischen Nutzbarmachung von Kultur durch, bzw. unter ausdrücklichem Verzicht auf, den Status Weltkulturerbe deutlich und ist dabei stets eng verwoben mit der Frage nach der ideellen Be- und Aufwertung der eigenen Kulturlandschaft. Die Dichotomisierung von ideellen und konjunkturellen Werten von Kultur findet sich dabei nicht nur in wissenschaftlichen Diskursen, sondern auch als Widerhall in der Praxis.

\section{Zur Herangehensweise}

Die Formierung sowohl eines Cultural Property, als auch die eines UNESCO Weltkulturerbes, verläuft in komplexen Interdependenzen, in denen auf normative Vorgaben ebenso rekurriert wird wie auf lokale Praxen. Dieser Aufsatz beleuchtet die beiden Phänomene aus einer juristischen und kulturanthropologischen Perspektive mit den jeweils fachspezifischen Erkenntnisinteressen und entsprechenden methodischen Instrumentarien.

Die Kulturanthropologie, die als hermeneutisch arbeitende Wissenschaft soziale und kulturelle Praxen in ihrem Kontext deskriptiv-analytisch untersucht, versteht die Heritagifizierung (Hemme, Tauschek und Bendix 2007a) kultureller Elemente als postmoderne kulturelle Praxis, die im Fall der geplanten binationalen UNESCO-Kulturlandschaft Montanregion Erzgebirge mittels qualitativ induktiver Methoden beforscht wird. Der Schwerpunkt liegt dabei auf der Akteursebene: Die mögliche UNESCO-Zertifizierung wird im Erzgebirge mit konkreten ökonomischen Erwartungen verknüpft, die insbesondere von der sächsischen Landesregierung, aber auch von lokalen Akteuren fokussiert werden. In unserem interdisziplinären Aufsatz liegt der Blickpunkt auf der Verflechtung wirtschaftlicher und 
ideeller Interessen lokaler und regionaler Akteure, die sich für oder gegen eine Nominierung als Welterbe aussprechen. Eingebettet aber nur marginal behandelt wird die Untersuchung in historische Inwertsetzungsprozesse, wie sie sich an der Entwicklung der erzgebirgischen Volkskunst nachvollziehen lassen, die bis heute die regionale Wirtschaft und Identitätskonstruktionen prägt. Darüber hinaus interessiert aber auch die Frage, wer die offizielle Deutungsmacht über die zu ernennenden Weltkulturerbestätten hat und wem sie in der von Vertreibung und (Zwangs-)Ansiedlung geprägten Region welches Identifikationspotential bieten.

Das Vergleichsbeispiel generiert sich aus Teilen der Debatte um den Bau der Dresdner Waldschlößchenbrücke und die Aberkennung des UNESCO-Status für das Dresdner Elbtal. Der Konflikt beeinflusst einerseits die Diskussionen um eine mögliche Erzgebirgsnominierung, andererseits zeigt eine eigenständige Betrachtung des Dresdner Falls das Spannungsverhältnis von ökonomischen Argumenten und Fragen von kollektiver Identität im Zusammenhang mit Weltkulturerbe. Diese Perspektive soll dem Blick auf das Erzgebirge vorangestellt werden, da die Kontroversen und die letztendliche Aberkennung des Dresdner Welterbetitels wesentlichen Einfluss auf die Diskussionen rund um die geplante UNESCO Kulturlandschaft Montanregion Erzgebirge haben.

Bei der Untersuchung der rechtlichen Regelungen im Kontext von Weltkulturerbestätten weisen die einschlägigen normativen Quellen der völkerrechtlichen und nationalstaatlichen Ebene gemeinsame Charaktere auf. Während die Regelungen der UNESCO im Sinne der Präambel der Konvention von 1972 den Schutz- und Konservierungsgedanken in den Vordergrund stellen und auch das Begutachtungsund Ernennungsverfahren entsprechend ausgestaltet haben, obliegt es jedem Staat selbst, potentielle Welterbestätten auf die Tentative List zu setzen und entsprechende interne Regelungen zu bilden. Die für die genannten Beispiele einschlägige deutsche Rechtslage weist dabei ebenso einen starken Bezug zum Denkmalschutzrecht auf. Vorschläge zur Tentative List sind vollkommen im Bereich der Exekutive gehalten. Dabei obliegt den zuständigen Landesministerien ein weiter Ermessensspielraum, der kaum durch rechtliche Regelungen begrenzt wird. Dies ist problematisch, weil der Status Weltkulturerbe weitreichende Folgen hat. Insbesondere die ökonomischen Auswirkungen, spiegeln sich nicht im bestehenden juristischen Rahmen wieder. Vielmehr obliegt es dem weiten Ermessen der Exekutive, wie mit Weltkulturerbe verfahren wird. Aus juristischer Sicht soll daher mit diesem Beitrag der Mangel an notwendigen Regelungen sowie die Diskrepanz zwischen den bestehenden Regelungen und den eigentlichen Beweggründen der Entscheidungsorgane aufgezeigt werden.

Die Verbindung kulturanthropologischer mit rechtswissenschaftlicher Erkenntnisinteressen schlägt eine Brücke zwischen, einerseits dem sinnverstehenden Ansatz, der empirisch nachvollziehbar die Vielstimmigkeit eines Feldes verdeutlicht und die de facto Interpretation und Anwendung gesetzlicher Rahmenbedingungen zeigt, und andererseits dem normativen Verständnis der Rechtswissenschaft, 
Arnika Peselmann und Philipp Socha

welches verschiedene Werte, private Interessen und öffentliche Belange versucht abstrakt in Ausgleich zu bringen.

Im Kontext von Welterbezertifizierungen nehmen Akteure Bezug auf bestehende nationale und völkerrechtliche Regelungen. Diese Referenzen machen auch für eine kulturanthropologische Forschung ein erweitertes Verständnis von entsprechenden Rechtsregimen nötig. Die Rechtswissenschaft hingegen bedarf der kritischen Reflektion der abstrakten Regelungen vor dem Hintergrund empirischer Untersuchungen, um Kon- und Divergenzen aufzuzeigen.

\section{Cultural Property (Rights), Weltkulturerbe, Identität}

\subsection{Cultural Property - Ein Überblick}

Die gegenwärtige Debatte um Cultural Property bezieht ihren Namen zwar von der Haager Konvention zum Schutz von Kulturgut bei bewaffneten Konflikten von 1954, geht aber weit über den dort definierten Begriff von Cultural Property hinaus. Autoren wie Michael Brown und Rosemary Coombe beziehen sich heute vielmehr auf die immaterielle Ebene von Kulturgut und rücken damit die Rechtsinstrumente des geistigen Eigentums in den Mittelpunkt der Debatte. Diese befasst sich in kritischer Auseinandersetzung im sozialen und historischen Kontext mit der kulturellen Dimension von Rechtsinstrumenten und bezieht sich dabei auf Diskussionen um Subjektivität, Identität und Gesellschaft, sowie der Kommerzialisierung von Kultur (Coombe 1998). Insbesondere die Ökonomisierung von Kultur und das Zusammenwirken mit bestimmten Rechtsinstrumenten stehen für diesen Beitrag im Fokus.

Für ein differenziertes Verständnis von Cultural Property ist zwischen Cultural Property an sich und konkreten Cultural Property-Rights, also tatsächlichen Rechtsinstrumenten, deren Regelungsbereich Auswirkungen auf Cultural Property hat, zu unterscheiden. So beschäftigt sich die Diskussion um Cultural Property mit verschiedenen Rechtsinstrumenten, wie dem Eigentum an Mobilien und Immobilien und insbesondere auch Rechten des geistigen Eigentums, sowie Rechtsinstrumenten sui generis speziell in Bezug auf Cultural Property. Schließlich sind auch öffentlich rechtliche Instrumente, wie der Zertifikationsprozess zum Weltkulturerbe, Teil der Diskussion. Historisch wurde der Begriff Cultural Property erstmalig in der Haager Konvention zum Schutz von Cultural Property in bewaffneten Konflikten von 1954 geprägt, in der der völkerrechtliche Schutz von „beweglichem und unbeweglichem Eigentum von großer Bedeutung für das kulturelle Erbe der Menschheit“ (UNESCO 1954:Art. 1a) geregelt wird. Auch das Übereinkommen über Maßnahmen zum Verbot und zur Verhütung der unzulässigen Einfuhr, Ausfuhr und Übereignung von Kulturgut von 1970 bezieht sich in Artikel 1 ausschließlich auf physische Objekte, die ,jedem Staat aus religiösen oder weltlichen Gründen als für Archäologie, Vorgeschichte, Literatur, Kunst oder Wissenschaft besonders wichtig sind.“ Expli- 
zit herausgestellt werden dabei unter anderem insbesondere „Antiquitäten [...und] Gut von künstlerischem Interesse wie Bilder, Gemälde und Zeichnungen [...oder] Originalwerke der Bildhauerkunst.“

Im kulturvölkerrechtlichen Regime der UNESCO zeichnete sich im Lauf der zweiten Hälfte des 20. Jahrhunderts die Entwicklung hin zum Begriff des Cultural Heritage ab, der im Übereinkommen zum Schutz des Kultur- und Naturerbes von 1972 in Artikel 1 und 2 als Denkmäler, Gebäude Ensembles und Stätten sowie Naturgebilde, geologische und physiographische Erscheinungsformen und Naturstätten konkretisiert wird. Mit dem Übereinkommen zur Bewahrung des immateriellen Kulturerbes und dem Übereinkommen über den Schutz und die Förderung der Vielfalt kultureller Ausdrucksformen wurde Anfang dieses Jahrhunderts die immaterielle Ebene normativ anerkannt. Diese beiden Instrumente beziehen sich auf „Praktiken, Darbietungen, Ausdrucksformen, Kenntnisse und Fähigkeiten [...], die Gemeinschaften, Gruppen und gegebenenfalls Individuen als Bestandteil ihres Kulturerbes ansehen“ (Art. 2, UNESCO 2003) sowie auf „Ausdrucksformen, die durch die Kreativität von Einzelpersonen, Gruppen und Gesellschaften entstehen und einen kulturellen Inhalt haben" (Art. 4, Abs. 3, UNESCO 2005a). Dabei verdeutlichen sie die Entwicklung des normativen Kulturbegriffs im Völkerrecht von Cultural Property, zu Heritage und Diversity. Formell stehen diese Dokumente gleichrangig nebeneinander. Aus den verschiedenen Schutzgütern und Regelungsstrukturen sowie entsprechenden Formulierungen in den Präambeln lässt sich Cultural Property im Sinne der Konventionen von 1954 und 1970 als Teil des Kulturellen Erbes der Menschheit (Cultural Property of Mankind) einordnen (Roussin 2003).

Als Teil der von den Critical Legal Studies angestoßenen Auseinandersetzungen untersucht die gegenwärtige wissenschaftliche Debatte um Cultural Property, verschiedene Rechtsinstrumente auf ihre Implikationen auf Kultur und umgekehrt. Gegenstand sind dabei unter anderem die geistigen Eigentumsrechte. Kontextualisiert und analysiert werden zum Beispiel das Rechtsinstrument des Urheberrechts in Bezug auf seinen Schutzbereich, der sich nur auf physisch materialisierte Gegenstände bezieht und bis zu 70 Jahren nach Tod des Autors gewährt wird. Im Kontext eines romantisierten Verständnisses eines Autors, der in seiner Stube sitzt und ein Gedicht verfasst, eignet sich dieses Instrument um eine überzeugende Interessenabwägung vorzunehmen. Dies sind zum einen die Interessen der Gesellschaft auf dieses Werk zugreifen zu können und zum anderen die des Autors, der für eine gewisse Zeit die Möglichkeit erhält, sein Werk exklusiv zu vermarkten, um für die Schaffung entsprechend entlohnt zu werden. In anderen Kontexten, wie beispielsweise den Traumgeschichten der Indigenen Australiens, zeigen sich allerdings Probleme mit der Anwendbarkeit dieses Rechtsinstruments. Diese Geschichten werden nur mündlich von Generation zu Generation überliefert und dabei geringfügig abgeändert. Der Schutz des Urheberrechts greift also nicht, da das Werk weder materialisiert wurde noch von einem bestimmten Autor stammt, der vor weniger als 70 Jahren gestorben ist. Somit sind diese Geschichten schutzlos der Appropri- 
Arnika Peselmann und Philipp Socha

sierung und Nutzung in anderen Kontexten ausgeliefert, die möglicherweise die Integrität dieser kulturellen Ausdrucksform verletzt, obwohl eine kollektive Autorenschaft zu identifizieren ist (Brown 2003). Die vor dem Hintergrund ihrer historischen Entwicklung zu verstehenden Rechte geistigen Eigentums führen in ihrer globalen Anwendung heute zu politischen, ökonomischen und gesellschaftlichen Spannungen.

Um dieser Problematik in nicht-westlichen Gesellschaften mit juristischen Mitteln zu begegnen, wurden bereits seit den 70er Jahren spezielle Rechtsinstrumente für den Schutz von kulturellen Ausdrucksformen und Folklore geschaffen (sog. Rechte sui generis, d.h. Rechte eigener Art). Beispielhaft seien hier die internationalen Instrumente des Tunis Model Law von 1976 und das Bangui Agreement von 1999 genannt. Des Weiteren wurden insbesondere in Südamerika verschiedene Rechtsinstitute auf nationaler Ebene geschaffen. Diese Rechte an kulturellen Ausdrucksformen und Folklore sui generis weisen starke Parallelen zum Urheberrecht auf. Unterschiede finden sich beim Originalitätserfordernis, der Dauer des Rechtsschutzes und der Zuordnung zu bestimmten Rechtsträgern. ${ }^{1}$ In Reaktion zu Problemen mit den bestehenden Rechten geistigen Eigentums in nicht-westlichen Gesellschaften bemüht sich auch das so genannte Intergouvernmental Committee der Weltorganisation für geistiges Eigentum (WIPO) mit starken Initiativen der afrikanischen und äquatornahen Staaten um ein neues Rechtsinstrument, welches den Schutz von traditionellen kulturellen Ausdrucksformen, traditionellem Wissen und Folklore sowie genetischen Ressourcen gewährleisten soll. ${ }^{2}$ Die vom Sekretariat angefertigten Arbeitsdokumente beschreiben den Schutzgegenstand als jegliche Form von traditionellen kulturellen Ausdrucksformen, Ausdruck von Folklore oder jedwede andere materielle oder immaterielle Form, in der traditionelle Kultur und Wissen ausgedrückt wird, und nennt explizit mündliche Ausdrucksformen, wie Geschichten, Epen, Legenden, Gedichte, Wörter, Zeichen und Symbole. Begünstigte sollen indigene Gruppen und traditionelle und andere kulturelle Gemeinschaften sein, die mit ,angemessenen und effektiven rechtlichen und praktische Mechanismen [...] verhindern können“ (WIPO 2006b) dass ihre Werke ohne vorherige Zustimmung unter anderem ,reproduziert veröffentlicht, adaptiert, im Radio und Fernsehen ausgestrahlt oder öffentlich aufgeführt, verbreitet, vermietet oder fixiert (Fotografie)“ (ebd.) werden.

Inwieweit ein solches Rechtsinstrument bei den Staatenvertretern Konsens und durch einen entsprechenden völkerrechtlichen Vertrag rechtliche Geltung gewinnt, ist ungewiss. Die gegenwärtigen Entwürfe verdeutlichen allerdings, wo und in welcher Form von einigen Staaten Handlungsbedarf zum Schutz von kulturellen Ausdrucksformen gesehen wird. Eine der Kernforderungen der Staaten, die bei den Verhandlungen die Initiative ergreifen, liegt insbesondere auf der rechtlichen Bestimmtheit und Durchsetzbarkeit eines solchen neuen Instruments. Hin-

\footnotetext{
${ }^{1}$ Vgl. dazu Zimbehl in diesem Band.

${ }^{2}$ Vgl. die Beiträge von Groth und Lankau in diesem Band.
} 
tergrund ist dabei ein verbesserter rechtlicher Schutz für eine exklusive globale ökonomische Nutzbarmachung der geschützten kulturellen Ausdrucksformen.

Bei der Entwicklung eines völkerrechtlichen Instruments zur Regelung kollektiver Eigentumsansprüche an kulturellen Elementen stellt sich die Frage nach der Konstituierung einer Gruppe als Rechtsträger und ihrer Legitimierung. Eine semantische Auffächerung des deutschen Eigentumsbegriffs verweist auf Eigen auch im Sinne eines Attributes, dass jemandem etwas zu eigen ist, die Person oder die Gruppe in ihrer Art ausmacht und zu ihr gehört. Aus diesem breiten Verständnis leitet sich die Vorstellung ab, dass sich Eigentum durch ein Zugehörigkeitsgefühl konstituiert und die Formulierung von individuellen oder im Falle von Cultural Property auch kollektiven Ansprüchen zulässt. Relevant ist dabei auch die Akzeptanz und Anerkennung einer kollektiven Identität durch außenstehende Akteure. Konstitutiv für die Identität von sozialen und kulturellen Gruppen wie Communities, Ethnien, Nationen und anderen Konstellationen sind kollektive Erinnerungen und Narrative (vgl. Assmann 2009, Binder et al. 2001, Francois und Schulze 2002), wie sie Pierre Nora für nationale Gedächtnisorte in Frankreich erforscht hat (1984). ${ }^{3}$ Eine Sache, ein Ort oder ein so genanntes Intangible wird durch ein Narrativ zum Kristallisationspunkt kollektiver Identität aufgewertet. Das Verhältnis unterschiedlicher Narrative ist durch spezifische (Macht)Strukturen bestimmt, wie es im Fall nationalstaatlicher Bezüge und der Ausbildung ethnisch konturierter Identitäten besonders deutlich wird. ${ }^{4}$ Gerade vor dem Hintergrund politischer Interessenlagen zeigt sich die komplexe Beziehung gegenseitiger Beeinflussung zwischen lokalen Gruppenbildungsprozessen und internationalen rechtlichen Rahmenbedingungen. Ein Beispiel dafür ist das konvergente Verständnis von Kultur und der primordialen Definition von ethnischer Zugehörigkeit, wie sie in der United Nations Declaration on the Rights of Indigenous Peoples der UN Generalversammlung von 2007 niedergeschrieben ist und wie sie dem Selbstverständnis zahlreicher indigener Interessenverbände entspricht (vgl. Huber 2005:44).

Eigentum in einem kulturanthropologischen Verständnis wird als Resultat von Verhandlungsprozessen auf Basis sozialer Beziehungen gefasst (vgl. Hann 1998), als eine kulturelle Praxis, die die Beziehung von Akteuren und materiellen oder immateriellen kulturellen Ausdrucksweisen regelt (Huber 2005:43). Eine Analyse der Formierung von Cultural Property muss zwei Dimensionen berücksichtigen: Zum einen die Dimension von Cultural Property als einem Paradigma politischer und (rechts)wissenschaftlicher Betrachtung unter kritischer Hinterfragung seiner

\footnotetext{
${ }^{3}$ Hier kann nur die schematische Verkürzung eines Gruppenbildungsprozesses dargelegt werden. Die Heterogenität von Gruppen, insbesondere in nicht-totalitären Kontexten, und die stetig andauernden Aushandlungen von kollektiven Identitäten werden am empirischen Material deutlich.

4 Vgl. Carolin Kollewes Arbeiten (2007) zur Aufwertung kultureller Artefakte einer stigmatisierten indigenen Gruppe, den Chichihualtepcs in Mexiko, die als prähispanische Objekte als kulturelles Erbe der mexikanischen Mehrheitsgesellschaft umgedeutet wurden und nun für die Chichihualtepcs identitätsstiftendes Potential entwickelt haben. Außerdem: Hafstein 2007.
} 
politischen und wirtschaftlichen Implikationen. Zum anderen als eine diskursive Strategie und Praxis eines Kollektivs (vgl. Tauschek 2009:69), die zu einem vorrechtlichen Anspruch an einem kulturellem Element führen kann.

\subsection{Der normative Charakter des UNESCO Weltkulturerberegimes}

Das Rechtsinstitut des UNESCO Weltkulturerbes mit seinem Zertifizierungsprozess und den normativen Regelungen zum Schutz und Erhalt von Weltkulturerbestätten kann in Wechselwirkung mit lokalen Identitätskonstruktionen treten und muss daher als Teil der Debatte um Cultural Property betrachtet werden.

Das Rechtsinstitut des UNESCO Weltkulturerbes ist als völkerrechtliches Instrument das einzige globale normative System zum Schutz von Weltkultur- und Naturerbe. Als völkerrechtliches Regime mit regelmäßigen Sitzungen von offiziellen Regierungsvertretern bindet es die Nationalstaaten unmittelbar in den Zertifizierungs- und Überprüfungsprozess von Weltkulturerbe mit ein. Diese Schlüsselfunktion von Regierungen bestimmt auch den einzigartigen Charakter des Welterbesystems. So sind es die Staaten selbst, die mögliche Welterbestätten auf die Tentative List der UNESCO setzen und deren Einschreibungsprozess vorantreiben. Somit kann man auch davon ausgehen, dass Welterbestätten ein großes Maß an Unterstützung seitens der jeweiligen Staatsregierung erhalten. Als völkerrechtlicher Vertrag begründet die Konvention reziproke Verpflichtungen unter den Mitgliedsstaaten, Weltkulturerbestätten auf ihrem Territorium im Sinne der Konvention zu schützen. Dies hat entsprechende innerstaatliche gesetzliche Regelungen und möglicherweise auch finanzielle Verpflichtungen seitens der Regierung zur Folge. Diese enge Verknüpfung von Regierungen und Kulturerbe führt allerdings auch zu Spannungen, die kritisch zu betrachten sind. Die in der UNESCO Konvention nicht vorgesehene Verknüpfung zur lokalen Bevölkerung in und um die Welterbestätten führte bereits mehrfach zu Interessenkonflikten. ${ }^{5}$ Staatsgelenkte Kulturpolitik birgt auch immer die Gefahr zur Manipulation der öffentlichen Meinung und Beeinträchtigung von Minderheitenrechten. Der normative Charakter des Welterbesystems, wie er in der UNESCO Konvention 1972 verankert ist, wird von der Idee des Schützens und Bewahrens von Kulturgut als Erbe der Menschheit geprägt. So sind die Mitgliedsstaaten der Konvention verpflichtet, Kultur- und Naturerbe zu ,identifizieren, schützen, bewahren, zugänglich zu machen und weiterzugeben“. (Art. 3, UNESCO 1972). Bereits die Präambel verdeutlicht, dass die Mitgliedsstaaten die Konvention in der Erwägung schlossen, dass der Verfall oder der Untergang jedes einzelnen Bestandteils des Kultur- oder Naturerbes eine beklagenswerte Schmälerung des Erbes aller Völker der Welt darstellt. Artikel 4 verdeutlicht den Anspruch, dass jeder Vertragsstaat die Weitergabe von Kultur- und Naturerbe an künftige Generationen sicherzustellen hat. Artikel 6 stellt fest, „dass dieses Erbe ein Welterbe darstellt, zu dessen Schutz die internationale Staatenge-

\footnotetext{
${ }^{5}$ Vgl. Beitrag Hauser-Schäublin/Klenke in diesem Band.
} 
meinschaft als Gesamtheit zusammenarbeiten muss“. Schließlich verpflichtet Artikel 5, „dass wirksame und tatkräftige Maßnahmen zum Schutz und zur Erhaltung“ ergriffen werden sollen. Dies umfasst insbesondere das Ergreifen einer allgemeinen Politik zum Schutz des Erbes, die Einrichtung von Dienststellen mit geeignetem Personal und den erforderlichen Mitteln, wissenschaftliche und technische Untersuchungen und Forschung, geeignete rechtliche, wissenschaftliche, technische, Verwaltungs- und Finanzmaßnahmen sowie die Errichtung nationaler und regionaler Zentren zur Ausbildung (Art. 5, UNESCO 1972).

Die Rhetorik des ersten Teils der Konvention betont damit den ideellen Charakter der moralischen Verpflichtung, Weltkultur- und Naturerbe für zukünftige Generationen zu erhalten. Der Schwerpunkt liegt dabei auf den Aspekten des Schützens und Bewahrens. Diese Verpflichtungen zum Schutz, sowie die genannten Maßnahmen die ergriffen werden sollen, stehen dabei allerdings unter dem Vorbehalt der staatlichen Souveränität. So betont beispielsweise Artikel 6 die ,volle [...] Achtung der Souveränität der Staaten, in deren Hoheitsgebiet sich das in den Artikeln 1 und 2 bezeichnete Kultur- und Naturerbe befindet“ und erklärt, dass die Schutzansprüche die „durch das innerstaatliche Recht gewährten Eigentumsrechte" unbeschadet lässt. Die weiten Formulierungen der Konvention haben somit keinen self-executing Charakter, sind also nicht unmittelbar in den Rechtsordnungen der Mitgliedsstaaten anwendbar. Vielmehr muss eine Ausgestaltung durch entsprechende nationale Gesetze erfolgen (Gutachten der Bundesregierung zur Bindungswirkung des UNESCOÜbereinkommens zum Schutz des Natur- und Kulturerbes der Welt).

Im Sinne dieses normativen Charakters der Konvention gestaltet sich auch das Begutachtungsverfahren durch die unabhängigen Gutachterorganisationen des Internationalen Rats für Denkmalpflege (ICOMOS) und die internationale Naturschutrunion (IUCN). Im Verfahren bei der Nichtregierungsorganisation ICOMOS begutachten Expert/innen, ob das in der Konvention niedergelegte Kriterium des ,außergewöhnlichen universellen Wertes“ einer Welterbestätte erfüllt ist. Am Ende des Bewertungsverfahrens steht ein kurzer Bericht des Expertengremiums, welches eine Beschreibung der Geschichte der Stätte, eine Zusammenfassung des rechtlichen Schutzrahmens, des Managements, des Zustands der Erhaltung und entsprechende Kommentare zu diesen Punkten sowie Empfehlungen für das Welterbekomitee enthält (ICOMOS 2007). Auch IUCN begutachtet Weltnatur- und gemischte Natur- und Kulturerbestätten nach vergleichbaren Kriterien. Diese sind insbesondere die Bewahrung der Biodiversität, nachhaltige Landnutzung, Erhöhung der landschaftlichen Schönheit, Sammlungen von ex situ Flora und Fauna, außerordentliche Wechselbeziehung von Mensch und Natur oder die historische Bedeutung als Ort von Entdeckungen in den Naturwissenschaften (IUCN 2001). Die Hinweise für Gutachter/innen enthalten dabei den ausdrücklichen Hinweis, dass sie über ihr IUCN Gutachten entsprechende Vorschläge zur Integrität und zum Management der Stätten einbringen sollen, welche als solche in vielen Fällen 
Arnika Peselmann und Philipp Socha

bereits „entscheidend für die Verstärkung der Bewahrung einer Stätte“ waren. ${ }^{6}$ Der normative Charakter des UNESCO Weltkultuerberegimes ist dominiert vom Gedanken des Beschützens und Bewahrens, welcher als Pflicht für die internationale Gemeinschaft festgeschrieben wird.

Beiden Konzepten, dem des Cultural Property und des Cultural Heritage, liegt ein reflexiver Umgang mit Kultur und Kultur als Ressource zu Grunde. Das Konzept des Welterbes erkennt den Wert von Kultur an und stellt diese in Form von zertifizierten Welterbestätten als Wert in das Erbe der gesamten Menschheit und normiert dabei die Pflicht des Erhaltens und Bewahrens. Die Debatte um Cultural Property befasst sich mit den Wechselwirkungen verschiedener Rechtsinstitute und Kultur insbesondere unter dem Gesichtspunkt der Ausschlusswirkung von Eigentumsrechten und der damit einhergehenden Inwertsetzung und Ökonomisierung. Beide Konzepte, das des Cultural Property und das des Cultural Heritage, beschreiben einen Inwertsetzungsprozess so dass der Begriff des Cultural Property teilweise auch synonym zum Cultural Heritage verwendet wird. ${ }^{7}$ Andererseits läuft eine Zertifizierung als Weltkulturerbe, die eine kulturelle Ausdrucksform formal und inklusiv in die Obhut der Menschheit stellt, dem exklusiven Cultural Property-Konzept entgegen (Bendix und Hafstein 2009).

\subsection{Dichotomisierung ideeller und ökonomischer Werte}

Die Verzahnung eines Cultural Property verstanden als diskursive Strategie mit dem UNESCO-Heritage-Regime drückt sich in den Inwertsetzungsprozessen kultureller Elemente im Zuge von Welterbeernennungen aus, die sich im Verständnis der Kulturanthropologin Barbara Kirschenblatt-Gimblett im Fall von Welterbestätten entlang einer spezifischen Verlaufsachse entwickeln:

The moment something is declared heritage, it enters a complex sphere of calculation. Valorization, ,the [re]appraisal of the heritage goods by means of deliberations. Pleas by art historians, debates in public media', and proclamation by UNESCO, is followed by valuation, ,the assessment of values that people actually attach to heritage goods.' based on what they spend to consume them or to ensure that they exist, even if they do not consume them. (Kirshenblatt-Gimblett 2006:193, zitiert in Klamer und Zuidhof 1999:31)

Die Unterscheidung in valorization und valuation, die ein kulturelles Element im Prozess seiner Heritage-Werdung erfährt, drückt die Gegenüberstellung von ideel-

\footnotetext{
6 "These recommendations have been, in many cases, critical to strengthening conservation in a site as they are eventually relayed back in an official letter from the World Heritage Centre" (IUCN 2001).

7 "The notion of cultural property is sometimes used synonymously with cultural heritage, but the two concepts nonetheless have distinct connotations and are associated with separate legal regimes and different manners of staking claims to culture ... [they] constitute parallel rather than identical modalities within the patrimonial regime." (Bendix und Hafstein 2009:5).
} 
len und ökonomischen Werten aus. In den Auswahl- und Verleihungsdiskursen liegt nach Auffassung von Regina Bendix die Marginalisierung potentieller wirtschaftlicher Gewinne eines Weltkulturerbes in dieser Dichotomisierung begründet, die ökonomische Nutzbarmachung abseits stellt, „als ob ihr etwas Unreines anhaf-

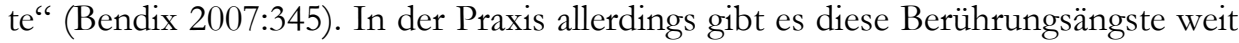
weniger: Ein Zusammenschluss aus Welterbestätten und Tourismusvertretern hat es sich in der Werbegemeinschaft der UNESCO-Welterbestätten in Deutschland e.V. zur Aufgabe gemacht, „einen behutsamen und hochqualifizierten Tourismus in den Welterbestätten im denkmalverträglichen Ausmaß zu fördern“ und die Stätten hinsichtlich der „touristischen Vermarktung zu beraten“. Die Gewinne sollten insbesondere zur Instandhaltung der Anlagen aufgewendet werden. Die Nutzung durch private Entrepreneure ist allerdings häufig weniger toleriert, wie das Beispiel des UNESCO-prämierten Karnevals in der belgischen Stadt Binche zeigt. Hier musste ein einheimischer Ladenbesitzer 500 Euro zahlen, weil er Anstecknadeln mit der für den Karneval typischen Wachsmaske hatte anfertigen lassen. Die Begründung lautete, dass die Gemeinde den Karneval schon immer ohne monetäre Interessen durchgeführt habe und eine ökonomische valuation des Spektakels abgelehnt werde, um die Besonderheit der lokalen Performanz zu sichern (Tauschek 2009:75). Die Besonderheit oder Einzigartigkeit als Merkmal ausgewählter kultureller Elemente verweist nicht nur auf den Kriterienkatalog der UNESCO, sondern ebenso auf die Echtheits- und Folklorismusdebatte: In den 1960er Jahren stellte der Volkskundler Hans Moser so genannte authentische kulturelle Praxen solchen Praxen gegenüber, die im Kontext touristischer Vermarktung performiert wurden (Moser 1962) und wurde dafür in volkskundlich-wissenschaftlichen Kreisen heftig kritisiert (Bausinger 1966). Die Unterscheidung von echten und imitierten kulturellen Ausdrucksformen, seien sie materiell oder intangibles, lässt sich historisch zurückverfolgen, insbesondere zu dem Zeitpunkt, der die technische Reproduktion ermöglichte (vgl. Benjamin 2006). Die puristische Vorstellung vom reinen und in seiner „Aura“ (ebd.) nicht durch massenweise Nachbildung korrumpierten kulturellen Element prägt auch gegenwärtige Debatten um die Vermarktung von Kultur. Dabei sieht George Yúdice (2003) Kultur als zweckdienliches Mittel und als Ressource für die Bewältigung ökonomischer und politischer Probleme. Die durch Kommodifizierung transformierten kulturellen Elemente erhalten einen Tauschwert $^{8}$, der strukturschwache Regionen wirtschaftliche Ressourcen bieten soll.

Im oben dargestellten Überblick der wissenschaftlichen Debatten um Cultural Property, die auch das Cultural Heritage-Regime miteinschließt, wurden die Parallelen und Wechselwirkungen in den Konstituierungsprozessen herausgestellt, aber auch die unterschiedlichen Konnotierungen, mit denen sie jeweils verbunden werden. Während das Konzept eines Cultural Property - verstanden als diskursive Strategie sowohl Exklusivität und Kontrolle als auch immer die Möglichkeit einer ökonomi-

\footnotetext{
8 Nicht im Sinne von Marx, sondern von Georg Simmel (2009) und in der theoretischen Weiterführung von Arjun Appadurai (1986).
} 
Arnika Peselmann und Philipp Socha

schen Inwertsetzung impliziert, ist die Idee eines Cultural Heritage im Sinne der Unesco Konvention von 1972 geprägt von der ideellen Intentionen des Schützens und Bewahrens, bei der die gesamte Menschheit in die Pflicht gestellt wird, kulturelle Errungenschaften für die Nachwelt zu erhalten. Soweit das normative Verständnis der Konvention, aber wie sieht ihre faktische Umsetzung aus? Zwei empirische Studien beleuchten den lokalen Umgang mit diesem völkerrechtlichen Regime, das in engem Bezug zur Formierung eines Cultural Property steht.

\section{Die Aberkennung des Weltkulturerbestatus des Dresdner Elbtals}

\subsection{Die Causa Waldschlößchenbrücke}

Die Debatte um den Verlust des Welterbetitels im Fall der Dresdner Waldschlösschenbrücke zeigt eine geradezu selbstverständliche Vermischung von Argumenten des ideellen Schutzes von Kultur für zukünftige Generationen und von ökonomischen Gesichtspunkten der infrastrukturellen Stadtentwicklung und des Tourismus. Nachdem das Elbtal im Juli 2004 als Welterbe in die Liste der UNESCO aufgenommen wurde, waren es das Referendum zum Bau der Waldschlößchenbrücke und die darauf folgende Einordnung in die Liste der gefährdeten Welterbestätten, die die intensive Diskussion um den Brückenbau und den Welterbetitel angestoßen haben. Nach mehrjährigen politischen Debatten und juristischen Auseinandersetzungen wurde Ende 2007 mit dem Bau der Brücke begonnen, was im Juni 2009 zur Aberkennung des Titels durch das Welterbekomitee der UNESCO führte. Neben juristischen, sozialen und kulturhistorischen Argumenten spielten in der Diskussion auch ökonomische Argumente auf beiden Seiten der Brückenbefürworter und -gegner eine Rolle. Im Lauf der Diskussion um den möglichen Verlust des Welterbestatus verwiesen die Brückengegner/innen auf die negativen Schlagzeilen, die Dresden national und international erhalten würde, sollte durch die Brücke die Landschaft des Naturerbes zerstört werden. Sie brachten somit das ökonomische Argument, dass der Verlust des Welterbetitels auch negative Auswirkungen auf die touristische Attraktivität der Stadt Dresden haben wird. Für die Auswahl von Reisezielen dient die Liste der Weltkulturerbestätten gerade asiatischen Reisenden und Tourismusbüros als Entscheidungsgrundlage. So merkt die kulturpolitische Sprecherin der Fraktion Bündnis 90/Die Grünen Christiane FiliusJehne folgendes an:

Der Titel Welterbe ist für das Dresdner Elbtal nicht nur ein unbezahlbarer Imagegewinn, er schlägt sich auch in barer Münze nieder. [...] Reisen zu Betonbrücken gibt es jedenfalls nachweislich nicht im Gegensatz zu Welterbe-Reisen, aus deren Programm Dresden bei Aberkennung des Titels schlicht gestrichen würde (Lerm 2005). 
Die Brückenbefürworter/innen argumentieren grundsätzlich mit der infrastrukturellen Notwendigkeit der Brücke für den Verkehr in der Stadt und dem entsprechenden infrastrukturellen Bedarf für die ökonomische Entwicklung als wettbewerbsfähiger Wirtschaftsstandort. Als Reaktion auf das Argument, dass wegen des Verlusts des Welterbetitels die Zahl der Tourist/innen zurückgehen könnte, betonen sie die Schönheit und Attraktivität der Stadt als Reiseziel, die unabhängig vom UNESCO Status als Touristenmagnet wirke. So konnte man Kommentare leseQwie diesen:

Und irgendwann werden alle diese internationalen UNESCO-Touristen auf der Waldschlößchen-Brücke stehen und sich der neuen Stadtansichten freuen und nicht mehr wissen, warum sie damals ihre Hand gegen Dresden erhoben haben. Die Dresdner sind ein hartes Völkchen und werden mit und ohne Weltkulturerbe diese Jahrhunderte währende Pracht zu verwalten und zu mehren wissen. Gäste Dresdens sind schon immer wegen des Flairs und nicht wegen eines Titels oder verlorener bundesdeutscher Fördertöpfe gekommen - vielleicht nun mehr denn je! ${ }^{?}$

Auch der sächsische Ministerpräsident bekannte mit seiner Meinung „Der Verlust des Welterbetitels ist verkraftbar" seine trotzige Meinung gegenüber der UNESCO (Friedrich 2009). Tatsächlich haben sich die Zahlen der Reisenden, nach Aberkennung des Titels und trotz der Finanzkrise in 2008/2009, sogar erhöht.

Der jahrelange Streit der Dresdner Bürger um ihr Weltkulturerbe kann als Konflikt zweier Narrative um die Deutungsmacht ihrer Stadt gelesen werden: In der Studie von Martina Jackenkroll (2008) zur Heritagifizierung des Dresdner Elbtals wurde sichtbar, wie der UNESCO Welterbe-Status von Dresdner Befürworter/innen in bereits bestehende lokale Deutungsmuster integriert wurde; so beispielsweise als das I-Tüpfelchen und Abschluss eines qualvoll langen Regenerierungsprozesses nach der Zerstörung Dresdens 1945 (Jackenkroll 2008:191). Allerdings wird Dresden auch als „Silicon Saxony“ erzählt, so zumindest im Jargon der Sächsischen Staatskanzlei (Friedreich 2009:176) mit Verweis auf eine über 200ährige Geschichte der Hochtechnologie. Die geplante Waldschlößchenbrücke wird als Notwendigkeit für eine ökonomische Weiterentwicklung des Wirtschaftsstandorts verstanden und fügt sich nach Ansicht ihrer Befürworter/innen ideal in die vorhandene Stadtlandschaft ein (vgl. Friedreich 2009:176). Die wirtschaftliche Nutzbarmachung des Welterbestatus wurde von Brückengegner/innen ins Feld geführt (Jackenkroll 2008:180). Der ökonomische Aspekt eines Weltkulturerbes ist trotz der primär schützenden und bewahrenden Funktion ein inhärenter Teil des Titels, der sich in einer Tourismusmarke (vgl. Groschwitz 2009) formiert.

9 Vgl. http://www.welt.de/kultur/article2176993/Der_lange_Kampf_um_die_Waldschloesschenbruecke.html (Zugriff am 05.06.2010). 


\subsection{Juristischer Rahmen des Weltkulturerbes in Deutschland}

Die Diskrepanz zwischen den Narrativen in Dresden, die die ökonomische Inwertsetzung durch den WKE Status als selbstverständlich behandeln, und dem normativen Charakter der rechtlichen Regelungen des WKE, wird auch in den juristischen Auseinandersetzungen im Zusammenhang mit dem Bau der Waldschlößchenbrücke deutlich.

Von den Entscheidungen der Gerichte im Fall Dresden ausgehend, hat die rechtswissenschaftliche Literatur den rechtlichen Status von Weltkulturerbe in Deutschland einer intensiven Prüfung unterzogen. Das Ergebnis, dass das WKE in Deutschland keine formelle Umsetzung in geltendes Recht gefunden hat, sei hier nur am Rande erwähnt. Nur über den Grundsatz der Bundestreue und die Völkerrechtsfreundlichkeit der deutschen Rechtsordnung sind die Behörden gehalten, den Schutz des WKE zu berücksichtigen. In welchem Umfang und mit welchem Gewicht der Schutz von Weltkulturerbe in Ermessensentscheidungen berücksichtigt wird, hängt dabei von den vollziehenden Behörden ab und unterliegt danach nur noch einer eingeschränkten Kontrolle durch die Gerichte (Wolf 2008).

Die Welterbekonvention wird im deutschen Recht im Kontext von denkmalund umweltschutzrechtlichen Bestimmungen behandelt. Als solche wird sie in entsprechenden Entscheidungen nur entgegen wirtschaftlichen Interessen Privater und der Öffentlichkeit abgewogen. Die positiven wirtschaftlichen Implikationen der Inwertsetzung als Kultur- oder Naturerbe müssen in dieser Zielrichtung nicht eingestellt werden. Dies zeigt sich beispielsweise in der Entscheidung des OVG Bautzen in seiner Entscheidung zur Waldschlößchenbrücke im Verfahren des einstweiligen Rechtsschutzes, wo festgestellt wird, dass

[m]anches dafür [spricht], dass die Welterbekonvention und die auf ihrer Grundlage ergangenen Entscheidungen des Welterbekomittees etwa im Rahmen von planerischen Abwägungen (vgl. \ 2 II Nr. 13 ROG), bei der Ausweisung von Schutzgebieten oder für die Beurteilung der Schutzbedürftigkeit und -würdigkeit von Kulturdenkmälern (so ausdrücklich etwa \2 II SachsAnhDenkmSchutzG) Berücksichtigung finden können.

Des Weiteren stellt das OVG Bautzen fest, dass nach einem entsprechenden Schreiben vom Landesamt für Denkmalpflege, welches die Unbedenklichkeit aus denkmalpflegerischer Sicht bescheinigte,

es sich der Planfeststellungsbehörde im Zeitpunkt der Abwägungsentscheidung auch nicht aufdrängen [musste], dass das Vorhaben einem - später anerkannten - Welterbestatus des Dresdner Elbtals möglicherweise entgegenstehen könnte.

Das Gericht stellt somit den Status Weltkulturerbe in eine Reihe mit den denkmalund umweltschutzrechtlichen Bestimmungen. Die ökonomischen Inwertsetzungseffekte des Weltkulturerbestatus, die durch seinen Erhalt hätten gewährleistet wer- 
den können, werden nicht berücksichtigt. Die Verfahren, die im Zusammenhang mit dem Bau der Waldschlößchenbrücke geführt wurden, zeigen somit ebenso wie der abstrakt normative Charakter der völkerrechtlichen Instrumente, dass die nationalen Regeln und Entscheidungsgründe der Gerichte hauptsächlich auf das Bewahren und Erhalten ausgerichtet sind und somit die eigentlichen Beweggründe der betroffenen Bürger und der zur Entscheidung berufenen Exekutive außer Acht lassen.

Es ist allerdings festzustellen, dass der Grund für die intensiven Auseinandersetzungen der Bürger/innen und Entscheidungsträger/innen mit „ihrem“ Dresden durch die aufgezeigte Diskrepanz zwischen den Selbstverständnissen als Kulturstadt und innovativer Standort von Hochtechnologie sowie den normativen Regelungen des Schützes und Bewahrens ausgelöst wurde. Die Auseinandersetzung mit dem Rechtsinstitut Weltkulturerbe, welches im Fall von Dresden zu nicht unerheblichen sozialen, politischen und juristischen Konflikten führte, und letztendlich die Ablehnung des Status Weltkulturerbe zur Folge hatte, war somit Anstoß einer intensiven Auseinandersetzung mit lokaler Identität. Diese Prozesse zeigten nicht nur Positionen und Machtkämpfe um Identitätskonstruktion von lokalen Akteuren, wie sie Sönke Friedrich aufzeigt, sondern haben auch unmittelbare Auswirkungen auf andere Weltkulturerbestätten. So nimmt der Fall Dresden Einfluss auf die Prozesse um die Bewerbung des Erzgebirges als Welterbe, wie im Folgenden ausgeführt wird.

\section{Cultural Heritage in the Making: Zeugnisse des erzgebirgi- schen Bergbauwesens als UNESCO-Weltkulturerbe}

\subsection{Metakulturelle Operationen im Erzgebirge}

Kulturelles Erbe ist nicht, es wird (Bendix 2007:340). Kulturelle Elemente werden unter bestimmten Maßgaben bewusst ausgewählt, sie werden symbolisch aufgeladen, museal präsentiert, gelistet und inventarisiert - diese reflexiven Prozesse fasst Barbara Kirshenblatt-Gimblett unter den Terminus der "metacultural operations" (Kirshenblatt-Gimblett 2004) und verweist dabei vor allem auf die Praxen der UNESCO-Weltkulturerbe Zertifizierung. Das Making of eines World Heritage soll hier in knappen Zügen anhand der laufenden Nominierungsvorbereitung für die geplante UNESCO-Industriekulturlandschaft Montanregion Erzgebirge skizziert werden. ${ }^{10}$ Der reflexive Umgang mit Kultur beginnt im Erzgebirge aber nicht erst mit dem Wunsch nach einer UNESCO-Zertifizierung, sondern hat historische Vorläufer in der so genannten Volkskunst, die als Teil der Regional-Identität Er₹gebirgisches Weibnachtsland vermarktet wird. Der Begriff Image unterscheidet sich von

${ }^{10}$ Das Beispiel in seinen vielschichtigen Verästelungen wird der Dissertationsfokus von Arnika Peselmann sein. 
Identität durch seine wirtschaftliche Prägung. Trotzdem sind beide Phänomene im Fall des Weihnachtslandes nicht klar voneinander abgrenzbar. Aus kulturanthropologischer Sicht sind gerade jene regionalen und lokalen Identitäten von Interesse, die in Wechselwirkung zwischen äußeren Einflüssen und lokaler Lebenswelt entstehen (vgl. Maase 1998:57-58, Friedreich 2005:226).

Gerade die Formierung erzgebirgischer Volkskunst wurde durch spezifische sozio-kulturellen Rahmenbedingungen ermöglicht, in dem sich konservatorische Bestrebungen ebenso ausprägen konnten, wie der Wunsch nach ,authentischem Erleben', der den touristisch-ökonomischen Aspekt ins Blickfeld führt. Die Verdichtung von schützenden Intentionen und den dazugehörigen institutionellen und gesetzlichen Strukturen können als Wegbereiter für metakulturelle Operationen im Welterbekontext gedeutet werden (Bendix 2007:342).

\subsection{Erzgebirgische Volkskunst: vom Warenartikel zum Sammelobjekt}

Seit dem 17. Jahrhundert begann im Erzgebirge verstärkt die Produktion von in Heimarbeit gefertigten hölzernen Haushaltsartikeln und später von Spielzeug und Raumdekoration, die motivisch vom bergbaulichen Umfeld geprägt waren. Ökonomische Krisen am Ende des 19. Jahrhunderts ließen die Spielzeugproduktion jedoch Gegenstand von Förderungsprogrammen der sächsischen Regierung und der so genannten „Volkskunstbewegung““ (vgl. Korff 1992, Schürch 2008) werden. Entwickelt hat sich der Begriff der Volkskunst in gesellschaftlichen und wissenschaftlichen Diskursen Ende des 19. Jahrhunderts als Teil der nationalstaatlichen Formierung (vgl. Schneider 2009a). Der Vertrieb handwerklicher Produkte als Volkskunst diente als sozialpolitische Maßnahme zur Förderung krisengeschüttelter Kleinbetriebe, zu denen auch die erzgebirgischen Holzschnitzer und Drechsler gehörten. In musealen Sammlungen erzgebirgischer Volkskunst ${ }^{11}$ drückt sich der Impetus des Schützens und Bewahrens aus, der nicht allein Objekte oberschichtiger Provenienz, sondern auch Elemente der Arbeiter- und Industriekultur als ,erbträchtig“" und für die Nachwelt erhaltenswert erachtet (vgl. Bendix 2007:342).

Die erzgebirgische Volkskunst macht auch einen wesentlichen Bestandteil des wirtschaftlich sehr einträglichen Regional-Images Erzgebirgisches Weihnachtsland aus. Es reicht jedoch nicht, das von professioneller Hand sorgfältig gepflegte Image einzig als Konsuminszenierung zu deuten. Der Kulturanthropologe Sönke Friedreich verweist auf den in der Region tief verwurzelten Pietismus, der in Glaubens- und Alltagspraxen Ausdruck findet. ${ }^{12}$ Welche reflexiven Praxen an der Eta-

11 Der frühere stellvertretende Vorsitzende des Landesvereins Sächsischer Heimatschutz, Oskar Seyffert, gründete 1913 das Sächsische Volkskunst Museum, in dem die erzgebirgische Volkskunst einen prominenten Platz einnahm (vgl. Jenzen 2006:582, Schramm 2003:121).

12 Friedreich fragt, inwieweit die Weihnachtsfolklore als das herausragendste Symbol regionaler Selbstetikettierung auf spezifische Frömmigkeitsformen zurückgeht. An der Ausprägung regionaler Identitäten seien zwar auch immer Agenten und Institutionen beteiligt, aber über die lebensweltliche Verwurzelung, wie sie für die religiösen Interpretationsmuster gegeben seien, könne man nicht ohne weiteres hinweggehen (Friedreich 2005:226). Die „Anleihen an pietistischer Sentimentalität“ (Fried- 
blierung des Images und regionaler Identitäten involviert waren/sind, kann an dieser Stelle nicht vertieft werden. Das Beispiel des Weihnachtslandes und der erzgebirgischen Volkskunst liefern aber Perspektiven auf das Wechselspiel reflexiver Praxen, strukturpolitischer und individuell, ökonomischer Interessenlagen und Lebenswelt im Inwertsetzungsprozess von kulturellen Elementen, die für die Betrachtung der geplanten UNESCO-Kulturlandschaft von Belang sind.

\subsection{Heritage in the Making}

Der geplanten Status als UNESCO Weltkulturerbe Montanregion Erzgebirge bezieht sich auf die 800jährige Bergbaugeschichte der Region, die mit Silbererzen ihren Anfang nahm, zuletzt wurden Uranerze gefördert wurden. Das Montanwesen hat die Landschaft über die spezifische Architektur von Bergbauanlagen und -städten hinaus geprägt: Die Fauna und Flora ebenso wie die Kultur der Region, angefangen von der gewerblichen Ausrichtung bis hin zum Kunsthandwerk und der wissenschaftlichen Beschäftigung mit dem Bergbau. So sind selbst die als Volkskunst bekanntgewordenen Schnitzereien und Drechslerarbeiten Erzeugnisse einer so genannten Bergbaufolgeindustrie, da sie in Folge abnehmender Erzausbeuten den Bergarbeiter und ihren Familien alternative Erwerbsquellen ermöglichte (Auerbach 2000).

Die Summe aller Facetten, durch die die montane Prägung der Region sichtbar wird und verbunden mit dem jahrhundertelangen und bis in die Gegenwart reichenden Kontinuum bergbaulicher Aktivitäten, stellt einen einmaligen Wert der Montanregion Erzgebirge für die Menschheit dar (vgl. Realisierungsstudie ${ }^{13} 2007$ : 16). $\mathrm{Zu}$ dieser Auffassung gelangte die Arbeitsgruppe UNESCO-Welterbe-Projek.t Montanregion Erzgebirge am Institut für Wissenschafts- und Technikgeschichte (IWTG) der Bergakademie Freiberg, die die wissenschaftliche Vorbereitung der bislang für 2013/14 geplanten Einreichung der seriellen Nominierung ${ }^{14}$ leisten. Gemeinsam mit dem Dresdner Elbtal hatte das Sächsische Ministerium für Wissenschaft und Kunst (SMWK) 1998 die Montanregion Erzgebirge für die Aufnahme in die Tentativliste vorgeschlagen. Dieses Anliegen wurde für beide Projekte von der Kultusministerkonferenz der Länder positiv entschieden. Während die

reich 2005:230) im Konstituierungsprozess des Images Erzgebirgisches Weihnachtsland können als transformierte Glaubenspraxen verstanden werden, die als Teil eines touristisch verwertbaren ,authentischen Weihnachtserlebnisses“ ökonomischen Wert entwickelt haben.

${ }^{13}$ Die Realisierungsstudie wurde im Auftrag des Fördervereins Montanregion Erzgebirge e.V. von der Arbeitsgruppe UNESCO-Welterbe-Projekt Montanregion Erzgebirge am Institut für Wissenschaftsund Technikgeschichte (IWTG) der Bergakademie Freiberg im Jahr 2007 erstellt. Sie stellt eine Fortschreibung der bereits 2001 vom Sächsischen Staatsministerium für Wissenschaft und Kunst initiierten und ebenfalls vom IWTG ausgearbeiteten Machbarkeitsstudie dar. Sowohl die Machbarkeits- als auch die Realisierungsstudie sind auf der Homepage des Fördervereins Montanregion Erzgebirge abrufbar.

$14 \mathrm{Vgl}$. Kriterien für eine serielle Nominierung in den Operational Guidelines (\$19) der UNESCO für das Welterbe. 
Vorbereitung für die Nominierung des Dresdner Elbtals einen zügigen Verlauf nahm und mit der Zertifizierung im Juli 2004 von - vorläufigem - Erfolg gekrönt war, wurde für die Montanregion Erzgebirge im Jahr 2000 vom SMWK erst einmal eine Machbarkeitsstudie in Auftrag gegeben, die am Institut für Wissenschafts- und Technikgeschichte der Bergakademie Freiberg unter der Leitung des dortigen Direktors durchgeführt wurde. Im Jahr 2001 wurde die Studie mit folgenden drei Ergebnissen vorgelegt: Die Montanregion Erzgebirge besitzt erstens das Potential zur Aufnahme in die Welterbeliste, sie stellt zweitens im Sinne der UNESCO eine Kulturlandschaft dar, deren Entwicklungsprozess noch nicht abgeschlossen ist. ${ }^{15}$ Als letzten Punkt verwiesen die Autor/innen darauf, dass das Erzgebirge als mitteleuropäische Kulturlandschaft nicht nur die sächsische, sondern auch die tschechische Seite als zweistaatliches Projekt unter sächsischer Federführung einschlieBen sollte (vgl. Realisierungsstudie 2007:3-4).

Die Reaktionen des damals noch zuständigen Ministeriums für Wissenschaft und Kunst ${ }^{16}$ waren zurückhaltend, da sich bereits erste Schwierigkeiten im Zusammenhang mit dem Dresdner Elbtal abzeichneten. Anregungen seitens des SMWK bezogen sich darauf, in weiteren Untersuchungen die wirtschaftlichen und touristischen Auswirkungen des Projekts zu untersuchen und den Nachweis zu erbringen, dass die Region das Welterbe-Projekt selbst befürworte und auch zu dessen Unterstützung bereit sei (Realisierungsstudie 2007:4). Trotz positiver Ergebnisse blieb die Landesregierung auf Distanz des von ihr initiierten Projekts. Deren fehlende Unterstützung führte 2003 schließlich zu einer Initiative des Regionalmanagements Erzgebirge, der SAXONIA Standortentwicklungs- und verwaltungsgesellschaft mgH und des IWTG der TU Bergakademie Freiberg der Förderverein Montanregion Eragebirge e.V. ${ }^{17}$ gegründet wurde. Das Ziel von Vertreter/innen aus Wirtschaft, Kommunalpolitik, Wissenschaft und private Spender/innen ist es, „Das Welterbe-Projekt Montanregion Erzgebirge ideell und materiell zu fördern, sowie für dieses Projekt in der Region zu werben“" (Realisierungsstudie 2007:4). Der vom SMWK eingeforderte Nachweis, dass die Region das Projekt nachhaltig unterstützt, sahen die Autor/innen der 2007 veröffentlichten Realisierungsstudie mit der Grundsatzentscheidung des Regionalkonvents Erzgebirge im Jahr 2005 als erbracht an, da es als ein Schlüsselthema des Regionalmanagements Erzgebirge erhoben wurde.

\footnotetext{
15Seit dem Jahr 1992 versieht das UNESCO-Welterbekommittee bestimmte Stätten mit dem Zusatz Kulturlandschaft, wenn sie einem bestimmten Kriterienkatalog entsprechen (vgl. UNESCO zu Cultural Landscape).

16Die Betreuung des Projekts ist nun nicht mehr Angelegenheit des SMWKs, sondern wurde ans sächsische Ministerium des Inneren übertragen. In Sachsen gehört die Denkmalpflege in den Bereich des Innenministeriums, das Ressort Archäologie und Bodendenkmalpflege ist beim Ministerium für Wissenschaft und Kunst angesiedelt.

${ }^{17}$ Vgl. http://www.wiwi.tu-freiberg.de/iwtg/monte/ (Zugriff am 04.04.2010).
} 


\subsection{Demokratische Legitimation und ökonomische Nutzbarmachung: Ein Gegenentwurf zu Dresden}

Auf Basis der Datenbanken des sächsischen Landesdenkmalamts sowie des Landesamts für Umwelt und Geologie und unter Maßgabe eines Kriterienkatalogs, wurden auf sächsischer Seite 34 Objekte und Ensembles ausgewählt, die in Verbindung mit den bislang sechs tschechischen Nominierungen eine UNESCO zertifizierte industriekulturelle Landschaft bilden sollen. Der Facettenreichtum ist es, der sich in der Serie der Objekte/Ensembles ausdrückt und der nach Einschätzung der Projektgruppe den Ansprüchen der UNESCO auf außergewöhnlichen universellen Wert genügt. Für die Bestandsaufnahme ist nicht nur die Dokumentation der Objekte/ Ensembles wichtig, sondern auch das nachgewiesene Potential für deren touristische Nutzbarmachung. Im Vorwort der Realisierungsstudie betonte der Vorsitzende des Fördervereins und Landrat des Landkreises Freiberg (heute Mittelsachsen), Volker Uhlig, dass ,bewusst Tradition und Zukunft verbunden werden sollen“, was bedeutet, „dass die Objekte von außergewöhnlichem universellen Wert gezielt mit der touristischen Inwertsetzung der Region verbunden werden und die Objektauswahl abgestimmt mit den Erfordernissen der Regionalund Wirtschaftsentwicklung geschieht" (Realisierungsstudie 2007, Vorwort). Um die Zielsetzung der Verträglichkeit von denkmalpflegerischen und ökonomischen Interessen in allen ausgewählten Stätten zu gewährleisten, führt die Freiberger UNESCO-Projektgruppe so genannte Pilotstudien durch: Eine im Welterbekontext „einmalige Methodik“"18, die einen möglichst breiten Rückhalt für die UNESCO-Nominierung in der Bevölkerung gewährleisten soll. Die Freiberger Projektgruppe versteht die Pilotstudien als positives Gegenbeispiel zum DresdnerWeltkulturerbe-Debakel, das potentielle Interessenkonflikte aufdecken und präventiv Alternativansätze entwickeln soll. Aber neben der Harmonisierung unterschiedlicher Interessenlagen hoffen die Nominierungsbefürworter durch die demokratisch legitimierten Pilotstudien „Druck von unten aufzubauen“19, der die derzeit noch distanzierte Landesregierung schließlich zur Antragsstellung bewegen soll.

\subsection{Industrielle Hinterlassenschaften oder kulturelles Erbe?}

Das Bekenntnis „zur Wahrung und Pflege des industriellen Erbe Sachsens“, das im Koalitionsvertrag der derzeitigen Landesregierung abgelegt wird und in einer Stiftung Sächsische Industriekultur Gestalt annehmen soll, ${ }^{20}$ weckt bei den Befürwortern ebenso Hoffnung, wie die Ernennung der neuen sächsischen Staatsministerin für Wissenschaft und Kunst, Frau Prof. Sabine von Schorlemer, die den geplanten Bau

\footnotetext{
18 Interview mit dem Leiter der UNESCO-Projektgruppe an der Technischen Universität Freiberg und stellvertretendem Vorsitzenden des Fördervereins im Dezember 2009.

19 Ebenda.

${ }^{20}$ Vgl. Koalitionsvertrag der sächsischen CDU und FDP über die Bildung der Staatsregierung für die 5. Legislaturperiode des Sächsischen Landtags.
} 
der Waldschlößchenbrücke als „Akt kultureller Selbstverstümmelung“ (Schorlemer 2006:1312) brandmarkte und dem UNESCO-Welterbeprogramm grundsätzlich positiv gegenübersteht. Allerdings ist es nicht nur die Landesregierung, der „die Angst vor dem Reinregieren“"21 durch die UNESCO genommen werden soll. Auch einige lokale Befürchtungen vor einem wirtschaftlichen Stillstand durch das „Leichentuch der Denkmalpflege“ möchte die UNESCO-Projektgruppe mittels öffentlicher Diskussionsrunden zerstreuen.

Widerstand regt sich aber auch bezüglich des Labels Montanregion: Über Ängste vor der wirtschaftlichen Stagnation hinaus wurde im Jahr 2003 der damalige sächsische Umweltminister und derzeitige CDU-Fraktionsvorsitzende Steffen Flath in der Freien Presse online wie folgt zitiert: „Nicht im Traum wäre es mir eingefallen, meine Heimat als Montanregion zu bezeichnen." Assoziationen mit dem Bergbauwesen halte er aus touristischer Sicht für kontraproduktiv und verweist auf das positive Image des erzgebirgischen Weihnachtslandes. Statt einer Rückbesinnung möchte er zukunftsweisende Wirtschaftszweige wie die ansässige Recyclingund Solarindustrie hervorheben: „Silicon Mountains ist ein Begriff, der nicht zu Unrecht den erfolgreichen Wandel beschreibt.“ Die Befürworter der UNESCOMontanregion halten Flath entgegen, dass das Bergbauwesen elementarer Bestandteil der lokalen Traditionspflege und regionalen Identität sei, wie sich an den stetig wachsenden Bergparaden und -aufzügen aufzeigen ließe. Als fester Veranstaltungspunkt an allen vier Adventssonntagen sei die Integrität in das erzgebirgische Weihnachtsimage gut ablesbar und die Besucherzahlen sprächen für sich. ${ }^{22}$ In der Annaberger-Zeitung kommen Bewohner/innen der geplanten Welterbestätte zu Wort, die trotz einer grundsätzlichen positiven Einstellung zum geplanten UNESCO-Projekt Zweifel hegen, ob der Begriff Montanregion glücklich gewählt ist: „Denn so mancher mag dabei zuerst an triste Haldenlandschaften denken“(Müller 2010).

\subsection{Toxic Heritage als Weltkulturerbe?}

Die 800 jährige Bergbaugeschichte der Region schließt auch die Uranerzförderung der sowjetisch-deutschen Aktiengesellschaft Wismut (SDAG Wismut) zum Zweck des atomaren Wettrüstens während des Kalten Krieges ein und die sich 1990 anschließende Sanierungswirtschaft. In der vorläufigen UNESCO-Nominierungsliste ist sie vertreten durch die Uranerzgrube Objekt 09 in der Nähe von Hartenstein mit den Schächten 371 und 382. Die Hinterlassenschaften des Uranbergbaus könnten als Stätten des Kalten Krieges gedeutet werden, die für die Nachwelt ein „dissonantes” (Tunbridge und Ashworth 1996) oder toxic heritage bereithalten. Der Verein zur Förderung, Bewabrung und Erforschung der Traditionen des sächsisch/thüringischen Uranbergbaus e.V. legt sein Augenmerk jedoch auf das „bergbautypische kulturelle

\footnotetext{
${ }^{21}$ Interview Leiter der UNESCO-Projektgruppe.

22 Interview Leiter der UNESCO-Projektgruppe.
} 
Erbe $^{\text {“23 }}$, das etwa in Festumzügen bei jährlichen Bergmannstagen seinen Ausdruck findet. ${ }^{24}$ Der 1993 gegründete Verein setzt sich neben einer Sanierung der geschädigten Umwelt vor allem für eine „Vertiefung der Verbundenheit der Beschäftigten und der Bevölkerung mit der Arbeit und den Leistungen der Wismut in ihren Einzugsbereichen" (ebd.) ein. Nicht das cultural, sondern das toxic heritage des Uranbergbaus hat 2001 zur Gründung des Vereins Atomopfer e.V. Selbsthilfe-Initiative Wismut geführt, in der sich frühere Wismut-Kumpels zusammengeschlossen haben, um für die Anerkennung ihrer durch radioaktive Verstrahlung verursachten Erkrankungen als Berufsunfälle zu kämpfen. Ihr Zorn richtet sich dabei weniger auf die SDAG Wismut, als vielmehr auf die Berufsgenossenschaften, die ihnen eine gesetzliche Unfallrente verweigern. Den durch Sanierungsarbeiten schwindenden Spuren des Uranbergbaus, wie beispielsweise die pyramidenförmigen Halden, hängen manche dagegen mit wehmütigen Erinnerungen nach. ${ }^{25}$

\subsection{UNESCO Montanregion Erzgebirge: Wessen Erbe?}

Auf der tschechischen Seite des Erzgebirges wurden bislang sechs Objekte oder Ensembles in den Regionen Usti nad Labem und Karlovy Vary auf die vorläufige Liste gesetzt. Das Bezirksmuseum in Most ist einer der tschechischen Partner für die binationale Nominierung und stellt auf einer zweisprachigen Homepage die potentiellen Welterbestätten vor. Dabei fällt auf, dass über die Beschreibung der Objekte oder Ensembles hinaus auch das deutsch-tschechische Verhältnis dieser Grenzregion unter den Überschriften „Was uns verbindet“ und „Was uns voneinander trennt" thematisiert wird. Die erste Kategorie bezieht sich vor allem auf die geographischen Gegebenheiten und die historische Beziehung seit dem Mittelalter bis zum Anfang des 20. Jahrhunderts. Die trennenden Aspekte beziehen sich vor allem auf die Zeit während und nach dem Zweiten Weltkrieg und der damit einhergehenden Vertreibung der deutschsprachigen Minderheit. Auf der Homepage des Moster Museums ist zu lesen:

Mit dem Austausch der Bevölkerung auf der tschechischen Seite kam es zum Abreißen der Bindungen an die Landschaft, an Grund und Boden und zum Handwerk. Auf sächsischer Seite hat es solche Entwicklungen nicht gegeben.

Der „Austausch der Bevölkerung“ bezieht sich zum einen auf die Vertreibung der so genannten Sudetendeutschen und zum anderen auf die mittels staatlicher Programme der damaligen Tschechoslowakei (zwangs-)angesiedelten Sinti und Roma, die größtenteils aus der Südslowakei stammten (Wiedemann 2007). Daran schließt sich die Frage, wem die potentiellen Welterbestätten Möglichkeit zur Identifikation

${ }^{23}$ Vgl. http:/ /www.bergbautraditionsverein-wismut.de/ (Zugriff am 05.05.2010).

${ }^{24}$ Das Uranbergbaustädtchen Bad Schlema veranstaltet beispielsweise seit 1996 Bergmannstage mit Festumzügen, deren Teilnehmer im bergmännischen Habit auftreten.

${ }^{25}$ Vgl. http:/ / www.atomopfer.de (Zugriff am 04.04.2010). 
und historischen Verortung bieten können/sollen? Und allgemeiner: Welche Interessen oder Widerstände lassen sich auf lokaler, regionaler oder nationaler Ebene im Zusammenhang mit einer UNESCO-Nominierung vorfinden? Diese Fragen werden meine Forschung auf tschechischer Seite weiterhin begleiten.

Die Betrachtung der Nominierungsvorbereitungen eines UNESCO-Titels im Erzgebirge erweiterte die Perspektive vom bereits verliehenen und nun wieder aberkannten Weltkulturerbe-Status des Dresdner Elbtals auf ein Heritage in the Making und die dabei angewandten metacultural operations. Das ,Dresdner Debakel' hat nicht nur starken Einfluss auf den Fortgang der Aushandlungsprozesse im Erzgebirge, sondern zeigt auch Parallelen in den argumentativen Schwerpunkten für oder gegen einen UNESCO-Status. Insbesondere die ökonomische Nutzbarmachung durch ideelle Aufwertung liegt noch vor dem Schutzgedanken im Hauptinteresse vieler Akteure. Aber ähnlich wie in Dresden hat die Diskussion über eine Weltkulturerbe-Nominierung auch eine Kontroverse über Zugehörigkeiten und Deutungsmacht über regionale Identitäten entfacht: Während manche ,ihre Heimat" nicht mit dem Begriff des Montanen assoziieren können oder wollen, fühlen andere ihre bergbauliche Tätigkeit aufgewertet.

\section{Zusammenführung der Untersuchungen}

In unserem interdisziplinären Beitrag haben wir das Cultural Heritage Regime und die Debatte um Cultural Property beleuchtet und im Zuge dessen auf die Parallelen in den Konstituierungsprozessen und auf die dabei möglichen Korrelationen hingewiesen. Das Changieren zwischen lokalen Strategien, wirtschaftlichen und rechtlichen Rahmenbedingungen bei der Inwertsetzung kultureller Elemente verstehen wir als kulturelle Praxis, die wir anhand empirischer Arbeiten auch in ihren Wechselwirkungen mit lokalen Identitätskonstruktionen untersuchen. Kultur als Ressource für ideelle und ökonomische Belange findet sich in beiden genannten Fallstudien wieder:

Im Entstehungsprozess der geplanten UNESCO-Industriekulturlandschaft Montanregion Erzgebirge lässt sich ein Wechselspiel diverser Interessenlagen ablesen, die sowohl die ökonomischen als auch ideellen Implikationen eines World Heritage umfassen. Im Spannungsfeld von Befürworter/innen und Gegner/innen der UNESCO-Nominierung ist die Frage nach der Deutungsmacht über die Vergangenheit und das Image der Region verknüpft mit wirtschaftlichen Belangen. Während die einen sich als progressiv verstehen und die montane Vergangenheit insbesondere des Uranbergbaus nicht noch international prämiert sehen möchten, sondern für die strukturschwache Region eine Zukunft in der Hochtechnologie als Silicon Mountain präferieren, wollen die anderen die Zeugnisse des jahrhundertelangen Bergbaus als universellen Wert für die Menschheit bewahren, verstehen es als Mittel der deutsch-tschechischen Völkerverständigung und als ökonomische Ressource. 
Gerade die wirtschaftliche Nutzbarmachung steht in der öffentlichen Argumentation für - als auch gegen - eine UNESCO-Nominierung an erster Stelle. Die von der sächsischen Regierung geforderte und in Studien positiv prognostizierte wirtschaftliche Rentabilität einer Zertifizierung ist den Befürworter/innen der Nominierung das stärkste Argument in der Auseinandersetzung mit den lokalen Akteuren, die eine wirtschaftliche Stagnation durch den konservatorischen Charakter des UNESCO-Heritage-Regimes befürchten. Die vor allem auf Basis wirtschaftlicher Interessen geführte Diskussion zeigt deutlich, dass in der derzeitigen Phase des Nominierungsprozesses wenige Berührungsängste mit der wirtschaftlichen Nutzbarmachung von Kultur bestehen. Der Hinweis des Leiters der UNESCO-Projektgruppe, dass das primäre Anliegen des UNESCO-Welterbegedankens doch das Schützen von Landschaften und Denkmälern ist, schließt nicht aus, dass die befürwortenden Akteure ein ausgeprägtes Bewusstsein für die wirtschaftliche Komponente einer UNESCO-Zertifizierung als Tourismusmarke haben.

Die ökonomischen Implikationen des Status Weltkulturerbe spielten auch in den Debatten um die Aberkennung des Dresdner Weltkulturerbetitels eine Rolle. Sowohl die Brückengegner/innen, die den Status als touristischen Standortfaktor als Argument gegen den Brückenbau einbrachten, als auch die Brückenbefürworter/innen, die auf die kulturelle Einmaligkeit und somit weltweite Anziehungskraft explizit ohne eines entsprechenden UNESCO Zertifikats hinwiesen, wurden in der Auseinandersetzung vorgebracht. Der rechtliche Rahmen auf internationaler und nationaler Ebene hingegen fokussiert den Anspruch des Schützens und Bewahrens ausgewählter Welterbestätten und nimmt die Mitgliedsstaaten in die Pflicht, Weltkulturerbe für zukünftige Generationen der gesamten Menschheit zu erhalten.

Die beiden Fallstudien zeigen, dass ein Potential des Status Weltkulturerbe darin besteht, dass Akteure oder Akteursgruppe durch die Auseinandersetzung mit dem Rechtsinstrument eine kulturellen Identität und Zugehörigkeit zu einer Kulturlandschaft oder Kulturerbe etablieren oder weiter konkretisieren. Auf dieser Basis können sich eigentumsähnliche Zugehörigkeitskonstruktionen bilden, die als Cultural Property diskutiert werden. 



\section{Teil 2}

Parameter des Schutzes von Cultural Property 



\title{
Die UNESCO-Konvention zum Schutz des immateriellen (Kultur-)Erbes der Menschheit von 2003: Öffnung des Welterbekonzepts oder Stärkung der kulturellen Hoheit des Staates?
}

\author{
Sven Mißling
}

Die UNESCO-Konvention zum Schutz des immateriellen Welterbes von 2003 wird als ein wichtiger Fortschritt im Bereich des internationalen Kulturgüterschutzes angesehen. Die mit ihr geschaffene Möglichkeit, neben materiellen Kulturgütern von herausragender Bedeutung für das kulturelle Erbe der Menschheit auch immaterielle kulturelle Ausdrucksformen von gleichem Rang und gleicher Bedeutung international rechtlich und institutionell zu schützen, wird als eine notwendige Anpassung des in der UNESCO bestehenden Kulturverständnisses an die Entwicklungen der Kulturwissenschaften in den letzten 20 Jahren und dementsprechend auch als eine konsequente Fortentwicklung der Wahrnehmung der der UNESCO durch ihre Verfassung ${ }^{1}$ zugewiesenen Aufgaben begriffen. $^{2}$

\footnotetext{
1 UNESCO Constitution vom 16.11.1945, in Kraft getreten am 04.11.1946. Siehe http://portal. unesco.org/en/ev.php-URL_ID=15244\&URL_DO=DO_TOPIC\&URL_SECTION=201.html (Zugriff am 04.04.2010).

2 Aikawa-Faure 2009, Odendahl 2005a. Vgl. auch: Sola 2008, Scovazzi 2008, sowie die Beiträge in: Bortolotto 2008, jeweils mit weiteren Nachweisen. Zur jüngeren Entwicklung des Welterbeschutzes unter dem Dach der UNESCO insgesamt: Bumbaru 2006.
} 


\section{Modernisierungstendenzen in der UNESCO: Die Öffnung des Welterbekonzepts zu Beginn des neuen Jahrtausends}

\subsection{Sektorielle Öffnung des Welterbekonzepts: Vom Schutz unbeweglicher Kulturgüter zur Anerkennung immaterieller Kulturpraxen}

Die Tätigkeit der UNESCO hat seit Beginn des neuen Jahrtausends starke Modernisierungsimpulse erfahren, die sich unter anderem in einer verstärkten Rechtsetzungstätigkeit der Organisation niedergeschlagen haben. Die bis dahin namentlich durch die UNESCO-Konvention zum Schutz des kulturellen und natürlichen Erbes der Menschbeit von 1972 geprägte Tätigkeit der $\mathrm{UNESCO}^{3}$ hat sich sowohl im Lichte kulturwissenschaftlicher Erkenntnisse als auch im Lichte der Entwicklung der Bedürfnisse der Staaten und der unterschiedlichen Träger von Kultur als sektoriell zu begrenzt erwiesen. Mit der Verabschiedung der Konvention zum Schutz des Unterwassererbes von $2001^{4}$, der Konvention zum Schutz des immateriellen Erbes der Menschbeit von $2003^{5}$ und schließlich der Konvention zum Schutz und zur Förderung der Vielfalt kultureller Ausdrucksformen von $2005^{6}$ haben die Mitgliedstaaten der UNESCO den rechtlichen, politischen und programmatischen Aktionsradius der Organisation nicht nur erweitert und an die modernen Bedürfnisse des Kultursektors angepasst, sondern zugleich wichtige neue international-rechtliche Schutzinstrumente geschaffen, die einen umfassenden Schutz von Kultur gewährleisten sollen.

Die Konvention zum Schutz des immateriellen Erbes der Menschheit von 2003 wird insoweit als ein wichtiger Baustein in einem modernen Konzept der UNESCO und vielfach auch als Meilenstein begriffen. ${ }^{7}$ Namentlich in außereuropäischen und nicht-westlich geprägten Kulturkreisen ist die Verabschiedung dieser

\footnotetext{
3 Vgl. Francioni 2008, Scovazzi 2008:37-78, Nafziger 2008:145-247, Musitelli 2002, Schorlemer 1992:128-149, Genius-Devime 1996. Auch: Berndt 1998:49-50, Odendahl 2005b:135-137, Fechner 1996.

${ }^{4}$ Convention on the Protection of the Underwater Cultural Heritage vom 02.11.2001, in Kraft getreten gem. ihrem Art. 27 am 2.1.2009 für diejenigen Staaten, die die Konvention vor dem 02.10.2008 ratifiziert haben. Zum aktuellen Ratifikationsstand und den derzeitigen Vertragsparteien siehe: http://portal.unesco.org/la/convention.asp?KO=1352\&language=E\&order=alpha\#1 (Stand: 03.03. 2010). Die Bundesrepublik Deutschland ist zzt. nicht Vertragspartei dieser Konvention.

${ }^{5}$ Convention for the Safeguarding of the Intangible Cultural Heritage vom 17.10.2003, in Kraft getreten am gem. ihrem Art. 34 am 20.04.2006 für diejenigen Staaten, die die Konvention vor dem 20.1.2006 ratifiziert haben. Zum aktuellen Ratifikationsstand und den derzeitigen Vertragsparteien siehe: http://portal.unesco.org/la/convention.asp?language $=\mathrm{E} \& \mathrm{KO}=17116 \# 1$. Die Bundesrepublik Deutschland ist z.Zt. nicht Vertragspartei der Konvention.

6 Convention on the Protection and Promotion of the Diversity of Cultural Expressions vom 20.10.2005, in Kraft getreten gem. ihrem Art. 29 am 18.03.2007 für diejenigen Staaten, die die Konvention bis zum 18.12.2006 ratifiziert haben. Zum aktuellen Ratifikationsstand und den derzeitigen Vertragsparteien siehe: http://portal.unesco.org/la/convention.asp?KO=31038 (Zugriff am 04.04. 2010). Die Bundesrepublik Deutschland hat die Konvention am 12.03.2007 ratifiziert, BGBl. II, S. 234.
}

${ }^{7}$ Aikawa-Faure 2009, Blake 2009, Blake 2008. 
Konvention vorangetrieben und begrüßt worden. ${ }^{8}$ Es ist wohl kein Zufall, dass zu den wichtigsten Promotoren dieser Konvention innerhalb der UNESCO namentlich ostasiatische Staaten wie beispielsweise Japan gehörten, deren Kulturverständnis - anders als in durch ein westlich-aufgeklärtes Kulturverständnis geprägten Gesellschaften - nicht auf der hegelianischen Vorstellung einer sich dialektischlinear vollziehenden Welt- und Kulturgeschichte, sondern auf dem Gedanken einer sich in sich stetig erneuernden, lebendigen und zeitgemäßen Ausdrucksformen manifestierenden, ideellen Überlieferung von Kultur beruht. ${ }^{9}$ Aus dieser Perspektive hat sich die Konzeption des in überlieferten Denkmälern und historischen Stätten („Erinnerungsorten“10) materialisierten Welterbes, wie sie in der UNESCOKonvention von 1972 ihren Ausdruck gefunden hat, als zu eng erwiesen. ${ }^{11}$

Das Konzept eines immateriellen Kulturerbes ist auch in solchen westlichen Staaten, deren Selbstverständnis in besonderem Maße auf der Idee einer spezifisch nationalen Kultur beruht, auf Zustimmung gestoßen. Staaten wie Frankreich, Spanien oder Italien, aber auch die baltischen und andere mittel- und osteuropäische Staaten, in denen vor dem Hintergrund der kommunistischen Geschichtserfahrungen immaterielle Kulturpraxen eine besondere Bedeutung für die (Wieder-) Herausbildung nationaler Identität haben dürften und die damit nicht zufällig zu den ersten Staaten zu zählen sind, die die UNESCO-Konvention von 2003 ratifiziert haben, können hierfür als Beispiele angeführt werden. ${ }^{12}$

${ }^{8}$ Srinivas 2008, Bortolotto 2008:7-42. Vgl. dazu auch die jeweiligen Darstellungen der Inhalte, Ziele
und der Entstehungsgeschichte der Konvention auf der Homepage der UNESCO, http://
www.unesco.org/culture/ich/index.php?pg=00002, und auf der Homepage der Deutschen UNES-
CO-Kommission e.V., http://www.unesco.de/immaterielles-kulturerbe.html?\&L=0 (Zugriff am
04.04.2010), jeweils mit weiteren Nachweisen.
9 Vgl. die Begriffsdefinition des "kulturellen Erbes" i.S.d. Konvention, Art. 2 Ziffer 1, insbes. Satz 2.
Dazu näher: Sola 2008:494-450. Insbesondere zum Einfluss Japans auf die Entstehung der UNES-
CO-Konvention von 2003 vgl.: Bortolotto 2008:37-40, Isomura 2004:41-48, Watanabe 2006, Ueki
2006.
${ }^{10}$ Vgl. dazu den von dem französischen Historiker und Sozialwissenschaftler Pierre Nora entwickel-
ten Begriff der „Erinnerungsorte“, welcher sich freilich schon in seiner Grundanlage nicht allein auf
geographische Orte begrenzt und insofern - wie nunmehr auch der Kulturbegriff im Rahmen der
UNESCO - ein weites intellektuelles Konzept darstellt. Dazu: Nora 1984, 1998, Nora und François
2005. Vgl. auch: Cornu 2004:197.
11 Dass nicht-westlich geprägte Kulturerbevorstellungen offensichtlich nur schwer mit der herge-
brachten Welterbekonzeption der Konvention von 1972 in Einklang zu bringen waren, lässt sich in
der geografischen und regionalen Verteilung der von der UNESCO bislang auf die Welterbeliste
aufgenommenen, 890 materiellen Kulturdenkmäler nachvollziehen. Die ganz überwiegende Anzahl
der anerkannten Welterbestätten befindet sich demnach in Europa sowie Nord- und Mittelamerika.
Eindrucksvoll nachgewiesen u.a. in der Liste und der interaktiven, globalen Karte des (materiellen)
Kulturerbes auf der UNESCO-Homepage: http://whc.unesco.org/en/list bzw. http://whc.unesco.
org/en/254/ (Zugriff am 04.04.2010).
12 Vgl. dazu http://portal.unesco.org/la/convention.asp?language=E\&KO=17116\#1 (Zugriff am
04.04.2010).
So hoben So haben seit der Annahme bzw. Ratifikation der Konvention bspw. Frankreich und Spanien je 4, Belgien 3, Italien 2, mittel- und osteuropäische Staaten wie Kroatien 7, Litauen 2, Estland 3, Lettland 1, Rumänien 2, Albanien 1, Bulgarien 2, Tschechien und die Slowakei jeweils 1, Ungarn 1, die Türkei 5, ehemalige Sowjet-Staaten wie Usbekistan 4, Aserbaidschan 3, Kirgisien 2, Georgien und Tadschiki- 


\subsection{Erweiterung des Weltkulturerbebegriffs nach der Welterbekonvention von 1972}

Parallel zu der sektoriellen Öffnung des Welterbekonzepts durch die angesprochenen Konventionen haben sich auch im Bereich des materiellen Kulturerbeschutzes Entwicklungen Bahn gebrochen, die ein modernes Kulturverständnis widerspiegeln: Der Begriff des materiellen Weltkulturerbes hat zuletzt eine Erweiterung erfahren, die sich in der Anpassung der Kriterien der Operational Guidelines zur Welterbekonvention von $1972^{13}$ manifestiert. Das Welterbe-Komitee der UNESCO erhebt unter dieser Konvention inzwischen nicht nur Denkmäler oder Stätten zum Weltkultur- oder Weltnaturerbe, die aus historischen, architektonischen, musealen oder naturhistorischen Gründen als solche von besonderem kulturellem Wert sind und daher erhaltenswert erscheinen. ${ }^{14}$ Mit der 2005 in den Operational Guidelines geschaffenen Möglichkeit, bestimmte Stätten als so genannte Cultural Landscapes zum Welterbe zu ernennen ${ }^{15}$, trägt die UNESCO der in den Kulturwissenschaften seit längerem etablierten Erkenntnis Rechnung, dass die Bewahrung von kulturellem und natürlichem Erbe sich nicht in der Musealisierung der Welterbestätten erschöpfen kann, sondern dass die „Patrimonialisierung“ von Kultur und Natur, wenn sie den Bedürfnissen der Menschen und der sozialen und politischen Realitäten entsprechen will, stets auch in lebendigen sozialen Kontexten gesehen und in solchen umgesetzt werden muss. ${ }^{16}$ Auch insofern kann von einer Modernisierung der Praxis der UNESCO seit dem Jahrtausendwechsel gesprochen werden. ${ }^{17}$

\section{3 Öffnung des Welterbekonzepts zugunsten der gesellschaftlichen Träger von Kultur in der Konvention von 2003}

Die UNESCO-Konvention zum Schutz des immateriellen Erbes der Menschheit von 2003 ist auch in anderer Hinsicht begrüßt worden: In der Präambel, im Begriff des „immateriellen Erbes“ im Sinne der Konvention (Art. 2 Ziffer 1 und 2) sowie

\footnotetext{
stan jeweils 1, Russland selbst 2, hingegen die VR China 26, Japan 16, Korea 8 und Indien 5 immaterielle Weltkulturerbegüter erfolgreich für die repräsentative Liste nominiert.

13 Operational Guidelines for the Implementation of the World Heritage Convention, UNESCODok. WHC.08/01, http://whc.unesco.org/en/guidelines (Stand: Januar 2008).

14 Vgl. Art. 1 und 2 des Übereinkommens zum Schutz des Kultur- und Naturerbes der Welt (Convention Concerning the Protection of the World Cultural and Natural Heritage, Welterbekonvention von 1972) vom 23.11.1972, in Kraft getreten am 17.12.1975, für die Bundesrepublik Deutschland am 23.11.1976, BGBl. 1977 II S. 213 und S. 215.

15 Vgl. \47 der Operational Guidelines zur Welterbekonvention von 1972 und Annex 3 zu den Operational Guidelines, „Guidelines on the Inscription of Specific Types of Properties on the World Heritage List", UNESCO-Dok. WHC.08/01, http://whc.unesco.org/en/guidelines (Zugriff am 04.04.2010).

16 Hönes 2009:118-121, Whitby-Last 2008.

17 Vgl. darüber hinaus auch zur Zukunft des Welterbesystems im Zusammenhang mit dem materiellen Kulturerbe i.S.d. UNESCO-Konvention von 1972: Schäfer 2009.
} 
auch in Art. 11 und 15 der Konvention ausdrücklich vorgesehenen Einbeziehung und Beteiligung lokaler, ethnischer oder kultureller Gruppen ${ }^{18}$ und anerkannter Nichtregierungsorganisationen (NGOs) im Zusammenhang mit der Identifizierung und Nominierung immateriellen Kulturerbes wird im Schrifttum heute ein bedeutender Fortschritt gegenüber der in der Konvention von 1972 zum Ausdruck kommenden Welterbe-Konzeption gesehen. ${ }^{19}$ Mitunter ist insoweit sogar ein Paradigmenwechsel der UNESCO reklamiert worden, der darin erkennbar werden soll, dass bei der Benennung und Ernennung kultureller Ausdrucksformen zum immateriellen Weltkulturerbe der territoriale und nationale Zusammenhang an Bedeutung verliere und die Bedeutung der kulturellen Praxen für die gesamte Menschheit in den Vordergrund gerückt werde. ${ }^{20}$

\section{Die starke Position der Staaten in der UNESCO: Völkerrechtliche Stärkung des Staates im Bereich der Kultur?}

Eine solche überschwängliche Bewertung der UNESCO-Konvention von 2003 kann aus einer völkerrechtlichen Perspektive, die auch die Praxis des Nominierungs- und Ernennungsprozesses innerhalb der UNESCO in Bezug auf das (immaterielle) Weltkulturerbe in den Blick nimmt, allerdings nicht einschränkungslos geteilt werden.

$\mathrm{Zu}$ betonen ist hier zunächst, dass die UNESCO-Konvention von 2003 - neben den weiteren genannten Neuerungen in Bezug auf das Welterbe-Konzept bereits als solche eine wichtige Fortentwicklung im Bereich des internationalen Kultur(-güter)-schutzrechts darstellt. Auch die in ihr an mehreren Stellen enthaltene Öffnung zugunsten der Träger und Akteure des immateriellen Kulturerbes und die ihr immanente, prinzipielle Berücksichtigung sozialer Bedingungen bei der Ernennung zum immateriellen Weltkulturerbe sind aus rechtlicher Perspektive als Fortschritt zu begrüßen. ${ }^{21}$ Fraglich bleibt jedoch, ob und ggf. inwiefern hierin eine

\footnotetext{
18 Art. 11 lit. a) der Konvention spricht insofern von den „relevanten Gemeinschaften, Gruppen und Nichtegierungsorganisationen“.

${ }^{19}$ Seeger 2009:124-126, Hafstein 2009, Blake 2009, Blake 2008.

20 Bortolotto 2008:16-37. Zum Paradigmenwechsel in Hinblick auf das Kulturerbe der UNESCO vgl. aber auch: Jacobs 2007.

${ }^{21}$ Nach Art. 2 Ziffer 1 Satz 1 sind unter „kulturellem Erbe“ i.S.d. Konvention die Praktiken, Darbietungen, Ausdrucksformen, Kenntnisse und Fähigkeiten - sowie die damit verbundenen Instrumente, Objekte, Artefakte und Kulturräume - zu verstehen, die Gemeinschaften, Gruppen und gegebenenfalls Individuen als Bestandteil ihres Kulturerbes ansehen. Art. 2 Ziffer 1 lautet weiter: „Dieses immaterielle Kulturerbe, das von einer Generation an die nächste weitergegeben wird, wird von Gemeinschaften und Gruppen in Auseinandersetzung mit ibrer Umwelt, ihrer Interaktion mit der Natur und ihrer Geschichte ständig neu geschaffen und vermittelt ihnen ein Gefühl von Identität und Kontinuität. Auf diese Weise trägt es zur Förderung des Respekts vor der kulturellen Vielfalt und der menschlichen Kreativität bei. Im Sinne dieser Konvention findet nur dasjenige immaterielle Kulturerbe Berücksichtigung,
} 
so weit reichende qualitative Änderung des Welterbekonzepts der UNESCO gesehen werden darf, dass von einem wirklichen Paradigmenwechsel die Rede sein kann.

Eine genauere Betrachtung der Konventionsinhalte zeigt, dass am Grundprinzip der UNESCO - nämlich dass in letzter Konsequenz die Staaten die Träger der Verantwortung für das Welterbe und damit die stärksten Akteure innerhalb der Organisation sind - durch die Konvention von 2003 nichts geändert worden ist. Zwar binden sich die Vertragsstaaten durch ihren Beitritt zu der Konvention nach Maßgabe ihrer Bestimmungen hinsichtlich der Ausübung ihrer (kulturellen) Hoheitsrechte. Im Ergebnis enthält die Konvention aber keineswegs eine umfassende Einschränkung oder gar Durchbrechung der staatlichen Souveränität im Kulturbereich zugunsten der UNESCO.

Der vorliegende Beitrag vertritt dementsprechend die These, dass nach der UNESCO-Konvention von 2003 die Rolle der Staaten und des Staates im Zusammenhang mit der Nominierung und Ernennung eines immateriellen Kulturerbes gegenüber dem Regime der Ernennung und des Schutzes eines materiellen Weltkulturerbes, wie er nach der Konvention von 1972 gewährleistet wird, keineswegs an Stärke eingebüßt hat. Dass mit der UNESCO-Konvention von 2003 die Bedeutung der Staaten zugunsten innerstaatlicher gesellschaftlicher, kultureller oder ethnischer Gruppen, die als Träger der von der Patrimonialisierung betroffenen Praxen angesehen werden können, effektiv zurückgedrängt wird, erscheint nur auf den ersten Blick so. Es wird hier vielmehr aufgezeigt werden, dass es nach wie vor die Staaten sind, denen in ihrer Eigenschaft als Vertragsparteien und damit als den unmittelbar aus der Konvention Berechtigten und Verpflichteten die zentrale Rolle bei der Identifizierung, der Benennung und der Durchsetzung des Schutzes zugunsten des immateriellen Weltkulturerbes zukommt. Der Text, die Systematik und die Konzeption der Konvention von 2003 selbst zeigen, dass in letzter Konsequenz die Staaten über das (immaterielle) Kulturerbe verfügen. Dies reicht soweit, dass im Kontrast zu der mitunter durchscheinenden Einschätzung, die WelterbeKonzeption erfahre durch die Konvention eine „Sozialisierung“ zugunsten der betroffenen Kulturträger ${ }^{22}$ - die UNESCO-Konvention von 2003 den Staten gerade erst eine völkerrechtliche Möglichkeit eröffnet, über das Instrumentarium des immateriellen Kulturerbes einen kulturellen Bereich für sich zu vereinnahmen, der bis dato der staatlichen Souveränität und damit auch der Kontrolle des Staates weitgehend entzogen war. Es darf nämlich nicht übersehen werden, dass zumindest auf der Ebene des Völkerrechts durch die Schaffung eines immateriellen Kul-

das mit den bestehenden internationalen Rechtsinstrumenten im Bereich der Menschenrechte sowie der Forderung nach gegenseitiger Achtung zwischen den Gemeinschaften, Gruppen und Individuen und einer nachhaltigen Entwicklung im Einklang steht" (sämtliche Hervorhebungen nicht im Original).

22 Zur Bewertung der Rolle der betroffenen Kulturträger vgl. u.a.: Blake 2008, Blake 2009, Hafstein 2009, Sola 2008:495. Vgl. auch: Marrie 2009. Vgl. außerdem: Grenet 2008:90-93. Außerdem: Schäfer 2009:247, dort jedoch in Bezug auf die Welterbekonvention von 1972. 
turerbes dem Staat erst vielfach die Möglichkeit eröffnet wird, über den Weg der Benennung und Ernennung bestimmter kultureller Ausdrucksformen und Praxen zum immateriellen Weltkulturerbe diese seiner Regulierung nach Maßgabe der Konvention zu unterwerfen. Dies soll im Folgenden näher beleuchtet werden. Es ist zu betonen, dass eine Tendenz, wie sie hier aufgezeigt werden wird, weder zwingend noch rechts- oder kulturpolitisch wünschenswert ist. Nichtsdestoweniger darf eine durch die Analyse der Konvention inspirierte, realistische Einschätzung der Zukunft des immateriellen Welterbes nicht darüber hinwegsehen, dass mit der Konvention von 2003 gewisse Verwerfungen auf diesem Feld vorprogrammiert sind.

\section{Die Rolle der Staaten bei der Ernennung und dem Schutz des immateriellen Kulturerbes nach den Bestimmungen der Konvention von 2003}

Die Rolle der Staaten bei der Ernennung und dem Schutz des immateriellen Kulturerbes wird an mehreren Stellen in der Konvention von 2003 selbst geregelt.

\subsection{Die Staaten als Herren der Konvention}

Zunächst ist hervorzuheben, dass den Vertragsstaaten gem. Art. 4 der Konvention bereits insoweit die zentrale Rolle zukommt, als sie mit der Vollversammlung das „souveräne Organ“ der Konvention stellen (Art. 4 Satz 2). ${ }^{23}$ Damit wird klargestellt, dass die Staaten die Herren der Konvention sind und als solche alle zentralen Entscheidungen treffen. Die Vollversammlung entscheidet namentlich über Änderungen der Konvention (Art. 38), nimmt regelmäßig die Rechenschaftsberichte des Komitees ${ }^{24}$ entgegen (Art. 8 Ziffer 1), das durch entsandte Vertreter der Vertragsstaaten besetzt wird und dessen Mitglieder von der Vollversammlung nach den Grundsätzen einer gleichgewichtigen geographischen Verteilung und ausgewogenen Rotation für jeweils vier Jahre gewählt werden (Art. 6). Die Vollversammlung genehmigt insbesondere auch die operationellen Richtlinien zur Umsetzung der Konvention (Art. 7 lit. e)) sowie die Verwendung der Mittel des Fonds gem. Art. 25 der Konvention (Art. 7 lit. c)) und übt damit in letzter Instanz eine effektive Kontrolle über die Arbeit des Komitees und die Vergabe von finanziellen Mitteln nach Maßgabe der Konvention aus. Die Einzelheiten des Verfahrens in der Vollversammlung, die grundsätzlich in öffentlicher Sitzung tagt und in der jeder Vertragsstaat das gleiche Recht hat, durch einen Vertreter an den Entscheidungen und Abstimmungen teilzunehmen, werden durch ihre Geschäftsordnung näher gere-

\footnotetext{
23 Srinivas 2008:538.

${ }^{24}$ Gemeint ist das „Zwischenstaatliche Komitee für den Schutz des immateriellen Kulturerbes“ gem. Art. 5 der Konvention.
} 
gelt. ${ }^{25}$ In der Vollversammlung hat jeder Vertragsstaat eine Stimme (Art. 12.1 der Geschäftsordnung). Entscheidungen der Vollversammlung werden vorbehaltlich abweichender Bestimmungen, die ausdrücklich in der Geschäftsordnung geregelt sind, grundsätzlich mit der Mehrheit der Stimmen der anwesenden und an der Abstimmung teilnehmenden Vertragsstaaten getroffen (Art. 12.3 der Geschäftsordnung). Änderungen der Konvention selbst können allerdings nur mit Zweidrittelmehrheit der anwesenden und abstimmenden Vertragsstaaten beschlossen werden (Art. 38 Ziffer 2 der Konvention). ${ }^{26}$

\subsection{Aufgaben und Pflichten der Staaten beim Schutz des immateriellen Erbes}

Über ihre zentrale Stellung als Herren der Konvention hinaus wirken die Staaten beim Schutz des immateriellen Kulturerbes mit. Die Konvention von 2003 trifft Regelungen über die Aufgaben und die Pflichten der Staaten auf nationaler wie auch auf internationaler Ebene.

\section{Schutz des immateriellen Erbes auf nationaler Ebene}

Die Konvention verpflichtet die Vertragsstaaten zunächst allgemein, die erforderlichen Maßnahmen zum Schutz des in ihrem Hoheitsgebiet befindlichen immateriellen Kulturerbes zu ergreifen (Art. 11 lit. a)). Unter „Schutz“ im Sinne der Konvention sind gem. deren Art. 2 Ziffer 3 Maßnahmen zu verstehen, die auf die Sicherung der Lebensfähigkeit des immateriellen Kulturerbes gerichtet sind, was nach der Legaldefinition in dieser Vorschrift die Identifizierung, die Dokumentation, die Erforschung, die Bewahrung, den Schutz, die Förderung, die Aufwertung, die Weitergabe, insbesondere durch formale und informelle Bildung, sowie die Neubelebung der verschiedenen Aspekte dieses Erbes einschließt. Namentlich der Identifizierung und Benennung der Elemente des immateriellen Erbes, welche sich in ihrem Hoheitsgebiet befinden, durch die Vertragsstaaten kommt nach der Konvention besondere Bedeutung zu (Art. 11 lit. b)). Sie wird durch die Erstellung von regelmäßig zu aktualisierenden Inventarlisten und periodischen Berichten der Staaten an das Komitee nach Art. 29 der Konvention sichergestellt (Art. 12). ${ }^{27}$

\footnotetext{
25 General Assembly Rules of Procedure von 2006, http://www.unesco.org/culture/ich/index.php? pg=00071 (Zugriff am 04.04.2010).

${ }^{26}$ Das Verfahren der Vertragsänderung ist im Einzelnen in Art. 38 der Konvention ausführlich geregelt. Dabei ist darauf hinzuweisen, dass gem. Ziffer 2 dieses Artikels beschlossene Änderungen der Konvention der Annahme, der Genehmigung, des Beitritts oder der Ratifikation durch die Vertragsstaaten bedürfen, um für diese verbindlich zu werden (Art. 38 Ziffer 3 und 4). Sofern es hieran in Bezug auf einzelne Vertragsstaaten fehlt, ist für diese Staaten nur diejenige Fassung der Konvention bindend, welcher sie gemäß den Regeln des Völkerrechts und ihres nationalen (Verfassungs-)Rechts zugestimmt haben.

${ }^{27} \mathrm{Zu}$ den nationalen Inventarlisten beispielhaft näher: Grenet 2008.
} 
Art. 13 der Konvention legt weitere Pflichten der Vertragsstaaten zum Schutz des immateriellen Erbes fest. Danach trifft jeden Staat die Verpflichtung, zur Sicherstellung des Schutzes, der Entwicklung und der Erhaltung des Wertes des in seinem Hoheitsgebiet befindlichen immateriellen Kulturerbes Anstrengungen zu unternehmen, eine allgemeine Politik zu verfolgen, die darauf gerichtet ist, die Funktion des immateriellen Kulturerbes in der Gesellschaft aufzuwerten und den Schutz dieses Erbes in Programmplanungen einzubeziehen (Art. 13 lit. a)), eine oder mehrere Fachstellen zu benennen oder einzurichten, die für den Schutz des in seinem Hoheitsgebiet befindlichen immateriellen Kulturerbes zuständig ist/sind (Art. 13 lit. b)), wissenschaftliche, technische und künstlerische Untersuchungen sowie Forschungsmethoden im Hinblick auf den wirksamen Schutz des immateriellen Kulturerbes, insbesondere des gefährdeten immateriellen Kulturerbes, zu fördern (Art. 13 lit. c)), geeignete rechtliche, technische, administrative und finanzielle Maßnahmen zu ergreifen, die darauf gerichtet sind, den Auf- oder Ausbau von Ausbildungseinrichtungen für die Verwaltung des immateriellen Kulturerbes sowie die Weitergabe dieses Erbes im Rahmen von Foren und anderen Örtlichkeiten zu fördern, die dazu bestimmt sind, dieses Erbe darzustellen, wiederzugeben und zum Ausdruck zu bringen oder die darauf gerichtet sind, den Zugang zum immateriellen Kulturerbe zu gewährleisten. Gleichzeitig sind dabei die herkömmlichen Praktiken zu achten, die für den Zugang zu besonderen Aspekten dieses Erbes gelten. Außerdem sind Dokumentationszentren für das immaterielle Kulturerbe einzurichten und der Zugang zu diesen ist zu erleichtern (Art. 13 lit.d)). Art. 14 der Konvention verpflichtet die Staaten zu weiteren Maßnahmen auf dem Gebiet der Bildung und Erziehung sowie zur Sensibilisierung und Stärkung professioneller Kapazitäten im Bereich des immateriellen Kulturerbes. Art. 15 enthält schließlich eine allgemeine Verpflichtung der Vertragsstaaten, sich um eine möglichst weitreichende Beteiligung der Gemeinschaften, Gruppen und gegebenenfalls der Individuen, die das immaterielle Erbe schaffen, erhalten und weitergeben, und um ihre aktive Einbeziehung in das Management des Kulturerbes zu bemühen.

Neben der allgemeinen Schutzverpflichtung aus Art. 11 ist die Pflicht zur Beteiligung der relevanten Kulturträger eine der zentralen Regelungen der Konvention im Zusammenhang mit dem Schutz des immateriellen Erbes auf der nationalen Ebene. Ihre Tragweite muss richtig eingeschätzt werden: Zwar haben die Staaten schon bei der Identifizierung des immateriellen Erbes und seiner Elemente die relevanten Gemeinschaften, Gruppen und NGOs zu beteiligen ${ }^{28}$, jedoch wird aus Art. 11 der Konvention auch deutlich, dass unter Anwendung des Kulturerbebegriffs aus Art. 2 Ziffer 1 ein doppelter Einschätzungsspielraum der Staaten besteht. Wenngleich nämlich nach Art. 2 Ziffer 1 Satz 1 der Konvention das Selbstverständnis der relevanten Kulturträger für den Begriff und den Inhalt des immateriel-

\footnotetext{
28 Art. 11 lit. b) der Konvention. Vgl. dazu im Einzelnen $\$ \int 76$ bis 95 der Operational Directives for the Implementation of the Convention for the Safeguarding of the Intangible Cultural Heritage vom Juni 2008, http://www.unesco.org/culture/ich/doc/src/00410-EN.pdf (Zugriff am 04.04.2010).
} 
len Kulturerbes maßgeblich ist ${ }^{29}$, so wird dies im Ergebnis doch zugunsten der Staaten und ihrer Regierungen bzw. der Fachadministrationen als den für die Umsetzung der Konvention innerstaatlich zuständigen Stellen eingeschränkt. Und zwar gilt dies insofern, als es zum einen den Staaten obliegt, aufgrund der eigenen kulturpolitischen Einschätzungen die in ihrem Hoheitsgebiet insofern relevanten Kulturträger im Sinne der Konvention auszuwählen, und zum anderen in Art. 11 der Konvention wohl eine Verpflichtung der Staaten gesehen werden muss, der diese lediglich nach pflichtgemäßem Ermessen nachkommen müssen. Materiellinhaltliche Kriterien und konkrete sachliche Maßstäbe dafür, wann eine bestimmte kulturelle Praxis als ein Element des immaterielles Kulturerbes zu benennen und mit konkreten Maßnahmen zu schützen ist, stellen weder die Konvention selbst noch die 2008 von der Vollversammlung angenommenen operationellen Ricbtlinien zur Umsetzung der Konvention ${ }^{30}$ auf. Es ist auch hervorzuheben, dass die operationellen Richtlinien zwar die Beteiligung relevanter Kulturträger und NGOs als solche verpflichtend vorsehen und die Modalitäten der Beteiligung näher ausgestalten. Die Stellungnahmen und Empfehlungen der auf diese Weise Beteiligten binden die Vertragsstaaten jedoch nicht hinsichtlich der Umsetzung und Verwirklichung von Schutzmaßnahmen. Etwas anderes gilt nur, wenn dies durch das nationale Recht angeordnet wird.

Damit verbleibt prinzipiell ein nicht unerheblicher, kulturpolitischer Einschätzungsspielraum der Staaten und ihrer Autoritäten, dahingehend zu entscheiden, welche Elemente des immateriellen Erbes von ihnen als solche identifiziert und folglich überhaupt geschützt werden.

Zusätzlich räumt namentlich Art. 11 lit. a) der Konvention den Staaten einen äußerst weiten Ermessensspielraum ein, welche konkreten Schutzmaßnahmen sie auf der nationalen Ebene als erforderlich für den Schutz des immateriellen Erbes ansehen und daher ergreifen wollen. Die Spannbreite möglicher Maßnahmen ist weit und kann von den in den nachfolgenden Konventionsbestimmungen näher ausgeführten Instrumenten bis hin zu detaillierten, nationalen legislativen oder administrativen Maßnahmen (bspw. etwa durch Regelungen im Bereich des Rechts des Geistigen Eigentums, des Urheberrechts oder aber auch durch konkrete administrative Eingriffe oder Regulierungen bei der Ausübung kultureller Praxen bis hin zum Verbot der Aneignung durch Dritte oder Verfälschungsverbote) reichen. Die in Art. 11 lit b), 12 bis 14 der Konvention aufgeführten, weiteren Pflichten der Staaten benennen Minimalmaßnahmen, die die Staaten zum Schutz des immateriellen Erbes auf nationaler Ebene unter Beachtung der ihnen im Weiteren eingeräumten Spielräume pflichtgemäß ergreifen müssen. Eine nähere Betrachtung ihrer Inhalte und namentlich die Einsicht in die bei einer genaueren Lektüre ihres Wortlautes erkennbaren, unterschiedlichen Grade rechtlicher Verbindlichkeit hinsicht-

\footnotetext{
${ }^{29}$ Sola 2008:495.

30 Operational Directives for the Implementation of the Convention for the Safeguarding of the Intangible Cultural Heritage vom Juni 2008, http://www.unesco.org/culture/ich/doc/src/00410EN.pdf (Zugriff am 04.04.2010).
} 
lich der einzelnen Maßnahmen zeigen jedoch, dass die Konvention den Staaten weite Spielräume eröffnet. Während die Minimalverpflichtungen aus Art. 11 lit. b) und 12 der Konvention durchaus verbindlichen Charakter entfalten (sich indes der Sache nach lediglich auf grundlegende Maßnahmen der Staaten zur Prüfung, Identifizierung und Dokumentation von auf ihrem Hoheitsgebiet vorhandenem immateriellem Kulturerbe beziehen), trifft die Staaten nach Art. 13 und 14 der Konvention lediglich die Pflicht „Anstrengungen zu unternehmen“ bzw. sich unter Einsatz der geeigneten Mittel „zu bemühen“, die dort angesprochenen, entsprechenden Maßnahmen zu ergreifen. Die Beurteilung der „Eignung“ dieser Maßnahmen im konkreten Zusammenhang bleibt abermals den Staaten selbst überlassen.

Darüber hinaus fehlt es in der Konvention auch an Durchsetzungsmechanismen und Sanktionen, um die Staaten im Fall der Nichtbeachtung der Pflichten aus Art. 11 ff. der Konvention zur effektiven Umsetzung der Konventionspflichten auf der nationalen Ebene zu bewegen. Die in Abschnitt V der Konvention in Art. 19 ff. getroffenen Regelungen der internationalen Zusammenarbeit und der internationalen Unterstützung, die zwar als Beispiel für eine verstärkte Wahrnehmung gemeinsamer und gegenseitiger Verantwortung bei der Umsetzung der Konventionsinhalte durch die Vertragsstaaten angesehen werden können, sehen insoweit keine entsprechenden unmittelbaren oder mittelbaren Durchsetzungsmechanismen vor, die auch ohne Beteiligung oder gar gegen den Willen des betroffenen Staates von Seiten der UNESCO und der Konventionsorgane oder seitens der anderen Vertragsstaaten ergriffen werden könnten. ${ }^{31}$

\section{Schutz, des immateriellen Erbes auf internationaler Ebene}

Auf der internationalen Ebene stellt die repräsentative Kulturerbeliste nach Art. 16 das zentrale Instrument der UNESCO-Konvention von 2003 dar. ${ }^{32}$ Das Komitee stellt nach Art. 16 Ziffer 1 auf Vorschlag der Vertragsstaaten eine repräsentative Liste des immateriellen Kulturerbes der Menschheit zusammen, aktualisiert und veröffentlicht diese. Wie die Welterbeliste nach der UNESCO-Konvention zum Schutz des materiellen Kulturerbes von 1972 hat auch diese Liste vor allem eine symbolische und repräsentative Bedeutung, auch wenn sich hier wie dort aufgrund der weiteren Bestimmungen der Konventionen gewisse Rechte und Pflichten der Staaten und der UNESCO selbst an die Aufnahme in die Welterbelisten knüpfen. ${ }^{33}$ In struktureller Parallelität zur Welterbekonvention von 1972 wird die repräsenta-

\footnotetext{
31 Hönes 2009:168.

32 Sola 2008:500-502, Srinivas 2008:540-541, Blake 2008. Kritischer: Kirshenblatt-GimbleTt 2004:57, Hafstein 2009.

${ }^{33}$ Neben den in den Konventionen selbst genannten Schutzmaßnahmen, die die Staaten in pflichtgemäßem Ermessen zu ergreifen haben, sind insbesondere die ebenfalls direkt aus den Konventionen folgenden Berichtspflichten gegenüber den Konventionsorganen sowie die gleichfalls in pflichtgemäßem Ermessen, jedoch unter einem weiten Vorbehalt hinsichtlich des Entschließungsspielraums stehende Teilnahme an den vorgesehenen, internationalen Programmen der UNESCO zum Schutz des Kulturerbes zu erwähnen.
} 
tive Liste des immateriellen Kulturerbes durch eine weitere Liste, nämlich diejenige des dringend schutzbedürftigen immateriellen Erbes, ergänzt (Art. 17 der Konvention).

Die materiellen Kriterien für die Benennung und die Aufnahme kultureller Praxen oder Ausdrucksformen auf die repräsentative Liste werden vom Komitee erarbeitet und von der Vollversammlung genehmigt (Art. 16 Ziffer 2, Art. 7 lit. g) i)). Sie werden unter Bezugnahme auf die in Art. 2 Ziffer 1 der Konvention enthaltene Definition des ,immateriellen Kulturerbes“ in $\int 19$ der operationellen Richtlinien von 2008 (R.1 bis R.5) kumulativ aufgezählt ${ }^{34}$, wo auch Einzelheiten des Benennungs- und Aufnahmeverfahrens geregelt sind (\$S 20-33 der Richtlinien). Bei der Lektüre der einschlägigen Regelungen fällt abermals die starke Stellung der Vertragsstaaten auf: Ihnen kommt nach Art. 16 Ziffer 1 i.V.m. Art. 7 lit. g) i) das Vorschlagsmonopol bezüglich des auf die repräsentative Liste aufzunehmenden immateriellen Kulturerbes zu. Zwar entscheidet über die Aufnahme im Ergebnis das Komitee (Art. 7 lit. g) i) der Konvention). Dadurch, dass die Staaten zuvor das zu listende immaterielle Kulturerbe nach Art. 11 der Konvention identifizieren, dokumentieren, in die nationalen Inventarlisten nach Art. 12 der Konvention aufnehmen und schließlich dem Komitee formell zur Prüfung der Aufnahme in die repräsentative Liste vorschlagen müssen ${ }^{35}$, sind im Wesentlichen sie es, die bis zu einem gewissen Punkt die effektive Kontrolle über den Auswahl- und Ernennungsprozess haben. Dass sie auch insoweit zunächst uneingeschränkte Herren der Konvention und des Verfahrens sind, zeigt sich unter anderem daran, dass nach $\int$ 22 der operationellen Richtlinien von 2008 der benennende Staat solange die Nominierung zum immateriellen Kulturerbe jederzeit zurückziehen kann, bis die Evaluation der benannten kulturellen Praxis durch das Komitee begonnen hat. ${ }^{36}$ Aus dem Wortlaut dieser Bestimmung ist zu schließen, dass erst ab diesem Zeitpunkt die Kontrolle über die Aufnahme auf die repräsentative Liste und den Status als repräsentatives immaterielles Kulturerbe in die Hände des Komitees gelegt wird

\footnotetext{
34 \$19 der Operational Directives von 2008 lautet: "In nomination files, the submitting States Parties will be requested to demonstrate that an element proposed for inscription on the Representative List satisfies all of the following criteria: R.1: The element constitutes intangible cultural heritage as defined in Article 2 of the Convention. R.2: Inscription of the element will contribute to ensuring visibility and awareness of the significance of the intangible cultural heritage and to encouraging dialogue, thus reflecting cultural diversity worldwide and testifying to human creativity. R.3: Safeguarding measures are elaborated that may protect and promote the element. R.4: The element has been nominated following the widest possible participation of the community, group or, if applicable, individuals concerned and with their free, prior and informed consent. R.5: The element is included in an inventory of the intangible cultural heritage present in the territory(ies) of the submitting State(s) Party(ies), as defined in Articles 11 and 12."

${ }^{35}$ Das Ergreifen von Maßnahmen auf der nationalen Ebene gem. Art. 11 und 12 der Konvention ist gem. \19 R.5 der operationellen Richtlinien Voraussetzung für die Aufnahme auf die repräsentative Liste nach Art. 16 der Konvention. Die Form der Benennung wird durch $\ 21$ der operationellen Richtlinien geregelt.

36 Vgl. dazu \26 der operationellen Richtlinien. Dabei ist zu beachten, dass die Evaluation durch das Komitee im Sinne der Vorschrift noch nicht bereits mit der Erstellung der Gutachten und Empfehlungen der sogen. subsidiary bodies (Expertengruppen), sondern erst mit deren Evaluierung durch das Komitee beginnt.
} 
und namentlich letztere von dem benennenden Staat zumindest nicht mehr einseitig beeinflusst, insbesondere beseitigt werden kann. Aus dem Zusammenhang mit $\$ 29$ der operationellen Richtlinie (Streichung von der repräsentativen Liste) kann gefolgert werden, dass spiegelbildlich zur Entscheidung über die Aufnahme auf die Liste die Entscheidung über die Streichung nicht in der freien Willkür der Vertragsstaaten steht, sondern vom Wegfall der materiellen Voraussetzungen der Listung, welcher durch das Komitee festzustellen ist, abhängt. Allerdings dürfen auch in diesem Zusammenhang die Stellung der Staaten im Komitee und die Möglichkeiten ihrer politischen Einflussnahme auf das Komitee nicht unterschätzt werden. In der Praxis ist das zwischenstaatliche Komitee in einem weitaus höheren $\mathrm{Maße}$ den Staaten und ihrem Einfluss verbunden als dies bei den Organen anderer internationaler Organisationen der Fall ist.

Auch obliegt es den Staaten, jederzeit den Transfer eines immateriellen Kulturerbes von der repräsentativen Liste auf die Liste des dringend schutzbedürftigen Erbes nach Art. 17 der Konvention oder umgekehrt zu betreiben (\$S 30 und 14 der operationellen Richtlinien), so dass man in der Praxis insgesamt von einem entscheidenden Einfluss der Staaten auf den Status des immateriellen Kulturerbes ausgehen muss.

Im Rahmen der Aufnahme kultureller Praxen auf die Liste des dringend schutzbedürftigen immateriellen Kulturerbes nach Art. 17 der Konvention ist die Rolle der Staaten entsprechend zum Verfahren nach Art. 16 ausgestaltet. ${ }^{37}$ Der Unterschied zur repräsentativen Liste nach Art. 16 besteht darin, dass in Fällen höchster Dringlichkeit die Nominierung und Listung des immateriellen Erbes auch auf Initiative des Komitees, eines anderen Vertragsstaates oder - was besonders bemerkenswert ist - sogar auf Initiative der betroffenen Kulturträger (d.h. der betroffener Gemeinschaften, Gruppen oder Individuen sowie auch beratender Körperschaften), nicht aber gänzlich ohne die Beteiligung eines Vertragsstaates, in dessen Hoheitsgebiet sich das in Frage stehende, besonders bedrohte immaterielle Erbe befindet, erfolgen kann (vgl. Art. 17 Ziffer 3 der Konvention, $\$ \$ 1$ U.6, 11 und 12 der operationellen Richtlinien). Bei Vorliegen höchster Dringlichkeit kann eine Listung auch ohne aktives Tätigwerden des betroffenen Vertragsstaates vorgenommen werden. Jedoch ist auch in diesem Fall der betroffene Staat unverzüglich über die Nominierung zu informieren $(\$ 12$ Satz 2 der operationellen Richtlinien) und während der Evaluation durch das Komitee zu beteiligen ( $\$ 11$ der operationellen Richtlinien). Ohne eine Beteiligung des betroffenen Vertragsstaates kann selbst im Fall höchster Dringlichkeit eine Listung nach Art. 17 Ziffer 3 der Konvention also nicht erfolgen ( $\mathbb{1} 1$ U.6 der operationellen Richtlinien).

Schließlich spielen die Staaten auch bei der Ergreifung und Durchführung von internationalen Schutzmaßnahmen nach Art. 18 der Konvention die entscheidende Rolle, indem sie namentlich die entsprechenden Programme, Projekte und Aktivitäten, die vom Komitee ausgewählt und in Zusammenarbeit mit den Staaten

${ }^{37}$ Vgl. dazu im Einzelnen $\iint 1-10$ und 14-18 der operationellen Richtlinien von 2008. 
durchgeführt werden, vorschlagen und sie auch das in der Konvention vorgesehene Instrument der internationalen Unterstützung nutzen können, welches den Staaten weitere Handlungsmöglichkeiten und Gestaltungsspielräume beim Schutz des immateriellen Kulturerbes auf der internationalen Ebene eröffnet. In diesem Zusammenhang (Abschnitt V, Art. 19 bis 24 der Konvention) kommt dem Komitee eine koordinierende Funktion zu, während die konkreten Maßnahmen von den Vertragsstaaten nach ihrer Einschätzung und unter ihrer Verantwortung durchgeführt werden. ${ }^{38}$

\section{Bewertung der Rolle der Staaten beim Schutz des immateriellen Erbes}

Zusammenfassend lässt sich mit Blick auf die einzelnen Regelungen, die die Konvention zum Schutz des immateriellen Kulturerbes von 2003 auf nationaler wie auch auf internationaler Ebene vorsieht, sagen, dass die Vertragsstaaten die maßgeblichen Akteure des Kulturerbeschutzes sind. Ihre Position ist auf allen Ebenen und in allen Phasen des Schutzes des immateriellen Erbes nach der Konvention die ausschlaggebende. Es liegt bei den Staaten, die Initiative für konkrete Maßnahmen auf nationaler und internationaler Ebene zu ergreifen. Dabei dürfte den in der Konvention vorgesehenen Maßnahmen der nationalen Ebene in der Praxis wohl die kulturpolitisch größere Bedeutung zukommen. Zum einen sind die in der Konvention vorgesehenen nationalen Schutzmaßnahmen in ihrer Bandbreite deutlich breiter gefächert und überlassen den Vertragsstaaten somit ein weites Spektrum an aus ihrer Sicht geeigneten und erforderlichen Handlungsmöglichkeiten, über die sie weitgehend selbst bestimmen können, was eine gewisse Bereitschaft der Staaten, in diesem Bereich Maßnahmen zu ergreifen, fördern dürfte. Zum anderen lässt die gesamte Systematik der Konvention eine gewisse Tendenz zum Übergewicht nationaler Schutzmaßnahmen gegenüber dem internationalen Schutz

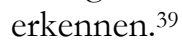

Auch die Verantwortung für die Umsetzung und Durchführung des Schutzes auf internationaler Ebene bleibt im Ergebnis, wie dargestellt, ganz überwiegend in der Hand und unter der effektiven Kontrolle der Staaten. Festzuhalten ist, dass ohne die Beteiligung der betroffenen Staaten - und erst recht nicht gegen ihren Willen - nach der UNESCO-Konvention von 2003 keinerlei Maßnahmen zum Schutz des immateriellen Kulturerbes denkbar sind.

In Hinblick auf diese starke Stellung der Staaten nach der UNESCOKonvention von 2003 sind die in ihr enthaltenen Beteiligungsrechte der betroffenen Kulturträger - namentlich der betroffenen Gemeinschaften, Gruppen, aber auch von Individuen und NGOs - zwar grundsätzlich begrüßenswert. Sie tragen sicherlich zu der gerade im Bereich des immateriellen Erbes, welches immer in gelebten kulturellen Praxen und Ausdrucksformen besteht, notwendigen Rückbin-

\footnotetext{
38 Sola 2008: 500-501, Srinivas 2008:538-539.

${ }^{39}$ Mariotti 2008:78-80.
} 
dung an ihre sozialen Voraussetzungen bei und sichern prinzipiell eine angemessene Berücksichtigung der Interessen der betroffenen Kulturträger, ohne die ein immaterielles Kulturerbe von vornherein nicht denkbar wäre. Allerdings besteht, wie aufgezeigt, unter dem Regime der Konvention insgesamt ein äußerst weiter kulturpolitischer Einschätzungs- und Ermessensspielraum der Vertragsstaaten. Die Bestimmungen der Konvention, welche die internationale Ebene betreffen, und insbesondere auch die dem Komitee als wichtigem Exekutivorgan nach der Konvention zugewiesenen Aufgaben in diesem Zusammenhang können - sofern sie nicht als von lediglich symbolischer oder repräsentativer Wirkung angesehen werden sollen - in erster Linie als eine besondere Form international strukturierter Koordinierung der einschlägigen Kulturpolitik der Vertragsstaaten begriffen werden.

Trotz der positiv zu bewertenden Fortschritte und Öffnungen, die aus einzelnen Bestimmungen der Konvention für das Kulturvölkerrecht folgen ${ }^{40}$, spricht aus der Konvention insgesamt ein nach wie vor traditionelles Völkerrechtsverständnis, in welchem die souveränen Staaten die maßgeblichen Akteure sind.

\section{Eröffnung von neuen staatlichen Einwirkungs- und Kontrollmöglichkeiten im Bereich der Kultur: Umfassende Kulturhoheit des Staates im Völkerrecht?}

Die starke Rolle der Staaten ergibt sich nicht allein aus den Inhalten der dargestellten Einzelregelungen der Konvention. Sie setzt weitaus grundsätzlicher an, indem bereits in der Konzeption der gesamten Konvention eine prinzipielle Reservefunktion der Staaten angelegt ist.

\subsection{Reservefunktion der Staaten bei der Bestimmung der Grundlagen der Konvention}

Dies mag insbesondere in Anbetracht der beschriebenen Öffnungen, die die Konvention enthält, zunächst nicht ohne weiteres erkennbar sein. Namentlich die in Art. 2 Ziffer 1 der Konvention enthaltene Definition des Begriffs des ,immateriellen Erbes" und die wiederholt angesprochenen Beteiligungsrechte der nichtstaatlichen Akteure scheinen eine andere Sprache zu sprechen. Allerdings kann die Rhetorik der entsprechenden Klauseln bei genauerem Hinsehen nicht darüber hinwegtäuschen, dass die Grundbedingungen eben dieser Konventionsbestimmungen letztlich der Interpretationsreserve der souveränen Vertragsstaaten unterliegen: Die UNESCO-Konvention von 2003 wird an den entscheidenden Stellen durch die Verwendung einer Vielzahl offener bzw. unbestimmter Rechtsbegriffe gekennzeichnet, die der Interpretation durch die Staaten als Herren der Konvention un-

40 Siehe oben, 1. 
terliegen. Bereits der Begriff des „immateriellen Erbes“ ist in viele Richtungen offen. Entscheidend ist in diesem Zusammenhang, dass die in Art. 2 Ziffer 1 angesprochenen „Gemeinschaften, Gruppen und gegebenenfalls Individuen“, nach deren kulturellem Selbstverständnis sich das immaterielle Kulturerbe i.S.d. Konvention definitionsgemäß bestimmen soll, von der Konvention selbst nicht näher bezeichnet werden. Damit ist der Begriff des immateriellen Erbes der Gefahr ausgesetzt, schon deshalb juristisch ungreifbar zu werden, weil die Anschauungen unterschiedlichster Personen bzw. Gruppen über die Bestandteile und immateriellen Elemente ihres kulturellen Erbes äußerst unbestimmt, subjektiv und heterogen sind und vor allem von Bezugsgruppe zu Bezugsgruppe oder gar von Individuum zu Individuum selbst in relativ abgrenzbaren sozialen Zusammenhängen in hohem Maße divergieren können. Dem Geist und den Intentionen der Schöpfer der Konvention ${ }^{41}$ dürfte es wohl am ehesten entsprechen, wenn die Staaten sich insofern (d.h. hier in Hinblick auf die Frage, was konventionserhebliches kulturelles Erbe ist) in ihren eigenen kulturpolitischen Wertungen weitgehend beschränken und ausschließlich auf das Selbstverständnis und die Anschauung der relevanten Kulturträger zurückgreifen. ${ }^{42}$

Somit wird notwendigerweise die Frage berührt, (1) wie, (2) anhand welcher mehr oder weniger objektiven Kriterien und insbesondere (3) von wem die insofern relevanten kulturellen Gemeinschaften, Gruppen oder Individuen, auf deren kulturelles Selbstverständnis in Art. 2 Ziffer 1 der Konvention Bezug genommen wird, überhaupt ausgewählt werden. ${ }^{43}$ Dieselbe Frage stellt sich im Zusammenhang mit den Beteiligungsrechten der „relevanten Gruppen“ i.R.d. Art. 11 lit. b) der Konvention. Auch hier stellt die Konvention selbst keine objektiven Maßstäbe dafür zur Verfügung, welche Gruppen als relevant anzusehen sind. An dieser Stelle besteht mithin eine Reservefunktion der Staaten, denn ihnen kommt aufgrund der Offenheit der Konvention an dieser grundlegenden Stelle die Rolle zu, die für die Auslegung des Kulturerbebegriffs i.S.d. Konvention relevanten Kulturträger auszuwählen. In letzter Konsequenz sind es also die Staaten, die entscheiden, welche der auf ihrem Hoheitsgebiet ansässigen oder aktiven Kulturträger mit ihren entsprechenden

\footnotetext{
${ }^{41}$ Zur Entstehungsgeschichte und den Intentionen der Konvention statt vieler: Sola 2008:491-494, Blake 2008, Jacobs 2007. Vgl. außerdem http://www.unesco.org/culture/ich/index.php?pg=00006 (Zugriff am 04.04.2010).

42 Sola 2008:495. Aus einer national-staatsrechtlichen Perspektive für die - durch die UNESCOKonvention von 2003 freilich nicht gebundene - Bundesrepublik Deutschland hierzu auch grundlegend: Mißling N.d..

Denkt man diesen Ansatz an diesem Punkt konsequent zu Ende, heißt dies für die Umsetzung und Anwendung der UNESCO-Konvention von 2003, dass die Regierungen auch durch innerstaatliches Recht verpflichtet werden müssen, das in ihrem Hoheitsgebiet befindliche immaterielle Erbe sowohl nach Maßgabe der kulturellen Selbstverständnisse der ihrer Hoheitsgewalt unterstehenden relevanten Kulturträger als bindend und grundsätzlich ohne eigene kulturpolitische Wertung aufzunehmen, als auch in einem zweiten Schritt der Umsetzung - wie angesprochen - nach pflichtgemäßen Ermessen die durch die Konvention vorgesehenen Schutzmaßnahmen der nationalen und der internationalen Ebene zu ergreifen bzw. zu initiieren.
}

${ }^{43}$ Siehe oben, 3.2 
Selbstverständnissen bezüglich ihres (immateriellen) kulturellen Erbes sie im Rahmen der Umsetzung der UNESCO-Konvention berücksichtigen.

Diese Auswahl der für das immaterielle Erbe relevanten Kulturträger kann auf unterschiedliche Weise geschehen und muss nicht in jedem Fall im Rahmen einer expliziten staatlichen Kulturpolitik erfolgen. ${ }^{44}$ Sie kann auch in gesellschaftlichen und kulturellen Prozessen direkt oder lediglich indirekt politisch diskursiv ausgehandelt werden. Günstigenfalls stellt die jeweilige nationale Rechtsordnung der Staaten (in diesem Zusammenhang namentlich die Grund- und Menschenrechte) hierfür den maßgeblichen Rahmen zur Verfügung; im ungünstigen Fall spielen die Ausübung von politischer Autorität oder der Einsatz anderer Machtinstrumente hierbei eine entscheidende Rolle. Wie auch immer der interne Auswahlprozess innerhalb der einzelnen Staaten diesbezüglich organisiert ist, stellt doch die innerstaatliche Anerkennung bestimmter kultureller Träger, denen eine zum immateriellen Erbe bestimmte kulturelle Praxis zugeordnet werden kann, durch den einzelnen Staat eine souveräne Entscheidung des Vertragsstaates dar. Damit lässt sich die hier beschriebene Reservefunktion der Staaten bezüglich der Bestimmung der relevanten Kulturträger und ihrer als immaterielles Kulturerbe infrage kommenden kulturellen Praxen letztlich nahezu in eine Reservekompetenz des Staates zu einer einzelfallbezogenen Entscheidung über die Tragweite der Konvention umformulieren.

\subsection{Das immaterielle Kulturerbe als Instrument staatlicher Kulturpolitik}

Das immaterielle Kulturerbe und sein Schutz nach der Konvention von 2003 können für die Vertragsstaaten daher ein wichtiges kulturpolitisches Instrument darstellen. Die Benennung von in ihrem Hoheitsgebiet befindlichem immateriellen Kulturerbe und insbesondere die Nominierung und die Aufnahme auf die repräsentative Liste gem. Art. 16 der Konvention haben nicht nur einen hohen, internationalen Prestigewert für die Staaten. Ihren Wert als praktisch bedeutsames kulturpolitisches Instrument in der Hand der Staaten und ihrer jeweiligen Regierungen können die in der Konvention von 2003 vorgesehenen Maßnahmen entfalten, indem sie einerseits zu einer staatlichen - mitunter nationalen - Vereinnahmung bestimmter kultureller Praxen führen und leicht als Mittel solcher staatlicher Kulturpolitiken eingesetzt werden können, die auf die Herausbildung, Stärkung oder Profilierung nationaler Identitäten abzielen. Andererseits können sie aber auch zur Schaffung oder Stärkung einer effektiven staatlichen Kontrolle der betroffenen kulturellen Praxen beitragen.

Die Wirkungsweise der zuerst genannten Konstellation ist besonders bedeutsam in Fällen, in denen dieselben oder inhaltlich verwandte kulturelle Praxen in grenzüberschreitenden kulturellen Zusammenhängen existieren und in denen Kulturträger in mehreren Staaten diese als ihr kulturelles Erbe für sich reklamieren,

44 Ebenda. 
gegebenenfalls sogar Exklusivität hierfür beanspruchen. Wenn in diesen Fallkonstellationen nur einer der Staaten, auf dessen Hoheitsgebiet die infrage stehende kulturelle Praxis geübt wird, diese für sich als ein staatliches (oder nationales) Kulturerbe reklamiert, nach Art. 11 lit. b) und 12 der Konvention identifiziert, dokumentiert und inventarisiert oder - erfolgreich - die Aufnahme in die repräsentative Liste des immateriellen Kulturerbes betreibt, muss dies nicht zwingend Ausdruck seiner Treue zur in der Präambel der Konvention niedergelegten Idee eines „kulturellen Erbes der [gesamten] Menschheit“ („Welterbes“) sein, sondern kann nicht zuletzt aufgrund der starken Symbolwirkung eines solchen Schrittes u.U. als eine kulturpolitische Vereinnahmung („Inbesitznahme“) der kulturellen Praxis durch den betreffenden Staat bewertet werden. Noch weiter gehende Möglichkeiten der staatlichen Vereinnahmung bietet insofern - zumindest theoretisch - Art. 11 lit. a) der Konvention. ${ }^{45}$ Es mag aus diesem Grunde sein, dass die operationellen Richtlinien zur UNESCO-Konvention von 2003 sowohl in ihren näheren Bestimmungen hinsichtlich der Listung nach Art. 16 der Konvention (\$ 20 der operationellen Richtlinien) als auch hinsichtlich der Listung nach Art. 17 ( $\int 3$ der operationellen Richtlinien) die Vertragsstaaten ausdrücklich dazu ermuntern, von der Möglichkeit der gemeinsamen Nominierung Gebrauch zu machen.

In der zweiten angesprochenen Konstellation sind Fälle anzunehmen, in denen Vertragsstaaten die durch die Konvention völkerrechtlich eröffneten Möglichkeiten, auf die Ausübung vorhandener kultureller Praxen auf ihrem Staatsgebiet regulierend einzuwirken, zur internen Durchsetzung staatlicher Kulturpolitiken benutzen. ${ }^{46}$ Die hiermit verbundenen Gefahren staatlicher Instrumentalisierung der Konvention scheinen weitaus größer als die bisher angesprochenen Effekte der anderen Konstellation. Es muss an dieser Stelle nicht im Detail ausgeführt werden, dass Staaten (insbesondere solche mit einer heterogenen ethnischen Struktur und einem hohen $\mathrm{Maß}$ an kultureller Diversität) namentlich die in der Konvention enthaltenen Instrumente auf der nationalen Ebene für bestimmte interne, kulturpolitische Interessen nutzbar machen können; entweder indem die kulturellen Praxen bestimmter kultureller Gruppen bevorzugt und besonders geschützt werden oder aber indem diejenigen bestimmter anderer Gruppe mithilfe der in der

\footnotetext{
45 An dieser Stelle unbehandelt muss indes die Frage bleiben, ob derartige Vereinnahmungen, wie sie hier aufgezeigt werden, in Übereinstimmung mit dem Geist und Sinn und Zweck der UNESCOKonvention von 2003, wie sie namentlich in deren Präambel und Art. 1 (Ziele der Konvention) niedergelegt sind, zu bringen sind. Die Frage, ob entsprechende Maßnahmen der Staaten, die insbesondere den Zielen der Konvention aus Art. 1 zumindest offenkundig widersprechen, nach der Konvention völkerrechtlich unzulässig sind, bleibt einer späteren Erörterung an anderer Stelle vorbehalten. Freilich spricht bei einer Auslegung der Konvention am Maßstab ihrer Ziele und Erwägungsgründe prima facie vieles dafür.

46 Auch insoweit stellt sich die angesprochene Frage der völkerrechtlichen Zulässigkeit eines solchen staatlichen Verhaltens. Schwierig ist in diesem Zusammenhang die ebenfalls an anderer Stelle näher zu erörternde Bestimmung, ob und ggf. inwiefern die Konvention es zulässt, dass die Vertragsstaaten bei der Ergreifung von Maßnahmen zum Schutz des immateriellen Erbes neben den in Art. 1 der Konvention genannten Zielen weitere Zwecke verfolgen.
} 
Konvention vorgesehenen Maßnahmen reguliert und somit indirekt staatlich kontrolliert oder sogar (missbräuchlich) behindert werden.

\subsection{Erweiterte kulturpolitische Handlungsmöglichkeiten der Staaten: Erweiterung der Kulturhoheit der Staaten}

Des Weiteren wird durch die UNESCO-Konvention von 2003 eine grundlegende Problematik des Kulturvölkerrechts berührt, die im Folgenden als solche und ungeachtet der angedeuteten Gefahren der Instrumentalisierung der Konvention von 2003 und ihrer Schutzbestimmungen durch die Staaten nähere Betrachtung verdient. Hierbei zeigt sich, dass die Konvention zum Schutz des immateriellen Kulturerbes nicht nur im Kontext und in der Systematik des Welterbeschutzes neues Terrain betritt, sondern dass sie - noch weitaus fundamentaler - den Staaten neue kulturpolitische Handlungsmöglichkeiten auf einem Gebiet einräumt, welches dem staatlichen Einfluss und erst recht der staatlichen Kontrolle, zumindest im Völkerrecht, bis dahin weitgehend entzogen war. Durch die UNESCO-Konvention von 2003 werden Rechte der Staaten im immateriellen Kulturbereich geschaffen oder doch wenigstens implizit vorausgesetzt.

Was damit gemeint ist, vermag der Vergleich zwischen den beiden UNESCOKonventionen zum Schutz des Kulturerbes von 1972 (materielles Kulturerbe) und 2003 (immaterielles Kulturerbe) zu verdeutlichen: Mit der Welterbekonvention zum Schutz des materiellen Kulturerbes von 1972 ist in der UNESCO der (völker-)rechtliche Schutz von unbeweglichen, körperlichen Kulturgütern (materielles Kulturerbe ${ }^{47}$ ) geregelt und ausgestaltet worden. Bei diesem materiellen Welterbe handelt es sich um Kulturgüter, die kraft ihrer dauerhaften und nicht ohne weiteres aufhebbaren Verbindung mit dem Ort, an welchem sie sich befinden, notwendigerweise der Territorialhoheit des Staates unterfallen, in welchem sie gelegen sind. ${ }^{48}$ In Hinblick auf diese materiellen Kulturgüter bestehen - wie im Übrigen auch in Hinblick auf bewegliche, materielle Kulturgüter - prinzipiell Hoheitsrechte desjenigen Staates, in welchem sie sich rechtmäßig befinden bzw. aus welchem sie rechtmäßig stammen. Weitaus schwieriger ist dies in Hinblick auf immaterielle kulturelle Praxen und/oder Ausdrucksformen. Diese Kulturgüter existieren lediglich ideell und werden nur in ihrer Performanz überhaupt erkennbar. Zwar gibt es für immaterielle Kulturgüter Träger, welche durch ihre Staatsangehörigkeit grundsätzlich unter die Hoheitsgewalt eines oder ggf. auch mehrerer Staaten fallen. Damit ist jedoch nichts über das Zuordnungsverhältnis der von ihnen ausgeübten, gepflegten, bewahrten oder vermittelten, immateriellen kulturellen Praxis zu die-

\footnotetext{
$47 \mathrm{Vgl}$. Art. 1 und 2 der Welterbekonvention von 1972.

$48 \mathrm{Zu}$ erinnern ist in diesem Zusammenhang daran, dass die Zuordnung von Welterbestätten unter die Territorialhoheit eines bestimmten Staates mitunter Gegenstand internationaler Streitfälle war. Vgl. nur den Fall des Tempels von Preah Vihear, IGH-Urteil v. vom 15.6.1962 (Kambodscha ./. Thailand), I.C.J. Reports 1962, S. 6. Vgl. Dazu u.a.: Johnson 1962, Singh 1962, Wagner 2008. Zuletzt auch: Mißling und Watermann 2009.
} 
sem/diesen Staat(en) gesagt. Es liegt im Charakter der Kultur als solcher und insbesondere im Wesen ihrer immateriellen Elemente, dass sie gerade nicht als solche einem Staat, sondern allenfalls ihren Trägern zugeordnet werden können - wobei selbst die Zuordnung zu den Kulturträgern sich auf lose temporäre oder auch nur punktuelle Verhältnisse beschränken kann.

Mit anderen Worten kann festgehalten werden, dass Kultur als solche und ihre immateriellen Elemente ihrer Natur nach nicht in die staatliche Souveränität fallen. Es liegt im Wesen immaterieller Kulturgüter, dass sie sich einer - zumindest dauerhaften und effektiven - staatlichen Kontrolle prinzipiell entziehen, wenngleich die Geschichte gezeigt hat, dass dies wiederholt und mit unterschiedlichen Mitteln versucht wurde. Ganz im Gegenteil tragen namentlich die nationalen und internationalen Grund- und Menschenrechte dazu bei, die Freiheit und die Unabhängigkeit „der Kultur“ (und hierbei auch ihrer immateriellen Elemente) vor zielgerichteten und willkürlichen Eingriffen oder Vereinnahmen seitens des Staates zu sichern. ${ }^{49}$

Dadurch, dass mit der UNESCO-Konvention von 2003 auf der Ebene der Vereinten Nationen zum ersten Mal mehr oder weniger weitreichende Regelungen zum Schutz immaterieller Kulturgüter getroffen worden sind, deren Umsetzung und Durchsetzung, wie gezeigt, in die ganz überwiegende Verantwortung der Staaten gestellt wurde ${ }^{50}$, sind im Kulturvölkerrecht erstmals auch rechtliche Kontrollmöglichkeiten der Staaten in Bezug auf immaterielle Kulturgüter (immaterielles Erbe) geschaffen worden. Insoweit ist es in der Tat berechtigt, von einem mit der UNESCO-Konvention von 2003 vollzogenen Paradigmenwechsel zu sprechen, denn durch sie wird ein mehr oder minder direkter Zugriff der Staaten auf Ele-

\footnotetext{
49 Zunächst ist hierzu in die einzelnen Rechts- und Verfassungsordnungen der Staaten zu blicken. Für die Bundesrepublik Deutschland wären insofern von den Grundrechten des Grundgesetzes, die in ihrer Gesamtheit dazu dienen, die freie Entfaltung von Kultur im weiten Sinne und kulturellem Leben in einem engeren Sinne zu gewährleisten, insbes. die sogen. „klassischen kulturellen Grundrechte" in Art. 4 Abs. 1 und Art. 5 Abs. 1 und 3 GG hervorzuheben. Daneben wären die Grundrechtskataloge sowie die objektiv-verfassungsrechtlichen Kulturstaatsklauseln der Landesverfassungen einschlägig. Auch auf der Ebene des Europarechts (d.h. sowohl der EU als auch des Europarates) sind entsprechende Kulturklauseln, namentlich Art. 167 AEUV, zu beachten. Auf der Ebene des Völkerrechts sind vor allem die Art. 27 der Allgemeinen Erklärung der Menschenrechte der Generalversammlung der Vereinten Nationen vom 10.12.1948, Art. 15 des Internationalen Paktes über wirtschaftliche, soziale und kulturelle Rechte vom 19.12.1966, auch Art. 19 des Internationalen Paktes über bürgerliche und politische Rechte vom 19.12.1966 sowie nicht zuletzt die Erklärung der Rechte indigener Völker der Generalversammlung der Vereinten Nationen vom 13.9.2007 und auch die ILO-Konvention 169 vom 27.6.1989 zu nennen. Zum Schutz indigener Kultur im internationalen Recht: Marrie 2009; Stoll und Hahn 2008. Zu den internationalen Menschenrechten und ihrem Verhältnis zum Kulturerbe im Völkerrecht allgemein: Sola 2008:488-489, Srinivas 2008:547-550.

${ }^{50} \mathrm{Zu}$ unterscheiden sind hiervon die Regelungen, die in der WIPO auf dem Gebiet des Geistigen Eigentums zugunsten immaterieller Güter bzw. kultureller Ausdrucksformen bislang geschaffen oder gegenwärtig diskutiert werden: Die dort in Rede stehenden internationalen Regelungen stellen Verpflichtungen der Staaten dar, die Rechte Privater, d.h. also der Kulturträger, an den immateriellen Gütern und/oder Ausdrucksformen zu sichern, während es im Rahmen der UNESCO-Konvention von 2003 um eine unmittelbare staatliche Verantwortung des Staates für bestimmte kulturelle Praxen (immaterielles Kulturerbe) geht. Zu dem Potenzial, das in der Konvention von 2003 für staatliches Kulturhandeln liegt, auch: Hönes 2009:169.
} 
mente „der Kultur“ eröffnet, die bislang weder im Kulturvölkerrecht noch in vielen nationalen Rechts- und Verfassungsordnungen einem entsprechenden oder überhaupt staatlichem Einfluss unterlegen haben. ${ }^{51}$ Auf den ersten Blick mag sich dies bei der Lektüre des Konventionstextes von 2003 nicht ohne weiteres aufdrängen, statuiert die Konvention doch dem Wortlaut ihrer einzelnen Bestimmungen (insbes. Art. 11 ff.) nach in erster Linie Aufgaben und Pflichten der Staaten zum Schutz des immateriellen Erbes im Lichte der Ziele der Konvention. Es darf jedoch nicht vergessen werden, dass die Erfüllung der benannten Aufgaben das Recht der Staaten voraussetzt, diese überhaupt wahrzunehmen.

In diesem Licht kann die UNESCO-Konvention mit einiger Berechtigung als wesentlicher Schritt hin zu einer mehr oder weniger umfassenden völkerrechtlichen Kulturhoheit der Staaten bezeichnet werden.

\section{$5 \quad$ Fazit und Ausblick}

Wenn nach dem Gesagten die Welterbekonzeption nicht zuletzt durch die Konvention zum Schutz des immateriellen Erbes der Menschheit von 2003 eine Modernisierung und auch eine gewisse Öffnung zugunsten nichtstaatlicher, d.h. vor allem lokaler und sozialer Kulturträger und Akteure - und somit zumindest auf den ersten Blick eine Verschiebung zu Lasten der Staaten - erfahren hat, so trägt sie nach den dargelegten Erwägungen umgekehrt gerade zu einer deutlichen Stärkung des Staates in Bereichen der Kultur bei, in denen seine Hoheitsrechte sowie seine rechtlichen und tatsächlichen Einwirkungsmöglichkeiten bislang grundsätzlich begrenzt waren. Es kann demnach nicht ausgeschlossen werden, dass - entgegen mancher Intention und Hoffnung bei der Schaffung dieser Konvention durch die in der Konvention niedergelegten Pflichten der Staaten, denen wie dargelegt immer auch Regelungsrechte in Bezug auf das immaterielle Kulturerbe derselben vorausgehen, und durch die konkrete Ausgestaltung des rechtlichen Instrumentariums, nach dem die letzte Entscheidung über die Patrimonialisierung von Kultur nach wie vor bei den Staaten liegt, die Staaten ihre Verantwortung für die Kultur (namentlich für die im Übrigen äußerst schwer rechtlich zu erfassenden und damit zu regulierenden, immateriellen kulturellen Praxen und Ausdrucksformen) deutlicher als bisher entdecken und entschiedener wahrnehmen werden.

Dass hiermit neben der nicht unberechtigten Befürchtung der Musealisierung von Kultur, die mit der Patrimonialisierung kultureller Ausdrucksformen und ihrer Ernennung zu einem immateriellen Weltkulturerbe durch die UNESCO stets verbunden ist, auch die beschriebene Gefahr einer Vereinnahmung, gar einer gezielten Kontrolle von Kultur durch den Staat einhergeht, lässt sich, wie ebenfalls dargetan wurde, nicht ohne weiteres von der Hand weisen. Es muss daher gefragt werden,

51 In diesem Zusammenhang wäre die - hier bereits aus Platzgründen nicht im Einzelnen zu erörternde - Problematik kultureller Schutz- und Förderungspflichten des Staates in ihrer gesamten Breite anzusprechen. 
wie den aufgezeigten Verwerfungen, die im Zusammenhang mit der Konvention von 2003 entstehen können, durch das Recht begegnet werden kann. Die Auslegung und konsequente Anwendung der Konvention von 2003 im Lichte der in ihrer Präambel niedergelegten Prinzipien und der in Art. 1 ausdrücklich genannten Ziele, d.h. also des Schutzes des immateriellen Erbes (lit. a)), der Sicherung des Respekts vor dem immateriellen Kulturerbe der betreffenden Gemeinschaften, Gruppen und Individuen (lit. b)), der Förderung des Bewusstseins für die Bedeutung des immateriellen Kulturerbes und seiner gegenseitigen Wertschätzung auf lokaler, nationaler und internationaler Ebene (lit. c)) sowie schließlich der Förderung der internationalen Zusammenarbeit und Unterstützung (lit. d)), durch die Staaten und die UNESCO selbst steht hierbei an erster Stelle. Es wurde die an anderer Stelle näher zu erörternde Frage aufgeworfen, ob eine Anwendung und Umsetzung der Konvention, insbes. ihrer Art. 11 ff., die nicht in Einklang mit diesen Zielen und Leitprinzipien stehen, zulässig ist. ${ }^{52}$

Daneben ist aber auch die richtige Einordnung der Konvention in das Regelwerk und den Maßnahmenkanon der UNESCO vorzunehmen: Die aufgezeigten Schwachstellen der Konvention werden insoweit durch die nur unwesentlich jüngere Konvention zum Schutz und zur Förderung der Vielfalt kultureller Ausdrucksformen von 2005 ausgeglichen, als in dieser die Vertragsstaaten auf eine Reihe nationaler wie internationaler Maßnahmen im Interesse und zum Schutz der kulturellen Vielfalt verpflichtet werden, die einer staatlichen Kulturpolitik, die bestimmte kulturelle Gruppen, ihre Praxen und Ausdrucksformen diskriminiert, prinzipiell entgegenstehen. ${ }^{53}$ Einzelheiten zum Verhältnis der beiden UNESCOKonventionen von 2003 und 2005 hinsichtlich dieses Punktes bedürfen der näheren Ausführung an anderer Stelle. Es kann hier aber die These gewagt werden, dass zwischen ihnen eine konzeptuelle Komplementarität besteht und dass sie damit als zwei miteinander in Bezug stehende Elemente in einem übergreifenden System des Kulturgüterschutzes im modernen Kulturvölkerrecht verstanden werden sollten. ${ }^{54}$ Des Weiteren schützen die einschlägigen, bestehenden Menschenrechtspakte und weitere, im Bereich der Kultur existierenden völkerrechtlichen Instrumente des Minderheiten- und Gruppenschutzes die Träger immateriellen Kulturerbes gegen die unangemessene Vereinnahmung ihrer Kultur durch den Staat, soweit sie Geltung haben. ${ }^{55}$ Abschließend sollte darauf hingewiesen werden, dass die angedeutete Kompensationswirkung der genannten völkerrechtlichen Konventionen auf der nationalen Ebene durch die interne Rechts- und Verfassungsordnung der Staaten durch ihre eigene Kulturverfassung und die kulturellen Grundrechte, soweit diese existieren - ergänzt wird. Diesen kulturellen Rechten kommt eine ganz besondere Bedeutung zu, denn je nach der infrage stehenden nationalen Rechtsordnung bin-

\footnotetext{
52 Siehe oben, Fn. 46.

53 Vgl. dazu u.a.: Musitelli 2005, Kolliopoulos 2005, Schorlemer 2005, Neuwirth 2006, Ruiz Fabri 2007, Nolte 2008.

54 Sola 2008:489-491.

55 Siehe oben, Fn. 50.
} 
den sie den betroffenen Staat ggf. mehr oder weniger direkt und unmittelbar im Verhältnis zu den seiner Hoheit unterworfenen kulturellen Gemeinschaften, Gruppen oder Individuen. Wenn hier festgestellt wurde, dass der Schutz des immateriellen Erbes nicht nur zuvorderst von den Staaten, sondern auch insbesondere auf der Ebene der Staaten gewährleistet wird ${ }^{56}$, so kann gleichzeitig festgehalten werden, dass der Schutz der unterschiedlichen Interessen der betroffenen Kulturträger, ebenfalls in erster Linie auf der nationalen Ebene durchgesetzt werden muss.

Die UNESCO-Konvention zum Schutz des immateriellen Kulturerbes von 2003 ist nach alledem durchaus ambivalent zu bewerten: Die Konvention stellt als solche unbestreitbar einen Fortschritt für das Kulturvölkerrecht dar. Die in ihr enthaltenen Öffnungen, namentlich soweit sie die Rolle und die Rechte der betroffenen Kulturträger betreffen, führen die UNESCO und ihr Regelwerk in der hier dargestellten Hinsicht durchaus in eine moderne Phase des Kulturgüterschutzes. Allerdings bleibt die Stellung der Staaten in diesem Zusammenhang nach wie vor die zentrale. Die Konvention von 2003 hat die Kulturhoheit der Staaten sogar noch gestärkt und eröffnet ihnen Handlungsmöglichkeiten, von denen derzeit noch nicht gesagt werden kann, wie sie zukünftig genutzt werden. Es erscheint daher angezeigt, dass die Staaten sich ihrer kulturellen Verantwortung - nicht nur in dem hier behandelten Bereich des immateriellen Erbes - bewusst werden und diese in angemessener Weise und im Licht der Leitprinzipien und Ziele der Konvention wahrnehmen. Genauso angeraten sind aber auch Wachsamkeit und die kritische Wahrung eines richtigen Verhältnisses zwischen Staat und Kultur - sowohl von Seiten der UNESCO selbst als auch seitens der relevanten Kulturträger, denn es darf nicht vergessen werden, dass Kultur zwar auch unter der Verantwortung des Staates steht, ihrem Wesen nach aber von einer richtigen Distanz zum Staat lebt.

${ }^{56}$ Siehe oben, 3. 



\title{
Ausdrucksformen der Folklore: Freie und abhängige Schöpfungen
}

\author{
Philipp Zimbehl
}

\section{Einleitung}

Der Streit um den adäquaten Schutz von Ausdrucksformen der Folklore wird seit vielen Jahren und auf vielen Ebenen geführt. Dieser Aufsatz wirft einen Blick auf die Frage des Schutzes indigener Kulturgüter im Verhältnis zur immaterialgüterrechtlichen Systematik. Ausgehend von der Tatsache, dass sich auf nationaler wie internationaler Ebene und auch in der wissenschaftlichen Literatur immer mehr die Überzeugung durchsetzt, dass es eines Rechtes eigener Art für den angemessenen Schutz bestimmter indigener Kulturgüter bedarf, sollen die neuesten sui generis Ansätze hier einem Vergleich mit ihren urheberrechtlichen Ursprüngen unterzogen werden.

\section{Immaterielle indigene Ausdrucksweisen als Gegenstand der Untersuchung}

Der Schutz immaterieller Güter basiert auf einem sehr diffizilen Gleichgewicht verschiedenster Interessen, die es in Ausgleich zu bringen gilt. Dies gilt insbesondere für die hier betrachteten Ausdrucksformen der Folklore. Kulturelle Praktiken, traditionelles Wissen und Ausdrucksformen haben oftmals einen ephemeren Charakter; sie sind nicht fixiert und allenfalls in ihrer Erscheinung dokumentiert, ohne dass dadurch jedoch der gesamte Umfang und die Bedeutung abgebildet werden 
würden. Diese Flüchtigkeit geht mit der Gefahr einher, dass das Wissen verschwindet, sobald sich kein Träger mehr findet und es damit nicht rekonstruierbar verloren geht. Auf der anderen Seite macht eben diese Liquidität auch das Wesen der Folklore als etwas Lebendiges, sich Entwickelndes aus (Wendland 2008:167).

Für diese Ausdrucksformen der Folklore einen angemessenen Schutz zu finden, ist das Ziel der Bemühungen sowohl auf der Ebene der World Intellectual Property Organisation (WIPO) ${ }^{1}$ als auch auf nationaler Ebene (Torsen 2008). Jedoch mehren sich die kritischen Stimmen in der Diskussion um die Einführung eines eigenen Schutzrechts für diese Folklore. An Bedenken werden insbesondere vorgebracht, dass eine zu starke Ausweitung eines Schutzes eine erhebliche Einschränkung der public domain mit sich bringe (Lewinski 2003:391).

Die Argumente beider Seiten sind nicht außer Acht zu lassen. Hier soll deshalb einem Rat von Hilty (2009) folgend ein Schritt zurückgetreten und die Ratio des immaterialgüterrechtlichen Schutzes und ihre Anwendbarkeit auf das spezielle Gebiet des Folkloreschutzes hinterfragt werden.

\section{Grundlagen des geistigen Eigentums}

Die Schwierigkeiten, die sich im Zusammenhang mit dem Schutz traditioneller kultureller Ausdrucksweisen stellen, lassen sich zumindest zum Teil direkt aus den Besonderheiten des geistigen Eigentums herleiten.

\subsection{Materielle und immaterielle Güter}

Geistiges Eigentum kann auch als Informationsinfrastruktur beschrieben werden; geschaffen durch menschliche Anstrengung und Einfallsreichtum (Mackaay 2007). Geistige Güter sind Immaterialgüter und können zwar auch eine körperliche Form finden, sind aber anders als materielle Güter von dieser unabhängig (Eingehend hierzu Schack 2007:14). Anders als im Falle des materiellen Eigentums kann Information nicht ohne weiteres gegen den Gebrauch durch Dritte abgeschirmt werden. Eine Idee etwa kann also grundsätzlich von jedem genutzt werden, der von ihr erfährt (Mackaay 2007).

In Abwesenheit von Immaterialgüterrechten gibt es zunächst keine Möglichkeit für denjenigen, der die Idee zuerst hatte, andere daran zu hindern, sie auch zu nutzen. ${ }^{2}$ Es kann niemand einen anderen von der Nutzung eines immateriellen Gutes ausschließen und es entsteht auch kein Nachteil für Mitnutzer, wenn mehr Menschen das Gut nutzen. Informationen, Wissen und Ideen sind im

\footnotetext{
1 Der Einheitlichkeit halber werde ich für nationale und internationale Organisationen, Normen und Verträge im Folgenden ihre englische Bezeichnung und deren Abkürzung verwenden.

2 Es gibt verschiedene Faktoren, die in diesem Fall dennoch wirken können, auf die ich hier jedoch zunächst nicht näher eingehen möchte. Eine Übersicht geben Landes und Posner (2003:41).
} 
ökonomischen Sinne nicht knapp. Es handelt sich zunächst um rein öffentliche Güter (Mackaay 2007).

\subsection{Knappheit und Freiheit als Eigenschaften des geistigen Eigentums}

Durch die Einführung eines Immaterialgüterrechts wird das eigentlich ubiquitäre Gut künstlich verknappt und einer Person als Rechtsobjekt zugeordnet (Schack 2007:9). Mit der Zuordnung des Gutes zu einer Person lässt sich aus juristischer Perspektive tatsächlich von Eigentum sprechen. Diese Verknappung und Zuweisung an eine bestimmte Person hat erhebliche Konsequenzen. Zum einen wird dem Schöpfer des geistigen Guts oder vielmehr demjenigen, dem das Gut durch das Recht zugewiesen ist, eine Möglichkeit an die Hand gegeben, Dritte von der Nutzung auszuschließen. Wichtiger noch ist, er kann einen Preis auf die Nutzung festsetzen. Hierdurch wird es dem Schöpfer möglich zumindest die versunkenen Kosten für die Schöpfung wieder einzufahren. Dies ist der wesentliche Aspekt geistigen Eigentums. Es verknappt ein Gut, um Anreiz für die Produktion von Gütern zu bieten (Landes und Posner 2003:20). Dies bedeutet jedoch auch, dass der Allgemeinheit der Zugang zu diesen Gütern erschwert wird. Darin liegt der grundlegende Konflikt, der das Immaterialgüterrecht prägt.

Geistige Güter, die keinem Schutzregime unterliegen, etwa weil ihr Schutz abgelaufen ist oder sie niemals schutzfähig waren, sind Bestandteil der Public Domain. Anders gesagt bilden solche Nutzungen die Public Domain, welche jedermann grundsätzlich ohne die Verletzung der Rechte anderer vornehmen kann (Benkler 1999:362). Dies hat eine besondere Bedeutung für die Schaffung neuer Geisteswerke. Geistige Schöpfung charakterisiert sich dadurch, dass sie nur selten isoliert stattfindet. Generell handelt es sich bei geistigem Schaffen um einen kumulativen Prozess; neue Geisteswerke bauen auf vorhandenen Informationen auf (Mackaay 2007). Für diesen Prozess ist der Zugang zu vorbestehenden Geisteswerken von entscheidender Bedeutung. Die Public Domain gewährt freien Zugang und senkt so die Transaktionskosten für die Schöpfer. Die Public Domain hat jedoch nicht nur eine ökonomische Bedeutung. Sie ist die Grundlage für den freien Austausch der Ideen, den freien Fluss der Gedanken und auch die Meinungsfreiheit (Benkler 1999:355, Boyle 2008).

\section{Das Urheberrecht als das Immaterialgüterrecht an künstlerischen Werken}

Das Urheberrecht als Immaterialgüterrecht der Wissenschaft, der Literatur und der Kunst (Schack 2007:29) ist aus juristischer Perspektive traditionell von zwei Begründungsansätzen geprägt. Die Unterschiede in den Begründungen für einen immaterialgüterrechtlichen Schutz von Geisteswerken sind nicht allein aus rechts- 
theoretischer Sicht bedeutend, sondern wirken unmittelbar auf das Verständnis des Urheberrechts.

\subsection{Der persönlichkeitsbezogene Ansatz}

Der schöpferische Geist, der kraft seiner individuellen Kreativität praktisch aus sich heraus ein geistiges Gut hervorbringt, steht seit der Aufklärung im Mittelpunkt der Betrachtung (Bappert 1962:155, Riley 2000:180). Auch wenn es aus heutiger Sicht selbstverständlich erscheinen mag, so war doch die Idee einer Persönlichkeit, die im Werk ihren Ausdruck findet, etwas grundlegend Neues. Die bestechende Konsequenz, die aus dieser Erkenntnis gezogen wurde, nämlich dass ein jedes geistiges Werk mit der Person des Urhebers unverbrüchlich verknüpft ist (Schack 2007:4), wirkt bis heute nicht nur nach, sondern prägt die Sicht, die ein erheblicher Teil der modernen Rechtsordnungen auf das Urheberrecht und insbesondere auch auf die Frage des Schutzes von traditionellen kulturellen Ausdrucksweisen hat (Riley 2000:179).

\subsection{Der Copyright Ansatz}

Demgegenüber steht das anglo-amerikanische Verständnis des Urheberrechts. Die Grundlagen dieses stark wirtschaftlich geprägten Ansatzes reichen bis in die USamerikanische Verfassung zurück. Schon diese sah vor, dass der Kongress die Macht haben sollte, den Fortschritt der Kunst dadurch zu fördern, dass er den Künstlern für eine begrenzte Zeit ein ausschließliches Recht an ihren Schriften und Entdeckungen sichert. ${ }^{3}$ Hier zeigt sich schon die Fokussierung auf Anreize, die für den anglo-amerikanischen Ansatz prägend sind. Eine wichtige Konsequenz aus der Fokussierung auf Anreize, die uns im Weiteren noch beschäftigen wird, besteht darin, dass das Copyright in erster Linie demjenigen zustehen soll, der die maßgeblichen Investitionen getätigt hat (Copinger et al. 2005).

\section{$5 \quad$ Folklore im System des Urheberrechts}

Das Urheberrecht mit seinem System aus wirtschaftlichen und ideellen Rechten scheint durchaus geeignet, viele der von den indigenen Gruppen vorgebrachten Anforderungen erfüllen zu können (WIPO 2003a:36). Entsprechend wird ein dem Urheberrecht ähnlicher Schutz auch in der Diskussion in den internationalen Foren immer wieder in Erwägung gezogen.

\footnotetext{
${ }^{3}$ Article 1, Section 8 - Powers of Congress: "The Congress shall have power ... To promote the progress of science and useful arts, by securing for limited times to authors and inventors the exclusive right to their respective writings and discoveries; [...]"
} 


\subsection{Folklore stricto sensu und ihre Ausübung}

Die Frage des Schutzes traditioneller kultureller Ausdrucksformen muss differenziert nach den zu schützenden Gegenständen beurteilt werden. Auf der einen Seite stehen die Ausdrucksformen von Folklore wie sie uns heute begegnen und wie sie heute von indigenen oder nicht indigenen Künstlern ausgeführt, gezeigt oder auch vermarktet werden. Auf der anderen Seite steht der Schutz der zu Grunde liegenden Kulturtechnik, also der Tradition an sich, so wie sie überliefert wurde (WIPO 2003a:26, Lewinski 2004:144). Diese Unterscheidung mag dem Verständnis einiger indigener Gruppen von ihren traditionellen Kulturgütern wiedersprechen, ist jedoch für eine Betrachtung aus Sicht des nationalen wie internationelen Zivilrechts absolut erforderlich (Wendland 2008:174).

\subsection{Der Schutz der Folklore stricto sensu}

Der Schutz der zu Grunde liegenden Folkloreform, der Folklore per se oder stricto sensu, durch das Urheberrecht ist eingehend und erschöpfend etwa von Riley (2000) und Lucas-Schloetter (2008) oder auch WIPO (2003a) behandelt worden, weswegen hier lediglich ein kurzer Überblick über die Probleme gegeben werden soll, die sich aus der Sicht des Urheberrechts für einen Schutz von Folklore ergeben.

\section{Das Originalitätserfordernis}

Welche Immaterialgüter durch ein Immaterialgüterrecht monopolisiert werden und welche nicht, wird durch den jeweiligen Gesetzgeber entschieden. Der genaue Fokus des Urheberrechts ist dabei seit jeher eher unklar und dem Wandel der Zeit unterworfen. Viele Rechtsordnungen und auch die Revised Berne Convention for the Protection of Literary and Artistic Workst (Berne Convention), als das grundlegende internationale Vertragswerk des Urheberrechts, vermeiden eine Festlegung daher (Schack 2007:82).

Zur Unterscheidung zwischen schutzfähigen und nicht schutzfähigen Werken hat sich jedoch eine Reihe von Merkmalen herausgebildet. Zwischen dem angloamerikanischen und dem kontinentaleuropäischen System zeigen sich hier indes erhebliche Unterschiede, die ihren Ursprung in den unterschiedlichen Begründungsansätzen für einen Schutz haben. So hat sich in Deutschland etwa der Begriff der Individualität als Unterscheidungsmerkmal herausgebildet. Ein Werk besitzt ausreichende Individualität, wenn es nicht lediglich vorhandene Ausdrucksformen wiederholt, sondern persönliche Züge trägt, die das Ergebnis eines Denkprozesses widerspiegeln (Wandtke et al. 2009). Das anglo-amerikanische Verständnis legt stärkeres Gewicht auf den Investitionsschutz. Nichtsdestotrotz,

\footnotetext{
${ }^{4}$ Siehe http://www.wipo.int/treaties/en/ip/berne/trtdocs_wo001.html (Zugriff am 04.05.2010).
} 
ist auch im US-Amerikanischen Urheberrecht die Originalität ein zentrales Kriterium. 5

Beide Ansätze sind in Hinsicht auf Folklore stricto sensu problematisch. Bei der Folklore soll im Normalfall gerade nicht der Geist des Schöpfers im Vordergrund stehen, sondern die Pflege und die Überlieferung bestehender Traditionen (Ramsauer 2005:65 mit weiteren Nachweisen). Die Ausübenden sind oft an strenge Vorgaben gebunden was die Ausübung ihrer Tradition angeht. Ein individueller Geist kann und soll hier nicht zum Ausdruck kommen (Farley 1997:21, LucasSchloetter 2008:293, weniger deutlich aber im Ergebnis ebenso Berryman 1993:316). Auch wenn man von den niedrigeren Anforderungen ausgeht, die das angloamerikanische Copyrightsystem vorsieht, so ist doch die unkünstlerische Wiedergabe oder Wiederholung von etwas Bestehendem nicht ausreichend, um die Anforderungen zu erfüllen. ${ }^{6}$

\section{Zeitlicher Umfang}

Weiteres grundlegendes Merkmal eines jeden Immaterialgüterrechts ist seine beschränkte zeitliche Gültigkeit. Die Berne Convention geht von einer Dauer von 50 Jahren post mortem auctoris aus, wobei es den Signaturstaaten durchaus frei steht, eine längere Dauer festzulegen. Die Funktion dieser zeitlichen Beschränkung lässt sich auf verschiedene Weise rechtfertigen. So verblasst ein jedes Persönlichkeitsrecht nach einer gewissen Dauer, so dass aus einer persönlichkeitsrechtlichen Perspektive eine Begrenzung zwingend erscheint (Rehbinder und Hubmann 2010).

Auch aus der ökonomischen Perspektive ist eine zeitliche Begrenzung geboten. Nach einer endlichen Dauer generiert ein Monopolrecht keinen Anreiz zur Schöpfung mehr, auf der anderen Seite steigen die sozialen Kosten, die durch das Monopol verursacht werden immer weiter (Landes und Posner 2003:214-216). Auch muss der Schöpfer, anders als bei materiellem Eigentum, keine weiteren Investitionen tätigen, um sein geistiges Eigentum funktionsfähig zu halten. Das heißt, dass seine Investitionen irgendwann mit entsprechenden Gewinnen wieder eingeholt sind, ohne dass ihm weitere Kosten entstehen. Damit entfällt die Rechtfertigung für einen weiteren Schutz nach einer gewissen Zeitspanne und der Allgemeinheit wird der Zugriff auf das monopolisierte Gut wieder gewährt.

Die Schöpfung von Folklore stricto sensu kann indes weit zurückreichen in die Vergangenheit - in manchen Fällen sogar so weit, dass, sofern das Werk jemals

\footnotetext{
${ }^{5}$ Bis 1991 wurde in den USA insbesondere von den Instanzgerichten die "sweat of the brow" Doctrine vertreten. Hiernach reichte die Anwendung von „knowledge, skill, labour and judgement" (Copinger, Skone James et al. 2005; Sherwood-Edwards 1994) aus, damit ein Schöpfer ein Urheberrecht auf das Werk erhielt. Mit dieser Rechtsprechung brach der US Supreme Court 1991 in Feist Publications v. Rural Telephone Service_(Feist Publications, Inc., v. Rural Telephone Service Co., 499 U.S. 340 (1991).

${ }^{6}$ Gerade dies war Gegenstand von Feist Publications, Inc., v. Rural Telephone Service Co., 499 U.S. 340 (1991).
} 
hätte Urheberrechtsschutz genießen können, dieser schon seit langem abgelaufen wäre (Nordmann 2001:141, Farley 1997:18).

\section{Autorenschaft/Rechtsinhaberschaft}

Mit dem vorherigen Punkt in Zusammenhang steht das Problem der Autorenschaft und hiermit wiederum in weiterer Konsequenz das Problem der Rechtsinhaberschaft. Sowohl die Dauer als auch die Rechtsinhaberschaft sind im Urheberrecht grundsätzlich an die Person des Schöpfers geknüpft (Rehbinder und Hubmann 2010:154). Es ist heute weitgehend anerkannt, dass die Vorstellungen einiger indigener Gemeinschaften in Konflikt mit dem individualisierten Verständnis von Rechtsinhaberschaft stehen, welches die modernen Urheberrechtssysteme abbilden (WIPO 2003a:36, Riley 2000:191).

Dieser Kontrast wird im Vergleich mit dem kontinentaleuropäischen Ansatz besonders deutlich. Während hier nach der Aufklärung gerade das Individuum in den Mittelpunkt rückte, ist dieses Verständnis anderen Kulturen fremd und wirkt auf diese auch befremdlich (Ramsauer 2005:68). Dies gilt nicht nur im Bereich des Immaterialgüterrechts, tritt hier aber besonders deutlich zu Tage (vgl. Herz 1993).

\subsection{Rechte an heutigen Ausdrucksformen der Folklore}

Auf der anderen Seite stehen die gegenwärtigen Ausdrucksformen der Folklore. Also das, was heutige Künstler auf Grund der Folklore stricto sensu schaffen. Folklore zeichnet sich insbesondere dadurch aus, dass sie sich in einem fortlaufenden Prozess entwickelt hat und durch ihre Träger in eben diesem Prozess am Leben gehalten wird (WIPO 2003a). So kommt den Rechten eben dieser Träger insbesondere im Zusammenhang mit der wirtschaftlichen Verwertung der Folklore erhebliche Bedeutung zu. Ein heutiger Künstler kann auf vielfältige Weise ein Recht an seinem von der Folklore stricto sensu abgeleiteten Werk erlangen.

\section{Bearbeitung eines Werkes in der Public Domain}

Wer ein Werk der Public Domain bearbeitet, kann an dieser Bearbeitung grundsätzlich ein Urheberrecht erlangen. Dieses Recht ist auf die Teile des Werkes beschränkt, welche nicht gemeinfrei sind. So kann sich derjenige, der ein Werk der Folklore stricto sensu bearbeitet, grundsätzlich dagegen wehren, dass die Bearbeitung etwa kopiert wird (Lucas-Schloetter 2008:302). Die Teile der Bearbeitung, welche schon zuvor in die Public Domain fielen, genießen indes keinen Schutz. Jeder Dritte könnte sie isoliert aus der Bearbeitung entnehmen und nutzen. 


\section{Leistungsschut:rechte}

Auch auf die Folklore stricto sensu anwendbar sind die Leistungsschutzrechte. In diesem Zusammenhang ist insbesondere das Leistungsschutzrecht des ausübenden Künstlers von Bedeutung. Führt jemand ein Werk auf, so kann er hieran ein sogenanntes right in performance erlangen. Die ausübenden Künstler stellen das Medium dar, durch welches das Werk in die Öffentlichkeit gelangt und werden hierfür mit einem eigenen Recht belohnt. Die Leistungsschutzrechte honorieren mithin nicht das Schöpferische, sondern die Wiedergabe von etwas Bestehendem (Schack 2007:267-268). Grundlagen für eine internationale Regelung wurden im Jahre 1961 durch die International Convention for the Protection of Performers, Producers of Phonograms and Broadcasting Organizations gelegt. Mit der ausdrücklichen Einbeziehung der Folklore in der WIPO Performances and Phonograms Treaty 1996 wurde klargestellt, dass auch derjenige, der ein Werk der Folklore aufführt, als ausübender Künstler im Sinne der Leistungsschutzrechte gesehen werden soll. Es ist zu erwarten, dass nach und nach immer mehr Länder die Folklore in die geschützten performances aufnehmen werden (Lewinski 2006:5).

\subsection{Das Verhältnis der Folklore per se zu ihrer Darstellung}

Wir rekapitulieren an dieser Stelle: Die Folklore stricto sensu kann keinen Schutz durch das Urheberrecht erlangen. Die individuelle Ausführung dagegen kann durch Bearbeitungsrechte oder Leistungsschutzrechte geschützt sein. Diese Situation führt zu einer Reihe von Problemen.

Zunächst steht die Aufführung der Folklore stricto sensu jedem zu. Solange diese in der Public Domain verbleibt, kann mithin niemand, ob Mitglied der indigenen Gemeinschaft oder nicht, daran gehindert werden, die Folklore aufzuführen und ein Leistungsschutzrecht an dieser Aufführung zu erwerben. Dies widerspricht indes in erheblichem Maße den Interessen der indigenen Gemeinschaften, die sich gerade durch eine unangemessene Nutzung oder Ausführung ihrer Folklore stricto sensu bedroht sehen (Farley 1997:8). Hinzu kommt, dass die Gewährung eines Leistungsschutzrechtes eine Ausbeutung der Folklore stricto sensu noch attraktiver zu machen droht. Auch die Bearbeitung eines Werkes der Public Domain steht grundsätzlich jedem frei.

Aus Sicht der indigenen Gruppen ist dies ein unhaltbarer Zustand. Weiter wird es als eine Frage der Gerechtigkeit betrachtet, die Kontrolle der Folklore in die Hände der Gemeinschaften zu legen, aus deren Mitte sie stammt. Auch darf die Tatsache, dass die Kontrolle über die Folklore auch die Kontrolle über die finanziellen Gewinne mit sich bringt, nicht außer Acht gelassen werden (WIPO 2003a). 


\subsection{Konstruktion einer Rechtsinhaberschaft der indigenen Gruppen}

Die individuelle Rechtsinhaberschaft ist das wohl drängendste Problem sowohl im Zusammenhang mit der Folklore stricto sensu als auch mit den Rechten an den heutigen Ausdrucksformen der Folklore. Es sind verschiedene Wege erwogen worden, um die Rechte den Gemeinschaften zuzuweisen, wie es dem Selbstverständnis der Gruppe entspräche (Nordmann 2001:177-179).

\section{Miturheberschaft}

Zunächst ließe sich daran denken, die dargestellte Form als ein gemeinsames Werk des individuellen Künstlers und der Gemeinschaft zu verstehen. Jedes moderne Urheberrechtsgesetz beinhaltet eine Regelung, die Schöpfern eines gemeinsamen Werkes auch das Urheberrecht gemeinsam zuweist. ${ }^{7}$ Gemeinsame Schöpfer haben das Urheberrecht auch gemeinsam auszuüben und bei der Ausübung der Rechte aufeinander Rücksicht zu nehmen. Erkennt man eine Miturheberschaft der Gruppe mithin an, müssten Dritte, die etwa die Folklore kommerziell nutzen wollen, die Zustimmung der Gemeinschaft einholen.

Eine solche Miturheberschaft setzt indes voraus, dass alle Beitragenden ihre Werke im Moment der Schöpfung bewusst untrennbar verbunden haben. Eine sukzessive Schöpfung stellt nach dem herrschenden Verständnis gerade kein gemeinsames Werk dar (Goldstein 2001:208, Nordmann 2001:119). Somit wären nur die Gruppenmitglieder als Schöpfer anzusehen, die zu dem Werk direkt beigetragen haben. Die Gruppe an sich und, wie es dem Verständnis der indigenen Gruppe entspräche, die Vorfahren der heutigen Schöpfer auch als Inhaber der Rechte zu betrachten, ist nach diesem Konzept indes nicht möglich (Farley 1997:34).

\section{Rechtsübertragung}

Weiterhin könnte man an einen freiwilligen Transfer der Rechte denken. Erwirbt ein indigener Künstler ein Recht an einem Werk der Folklore, steht es ihm, in dem durch seine Rechtsordnung vorgegebenen Rahmen, frei, das Recht zu übertragen. Sofern die indigene Gruppe eine entsprechende Rechtspersönlichkeit besitzt, ließe sich das Recht auch auf die Gruppe übertragen (Nordmann 2001:124-125, zu den hiermit einhergehenden Problemen auch Schlinkert 2007:91-92).

Dass dieses Konzept den Bedürfnissen der indigenen Gruppen nach einem Schutz gegen Ausbeutung nur unzureichend gerecht wird, ist indes evident. Dritte werden sich regelmäßig nicht an die internen Regeln der Gemeinschaften gebunden fühlen und das Recht übertragen. Auch bei Gemeinschaftsangehörigen steht keinesfalls fest, dass diese bereit sind, ihre Rechte zu übertragen.

\footnotetext{
7 Etwa Deutschland: \8 I UrhG; USA: Copyright Act 1968, Sec. 10 (1) “works of joint authorship”; Mexico: Copyright Law, Art. 12.
} 


\section{Abhängige Schöpfung}

Zuletzt soll an dieser Stelle das Konzept der abhängigen Schöpfung erwähnt werden. Es handelt sich hierbei um eine Konstruktion, die insbesondere im Rahmen der Arbeitsverhältnisse große Bedeutung genießt. In den vom Copyright geprägten Rechtsordnungen entsteht das Urheberrecht im Falle, dass das Werk in einem Abhängigkeitsverhältnis geschaffen wird, direkt in der Hand der, mitunter auch juristischen, Person des Arbeits- oder Auftragsgebers. In kontinentaleuropäischen Rechtsordnungen ist es wegen des strengen Schöpferprinzips zwar nicht möglich, das Recht direkt in der Hand des Auftraggebers entstehen zu lassen, jedoch kann der Urheber verpflichtet sein, seinem Auftraggeber die Nutzungsrechte an dem von ihm geschaffenen Werk einzuräumen. Es wurde erwogen, dass sich diese Konzepte auch auf das Verhältnis des indigenen Künstlers zu seiner Gruppe übertragen ließen (WIPO 2003a).

Abgesehen davon, dass eine solche Fiktion wohl dem Selbstverständnis vieler indigener Gruppen zuwider liefe, bedarf es eines sehr weiten Verständnisses des Abhängigkeitsverhältnisses, um die reine Gruppenzugehörigkeit schon als solches zu betrachten (Nordmann 2001:123-124).

\subsection{Zusammenfassung}

Bis zu diesem Punkt lässt sich feststellen, dass die Folklore per se keinen Schutz genießt. Ihr fehlt es an der nötigen Gestaltungshöhe und an einem identifizierbaren Autor, außerdem wird ihr Schutz oftmals bereits zeitlich abgelaufen sein, so er denn jemals bestand. Dagegen können heutige Ausdrucksformen der Folklore auf vielfältige Weise Schutz genießen; entweder als eigenschöpferische Bearbeitung oder zumindest als leistungsschutzrechtlich geschützte Auf- oder Ausführung. Für die indigenen Gemeinschaften ist dies in vielfältiger Hinsicht tragisch. Nicht nur, dass sie selbst keine Kontrolle ausüben können, sie müssen es unter Umständen auch noch hinnehmen, dass Dritte Rechte an ihrer Folklore erwerben. Die Möglichkeiten die Rechte den Gemeinschaften zuzuweisen wirken vor dem Hintergrund des Verständnisses der indigenen Gruppen von Schöpfung und Inhaberschaft von Folklore konstruiert und unzureichend. Aus diesem Grunde werden insbesondere im Rahmen der WIPO, aber auch auf nationaler Ebene, heute sui generis-Lösungen angestrebt, welche nicht an die klassische Systematik des Urheberrechts gebunden sind. Zwei der jüngsten Ansätze sollen im Folgenden untersucht werden.

\section{Systematik eines sui generis Schutzes}

Es fehlt, stärker vielleicht als in anderen Bereichen, auf dem Feld des geistigen Eigentums in vielen ehemaligen Kolonialstaaten an einer eigenständigen Rechts- 
tradition, die auf die kulturellen Besonderheiten zugeschnittene Begründungsansätze und Dogmatik hätte vorbringen können.

Dies wird zum Teil als derart unbefriedigend empfunden, dass eine Hinwendung zu einem ganz eigenen Recht des geistigen Eigentums gefordert wird (Forsyth 2003). Die Bemühungen der WIPO, der UNESCO und anderer nationaler wie regionaler Organisationen ein sui generis Recht zu schaffen, können in diesem Kontext als Versuch gesehen werden, eben dies zu erreichen. Hier sollen zwei der jüngsten Ansätze mit besonderer Hinsicht auf Folklore diskutiert werden.

\subsection{Moderne sui generis Ansätze}

Die Versuche, einen eigenen Rechtsschutz für Folklore mittels eines sui generis Systems zu schaffen, sind vielfältig. Bisher hat sich jedoch noch kein Vorstoß international durchsetzen können. Regelmäßig scheiterten die Bemühungen an der Umsetzung in den einzelnen Staaten. Inzwischen hat die Entwicklung indes wieder an Fahrt gewonnen und mit dem Pacific Model Law for National Laws so wie den WIPO Draft Provisions liegen nunmehr zwei sui generis Ansätze vor, die sich wesentlich differenzierter mit dem Schutz von Folklore auseinandersetzen als etwa noch das Tunis Model Law aus dem Jahre 1976, welches zwar als erstes Gesetz Folklore ausdrücklich in den Schutz einbezog, in wesentlichen Punkten jedoch nicht dem Interesse der indigenen Gemeinschaften entsprach (Nordmann 2001:28).

\section{Das Pacific Model Law}

Das Pacific Model Law wurde vom Sekretariat des Pacific Islands Forum als Modell für die regionale Gesetzgebung ausgestaltet. Es verfolgt explizit den Zweck, Ausdrucksformen der Folklore zu fördern. Jeder Staat, der das Gesetz als Ganzes oder Teile hiervon übernehmen will, ist gehalten dies zu tun. Entwickelt wurde das Modell in enger Zusammenarbeit des Sekretariats der Pazifischen Gemeinschaften mit der UNESCO. Das Pacific Model Law ist der Versuch, die großen Unterschiede in den nationalen Gesetzgebungen der Mitglieder auszugleichen (Forsyth 2003).

\section{Die WIPO Draft Provisions}

Die WIPO Draft Provisions wurden auf Anfrage des IGC auf Basis verschiedener Dokumente $^{8}$ erstellt und auf Grundlage der eingereichten Kommentare erweitert. Ziel war es, eine Grundlage zu schaffen, anhand derer ein Folkloreschutz implementiert werden kann (WIPO 2006b). Hierbei sollte besondere Rücksicht auf die Bedürfnisse der indigenen Völker auf der einen Seite und auf die Kompatibilität mit den Rechtssystemen verschiedener Länder andererseits gelegt werden. Eine

8 WIPO/GRTKF/IC/3/10; WIPO/GRTKF/IC/5/3; WIPO/GRTKF/IC/6/3; WIPO/GRTKF/IC/6/3; WIPO/GRTKF/IC/7/3; WIPO/GRTKF/IC/7/4; WIPO/GRTKF/IC/8/4. 
entscheidende Erkenntnis, die von der WIPO erstmals in dieser Klarheit geäußert wurde, war, dass ein universelles Regelungssystem, welches auf alle Bedürfnisse und Ansprüche der verschiedenen Gruppen, Länder und Jurisdiktionen eingeht, nicht ohne Weiteres zu erreichen ist (WIPO 2006b).

\subsection{Geschützte Güter}

Im Urheberrecht wird grundsätzlich nur eine konkrete Ausdrucksform geschützt. Die hinter dieser Ausdrucksform stehende Idee, Erkenntnis oder Information bleibt dagegen frei (Schack 2007:87). Das heißt, dass etwa eine Methode, ein Konzept oder ein bestimmter Stil an sich nicht schutzfähig ist, sondern nur ein konkretes Werk, welches nach diesem Stil geschaffen wird.

Die sui generis Regelwerke umfassen dagegen einen wesentlich weiteren Fokus. Unter anderem gehören Namen, Zeremonien und kulturelle Praktiken zu den vorgeschlagenen Schutzgütern nach dem Pacific Model Law. Entscheidend ist, dass jegliche Form, in der sich traditionelles Wissen zeigt, inklusive Motive und Geschichten geschützt sein soll (Secretariat of the Pacific Community 2002b: Explanatory Memorandum Clause 7). Ähnlich weitreichend sehen auch die Revised Draft Provisions den Schutz der relevanten Güter vor.

\subsection{Das Schutzsystem der sui generis Regeln}

Ein Blick auf das Schutz- und Abwehrsystem der sui generis Regelwerke, den eigentlichen Kern der Regelungen, zeigt deutlich, wie sehr ihre Systematik an derjenigen des Urheberrechts orientiert ist. So gewährt das Pacific Model Law die traditional cultural rights, die ihrem Umfang nach den klassischen urheberrechtlichen Verwertungsrechten entsprechen. Den moral rights, also den aus dem kontinentaleuropäischen Ansatz bekannten Urheberpersönlichkeitsrechten, ist ein eigener Abschnitt gewidmet. Alle in Art. 7 II Pacific Model Law beschriebenen Nutzungen bedürfen der vorherigen Zustimmung der Gemeinschaft, wenn sie außerhalb des traditionellen Kontextes vorgenommen werden. Hierdurch soll sichergestellt werden, dass die Nutzung von Folklore innerhalb der Gemeinschaften nicht durch das Recht behindert wird (Secretariat of the Pacific Community 2002a:39).

Das Schutz- und Abwehrsystem der Revised Draft Provisions ist ebenfalls an das Urheberrecht angelehnt. Jedoch wird hier zwischen verschiedenen Schutzniveaus differenziert. Die Revised Draft Provisions unterscheiden bzgl. des Umfangs der gewährten Rechte zwischen solchen Folkloreformen, die bei einer zuständigen Stelle registriert wurden, und solchen bei denen das nicht der Fall ist. Für die registrierten Formen gilt das höchste Schutzniveau. Die Nutzung einer solchen Form ist nur dann erlaubt, wenn die Zustimmung der Gemeinschaften vorliegt, unabhängig davon, ob die Nutzung mit Gewinnerzielungsabsicht erfolgt oder nicht. Hinzu kommt die explizite Möglichkeit, Dritte am Erwerb eines Immaterialgüterrechts an Folklore zu hindern. Hiermit soll einem Problem begegnet werden, das sich tradi- 
tionell im Bereich des Traditional Knowledge stellt. Die Gefahr, dass Dritte sich verfügbare Rechte an indigenen Zeichen oder Ähnlichem sichern. Bei den nicht registrierten Folkloreformen ist der Schutz dagegen auf die Rechte des Art. 3 (b) Revised Draft Provisions beschränkt. So kann in jedem Falle die Anerkennung der Gemeinschaft als Quelle der Folklore verlangt und jede herabsetzende Nutzung verhindert werden. Nur im Falle, dass die Verwendung mit Gewinnerzielungsabsicht erfolgt, soll der Gemeinschaft ein Anteil der Einnahmen oder eine andere Vergütung zukommen.

\subsection{Wesentliche Merkmale}

Die sui generis Ansätze sind vor dem Hintergrund der Schwäche des klassischen Urheberrechts für einen Schutz der Folklore stricto sensu entwickelt worden. Ihrer Struktur nach ähneln sie Urheberrechtsgesetzen. Sie weisen indes einige Besonderheiten auf, die es hier zu erschließen gilt.

\section{Originalitätserfordernis}

Auch im Rahmen des Folkloreschutzes stellt sich die Frage, welche Kriterien anzulegen sind, damit eine bestimmte Ausdrucksform dem Schutz unterfällt. Sowohl das Pacific Model Law als auch die Revised Draft Provisions verzichten auf das Kriterium der Originalität. Anstatt die Individualität oder Fähigkeit des Schöpfers als wesentliche Hürde für den Schutz in den Mittelpunkt zu stellen, wie es typisch für das Urheberrecht ist, werden im Pacific Model Law alle solchen "expressions of culture" geschützt, in denen sich traditionelles Wissen manifestiert (Art. 4 Pacific Model Law, Ramsauer 2005:100). Es soll ausdrücklich nicht auf die Originalität oder Neuheit ankommen, es muss sich lediglich um einen Ausdruck menschlicher Kreativität handeln (Secretariat of the Pacific Community 2002a:19). Auch die Revised Dradt Provisions wählen einen bewusst offenen Ansatz, der keine engeren Voraussetzungen vorsieht und verweisen explizit auf die nationalen Gesetzgeber, um die genauen Voraussetzungen festzulegen.

\section{Dauer des Schutzes}

Die sui generis Regelungen nehmen die Forderungen nach einem ewigen Schutz der Folkloreform auf. Sowohl das Pacific Model Law (Art. 9) als auch die Revised Draft Provisions (Art. 6) gehen davon aus, dass ein Recht der Folklore ewig gewährt werden soll.

\section{Rechtsinhaberschaft}

Das Bedürfnis einer kollektiven Rechtsinhaberschaft wird allgemein als die wesentliche Hürde für den Schutz der Folklore stricto sensu durch das Urheberrecht gese- 
hen. Sowohl die Revised Draft Provisions als auch das Pacific Model Law nehmen diese Frage auf und lösen sie auf differenzierte Weise. Das Pacific Model Law weist die Rechte an der Folklore stricto sensu entweder den Gruppen selbst oder den Individuen, die von den Gruppen in Übereinstimmung mit dem customary law als Verantwortliche für diese Rechte angesehen werden, zu (Art. 6 Pacific Model Law). Hilfsweise können die Rechte auch dem Staat zugewiesen werden (Ramsauer 2005: 105). Auch im Falle der Revised Draft Provisions liegen die Rechte bei den indigenen Gruppen. So soll der Schutz den Gruppen zu Gute kommen, in denen eine bestimmte Folkloreform ihren Ursprung hat, die sie pflegen, bewahren oder ausführen (Art. 2 Revised Draft Provisions). Von eben diesen Gruppen muss die Zustimmung zur Verwertung eingeholt werden (Art. 4 Revised Draft Provisions) und diese sind berechtigt sie zu registrieren (Art. 7 Revised Draft Provisions).

\section{Die sui generis Regelwerke und die kumulierte Schöpfung}

An dieser Stelle soll ganz besonders der Rat von Hilty (2009) ernst genommen werden, sich auf die Erfahrungen zu stützen, die wir bereits haben. In diesem Zusammenhang kann dies insbesondere bedeuten, die Lehren, die wir aus dem Urheberrecht ziehen können, auf das sui generis System, welches dem Urheberrecht in seinen Rechtsfolgen derart ähnlich ist, zu übertragen. Da Geisteswerke regelmäßig auf etwas Vorbestehendem aufbauen, stellt sich die Frage, wie dieser Prozess durch die sui generis Regelwerke beeinflusst wird. Im Wesentlichen lassen sich aus Sicht des Urheberrechts zwei Wege der Schöpfung unterscheiden.

In einem Fall hängt das neuere Werk vom älteren ab. Der Schöpfer übernimmt wesentliche Teile des alten Werkes und verwendet sie für sein Werk. Diese Art der Nutzung stellt als Bearbeitung eine Verletzung des Urheberrechts am Ausgangswerk dar, sofern der Schöpfer des abgeleiteten Werks nicht die notwendige Zustimmung des Schöpfers des Ausgangswerkes eingeholt hat. Der entgegengesetzte Fall ist die sogenannte freie Benutzung. Hier lässt sich der Schöpfer lediglich von einem anderen Werk inspirieren, ohne dieses jedoch direkt zu übernehmen. In diesem Falle bedarf es keiner Zustimmung durch den Schöpfer des Ausgangswerkes.

Wo die Grenze zwischen freier Benutzung und unfreier Bearbeitung verläuft, ist nicht selten schwierig zu bestimmen und regelmäßig Gegenstand heftiger Diskussionen. So hat sich im deutschen Recht die Lehre vom inneren Abstand entwickelt. Nach dieser Faustformel liegt eine freie Bearbeitung dann vor, wenn das Werk sich innerlich vom Ausgangswerk so weit entfernt, dass das Originalwerk mit seinen Eigenarten hinter den Eigenarten der Bearbeitung verblasst (Schack 2007:118). Ähnliche Ansätze finden sich in vielen Rechtsordnungen (Für die USA: Landes und Posner 1989:347).

Auch das Pacific Model Law so wie die Draft Provisions sehen es vor, dass die Folklore stricto sensu genutzt wird, um derivative Werke zu schaffen, stellen jedoch 
die "adaptation" und die "creation of derivative works", also die Nutzung einer bestehenden Folkloreform zum Zwecke der weiteren Schöpfung, unter den Zustimmungsvorbehalt. Offen ist indes, ob eine freie Benutzung überhaupt noch vorgesehen ist. Die Model Provisions von 1982 sahen eine Schranke für die freie Benutzung (in Form des "borrowing") ausdrücklich vor (Nordmann 2001:206). Eine solche Freistellung fehlt indes in den Revised Draft Provisions. Auch das Pacific Model Law sieht eine entsprechende Schranke nicht vor.

Angesichts des weiten Schutzbereiches und des Fehlens einer ausdrücklichen Freistellung der freien Benutzung ist hier von einem sehr geringen Raum für bloße Inspiration auszugehen. Ausgleichsregeln, die den freien Fluss der Ideen in Einklang mit den Schutzzielen bringen sollen, sind im Gegensatz zu den WIPO Model Provision von 1982 (Ramsauer 2005:120) nicht zu erkennen. Das heißt indes nicht zwingend, dass sich ein Künstler nicht von indigener Kunst inspirieren lassen kann. Jedoch ist der Rahmen, in dem er sich bewegt, wesentlich enger gesteckt. Nicht nur Werke, die nach dem klassischen urheberrechtlichen Verständnis vom Vorbestehenden abhängig sind, bedürfen der Zustimmung, sondern auch solche, die sich lediglich an etwas Vorbestehendem orientieren. Der Raum für Inspiration ist also wesentlich verengt. Der Schutz durch die sui generis Regelwerke soll nach Vorstellung ihrer Schöpfer komplementär zum Urheberrecht und anderen Immaterialgüterrechten sein (Secretariat of the Pacific Community 2002b, WIPO 2003a, WIPO 2006c Annex I:45). Das bedeutet indes, dass Bearbeiter und ausführende Künstler weiterhin die oben genannten Rechte erwerben können. Nunmehr sind diese in ihrer Schöpfung jedoch von der Zustimmung der indigenen Gemeinschaften abhängig, wie sie es bei der Nutzung eines urheberrechtlich geschützten Werks wären.

\section{Die sui generis Regeln und ihre Konsequenzen für die Allgemeinheit}

Die vorgestellten sui generis Ansätze haben erhebliche Auswirkung auf die Schöpfung abgeleiteter Werke. Ohne hier zu sehr ins Detail zu gehen, lassen sich hier doch einige mögliche negative Folgen vorhersagen, die ein Schutz nach dem Vorbild der sui generis Regeln haben könnte.

\subsection{Transaktionskosten}

Der Künstler, der ein traditionelles Motiv verwenden will, müsste regelmäßig eine Lizenz zur Nutzung eben diesen Motivs erwerben. Dies kann insbesondere in dem Fall zu erheblichen Problemen führen, in dem sich verschiedene Gruppen uneinig über die Inhaberschaft einer bestimmten Folkloreform sind. Für einen Dritten, der eine Ausdrucksform der Folklore nutzen möchte, entsteht so erhebliche Rechtsunsicherheit. Um nicht Gefahr zu laufen, mit seiner Schöpfung das Recht einer der 
indigenen Gemeinschaften zu verletzen, müsste er sicherstellen, alle relevanten Akteure einbezogen zu haben. Dies ist grundsätzlich ein Problem, welches sich auch im Zusammenhang mit dem Urheberrecht stellt. Jedoch ist hier die Zahl der beteiligten Akteure wesentlich geringer. Auch zeigt sich hier einer der wesentlichsten Aspekte der zeitlichen Beschränkung des Urheberrechts. Die Berechtigung von Akteuren zurückzuverfolgen, die schon seit mehreren hundert Jahren verstorben sind, dürfte jeden Erlaubnissuchenden vor erhebliche Probleme stellen.

\subsection{Meinungsfreiheit}

Ein Blick auf die Ausdrucksformen, die letztendlich monopolisiert werden sollen, zeigt, dass es sich hier auch um ganz grundlegende Mittel der kulturellen Kommunikation handelt. Dies ist insbesondere dort kritisch zu sehen, wo indigene Kunst als Ausdrucksmittel des Protestes, etwa gegen Eliten innerhalb der eigenen Gruppe oder Gesellschaft, genutzt wird. ${ }^{9}$ Den indigenen Künstlern stehen in erster Linie die Ausdrucksformen ihrer eigenen kulturellen Gruppe zur Verfügung, um sich zu artikulieren. Eine Monopolisierung könnte hier zu einer Art Zensurinstrument werden.

\subsection{Fortentwicklung innerhalb der Gemeinschaften}

Die erhöhten Transaktionskosten haben indes nicht nur Auswirkungen auf Auswärtige. Auch Gruppenangehörige wären von solchen Folgen betroffen. Insbesondere sind hier zwei Situationen zu erwägen. Zum einen sind auch indigene Künstler nunmehr an die Vorgaben der Gemeinschaften gebunden, die nicht zwangsläufig ihren eigenen Vorstellungen entsprechen. Sie würden somit der definitorischen Hoheit einer Autorität unterworfen, die andere Vorstellungen von kulturellem Kontext und Authentizität hat. Dies könnte zum einen dazu führen, dass eine bestimmte Art der Ausdrucksform als Standard definiert wird und die grundsätzlich so wichtige Fortentwicklung und Lebendigkeit der Ausdrucksformen der Folklore nicht mehr stattfindet. Es drohe die Gefahr von Zensur durch Eliten innerhalb der Gemeinschaften, ein Einfrieren der Kultur und ein Abwürgen der kulturellen Entwicklung (Brown 1998).

\section{Alternative Wege zum Schutz}

Coombe (2003a) attestiert einigen Kritikern eines stärkeren Schutzes indigener Kultur mangelndes Verständnis der indigenen Gemeinschaften und stellt fest, dass das westliche Verständnis der inneren Strukturen der indigenen Gemeinschaften sich weiterhin auf das Bild autoritärer patriarchalischer Herrschaft stützt. Sie zeigt

\footnotetext{
${ }_{9}^{9}$ Vergleiche das interessante Beispiel unter http://www.goethe.de/Ins/id/lp/prj/art/arc/pla/ess/ de166391.htm (Zugriff am 04.05.2010).
} 
an einer Reihe von Beispielen, dass es den indigenen Gruppen keineswegs darum geht, die Nutzung von Folklore als Ganzes zu verbieten. Vielmehr sei die wichtigste Forderung, wichtiger noch als ökonomischer Gewinn, eine Anerkennung der indigenen Gemeinschaften als Ursprung der betreffenden Folklore. Auf der anderen Seite sind die Vorbehalte, die gegen einen zu starren Folkloreschutz vorgebracht werden, nicht von der Hand zu weisen. Die von Coombe erwähnten Beispiele zeigen indes auch mögliche alternative Wege auf, die sich zwar weiterhin im Spannungsfeld zwischen Schutz und Freiheit bewegen, die größten Probleme indes zu lindern vermögen. Auch die Arbeit von Bicksei in diesem Band kann Anregung für weitere Untersuchung eines alternativen Ansatzes sein. Die wesentlichen Merkmale lassen sich hier kurz skizzieren.

\subsection{Schutz besonders heiligem Wissens}

Es ist keineswegs nur ein Merkmal indigener Kultur, dass es bestimmte immaterielle Güter gibt, deren Missbrauch nicht hingenommen werden kann. ${ }^{10}$ So wird in vielen europäischen Staaten die Verunglimpfung von religiösen Bekenntnissen sogar strafrechtlich verfolgt. ${ }^{11}$ Dieser Weg muss indes mit aller Vorsicht und Bedacht eingeschlagen werden, um hier nicht eben die kulturelle Zensur zu fördern, die auch als Vorbehalt gegen einen zu inflexiblen Schutz von Ausdrucksformen der Folklore besteht.

\subsection{Anleitung und Aufklärung der Träger der Kultur}

Ein zweiter wichtiger Aspekt, der auch in den sui generis Regeln zum Ausdruck kommt, ist die Anleitung und Aufklärung der Akteure über ihre Rechte. In dem von Coombe (2003a) vorgestellten Fall der Ami war ein Schutz durch ein right in performance durchaus zu gewähren. Hier stellt sich mithin die Aufgabe, die ausführenden Gruppenmitglieder über ihre Rechte aufzuklären und ihnen Mittel an die Hand zu geben, ihre Rechte auch transnational durchzusetzen.

\subsection{Anerkennung der Gruppe}

Ein dritter Aspekt ist die Anerkennung der Gruppe als Quelle einer bestimmten Ausdrucksform. Dies ist zum einen eine der wesentlichen Forderungen indigener Gruppen, hat zum anderen jedoch auch ökonomische Konsequenzen. Eine explizite Anerkennung der Authentizität hat an sich einen ökonomischen Wert (LucasSchloetter 2008:309 mit weiteren Nachweisen). Durch die Einführung von Authentizitätsmarken könnte besonders im Bereich der künstlerischen Ausdrucks-

10 Zur ökonomischen Begründung dieses Minimalschutzes siehe Bicskei, Bizer und Gubaydullina in diesem Band.

11 Siehe etwa den $\$ 166$ StGB, welcher indes sehr umstritten ist. Informativ zur ganzen Problematik Renzikowski (2002). 
formen, in dem Authentizität von besonderer Bedeutung ist, ein System geschaffen werden, welches den indigenen Gemeinschaften eine bessere Position auf dem Markt der Kulturgüter einräumt.

\section{Schlussbetrachtung}

Ein klares Ergebnis lässt sich nur schwerlich formulieren. Zwei Erkenntnisse sind substantiell. Zum einen bieten die sui generis Systeme einen weitreichenden Schutz der Folklore stricto sensu, zum anderen sind die Auswirkungen eines starren Folkloreschutzes nicht absehbar. Alternativen sind indes nicht leicht $\mathrm{zu}$ finden. Selbst enthusiastische Befürworter eines Schutzes sehen die ganz grundlegenden Probleme, die eine Monopolisierung mit sich bringt. Jedoch sollte dies nicht dazu führen, einen Schutz für unmöglich zu halten. In diesem Zusammenhang sollten die hier gemachten Vorschläge verstanden werden. Sie sind fragmentarisch und erheben keinen Anspruch, vollständig zu sein. Vielmehr sind sie der Ausgangspunkt der weiteren Arbeit zu diesem Thema. Insbesondere sei darauf hingewiesen, dass auch die Alternativvorschläge eine Reihe von Problemen mit sich bringen, die teilweise einer juristischen, teilweise einer anthropologischen Herangehensweise bedürfen. Die Frage was authentisch ist, wird von Juristen schwer zu beantworten sein. Die Frage, wie eine Rechtsregel in ein bestehendes System zu integrieren ist, liegt auBerhalb des anthropologischen Kontextes. So werden auch weitere Arbeiten zu diesem Thema stets die Expertise verschiedener Disziplinen benötigen. 




\title{
Der Schutz kultureller Güter: Die Ökonomie der Identität
}

\author{
Marianna Bicskei, Kilian Bizer, Zulia Gubaydullina
}

\section{Einleitung}

Das Intergovernmental Committee on Traditional Knowledge, Genetic Resources and Traditional Cultural Expressions der World Intellectual Property Organization (WIPO) bietet Ländern aus allen Teilen der Welt ein Forum, um darüber zu beraten, ob mehr Schutz für kulturelle Güter notwendig ist. Die Convention on the Protection and Promotion of the Diversity of Cultural Expressions der Vereinten Nationen legt ergänzend fest "to protect and promote the diversity of cultural expressions, [...] and to give recognition to the distinctive nature of cultural activities, goods and services as vehicles of identity, values and meaning" (UNESCO 2005, Article 1(a) und (g)). Der Schutz der Kultur in bestimmten Aspekten und Konstellationen steht also auf der internationalen Agenda. Was ist aus ökonomischer Sicht davon zu halten?1

Kultur äußert sich auf vielfältige Art und Weise, z.B. in sakralen Ritualen oder in Bildern, die einen wichtigen Teil der Identität bestimmter Gruppen ausmachen und vor widerrechtlicher Aneignung und Missbrauch geschützt werden sollten. Dies könnte spezifische Maßnahmen erfordern, die bestimmte Kulturgüter unter Schutz stellen.

\footnotetext{
1 Wir erhielten wertvolle Kommentare zu früheren Versionen dieses Papiers von Prof. Ejan Mackaay, sowie von Prof. Dr. Gerald Spindler und PD Dr. Christoph Brumann.
} 
Während tatsächlich einige Länder mehr Schutz für bestimmte kulturelle Güter fordern, sehen aber andere diese eher als öffentliche Güter. Letztere argumentieren, dass die meisten kulturellen Güter frei zugänglich sein sollten, solange grundlegende intellektuelle Eigentumsrechte nicht verletzt sind. So ist es beispielsweise beim Yoga als einer Meditationsform, die zwar weltweit praktiziert wird, ursprünglich aber aus Indien stammt. Sollte Yoga nur der indischen Bevölkerung zugänglich sein? Oder sollte jeder nichtindische Yoganutzer eine Genehmigung erwerben müssen oder ein Entgelt zahlen? Wie verhält es sich bei jemandem, der eine fremde Sprache lernen möchte: Sollte man auch dafür einen bestimmten Betrag entrichten müssen, weil die Sprache zu einer anderen Kultur gehört?

In diesem Beitrag analysieren wir, ob kulturelle Güter aus einer ökonomischen Perspektive, die ausdrücklich die Effekte auf die Identität einschließt, einen weitergehenden Schutz verlangen. Wir entwickeln eine Struktur, die erlaubt, verschiedene Effekte auf drei der relevanten Akteursgruppen zu bewerten. Wir unterscheiden die Akteursgruppen Kulturträger, Kulturkonsumenten und Kulturproduzenten, die alle durch kulturelle Güter betroffen sind. Auf der Basis dieser Bewertungsgrundlage können wir einen Einblick in das Regulatory Choice Problem nationaler Gesetzgeber sowie der internationalen Gemeinschaft schaffen, der eine Antwort auf die Frage gibt, wie viel Schutz kultureller Güter generell anzustreben ist.

Dieser Beitrag gliedert sich wie folgt: Abschnitt 2 diskutiert Identität als ökonomische Variable, die wir für entscheidend für die Bewertung des Schutzes kultureller Güter halten. Abschnitt 3 betrachtet den Nutzen, der durch Konsum und Produktion kultureller Güter entsteht, und entwickelt einen Vorschlag, wie diese Güter nach direkten und indirekten Effekten klassifiziert werden können. In Abschnitt 4 behandeln wir verschiedene Fragestellungen in Bezug auf den Schutz kultureller Güter. Abschnitt 5 präsentiert darauf aufbauend einige Schlussfolgerungen.

\section{Schutz kultureller Güter: Ein Literaturüberblick}

Um unsere auf Identität basierende Argumentation zum Schutz kultureller Güter zu entwickeln, betrachten wir zuerst Identität aus ökonomischer Sicht und gliedern diese in die ökonomische Nutzenfunktion ein. Die ökonomische Literatur zu kulturellen Gütern bietet dafür mehrere Ansätze, die sich hauptsächlich auf internationalen Handel gründen. Die deskriptive Literatur über Identität stützt sich zum Großteil auf die Thesen von Akerlof und Kranton (2000), deren Beitrag zur Beziehung zwischen Identität und ökonomischen „Ergebnissen“ breite Akzeptanz gefunden hat, dass nämlich Identität als ein determinierender Faktor der Nutzenfunktion zu sehen sei.

Die Nutzenfunktion in ihrem Modell besteht aus folgenden drei Variablen: Identität, die durch soziale Kategorien wie Geschlecht oder Beschäftigung und deren typische Charakteristiken bestimmt wird. Dabei sind die sozialen Kategorien, 
die als eine Form von Gruppierung betrachtet werden können, mit unterschiedlichen optimalen physischen Merkmalen und vorgeschriebenen Verhaltensweisen verknüpft. Die zweite Variable bezieht sich auf die Handlungen einer Person zur Nutzenmaximierung, während sie versucht, nicht von den Vorschriften sozialer Kategorien abzuweichen. Die dritte Variable besteht schließlich aus den Handlungen anderer Menschen, die zusammen mit der zweiten Variable den Güter- und Dienstleistungskonsum einer Person entscheidend beeinflussen. Es wird dabei angenommen, dass eine bestimmte soziale Kategorie die Identität einer Person prägt und so das identitätsbezogene Verhalten mitbestimmt. Dies bedeutet, dass Identität eine wichtige Rolle für die Nutzenfunktion spielt, da die Handlungen einer Person ihren Nutzen verändern können, abhängig davon, wie stark diese Handlungen auch die Identität des Handelnden beeinflussen. Im Modell von Akerlof und Kranton entstehen identitätsbasierte Payoffs sowohl durch die eigenen Handlungen als auch die anderer Individuen. Das bedeutet, dass die Handlungen anderer Personen den eigenen Nutzen und die eigene Identität verändern können. Dieser Sachverhalt soll im Folgenden näher untersucht werden.

In diesem Beitrag konzentrieren wir uns vor allem auf Nutzenveränderungen, die durch die Handlungen anderer ausgelöst werden. Akerlof und Kranton (2000:725) argumentieren, dass “...those who try to change social categories and prescriptions may face similar derision because the change may devalue other's identity...". Im Akerlof-Kranton-Modell haben Personen die Möglichkeit, begrenzt Einfluss auf ihre Identität zu nehmen. Grundsätzlich ist es möglich, sich anderen sozialen Kategorien anzuschließen, auch wenn dies z.B. durch Aussehen oder Akzent oder andere Charakteristika beschränkt sein kann. Wir nehmen zusätzlich an, dass sich unterschiedliche Kulturen in Form sozialer Kategorien auf kulturelle Güter gründen. Konsum und Produktion dieser Güter innerhalb einer Kultur werden durch die Handlungen und Entscheidungen von Individuen bestimmt, die dadurch ihre Nutzenfunktion und somit auch ihre Identität verändern. Dabei bedürfen vor allem die Auswirkungen der Handlungen Dritter (Angehörige einer anderen Kultur) in Bezug auf kulturelle Güter und auf die Identität einer Person besondere Aufmerksamkeit. Akerlof und Kranton nennen in diesem Zusammenhang einen Identitätsverlust, wenn jemand die internalisierten Werte ("prescriptions") einer Person verletzt. So erzeugen die Handlungen Dritter Externalitäten, gegen die keine Schutzmöglichkeiten bestehen. Diese Handlungen bezogen auf kulturelle Güter sind die Basis für unsere Analyse. Wir meinen, dass diese Kategorien kultureller Güter möglicherweise von Institutionen wie der WIPO einem gewissen Schutz unterstellt werden könnten.

Francois und van Ypersele (2002) verfolgen einen anderen Ansatz in Bezug auf den Schutz kultureller Güter und ihren Einfluss auf die Wohlfahrt. Dieser basiert auf einer unterschiedlichen Wertschätzung kultureller Güter durch Einheimische und Ausländer. Die Kulturgüterproduktion weist bei ihnen Skalenerträge auf. Die Autoren betrachten exemplarisch den Handel mit Filmen. In ihrem Beispiel erhalten Hollywoodfilme, die unbeabsichtigt zur Schädigung traditioneller bzw. indige- 
ner Kulturen beitragen, von Einheimischen und Ausländern die gleiche Wertschätzung. Das bedeutet, dass beispielsweise die Konsumenten in den USA und in Frankreich bereit sind, für den Konsum von Hollywood-Filmen zu bezahlen. Im Gegensatz dazu erhalten indigene Filme jedoch innerhalb der Länder unterschiedliche Wertschätzungen. So präferieren nicht alle Konsumenten in Frankreich die französische Autorenfilme und würden dafür ein Premium bezahlen. In dem Modell mit zwei Ländern (Frankreich, USA) und drei kulturellen Gütern (Hollywoodfilme, französische und US Autorenfilme) untersuchen Francois und van Ypersele, ob der Schutz kultureller Güter (in diesem Fall Autorenfilme) wohlfahrtssteigernd und ob ein Kulturzoll auf Hollywoodfilme pareto-verbessernd ${ }^{2}$ wirken könnte. Würde ein Zoll (oder eine Quotenregelung) eingeführt, würden sogenannte „Blockbuster“ mit einem weltweiten hohen Marktanteil nicht traditionelle Filmproduktionen, die einen hohen Anteil an Fixkosten haben, vom Markt verdrängen. Für einen Teil der Einheimischen hätte dies einen positiven Effekt, da sie auch weiterhin lokale Autorenfilme konsumieren könnten, die sie sehr schätzen. Darüber hinaus ist der Konsum heimisch produzierter, kultureller Güter notwendig, um einerseits die einheimische Kultur zu erhalten, und andererseits genug Exportpotential zu bieten. Ein wichtiger Aspekt in diesem Beitrag ist die Wertschätzung kultureller Güter. Diese ist grundlegend für die verschiedenen kulturellen Gruppen und muss daher auch bei der Untersuchung von Schutzmaßnahmen für kulturelle Güter adressiert werden.

In einem weiteren wichtigen Beitrag in der Diskussion um den Schutz kultureller Güter untersucht Janeba (2004), wie sich der freie Handel mit kulturellen Gütern auf kulturelle Identität auswirkt. Kulturelle Güter unterscheiden sich bei ihm von anderen Gütern dadurch, dass sie Interdependenzen zwischen individuellen Konsumentscheidungen herstellen, was letztlich zu kultureller Diversität führt. So könnte der Konsum eines kulturellen Gutes dieses für andere attraktiver machen. Eine Identität bildet sich heraus, wenn alle Individuen in einer Gesellschaft das gleiche kulturelle Gut konsumieren (ein kulturell homogenes Gut). Die Identitätsfunktion kann so als Netzwerkexternalität beschrieben werden. Deutsche etwa trinken Bier, was als gemeinsame Tradition gilt. Wenn jeder in der Gesellschaft das gleiche Gut konsumiert, ist der Identitätsverlust gleich null, und der Konsum wird durch die beiden Faktoren „Preis“ und ,gesellschaftliche Bestrafung" bestimmt. Der Konsum mag nun durch Handelsliberalisierung beeinflusst werden, was zu einer Veränderung des homogenen Konsumverhaltens einer Gesellschaft und somit zum Verlust der kulturellen Identität führen kann. Janeba bevorzugt daher einen protektionistischen Ansatz hinsichtlich kultureller Güter, den er auf eine Wohlfahrtsanalyse sowohl in geschlossenen als auch in offenen Volkswirtschaften stützt. Unter Berücksichtigung des eines Handelsmodells, das unterschiedliche Technologien und damit Skalenerträge unterstellt, leitet er daraus inter alia ab, dass

2 Von einer Pareto-Verbesserung spricht man, wenn es mindestens einem Individuum besser geht und keinem schlechter. Einer solchen Pareto-Verbesserung sollten theoretisch alle zustimmen können. 
im Falle kultureller Homogenität (gleiche Geschmäcker und Konsum kultureller Güter), Handel nicht zu Paretoverbesserungen in geschlossenen Volkswirtschaften (Autarkie) führen kann. Zusammengefasst zeigt das Modell, dass Individuen, die das exportierte kulturelle Gut konsumieren, während sich das gesellschaftliche Konsumverhalten durch den Import anderer kultureller Güter ändert, durch die Handelsliberalisierung schlechter gestellt werden. Daher sind laut Janeba diese auch die größten Gegner einer Handelsliberalisierung. Weiterhin hat Globalisierung einen negativen Einfluss sowohl auf nationale Kulturen als auch auf individuelle Identitäten. Daher sei der Konsum einheimischer Güter zu unterstützen, um Kultur zu bewahren.

Im Fokus der öffentlichen internationalen Debatte stehen die Bewahrung nationaler Kulturen und kultureller Identitäten sowie der Schutz kultureller Güter. Die ökonomische Diskussion greift dies auf, indem sie nach dem Nutzen stiftenden Effekt kultureller Güter fragt. Vor diesem Hintergrund fragen wir uns, was genau eigentlich zu schützen ist. Um diese Frage zu beantworten, wählen wir einen ökonomischen Ansatz in Bezug auf kulturelle Güter und ihren Schutz. Wir schlagen verschiedene Kriterien vor, die dabei helfen, zu schützende kulturelle Güter zu identifizieren und von denen zu unterscheiden, die nicht zu schützen sind. Diese Kriterien beinhalten Überlegungen zu positiven und negativen, direkten und indirekten Effekten des Konsums kultureller Güter. Wir unterscheiden, wie verschiedene Gruppen vom Konsum kultureller Güter betroffen sind.

\section{Nutzen aus kulturellen Gütern}

Kultur ist ein nicht leicht zu fassendes Phänomen. Die Vereinten Nationen definieren Kultur als "diverse forms across time and space; this diversity is embodied in the uniqueness and plurality of the identities and cultural expressions of the people and societies making up humanity" (UNESCO 2005, 1). Innerhalb dieses Beitrags soll Kultur als aus verschiedenen Gütern (kulturelle Güter) bestehend, die sich wiederum aus materiellen und immateriellen Teilen mit kultureller Bedeutung zusammensetzen, verstanden werden (siehe Cheng 2006). Die Besonderheit der kulturellen Güter liegt vor allem darin, dass sie als Vektoren der Identität und nicht als bloße Güter oder Konsumgüter behandelt werden müssen (UNESCO 2001, Article 8). Bei diesen Gütern stellen die immateriellen Güter ein größeres Problem dar, da die materiellen Güter größtenteils durch klassische Eigentumsrechte geschützt sind. Dennoch betrachten wir beide zusammen.

Für unsere Analyse unterscheiden wir zwischen den drei Akteursgruppen der Kulturträger, der Kulturkonsumenten und der Kulturproduzenten. Kulturträger sind die Individuen oder Gruppen, von denen ein Kulturgut stammt. Sie können auch als Insider bezeichnet werden, da sie zu der Gruppe bzw. Gesellschaft ${ }^{3}$ gehö-

\footnotetext{
${ }^{3}$ In diesem Beitrag wird die Frage, wer zu besagter Gruppe gehört, nicht behandelt. Es wird angenommen, dass die Gruppe homogen ist und eine homogene Wertschätzung in Bezug auf ein be-
} 
ren, in der das Kulturgut sich entwickelt hat. Sie können gleichzeitig das Kulturgut produzieren und konsumieren. Zu der Gruppe der Outsider gehören die sogenannten Kulturkonsumenten und Kulturproduzenten, die als Gesellschaften, Gruppen oder Individuen definiert sind, die ein bestimmtes Kulturgut benutzen oder produzieren, gleichzeitig aber nicht an seiner Entwicklung beteiligt waren. Beide Gruppen, die Insider und Outsider (siehe Abbildung 1), ziehen Nutzen aus dem Kulturgut und haben für das Gut eine positive Wertschätzung.

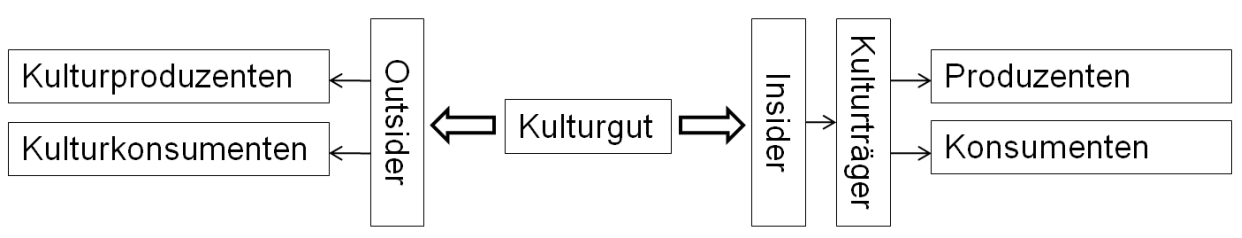

Abbildung 1: Unterteilung der Akteure auf Insider und Outsider

In der weiteren Analyse spielt (aus regulativer Sicht) auch der Nutzen der Kulturkonsumenten und der Kulturproduzenten eine Rolle. Zuerst betrachten wir aber das Konzept der Kulturnutzenfunktion (cultural utility function) des Kulturträgers. Wir treffen die Annahme, dass der Kulturträger sowohl direkt als auch indirekt Nutzen aus kulturellen Gütern zieht. Der direkte Nutzen besteht hierbei aus monetären Erträgen, die immer dann entstehen, wenn ein Kulturkonsument bereit ist, für ein Kulturgut, wie etwa Tanzstunden, das Kopieren eines traditionellen Musters oder ein Bild eines historischen Monuments, zu bezahlen. Dieser Nutzen kann alternativ auch als direkter Konsumertrag oder direkter Marktertrag beschrieben werden (Frey 2008). Daraus folgt, dass wenn Kulturkonsumenten für ein Kulturgut nicht bezahlen, dies negative Auswirkungen auf den direkten Nutzen des Kulturträgers hat. Bei einem Ausfall der Erträge könnte in einigen Fällen das Problem vorkommen, dass die Kulturträger, die in die Herstellung eines Gutes investiert haben, ihre Kosten nicht decken können, was wiederum langfristig eine negative Wirkung auf den Anreiz zur Schöpfung kultureller Güter haben kann (siehe Koppel 2008, Liebig 2008). Dies kann einen gesamtwirtschaftlichen Verlust bedeuten. Dieser direkte Nutzen für die Kulturträger könnte noch weiter reduziert werden, wenn andere Individuen oder Gruppen, die ursprünglich keine Kulturträger waren, anfangen, das gleiche Gut ohne Erlaubnis zu produzieren und daraus Profit zu ziehen. Diese Faktoren spielen vor allem dann eine Rolle, wenn z.B. immaterielle Eigentumsrechte verletzt werden. Bei dem direkten Teil der kulturellen Nutzen-

stimmtes Kulturgut besitzt. Abweichendes Verhalten und dessen Konsequenzen werden hier nicht analysiert. Die in der empirischen Forschung aus den Kulturwissenschaften belegbare Heterogenität der meisten Gruppen bedarf der weiteren, zukünftigen Analyse. Da die internationalen Verhandlungen, auf deren Beobachtung unser Beitrag u.a. aufbaut, oft ebenfalls von homogenen Gruppen ausgehen, mindert dies nicht die Relevanz unsere Ergebnisse in diesem Rahmen. 
funktion müssen andere ökonomischen Gesichtspunkte weiter untersucht werden. Wenn etwa der Verlust direkten Nutzens der Kulturträger durch Nutzengewinn der Kulturkonsumenten überkompensiert wird, erscheint es ökonomisch sinnvoll, den Konsum zuzulassen. Die Argumentation ist die gleiche wie im Fall eines Monopols. Selbst wenn die Einkünfte eines Monopolisten schrumpfen, so ist es doch aus ökonomischer Sicht rational, seinen Handlungsspielraum zu begrenzen.

Der indirekte Nutzen setzt sich aus verschiedenen Effekten zusammen, die zusammen die Identität des Kulturträgers bilden. Diese Effekte beinhalten intrinsische Werte wie etwa „option value“, „existence value“, „,bequest value“, „prestige value“, „education value“. Sie werden als Nichtmarkt- bzw. Nichtbenutzererträge bezeichnet (Frey und Meier 2006:1022). Der Nutzen, der aus der auf diese Weise definierten Identität gezogen wird, findet so Eingang in die kulturelle Nutzenfunktion des Kulturträgers. Identität als Teil der Nutzenfunktion wurde bereits in Akerlof und Kranton (2000) in Bezug auf den Arbeitsmarkt diskutiert und kann auch auf die kulturelle Nutzenfunktion angewendet werden. Throsby (2003) hält den Wert der Identität in einem monetären Sinn für schwer bestimmbar, da Identität als ein nicht handelbares Gut zu betrachten ist.

Dieser Teil der Nutzenfunktion ist kompliziert, da er von verschiedenen Effekten bestimmt wird. Einerseits beeinflusst Identität individuelle Konsumentscheidungen und kann daher, ähnlich einer Netzwerkexternalität, als Konsumexternalität gesehen werden (siehe Janeba 2004). Andererseits können manche Kulturgüter die grundlegende Identität (Würde) einer Person entscheidend prägen, sodass sich die Schädigung dieser Güter negativ auf die Identität und somit auch auf den Nutzen dieser Person auswirkt. Laut Harrison (Harrison 1999:240ff.) gelten Kulturgüter als identitätsstiftende Symbole, die sich auf alle Gegenstände oder Gewohnheiten beziehen, die als Merkmale oder Repräsentant der sozialen Identität benutzt werden, als authentisch, wenn diese von Inhabern gepflegt werden. ${ }^{4}$ Die gleichen Handlungen durch Außenstehende sieht man hingegen als Imitationen oder als Entwendung von Charakteristiken und Eigenschaften der Inhabergruppe an, die als eine Entwertung der Identität interpretiert werden kann.

Aus ökonomischer Sicht lassen sich Kulturgüter danach klassifizieren, wie stark Kulturkonsumenten Gebrauch von ihnen machen (und so den Nutzen der Kulturträger beeinflussen). Die Frage, ob ein Schutz kultureller Güter notwendig ist, sollte mit Hilfe der oben beschriebenen Nutzenfunktion analysiert werden. Basierend auf den beschriebenen Nutzeneffekten der Kulturgüter können sie so in verschiedene Gruppen eingeteilt werden, je nachdem, wie groß der Effekt des Kulturkonsumenten auf den Kulturträger ist. Demnach kann der direkte Nutzen zunehmen, abnehmen oder gleich bleiben, wenn Kulturkonsumenten kulturelle Güter in Anspruch nehmen.

\footnotetext{
${ }^{4}$ Auch hier ist uns bewusst, dass die begriffliche Festlegung der Operationalisierbarkeit dienen, in der ethnographisch dokumentierbaren Lebenswelt jedoch mit weit weniger deutlichen Grenzziehungen versehen ist.
} 
Im Falle des identitätsstiftenden, indirekten Teils der Nutzenfunktion untersuchen wir den Effekt, den der Konsum von Kulturgütern auf den indirekten Nutzen ausübt. Basierend auf den Einfluss, den der Kulturgüterkonsum auf den Gesamtnutzen der Kulturträger hat, unterscheiden wir drei Typen von Kulturgütern:

(1) Kulturkonsum wirkt sich positiv auf den Nutzen der Kulturträger aus, weil der indirekte Kulturgüternutzen sich gemäß der Zahl der Nutzer (Kulturkonsumenten) erhöht. Ein Kulturgut fällt in diese Kategorie, wenn die Gruppe, die dieses Gut "besitzt", bestrebt ist, es unter Personen, in Gruppen oder Gesellschaften (Kulturkonsumenten) zu verbreiten. So erzeugt z.B. eine Sprache, die von anderen als den Trägern gesprochen wird, positive Netzwerkexternalitäten (siehe Janeba 2004); die Übernahme einer Religion durch eine Gesellschaft schafft vergleichbare Normen und Wertvorstellungen. Auch das Christentum, das durch Missionen verbreitet wurde, kann aus der Perspektive der Christen als ein Beispiel für ein solches Kulturgut zählen. ${ }^{5}$

(2) Der Kulturgüternutzen kann auch unabhängig vom Gebrauch durch Kulturkonsumenten sein, aber diese Art Kulturgut ist äußerst rar. Der Buddhismus beispielsweise ist als Religion bzw. spirituelle Philosophie friedlich. Nach der Lehre der Buddhisten ist es wichtig, den Mittelweg in sich zu finden und sich von den negativen Gefühlen zu befreien. Das Ziel ist es, in das Nirwana zu kommen, aber diesen Weg kann man nur allein gehen. Daher hat die Ausübung durch andere "Konsumenten" in diesem Fall weder auf den direkten, noch auf den indirekten Nutzen der Buddhisten Einfluss.

(3) Der Kulturgüternutzen verringert sich durch den Konsum, wenn z.B. heilige Rituale von Kulturkonsumenten zelebriert werden, die dadurch für die Kulturträger ihre ursprüngliche Bedeutung verlieren. Laut De Beus (1996), der den Einfluss nationaler Identität untersucht, kann dies sogar das Zugehörigkeitsgefühl, die Würde und letztlich die Identität der Träger (negativ) verändern. Welche Kulturgüter solche starken identitätsstiftenden bzw. würdestiftenden Effekte besitzen, lässt sich nicht ohne Einzelfallanalyse klären. Ein Anzeichen für einen derartigen Effekt könnte die Geheimhaltung bezüglich dieser Kulturgüter fördern: Kulturträger, die versuchen, den Zugang anderer (Kulturkonsumenten oder Gruppen, die das gleiche Gut "produzieren" möchten) zu einem Gut zu beschränken (siehe (Harrison 1999), (Brown 2005)), können als Beweis für Schutzbedürftigkeit gesehen werden. Da laut Throsby (2003) Individuen ihre eigene Wohlfahrt am besten einzuschätzen in der Lage sind, signalisieren diese Bestrebungen, besagtes Gut zu verbergen, weil die Nutzung durch andere Einfluss auf die Identität der Kulturträger hat. Als Beispiel für ein solches Kulturgut lässt sich die Schildkröte, die bei den Ganalbingu in Australien als religiöses Symbol gilt, anführen. Diese ist von einem Textilproduzenten verwendet worden (Janke 2003, Fallstudie 3), wodurch die Identität dieser Gruppe geschädigt wurde.

\footnotetext{
${ }^{5}$ Wir vernachlässigen an dieser Stelle, dass es auch zu negativen Wirkungen kommen kann, weil in der Regel die „neuen Christen“ früher einer anderen Religion angehörten, bei denen sie dann einen entsprechenden Nutzenverlust verursachten.
} 
Eine weiter gehende Differenzierung zwischen direktem und indirektem Nutzen der Kulturträger, der durch Kulturgüterkonsum positiv oder negativ beeinflusst wird, führt zu der in der folgenden Abbildung dargestellten Klassifizierung.

Die in Abbildung 2 dargestellte Matrix kombiniert die Effekte auf den direkten und den indirekten Nutzen der Kulturträger, die durch Kulturkonsum und Produktion Außenstehender hervorgerufen werden, wobei der Effekt auf den direkten Nutzen auf der X-Achse, der auf den indirekten Nutzen auf der Y-Achse abgetragen ist.

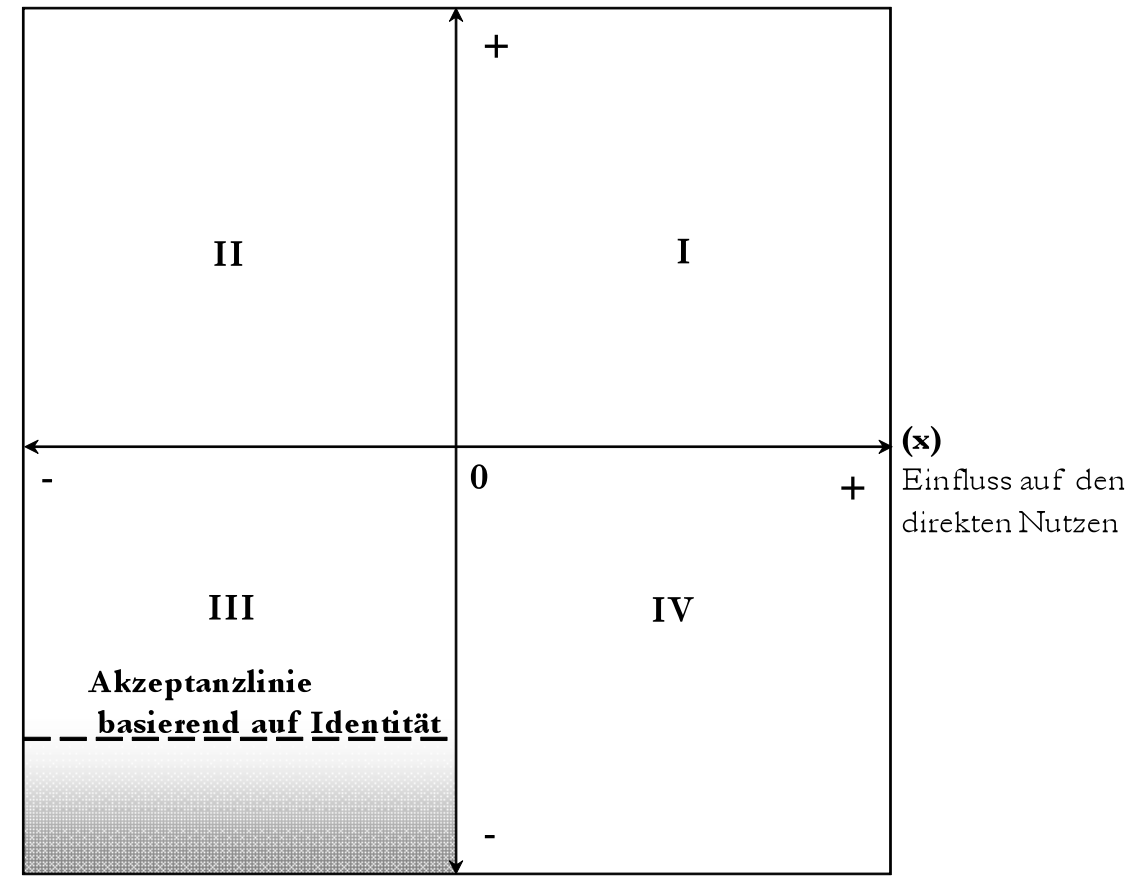

(y)

Einfluss auf den indirekten Nutzen

(Identität)

\section{Abbildung 2: Durch Fremdkonsum und -produktion erzeugte Nutzeneffekte für Kulturträger und der Schutzbereich für Kulturgüter (schattiert)}

Im ersten Quadranten (I) ist kein regulierender Eingriff erforderlich, da sich der Konsum kultureller Güter sowohl auf den direkten als auch auf den indirekten Nutzen positiv auswirkt. Eine erhöhte Produktion durch Nachahmer würde diesen Effekt nur verstärken, da auch hierdurch positiver Nutzen erzeugt wird.

Ein Beispiel ist Sprache als Teil einer Kultur. Die "Besitzer" einer bestimmten Sprache könnten an ihrer Verbreitung interessiert sein, um so z.B. den Handel 
zwischen unterschiedlichen Kulturen zu erleichtern oder mit Dritten einfacher zu interagieren (Lazear 1999). Eine gemeinsame Sprache ist die Grundlage für an Bedeutung zunehmende internationale und auch nationale Kommunikation sowie den Handel (Ridler 1986). Schon dadurch kommt es zu langfristig positiven Effekten.

Im zweiten Quadranten (II) wirkt sich der Konsum von Kulturgütern positiv auf den indirekten, aber negativ auf den direkten Nutzen der Kulturträger aus. Deshalb ist der direkte Nutzen der Kulturträger, der Nutzen der Kulturkonsumenten und der der Kulturproduzenten im Detail zu analysieren. Außerdem sind die langfristigen Wohlfahrtseffekte des Konsums und der zusätzlichen Produktion zu betrachtet. In dem Fall, dass die Kulturträger, wenn sie einen erhöhten Schutz für ihre Kulturgüter fordern, nur ihre Monopolposition bei der „Produktion“ der entsprechenden Güter erhalten und sichern wollen, könnte es bei ökonomischer Analyse rational erscheinen, anderen „Produzenten“ den Markteintritt zu ermöglichen und so zusätzliche Erträge für die Gesellschaft zu erzeugen. Zusätzliche Produktion könnte allerdings auch in einer anhaltenden Preisreduktion der betreffenden Güter resultieren oder sogar ihren kulturellen Charakter zerstören. Dann geht die Produktion nicht mit einem langfristigen Nutzenzuwachs für die Gesellschaft einher.

Es ist auch denkbar, dass Kulturkonsumenten nur Nutzen aus dem Konsum eines bestimmten Kulturguts ziehen können, wenn dieses von den Kulturträgern produziert wird. In dieser Situation wäre ein regulierender Eingriff unnötig, da die eben beschriebene Eigenschaft der Nutzenfunktion als Markteintrittsbarriere für weitere Produzenten wirkt. Laut Mas-Collel (1999) ist bei Kulturgütern der Unterschied zwischen Original (Produktion durch Kulturträger) und identischer Kopie (Produktion durch Außenstehende) jedoch fragwürdig. Sein Argument ist, dass man ein Objekt unmöglich perfekt beschreiben kann, und entsprechend nur schwer geklärt werden kann, was die einzigartigen Merkmale eines Originals sind. Die Bewertungen hängen dann von der intrinsischen Motivation der Kulturträger und der -konsumenten ab. Er argumentiert jedoch, dass die "Besitzer" der Güter nicht immer daran interessiert sind, andere am Kopieren zu hindern, weil die Verbreitung von Kopien den Wert des Originals noch erhöhen kann.

Für Fälle innerhalb dieses Quadranten existieren rechtliche Mittel, die zur Lösung der beschriebenen Streitfragen herangezogen werden können. Das System des fairen Handels z. B. stellt sicher, dass Güter, die aus einem bestimmten Erdteil stammen und gemäß der Gesetzmäßigkeiten des fairen Handels hergestellt wurden, von Außenstehenden weder produziert noch verkauft werden können (World Fair Trade Organization 2007). Die Werke kreativer Menschen werden beispielsweise in Australien und Großbritannien durch Moral Rights vor Urheberrechtsverletzung und inhaltlichem Missbrauch geschützt (Janke 2003:20). Somit sind Reproduktion und Abänderungen nur mit ausdrücklicher Erlaubnis des Autors möglich.

Als Beispiel kann die Gründungslegende der Quileute in Amerika dienen. Diese sind durch Stephenie Meyer's Vampir Chronik weitgehend bekannt und be- 
rühmt geworden (Riley 2010). Die „Twilight“-Geschichte, die auf einer Erzählung der Schöpfung der Quileute basiert, hat Millionen Dollar durch Film und Bücher, etc. eingebracht. Die Quileute selbst haben bislang nicht davon profitiert, obwohl der Kern dieser Produkte auf ihrem kulturellen Eigentum beruht. Diese Berühmtheit der Quileute hat dazu geführt, dass andere Produzenten den Namen „Quileute" als Symbol für ihre Produkte (z.B. Kapuzenpullover, Schmuck) zu verwenden angefangen haben, ohne die Erlaubnis dieser Gruppe eingeholt zu haben. Die Quileute sind ihrerseits bereit, weite Teile ihrer Kultur mit den Außenstehenden zu teilen, denn ihrem Stammesrecht zufolge sind nur die Friedhöfe und religiöse Zeremonien Ausnahmen, die als heilig und deshalb für Außenstehende als verboten gelten (Riley 2010).

Die Situation, dass die Quileute ihre Kulturgüter öffentlich zugänglich machen und vermarkten wollen, um damit Geld zu verdienen, bedeutet, dass der Einfluss der Outsider keine identitätsverletzende Wirkung hat. Das Interesse dieser Menschen liegt vor allem darin, an dem Gewinn, der mit ihrer Kultur verdient wurde, beteiligt zu werden. Zusätzliche Schutzmaßnahmen sind an dieser Stelle nicht erforderlich.

Im dritten Quadranten (III) sind die kompliziertesten Fälle dieser Analyse angesiedelt, bei denen der Konsum oder die zusätzliche Produktion eine negative Wirkung auf den direkten und indirekten Nutzen der Kulturträger hat.

Dabei ist besonders wichtig, die Wirkungen auf den indirekten Nutzen im Allgemeinen von denen auf die Identität der Kulturträger im Besonderen zu unterscheiden. Denn nicht jede Beeinträchtigung der Identität einer Person ist gleichbedeutend mit einer Beeinträchtigung der Würde. Anders ausgedrückt verletzt nicht jede den indirekten Nutzen negativ beeinflussende Art von Kulturkonsum und Produktion gleichzeitig die Würde des Kulturträgers. Auf Grund dessen teilt die Akzeptanzlinie die indirekten Nutzeneffekte in zwei Bereiche. Diese Akzeptanzlinie ist national variabel durch entsprechende Verfassungsnormen und zugehörige Rechtsprechung. Die Position und Bedeutung der Linie spiegeln also vorherrschende gesellschaftliche Normen wider, die in verschiedenen Gesellschaften unterschiedlich ausgeprägt sein können. Sie unterliegen der Rechtsprechung und Verhandlungen in der jeweiligen Gesellschaft. Kurz gesagt, "dignity does not depend on personal favors and occasional balances of power, but on social openness guaranteed by the rule of law, vigilant public debate, the plurality of associations, and social rights" (Beus 1996:177).

Die Selbstbestimmung der eigenen Identität einer Kulturgruppe in Zusammenhang mit deren Tradition und Gewohnheiten wurde in der Declaration on the Rights of Indigenous Peoples der Vereinten Nationen anerkannt (United Nations 2007, Article 33). Ferner wurde der Zusammenhang zwischen der Kultur und der Würde der Kulturträger berücksichtigt. Laut dieser Deklaration haben diese Gruppen das Recht auf die Würde und Diversität ihrer Kultur, Tradition, Geschichte und Aspiration (United Nations 2007, Article 15). Die Würde zählt zu den Grundrechten einer Person schlechthin. Andere Grundrechte, wie etwa das Recht auf Privatsphä- 
re oder das Recht auf freie Religionsausübung, gründen sich auf das Konzept der Würde. Wenn ein Kulturgut ein untrennbarer Bestandteil einer Kultur ist und die grundlegende Identität des Kulturträgers dadurch beeinflusst wird, sei an dieser Stelle die Schutzwürdigkeit dieses Gutes nachgewiesen. Die Lage der Linie, die diese identitäts- und würdestiftende Eigenschaft der Kulturgüter widerspiegelt, unterliegt einer Akzeptanz der Beteiligten und wird bei den unterschiedlichen Kulturen und Kulturgruppen andere Positionen einnehmen. Diese Annahme unterliegt der folgenden Richtlinie der UN in Bezug auf die Human Rights, nämlich, "recognizing, respecting, valuing their customs, rules is essential to their identity, dignity” (United Nations 2000, Principle 4).

In dem schattierten Bereich unterhalb der „Akzeptanzlinie“ sind demnach die Kulturgüter enthalten, die basierend auf den indirekten Nutzen als schutzbedürftig einzustufen sind. Der Effekt von Außenseiter wirkt hier so stark auf die Identität und die Würde der Kulturträger, dass die damit einhergehenden Nutzeneinbußen bei den Kulturträgern nicht mehr zu tolerieren sind. Weil in diesen Fällen massiv die Würde einer Person beeinträchtigt ist, sind auch Schutzmaßnahmen angebracht.

Ein Beispiel dafür ist die ursprünglich indianische Tradition der Navajo Schwitzhütten, die für die zeremonielle Reinigung aber auch für die Heilung eingesetzt werden. Nach dem Navajo Gewohnheitsrecht war es verboten, Geld für diese heilige, intime Zeremonie zu verlangen (Rehfeld 2009). Allein die Teilnahme von Außenstehenden an diesem sakralen Ritual, und noch viel mehr dessen Nachahmung riefen einen Aufschrei in der indigenen Bevölkerung hervor. Da es sich um ein Ritual handelt, verlieren die Navajos an Identität, wenn andere dasselbe Ritual als Wellness-Maßnahme vollziehen. In diesem Quadranten unterhalb der Akzeptanzlinie angesiedelte Kulturgüter sind schutzbedürftig.

Im Bereich oberhalb der Akzeptanzlinie, wenn die Würde bzw. die grundlegende Identität nicht im selben $\mathrm{Maß}$ beeinträchtigt ist, kann eine ökonomische Analyse dazu dienen, eine optimale Lösung zu finden. Bei dieser Betrachtung sind alle drei Gruppen relevant ${ }^{6}$ : Kulturträger, Kulturproduzenten und Kulturkonsumenten. Im Zusammenhang mit dem direkten, wirtschaftlichen Nutzen betonen die Vereinten Nationen aber, dass die Kulturgruppen die Begünstigten einer kommerziellen Anwendung ihrer Kulturgüter sein sollten (United Nations 2000).

Eine positive Korrelation des Nutzens der Kulturkonsumenten mit der Bedeutung eines Gutes für die Kulturträger könnte dabei ein besonderes Problem darstellen, da diese potentiell identitätszerstörenden Kulturgüter für die Konsumenten auch besonders wertvoll sein könnten (Harrison 1999). Es ist daher notwendig, zwischen den verschiedenen Teilen der Nutzenfunktion der Kulturträger zu unterscheiden. Nur wenn eine identitätszerstörende Wirkung des Kulturkonsums bzw. der zusätzlichen Produktion eines Kulturguts ausgeschlossen werden kann (Fälle

6 Eine weiter gehende Unterteilung der Kulturkonsumenten etwa in nationale oder internationale Gruppen ist ebenfalls möglich und würde in weiteren Parametern für die Maximierung resultieren. 
oberhalb der Akzeptanzlinie), ist der Fall im zweiten Quadranten zu verorten. So ist die Zugehörigkeit zur Oberschicht der in Kambodscha beheimateten Toraja, marapuan, durch den Besitz eines traditionellen torajanischen Hauses, tongkonan, ersichtlich. Das tongkonan ist ein beeindruckendes Gebäude mit komplexen Strukturen, dessen Bau erheblicher Ressourcen und handwerklicher Fähigkeiten bedarf (Scarduelli 2005:390ff.). Es ist das Privileg der marapuan, ihr Haus mit speziellen Malereinen und Schnitzereien zu verzieren, und in diesen Häusern Beerdigungsrituale durchzuführen. Die Größe des tongkonan und die Qualität der Verzierungen spiegeln Rang, Ehre und gesellschaftliches Ansehen wider (Scarduelli 2005:392). Die Emigrationswelle in den 1970ern und die damit einhergehenden Geldtransfers nach Hause haben die gesellschaftlichen Strukturen stark verändert. Diese Transfers ermöglichten auch sozial schwächeren Familien, selbst große tongkonans zu bauen und teure, eindrucksvolle Beerdingungszeremonien abzuhalten (und so als zusätzliche Produzenten dieses Kulturgutes aufzutreten). Das führte zu einem stark wachsendem Tourismus und so auch Kulturkonsum. Die marapuan stehen dieser Entwicklung kritisch gegenüber und streben danach, ihre traditionelle gesellschaftliche Position zu erhalten (Adams 1997), die eng mit dem oben erwähnten Recht zusammenhängt, tongkonans zu bauen und teure, extravagante Beerdingungszeremonien abzuhalten. Der bisherige Stand der Forschung lässt jedoch keine Rückschlüsse darauf zu, in welchem Maße die Oberschicht Schutz für ihre Privilegien erreichen möchte. ${ }^{7}$ Der identitätsstiftende Effekt nimmt mit der Zahl der nicht-adligen Produzenten ab, aber eine Wirkung auf die grundlegende Identität bzw. Würde dieser adligen Gruppe konnte bisher nicht festgestellt werden. Für die ganze Region entsteht demnach ein positiver Wohlfahrtseffekt, weil zusätzliche Kulturgutproduzenten in den Markt eintreten und mehr Tourismus erzeugen. Die Verteilungswirkung für die Gesellschaft und das Wiederaufleben der Tradition könnten als zusätzliche positive Effekte des „Nichtschützens“ betrachtet werden.

Fälle im vierten Quadranten (IV), in denen Außenstehende einen positiven Einfluss auf den direkten Nutzen der Träger, gleichzeitig jedoch einen negativen Einfluss auf ihre Identität ausüben, scheinen jedoch schwer vorstellbar. Ein Beispiel wäre das sprichwörtliche „Verkaufen der Seele an den Teufel“, tätszerstörend wirkt aber aufgrund der Zahlung dennoch auch positive Wirkungen entfaltet. Selbst in der Geschichte von "Timm Thaler oder das verkaufte Lachen“ (Krüss 1962) verkauft der jugendliche Protagonist zwar sein Lachen an den Teufel und gewinnt dafür alle Wetten, entdeckt aber dadurch schnell den Wert seines Lachens und will die Transaktion rückgängig machen. Zumindest so lange Transaktionen vollkommen freiwillig und ohne Ausnutzen der Situation (Teufel verführt Jugendlichen zum Verkauf des Lachens) zustanden kommen, dürften Fälle im vierten Quadranten die seltene Ausnahme bleiben. Deshalb sind auch keine regulativen Eingriffe notwendig.

${ }^{7}$ Feldforschungsbericht aus Indonesien von Beate Engelbrecht (Oktober 2009).

8 Wir bedanken uns bei Prof. Ejan Mackaay, der uns diese Gedanken während der Konferenz vom 12.-15. November 2009 in der Nähe von Göttingen mitgeteilt hat. 
Aus der Klassifizierung nach direkten und indirekten Nutzeneffekten wie in Abbildung 2 dargestellt ist abzuleiten, dass nur Kulturgüter des dritten Quadranten zu schützen sind. Innerhalb des Quadranten haben wir unterschieden zwischen den kulturellen Gütern, die einen Einfluss auf die grundlegende Identität einer Gruppe (Würde) haben und deshalb einen besonderen Schutz erfordern, und denen, bei denen durch eine Kosten-Nutzen-Abwägung festzustellen ist, ob eine Produktion oder der Konsum der Kulturgüter durch andere zuzulassen ist. Auch in diesen Fällen kann eine zusätzliche Regulierung erforderlich sein, um die Interessen der Gruppen angemessen zu repräsentieren und eine entsprechende Abwägungsentscheidung herbeizuführen.

\section{Der Schutz der Kulturgüter}

Bei der Debatte um den Schutz kultureller Güter spielen zumeist Argumente eine Rolle, die in erster Linie den Handel mit Kulturgütern und dessen gesamtwirtschaftliche Wirkungen analysieren und nicht die Schutzwürdigkeit der Kulturgüter an sich. Unser Ansatz basiert hingegen auf der Eigenschaft der kulturellen Güter, auch einen indirekten Nutzen stiften zu können, der wiederum auf dem Zusammenhang von Identität und Würde mit kulturellen Gütern beruht. Dies führt dazu, dass wir unabhängig von den unterschiedlichen Interessenparteien (z.B. Industrieländer, Entwicklungsländer), erst einmal nur für wenige Kulturgüter eine Schutzbedürftigkeit feststellen. Zu diesem Ergebnis kommen wir, weil wir einen Zusammenhang zwischen der Würde des Menschen und bestimmten kulturellen Gütern herstellen. Allerdings haben keineswegs alle kulturellen Güter einen direkten Einfluss auf die Identität und damit die Würde von Individuen. Deshalb liegen nur wenige Güter unterhalb der Akzeptanzlinie, für die dann auch zusätzliche Schutzmaßnahmen einzuführen sind. Für diese Güter ist allerdings ein geeigneter Schutz zu schaffen. Das gilt auch für die übrigen Fälle, die in diesem Quadranten anzusiedeln sind, wenn sie auch vermutlich andere Regulierungsansätze erfordern.

$\mathrm{Da}$ nur ein kleiner Teil der kulturellen Güter unmittelbar schutzbedürftig ist, nämlich der, der die grundlegende Identität bzw. Würde der Kulturträger berührt, stellen sich drei weitergehende Fragen:

(1) Wie können wir feststellen, ob ein Kulturgut einen grundlegenden identitäts- bzw. würdestiftenden Effekt hat und entsprechend gesetzlich geschützt werden sollte? Es existiert keine Entscheidungsregel, die sich auf unterschiedliche Kulturgüter, die zu unterschiedlichen Gesellschaften gehören und diverse Gruppen betreffen, anwenden lässt. Nur auf Basis einer Einzelfallanalyse kann ein Gericht, welches die Interessen der Kulturträger gegen die Meinung unabhängiger Experten und anderer interessierter bzw. betroffener Gruppen abwägen sollte, determinieren, ob ein Gut die entsprechenden Charakteristika besitzt. Die Festlegung, welche Güter identitätskritisch sind, lässt sich von den Gruppen selbst bestimmen. Laut der United Nations haben die Kulturgruppen das Recht, ihre eigenen Rechte aus- 
zuüben und sich an den ihre Rechte betreffenden Entscheidungsprozesse beteiligen (United Nations 2007, Article 2, 18). Das bedeutet im Grunde genommen, dass die Kulturträger oder ihre Repräsentanten bei der Feststellung und Anerkennung dieser Güter zu wichtigsten Akteuren werden müssen. Die sakralen Güter, die vermutlich die Mehrheit der schutzwürdigen Güter unterhalb der Akzeptanzlinie bilden, sollten im Mittelpunkt dieser Untersuchung stehen.

(2) Wenn ein Kulturgut keine grundlegenden identitäts- bzw. würdestiftenden Effekte besitzt, wie sollte der Markt dann reguliert werden? Was ist der Nutzen einer entsprechenden Regulierung, verglichen mit den Kosten? Kulturgüter weisen einige Ähnlichkeiten mit geistigem Eigentum auf, was auf ihren auch immateriellen Charakter zurückzuführen ist. Es ist aber kaum sinnvoll, die gleichen Instrumente wie für den Schutz geistigen Eigentums anzuwenden, da Kriterien wie Neuheit oder Individualität des Autors hier nicht zutreffen. Einige Güter, wie z.B. Bilder, Skulpturen, Gebäude oder Monumentalbauten, sind durch den Status als UNESCO Weltkulturerbe geschützt. Dieser ist aber nicht immer auch gleichbedeutend mit ausreichendem Schutz, sondern bietet oft eher Möglichkeiten zur Kommerzialisierung, die dann auch identitätsbeeinträchtigende Wirkungen haben können (siehe Diskussion in Berger 2009).

(3) Welche Rolle spielt die internationale Gemeinschaft innerhalb des regulativen Prozesses? Die internationale Gemeinschaft kann einen Rechtsrahmen herstellen, innerhalb dessen sich die nationalen Staaten zur Untersuchung von Einzelfalllösungen verpflichten. Dies würde helfen, die Bedeutung der Rolle von Kultur und Kulturgütern für indigene Gruppen innerhalb der jeweiligen Gesellschaften deutlich zu machen.

Darüber hinaus ist der Kulturgütermarkt nicht auf ein bestimmtes Land beschränkt. So wäre z.B. eine Situation denkbar, in der einige Akteure, wie etwa Konsumenten und Produzenten, im Ausland angesiedelt sind, was als Konsequenz internationale Verhandlungen erfordert, die bilateral oder multilateral ausgestaltet sein können. Brown (2005) argumentiert, dass ein globaler Markt auch ein globales Kontroll-Regime erfordert und geht damit über die bilaterale Lösung hinaus.

Eine weitere Herausforderung besteht darin, dass nicht alle gesellschaftlichen oder indigenen Gruppen in ihrem jeweiligen Land anerkannt sind und daher ihre eigenen Interessen nicht vertreten können. Die betroffenen Gruppen sollten, wenn sie keinen entsprechenden Schutz durch ihre Regierung erhalten, auch international die Initiative ergreifen und auf die Schutzwürdigkeit dieser Güter aufmerksam machen. Ein Instrument internationale Aufmerksamkeit zu erzeugen, ist der "indigenous caucus" der World Intellectual Property Organization (WIPO), bei dem NGOs und indigene Gruppen aus der ganzen Welt auf das Problem unzureichenden Schutzes ihrer Kulturgüter hinweisen. 


\section{$5 \quad$ Fazit}

Die internationale Debatte zum Schutz kultureller Güter bedarf einer Antwort auf die Frage, welche spezifischen Maßnahmen über bereits existierende Eigentumsrechte hinaus ergriffen werden müssen. Da sich diese Debatte auf sogenannte suigeneris-Rechte konzentriert (siehe Brown 2005), schlagen die Autoren in diesem Beitrag vor, zwischen direkten und indirekten Effekten von Kulturgütern zu unterscheiden. Direkte Effekte sind finanziell messbar und ergeben sich aus der Verbreitung eines Kulturgutes in Märkten. Auch wenn diese Quantifizierung oft nur schwer durchzuführen ist, so existiert doch eine breite Literatur bezüglich des Wertes von Kulturgütern. Indirekte Effekte treten in Form von positivem oder negativem Einfluss eines Kulturgutes auf die Identiät seiner Träger auf. Diese Effekte schließen auch option values, prestige values, etc. ein, die sich erheblich schwerer monetarisieren lassen.

Falls nur direkte Effekte auftreten, sollten positive und negative Effekte gegeneinander abgewogen werden. Kulturgüter sollten regulativ geschützt werden, wenn die durch den Schutz entstehenden Nettoerträge die Kosten übersteigen, der Ertrag also höher ist als ohne regulativen Eingriff. Solch eine Bewertung kann nur auf Basis einer Einzelfallanalyse vorgenommen und somit nicht durch internationale Standards geregelt werden.

Treten neben den direkten auch indirekte Effekte auf, so ist ihr Einfluss auf die Identität der Kulturträger zu untersuchen. Kann ein Einfluss festgestellt werden, so ist es wichtig herauszufinden, ob dieser das Potenzial hat, die grundlegende Identität bzw. Würde der Träger nachhaltig zu schädigen. Nur wenn dies zutrifft, sollten zum Schutz der betroffenen Güter internationale Normen in Form von sui generis Eigentumsrechten eingeführt werden. Ein nachhaltiger Einfluss eines Kulturgutes auf Identität kann etwa dann angenommen werden, wenn die Träger viele Ressourcen darauf verwenden, das betroffene Gut geheim zu halten. Die Akzeptanzlinie, die identitätsrelevante Güter auf der Basis der Würde des Menschen abgrenzt, müssen die jeweiligen Staaten in Abstimmung mit den gesellschaftlich relevanten Gruppen festlegen.

Zusammengefasst stellen wir fest, dass nur in relativ wenigen Fällen Kulturgüter spezielle Schutzmaßnahmen in Form von Rechten eigener Form (sui generis) bedürfen. Kulturelle Eigentumsrechte sollten auf internationaler Ebene nur dann eingeführt werden, wenn die Würde der Kulturträger ernsthaft gefährdet ist. Neben dieser Kategorie gibt es aber ein weites Feld, in dem regulatorische Eingriffe dafür sorgen können, dass Marktakteure zu sinnvollen und nutzensteigernden Entscheidungen kommen. 




\title{
Ethnographische Filmarbeit und Copyright: Überlegungen zur Situation in Indonesien
}

\author{
Beate Engelbrecht
}

Beim Filmen von Kultur sind immer Menschen involviert: Menschen vor der Kamera, Menschen hinter der Kamera, Menschen neben der Kamera, sogar Menschen, die nicht anwesend sind. Wie arbeiten diese Menschen zusammen? Wer hat die Autorität darüber zu entscheiden, was gefilmt wird, wie gefilmt wird, was veröffentlicht wird, wie das Endprodukt aussieht? Wer ist der Autor eines Films, wer hat das Copyright und wer hat das Copyright für die im Film dokumentierten kulturellen Darbietungen? Autorität konstituiert sich lokal. Copyright wird in der nationalen Gesetzgebung geregelt. Autorenschaft hat etwas von beidem: Autorität über die Schöpfung eines Werkes und Erwerb des Copyright am Werk. An dem hier diskutierten Beispiel sind Deutsche, Indonesier und Toraja beteiligt. Wie kann in diesem transkulturellen und transnationalen Kontext zusammen gearbeitet werden? Wie verhandeln die Beteiligten ihre Rechte? Und wie stehen die lokalen Vorstellungen und die nationalen Gesetzgebungen zueinander?

\section{Ethnographischer Filmprozess}

Der ethnographische Filmprozess wurde immer wieder analysiert. ${ }^{1}$ Wesentlich dabei ist die Frage wie zusammengearbeitet wird. An jedem Punkt des Prozesses

\footnotetext{
1 Vgl. Bibliographie in Husmann und Wellinger 1992, Crawford und Turton 1992, Ballhaus und Engelbrecht 1995, MacDougall 1998, Ruby 2000, Postma und Crawford 2006.
} 
sind verschiedene Personen involviert. Alles beginnt damit, dass jemand eine Idee hat. Es folgen Untersuchungen über das Thema und Diskussionen darüber, was aufgezeichnet werden soll. Am Anfang mag ein Ethnologe ${ }^{2}$ alleine arbeiten, Fragen stellen, seine Schlussfolgerungen ziehen, ein Konzept entwickeln. In einem zweiten Schritt mögen ein Filmemacher und Vertreter der Gefilmten miteinbezogen werden. Offizielle und inoffizielle Genehmigungen werden eingeholt. Beim Filmen sind des Weiteren der Kameramann und der Tonmann, die Darsteller und die lokalen Berater beteiligt. Filmemacher, Ethnologe, Kameramann können eine Person sein oder ein Filmteam. Ein möglicher Vertrag regelt die Beziehungen, Aufgaben und Rechte (Asch 1988). Die Darsteller und Berater kommen meist von der Lokalität, wo gefilmt wird. In einem multi-sited Filmprojekt sind Darsteller und Berater von verschiedenen Örtlichkeiten involviert. Die Beziehung zur lokalen Bevölkerung, zu den Darstellern und Beratern ist meist nicht sehr klar: es ist eine Zusammenarbeit, doch deren Qualität ist oft schwer zu definieren. Nach dem Filmen nimmt der Filmemacher/Ethnologe das Filmmaterial mit nach Hause, um es zu analysieren und zu schneiden. Beim Schnitt mögen neue Personen in den Prozess eintreten, z.B. ein Cutter, ein Redakteur. Es mag sein, dass Leute von vor Ort hinsichtlich der Übersetzung, der Auswahl der Einstellungen und/oder der Filmstruktur um Mitarbeit gebeten werden. Schließlich werden bei der Vorführung vor Ort die Zuschauer gebeten, ihre Kommentare abzugeben und der Veröffentlichung zuzustimmen. Dies kann wiederum gefilmt und am Ende des Films eingeschnitten werden, so dass die Vereinbarung sichtbar wird. Letztlich entscheidet aber nur eine Person darüber, was im Film sein wird: der Autor (Filmemacher/Ethnologe).

\section{Filmische Dokumentation eines merauk-Festes in Buntao}

Im Juli 2009 fanden in Tana Toraja (Süd-Sulawesi, Indonesien) zwei Veranstaltungen statt, die miteinander in Beziehung standen: eine Internationale Konferenz zur Zukunft der Toraja-Kultur mit Ethnologen, Politikern, studierten Toraja und Interessierten aus der Region, und ein merauk-Fest zur Einweihung eines tongkonan.

Der tongkonan ist weit mehr als ein schönes und beeindruckendes Haus. ${ }^{3}$ Es ist ein "origin house", das Ahnenhaus einer Familiengruppe. Der tongkonan repräsentiert deren sozialen Status. Das ursprüngliche Haus mag zerfallen oder zerstört sein, aber die Familie besteht weiter und kann deshalb auch das Haus wieder neu bauen.

\footnotetext{
2 Im vorliegenden Text wird durchgängig die männliche Form benutzt. Im Sinne des Gleichbehandlungsgesetzes sind diese Bezeichnungen als nicht geschlechtsspezifisch zu betrachten.

${ }^{3}$ Siehe Waterson 1990, 2003, 2009, Kis-Jovak et al. 1988, Nooy-Palm 2001.
} 
Das merauk-Fest ist eines der Feste der „,aufgehenden Sonne“, die von den protestantischen Missionaren verboten worden waren. ${ }^{4}$ Es ist das wichtigste der lebensspendenden Rituale (Waterson 2009:177) und wird heute nur noch selten (in traditioneller Weise) durchgeführt. Es ist nicht nur ein Familienfest, es ist auch ein Verdienstfest (Nooy-Palm 1986:62). Das hier zur Diskussion stehende meraukFest wurde zur Einweihung des neu gebauten tongkonan BARA'BA in Lentenan (Buntao) veranstaltet. Die Familie, die das Fest durchführte, die selbst römischkatholisch ist, hatte entschieden, das Fest in traditioneller Weise zu zelebrieren, d.h. in der Tradition der alten Religion aluk to dolo. Sie sagten, dass das letzte traditionelle merauk-Fest vor rund 30 Jahren in dieser Region veranstaltet wurde. ${ }^{5}$ Viele der Organisatoren hatten das Fest noch nie zuvor gesehen oder erinnerten sich nur noch schlecht. Sie luden deshalb ältere Männer, die noch die alte Religion aluk to dolo praktizierten, zur Mithilfe ein. Sie konnten sich an die alten Zeremonien erinnern und bei der Durchführung beistehen. Schritt für Schritt wurden die Reihenfolge der Opfer und die einzelnen Arrangements ausgearbeitet. Das Fest begann mit einer Reihe von elf Opfern, bei denen die verschiedenen Ahnen und Geister um Verzeihung und Hilfe gebeten wurden, und endete mit einer großen Zeremonie am letzten Tag. Während bei den Opfern 15 bis 40 Personen anwesend waren, nahmen an den letzten Festlichkeiten rund 500 Leute teil.

\subsection{Akteure}

Die bei den Entscheidungen ausschlaggebenden Akteure sind alle Familienmitglieder des tongkonan. Die Entscheidung, ein merauk-Fest zu machen, war vornehmlich von den neun Pong Masak-Geschwistern getroffen worden. Sie fühlten sich dazu verpflichtet, war es doch der letzte Wille ihres Vaters, der vor 20 Jahren gestorben war. Der älteste Bruder, Leo Kala Pong Masak, ein pensionierter Regierungsangestellter und aktiver Politiker im neuen Parlament von Nord-Toraja, war für das Fest verantwortlich. Während der Opfer war er eher selten zu sehen, aber während der abschließenden Zeremonien trat er eindeutig als verantwortlicher Gastgeber auf. Zwei seiner Onkel und einer deren Cousins leiteten in seiner Abwesenheit die Aktivitäten vor Ort. Sie diskutierten die Reihenfolge der Opfer und die einzelnen Tätigkeiten mit einigen Beratern, die auch ausführende Akteure waren. Einer der Onkel war auch der toparengnge', das traditionelle politische Oberhaupt des betroffenen Gebietes.

Die wichtigsten ausführenden Akteure bei den Opfern waren zu Beginn Personen, die noch der traditionellen Religion aluk to dolo anhingen. Bestimmte Handlungen konnten nur von ihnen ausgeführt werden. Andere, wie z.B. die Darbringung der Opfer, sollten von bestimmten Familienmitgliedern vorgenom-

\footnotetext{
${ }^{4}$ Die protestantischen Missionare tolerierten dahingegen die Totenfeste, die heute ein Kennzeichen von Tanah Toraja sind.

${ }^{5}$ Nooy-Palm beschreibt ein Merok-Fest in Buntao, an dem sie 1969 teilnahm (1986:69-83).
} 
men werden. Weitere Familienmitglieder, Freunde und Kinder nahmen an den Opfern als Zuschauer teil. Für bestimmte Handlungen und Gebete wurde Ne'Wei gerufen, der die lokalen Zeremonien des aluk to dolo kennt und die Familie vor und während des Festes beriet. Tato' Dena', ein tominaa, ein spezieller Priester, der seine Position von seinem Vater geerbt hat, war der religiöse Hauptakteur während der letzten zwei Tage des Festes: er betete, er überwachte und leitete die Personen an, die die Opfer durchführten, und er spendete den Segen am Ende. Er respektierte jedoch die lokalen Variationen und leitete diesbezügliche Fragen an Ne'Wei weiter.

Am letzten Tag des Festes waren alle Pong Masak-Geschwister anwesend. Sie schauten nach den Gästen und sorgten für einen reibungslosen Ablauf. Die Familie hatte viele Personen benannt, die sich um die einzelnen Aufgaben kümmerten. Außerdem hatte die Familie drei Tanzgruppen eingeladen, zwei professionelle und eine Laiengruppe aus Lentenan, wie auch Schüler einer lokalen Schule, die auf traditionellen Bambusflöten, für die Buntao berühmt ist, spielten.

Die Gäste und Besucher waren aus unterschiedlichen Gründen zum Fest gekommen. Die Familie Pong Masak hatte die Familien des tongkonan zum meraukFest eingeladen. Jede Familie beteiligte sich mit einem Schwein am Fest und mit jedem Schwein erhöhte sich das Ansehen des tongkonan. Auch die anwesenden Repräsentanten wichtiger Organisationen und politischer Institutionen wie z.B. der bupati (der Präsident der Provinz), verschiedene puangs' und toparengnge' und der katholische Priester steigerten das Prestige der Familie.

Auch Touristen besuchten das Fest. Normalerweise kommen sie nach Tana Toraja, um Totenrituale zu sehen. Diesmal hatte ihnen jedoch das Tourismus-Büro in Rantepao etwas Besonderes angeboten: ein merauk-Fest. Die Touristen wurden von der Familie willkommen geheißen, es wurde ihnen erlaubt, fast überall herumzulaufen und sie konnten fotografieren und filmen, wann und was auch immer sie wollten. Eine spezielle Gruppe von „Touristen“ waren die Teilnehmer der eingangs erwähnten Konferenz, die Tanete A. Pong Masak, der Bruder von Leo Kala und Dozent an einer Universität in Jakarta, organisiert hatte. Sie kamen während der letzten zwei Tage des Festes und wurden wie Ehrengäste behandelt. Auch sie fotografierten und filmten dieses besondere Ereignis.

In Tana Toraja werden wichtige Feste üblicherweise mit Video dokumentiert. In diesem Fall hatte die Familie einen lokalen Videographen beauftragt, der ursprünglich aus Buntao stammte. Die Familie fragte weiterhin ein Familienmitglied, die Feierlichkeiten der letzten zwei Tage aufzunehmen. Ein professioneller Fotograf und Filmemacher aus Yogyakarata, der zur Konferenz gekommen war, machte eine Foto-Dokumentation des Festes. Und schließlich hatte Tanete Pong Masak vorgeschlagen, dass auch ich das Fest filmen sollte.

Wenn man diese verschiedenen Gruppen betrachtet, die alle fotografiert und gefilmt haben, stellt sich die Frage: Hat man das Recht, alles zu filmen, jeden, überall, und darf man das Material danach so benutzen, wie es einem gefällt? In- 
wieweit gibt das nationale Gesetz bestimmte Regeln vor, inwieweit berührt dies welche Filmer?

\subsection{Ziele}

Die Familie fühlte sich verpflichtet, das merauk-Fest durchzuführen. Aber sie hätten es sicher nicht getan, sie hätten sicher nicht soviel Geld ausgegeben, wenn es nicht für ihre Zukunft wichtig gewesen wäre. Die Familie ist stolz auf ihren tongkonan. Das merauk-Fest bot eine gute Gelegenheit, die Wichtigkeit der Familie zu demonstrieren und die Familie weiter voranzubringen. Sie hießen ihre Gäste, Touristen und Konferenzteilnehmer willkommen. Sie wollten eine Video-Dokumentation. Sie sind auch an der Publikation der Aufnahmen interessiert, nicht nur an der langen ungeschnittenen Fassung für die Familie, sondern auch an einer geschnittenen Filmfassung, die in Indonesien und außerhalb zirkulieren kann.

Die Besucher fotografierten und filmten mit Kameras und sogar mit ihren Mobiltelefonen. Sie wollten sich an das Ereignis erinnern, ihre Erlebnisse und Begeisterung mit ihren Familien und Freunden teilen. Die Konferenzteilnehmer fotografierten und filmten auch, aus professionellen Gründen, in ihrer Eigenschaft als Ethnologen, als Wissenschaftler, als Filmemacher und Fotografen.

Der lokale Videograph konnte mit seinen Aufnahmen Geld verdienen. Sein Interesse am Fest und am Film endete mit der Übergabe der DVD-Kopien und der DV-Kassetten an den Auftrageber, die Pong Masak Familie. Die Familie hatte ihm das Recht eingeräumt, die DVDs auch auf eigene Rechnung zu verkaufen.

Meine Ziele waren anders ausgerichtet. Ich nutzte die Filmaufnahmen, um Fragen hinsichtlich Autorität, Autorenschaft und Copyright im Zusammenhang mit ethnographischen Filmaufnahmen nachgehen zu können.

\subsection{Traditionelle kulturelle Ausdrucksformen}

Feste zu filmen heißt immer auch traditionelle kulturelle Ausdrucksformen (TCE, Traditional Cultural Expressions $)^{6}$ aufzunehmen. Im Fall des merauk-Fests ist dies der tongkonan, das "origin house" selbst, mit seinen wundervollen Schnitzereien. Dann sind da die Opfer. Sie werden von lokalen Spezialisten durchgeführt und unterscheiden sich von Ort zu Ort. Es sind Traditionen, die zugleich auch immer wieder neu erschaffen werden. Sie bestehen aus diversen Handlungen: Vorbereitung der Opfer, Tötung der Tiere, Gebete, Darbietung der Opfer und schließlich einem gemeinsamen Essen. Während der Zeremonien des letzten Tages traten mehrere Personen auf und sprachen: sie beteten, sie präsentierten den family tree, die Abstammung der Familie, und erinnerten sich an die Geschichte der Familie und des tongkonan. Und da waren die Tänzer, Musiker und Sänger.

${ }^{6}$ Vgl. S. 164. 
Für mich stellte sich die Frage, wem die TCE gehören, wen man fragen könnte, um mehr über sie zu erfahren, um die Genehmigung der Nutzung in einem Film zu erhalten. Die Toraja kennen Eigentum vor allem in Zusammenhang mit materiellen Dingen. Insbesondere bei der Verteilung des Erbes wird deutlich, wem was gehört und wie was weitergegeben wird (Idrus 2007, Waterson 2009). Die Feste spielen dabei eine zentrale Rolle: Je mehr sich die einzelnen Mitgliedern an den Ausgaben bei den Festen beteiligt haben, desto mehr werden sie am Erbe partizipieren. Bezüglich nicht-materieller kultureller Ausdrucksformen ist dies bedeutend unklarer. Grundsätzlich kann man davon ausgehen, dass es hier kein Individualoder Familieneigentum gibt. Allerdings werden bestimmte Tänze, Musik und Gesänge bestimmten Regionen in Tana Toraja zugeordnet.

\section{Zusammenarbeit und Verhandlungen}

Ruby (1991) diskutiert die unterschiedlichen Formen der Zusammenarbeit bei der ethnographischen Filmarbeit. Er unterscheidet entsprechend des Grades der Zusammenarbeit in einem Filmprojekt zwischen kooperativen, kollaborativen und subject-generated Filmen. Bei kooperativen Filmen arbeitet der ethnographische Filmemacher mit den gefilmten Leuten zusammen. In kollaborativen Filmen teilen sie sich die Autorität im Filmprozess. In den subject-generated Filmen mag ein Ethnologe involviert sein, aber er hat keinerlei Autorität über Entscheidungen im Filmprozess.

Die Organisatoren des merauk-Festes haben einen lokalen Videographen mit der filmischen Dokumentation beauftragt und dafür bezahlt. Er entschied selbst, was und wie er filmte. Beide, Videograph und Auftraggeber, sind Toraja, beide kommen aus dem gleichen Distrikt, beide waren sich einig über den Inhalt des Films. So könnte man sagen, dass das entstandene Filmdokument subjectgenerated ist.

Tanete Pong Masak hatte mich eingeladen, bei dem merauk-Fest zu drehen. Er hatte mir geschrieben, dass wir zusammen einen Film über das Hauseinweihungsfest in Buntao machen würden (E-Mail, 06.06.2009). Als ich in Jakarta ankam, dachte ich, dass wir bei diesem Filmprojekt zusammen arbeiten würden. Wir sprachen jedoch nur kurz über den Inhalt und dann sagte er:

You should try to capture the image system of the Torajanese and [...] But

I think you know much better about that, you know, as you have a style

[...] (Gespräch geführt am 13.7.2009)

Er gab mir die Telefonnummer seines Bruders Leo Kala und rief ihn später an, um ihm mitzuteilen, dass ich komme. An meinem ersten Tag in Rantepao telefonierte ich mit Leo Kala Pong Masak. Er sagte mir, dass ich am Nachmittag zum Haus seiner Mutter kommen solle, um das dort stattfindende Opfer zu filmen. Als ich dort ankam, war er nicht da, aber er hatte seine Onkel instruiert mir beim Filmen 
zu helfen. Mitten im Opfer rief er einen der Onkel an, um zu fragen, ob alles geklappt habe, und sich zu entschuldigen, dass es ihm nicht möglich sei, an diesem Tag zu kommen. Am nächsten Tag kam er dann, als ich dabei war, das nächste Opfer zu filmen. Einen Tag später wurde mir gesagt, dass die Familie mich als ein Mitglied des tongkonan BARA'BA adoptiert hatte. Während der ganzen Filmzeit war immer ein Familienmitglied anwesend, half, schaute, was ich (und die anderen) tat, griff aber nie direkt ein.

Nach dem Fest verhandelten Leo Kala und Tanete Pong Masak mit dem lokalen Videographen und mit mir über dessen und mein Videomaterial. Der Videograph hatte ein Acht-Stunden-Video hergestellt. Leo Kala prüfte das Ergebnis und billigte es. Der Videograph hat ihm dann die DVDs und die Originalbänder gegen Bezahlung ausgehändigt. Leo Kala und ich kamen überein, dass ich, als adoptiertes Familienmitglied, einen Festbeitrag bezahle. Ich erhielt dann das Recht, mein Filmmaterial und das des lokalen Videographen frei verwenden zu dürfen, womit auch der Videograph einverstanden war. ${ }^{7}$ Die Familie erwartet nun von mir ein vollständiges Videodokument des merauk-Festes unter Verwendung meiner Aufnahmen und den Aufnahmen des lokalen Videographen. Auch haben sie nichts dagegen, dass ich das Filmmaterial für meine eigenen Publikationen nutze. Tanete Pong Masak hat zudem die Idee, einen Film für das indonesische Fernsehen zu machen.

Hinsichtlich der Rechte am eigenen Bild war es ein Vorteil, dass die ersten Opfer von nur wenigen Personen besucht wurden. So hatte ich Zeit, sie einer nach dem anderen zu fragen, ob sie damit einverstanden sind, gefilmt zu werden. Sie waren irgendwie erstaunt, dass ich sie fragte, lächelten und nickten. Später diskutierten sie darüber, ob es eine Chance gibt, den Film jemals zu sehen. Sie waren sich bewusst, dass der Film in Deutschland, in Europa etc. gezeigt würde. Aber würde der Film auch nach Tana Toraja kommen? Es gab aber keine Möglichkeit, die vielen Leute, die am Schluss am Fest teilnahmen, um Filmerlaubnis zu bitten. Da das Filmen mit Video bei diesen Gelegenheiten normal und akzeptiert ist, und da niemand negativ auf die Kamera reagierte, kann man folgern, dass niemand tatsächlich etwas gegen das Filmen hatte.

Wenn ich mir den Beispielfall näher betrachte, stellen sich mir zahlreiche Fragen hinsichtlich der ethnographischen Filmarbeit, der Entscheidungsautorität, der Autorenschaft und des Copyright. Wir bewegen uns dabei auf zwei komplexen Feldern: dem der Art der Zusammenarbeit, die variieren kann, und dem der lokalen, nationalen und internationalen Eigentumskonzeptionen. Bei meiner ethnographischen Filmarbeit arbeitete ich mit den Entscheidungsträgern und den Darstellern reibungslos zusammen. Es kann also von einer kooperativen Zusammenarbeit im Sinne Rubys gesprochen werden. Doch wie ist diese Zusammenarbeit im Hinblick

\footnotetext{
7 Persönliche Mitteilung des Videographen am 04.08.2009.
} 
auf das indonesische Copyright einzuschätzen? Inwieweit beeinflusst dieses die weitere Nutzung der Aufnahmen?

\section{Copyright in Indonesien}

In vorkolonialer Zeit wurden alle Fragen des sozialen Zusammenlebens nach bestimmten Regeln und Grundsätzen, dem Adat, auf lokaler Ebene behandelt. Angesichts der lokalen Gebundenheit, der oralen Überlieferung und der Flexibilität in der Handhabung hatte sich daraus kein überregionales Rechtssystem entwickelt. In der Kolonialzeit wurde 1911-13 in ganz Niederländisch-Ostindien ein Urheberrecht, das auf dem niederländischen Recht basierte, eingeführt. Allerdings blieb das lokale adat-,,Recht“ weiterhin bestehen, insbesondere weil es in der Lage war, neue Elemente aufzunehmen (Kusumadara 2008).

Bei der Unabhängigkeit 1945 übernahm der Staat Indonesien zunächst einmal das niederländische Rechtssystem. Neue Gesetze wurden dann eingeführt, wenn sie gebraucht wurden, wie z.B. 1961 der Trademark Act. Umfassendere Reformen des Urheberrechts wurden erst 1987 mit dem Copyright Act, 1989/1991 mit dem Patent Act und 1992/1993 auf Druck der USA und der EU mit einem neuen Trademark Act vorgenommen. Letzteres betraf insbesondere den wachsenden Raubkopien-Markt in Indonesien (Kusumadara 2008, Sardjono 2007:10).

Ein grundlegendes Problem bestand darin, dass diese Gesetze von der Bevölkerung meist ignoriert wurden. Das aus den westlichen, industrialisierten Ländern stammende Konzept des geistigen Eigentums (Intellectual Property Right) lag nicht im Interesse der lokalen Bevölkerung und wurde vor allem nicht von den adatNormen unterstützt (Kusumadara 2008).

Mit dem Beitritt Indonesiens zur World Trade Organisation (WTO) $1995^{8}$ und damit der Übernahme der TRIPS-Vereinbarungen sowie der Unterzeichung der Berner Übereinkunft zum Schutz von Werken der Literatur und Kunst (Berne Convention) 199710 war die indonesische Regierung gezwungen, ihr Urheberrecht bis 2005 zu überarbeiten (Aragon and Leach 2008:612, Kusumadara 2008). Indonesien verabschiedete deshalb 2002 ein neues Copyright Law. Im Kontext der Diskussionen in der World Intellectual Property Organisation (WIPO) und der UNESCO über die Urheberrechte sah sich Indonesien weiterhin veranlasst, seinen Reichtum an inmateriellem kulturellem Erbe (kekayaan warisan budaya) durch ein "Law on the Protection of Traditional Cultural Expressions (TCE)" zu schützen und den ökonomischen Nutzen für die lokalen Gemeinden wie auch für den Staat zu erhöhen. 2006

\footnotetext{
${ }^{8}$ http://www.wto.org/english/theWTO_e/countries_e/indonesia_e.htm (Zugriff am 01.02.2010).

9 TRIPs steht für die "trade-related aspects of intellectual property rights", das heisst das Übereinkommen über handelsbezogenene Aspekte der Rechte über geistiges Eigentum von 1994.

$10 \mathrm{http}: / /$ www.wipo.int/treaties/en/Remarks.jsp?cnty_id=970C (Zugriff am 08.11.2009). Sie trat am 05.09.1997 in Kraft.
} 
wurde ein Entwurf dieses Gesetzes an diverse Gremien zur Diskussion gegeben. (Aragon and Leach 2008:613).

\subsection{Traditionelles Recht: Adat}

Adat, wie das indonesische Gewohnheitsrecht genannt wird, ist in den lokalen Traditionen verankert. Es wird von Generation zu Generation weitergegeben und den aktuellen Gegebenheiten angepasst. Es regelt das Zusammenleben auf lokaler Ebene. Im adat-System zählen einstimmige, harmonische Entscheidungen mehr als die strikte Anwendung der vorhandenen Regeln (Holznagel 1999:7-8, Kurniawan 2008:10-12). Es gab in jedem Ort adat-Autoritäten (Li 2007:341), die von der Bevölkerung anerkannt wurden. Ihre politische Stellung war von Person zu Person, von Ort zu Ort, von Region zu Region unterschiedlich. Mit Beginn der Kolonialzeit wurde das auf dem adat basierende politische System aufgelöst.

Adat erkennt das individuelle Eigentum an materiellen Gütern an, allerdings steht dabei das Interesse der Gemeinschaft im Vordergrund (Kusumadara 2008). Dies wird bei den Toraja u.a. bei der Verteilung des Erbes sichtbar. ${ }^{11}$ In den letzen Jahren haben sich überregionale, moderne adat-Vereinigungen gebildet, die sich insbesondere für die Wahrung bzw. Rückgabe der Landrechte an die traditionellen Gemeinschaften einsetzen. ${ }^{12}$ So scheint adat allerdings kein privates, individuelles Eigentumsrecht an intellektuellen Werken oder Erfindungen zu kennen. In ganz Indonesien herrscht die Ansicht vor, dass das Wissen eines Menschen und seine Schöpfungen auf dem Wissen und den Erfahrungen seiner Vorfahren basieren und daher allen zur Verfügung stehen. Wissen wird also als Gemeineigentum betrachtet, das dem Nutzen aller dient. Im Gegensatz zum westlichen Verständnis ist eine breite Nutzung gewollt (Kusumadara 2008).

Dana Rappaport hat jahrelang über die Musik und Gesänge der Toraja gearbeitet und hat eine umfangreiche Sammlung an Tondokumenten angelegt (Interview mit Rappaport, Juli 2009). Die Darsteller (performer) waren mit der Aufzeichnung einverstanden. Doch auch ihr ist unklar, wer hinsichtlich der weiteren Verwendung der Aufzeichnungen noch sein Einverständnis geben sollte.

\subsection{2: Reform des indonesischen Urheberrechts}

Das indonesische Urheberrecht orientierte sich von Anbeginn an westlichen Vorlagen. Dies gilt auch für das 2002 neu verabschiedete Copyright: Werke wie Bücher, Artikel, Musik und Kunst, deren Schöpfer bekannt ist, sind bis 50 Jahre nach dem Tod des Schöpfers urheberrechtlich geschützt ("Law of The Republic of Indonesia Number 19 Year 2002 Regarding Copyrights“ Artikel 29.113). Werke wie

\footnotetext{
11 Siehe S. 157.

12 Vgl. Davidson und Henley 2007.

13 Wenn nicht anders angegeben, stammen alle folgende Artikel dieses Kapitels aus diesem Gesetz. Siehe auch: http://www.dgip.go.id/ebhtml/hki/filecontent.php?fid=9164 (Zugriff am 14.02.2010).
} 
Computerprogramme, fotografische und filmische Werke sind für 50 Jahre nach Veröffentlichung urheberrechtlich geschützt. Danach scheinen sie in die public domain überzugehen (Artikel 30(1)).

Für kulturelle Produkte und Folklore (folklor dan hasil kebudayaan rakyat), bei denen es keine bekannten Urheber gibt, gelten besondere Regelungen. Hier verfügt der Staat über das zeitlich unbegrenzte Copyright (Aragon and Leach 2008:613, Utomo 2009:4). In Artikel 10 heißt es:

(2) The State shall hold the Copyright for folklores and works of popular culture that are commonly owned, such as stories, legends, folk tales, epics, songs, handicrafts, choreography, dances, calligraphies and other artistic works.

(3) To publish or reproduce the works as referred to in paragraph (2), any person who is not the citizen of Indonesia shall, firstly, seek permission from the institution related to the matter.

Die Ergebnisse einer wissenschaftlichen, künstlerischen oder literarischen Arbeit, wenn sie einen veröffentlichbaren Stand erreicht haben, haben urheberrechtlichen Schutz (Artikel 12.1). Dies gilt auch für Fotografien und Filme.

Ein „Autor“ kann eine oder mehrere Personen sein, durch deren Inspiration ein Werk hergestellt wurde, das deren intellektuelle Fähigkeit, Einbildungskraft, Können oder Expertise ausdrücklich offenbart (Artikel 1.2). Als Autor anerkannt wird man, indem man sich bei der Generaldirektion in das Werkregister eintragen lässt oder der Verlag jemanden bei der Publikation eines Werkes als Autor nennt (Artikel 5.1). Hinzukommen die verwandten Rechte, zum Beispiel die Rechte eines Darstellers hinsichtlich der Reproduktion seines Auftritts (Artikel 5.9). Darsteller sind zum Beispiel Schauspieler, Musiker, Tänzer (Artikel 5.10). Sie haben das exklusive Recht, einer anderen Person ihre Einwilligung zu geben oder zu verweigern, ihren Auftritt zu senden bzw. zu reproduzieren (Artikel 49). Sie haben dieses Recht für 50 Jahre ab dem Tag des Auftritts bzw. der audio(-visuellen) Aufzeichnung (Artikel 50).

\subsection{6: Entwurf eines Gesetzes zum Schutz der Traditional Cultural Expressions}

Infolge der Diskussionen über die Traditional Cultural Expressions (TCE) und über die Sicherung von Intangible Cultural Heritage (ICH) in der WIPO und der UNESCO hat die indonesische Regierung 2006 einen Entwurf eines sui generis Gesetzes zum Schutz der TCE in Umlauf gebracht, das über die Regulierungen im Urhebergesetz von 2002 hinausgeht.

Gemäß der WIPO verbindet das Kulturerbe einer Gemeinschaft oder einer Nation ihre Vergangenheit mit ihrer Zukunft. Kulturerbe ist lebendig, es wird ständig erneuert, da die traditionellen Künstler (traditional artists) neue Vorstellun- 
gen und Erfahrungen in ihre Produktion einbringen. ${ }^{14}$ Doch geht die WIPO davon aus, dass die Kreativität durch die bestehenden Urheberrechte nicht adäquat geschützt wird. Besonders indigene und traditionelle Gemeinschaften verweisen immer wieder auf nicht-autorisierte Adaptionen und Reproduktionen, die dann kommerziell genutzt werden, bzw. auf die despektierliche Nutzung ihrer TCE und auf das Fehlen der Quellenverweise (WIPO 2003b:31).

Im Wesentlichen geht es bei dem indonesischen Gesetzesentwurf ${ }^{15}$ um folgende Punkte:

(1) Schutz von TCE, die von Gemeinschaften und traditionellen Gesellschaften erhalten (masyarakat tradisional) und praktiziert werden (Aragon and Leach 2008:613).

(2) Regulierung der Reproduktionen und Adaptionen regionaler, indonesischer Kunst, Musik, Theater, und Tänze wie auch Geschichten und rituelle Zeremonien unabhängig ihres Ursprungsdatums.

(3) Ausnahmen soll es für den Bereich der Bildung, Forschung, des Journalismus und der Wohltätigkeit geben, solange kein wirtschaftlicher Zweck verfolgt wird. Das Gesetz verlangt von Indonesiern und Ausländern, mit den owner communities eine Nutzungsvereinbarung zu schließen, diese auf Distriktsebene anzumelden, und manchmal auch bei den Büros der Provinz oder der Nationalregierung. Ausländer müssen zudem von den Distrikt-, Provinz- oder den NationalAgenturen, die sich um die Verteilung des Erlöses kümmern, eine Erlaubnis erhalten. Die Beteiligung der owner communities am Erlös ist nicht zwingend vorgeschrieben. Vergehen gegen dieses Gesetz sollen zivil- oder strafrechtlich verfolgt werden (Aragon and Leach 2008:626, Fußnote 17).

(4) Verhinderung der transnationalen Aneignung. In Reaktion auf den globalen Druck fürchten indonesische Führer verständlicherweise, dass ihr „nationales“ Cultural Property ausländisches intellectual property wird (Aragon und Leach 2008:608). Edi Sedyawati erklärt dies bei einer WIPO-Konferenz in Bandung 2007 (WIPO 2007) ausführlicher. Sie weist darauf hin, dass Borgen und Teilen von Schöpfungen bei den indonesischen ethnischen Gruppen eine allgemeine Praxis sei, was auch das Kopieren von Kunstwerken und der Austausch von Ideen beinhaltet. Jazsi (2009) ruft in Erinnerung, dass traditionelle Künstler/Handwerker insbesondere durch das Kopieren von Kunstwerken ihren Beruf erlernen. Edi Sedyawati erklärt weiter, dass sich die Fähigkeiten durch die Migration weiter verbreitet haben. Für sie ist diese Art der Verbreitung etwas grundlegend anderes, als die transnationale Aneignung aus kommerziellen Interessen. Wenn traditionelle Kunst der public domain zugeschrieben würde, könnten sich Ausländer diese aneignen und kommerziell verwenden.

\footnotetext{
${ }^{14}$ http://www.wipo.int/export/sites/www/about-ip/en/iprm/pdf/ch2.pdf (Zugriff am 04.04.2010). ${ }^{15}$ Der Gesetzentwurf selbst lag mir nicht vor. Im Folgenden stütze ich mich auf Aragon und Leach 2008 und Jaszi 2009.
} 


\section{Entscheidungsträger und Nutznießer}

Das indonesischen Copyright und das geplante Gesetz zum Schutz der TCE werfen insbesondere in Zusammenhang mit den lokalen adat-Regeln neue Fragen auf. Vier Punkte sind hier näher zu betrachten: Die verwendeten Begrifflichkeiten, die Frage nach der Autorenschaft und der Position der betroffenen Darsteller und Künstler, die Frage nach dem Bestimmungsrecht (Autorität) und die Frage nach der Umsetzung der Gesetze. Letztlich handelt es sich um die Fragen: Wer hat welche Interessen, Rechte und wer zieht wann welchen Nutzen?

\subsection{Begrifflichkeiten}

In den Gesetzestexten und Erklärungen werden oft Begriffe verwendet, die als terminus technicus hingenommen werden müssen, auch wenn sie als unzureichend anzusehen sind und/oder auf Probleme hinweisen, die den breiteren Kontext betreffen.

Immer wieder wird insbesondere in WIPO-, UNESCO- und nationalen Copyright-Dokumenten von Ausdrucksformen der Folklore (expressions of folklore) bzw. traditionellen kulturellen Ausdrucksformen (traditional cultural expressions) gesprochen. WIPO und UNESCO beschreiben sie wie folgt:

Traditional cultural expressions (or, "expressions of folklore") include music, art, designs, names, signs and symbols, performances, architectural forms, handicrafts and narratives.

TCEs are integral to the cultural and social identities of indigenous and local communities, they embody know-how and skills, and they transmit core values and beliefs. Their protection is related to the promotion of creativity, enhanced cultural diversity and the preservation of cultural heritage. ${ }^{16}$

An anderer Stelle macht die WIPO (2006a) deutlich, dass TCE von einer Generation zur nächsten entweder mündlich oder durch Imitation weitergereicht werden, dass es keine Autoren gibt, dass die Gemeinschaft (community) der Bezugspunkt ist und dass es sich nicht um ein statisches Element handelt, d.h. Veränderungen sind über die Zeit möglich und gewollt. ${ }^{17}$ Aragon and Leach (2008:607) betonen dahingegen, dass indigene Gruppen und Nationalstaaten dazu neigen, eine Auffassung von stabiler und einheitlicher Kultur neu zu erfinden. Ethnologen versuchen bereits seit Jahrzehnten, diese Vorstellung zu korrigieren bzw. sie durch das fachgebräuchliche dynamische, permeablen Kulturkonzept zu ersetzen.

\footnotetext{
16 http://www.wipo.int/tk/en/folklore/, Zugriff am 02.06.2010.

$17 \mathrm{Vgl}$. Antons 2009.
} 
Nach Aragon und Leach (2008:614) hat die indonesische Regierung Probleme mit den Begriffen „,indigen“ und „traditionell“, wie sie in den internationalen Gremien benutzt werden. Sie weigern sich, anzuerkennen, dass es in ihrem Land indigene Gruppen gibt, oder wie es Basuki Antariska ${ }^{18}$ formuliert: alle Indonesier sind Indigene. Zu beachten ist aber der feine Unterschied, der zwischen indigen und traditionell gemacht wird:

Being labeled "indigenous" might qualify minority groups to make claims for the return of lost land. Being labeled "traditional" instead qualifies them for tourism or development projects. (Aragon und Leach 2008:614)

Dies erlaubt der indonesischen Regierung die „traditionellen Gemeinschaften“ in ihre Aktivitäten einzuschließen, für sie zu sprechen, ihre Interessen zu vertreten. Viele ethnische Gruppen haben in Indonesien deshalb Schwierigkeiten, sich des internationalen Diskurses des „Indigenen“ zu bedienen, um ihre Interessen besser vertreten zu können. Dies wird bei der Entwicklung der überregionalen Allianzen von adat-Gesellschaften deutlich, die sich insbesondere für die Landrechte einsetzen. ${ }^{19}$ Der Begriff adat-Gesellschaft (masyarakat adat) wurde 1993 von NGOs eingeführt, um sich auf die im internationalen Gesetz verankerte Kategorie der indigenous people beziehen zu können (Li 2007:341).

Unabhängig von der häufigen Verwendung in Gesetzestexten und damit verbundenen Dokumenten ist der Begriff traditional community grundsätzlich problematisch. Dabei impliziert der Begriff ,traditionelle Gemeinschaft" nicht das gemeinschaftliche Eigentum an Gütern, sondern bedeutet, dass die individuellen Rechte, die individuellen und kollektiven Ressourcen zu nutzen, von der umgebenden Gemeinschaft anerkannt werden (Li 2007:341). Mit der Unabhängigkeit Indonesiens wurden die traditionellen politischen Strukturen in dem Sinne endgültig aufgelöst, dass traditionelle lokale Vertreter offiziell keine Anerkennung mehr fanden. Was ist also heute eine traditional community und wer ist vor allem ihr legitimer Vertreter? So fragt Bräuchler in Hinblick auf die Dezentralisierungspolitik: „[...] wer denn wie entscheidet, ob es sich überhaupt um ein adat-Dorf handelt oder nicht"، (2007:48).

Im Gesetzentwurf von $2006^{20}$ wird auch zwischen ,traditioneller Gemeinschaft" (traditional community) und „traditioneller Gesellschaft” (traditional society) unterschieden. Basuki Antariska ${ }^{21}$ erklärt dies folgendermaßen: Eine traditionelle Gemeinschaft ist eine Gruppe von Leuten, die an einen bestimmten Platz leben

\footnotetext{
18 Basuki Antariska (Pak Basuki), Head Section on UNESCO, Department of Culture and Tourism, Jakarta in einem nicht veröffentlichten Gespräch mit Brigitta Hauser-Schäublin anlässlich einer WIPO IGC-Kommissionssitzung in Genf 2009.

${ }^{19}$ Vgl. http://www.aman.or.id (Zugriff am 04.04.2010).

20 Vgl. Aragon und Leach 2008:613-614.

${ }^{21}$ Basuki Antariska in einem nicht veröffentlichten Gespräch mit Brigitta Hauser-Schäublin anlässlich eine WIPO-Konferenz in Geneva 2009.
} 
und die gleiche Kultur haben. Eine traditionelle Gesellschaft ist eine Gruppe von Leuten, die die gleiche Kultur haben, aber in verschiedenen Regionen leben. Andere sprechen auch von adat-Gesellschaft (Utomo 2009). Ungeklärt bleibt die sich daran anschließende Frage, wer denn dann legaler Rechtsvertreter und Nutznießer im traditionellen Kontext sein kann (Li 2007:338).

\subsection{Autoren, Darsteller, Künstler}

Welche Rolle spielen eigentlich die Menschen bei kulturellen Produktionen: Sind sie Autoren, Darsteller oder Künstler?22 Auch wenn das indonesische Copyright den Begriff des Autors ${ }^{23}$ eines Werkes definiert, stellt sich bei näherer Betrachtung heraus, dass nicht eindeutig geklärt ist, wie Autoren (author/pencipta) und Darsteller (performer/pelaku) gemäß des Copyright Law zueinander stehen und ob „Künstler“ nicht etwas von beidem haben, im Sinne eines Schöpfers (creator).

Donzelli (2007) weist darauf hin, dass in Tana Toraja die traditionellen Priester (tominaa) eine spezielle Sprache (basa tominaa) sprechen, welche sie an ihre Söhne weiter geben. Die traditionellen rituellen Texte müssen bei den Auftritten immer in der gleichen Weise rezitiert werden. Demnach gehören die rituellen Texte zu den traditionellen kulturellen Ausdrucksformen der Toraja, die Priester selbst haben also daran kein Copyright. Dennoch, durch die spezielle Sprache und die kontrollierte Weitergabe des Wissens in der Familie, sind die Priester mehr als nur Darsteller. Sie sichern das traditionelle Wissen und haben darauf einen direkten und monopolartigen Zugriff, der sie zumindest zu Besitzern des Wissens macht. Seit neuestem gibt es aber junge Männer, die die rituellen Texte studieren und sie in einem neuen Stil arrangieren. Sie bieten ihre Dienste als Sprecher z.B. bei Totenfesten an und werden als "protocols" gebucht und dafür bezahlt. ${ }^{24}$

The emergent generation of ritual specialists displays a new way of conceiving the notion of authorship in ritual speech, which results in a tendency to emphasize personal oratorical styles. [...] their attitudes gesture towards a form of stylistic copyright in which the emphasis on individual styles fades into a conception of personal ownership of ritual words and formulas. (Donzelli 2007:145-6)

Interessanterweise sprechen Aragon und Leach (2008) in ihrem Artikel von Künstlern und nicht von Autoren oder Darstellern. Kunst wird hier in einem breiten Sinn als kulturelle Aktivität verstanden. Die Künstler verstehen sich als Schöpfer, Imitatoren und Bewahrer zugleich. Sie fühlen sich dem kulturellen Erbe der Vor-

\footnotetext{
22 Zimbehl gibt in diesem Band einen Einblick in die Diskussion um die Rechte der Darsteller (ausführenden Akteure) und der Produzenten (bestimmenden Akteure).

$23 \mathrm{Vgl}$. Seite 162.

${ }^{24}$ Die traditionellen Priester erhalten für ihren Einsatz größere Mengen Fleisch von den geopferten Tieren.
} 
fahren verpflichtet, verstehen sich jedoch nicht als Eigentümer (Aragon und Leach 2008:618).

Im Rahmen des Projekts "The Impact of Intellectual Property Laws on Indonesian Traditional Arts" 25 haben Wissenschaftler aus Indonesien, den USA und Europa untersucht, wie die indonesischen Künstler und Autoren das indonesische Urheberrecht wahrnehmen. Auch andere Wissenschaftler haben sich mit dieser Frage beschäftigt.

Die Künstler weisen die Idee, alleiniger Schöpfer eines Werkes zu sein, zurück (617); Sie haben viele Werke geschaffen, ohne ihre Werke als ihr privates Eigentum zurückzuhalten. Früher haben die Künstler ihre Werke nicht signiert. Andere Künstler konnten sie frei nutzen, sie waren Teil der public domain (Kusumadara 2008:4). Auch Edi Sedyawati (in Kamil 2000:3) hat auf einer WIPO-Konferenz 2000 in Bali darauf hingewiesen, dass bei alten Werken die Künstler manchmal auch bekannt sind. Sie hatten aber nichts dagegen, dass ihre Werke von vielen anderen kopiert und als Souvenirs verkauft werden. Das Kopieren von Werken wird vielmehr als eine Form des Respekts und der Anerkennung betrachtet. Die Künstler fühlen sich sogar verpflichtet, die Vervielfältigung ihrer Kunst zu unterstützen und nicht zu behindern (Aragon und Leach 2008:623). Viele Künstler Batik-Designer, Musiker, Maler, Weber, Tänzer und Puppenspieler - waren stolz und erfreut, wenn andere ihren Stil kopieren wollten (619).

Immer wieder taucht in Texten und Konversationen das Wort style auf, es wird anerkannt, dass die Autoren, Darsteller und Künstler eine besondere Art und Weise der Repräsentation haben. Tanete Pong Masak sprach über „meinen Filmstil“, den er respektiere. Aragon und Leach (2008:220) berichten von Künstlern, die erklärten, dass ihre gamelan-Musik eine kollektive, nicht individuelle kulturelle Praktik sei, und dass sie stolz darauf sind, wenn ihr Stil von anderen imitiert wird. Ein Puppenspieler sprach über die Möglichkeiten der Variation eines Puppenspiels unter Wahrung des grundlegenden Musters. Er beansprucht für sich eine individuelle Vorgehensweise, ohne jedoch hinsichtlich des Kanons einen Eigentumsanspruch zu formulieren (Aragon und Leach 2008:618). Donzelli (2007) berichtet von dem Bestreben der jungen Leute, für ihren Stil Copyright zu verlangen.

Edi Sedyawati (in Kamil 2000:3) meint, dass das Kopieren und Imitieren von TCE im Rahmen der eigenen Gesellschaft in Ordnung ist, aber bei kommerzieller Verwertung durch Männer anderer Gesellschaften als „nachteilig“ zu betrachten ist. Die Künstler lehnen jedoch die Idee, dass ihre lokalen sozialen Aktivitäten durch die Regierung als kommerzielles Eigentum behandelt werden, ab (Aragon und Leach 2008:624). Sie haben keine Befürchtungen, dass ihre traditionellen Künste in Gefahr stehen, verzerrt oder entwertet zu werden, oder dass die TCE, die in einem religiösen Kontext stehen, in profanen Kontexten aufgeführt und somit mißbraucht werden. Sie wollen ihre neuen Kreationen vor Nutzung durch Andere schützen (Indonesian Media Law and Policy Centre, Social Science Research

${ }^{25}$ Förderung durch die Ford Foundation. Für eine Projektbeschreibung siehe Jaszi 2009. 
Council 2005). Die lokalen Gruppen haben zudem kein Problem mit dem Missbrauch ihrer Kultur durch Fremde (Aragon und Leach 2008:610). Die Künstler haben vielmehr Angst, dass sie wegen der Gesetze in Zukunft nicht mehr auf das Erbe ihrer Gruppe zugreifen können (608).

\subsection{Autorität über traditionelle kulturelle Ausdrucksformen}

Es stellt sich also die Frage, wer denn die Entscheidungsautorität über die Nutzung von traditionellen kulturellen Ausdrucksformen hat. Der Staat, die Künstler, die traditionelle Gemeinschaft (traditional community)? Zimbehl ${ }^{26}$ untersucht folklore als Gegenstand des Urheberrechts und zeigt auf, dass es in Bezug auf die traditionellen kulturellen Ausdrucksformen ziemlich unklar ist, wer über die Nutzung entscheiden kann. Bei ihm wie im indonesischen Gesetzentwurf von 2006 wird immer wieder auf die traditionellen Gemeinschaften Bezug genommen. Aber wer ist die Gemeinschaft bzw. die kompetente Autorität in einem speziellen Fall? „Wem gehört Kultur?" 27

In meinem Filmprojekt habe ich versucht, herauszufinden, wer mir in Tana Toraja darüber Auskunft geben kann, wer mir sagen kann, ob ich etwas filmen und veröffentlichen darf. Dabei stieß ich auf ein Bündel von Problemen: Wer ist die traditionelle Gemeinschaft, die über die Nutzung einer TCE entscheidet? Ist es immer die gleiche Gruppe für verschiedene TCE? Durch wen wird entschieden? Und wie soll das dokumentiert werden? In meinem Fall ließen sich diverse TCE feststellen, die möglicherweise unterschiedlichen traditionellen Gemeinschaften zugeordnet werden könnten: Das Haus, der tongkonan, gehört der Familie, sie hat gemeinsam entschieden, wie er aussehen soll, welche Schnitzereien verwendet werden sollen. Das Fest, die Opfer, die Abschlussveranstaltung sind schwer einzuschätzen. Teilweise wurden sie durch Ne'wei nach lokalen Mustern gestaltet, teilweise durch Tato Dena nach doch eher regionalen, und die Einbeziehung bestimmter Kultobjekte, die in Besitz des puang von Sangalla' sind, brachte nochmals eine andere Region mit ins Spiel. Die drei genannten Wissensträger haben den Aufnahmen zugestimmt, aber haben sie dazu auch die notwendige Autorität? Des Weiteren gab es die Tanzgruppen und das Kinderorchester. Letzteres spielt auf für Buntao typischen Musikinstrumenten, also könnte diese Musik Buntao zugeordnet werden. Doch wer wäre dann hier anzusprechen? Die Tanzgruppen kamen aus verschiedenen Dörfern, waren als Gruppe zumindest ansprechbar. Aber wem gehören die Tänze an sich, handelt es sich hier um weit verbreitete TorajaChoreographien oder hat jede Tanzgruppe eine eigene?

Diesen und ähnlichen Fragen wurde auch in dem oben genannten Forschungsprojekt nachgegangen (vgl. Indonesian Media Law and Policy Centre, Social Science Research Council 2005, Sardjono 2007, Aragon und Leach 2008).

\footnotetext{
${ }^{26} \mathrm{Vgl}$. Beitrag in diesem Band.

27 Vgl. Brown 2003, Kasten 2004, McLeod 2001, Scafidi 2005.
} 
Befragt wurden Künstler - Handwerker, Musiker, Darsteller - das heisst Menschen, die tagtäglich mit TCE umgehen, sie professionell nutzen, davon leben. Viele der Künstler wissen, dass ihre Schöpfungen auf den Traditionen ihrer Vorfahren basieren. Sie sehen sich allerdings nicht primär als Nutzer dieser Quelle der Kreativität, wie es das Gesetz formuliert, sondern vielmehr als autorisierte Träger dieser Traditionen (Aragon und Leach 2008:623).

Beim Puppenspiel handelt es sich z.B. um eine spezielle Form des TCE dahingehend, dass es doch eher auf einer künstlerischen Tradition basiert als auf den Traditionen einer Lebensgemeinschaft. Die Puppenspieler sind deshalb der Meinung, dass es weniger die Aufgabe einzelner Personen, eines Ältestenrates oder gar des Staates sei, auf die Einhaltung der Tradition zu achten. Vielmehr handelt es sich dabei um eine Diskussion zwischen herausragenden Künstlern und ihrem lokalen Publikum, die den vererbten Status und die Richtlinien für das Repertoire respektieren (618).

Die neuen Gesetze und internationalen Richtlinien bedrohen die lokalen Autoritätsstrukturen hinsichtlich des lokalen Wissens und der Künste (620). Die Autorität der Gruppen und Individuen, die bisher die Interessen gelenkt haben, würde dann nicht mehr anerkannt. Eine irreführende Unterscheidung zwischen ,traditionellen" (statischer, irrelevanter) und lebenden künstlerischen Praktiken würde gemacht werden. Außerdem würde eine starke Nachfrage nach individualisierten Rechten ausgelöst, weil die Anspruchsberechtigten verhindern wollen, dass ihre Werke als „anonym“ bezeichnet werden und dem Staat anheim fallen (Indonesian Media Law and Policy Centre, Social Science Research Council 2005).

\subsection{Politische Zuständigkeiten}

Mit dem Copyright Law von 2002 und dem Gesetzentwurf von 2006 gibt es auch noch rein organisatorische Probleme. Insbesondere im Gesetzentwurf von 2006 werden verschiedene politische Ebenen angesprochen, die im Bewilligungsprozess berücksichtigt werden sollen bzw. müssen. Bei der Reproduktion von TCE sollen primär die Genehmigungen bei den traditionellen Gemeinschaften eingeholt werden, ohne jedoch zu präzisieren, wer hier der Ansprechpartner sein könnte. Auch bei den diversen politischen Organen auf Dorf-, Distrikts- oder Provinzebene fehlen die Ansprechpartner. Unklar ist auch, wer schließlich das Sagen hat. Das gleiche Problem besteht bei der Verteilung möglicher Einnahmen unter den diversen Parteien. Im Falle der Neu-Inszenierung des Bugi-Eposes La Galigo, das mit Genehmigung der lokalen Bevölkerung in Großbritannien sehr erfolgreich umgesetzt wurde, entbrannte genau eine solche Diskussion, verlangten die Behörden doch, dass die ausländischen Künstler für die Nutzung des TCE bezahlen sollten, während die lokale Bevölkerung dies nicht tat (Aragon und Leach 2008:609-611). Der Hinweis im Gesetzentwurf, dass die traditionellen Gemeinschaften nicht unbedingt an den Einnahmen beteiligt werden sollen, ist somit besonders bemerkenswert. Nach Aragon sind dies einige der Gründe, warum für das Gesetz von 
2002 noch keine Umsetzungsbestimmungen verabschiedet wurden (Mündliche Mitteilung, Dezember 2009). Ohne diese Bestimmungen gilt ein Gesetz in Indonesien als noch nicht in Kraft getreten. Ein gleiches Schicksal könnte auch das Gesetz von 2006 ereilen, sollte es beschlossen werden.

It is unclear [...] how Indonesia’s 2002 Copyright Law will fit with, or be superseded by, the TK and TCE law, if the latter is ever passed. What is clear is that, if enacted, the law would entail escalated bureaucratic supervision of local expressive practices plus many practical challenges based on its arguable concepts of culturally bounded, homogenous ethnic communities. (2008:613-14)

Die unterschiedliche Behandlung von inländischen und ausländischen Nutzern von indonesischen TCE bei kommerzieller Verwertung ist augenfällig, wobei im Ausland lebende Indonesier gemäß der Definition von traditioneller Gesellschaft wohl als Inländer zu betrachten sind. Unklar ist auch, was kommerzielle Nutzung heißt. Für Fernsehproduktionen gibt es schon jetzt eine Regelung. Sie müssen bei der indonesischen Botschaft eine Einreise- und Drehgenehmigung einholen. ${ }^{28}$ Der Gesetzentwurf sieht allerdings vor, dass jegliche finanzielle Einnahme, auch wenn sie nur zur Deckung der Produktionskosten dienen sollte, als kommerzielle Nutzung betrachtet wird. Was geschieht mit einem Ausländer, Touristen oder Wissenschaftler, der mit der Einwilligung der Leute vor Ort Filmaufnahmen macht, sie nach Hause mitnimmt und erst dort einen Film veröffentlicht und verbreitet? Es scheint rechtlich vollkommen unproblematisch $\mathrm{zu}$ sein, die Filmaufnahmen im Internet zu veröffentlichen, auch wenn dadurch das Bild einer traditionellen Gemeinschaft negativ beeinflusst wird, wie dies angesichts der zahlreichen Büffelopfer bei den Totenfesten der Toraja der Fall ist. Aber sobald auch hier irgendwie Geld fließt, scheint dies als kommerzielle Nutzung betrachtet zu werden und wäre dann auf allen Ebenen zu verhandeln. Und was geschieht, wenn ein Film gemeinsam mit einem Indonesier veröffentlicht wird? Wenn wir keine klar getrennte Welten haben, was in einer transnationalen Gesellschaft zunehmend der Fall sein wird?

In der Abschlussdiskussion des obengenannten Forschungsprojektes (Indonesian Media Law and Policy Centre, Social Science Research Council 2005) wurde darauf hingewiesen, dass, sollte der Gesetzentwurf verabschiedet und zusammen mit dem Copyright Law von 2002 in Kraft treten, mit einer erheblichen Bürokratisierung zu rechnen wäre. Des Weiteren wird eine zunehmende Kontrolle des Staates über die Künste befürchtet. Der Staat könnte von Darstellern und Künstlern, die mit TCE-Werken arbeiten wollen, Gebühren erheben.

28 http://indonesian-embassy.de/en/consular_immigration/form_visa/ANTRAG-DREHGENEHMIGUNG.pdf (Zugriff am 04.03.2010). 


\section{Fazit}

Das merauk-Fest war für Viele ein Erlebnis, das sie auch mit Film - mit Videooder Handy-Kameras - festzuhalten versuchten. Bezüglich der eingangs gestellten Fragen sind vier unterschiedliche Arten des Filmemachens näher zu betrachten: Familienfilme, Touristenfilme, lokale Dokumentationsfilme und ethnographische Filme. Beim merauk-Fest gab es keine Fernsehproduktionen, weder inländisch noch ausländisch, die das Bild abgerundet hätte.

Die Familie, die das merauk-Fest veranstaltete, hatte grundsätzlich nichts gegen das Filmen einzuwenden. Ihre Gäste wie auch die Touristen konnten frei filmen und mit ihren Aufnahmen machen, was sie wollten. Der lokale Videograph, der einen Dokumentationsfilm drehte, und ich, die als Ethnologin einen ethnographischen Film machen wollte, waren wesentlich länger vor Ort, hatten eine engere Beziehung zur Gastgeberfamilie, und arbeiteten ja auch in deren Auftrag bzw. mit deren expliziter Einwilligung. Nicht nur, dass wir von ihnen die Drehgenehmigung bekommen haben, wie es im Copyright Law (2002) und im Gesetzentwurf (2006) vorgesehen ist, haben sie sich auch durch zusätzliche Informationen, Rücksichtnahmen, Äußerungen an den Filmen partizipiert. In beiden Fällen könnte somit von einer kooperativen Filmarbeit nach Ruby gesprochen werden. Allerdings bin ich von außen mit einer Filmidee gekommen, während der lokale Videograph von vor Ort ist und somit der Kategorie der subject-generated films zugeordnet werden müsste. Bei seiner Arbeit stellt sich allerdings die Frage, wie das entstandene Endprodukt einzuordnen ist, wer Autor ist, oder ob es überhaupt einen gibt. Ob es sich bei der Filmdokumentation überhaupt um ein Werk handelt (das durch ein Copyright geschützt ist). Hier wurden ja die Geschehnisse „nur“ aufgenommen und eins zu eins auf DVDs zugänglich gemacht. Angesichts des gegebenen Auftrags und der Übergabe des ganzen Materials an die auftraggebende Familie am Schluss, kann man davon ausgehen, dass die Familie das Recht hat, über die weitere Nutzung des Materials zu bestimmen.

Im Fall des ethnographischen Films, der durch mich, eine Ausländerin, hergestellt wird, stellt sich alles etwas anders dar. Mein Plan ist es, nicht nur eine Langfassung, ähnlich der des lokalen Videographen, für die Familie zu machen, sondern auch einen Film über die beiden Ereignisse, merauk-Fest und Konferenz, herzustellen. Wie ist nun dieser Film einzuordnen? Die Familie hat ihr Einverständnis für die Aufnahme bei beiden Ereignissen gegeben. Die Darsteller, die am Fest Beteiligten wie auch die Konferenzteilnehmer, haben den Aufnahmen zugestimmt. Bezüglich der Aufzeichnung der Tänze und Musik kann man davon ausgehen, dass die Darsteller der Aufzeichnung still schweigend zustimmten. Nach dem indonesischen Recht wären die Tänze und die Musik eindeutig als traditionelle kulturelle Ausdrucksformen zu klassifizieren, bei denen der Gesetzentwurf von 2006 besondere Regelungen vorsieht. Bei einer Umsetzung dieses Gesetzentwurfs würde sich mir die Frage stellen, wer denn welche Entscheidungskompetenz hat, inwieweit die 
lokale Bevölkerung hier zwar zustimmen oder ablehnen darf, aber nicht entscheiden kann, wie im Fall der Neuinszenierung des Epos La Galigo zu sehen war.

Bei Veröffentlichung des Films, wäre ich der Autor, im Sinne des indonesischen und des deutschen Copyrights. Die Veröffentlichung selbst würde unter deutschem Recht stehen. Das Copyright Law von 2002 sieht vor, dass Ausländer bei kommerzieller Nutzung der Aufnahmen, dafür von Indonesien eine Genehmigung brauchen. Wie würde es jedoch aussehen, wenn Tanete Pong Masak und ich gemeinsam einen Film veröffentlichen und vermarkten, oder wenn ich einen Film über einen indonesischen Partner veröffentlichen und vermarkten würde?

Die Fragen nach Autorität, Autorenschaft und Copyright müssen noch vertiefend untersucht werden. Welche Eigentumskonzepte liegen für TCE vor und sind TCE generell als Cultural Property zu betrachten? Und wie steht es mit der Zusammenarbeit in Filmprojekten, welche Konsequenzen hat es hinsichtlich der Autorenschaft und des Copyright. Angesichts der vielen offenen und teilweise unklärbaren Fragen - eine Situation, die für Ethnologen eher alltäglich ist - ist zu überlegen, ob es nicht auch bei Filmaufnahmen allgemeine Richtlinien geben kann, wie sie in kollaborativen Forschungsprojekten schon diskutiert werden. ${ }^{29}$ Amerikanische Indigene und Australische Aborigines fordern den Schutz ihres traditionellen Wissens und ihrer Kultur wesentlich nachdrücklicher als andere. Von der Australian Film Commission wurden deshalb Richtlinien für Filmemacher, die mit indigenen Gruppen zusammenarbeiten, veröffentlicht (2003), allerdings basieren diese auf der spezifischen juristischen Situation in Australien. So müssen wir fragen, ob es möglich sein wird, Richtlinien zu entwickeln, welche auch in weniger regulierten Arbeitssituation wie in Tana Toraja anwendbar sind. Bis dahin werde ich mich in den Verhandlungen an meinen Erfahrungen bei Dreharbeiten in anderen Ländern und an dem mir bekannten deutschen Urheberrecht orientieren, unter dem ja meine Filme veröffentlicht werden.

${ }^{29}$ Vgl. die Cross-Cultural Partnership Working Group: http://connected-knowledge.net/ (Zugriff am 17.10.2009). 




\section{Teil 3}

Muster und Motivationen im Verhandeln von Cultural Property 



\title{
Perspektiven der Differenzierung: Multiple Ausdeutungen von traditionellem Wissen indigener Gemeinschaften in WIPO-Verhandlungen
}

\author{
Stefan Groth
}

\section{Einleitung}

Für das Verständnis von Verhandlungen um Cultural Property ist ein Überblick über unterschiedliche Perspektiven auf den Gegenstand - sei es nun traditionelles Wissen, Folklore, traditionelles Kunsthandwerk oder aber auch Weltkulturerbe im Sinne der UNESCO-Konventionen ${ }^{1}$ - unabdingbar. Die Analyse solcher Perspektiven auf internationaler Ebene stellt dabei eine spezifische Konstellation dar, in der sich Akteure und Themen aus verschiedenen räumlich-zeitlichen Kontexten mit je spezifischen soziokulturellen und professionellen Hintergründen verständigen. Eine gemeinsame Terminologieverwendung kann dabei als conditio sine qua non betrachtet werden: ohne grundlegendes geteiltes Verständnis über den Gegenstand und die Probleme, die er in konkreten Verhandlungssituationen impliziert, sind solche Verhandlungen nicht zu denken. Dies heisst jedoch nicht, dass Terminologie in jenen Arenen frei von Kontroversen, eindeutig oder unifunktional ist. Vielmehr liegt bereits in der Terminologieverwendung Ambiguität, die in pragmatischen Strategien (Silverstein 1976:47-48) ihren Ausdruck findet. Konstellationen um Terminologie vor dem Hintergrund unterschiedlicher ideologischer oder strategischer Interpretationen des Gegenstandes können dabei als Perspektiven be-

\footnotetext{
${ }^{1}$ Vgl. Peselmann und Socha, Eggert, Hauser-Schäublin und Klenke in diesem Band.
} 
zeichnet werden. Im vorliegenden Beitrag soll eine Bestandsaufnahme solcher Perspektiven um traditionelles Wissen von indigenen Gemeinschaften und der Verknüpfungen und Wechselwirkungen zwischen ihnen am Beispiel internationaler Verhandlungen der World Intellectual Property Organization (WIPO) geleistet werden.

In seinem wegweisendem Artikel "Diplomacy and Domestic Policy: The Logic of Two-Level Games" schreibt der Politikwissenschaftler Robert D. Putnam:

Domestic politics and international relations are often somehow entangled, but our theories have not yet sorted out the puzzling tangle. It is fruitless to debate whether domestic politics really determine international relations, or the reverse. The answer to that question is clearly "Both, sometimes." The more interesting questions are "When?" and "How?" (Putnam 1988:427)

Mit diesem Verweis auf die Beziehung zwischen verschiedenen räumlichen Ebenen, die beide jeweils die Sphäre des Politischen wie auch sich gegenseitig beeinflussen, stellt Putnam die Frage nach dem Wechselspiel zweier Variablen, die für die Ethnologie der annähernd letzten beiden Jahrzehnte zentral waren: das „Lokale" und das "Globale“2. Als Axiom in den Politikwissenschaften verweist ihr Zusammenhang darauf, dass die Gestaltung politischer Richtlinien auf nationaler Ebene zeitweise von Entwicklungen auf der internationalen Ebene informiert und beeinflusst wird - und ebenso andersherum. ${ }^{3}$ Als mittlerweile allgegenwärtiges Paradigma in der Ethnologie ist die Beziehung zwischen dem „Lokalen“ und dem „Globalen“ ein Hinweis auf ein verlagertes Verständnis des Forschungsgegenstandes: nicht mehr die Erforschung von lokaler Bevölkerung und deren sozialen Beziehungen und „Kultur“, sondern oftmals vordergründig deren Einbettung in "global flows" (Appadurai 1990, 1996, vgl. in Abgrenzung auch Tsing 2005) wird thematisiert. „Kultur" und deren Träger werden dabei als "moving target” (Welz 1998) konzeptualisiert, die in ihrer "interconnectedness in space" (Hannerz 1996) einer "multi-sited" (Marcus 1995) Methodologie bedürfen. Der globale Turn der Ethnologie bringt trans- und internationale Prozesse, die die lokale Ebene transzendieren, in den Fokus von Ethnographie und Theoriebildung und wirft damit Licht auf die Verknüpfung von lokalen Situationen mit der globalen Sphäre. Neben der Vernetzung und Mobilität von Menschen (vgl. Tsing 2005, Ong und Collier 2004, Clarke 2004, Welz 2004) ist auch die Zirkulation von kulturellen Artefakten Forschungsthema (vgl. Appaduari 1986, Brown 2003, Marcus und Myers 1995, Myers 2001, 2002). Ganz ähnlich dem obenstehenden Zitat von Putnam ist dabei ebenso in der Ethnologie die interessantere Frage die nach dem „Wann“ und

\footnotetext{
2 Für einen frühen Überblick über die (vornehmlich US-amerikanischen) ethnologischen Diskussionen um die Beziehung dieser beiden Variablen siehe Kearney 1995.

3 Putnam illustriert dies am Beispiel der Ergebnisse des G7-Gipfels in Bonn 1978 und gleichzeitiger innenpolitischer Entscheidungen als Reaktion auf die Erste Ölkrise (Putnam 1988:1-2).
} 
„Wie“ der Verschränkung beider Variablen. Wie sind zum Beispiel internationale Diskurse um Menschrechte von Normen und Praktiken auf nationaler und lokaler Ebene beeinflusst, und wann ist es für Akteure auf der nationalen und lokalen Ebene ratsam, sich auf solche internationalen Diskurse zu beziehen, und wann nicht (vgl. Schneider 2009b)? Und: wie und wann treffen im Konstituierungsprozess von Cultural Property die lokale Produktion kultureller Artefakte und internationale Zertifizierungs- und Reglementierungspraxen aufeinander (vgl. Peselmann und Socha in diesem Band)?

Die Erforschung von internationalen Organisationen ist dabei ein wesentlicher Aspekt ethnologischer Forschung der sich mit dieser Verschränkung beschäftigt. In solchen internationalen Arenen verhandeln zum einen NGOs und indigene Gruppen globale Probleme und bringen Argumente, Dokumente und Rhetoriken aus nationalen und lokalen Ebenen mit ein; zum anderen beziehen sich Diplomaten und civil servants auf lokale Erfahrungen und stimmen Richtlinien und politische Agenden auf nationale und organisationale Interessen ab. Global flows verdichten sich in internationalen Institutionen und wirken von dort zurück auf lokale, nationale und regionale Zusammenhänge. Durch die Zusammenkunft von lokalen Aktivisten, offiziellen Staatsvertretern und zivilgesellschaftlichen Organisationen in Verhandlungen über lokale Anliegen und globale Probleme sind solche Institutionen ein wesentlicher Ansatzpunkt für die ethnologische Erforschung globaler Prozesse.

Um nun den ethnologischen Paradigmenwechsel mit Putnam zu verknüpfen muss eine Analyse solcher Institutionen und Prozesse sowohl der Multiplizität von Akteuren und Anliegen gerecht werden, wie sie in der Verdichtung globaler Diskurse zu finden sind, als auch die Beziehung zwischen verschiedenen Ebenen der politischen Willensbildung - bei Putnam domestic politics und international relations; aus ethnologischer Sicht aber auch lokale und regionale Arenen - berücksichtigen. Multiperspektivität im Kontext internationaler Institutionen ist damit nie alleine eine Frage der spezifischen Akteure und ihrer Intentionen wie Interpretationen, sondern bedeutet immer auch die Vermittlung zwischen verschiedenen Ebenen. Sowohl rechtliche, institutionelle oder sprachliche Rahmenbedingungen der jeweiligen Bezugssysteme als auch die konkreten Aushandlungspraxen am spezifischen Gegenstand haben Einfluss auf die Art und Weise, wie, über was, und mit welchem Ausgang verhandelt wird. In der Konvergenz verschiedener Ebenen und Akteure liegt dabei zum einen eine hohe Komplexität, die bisweilen nur schwer zu durchschauen ist. Betrachtet wird nicht lediglich ein einzelnes Bezugssystem - so zum Beispiel ein nationaler Diskurs einschließlich seiner spezifischen Rahmenbedingungen und Akteure -, sondern das Aufeinandertreffen einer Vielzahl von Diskursen. Zum anderen bietet diese Komplexität aber auch die Möglichkeit, Positionen und Strategien zu kartieren - ohne zunächst den Anspruch zu stellen, jeweils die exakte Genese jener bestimmen, geschweige denn, aus der beobachtbaren Praxis die genauen Intentionen (und intentionalen Konstellationen) der Akteure ableiten zu können. 
Hilfreich ist dieses kartierende Vorgehen beispielsweise bei der Analyse von kontingenter Terminologie: wie und in welchen changierenden Kontexten werden bestimmte Begriffe gebraucht, und was impliziert der je spezifische kontextualisierte Gebrauch von Begriffen? Gibt es bei der Ausdeutung von Terminologie multiple Möglichkeiten, oder hat Terminologie eindeutige Signifikanten? Kann die Kontingenz von Terminologie strategisch gefüllt und damit in variablen Interessenkonstellationen intentional genutzt werden? In der Rechtslinguistik sind solche Fragen beispielsweise unter den Aspekten „Vagheit” und „Ambiguität“ verhandelt worden (Bhatia et al. 2005, Hutton 2009), wobei hier vor allem ein Fokus auf die pragmatistische Dimension des Umgangs mit solchen Unsicherheiten in der konkreten juristischen Auslegung gelegt wurde (Münch 1995). Dabei wurden zwar auch transkulturelle (Bhatia et al. 2008) und mehrsprachige (Hilf 1973, Jansen 1999, Luttermann 1999, Triebel 2004) Kontexte in den Blick genommen, im Vordergrund standen aber zumeist Fragen der Rechtssicherheit und Auslegungsverfahren. Vernachlässigt wurden hingegen die strategischen und ideologischen Implikationen kontingenter (und damit mehrdeutiger) Terminologie in verschiedenen soziokulturellen und politischen Kontexten. Nennenswerte Ausnahmen liegen im Bereich der Rechtssemiotik - so beispielsweise Alan Audis "A Semiotics of Cultural Property Argument” (2007) - in denen neben formalen Betrachtungen eine Re-Kontextualisierung von Begriffen in linguistische und ideologische Systeme vollzogen wird, die sowohl eine Verortung als auch eine Bewertung von Terminologie in Bezug auf ihre soziale und kulturelle Eingebettetheit (embeddedness) gestattet. Der Blick auf die Eingebettetheit von transnationalen Entscheidungsprozessen und ihrer Terminologie in lokal, national, regional und global situierte Wert- und Bewertungssysteme gestattet dabei die Rekonstruktion der Perspektivbildung auf solche Prozesse und ihre Themen. So stellen Garsten und Hasselström für das Beispiel transnationaler Finanzmärkte heraus, dass

[...] transnational financial trading and the positioning of corporations as socially responsible actors are fields that merit anthropological attention for several reasons. [...] the ideas and actions of financial traders and corporate leaders contribute in significant ways to the structuring of market transactions across the world. [...] they contribute to the diffusion of perspectives on markets and market actors and influence our understandings of the scope of individual action in market transactions. All in all, we argue, they play a vital role in the development and organization of contemporary markets. (2003:250)

Über die konkrete Sphäre der Praxis hinaus wirken die von Akteuren, ihren Ideen und Handlungen fabrizierten Perspektiven auf ein Objekt $\square$ auf dessen Rezeption und Konstituierung ein und rekontextualisieren es in soziokulturelle Systeme. Damit einher geht eine Naturalisierung solcher Diskurse - einschließlich der ihnen eigenen Terminologien -, die ob ihrer Eingebettetheit als unpolitisch und gegeben konstruiert werden (vgl. beispielsweise Barthes 1972:128, Hill 2008:154; für die 
Debatte um Cultural Property Audi 2007:142, Aragon und Leach 2008). Die Einbettung transnationaler politischer und ökonomischer Prozesse in moralische Diskurse kann über eine Naturalisierung hinaus zudem zur Wertaufladung bestimmter Praxen und somit zu einer (De-)Legitimierung führen (vgl. Beckert 2005, 2007), die wiederum geknüpft ist an die für die Diskurse je adäquate Rhetorik. Terminologie erfüllt damit in internationalen Arenen nicht lediglich eine definitorische Rolle, sondern verweist auf ideologische Perspektiven, die Themen und Positionen in spezifische soziale und kulturelle Kontexte einbetten und somit einen Einfluss auf deren Konzeptualisierung haben.

Eine Bestandsaufnahme multipler Perspektiven in internationalen Verhandlungen, die die soziokulturelle Dimension von kontingenter Terminologie in die Analyse miteinbezieht, bietet die Möglichkeit über die Feststellung der Differenz im Terminologiegebrauch hinaus ideologische und strategische Verankerungen ${ }^{4}$ von Begriffen herauszuarbeiten. Am Beispiel des Intergovernmental Committee on Intellectual Property and Genetic Resources, Traditonal Knowlegde and Tradtional Cultural Expressions (IGC) der WIPO (eine der 16 specialized agencies der Vereinten Nationen) sollen in diesem Artikel die verschiedenen Perspektiven auf indigene Gemeinschaften (indigenous communities) ${ }^{5}$ und deren traditionelles Wissen (traditional knowledge) als terminologische Konstellationen der Differenzierung in dieser internationalen Arena untersucht werden. Im Sinne einer Kartierung von Perspektiven stellt dieses Vorgehen einen ersten Schritt zur Analyse von Ideologien über traditionelles Wissen dar, das für ein Verständnis von strategischer und intentionaler Terminologieverwendung - und damit auch für ein Verständnis unterschiedlicher Konzeptionen von Cultural Property - unabdingbar ist.

\section{Das Feld: WIPOs Komitee zu Geistigem Eigentum und Traditionellem Wissen}

Das IGC der WIPO bringt 184 Nationalstaaten ${ }^{6}$ und eine stetig wachsende Zahl von NGOs und indigenous and local communities (ILCs) zusammen. Im Vordergrund

\footnotetext{
${ }^{4}$ Das Konzept des anchoring von Terminologie in ideologischen Regimen als semiotischer Prozess geht auf Irvine und Gal (2000) zurück. Dabei werden beispielsweise Ideologien der Differenzierung über verschiedene Rekursionsstufen (fractal recursivity) und Löschprozesse (erasure) zu ihrem Ursprung - ihrer tatsächlichen Verankerung in soziokulturellen Kontexten - zurückverfolgt (Gal 2005:24-27), was direkte Fehlschlüsse auf Intention aus Performanz zu vermeiden hilft. Diese Rückverfolgung kann in diesem Artikel nicht geleistet werden, da zunächst existierende Perspektiven kartiert werden müssen, die erst in einem weiterem Schritt auf ihre Verankerung hin untersucht werden können.

${ }^{5}$ Für die vorwiegend negativen Konnotationen des community-Begriffes (und auch des Begriffes der Gemeinschaft) siehe Noyes 2006 und weiter unten. In den untersuchten Verhandlungen wird er verwendet - im Prinzip ein weiteres Beispiel der intentionalen Terminologieverwendung - und wird hier entsprechend (in Übersetzung) beibehalten.

${ }^{6}$ Für einen Überblick über die Mitgliedsländer der WIPO siehe http://www.wipo.int/members/en (letzter Zugriff am 17.04.2010). Für einen Überblick über die für den IGC-Prozess akkreditierten
} 
der Verhandlungen steht dabei die Diskussion über die Möglichkeit und Erwünschtheit der Entwicklung eines internationalen Rechtsinstrumentes für den Schutz von immateriellen kulturellen Ressourcen im Rahmen des Systems des geistigen Eigentums (intellectual property). Der Gründung des Komitees ging ein gesteigerter Druck von Entwicklungsländern und indigenen Gruppen innerhalb der WIPO und des Systems der Vereinten Nationen voraus. ${ }^{7}$ Deren Unzufriedenheit mit der Handhabung von „Kultur“8 im Rahmen internationaler Intellectual Property-Gesetzgebung lag beispielsweise in Konflikten mit pharmazeutischen Konzernen begründet, die aus traditionellem Wissen über Heilpflanzen Kapital schlugen; einen weiteren Streitpunkt stellte die unauthorisierte Entwendung von indigenen Kunstwerken oder traditionellen kulturellen Ausdrucksformen für kommerzielle Zwecke dar. ${ }^{9}$ Die Erwartungen der Mitgliedsstaaten des WIPO IGCs und der zahlreichen Beobachter bezüglich der Ergebnisse dieser Unternehmung könnten unterschiedlicher nicht sein: westliche Industrienationen sind mit der Ausgestaltung des Patent- und Copyrightregimes weitestgehend zufrieden und an einer Änderung oder der Schaffung eines neuen Rechtsinstruments für geistiges Eigentum wenig interessiert, da das derzeitige System zu ihrem Vorteil arbeitet - die „klassischen" Patent- und Urheberrechtsbestände sind auch über die nationalen Grenzen hinweg geschützt. Für die im IGC diskutierten Ressourcen sieht dies jedoch anders aus. Gegen die grenzübergreifende widerrechtliche Aneignung und Verwendung von kulturellen Ressourcen gibt es zur Zeit kein wirksames Mittel im internationalen Immaterialgüterrecht. Länder mit einem hohen Anteil indigener Bevölkerung und einer großen Bandbreite an traditionellen Wissensbeständen wie Indien, Brasilien, Peru oder viele afrikanische Staaten drängen daher auf die Einrichtung eines rechtsverbindlichen Übereinkommens auf der internationalen Ebene, das die Träger dieser Wissensbestände schützt oder entschädigt.

Die Zusammensetzung des IGC aus Teilnehmern mit einer Vielzahl von nationalen, soziokulturellen, professionellen und linguistischen Hintergründen führt dabei nicht nur zu einer Vielzahl unterschiedlicher strategischer Agenden, sondern auch zu einer großen Anzahl divergenter Verständnisse der für diesen Komplex grundlegenden Konzepte von „Kultur“, „Eigentum“ oder „Gemeinschaft“. Während die zahlreichen NGOs und indigenen Gemeinschaften zwar nur als Beobachter zugelassen sind und kein Wahlrecht in den Verhandlungen haben, so ist ihnen doch gestattet, an den offiziellen Diskussionen teilzunehmen und ihre Ansichten in den Prozess mit einzubringen. Dies führt zum einen zu einer Multiplikation von Perspektiven auf den verhandelten Gegenstand (der dadurch freilich nicht eindeu-

adhoc observer siehe http://www.wipo.int/export/sites/www/tk/en/igc/ngo/accreditedlist.pdf (letzter Zugriff am 17.04.2010).

7 Vgl. Hafstein 2004:312, Wendland 2002a, 2002b; für eine breitere Sicht auf traditionelles Wissen im globalen Diskurs über geistiges Eigentum Dutfield 2008:327-355.

${ }^{8}$ Für eine Diskussion über verschiedene Kultur- und Traditionsbegriffe in solchen Kontexten vergleiche Carneiro da Cunha 2009, Groth 2009.

${ }^{9}$ Für weitere Beispiele siehe Brown 2003, Janke 2003. 
tiger wird) und in der Konsequenz zum anderen dazu, dass es bezüglich der gebrauchten Terminologie und ihrer spezifischen Ausfüllung nur wenig geteiltes Verständnis zwischen den verschiedenen Akteuren gibt. Besonders deutlich wird das durch die Tatsache, dass es, nach fast einem Jahrzehnt IGC-Verhandlungen, immer noch keine konsensuale und verbindliche Definition der Begriffe "traditional knowledge" und "traditional cultural expressions" gibt".

Das IGC ist eine Momentaufnahme eines global flows: als Teil eines größeren globalen Prozesses - unter anderem bestehend aus Debatten über den Schutz, die Erhaltung oder Kommerzialisierung von (indigener) Kultur, die Restrukturierung des Systems des geistigen Eigentums oder den anhaltenden Konflikt zwischen dem Globalen Norden und dem Globalen Süden - erlaubt es die Nachverfolgung von Perspektiven auf den verhandelten Gegenstand sowie auf inhaltlich wie strategisch angrenzende Themengebiete. In diesem Artikel soll eine Bestandsaufnahme solcher Perspektiven um traditionelles Wissen von indigenen Gemeinschaften und der Verknüpfungen und Wechselwirkungen zwischen ihnen geleistet werden. Es soll gezeigt werden, wie sich im Rahmen des WIPO IGC mithilfe unterschiedlicher Ausdeutungen von Begriffen terminologische Konstellationen konstituieren, denen je spezifische kulturelle und soziale Konzeptionen und ideologische Verzerrungen (distortions; vgl. Silverstein 1979) implizit sind. Diese wiederum können auf unterschiedlichen Ebenen Auswirkungen auf den Verlauf von Verhandlungen sowie auf die Bewertung von verhandelten Themen und Problemen haben.

\section{Perspektiven der Differenzierung}

Im Folgenden sollen nun insgesamt fünf Perspektiven der Differenzierung und Angleichung auf traditionelles Wissen herausgearbeitet werden. Dabei sind zwei Dinge gesondert herauszustellen: die enge Verzahnung der Verwendung der Konzepte traditional knowledge und traditional cultural expressions mit dem Attribut indigenous in den IGC-Verhandlungen - zeitweise wird auch von indigenous knowledge gesprochen - führt in den nachfolgenden Schilderungen unterschiedlicher Perspektiven zu einer Verquickung dieser Begriffe. Träger (bearers; vgl. Carneiro da Cunha 2009:9) von traditionellen Wissen sind im IGC-Kontext in der Mehrzahl der Fälle indigene Gemeinschaften. Eine Betrachtung von Perspektiven auf traditionelles Wissen ist damit auch immer zwangsläufig eine Betrachtung des IndigenitätKonzeptes. Dabei soll hier keineswegs der Versuch unternommen werden, der Komplexität der Verwendung oder Bedeutung von Indigenität in Gänze gerecht zu werden ${ }^{11}$. Vielmehr sollen auf Grundlage der konkreten Verwendung bestimmter

\footnotetext{
10 Auf eine solche Definition wird in diesem Artikel ebenso verzichtet, da sie dem hier angestrebten Ziel der Explikation multipler Perspektiven auf einen Gegenstand widersprechen würde, und zudem gerade die Kontingenz von Terminologie - nicht deren definitorische Abgrenzung - zum Thema hat. ${ }^{11}$ Für Überblicke zu Forschungsstand und diskursiver Verwendung des Indigenitäts-Begriffes vergleiche zum Beispiel Lee 2006, Cadena 2007, Niezen 2003.
} 
Terminologie im Rahmen der internationalen Verhandlungen des IGC gezeigt werden, wie sich die Verbindung von traditionellem Wissen mit dem Attribut der Indigenität auf die unterschiedlichen zu skizzierenden Perspektiven auswirkt.

Zum anderen ist bei der Betrachtung von Multiperspektivität - zumal in diplomatischen und damit strategischen Kontexten - die Frage nach der Intentionalität und agency sowohl sprachlicher Äußerungen als auch generell von Verhandlungsstrategien zu stellen. So hat Elizabeth Povinellis Arbeit zu Anerkennungspraxen im Kontext von Indigenität in Australien beispielhaft gezeigt, dass allzu direkte Forderungen dem Interesse von indigenen Gruppen gegenläufig sein können, da sie als nicht „authentisch“ gewertet werden (Povinelli 2002, vgl. zu Fragen der Limitierung qua Anerkennung von Gruppen als indigen auch Trouillot 2003). Neben solchen Diskurs-Limitierungen, die eine allzu offensichtliche strategische Positionierung beispielsweise über den Indigenitäts-Begriff unwahrscheinlich machen, steht der Aspekt der ideologischen Verzerrung im Gebrauch von Terminologie. Intentionen können dadurch nicht direkt aus der Performanz abgeleitet werden, sondern müssen zunächst kontextualisiert und auf etwaige Verzerrungen hin analysiert werden. Während eine Kontextualisierung der extensiven Anreicherung der jeweiligen Perspektiven durch Beobachtungsdaten bedürfte - die hier zunächst hinter die Feststellung solcher Perspektiven zurückgestellt werden soll - würde die Analyse von ideologischen Verzerrungen die theoretische Einbettung in Diskurse um Sprachideologie (vgl. Silverstein 1976, 2004, Briggs 1993, Woolard und Schieffelin 1994, Kroskrity 2000, Schieffelin et al. 1998, van Dijk 1998) voraussetzen, die allerdings den Rahmen dieses Artikels sprengt. Es soll hier deswegen zunächst eine „Bestandsaufnahme“ unterschiedlicher Perspektiven geleistet werden, die notwenige Voraussetzung sowohl für Kontextualisierungen wie auch für damit zusammenhängende theoretische Einbettungen ist.

\subsection{Stigma}

In vielen afrikanischen Ländern ist der Begriff ,indigen“ ein Verweis auf diejenigen Menschen, die „ursprünglich“ an einem bestimmten Ort gelebt haben - er drückt eine zeitliche ${ }^{12}$ Differenz zu etwas oder jemand Anderem aus, eine Dichotomie, die nur mit einem Gegenstück in der Vergangenheit funktional ist. In vielen (aber durchaus nicht allen) Fällen ist dieses Gegenstück der Kolonialismus. Als diskursiver Marker ist das Attribut "indigenous" offen für Interpretation wie auch für

\footnotetext{
12 Kamusella zeigt am Beispiel Europas zudem, dass sich eine solche Differenzierung - vermittelt durch sprachliche Kommunikation oder Sprachpolitik - auch in der räumlichen Imagination vollziehen kann: "When I did research in Vienna in 2005, I ran a small experiment. I asked Austrian, German, and other Western colleagues in the Institute of Human Sciences (Institut für die Wissenschaften vom Menschen) how far Vienna was from Bratislava. The usual guesses were 200 to 500 kilometers. In reality, it is 66 kilometers by car from city center to city center. This clearly shows how much even an educated Austrian or German sees her or his country as part of the West, even to the defiance of actual geography." (Kamusella 2009:2)
} 
strategische und ideologische Aufladungen. Es findet Verwendung in den verschiedensten Kontexten und für unterschiedliche Zwecke. Die Dichotomie zwischen Kolonialismus in der Vergangenheit und soziopolitischen Regimen in der Gegenwart ist dabei nur ein Beispiel, wiewohl ein sehr wirkmächtiges. Damit ist Indigenität ein relationales Konzept:

[S]ocial groups become indigenous or aboriginal or native by virtue of the recognition that someone else arrived in a place and found them or their ancestors 'already' there. (Pratt 2007:398)

Indigenität exisitiert und konstitutiert sich kraft einer Differenz, und die Repräsentation der Differenz existiert und konstituiert sich über Opposition und Abgrenzung. Als semantisches Label verweisen die Pole dieser Dichotomie auf Ausdeutungen und Ideologien, die ihr Bedeutung verleihen, sei es positiv oder negativ. Um also diese verschiedenen potentiellen Bedeutungen zu verstehen, müssen die verschiedenen Konstellationen der Differenzierung zwischen Vergangenheit und Gegenwart untersucht werden.

Auf einer Ebene ist diese Dichotomie zwischen indigen und nicht-indigen eine nationale, eine Instanz von Innenpolitik, indem sie in Beziehung zu Geschichte und ihrer Materialisierung in einem begrenzten Raum, demarkiert auf Karten (wie artifiziell auch immer) steht. Als solche beeinflusst sie soziale Beziehungen oder die institutionalisierten Formen der gegenseitigen Anerkennung (Hegel). Sie gestaltet Machtbeziehungen aus zwischen urbanen Eliten und Trägern von traditionellem Wissen sowie zwischen jedem dazwischen und darüber hinaus. Als politisches Konzept weist diese Dichotomie zurück auf die Vergangenheit um einen Einfluss auf die Gegenwart zu haben und um als politisches Druckmittel Verwendung finden zu können. Eine beachtliche Anzahl von NGOs (innerhalb des IGC, in benachbarten Arenen, sowie auf regionaler und lokaler Ebene), die sich mit diesen Machtbeziehungen und den daraus entstehenden Ungleichheiten auseinandersetzen, ist eine anschauliche Demonstration der Wirkmächtigkeit dieses Konzeptes.

In seiner negativen Konnotation ist „,indigen“ ein Attribut das einer sozialen Gruppe in der Funktion eines Stigmas auferlegt wird: eine Gruppe ist indigen und damit gleichsam rückschrittlich und nicht modern. Seine semantische Nähe und häufige Verknüpfung mit dem Begriff der community13 - die sich in den WIPOVerhandlungen beispielsweise als "indigenous and local communities" wiederfindet - als überholte Form von Sozialstruktur deutet darauf, wie er als negatives ideologisches Residuum der Vormoderne konzeptualisiert wird:

\footnotetext{
13 Der englische Begriff der community wird hier beibehalten, da er sich gegenüber dem deutschen Äquivalent der Gemeinschaft (vgl. zum Stand der soziologischen Debatte um Gemeinschaft und Gesellschaft Opielka 2006) in einigen Nuancen unterscheidet und desweiteren als quasi feststehender Terminus im Rahmen des IGC und in darüberhinausweisenden Diskursen um Cultural Property Verwendung findet.
} 
As a rule, groups represented as "communities" are comparatively isolated, subaltern, and not considered to be viable autonomous collective subjects. Indeed, "community" is in part a euphemism conferring dignity and value on groups in a negative position: it is a verbal gift from the rich to the poor. At the same time, insofar as the label implies a refusal of individualism, it distances its referent from modernity. (Noyes 2006:29)

Indigene Communities werden in dieser terminologischen Konstellation so gedeutet, als distanzierten sie sich von den Versprechungen der Moderne und hingen der Vergangenheit nach ohne die Gegenwart wertzuschätzen und zu ihr beizutragen. Während die Konzepte des Traditionalismus und Konservatismus im Allgemeinen mit der Idee verknüpft sind, dass etwas aus der Vergangenheit verfolgt wird um zum Wohl der Gesellschaft beizutragen, so ist der Begriff der community als in der Vergangenheit verankerter sozialer Akteur häufig mit Konzepten wie Isolation, Subalternität und Reproduktion an Stelle von Innovation verknüpft. Dies trifft insbesondere für den Bereich der kulturellen Kreativität zu. Wie Valdimar Hafstein beobachtet, wurde (und wird) nicht nur der europäischen Landbevölkerung, sondern auch kolonialen Subjekten die Fähigkeit zum künstlerischen Ausdruck abgesprochen: "The subaltern do not produce, they reproduce" (2007:89). In der Konsequenz sind indigene Gemeinschaften in einer paradoxen Situation, da ihre Indigenität Anschuldigung und Hindernis zur gleichen Zeit ist. Als Alter in einer external eingerichteten Position der Differenz werden sie mit Opposition und Abgrenzung konfrontiert. Gleichzeitig ist diese aufgezwungene Abgrenzung aber auch als Vorwurf konstruiert: eine Gruppe ist indigen, und deswegen nicht modern. Daraus folgt zudem der Anspruch Egos, selbst modern zu sein. Als Exklusionsideologie wird hier Alter eine negative soziale Position zugeschrieben, die ihn auf eine andere zeitliche Ebene in der Vergangenheit versetzt (vgl. Fabian 1983). Gleichsam bedeutet dies eine Verorterung von Ego am anderen Pol dieser Dichotomie in der Gegenwart und als Teil der Moderne. Um zur Frage um kulturelle Kreativität zurückzukehren: mit diesem Verortungsprozess und damit auch mit der Zustimmung zum Individualismus geht die Anerkennung von individueller Innovation einher, die Alter zur gleichen Zeit abgesprochen wird:

[...] creativity and originality were the privilege of the bourgeoisie, while the masses were unoriginal and could only transmit the songs and tales of earlier generations. The art of the common people consisted only of copies. (Hafstein 2007:89)

Was Hafstein für das europäische Konzept des originalen Autors im Kontrast zum unoriginalen kommunalen Subjekt beschreibt entspricht der Beziehung zwischen indigenen Communities und ihrem selbsternannten modernen Gegenspieler, zumindest in dieser spezifischen Konstellation. Dies wird zum Beispiel aus einer Passage aus einer Auswahl von Fallstudien deutlich, die als konstitutives Dokument des WIPO IGC Prozesses gesehen werden kann (Janke 2003). Im konkreten 
Fall wurde bei einem australischen Gericht im Jahr 1989 Klage von einem indigenen Künstler gegen einen T-Shirt Hersteller eingereicht, der dessen Kunstwerke ohne Erlaubnis nutzte. Vor diesem Fall wurde, so argumentiert Janke, im Allgemeinen angenommen dass indigene Kunstwerke nicht durch das Copyright geschützt wären:

This assumption considered that Indigenous artworks were not "original" because they are based on traditional creation designs; they are passed on through the generations; and, are not the independent creative effort of the individual artist. (Janke 2003:52)

Der Fall wurde zu Gunsten des indigenen Klägers entschieden, wobei der wesentliche Anteil an individueller Kreativität betont wurde, der in das entsprechende Kunstwerk miteingeflossen war. Dennoch - die zugrunde liegende Auffassung, dass traditional knowledge und folklore den Anforderungen an Originalität nicht genügen findet sich weiterhin in WIPO Verhandlungen wie auch in nationalen Kontexten. Der Einfluss dieser Perspektive der Differenzierung und die Negation von Innovationsfähigkeit auf internationale Verhandlungsprozesse (wie zum Beispiel das IGC), die Übereinkommen über den Schutz von traditionellem Wissen und Folklore anstreben ist dabei dadurch bedeutsam, wie weiter unten noch ausgeführt werden wird.

Die Praxen der Differenzierung die in dieser Konstellation zum Tragen kommen sind dabei nicht auf die internationale und nationale Ebene beschränkt. Solche soziokulturellen Dynamiken sind ebenso lokal wirkmächtig, indem soziale Prozesse auf breitere Narrative und Diskurse rekurrieren. Und, obgleich der Status einer indigenen Gemeinschaft als "viable autonomous collective subjects" (Noyes 2006) temporär und sporadisch aberkannt werden mag, gibt es trotzdem Sozialisation. Gegenseitige Anerkennung ist die Bedingung für Intersubjektivität: um als selbstständiges Subjekt zu existieren, bedarf es der Anerkennung der eigenen Abhängigkeit im Verhältnis zu einem selbstständigen Gegenüber (Hegel 2006:120_ 136). Die Attribution von Andersartigkeit, so abgrenzend sie auch sein mag, ist immer auch die Anerkennung der Verschränkung und Abhängigkeit mit anderen selbstbewussten Subjekten; damit ist die hier aktive Dichotomie eher Fakt als Fiktion oder, um es anders zu formulieren, mehr soziale Praxis als nur Narrativ. ${ }^{14}$

Wie Eigentumsbeziehungen sozial konstituiert sind (das heißt als Prozess der Anerkennung und Abgrenzung von auf ein Objekt gerichteten Begierden), so sind es auch die Praxen der Differenzierung. Sie sind manifest in sozialen Ritualen (im Sinne der Verwendung des Ritualsbegriffes in Silverstein 2004) und beeinflussen als solche kommunikative Praxen in und zwischen sozialen Gruppen. Um ein Beispiel zu geben: im Rahmen eines side events einer IGC-Sitzung im Oktober 2008 wurde ein gemeinsames Pilotprojekt zwischen WIPOs Abteilung um traditionelles

\footnotetext{
14 Wobei die pragmatische Dimension von Narrativen hier nicht unterschlagen, sondern eher der Dimension der direkten sozialen Praxis nachgeordnet werden soll.
} 
Wissen und Folklore, dem American Folklife Center der US-amerikanischen Library of Congress, dem Duke Centre for Documentary Studies, der Maasai Cultural Heritage Foundation und den National Museums of Kenya vorgestellt. Ziel des Projektes ist die Vermittlung der notwendigen Kompetenz zur Dokumentation und Digitalisierung von traditionellem Wissen und Folklore der Maasai-Gemeinschaft und Süd-Kenias ein Musterbeispiel für die sogenannten "capacity building”-Programme, die einen großen Teil der Entwicklungsinitiativen der Vereinten Nationen ausmachen. Während der anschließenden Diskussion brachte eine indigene Delegierte der Samburu-Gemeinschaft Nord- und Zentral-Kenias ihre Bedenken zum Ausdruck, dass mit der Dokumentation des traditionellen Wissens mit dem Ziel eines wie auch immer gearteten Schutzes dieser Bestände andere kulturelle oder indigene Gemeinschaften gesetzlich von ihren Rechten ausgeschlossen oder diskriminiert werden könnten (Aufzeichnungen des Autors, 16.10.2008) - die Maasai und die Samburu haben einen erheblichen Teil kultureller Artefakte und tradtionellen Wissens gemein. Als zum Teil nationale Initiative - die kenianische Regierung ist über die Beteiligung des National Museums of Kenya in das Projekt involviert -, um für solche Ressourcen Schutzmechanismen zu etablieren, würden automatisch andere Ansprüche - zum Beispiel von den Samburu - auf diese angefochten werden. Solche Formen der nationalen oder lokalen Verwicklungen multiplizieren daher Differenz: internationale Prozesse wie die beschriebene gemeinsame Initative distinkter Akteure können zur Wertschätzung einer indigenen Gemeinschaft (und ihrer Ressourcen) und im gleichen Zug zur Abwertung und fortwährenden Stigmatisierung einer anderen indigenen Gemeinschaft führen; Differenz, in diesem Fall, wird ausgeweitet auf eine area of contestation zwischen verschiedenen Interessengruppen, die jeweils an einer Verbesserung nicht nur ihres sozialen, sondern oftmals auch ihres ökonomischen Status interessiert sind: zusätzlich zu den Bedenken, die die Vertreterin der Samburu äußerte, stellte sie auch Nachfragen bezüglich eines möglichen Einbezugs ihrer Gemeinschaft in die Dokumentations- und Preservationsbemühungen des Projektes, da dieser Einbezug höchstwahrscheinlich eine Neubewertung und Stärkung derer Situation bedeuten würde. Dieser Wandel in der Einstellung gegenüber indigenen Gemeinschaften auf nationaler Ebene ist jedoch selektiv und mehrdeutig, wie beispielsweise durch eine Stellungnahme eines Repräsentanten der Maasai Cultural Heritage Foundation während eines WIPO-Panels zu traditionellem Wissen illustriert wird:

The Maasai heritage in all its forms is facing serious problems and challenges. In most circumstances, the indigenous governance systems are not recognised by the Government as most of these cultural practices are considered to be primitive and do contribute to underdevelopment of the Maasai people. (Ole Kaunga 2006:4)

Insofern es als opportun angesehen wird - man denke an Tourismus oder das oben erwähnte internationale Prestige-Projekt - werden indigene Gemeinschaften 
unterstützt; von solchen Fällen abgesehen ist eine Stigmatisierung indigener Gemeinschaften jedoch eher Regel als Ausnahme.

\subsection{Potential}

Zudem ist das hier beschriebene „Stigma der Indigenität“ nicht nur auf individuelle und kommunale Subjekte anwendbar, sondern darüber hinaus auch auf materielle Artefakte (Martínez Novo 2005), biologische Ressourcen (Pilcher 1998) und traditionelles Wissen selbst. Gerade traditionelles Wissen wird häufig als etwas von potientellem Wert konzeptualisiert, das in einem archaischen, irrationalen und mythischen Glaubenssystem „feststeckt“; damit wird es als prämodernes Artefakt konstruiert, das der modernen Wissenschaft oder der rationalen Bewertung unterworfen werden muss, um verwend- und verwertbar zu sein (vgl. Latour 1997). Demgegenüber ist es

[...] not considered so when non Maasai have expropriated and used the same culture and used it for economic gains. The Maasai culture is a resource and it is being used by un-authorized non-Maasai for their own benefits. (Ole Kaunga 2006:4)

Diese Devaluierung - oder eben Inwertsetzung - von traditionellem Wissen geht Hand in Hand mit seinem wahrgenommenen Potential, sei es für Tourismus, die Vermarktung von Kunsthandwerk, Biodiversität oder die Entwicklung von Pharmazeutika. Sein Potential muss zunächst offengelegt werden und zu einem gewissen Grad von seinem ursprünglichen Kontext separiert werden: für den Tourismus ist eine Aura von „Authentizität“" wesentlich, doch es sollte dabei „sauber“ und frei von Konflikten sein (Graburn 1976); für Kunsthandwerk sollte es möglichst standardisiert (und damit kompatibel für Konsumptionsanforderungen) und mit einer positiven Konnotation belegt sein (Chibnik 2003); für technologische und agrikulturelle Innovation muss es in Datenbanken eingepflegt und mit modernem Wissen verknüpft werden (Seleti 2009); für Biodiversität und seine Nutzung als medizinisches Wissen müssen seine Komponenten von störender Folklore getrennt und ins Labor übertragen werden (Hayden 2003). Dieses Stigma des unausgeschöpften Potentials tritt in WIPO-Prozessen in unterschiedlichen Formen auf:

Traditional Medicine is a source of prosperity proper to Oman. However this intellectual asset has so far not been fully exploited, mainly because Omanis are not yet fully aware of the value of the wealth they own. (Ghafele 2005)

Ähnlich der marxistischen Doktrin, das Proletariat müsse von einer proletarischen Elite angeführt werden um sich von den Fesseln der Unterdrückung zu befreien, kann, nach dieser Passage aus WIPO-Verhandlungen um traditonelles Wissen, der „Reichtum“, der in einem archaischen Wissenssystem verborgen liegt, nur dann intellektuell und ökonomisch verwertet werden, indem er wissenschaftlichen Pro- 
zessen außerhalb dieses Systems zugeführt wird. In ähnlicher Diktion verweist der folgende Ausschnitt aus einem WIPO-Report auf das Potential von ägyptischem traditionellem Wissen für die Entwicklung und Produktion von Pharmazeutika:

The (Egyptian, S.G.) Delegation stated that traditional knowledge and its experiences were of paramount importance to many species, particularly to consumers, producers and breeders in general. In addition, the Delegation stressed the importance and potential of traditional knowledge in the field of pharmaceutical production. (WIPO 2001b)

Nicht so sehr der gegenwärtige Gebrauch und die traditionelle Praxis ist es, die den Wert von traditionellem Wissen bestimmt, als vielmehr die Aussicht auf die Möglichkeit der Transformation dieses Wissen um es für "[...] the development of scientifically acceptable products and processes" (Satish 2003) nutzbar zu machen, wie der Abstract zu einem Artikel über das Potential von traditonellem Wissen über den Neem-Baum suggeriert. Das „Stigma der Indigenität“ ist, wird es auf die Sphäre des Wissens ausgeweitet, eine Rhetorik die die Übersetzung von etwas vormodernem und irrationalem in eine Sprache der Wissenschaft und Rationalität (er-)fordert. Damit wird dieses Wissen von seinem Ursprung in indigenen Gemeinschaften getrennt, deren Fähigkeit zur Kreation und Innovation negiert wird (siehe oben). Übersetzung ist in diesem Sinn auch gleichsam Aneignung: durch die Überführung von Wissen aus einem „nutzlosen“ oder „wertlosen“ Status in etwas Verwertbares wird gleichzeitig das Recht zu dieser neuen Form des Wissens erworben. Bioprospecting, also die Patentierung von genetischen Ressourcen die durch die Erforschung von traditionellen kulturellen Ausdrucksformen und dem Gebrauch von Heilpflanzen in traditoneller Medizin entdeckt wurden (Hayden 2003), ist ein pointiertes Beispiel für diese Praxis.

\subsection{Recht}

Aus einer anderen Perspektive betrachtet werden soziale Kämpfe mit Terminologie geführt: wir sind indigen, und deshalb haben wir Rechte. In zahlreichen Instanzen haben indigene Repräsentanten in WIPO Verhandlungen sich auf die Declaration on the Rights of Indigenous Peoples (2007) der Vereinten Nationen bezogen, um ihre Ansprüche als indigene Völker zu legitimieren. Die Perspektiven der Differenzierung sind in diesem Fall umgekehrt: in seiner positiven Konnotation wird das Attribut „indigen“ von sozialen Gruppen als politisches und rechtliches Druckmittel verwendet. Es verweist auf bestehende Regimes auf nationaler und internationaler Ebene, die die Zuerkennung von bestehenden Rechten gewährleisten sollen Rechte auf Eigentum, Rechte auf Land, und Menschenrechte. Um Zugang zu diesen Rechten zu erlangen bedarf es der Verknüpfung mit deren entsprechenden Diskursen. Für indigene Gemeinschaften und NGOs gibt es im Rahmen des Systems der Vereinten Nationen verschiedene Möglichkeiten, um ein Anliegen zu rahmen. So haben zum Beispiel UNESCO-Prozesse häufig Sprache - oder viel- 
mehr bedrohte Sprachen - als distinktes Anzeichen herausgehoben, um zu bestimmen ob eine Gruppe indigen ist und Schutzmaßnahmen „verdient“ oder nicht. NGOs oder indigene Gemeinschaften wären daher gut beraten, in solchen Prozessen ihre Anliegen aus der Perspektive einer (bedrohten) Sprachgemeinschaft zu formulieren (Toivanen 2007). WIPO hingegen beschäftigt sich zuvorderst mit geistigem Eigentum, das IGC mit Rechten an geistigem Eigentum im Kontext von traditionellem Wissen. Daher wäre es ratsam, den Aspekt der Sprache hintanzustellen und den Fokus auf traditionelles Wissen und Folklore zu legen. Unterschiedliche organisationale Kontexte oder Konstellationen erfordern die unterschiedliche und je an diese Kontexte und Konstellationen angepasste Rahmung von Anliegen, in gewissem Sinne eine Übersetzung der eigenen Gruppe oder Gemeinschaft und derer Interessen in die jeweiligen Rahmenbedingungen der Organisationen und Prozesse. Zudem besteht ein Versuch verschiedener indigener Gemeinschaften im WIPO IGC-Prozess darin, "to bring human rights language into the IGC" (Aufzeichnungen des Autors, Juli 2009); also Anliegen und Interessen um traditonelles Wissen als Anliegen um Menschenrechte zu rahmen und zu übersetzen, um so von internationalen Gremien, die sich mit Menschenrechten beschäftigen, anerkannt zu werden und damit den Druck auf solche Parteien im IGC-Prozess zu erhöhen, die kein Interesse an substantiellen Ergebnissen haben. Ein Einklinken in solche Diskurse ist dabei jedoch nicht auf die internationale Ebene beschränkt. Globale Diskurse um Rechte sind verwoben mit lokalen Praxen: internationale Prozesse werden in nationalen Verhandlungen referenziert, indem nationale legislative oder administrative Gremien mit Konventionen aus dem System der Vereinten Nationen konfrontiert werden. Während also Indigenität als dichotomisierende Markierung Verwendung findet, kann sie zur gleichen Zeit auch als Druckmittel gebraucht werden. Die Terminologie, die zur Stigmatisierung einer sozialen Gruppe dienen kann, lässt sich in einer umgekehrten Verwendung durch die Übersetzung von einer negativen Perspektive in eine Konstellation, in der ihr eine positive (oder produktive) Konnotation zukommt, benutzen. Die Voraussetzungen für diesen Übersetzungsprozess ist dabei ein Wissen über verschiedene Rechtsdiskurse und Konstellationen der Differenzierung sowie die Kompetenz, eigene Anliegen so zu formulieren, dass sie zu den jeweiligen Diskursen und Konstellationen „,passen“.

\section{Perspektiven der Gemeinsamkeit}

Bislang wurde in diesem Artikel ein Blick auf drei verschiedene Perspektiven auf traditionelles Wissen geworfen: Stigma, Potential und Recht. Sie alle betonen - in unterschiedlichen Ausprägungen - Differenz. Als Stigma werden indigene Gemeinschaften abgewertet, während ihre Antonyme positiv aufgewertet werden. Als Potential wird ihr traditionelles Wissen entwertet und von „verwertbarem“ Wissen getrennt, solange es nicht übersetzt worden ist. Und letztlich wird aus der Perspek- 
tive eines Rechts von indigenen Gemeinschaften auf ihre Differenz verwiesen, um Zugriff auf Diskurse zu erlangen, die ihnen Rechte garantieren können.

\subsection{Einheit}

Darüber hinaus gibt es jedoch auch Perspektiven auf traditionelles Wissen indigener Gemeinschaften, die auf nationaler Ebene Gemeinsamkeiten unterstreichen und Differenz externalisieren: während die Declaration on the Rights of Indigenous Peoples (2007) keine Definition des Begriffes „indigen“ enthält (ebensowenig das Mandat des United Nations Permanent Forum on Indigenous Issues, UNPFII), fokussieren aktuellere und kritische Annäherungen an den Begriff die soziale und ökonomische Marginalisierung einer Gruppe von Personen (Lee 2006, Cadena 2007). Aus einer Perspektive der „,nationalen Einheit“ ist eine solche Konzeptualisierung von Indigenität problematisch: Prinzipien der nationalen Souveränität und innenpolitische Diskurse würden in aller Wahrscheinlichkeit den einer solchen Marginalität, die einer Gruppe aufgrund ihres ethnischen oder sozialen Hintergrundes aufgezwungen wird, impliziten Vorwurf des Rassismus abweisen. Eine solche Perspektive würde nationalen Identitätsbildungsprozessen sowie Bemühungen um nationale Einheit widersprechen (vgl. Anderson 1983). Wiederum ist der Abwehr gegen ein solches politisches Konzept, wie er beispielsweise in WIPOs IGC von NGOs und indigenen Gemeinschaften zum Ausdruck gebracht werden, eine anschauliche Demonstration seiner Wirkmacht.

Eine Möglichkeit, um diese ungewollte Dichotomie „aufzulösen“ ist die Externalisierung von Differenz. Der Begriff ,indigen“ ist, wie weiter oben gezeigt wurde, eine zeitliche Differenz zu etwas anderem, eine Dichotomie die nur funktional ist, wenn sie ein Gegenstück in der Vergangenheit hat. Referenzpunkte können hier konkrete historische Ereignisse (wie nationale Unabhängigkeit) und, genereller, kolonialistische Phasen sein. Dabei wird der Kolonialismus als geteilte Erfahrung genutzt, als nationale Erinnerung, die in ihrer vereinigenden Konnotation zeitweise soziale Unterschiede überbrückt. Susan U. Philips Analyse von Sprachideologien vor tongaischen Gerichten (2000) ist ein Beispiel, wie linguistische Regime und Terminologie genutzt werden, um eine nationale Identität herzustellen die über Referenz auf die Vergangenheit soziale Stratifikation vorübergehend verschleiert:

[...] they are invoking a relationship that establishes continuity between past and present political regimes. The distinctive features of the Tongan sister-brother relationship are viewed by Togans as having existed prior to European contact $[\ldots](254)$

Terminologie wird benutzt, um eine nationale Identität zu konstruieren, die sich um Differenz zu externalisieren - auf die Vergangenheit bezieht: (nationale) Einheit wird möglich, da der negative Pol der Dichotomie jemand oder etwas anderes ist. Damit weicht die soziale Stratifikation einer imaginierten nationalen und egali- 
tären Identität. Die Rede von Zimbabwes Präsident Robert Mugabe, die Feststellung nämlich, dass " $[\mathrm{t}]$ he white man is not indigenous to Africa. Africa is for Africans. Zimbabwe is for Zimbabweans”, (CBC 2000) illustriert zudem das gewaltsame Potential dieses Konzeptes (vgl. Anderson 1983). Während der Aufstände gegen weiße Farmer in Zimbabwe in 2000 gehalten, wird hier Kolonialismus von Mugabe nicht lediglich als Markierung in der Vergangenheit, sondern auch als politisches und ideologisches Werkzeug in der Gegenwart genutzt. Der Indigenisation and Economic Empowerment Act, ein 2007 verabschiedetes und mittlerweile in Kraft getretenes - wenn auch derzeit nicht konsequent durchgesetztes (The Economist 2010) - Gesetz, das unter anderem vorsieht, dass 51 Prozent aller ausländischer oder von Weißen besessenen Unternehmen auf “indigenous Zimbabweans" übertragen werden müssen, stellt eine Fortsetzung dieser Politik dar, die über eine Referenz auf die Vergangenheit (im spezifischen Fall gar auf ein bestimmtes Datum, den Unabhängigkeitstag) Exklusionspraktiken über den Marker der Indigenität nicht nur terminologisch konstituiert, sondern pragmatisch aktualisiert. Dem Gesetz zu Grunde liegt dabei folgende Definition:

"[] ndigenous Zimbabwean" means any person who, before the $18^{\text {th }}$ April, 1980, was disadvantaged by unfair discrimination on the grounds of his or her race, and any descendant of such person, and includes any company, association, syndicate or partnership of which indigenous Zimbabweans form the majority of the members or hold the controlling interest. (Indigenisation and Economic Empowerment Act, 1.2, 2007)

Das Ende des Kolonialismus erzeugt diese Wende. Die zeitliche Differenz dieser Dichotomie hat sich damit geändert: wenn der Kolonialismus als negatives Objekt konzeptualisiert wird, als Markierung oder als Schnitt zur Bewertung der Vergangenheit und Gegenwart, dann sind die, die ursprünglich in einer bestimmten Gegend gelebt haben, eine andere Gruppe von Personen, die nun nicht mehr anhand soziokultureller Realitäten, sondern mithilfe einer externalisierten Entität in der Vergangenheit bewertet werden. Jeder, der vor dem Kolonialismus auf dem Land gelebt hat, wird damit indigen - und dies ist eine Aussage, die in der Diplomatie und auf der internationalen Ebene, im besonderen in WIPOs IGC, von Repräsentanten afrikanischer Nationen getroffen wird (Aufzeichnungen des Autors, Februar 2008 und Juli 2009). NGOs und indigene Gemeinschaften fechten diese Konzeption auf internationaler Ebene an: es gibt einen Kampf um Anerkennung (Honneth 1995), da die Interessen von Trägern traditionellen Wissens nicht mit denen nationaler Innen- und Außenpolitik übereinstimmen. Die Monopolosierung der Repräsentation von nationalstaatlichen Interessen ist letztlich eine Fortführung der Differenzierungspraxen der nationalen Ebene. Sie verwehrt indigenen Gemeinschaften das Recht zur Repräsentation und Argumentation ihrer eigenen Interessen und Ansprüche in internationalen Prozessen. Gleichzeitig appropriiert sie das traditionelle Wissen dieser indigenen Gemeinschaften, da ja jeder in Afrika indigen sei. Es wird daher argumentiert, dass die Verwaltung der Rechte um traditionelles 
Wissen nicht in der Hand vereinzelter indigener Gemeinschaften sondern in den Händen der nationalen Regierung liegt. Die Perspektive der nationalen Einheit fungiert damit als politisches Druckmittel von Nationalstaaten in internationalen Verhandlungen wie denen des WIPO IGCs.

\subsection{Gerechtigkeit}

Um diesem Argument mehr Gewicht zu verleihen, wird eine weitere Dichotomie referenziert: der Globale Süden gegen den Globalen Norden, und in Konsequenz dieser Gegenüberstellung die Frage um (transnationale) Gerechtigkeit. Ungleichheiten zwischen entwickelten und Entwicklungsländern werden in zahlreichen (wenn nicht allen) Gremien der Vereinten Nationen unter den unterschiedlichsten Aspekten - Technologietransfer, Patente auf lebenswichtige Medikamente, Bildung und so weiter - verhandelt. Es wird danach gefragt, ob das System der Vereinten Nationen in seiner derzeitigen Ausgestaltung ,gerecht“ im Sinne einer ausgewogenen Repräsentation zwischen Entwicklungsländern und Industrienationen ${ }^{15}$ ist (Gad 2006) und wie es reformiert werden könnte. Die Kombinationen dieser „Teilung“ zwischen Nord und Süd mit den Anliegen indigener Völker und deren Rechten ist dabei besonders gewichtig, werden darin doch die Domänen der ökonomischen/moralischen Rechte und der Menschenrechte miteinander verknüpft. Im Rahmen der WIPO IGC-Verhandlungen wurde von einem Repräsentanten eines Entwicklungslandes die Forderung geäußert, dass das derzeitige System (in diesem Fall das des geistigen Eigentums) in den meisten Fällen den Industrienationen (also dem Norden) zu Gute kam und weiterhin kommt, es jedoch nun Zeit sei "[...] for us to finally get something" (Aufzeichnungen des Autors, Juli 2009). In dieser Konstellation werden die Praxen der Differenzierung auf Ungleichheiten der Vergangenheit und Gegenwart gerichtet und es wird eine (trans-)nationale und regionale Einheit (des Südens) konstruiert um ein stärkeres Argument zu machen. Indigene Gemeinschaften und ihr traditionelles Wissen werden hierbei unter einen der beiden Pole der Dichotomie zwischen Nord und Süd subsummiert und als „Kronzeugen" der ungleichen Behandlung und Ungerechtigkeiten herangezogen, und nicht als autonome kommunale Subjekte betrachtet.

\section{Fazit}

In diesen letzten beiden Perspektiven auf traditionelles Wissen indigener Gemeinschaften - nationale Einheit und Gerechtigkeit - subsummieren die Praxen der Differenzierung vermittels des Staates auf der einen, regionaler und transnationaler Koalitionen in internationalen Verhandlungen auf der anderen Seite, das indigene

15 Der terminologische „Shift“ zwischen den Komponenten -länder und -nationen ist bereits ein Indikator für eine ungleichmäßige Denotation der jeweiligen Entitäten. 
Subjekt und inkorporieren es gegen eine externalisierte Entität in der Vergangenheit (Kolonialismus und nationale Einheit) oder Gegenwart (Globaler Süden versus Globaler Norden und internationale Gerechtigkeit).

Zusammengenommen bestehen die in diesem Beitrag skizzierten Perspektiven aus verschiedenen räumlich-zeitlich und sozio-kulturellen Parametern. Sie alle beziehen sich in unterschiedlicher Ausprägung auf die Vergangenheit und strukturieren die Gegenwart durch die Konstruktion von Dichotomien und differenzierenden Perspektiven. Die Multiplizität von Perspektiven auf traditionelles Wissen indigener Gemeinschaften in internationalen Verhandlungen - hier am Beispiel des WIPO IGC - wird darüber hinaus durch weitere isofunktionale Terminologiefragmente angereichert, die sich beispielsweise auf Konzeptionen von „Eigentum“ (vgl. Carpenter 2009), „Erbe“ (vgl. Bendix 2009b) oder „Wert“ (vgl. Myers 2001, 2004) beziehen. Die Erforschung solcher Verhandlungen im allgemeinen und die Analyse von Konstitutionsprozessen um Cultural Property im besonderen sind auf eine (letztlich ethnographische) Bestandsaufnahme solcher Perspektiven angewiesen, um Kontingenzen und umstrittene Denotationen (Silverstein 2004) in der Begriffsverwendung aufdecken zu können.

Die „Kartierung“ eines solchen semiotischen „Raumes“ muss dabei in einem weiteren Schritt von einer (meta-)pragmatischen Analyse ergänzt werden, die kulturelle oder ideologische Konzeptionen von Sprache und Sozialstruktur auf ihre intersubjektive Verteiltheit in Verhandlungsprozessen überprüfen und somit letztlich auch Intentionalität und (metapragmatische) Strategien über terminologische Konstellationen offenlegen kann. 



\title{
Die verborgene Effektivität minimaler Resultate in internationalen Verhandlungen: Der Fall der WIPO
}

\author{
Matthias Lankau, Kilian Bizer, Zulia Gubaydullina
}

\section{Einleitung}

Stellen Sie sich vor, Sie sitzen in einem Konferenzsaal, in dem sich Delegierte aus mehr als 100 Nationen feierlich versammeln, um einwöchige diplomatische Verhandlungen zu führen. Auf der Agenda steht ein mögliches neues Schutzregime intellektueller kultureller Güter in den Bereichen genetische Ressourcen, traditionelles Wissen und Folklore. Trotz des scheinbar großen Interesses an dieser Thematik wird die knappe Zeit mehr oder weniger dadurch verschwendet, dass die Delegierten vorgefertigte Statements ihrer nationalen Interessen verlesen, die oftmals in erheblichem Gegensatz zueinander stehen. Einige Delegierte bestehen auf einen Schutz ihrer intellektuellen kulturellen Güter vor dem Missbrauch von außen. Andere Länder, die eine große Nachfrage nach diesen kulturellen Gütern haben, sprechen sich dafür aus, diese frei verfügbar zu belassen, was ihnen substantielle ökonomische Vorteile bietet. Diese Pattsituation führt zu einem andauernden Austausch von Interventionen, der keinen Spielraum für substantiellen Fortschritt zu lassen scheint.

Dies ist der Fall des Intergovernmental Committee on Intellectual Property and Genetic Ressources, Traditional Knowledge and Folkelore (IGC) der World Intellectual Property Organization (WIPO) in Genf. Das IGC wurde 2000 per Mandat durch die Generalversammlung der WIPO zum Zweck ergebnisorientierter Verhandlungen eingerichtet. Jedoch schaffte es das Gremium seit dem Beginn der Verhandlungen nicht, sich auf bedeutende Fortschritte einigen zu können. Im Gegenteil, sämtliche Beschlüs- 
se hatten eher minimalen Charakter und nichts deutete darauf hin, dass ein bindender internationaler Vertrag oder ein vergleichbares Instrument in absehbarer Zeit beschlossen werden könne. Als Außenstehender nimmt man diese Verhandlungen deshalb auch als höchst ineffektiv wahr.

Nun wissen jedoch die meisten, die sich mit Internationalen Verhandlungen auskennen, dass der Prozess der Einigung auf einen internationalen Vertrag in den meisten Fällen sehr langsam ist. Trotzdem kann man mit Fug und Recht behaupten, dass der Fall des IGC diesbezüglich außergewöhnlich ist. ${ }^{1}$ Wohl aus diesem Grund hat sich der erst kürzlich ernannte neue Generaldirektor der WIPO, Francis Gurry, auf die Fahnen geschrieben die Effektivität der IGC Verhandlungen zu steigern. In seiner Antrittsrede bezog er sich direkt auf das IGC und mahnte an:

$[\mathrm{I}] \mathrm{t}$ has become apparent that there is a need to recognize explicitly the contribution to human society of collectively generated and maintained innovation and creativity and to protect the artifacts of that innovation and creativity. The Organization has undertaken a long process of discussion and negotiation on the means of meeting this need. I believe that it is time to move this process to concrete outcomes that will see WIPO embrace a broader base of constituents and a more universal mission. (WIPO 2008b)

Hieraus lässt sich schließen, dass nicht nur Außenstehende das Erfordernis konkreter Resultate sehen. Gurrys Ruf nach Effektivität zeigt jedoch deutlich, wie unwahrscheinlich es ist, dass die IGC Verhandlungen jemals zu handfesten Resultaten führen.

Deshalb drängt sich aus ökonomischer Sicht die Frage auf, warum Länder an diesem Prozess überhaupt teilnehmen, wenn er doch kostenintensiv ist. Vorsichtige Schätzungen ergeben, dass ein Land im Durchschnitt direkte Teilnahmekosten von 13.600€ für eine Verhandlungswoche ${ }^{2}$ aufwenden muss. Insgesamt gibt die internationale Gemeinschaft somit 1,6 Mio. $€$ für eine Verhandlungsrunde aus. ${ }^{3}$ Durch eine ökonomische Brille betrachtet, erscheint dies höchst paradox. Würden sich nicht alle Beteiligten besser stellen, wenn sie einfach zu Hause blieben? Die zentrale Frage dieses Beitrags ist demzufolge, warum Länder an internationalen

\footnotetext{
${ }^{1}$ Eigentlich bezieht sich der Begriff Verhandlung nur auf das Interagieren mehrerer Regierungen innerhalb einer diplomatischen Konferenz, in der diese Länder versuchen, einen internationalen Vertrag zu verabschieden. Das IGC könnte somit als vorbereitendes Forum für eine Ministerialkonferenz gesehen werden.

${ }^{2}$ Diese Zahl enthält direkte Personalkosten für eine IGC-Runde, Vor- und Nachbereitungszeit sowie Reise- und Unterbringungskosten. Nicht bestimmbare, indirekte Ministerialkosten sind darin nicht berücksichtigt. Die Berechnung dieser Zahl basiert auf persönlichen Interviews mit Regierungsabgesandten, die an den IGC-Verhandlungen teilgenommen haben.

3 1,43 Mio. $€$ dieses Betrags sind durch eine IGC-Runde entstehende direkte Kosten für alle Mitgliedsländer, inkl. freiwilliger Zuschüsse (67.000 €) für die Reisekosten von Abgesandten aus indigenen Gemeinschaften (siehe (WIPO 2008a). Dem WIPO Generalsekretariat entstehen bei einer IGCRunde ca. 0,18 Mio. € an direkten Kosten für unterstützende Aktivitäten. Diese Zahlen beruhen auf einem persönlichen Interview mit Wend Wendland (WIPO, Head of Traditional Creativity, Cultural Expressions and Cultural Heritage Section, and Deputy Director of The Global IP Issues Division).
} 
Verhandlungen, die ineffektiv erscheinen, überhaupt teilnehmen. Die Autor/innen schlagen hierzu die verborgene Effektivität internationaler Verhandlungen als einen möglichen Erklärungsansatz vor und identifizieren drohende Mandatsverlustkosten als das grundlegende funktionale Prinzip.

\section{Die Effektivität Internationaler Verhandlungen - Eine Literaturübersicht}

In der Theorie wird traditionell angenommen, dass Länder an internationalen Verhandlungen teilnehmen, wenn sie sich durch den entstehenden Vertrag gegenseitig besser stellen können. Es erfolgt also eine Pareto-Verbesserung. Obwohl die exakte Position auf der Pareto-Grenze von der jeweiligen Verhandlungsmacht der Länder beeinflusst werden kann ${ }^{4}$, erzielt jedes Land prinzipiell eine Nutzensteigerung relativ zum Status Quo (Gruber 2000:27-32). Darüber hinaus kann es, so Gruber, jedoch auch Fälle geben, in denen eine Vertragspartei Nutzensteigerungen erfährt und die andere Nutzenverluste, obwohl dies auf den ersten Blick paradox erscheinen mag (2000:38-43). Wenn beispielsweise Land Y den Status Quo unilateral zu einem neuen Status Quo ändern kann, welcher Nutzensteigerungen für Y, jedoch Nutzenverluste für Land X bedeutet, eliminiert Y „Nicht-Kooperation“ als sinnvolle Verhaltensalternative von X. Aus diesem Grund wäre es für X rational, sich einem Vertrag zu unterwerfen, der weniger Verluste bedeutet als der unilateral geänderte Status Quo. Gruber nennt diese Fähigkeit eines Landes „Go-It-AlonePower". 5

Demzufolge wäre die Teilnahme am Verhandlungsprozess dann rational, wenn die ausgehandelte Einigung ${ }^{6}$ in dem Sinne effektiv ist, als dass die teilnehmenden Länder dadurch einen höheren Nutzen als im alten oder unilateral geänderten Status Quo erfahren. Typischerweise wird in Studien, die sich mit der Effektivität des Verhandlungsprozesses an sich beschäftigen, implizit angenommen, dass eine solche wohlfahrtssteigernde Situation erreicht wird. Aus diesem Grund wird die Effektivität des Verhandlungsprozesses mit dem Kriterium der Einigung auf einen Vertrag bewertet, was jedoch andere mögliche Erklärungen der Effektivität vernachlässigt. Wenn beispielsweise die Arbeit internationaler Organisationen beurteilt wird, wird dies meist an diesem traditionellen Kriterium festgemacht. Exemp-

\footnotetext{
${ }^{4}$ Für den Effekt von Verhandlungsmacht auf die Verteilung von Vertragsnutzen siehe beispielsweise Hirschmann (1945), Tollison und Willet (1979) und Hug (2008).

${ }^{5}$ Es wäre auch denkbar, dass Y X mit einem erheblichen Verlust für beide droht und X sich deshalb zur Unterzeichnung eines Abkommens mit geringeren Verlusten entschließt. Dazu muss die Drohung jedoch glaubhaft sein, was laut Gruber (2000:34-38) in der Theorie oft kritisiert wurde. Jedoch identifizieren z.B. Braithwaite und Drahos (2000) militärische und ökonomische Zwänge als einen Hauptmechanismus in internationalen Verhandlungen.

${ }^{6}$ Hierbei ist zu erwähnen, dass Verhandlungsergebnisse neben legal bindenden Verträgen auch noch nicht-bindende normative Instrumente, Resolutionen, Erklärungen, Entscheidungen, Richtlinien oder Modellgesetze enthalten können.
} 
larisch seien hier die Vereinten Nationen (Szasz 1989:915-916, UN 1980), aber auch die WIPO selbst angeführt, in denen das Sekretariat lang andauernde Blockaden in einigen ihrer permanenten Foren angeprangert hat (New 2009).

Aber auch in der wissenschaftlichen Auseinandersetzung wird die Effektivität des Verhandlungsprozesses ausschließlich im Hinblick auf das traditionelle Kriterium der Einigung auf einen Vertrag beschrieben. Hier argumentiert beispielsweise Szasz (1992:42-43), dass der internationale Verhandlungsprozess im höchsten Maße effektiv sei, weil er stets zu Einigungen geführt hat, wenn eine klare Notwendigkeit bestand.

In "Negotiation as a learning process" modelliert Cross Verhandlungen als notwendige Interaktion zwischen verschiedenen Parteien, um eine Einigung zu erzielen (Cross 1977:35-41). Er analysiert dabei Verhandlungen vereinfacht mit Hilfe eines Zwei-Personen-Spiels, in dem nur eine Thematik betrachtet wird, etwa das Aufteilen einer bestimmten Geldsumme zwischen zwei Parteien. Innerhalb dieses Spiels entscheidet sich jeder Spieler für eine Verhandlungsstrategie (S), die eine Funktion der Strategie des Verhandlungspartners (R), und der Unsicherheit über diese Strategie (V) darstellt. Der Spieler wählt die Strategie so, dass der erwartete Gewinn aus den Verhandlungen $\mathrm{U}_{A}\left(\mathrm{~S}_{\mathrm{A}}, \mathrm{S}_{\mathrm{B}}\right)$ maximiert wird. Durch strategische Manöver („Bluffen“) mit überzogenen Payoff-Forderungen und daraus resultierender hoher Unsicherheit (V) bilden die Spieler zu Beginn der Verhandlungen keine akkuraten Erwartungen (R) aus, so dass ihre Strategieentscheidungen demnach auch nicht optimal sind. Während des Verhandlungsprozesses passen die Spieler jedoch ihre Erwartungen $\left(\mathrm{R}_{\mathrm{A}}, \mathrm{R}_{\mathrm{B}}\right)$ und somit auch ihre Strategien auf Grund wahrnehmbarer Signale - zum Beispiel geäußerte Forderungen bezüglich der Geldaufteilung - des jeweils anderen Spielers $\left(r_{A}, r_{B}\right)$ an. Wenn sich die Verhandlungen im weiteren Verlauf dem erwarteten Ende nähern werden die Forderungen immer realistischer, was die Unsicherheit reduziert und den Spielern genauere Annahmen bezüglich der Strategie des Partners erlaubt. Dieser Lernprozess garantiert letztlich eine Einigung, was Cross schlussfolgern lässt, dass der Verhandlungsprozess eine notwendige und effektive Aktivität ist, um sich zu einigen, da er einen gehaltvollen Lernprozess hervorruft.

Sjöstedt, Spector und Zartman (1994:13-16) greifen den Lernaspekt auf und entwickeln ein umfassenderes, jedoch weniger formales Modell eines internationalen Verhandlungsprozesses, was indirekte Erkenntnisse bezüglich deren Effektivität erlaubt. Sie heben besonders hervor, wie wichtig Flexibilität als Voraussetzung ist, um sich als Delegierter von festen Positionen zu lösen und nach Einigung bzw. kooperativer Problemlösung zu streben. Abbildung 1 macht deutlich, wie für Sjöstedt et al. diese Flexibilität durch einen iterativen Anpassungsprozess in zwei Bereichen hervorgerufen wird. Zum einen nennen die Autoren hier das "issue reframing" - also im Verhandlungsverlauf verschiedene Auslegungen der Verhandlungsgegenstände - und zum anderen die iterative Klarstellung der jeweiligen Interessenlagen. Der Verhandlungsgegenstand wird im Laufe der Beratungen deutlicher, was zu einer Anpassung in Bezug auf die Wahrnehmung nationaler Interes- 
sen führt und so wiederum auf den Verhandlungsgegenstand zurückwirkt. Als Resultat dieses gemeinsamen Lernprozesses kommt so möglicherweise eine Lösung zu Stande, die von allen Beteiligten akzeptiert wird, weil sich widersprechende Auslegungen und nationale Interessen annähern. Die Entwicklung dieses Anpassungsprozesses hängt entscheidend von neu in die Verhandlungen eingebrachten Informationen, allerdings auch nationalen Zielen sowie nationalen politischen Debatten ab. Darüber hinaus können auch Macht und Überzeugungstaktiken beeinflussen, wie die Thematiken und Interessen wahrgenommen werden. Somit sehen die Autoren den Verhandlungsprozess als entscheidendes Element, um eine effektive Annäherung der Sichtweisen und letztlich eine Einigung der Beteiligten $\mathrm{zu}$ erzielen. Immer neue Verdeutlichungen von Thematiken und Interessen führen die Beteiligten zu einem gemeinsamen Verständnis der grundlegenden Problematik und helfen bei der Auslotung einer Basis für eine gemeinsame Einigung.

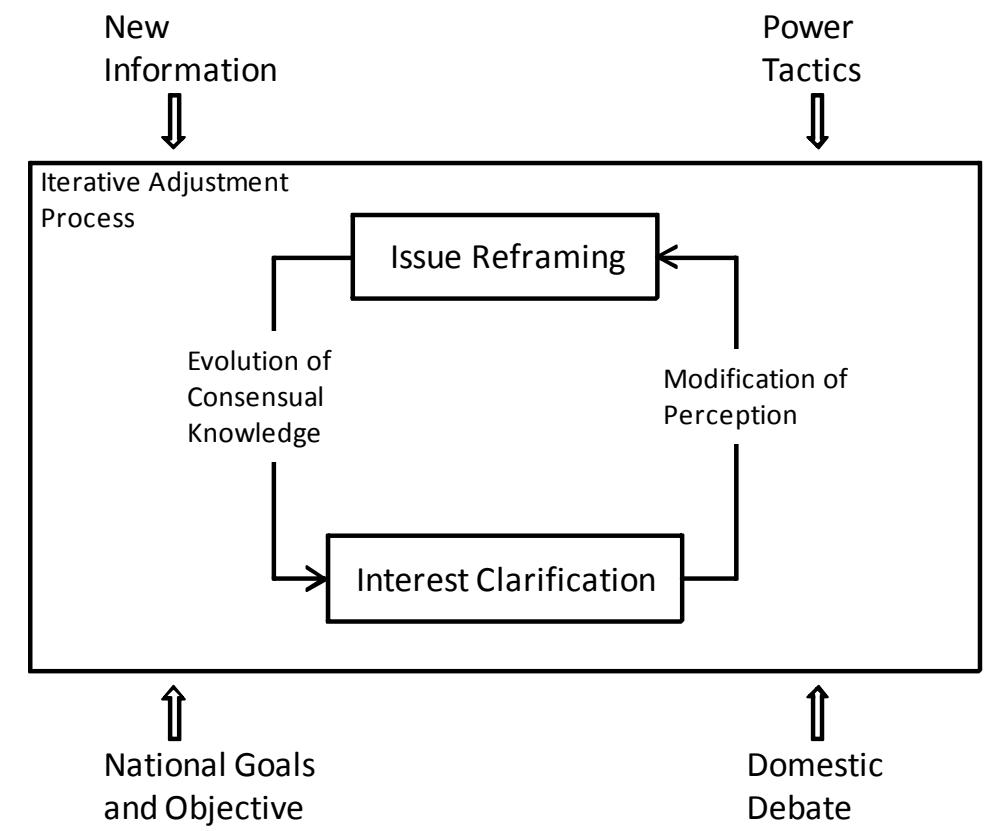

Abbildung 1: Iterative Verhandlungsdynamiken (Sjöstedt et al. 1994:15)

Druckman liefert in seinem Beitrag Erkenntnisse zur Effektivität internationaler Verhandlungen, indem er Wendepunkte zur Erzielung einer Einigung hervorhebt - z.B. Prozessabweichungen, die den Übergang von einem Verhandlungsstadium ins Nächste markieren. In seiner Analyse zeigt sich, dass entscheidende Wendungen in den Bereichen Handel, Umwelt und Politik meist aus dem Prozess selbst heraus ausgelöst werden, was darauf hindeutet, dass Delegierte einen entscheiden- 
den Fortschritt im Hinblick auf eine Einigung durch inhaltliche oder prozedurale Änderungen in den Beratungen erreichen können (Druckman 2001:537-541).

Trotz der Erkenntnisse, die diese Beiträge für die Effektivität im Sinne der Erreichung einer Einigung liefern, können sie doch kaum die Teilnahme an den IGC Verhandlungen erklären. Dies liegt darin begründet, dass das traditionelle Kriterium einer Pareto Verbesserung in diesem Kontext schlichtweg nicht anwendbar scheint. Es drängt sich eher der Verdacht auf, dass die potentiellen Gewinne einer Verhandlungspartei die Verluste der anderen sind. Die theoretische Erklärung der Teilnahme an Verhandlungen dieser Art erscheint somit von vornherein schwierig, da die herkömmlichen Ansätze bisher vernachlässigen, dass der Verhandlungsprozess selbst, unabhängig von den Nutzenauswirkungen eines Vertrages, für jedes Land effektiv sein kann. Dies ist die verborgene Effektivität der Minimal Results in internationalen Verhandlungen.

\section{Das Modell der Minimal Results in der internationalen Gesetzgebung}

Ziel dieses Teilabschnittes ist es, ein ökonomisches Modell zum Verständnis des Verhandlungsprozesses im IGC zu entwickeln, um daraus die entsprechenden Schlüsse zu dessen Effektivität zu ziehen. Basis dieser Analyse ist die Teilnahme der Autor/innen an den IGC Konferenzen 13-15 im Oktober 2008, sowie Juli und Dezember 2009. Dabei sei an dieser Stelle darauf hingewiesen, dass die Autor/innen sich auf die Darstellung der Hauptwirkungszusammenhänge beschränken und von einer mathematisch-detaillierten Darstellung des Verhandlungsprozesses absehen, da dies den Rahmen dieses Beitrags sprengen würde. Zunächst werden in Abschnitt 3.1 die wichtigsten formellen und informellen Regeln, die den Verhandlungsverlauf im IGC steuern, reflektiert. Auf dieser Grundlage kann geschlossen werden, dass das IGC unter konstantem Druck steht, zum Ende des Mandats einen sichtbaren Fortschritt zu erzielen.

\subsection{Formelle und Informelle Verhandlungsregeln im IGC}

Das IGC arbeitet auf Basis eines Mandats, welches von der WIPO Generalversammlung regelmäßig für einen Zeitraum von zwei Jahren beschlossen werden muss (WIPO 2000). Mandate dieser Art werden selten komplett entzogen, jedoch ist es möglich, dass sie für eine gewisse Zeit ausgesetzt werden. Dies kann als Worst-Case-Szenario betrachten werden. Im Allgemeinen streben die Delegierten eine Mandatsverlängerung selbst dann an, wenn sie sich inhaltlich nicht einig sind. Natürlich gibt es kein klares Kriterium für die Verlängerung des Mandats. Wenn jedoch innerhalb des Zweijahreszeitraumes kein Fortschritt erzielt würde, wäre es schwierig zu argumentieren, dass sich dies während einer neuen Mandatsperiode ändern würde. Unzureichender Fortschritt in Verhandlungen kann dazu führen, 
dass die Länder, die an bestimmten Themen besonders interessiert sind, diese in andere existierende bzw. neue Foren auslagern. Daraus resultiert die für diesen Beitrag zentrale Minimal Result Bedingung. Das IGC steht unter ständigem Druck gegen Ende seines Mandats ein Minimal Result zu präsentieren, um eine Mandatsverlängerung zu erwirken.

Die formellen Verhandlungsregeln im IGC sind hauptsächlich in den General Rules of Procedure der WIPO festgelegt (WIPO 1998). Hierbei handelt es sich um prozedurale Richtlinien, die in sämtlichen untergeordneten Gremien oder ad hoc Komitees der WIPO gültig sind. Insgesamt gesehen bestimmen diese Regeln sehr stark den Verhandlungsverlauf, weil sie wichtige Punkte wie Mitgliedschaft, Kompetenzen einzelner Akteure sowie Abstimmungsrechte festlegen. Beispielsweise ist es nur Mitgliedsstaaten des IGC gestattet dem Plenum Vorschläge bzw. Gegenvorschläge zu präsentieren. Diese können sowohl substantieller als auch prozeduraler Art sein, da jedes Untergremium der WIPO spezielle Verfahrensordnungen bestimmen kann (WIPO 2001a). Sollten IGOs, NGOs und andere Beobachter der Verhandlungen Vorschläge machen wollen, müssen sie demzufolge mindestens ein Land gewinnen, welches diese unterstützt und formal ins IGC einbringt. Darüber hinaus genießen nur Mitgliedsstaaten des IGC das Recht abzustimmen, wobei jedes Land über genau eine Stimme verfügt. Im Gegensatz zu der Generalversammlung der WIPO, in der die Mehrzahl der Sachverhalte durch eine Zweidrittelmehrheit beschlossen wird, beschließt das IGC formell gesehen mit einer einfachen Mehrheit der abgegebenen Stimmen. Nichtsdestotrotz werden Entscheidungen faktisch mit Einstimmigkeit getroffen. Chrispeels (1998:132-133) bemerkt hierzu, dass dies in fast jedem multilateralen Verhandlungsforum der Fall ist und der eine-Stimme-pro-Staat-Regel geschuldet ist. Demzufolge finden im IGC faktisch keine Stimmauszählungen statt, und Beschlüsse werden dann gefasst, wenn kein Land interveniert. Darüber hinaus ist im IGC, wie auch in vielen anderen internationalen Gremien, zu beobachten, dass Mitgliedsstaaten sich zu Koalitionen in Ländergruppen zusammenschließen (Behnam 1998). Im IGC existieren im Moment folgende sieben Ländergruppen ${ }^{7}$ : (1) die Afrikanische Gruppe; (2) die Gruppe B, in welcher die Industriestaaten organisiert sind; (3) die Europäische Union; (4) die Asiatische Gruppe; (5) die Gruppe der Osteuropäischen und Baltischen Staaten; (6) die Gruppe der Zentralasiatischen, Kaukasischen und Osteuropäischen Länder sowie (7) die Gruppe der Lateinamerikanischen und Karibischen Staaten (GRULAC).

Auf Basis dieser grundlegenden Verhandlungsregeln soll in den nächsten Abschnitten das Minimal Results Verhandlungsspiel dargestellt werden.

\footnotetext{
${ }^{7}$ Obwohl es sich um keinen Zusammenschluss von Ländern handelt, wird über die hier genannten Ländergruppen hinaus auch China als eine eigene Gruppe gesehen.
} 


\subsection{Modellannahmen}

Eine spieltheoretische Analyse der Verhandlungen im IGC müsste im Prinzip berücksichtigen, dass diese multilateralen sowie multithematischen Charakter haben. Dennoch sind die Autor/innen der Meinung, dass erste Erkenntnisse in Bezug auf die Hauptwirkungszusammenhänge der Verhandlungen auch aus einem Modell zu gewinnen sind, in dem vereinfachende Annahmen getroffen werden.

Hierzu wird als erstes von bilateralen Verhandlungen zu einem einzigen Sachverhalt innerhalb einer Mandatsperiode ausgegangen. Der Sachverhalt kann dabei sowohl inhaltlicher als auch prozeduraler Natur sein und beim Übergang in ein neues Mandat wechseln. Zusätzlich wird davon ausgegangen, dass beide Spieler perfekte Repräsentanten ihrer jeweiligen Länder sind, welche rational agieren, indem sie ihren Verhandlungsnutzen maximieren. Demzufolge haben die Spieler keine persönlichen Motive, an den Verhandlungen teilzunehmen. Land A ist wirtschaftlich schwach, jedoch reich an Cultural Property, welches durch wirtschaftliche Akteure des Landes B ausgebeutet wird. Demzufolge liegt es im Interesse von A durch die Verhandlungen eine Verbesserung des Status Quo zu erzielen, weshalb A als Mover bezeichnet wird. Im Gegensatz dazu ist Land B wirtschaftlich stark. Darüber hinaus überwiegen seine ökonomischen Interessen der Verwertung des Cultural Property aus A seine Interessen, dieses zu schützen. Aus diesem Grund möchte B den Status Quo seines Landes bewahren, weshalb B auch Blocker genannt wird. Folglich wird in diesem Beitrag angenommen, dass sich die eigentlich multilateralen IGC Verhandlungen auf die beiden Parteien reduzieren, deren Interessen in Bezug auf Cultural Property am weitesten auseinander liegen.

Zweitens realisieren beide Länder im Status Quo bestimmte Payoffs (P $\mathrm{SQ}_{\mathrm{A}}^{\mathrm{A}}, \mathrm{P}_{\mathrm{SQ}}^{\mathrm{B}}$; $\mathrm{P}_{\mathrm{SQ}}^{\mathrm{B}}>0$ ). Wie in Abbildung 1 zu sehen, wird dabei von Symmetrie ausgegangen, sodass $\mathrm{P} A \mathrm{SQ}=-\mathrm{P}_{\mathrm{SQ}}^{\mathrm{B}}$ gilt, was zum Ausdruck bringt, dass Bs Aktivitäten im thematischen Bereich der IGC Verhandlungen Gewinne auf Kosten von A generieren. Interpretiert man diese als negative Externalitäten für $A$, ist diese Symmetrie der zentrale Grund weswegen A die Schaffung des Verhandlungsforums gefordert hat. Demzufolge bedeutet ein Payoff von Null in diesem Kontext, dass eine verteilungsgleiche Situation realisiert wäre, in dem kein Land Gewinne auf Kosten des anderen realisiert. Aus diesem Grund ist es in As Interesse, innerhalb der Verhandlungen eine Einigung zu erzielen, die einen Payoff so dicht wie möglich an Null impliziert.

Als nächstes wird das IGC als eines von vielen internationalen Verhandlungsforen definiert. Dadurch wird explizit erlaubt, dass die Spieler auch außerhalb des IGC Verhandlungskontextes Einfluss auf die Position des jeweils anderen ausüben können (Kaufmann 1998).

Viertens liegt es, wie bereits angedeutet, im Interesse der Delegierten, das IGC Mandat zu erhalten. Dies wird durch die Annahme von Mandatsverlustkosten (MVK) formalisiert. Hierbei wird argumentiert, dass wenn es den Verhandlungs- 
partnern nicht gelingen sollte, sich auf ein sichtbares Zeichen des Fortschritts zu einigen, das Mandat nicht verlängert und $\mathrm{B}$ eine Verringerung seines Payoffs $\left(\mathrm{P}_{\mathrm{S}}^{\mathrm{B}}\right)$ durch $\mathrm{MVK}^{\mathrm{B}}$ erfahren würde. Diese Kosten sind durch die folgenden Elemente determiniert:

Zunächst reflektieren die $\mathrm{MVK}^{\mathrm{B}}$ die Verhandlungsmacht von A, also in welchem Ausmaß er B zu Zugeständnissen drängen kann. Diese Verhandlungsmacht kann beispielsweise aus Themenverknüpfungen mit anderen internationalen Foren resultieren (Tollison und Willet 1979). Da das IGC eines von vielen internationalen Foren ist, könnte A etwa Zugeständnisse in anderen Foren, die in Bs Interesse liegen, benutzen, um im IGC Fortschritte zu verlangen. Sollten dagegen keine Fortschritte erzielt werden, könnte A Verhandlungen in anderen Foren ebenfalls blockieren. Sollte A darüber hinaus über die in Abschnitt 2 beschriebene Go-ItAlone-Power verfügen, so wäre er in der Lage, Zugeständnisse von B zu verlangen, die dessen Status Quo reduzierten.

Als nächstes reflektieren die MVK ${ }^{B}$ die Verhandlungsziele von B. Diese können beispielsweise durch normative Sichtweisen auf die internationale Verteilungsgleichheit bestimmt sein, die wiederum von der politischen Aufmerksamkeit in Bezug auf die Verhandlungssachverhalte abhängt (Trumbore 1998). So wäre es mitunter denkbar, dass die Verhandlungsziele von B beeinflusst würden, sollten die Sachverhalte rund um den Schutz von Genetic Resources, Traditional Knowledge und Folklore an innenpolitischem Gewicht gewinnen, was in der Folge B dazu verleiten würde, größere Zugeständnisse zu gewähren (ein Anstieg der $\left.\mathrm{MVK}^{\mathrm{B}}\right)$.

Die Mandatsverlustkosten werden auch noch auf eine andere Weise durch Verhandlungen in anderen internationalen Foren beeinflusst. Diese würden zum Beispiel fallen, sollte ein anderes Forum ein ähnliches Verhandlungsthema aufgreifen, da die Verhandlungen potentiell auch dorthin verlagert werden könnten.

Letztendlich werden die Mandatsverlustkosten von B von einem drohenden Prestigeverlust in der internationalen Gemeinschaft bestimmt, sollte sich das Land zu keinen Zugeständnissen bewegen lassen.

Im Gegensatz zu B wird bei A davon ausgegangen, dass keine Mandatsverlustkosten existieren $\left(\mathrm{MVK}^{\mathrm{B}}=0\right)$. Dies liegt vor allem darin begründet, dass A keine Vergeltung in anderen Foren befürchten muss, sollten die IGC Verhandlungen zum Stillstand kommen. Darüber hinaus ist es unwahrscheinlich, dass A in einem solchen Fall politisches Prestige einbüßen muss oder innerstaatlich abgestraft wird. Das Gravierendste, was A durch einen Abbruch der Verhandlungen passieren könnte, wäre, dass das Land seinen Status Quo Payoff beibehält. ${ }^{8}$ Die resultierende Diskrepanz in den Mandatsverlustkosten versetzt A demzufolge in die Lage, B Zugeständnisse abzuverlangen, da A jederzeit damit drohen kann, Bs Payoffs durch einen Abbruch der Verhandlungen zu reduzieren.

${ }^{8}$ Transaktionskosten, die bei der Schaffung eines neuen Verhandlungsforums entstehen, werden zum jetzigen Zeitpunkt vernachlässigt. 
Nun kann man in diesem Verhandlungskontext jedoch davon ausgehen, dass die aus Bs Mandatsverlustkosten resultierenden Möglichkeiten von A eher gering sind. Dies liegt hauptsächlich in As geringer Verhandlungsmacht begründet. Durch seine wirtschaftliche Schwäche vermag er es nicht, Themenverknüpfungen derart zu gestalten, dass er B substantielle Zugeständnisse abverlangen kann. Auch ist es wahrscheinlich, dass A unzureichende „Go-it-alone-Power“ besitzt. Darüber hinaus wird angenommen, dass der innenpolitische Druck im Land des B sowie die drohenden Kosten durch einen Prestigeverlust zurzeit eher gering sind. Fasst man diese Annahmen zusammen, so stellt man fest, dass die MVKB hier zwar positiv, jedoch verglichen mit dem $\mathrm{P}_{\mathrm{SQ}}^{\mathrm{B}}$ eher gering sind. Diese können jedoch variieren, sollte sich eines der Elemente, aus denen sich die MVK zusammensetzen, ändern.

Abbildung 2 fasst die angenommene Payoffsituation beider Länder im Status Quo sowie die Situation, in der das Mandat ausgesetzt wird, graphisch zusammen. Dabei wird zunächst von konstanten Mandatsverlustkosten ausgegangen.

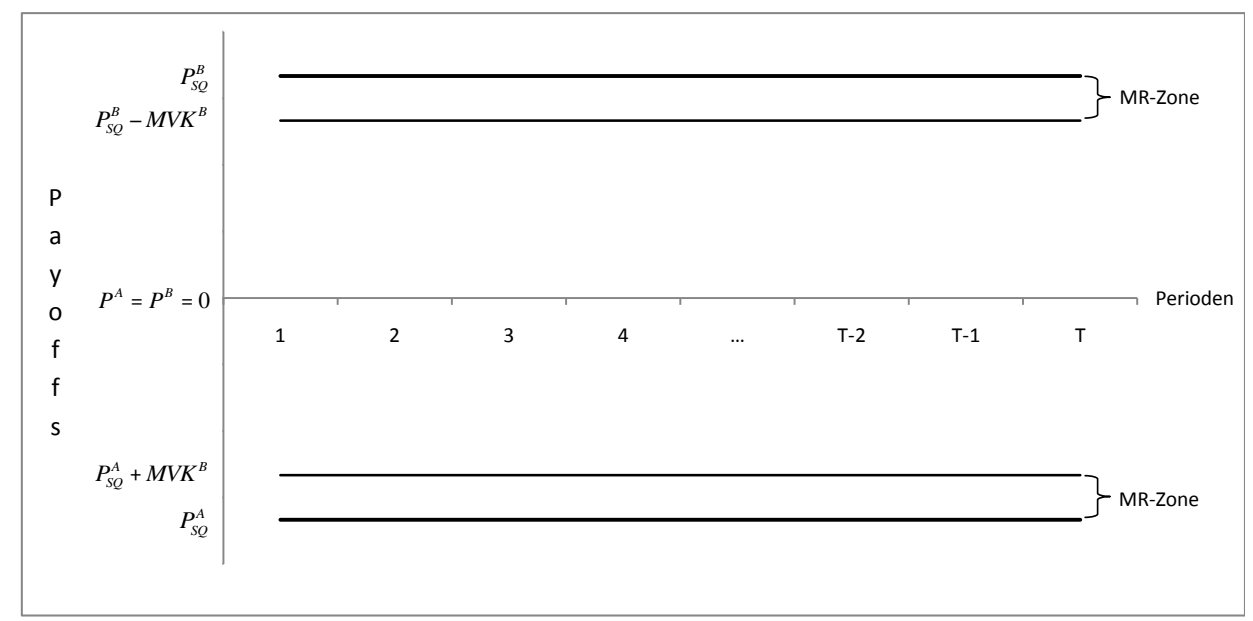

\section{Abbildung 2: Payoffs im Minimal Results Spiel}

Im Rahmen dieses Modells wäre ein Minimal Result dann erreicht, wenn die Verhandlungen in einer erwarteten Verbesserung von As Status Quo resultieren. ${ }^{9}$ Dabei ist es unerheblich, wie schwach diese erwartete Verbesserung wäre. Nun sind jedoch die maximalen Zugeständnisse von B durch die Höhe seiner Mandatsverlustkosten determiniert, da diese auch als seine maximale Zahlungsbereitschaft zur Erhaltung des Mandats interpretiert werden können. Geometrisch gesehen übersetzt sich dies in einen schmalen Korridor, der Minimal Result Zone (MR-

9 Da von einer Symmetrie ausgegangen wird, wäre eine erwartete minimal Verbesserung von $\mathrm{A}$ gleichbedeutend mit einer erwarteten minimalen Verschlechterung von B. 
Zone), innerhalb welcher Einigungen erzielt werden können, die zu einer Verlängerung des Mandats führen.

Während eines Mandats existieren T Verhandlungsperioden, wobei die genaue Anzahl keinem der Spieler bekannt ist. In jeder Periode, die T vorausgeht, können die Spieler Vorschläge machen, die entweder angenommen oder von einem Gegenvorschlag gefolgt werden. Darüber hinaus haben die Spieler immer die Option die Verhandlungen abzubrechen. Das Spiel startet mit einem Vorschlag von A, da es in seinem Interesse ist, den Status Quo zu verbessern. Die Identität des letzten Spielers wird erst in Periode T enthüllt, ohne dass die Spieler darüber Erwartungen bilden könnten. Da T die letzte Periode des Spiels ist, haben die Spieler hier nur die Möglichkeit, den letzten Vorschlag zu akzeptieren oder die Verhandlungen abzubrechen. Abbildung 3 fasst die Handlungsoptionen beider Spieler mit den assoziierten Payoffs graphisch zusammen.

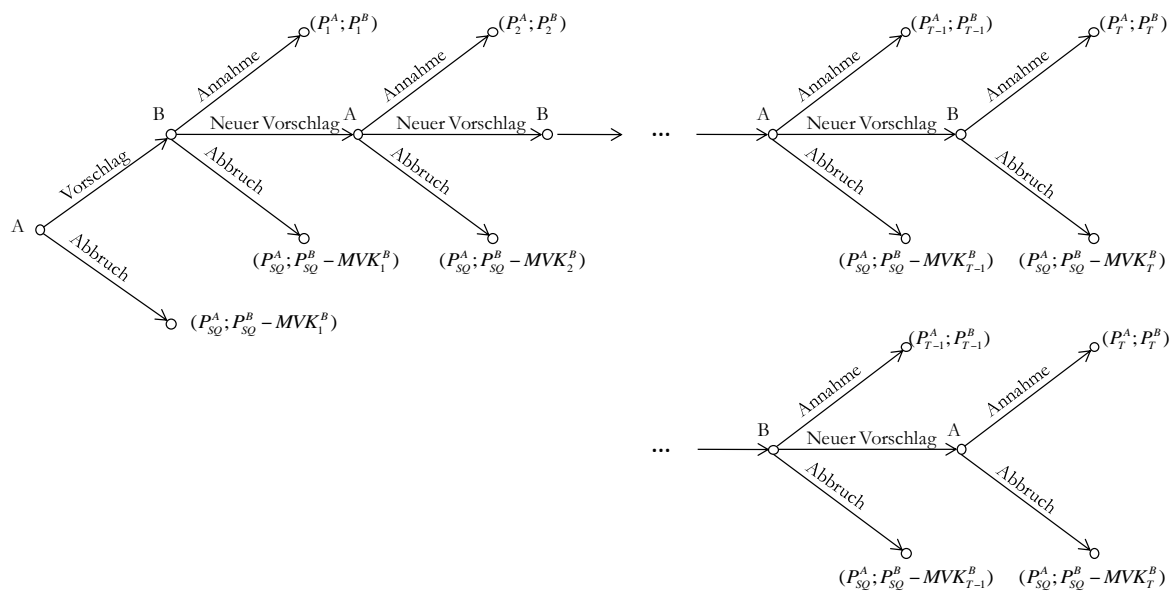

Abbildung 3: Entscheidungsbaum des Minimal Results Spiels

Während beiden Spielern die Payoffs im Status Quo bekannt sind, ist die exakte Höhe der MVK ${ }^{B}$ private Information des B. Des Weiteren wird angenommen, dass den Spielern die konkreten Payoffkonsequenzen ihrer Vorschläge unbekannt sind, weshalb sie ihre Entscheidungen auf Erwartungen ${ }^{10}$ hierüber $\left(\mathrm{P}_{\mathrm{t}}^{\mathrm{A}}, \mathrm{P}_{\mathrm{t}}^{\mathrm{B}}\right)$ stützen. Hierbei wird von Symmetrie ausgegangen, sodass $\mathrm{P}_{\mathrm{t}}^{\mathrm{B}}=-\mathrm{P}_{\mathrm{t}}^{\mathrm{A}}$ gilt. Demzufolge würde ein Vorschlag von A, welcher seinen Status Quo verbessert, in einer symmetrischen Verschlechterung des Status Quo von B resultieren. Zeitpräferenzen werden hier vernachlässigt.

10 Es sei an dieser Stelle darauf hingewiesen, dass das hier verwendete Konzept der PayoffErwartungen sich vom traditionellen Verständnis, also mit ihrer Eintrittswahrscheinlichkeit gewichtete Ereignisse, unterscheidet. In diesem Beitrag wird angenommen, dass den Ländern ihre Payoffs nicht exakt bekannt sind, und sie deshalb Erwartungen bezüglich dieser bilden. 


\subsection{Verhandlungsdynamiken}

Obwohl beide Spieler wissen, dass sie zum Ende des Mandats mindestens einem Minimal Result zustimmen müssen, ist keinem bekannt, wer den letzten Vorschlag macht. Aus diesem Grund kann das Spiel auch nicht durch Rückwärtsinduktion gelöst werden. In der Folge wird gezeigt, wie diese Konstellation die fortschreitenden Verhandlungen bezüglich der erwarteten Vorschlagspayoffs beeinflusst.

\section{Konstante Mandatsverlustkosten}

Zunächst wird angenommen, dass die $\mathrm{MVK}^{\mathrm{B}}$ das Spiel über konstant bleiben, und dass B der letzte Spieler ist. Da beide Spieler das Mandat behalten wollen, starten sie in die Verhandlungen mit dem Ziel Kompromisse zu schließen.

A startet die Verhandlungen, da es im nationalen Interesse liegt seinen $P_{S Q}^{A} z u$ verbessern. Folglich schlägt er mit einem erwarteten Payoff von $\mathrm{P}_{1}^{\mathrm{A}}$ vor. Dieser Vorschlag würde zwar immer noch in einem negativen Payoff resultieren, seinen Status Quo jedoch erheblich verbessern. Aufgrund der Symmetrieannahme würde $\mathrm{B}$ somit $\mathrm{P}_{1}^{\mathrm{B}}$ realisieren, was wiederum eine erhebliche Reduktion seines Status Quo bedeutet. Es ist demzufolge rational, den Vorschlag des A abzulehnen, da die erwarteten Kosten eines Minimal Results in Periode $\mathrm{T}$ geringer wären als die des Vorschlags des A. Der Abbruch der Verhandlungen ist jedoch keine Option, da die erwarteten Kosten des Minimal Results ebenfalls geringer als die Mandatsverlustkosten einzuschätzen sind.

In Periode 2 wird B demzufolge seinen eigenen Vorschlag vorbringen von dem er nur eine infinitesimale Verringerung seines Status Quo $\left(\mathrm{P}_{2}^{\mathrm{B}}=\mathrm{P}_{\mathrm{SQ}}^{\mathrm{B}}-\mu\right)$ erwartet. ${ }^{11}$ Diesen Vorschlag wird A jedoch ablehnen, obwohl er eine leichte Verbesserung bedeuten würde $\left(\mathrm{P}_{2}^{\mathrm{A}}=\mathrm{P}_{\mathrm{SQ}}^{\mathrm{A}}+\mu\right)$, da er im Laufe der Verhandlungen dichter an Bs maximale Zahlungsbereitschaft für das Mandat gelangen kann. In Periode 3 bringt er demzufolge einen Gegenvorschlag $\mathrm{P}_{3}^{\mathrm{A}}$, mit $\mathrm{P}_{3}^{\mathrm{A}}<\mathrm{P}_{1}^{\mathrm{A}}$, welcher demzufolge auch seine Kompromissbereitschaft zum Ausdruck bringt. Dennoch wird B wieder ablehnen, weil As Vorschlag immer noch zu kostenintensiv im Vergleich zu seiner maximalen Zahlungsbereitschaft ist.

Von hier an dürfte klar sein, dass die Regeln des Spiels eine Serie von Vorschlägen und Gegenvorschlägen in Bewegung setzen, durch die beide Spieler Kompromissbereitschaft zeigen. Hierbei ist der Vorschlagspfad auf dem B sukzessive von seinem Status Quo abweicht, klar festgelegt. ${ }^{12}$ In jeder seiner Vorschlagsperioden wird er jeweils nur infinitesimal von seiner vorigen Position abrücken, um so Kooperationsbereitschaft zu signalisieren und A keinen Grund zu liefern, die Verhandlungen abzubrechen. Formal wird B demzufolge in Periode T

\footnotetext{
${ }^{11}$ Dabei kann er nicht mit einer Payofferwartung seines Status Quo vorschlagen, da A stets droht das Spiel abzubrechen, sollte B nicht den Willen zur Kooperation zeigen.

12 Der Pfad As wird hier vernachlässigt.
} 
mit einer Erwartung von $\left(\mathrm{P}_{\mathrm{T}}^{\mathrm{B}}=\mathrm{P}_{\mathrm{SQ}}^{\mathrm{B}}-\mathrm{T} / 2 \mu\right)$ vorschlagen. Obwohl dies für A nur ein Minimal Result darstellt, ist es dennoch rational, anzunehmen, da immerhin eine geringe Verbesserung seines Status Quo erwartet werden kann $\left(\mathrm{P}_{\mathrm{t}}^{\mathrm{A}}=\mathrm{P}_{\mathrm{SQ}}^{\mathrm{A}}+\right.$ $\mathrm{T} / 2 \mu$ ). Abbildung 4 fasst diese Prämissen graphisch zusammen. Dabei illustrieren die Zickzacklinien die aus den Vorschlägen beider Spieler entstehende Entwicklung der erwarteten Payoffs. Aufgrund der Symmetrieannahme stellt der positive Teil des Diagramms die Payoffs für B, der Negative die für A dar.

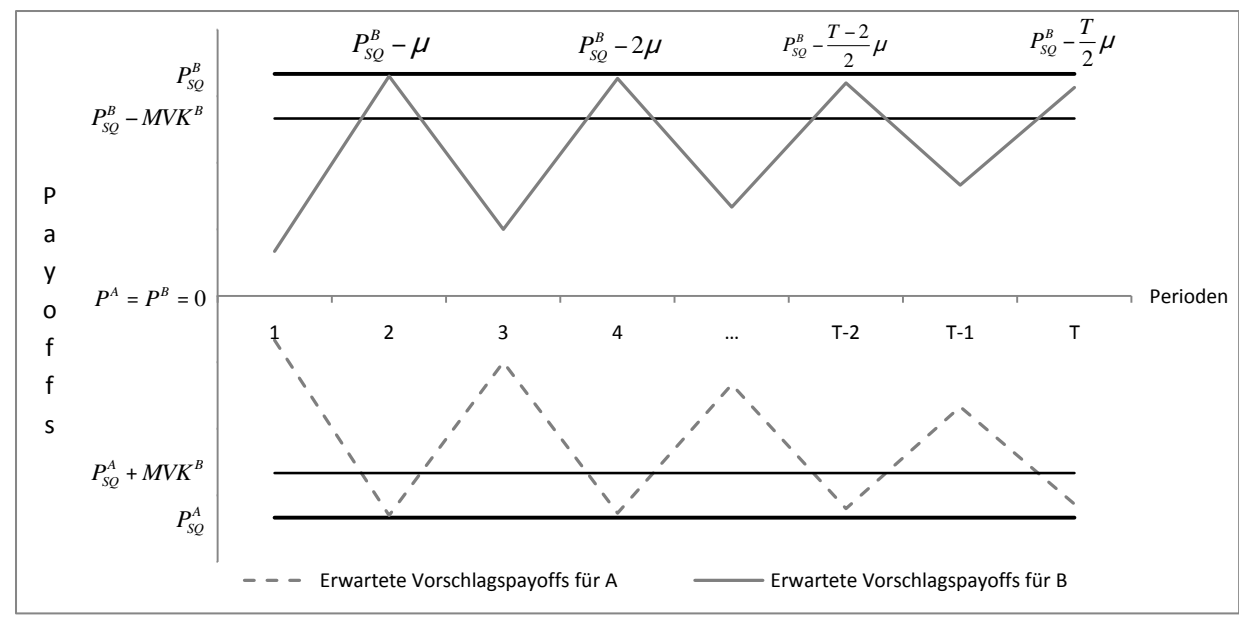

Abbildung 4: Verhandlungsverlauf mit konstanten Mandatsverlustkosten und B als letztem Spieler

Wie verändert sich der Verhandlungsverlauf wenn A den letzten Zug macht? Da keiner der Spieler diese Situation antizipieren kann, verläuft das Spiel zunächst genauso, als wenn B der letzte Spieler wäre, bis auf die letzte Periode. Wie aus Abbildung 5 ersichtlich, wäre A nun in der Lage Bs maximale Zahlungsbereitschaft auszunutzen. Könnte er mit $\mathrm{P}_{t}^{\mathrm{A}}=-\left(\mathrm{P}_{\mathrm{SQ}}^{\mathrm{B}}-\left(\mathrm{MVK}^{\mathrm{B}}+\mu\right)\right)$ vorschlagen, so müsste $\mathrm{B}$ rationaler weise immer noch zustimmen. Jedoch ist A die exakte Höhe der MVK ${ }^{B}$ unbekannt. Sollte dieser zu anspruchsvoll vorschlagen, würde es B rationaler Weise vorziehen, die Verhandlungen zu beenden. Demzufolge wird A in Periode T auch nur infinitesimal mehr verlangen als B in T-1 vorgeschlagen hat.

Alles in allem macht es in diesem Spiel also keinen Unterschied, wer den letzten Zug ausführt, da in beiden Fällen nur ein Minimal Result als Endergebnis zu erwarten ist. Dies ist durch die Annahme vergleichsweise niedriger Mandatsverlustkosten für $\mathrm{B}$ determiniert. 


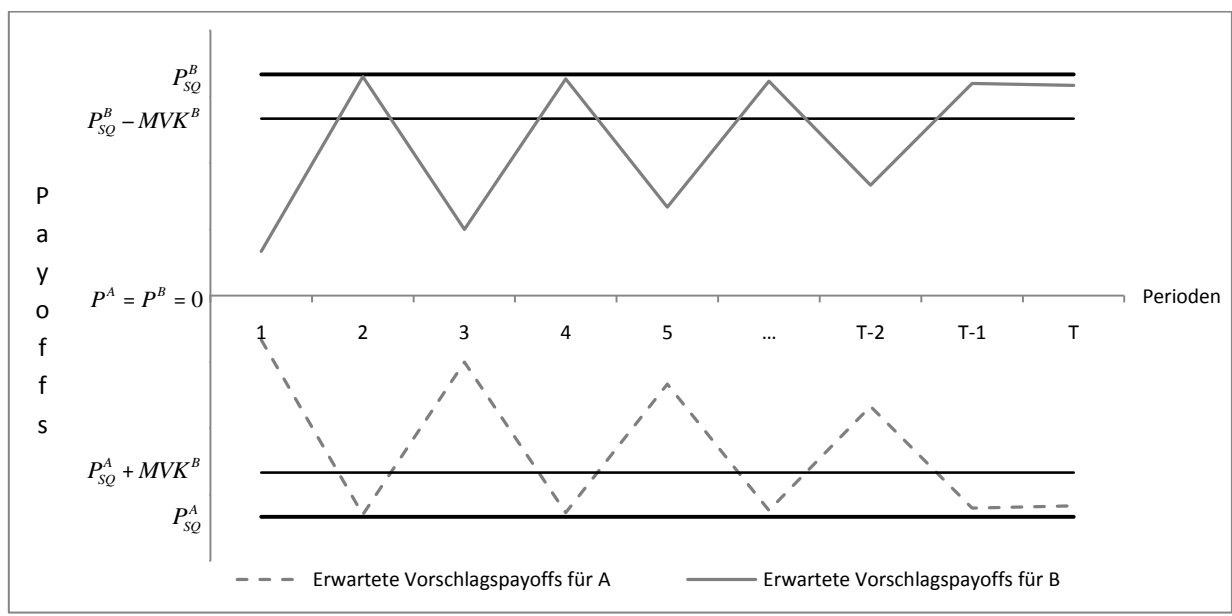

Abbildung 5: Verhandlungsverlauf mit konstanten Mandatsverlustkosten und A als letzten Spieler

\section{Steigende Mandatsverlustkosten}

Unter der Prämisse, dass die Klassifikation aus Kapitel 3.2 akzeptiert wird, können Veränderungen der Mandatsverlustkosten sowohl durch exogene, als auch endogene Faktoren des Verhandlungsprozesses ausgelöst werden. Ein exogener Faktor wäre in diesem Zusammenhang etwa ein Regierungswechsel in Land B, welcher eine substantielle Änderung der Sicht auf Verteilungsgerechtigkeit in diesem Land nach sich zieht. Endogen wäre eine Veränderung hingegen herbeigeführt, wenn z.B. durch Verhandlungsresultate (vom Generalsekretariat angefertigte Berichte) oder das Verhalten der Akteure die Öffentlichkeit dazu ermutigt wird, sich besser über die Situation zu informieren. Dies könnte zu einer veränderten Interessenslage führen aus der heraus eine Einigung erforderlich erscheint, die größere Zugeständnisse von B beinhaltet.

Trotzdem diese Veränderungen in beide Richtungen möglich sind, scheint es schlüssig, dass im Laufe der Zeit - wahrscheinlich im Verlauf mehrerer Mandatsperioden - ein leichter Anstieg der Mandatsverlustkosten zu erwarten ist (siehe Abbildung 6). 


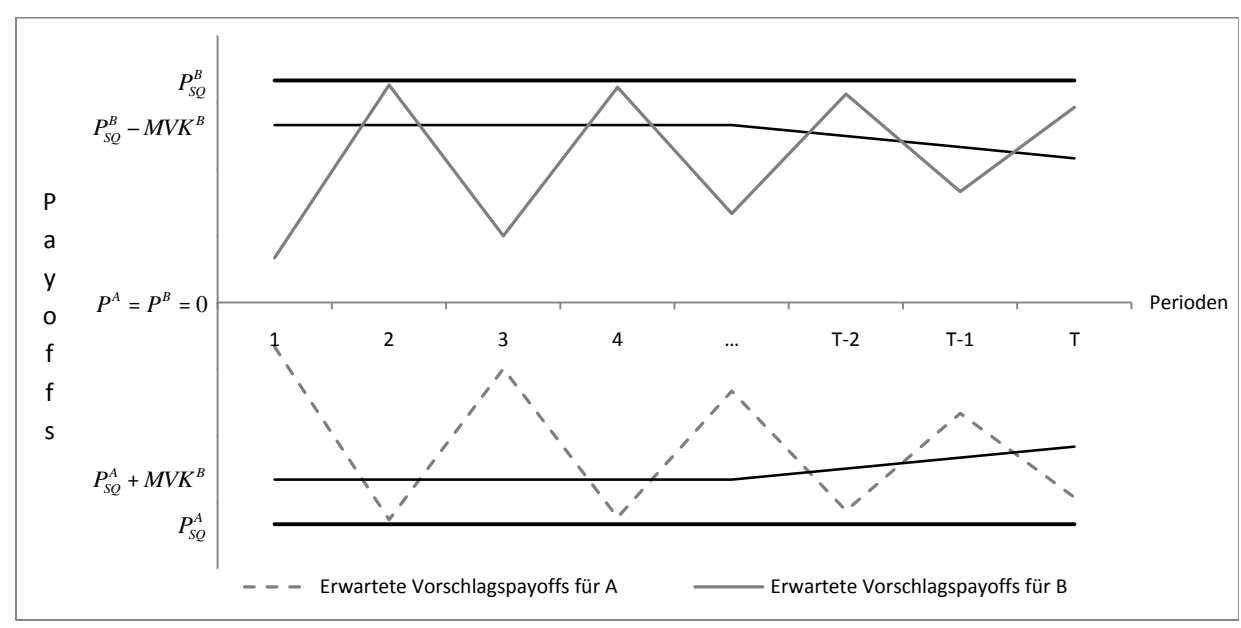

Abbildung 6: Verhandlungsverlauf mit steigenden Mandatsverlustkosten

In diesem Modell steigen die MVK ${ }^{B}$ relativ zum Status Quo beider Spieler in einer nicht näher bestimmten Periode vor T-2. Die Minimal Result Zone öffnet sich somit, was für B bedeutet, dass er nun Zugeständnisse als rational betrachten muss, die vorher außerhalb seiner maximalen Zahlungsbereitschaft lagen. Unter der Annahme, dass diese Entwicklung beiden Spielern zu Beginn nicht bekannt ist, starten die Verhandlungen wie oben bereits beschrieben. Die hier eingeführte Veränderung steigert jedoch As Drohpotenzial, und veranlasst B, größeren Zugeständnissen zuzustimmen. Da A Bs maximale Zahlungsbereitschaft jedoch nicht bekannt ist, wird er seine theoretisch maximal möglichen Zugeständnisse nicht voll ausschöpfen können. Dennoch ist eine Verbesserung seiner Situation gegenüber der bei konstanten Mandatsverlustkosten zu erwarten.

\subsection{Implikationen: Die verborgene Effektivität der Minimal Results}

Mit Hilfe der in den vorigen Abschnitten beschriebenen zwei Varianten des Minimal Results Spiels lässt sich nun die Frage beantworten, warum Länder an scheinbar ineffektiven internationalen Verhandlungen teilnehmen: Einige Länder sehen sich mit Mandatsverlustkosten konfrontiert, wovon andere Kenntnis haben, nicht aber, wie hoch diese Kosten sind.

Fangen wir mit einer kurzfristigen Betrachtung der aktuellen Mandatsperiode an. Spieler A hat auf Grund von Bs Verhalten im Status Quo einen erheblich negativen Payoff. Es ist demnach in As Interesse, auf die Errichtung eines Forums hinzuarbeiten und Verhandlungen aufrecht zu erhalten. Auch wenn diese Verhandlungen nur zu Minimal Results führen, so wird sich As erwartete Situation verglichen zum Status Quo doch verbessern. Er verhält sich also rational, da er Minimal Results überhaupt keinen vorzieht. 
Bs Partizipation an den Verhandlungen lässt sich hauptsächlich durch drohende Mandatsverlustkosten erklären. Zwar würde B gerne den Status Quo aufrechterhalten, da jedes Zugeständnis gegenüber A seinen Payoff schmälert. Jedoch zieht Nichtteilnahme Mandatsverlustkosten nach sich. B handelt demnach auch rational, da er diese für größer hält, als die Zugeständnisse, die er A gegenüber einräumt. So gesehen kann die Differenz zwischen den erwarteten Kosten eines Minimal Results und den gesamten Mandatsverlustkosten auch als Verhandlungsrente (VR) aufgefasst werden. Formal lässt sich Bs Ertrag am Ende der ersten Mandatsperiode mit folgender Formel beschreiben:

$$
V R_{T_{1}}^{B}=M V K^{B}-\left(P_{S Q}^{B}-P_{T_{1}}^{B, M R}\right), \text { wobei }
$$

$P_{T_{1}}^{B, M R}=$ Payoff, den B im Minimal Result der ersten Mandatsperiode erwartet $V R_{T_{1}}^{B} \quad=$ Ertrag, den $\mathrm{B}$ aus dem Minimal Result der ersten Mandatsperiode $\mathrm{T}_{1}$ erwartet

Bei längerfristiger Betrachtung des Spiels sagt das Modell voraus, dass am Ende jeder weiteren Mandatsperiode ebenfalls Minimal Results erzielt werden $\left(P_{T_{i}}^{B, M R}\right)$, solange die Mandatsverlustkosten nicht signifikant ansteigen. In jeder Periode wird B demnach einen Verhandlungsertrag erzielen. Weil sich jedoch die erwarteten Kosten, die diese Resultate für B bedeuten, aufaddieren, wird sich der Ertrag mit jeder neuen Periode Null annähern. Daher lässt sich auf lange Sicht Bs Teilnahme nur dadurch erklären, dass er sukzessive diese schrumpfenden Renten ausnutzt. Formal stellt sich diese Entwicklung wie folgt dar:

$$
V R_{T i}^{B}=M V K^{B}-\sum_{j=1}^{T i}\left(P_{S Q}^{B}-P_{T j}^{B, M R}\right)
$$

$P_{T_{j}}^{B, M R}=$ Payoff, den $\mathrm{B}$ aus dem Minimal Result der Periode $\mathrm{T}_{\mathrm{i}}$ erwartet

$V R_{T i}^{B} \quad=$ Verhandlungsertrag, den $\mathrm{B}$ aus dem Minimal Result der Periode $\mathrm{T}_{\mathrm{i}}$ erwartet

Am Ende einer bestimmten Mandatsperiode werden Bs erwartete Kosten aus den Minimal Results genauso hoch sein wie seine Mandatsverlustkosten. Gemäß dem hier vorgestellten Modell müssten die Verhandlungen zu diesem Zeitpunkt dieser Periode enden. Formal ist die Bedingung für ein solches Ereignis: 


$$
\sum_{j=1}^{T i}\left(P_{S Q}^{B}-P_{T j}^{B, M R}\right)=M V K^{B}
$$

Mit Bezug auf A sei an dieser Stelle noch einmal betont, dass er die exakte Höhe von Bs Mandatsverlustkosten nicht kennt. Dadurch erklärt sich auch, weshalb er Bs maximale Zahlungsbereitschaft nicht direkt ausnutzt. A wird also in den andauernden Verhandlungen ausloten, wie hoch diese Zahlungsbereitschaft ist. Sie mag, wie im Falle konstanter Mandatsverlustkosten, gering sein, nichtsdestotrotz wird er als rationaler Spieler versuchen, Zugeständnisse in dieser Höhe zu erwirken. Darüber hinaus kann A auch darauf hoffen, dass sich während der andauernden Verhandlungen Bs Mandatsverlustkosten - exogen oder endogen - erhöhen, und somit auch Bs Zahlungsbereitschaft wächst.

Es lässt sich an dieser Stelle festhalten, dass sich sowohl bei kurz- als auch bei längerfristiger Betrachtung beide Spieler rational verhalten, wenn sie an diesen (Nullsummen-)Verhandlungen teilnehmen. A kann durch Minimal Results eine leichte Verbesserung seines Status Quo erwarten, wohingegen B Verhandlungserträge erzielt. Das hier abgeleitete Resultat ist allerdings nur unter der Annahme gültig, dass A gegenüber B Drohpotential besitzt, das sich durch die Mandatsverlustkosten begründet. Da sich dies nicht aus klassischen Erklärungen für Verhandlungseffektivität ableiten lässt, nennen wir diesen Umstand die verborgene Effektivität der Minimal Results in internationale Verhandlungen.

\section{Zusammenfassung und Ausblick}

Die Motivation für diesen Artikel waren die Sitzungen 13-15 des Intergovernmental Committee on Intellectual Property and Genetic Resources, Traditional Knowledge and Folklore der WIPO, denen die Autor/innen in Genf beigewohnt haben. Seit das Komitee 2000 ins Leben gerufen wurde, haben die Verhandlungen nicht $\mathrm{zu}$ substantiellen Ergebnissen, sondern nur zu minimalen Fortschritten geführt. Darum ist es auch das Ziel dieses Artikels, einen Erklärungsansatz dafür zu finden, dass Länder an diesen kostspieligen und scheinbar ineffektiven Verhandlungen teilnehmen, da klassische ökonomische Theorien hier versagen.

Um dies zu bewerkstelligen, haben wir ein einfaches Verhandlungsmodell eingeführt. Der Eckpfeiler dieses Modells ist, dass sich „Blockadeländer“ mit Kosten konfrontiert sehen, wenn ein Mandat auf Grund fehlender Verhandlungsergebnisse ausläuft. Länder, die auf Fortschritt drängen, sehen sich keinen solchen Mandatsverlustkosten ausgesetzt, weswegen ihnen dies die Möglichkeit eröffnet, Zugeständnisse von den Blockadeländern zu fordern, obwohl hier angenommen wird, dass die Kosten gering sind. Abschnitt 3.3 zeigt, wie Verhandlungen zu Minimal Results führen, die auf den ersten Blick ineffektiv erscheinen. Hingegen wird in Abschnitt 3.4 deutlich, warum diese Verhandlungen am Ende doch als effektiv 
betrachtet werden können. Obwohl verborgen für einen Außenstehenden, kann Spieler A zunächst eine kleine, längerfristig sogar eine größere Verbesserung seines Status Quo erwarten, wohingegen sich für Spieler B Verhandlungserträge ergeben, da die aus den Zugeständnissen resultierenden erwarteten Kosten kleiner als seine Mandatsverlustkosten sind. Innerhalb dieses Modells ist es also für beide Spieler sinnvoll, an den Verhandlungen teilzunehmen, was aggregiert auf Blockade- und auf Fortschritt drängende Länder übertragen werden kann.

Wie viel Erklärungsgehalt besitzt solch ein Modell jedoch, wenn man Verhandlungen in der wirklichen Welt betrachtet? Wir wollen diese Einschätzung mit einer Betrachtung der Mandatsverlustkosten beginnen, da sie das Schlüsselkonzept dieses Modells darstellen. Das Konzept stützt sich auf Beobachtungen des Verhaltens von Abgeordneten in Verbindung mit den institutionellen Rahmenbedingungen der Verhandlungen. Im Laufe der IGC-Sitzungen stellte sich heraus, dass, obwohl die Abgeordneten aus Blockadeländern nicht an größeren Fortschritten im IGC interessiert waren, das Mandat doch erhalten bleiben sollte. Demnach scheint es folgerichtig anzunehmen, dass der Verlust dieses Mandats Kosten für diese Länder impliziert. Renninger (1989:252) spricht in diesem Zusammenhang von durch Verhandlungsabbruch entstehenden politischen Kosten. Darüber hinaus argumentiert er, dass diese für wirtschaftlich starke Länder relativ gering sind, was in diesem Modell Berücksichtigung findet. Er beschreibt dabei, dass es die Länder der Südhalbkugel in den 1970er und 1980er Jahren nicht geschafft haben, substantielle Zugeständnisse im Rahmen der Zusammenarbeit für wirtschaftliche Entwicklung zu bekommen, was während spezieller Zusammenkünfte der UN Vollversammlung verhandelt wurde. Daraus folgt, dass ein minimaler Fortschritt aus solchen Verhandlungen hervorgehen muss, damit ein Mandat erhalten bleibt, was wiederum geringere Kosten als der Verlust des Mandats bedeutet. In der Tat scheint Renningers Beispiel die Vorhersagen des hier vorgestellten Modells zu unterstützen:

The process has in some sense worked for the South in that certain concessions have been obtained and the issues of concern to the South have retained their prominent position on the international agenda. (Renninger 1989:252)

Weiterhin haben wir angenommen, dass Verhandlungen nur zwischen zwei Ländern und nur in Bezug auf ein Thema geführt werden. Als erstes sei hier darauf verwiesen, dass Verhandlungen im IGC natürlich aus mehr als nur einem Thema bestehen. Da jedes Thema eine andere Kostenfunktion in Bezug auf Verhandlungsabbruch besitzen könnte, müsste an dieser Stelle das abstrakte Konzept der Mandatsverlustkosten näher spezifiziert werden. Trotzdem konnten wir während der 14. Verhandlungsrunde beobachten, dass sich die Abgeordneten gegen Ende auf ein bestimmtes Themengebiet konzentrierten, um die Bedingungen für eine Mandatsverlängerung auszuhandeln. Daraus lässt sich schließen, dass die vereinfachende Annahme in diesem Modell zu einem gewissen Grad anwendbar ist. Dar- 
über hinaus nehmen an multilateralen Verhandlungen mehr als nur zwei Länder teil. Dabei wird es auch Länder geben, die zu bestimmten Themen keine klar formulierte Position besitzen und entsprechend eher als Zuschauer betrachtet werden können. Trotzdem war zu beobachten, dass die meisten Länder Gruppen bilden, die sich grob in Anhänger und Gegner neuer Schutzregimes für intellektuelle kulturelle Eigentümer unterschieden. Neben dieser Tendenz führt die informelle „Regel“ des einstimmigen Beschlusses dazu, dass sich die Verhandlungspartner auf die stärksten Pro- und Kontra-Argumente bei den meisten Themen konzentrieren, da diese zuerst Berücksichtigung finden müssen. ${ }^{13}$ Wenn ein Land substantiellen Verhandlungsfortschritt nicht unterstützt, wird es schwierig, es zu übergehen. Insgesamt lässt sich folgern, dass, obwohl das hier beschriebene Zwei-Länder-Modell keine Feindynamik innerhalb und zwischen Gruppen beinhaltet, es aber doch einige Schlussfolgerungen im Bezug auf wirkliche Verhandlungen erlaubt.

Drittens nehmen wir in dem Modell an, dass Länder rational im Sinne der Maximierung des eigenen Verhandlungsnutzens agieren. Diese Position haben verschiede Autoren wie Brams (1975), Snidal (1985) oder Sykes (2004) in Bezug auf spieltheoretische Analysen verteidigt. Aus diesem Grund halten wir diese Annahme auch in diesem Fall für anwendbar. Weiterhin wurden Abgeordnete als perfekte Repräsentanten ihres jeweiligen Landes modelliert. Diese Annahme ist eher problematisch, da ihre Verhandlungsführung sehr wohl durch eigene Interessen beeinflusst sein kann. Gemäß der New Political Economy streben Abgeordnete bei internationalen Verhandlungen nach einem möglichst konfliktfreien Leben im öffentlichen Dienst, das sowohl Prestige, als auch ein hohes monetäres und nichtmonetäres Einkommen mit sich bringt (Gygi 1990:12-13). Man kann somit annehmen, dass Abgeordnete für Auslandsreisen zusätzlich entlohnt werden, persönliche Vorteile aus diesen ziehen, wie etwa Freunde zu treffen und schöne Städte zu besuchen und vielleicht sogar aus stressbehafteten Situationen in ihren Ministerien entkommen. Auf diese Weise ließe sich die fortdauernde Teilnahme an Verhandlungen alleine durch eigennützige Motive erklären. Allerdings wäre ein solcher Erklärungsansatz der gleichen Kritik unterworfen, wie andere auch. Die meisten Abgeordneten im IGC scheinen ihre Positionen gut mit ihrem Heimatland zu koordinieren, da sie vorgefertigte Statements vortragen. Auch müssen einige sich zunächst mit Ministerien beraten, bevor sie Fortschritten zustimmen. Daher fällt es schwer, anzunehmen, dass sie genug persönliche Freiheit besitzen, um ungeachtet möglicher Kosten eigenmächtig der Fortführung der Verhandlungen zuzustimmen. Darüber hinaus lässt sich auch aus der Tatsache, dass Verhandlungen oft bis spät in die Nacht dauern, schließen, dass nationale Interessen eine Rolle für das Verhalten der Abgeordneten spielen. Diese Tatsachen rechtfertigen die Vorhersagen unseres Modells.

\footnotetext{
${ }^{13}$ McKelvey und Ordeshook (1984) argumentieren beispielsweise, dass trotz Mehrheitsregelungen in Komitees Parteien mit starken Interessen es oftmals schaffen, diese Regeln zu umgehen und ihre eigenen Interessen durchzusetzen.
} 
Zusammenfassend ist festzuhalten, dass das Minimal Results Modell trotz einiger vereinfachender Annahmen dazu geeignet ist, einen Erklärungsansatz dafür zu liefern, warum Länder freiwillig an scheinbar ineffektiven internationalen Verhandlungen teilnehmen. Über dieses Modell hinaus bleibt zu untersuchen, warum Länder sich mit einer bestimmten Position, dem Blockieren oder dem Fortschritt, identifizieren. Es könnte etwa versucht werden, nationale Charakteristika herauszuarbeiten, die das Verhalten in internationalen Verhandlungen bestimmen. Entsprechend erachten es die Autor/innen als notwendig, die Folgen möglicher internationaler Schutzregime, wie sie von ihren Verfechtern vorgeschlagen werden, aus ökonomischer Sicht zu untersuchen. 




\section{Teil 4 \\ Forschen über Cultural Property}





\title{
Zur technischen Dimension der Konstituierung von Cultural Property und Cultural Heritage: Perspektiven der kulturwissenschaftlichen Technik- forschung
}

\author{
Johannes Müske
}

Thema der folgenden Überlegungen ist, wie die Fragestellungen und Forschungsansätze der kulturwissenschaftlichen Technikforschung einen Zugang für die Untersuchung der Entstehung von Cultural Property und Cultural Heritage bieten können. Die Untersuchung der Rolle der Technik bei der Entstehung von Cultural Property (aus einer Metaperspektive) ist eine projektübergreifende Aufgabe, die sich die interdisziplinäre Forschergruppe gestellt hat. Technik spielt auf unterschiedlichste Weise eine wichtige Rolle in der alltäglichen Lebenswelt und also auch in den einzelnen Forschungsprojekten. Dies soll an den Beispielen von Kommunikations- und der Speichertechnik gezeigt werden. Die unterschiedlichen Ebenen, in denen Technik involviert ist, sollen daher am Beispiel der Teilprojekte der Forschergruppe beschrieben werden. Im Zusammenhang mit dem „technischen“ Frageinteresse werden die Phänomene Cultural Property und Kulturerbe nicht unterschieden: Die „Praxis der Herauslösung kultureller Artefakte“ (Hemme, Tauschek und Bendix 2007b:9) aus ihrem alltäglichen Kontext ist beiden Phänomenen inhärent, indem sie sowohl die Voraussetzung dafür ist, kulturelle Artefakte einem ( $\mathrm{He}$ ritage)-Schutzregime zuzuführen als auch dafür, aus ihnen (Eigentums-)Ansprüche abzuleiten. Dass die einzelnen Teilprojekte mit beiden Begriffen operieren und je- 
weils unterschiedliche theoretische Perspektiven auf die Inwertsetzung von Kultur haben (vgl. dazu Bendix und Hafstein 2009), bleibt davon unberührt.

Zum besseren interdisziplinären Verständnis wird das Thema entlang der Forschungstradition der volkskundlichen Disziplinen ${ }^{1}$ entwickelt. Weil Technik implizit in die von den Forschungsprojekten untersuchten Felder hineinspielt, muss sie stets mitgedacht werden. Entsprechend ist ein besseres Verständnis von Technik als einer „Querschnittsdimension“ (Schönberger 2007) bei der Entstehung von Cultural Property als eines der Forschungsinteressen definiert. ${ }^{2}$ Zunächst zeichnet der Artikel nach, wie das Entstehen der technischen Moderne eine Mitbedingung für die Herausbildung spezifischer Fragestellungen zur „Rettung“ der „,traditionellen“ Volkskultur waren, die in der Institutionalisierung des Fachs Volkskunde mündeten. In den 1960er und 1970er Jahren verabschiedete sich die Volkskunde vom Gegensatz von Technik und Volkskultur und begann damit, Technik als unhinterfragten Bestandteil der alltäglichen Lebenswelt zu sehen. Gleichwohl ist das „Gewohntsein“ (Hengartner 2004) an Technik nicht ,automatisch“ mit dem Auftreten neuer Technik gegeben. Technik und die mit ihr verbundenen Praxen müssen sich erst langsam herausbilden, wie erfahrungsgeschichtliche Studien zeigen (zum Beispiel Stadelmann und Hengartner 2002; Merki 2002). Hierbei sei neben der erfahrungsgeschichtlichen Dimension auf eine zweite Querschnittsdimension verwiesen: der Umgang mit der Technik, die intendierte und unintendierte Folgen haben kann. Dass manche Technik anders genutzt wird als vorhergesehen hängt mit ihrer Materialität zusammen, die unterschiedliche Nutzungen zulässt. So soll insbesondere auf die technische Verdinglichung von flüchtigen kulturellen Phänomenen eingegangen werden, die paradoxerweise durch die Digitalisierung mit einer gleichzeitigen Entdinglichung des Gespeicherten einhergeht. Abschließend wird angeregt, über Parallelen im Umgang mit der Volkskultur in der technischen Moderne und den traditional cultural expressions in der heutigen spätmodernen Lebenswelt nachzudenken.

\section{Die Rettung der Volkskultur vor der technischen Moderne}

Im Kontext der interdisziplinären Erforschung von Cultural Property sei in den folgenden Abschnitten zunächst an einige disziplinäre „Sichtweisen der Volkskunde“

\footnotetext{
${ }^{1}$ Neben der Fachbezeichnung Volkskunde trägt das Fach seit den 1970er Jahren auch die, gern miteinander kombinierten, Namen Kulturanthropologie, Europäische Ethnologie, Empirische Kulturwissenschaft. Auf die Fachnamensdiskussion und die unterschiedlichen theoretischen Begründungen soll hier nicht eingegangen werden. Im Folgenden wird daher der Name Volkskunde verwendet, den auch die deutschsprachigen Fachverbände und die von ihnen herausgegebenen Fachzeitschriften im Titel führen.

2 Die Fragestellung ist insbesondere im Hamburger Teilprojekt 5 „,Klänge und Töne als Cultural Property?" angesiedelt, weil dort ein sehr enger Technikbezug gegeben ist: dieser resultiert aus dem Forschungsthema, das die technische Fixierung von flüchtigen Phänomenen, Klängen, behandelt. Zum Gesamtprojekt vergleiche die Projektwebseite unter www.cultural-property.uni-goettingen.de (Zugriff am 09.05.2010).
} 
(Lehmann und Kuntz 1988) erinnert. In der Fachgeschichtsschreibung der Volkskunde und ihren Nachbarwissenschaften gilt die Feststellung, dass die Herausbildung und die Institutionalisierung des Faches eng verknüpft sind mit dem Erscheinen der industrialisierten Moderne als „Klassiker“. Enthusiastische Heimatforscher, oftmals Lehrer oder Pfarrer, begannen im 19. Jahrhundert damit, Sammlungen anzulegen, die die „bedrohte“ bäuerliche Volkskultur vor dem Untergang, zumindest vor dem Vergessen, bewahren sollten. Ein Gründungsboom von Heimatmuseen und volkskundlichen Vereinigungen ging damit einher. Die Entstehung des bürgerlich-romantischen Interesses am eigenen „Volk ${ }^{* 3}$ und seinen Ursprüngen entwickelte sich in Abgrenzung zum schon früher verbreiteten Interesse an fremden „Völkern“. In den „Wunderkammern“ versammelten und inventarisierten adelige und bürgerliche Sammler das, was aus ihrer europäischen Sicht als repräsentativ oder „eigentümlich“ für die „fremden Stämme“ galt. Die später entstandene Volkskunde führte dieses Forschungsinteresse am Fremden, gar „Primitiven“, fort, indem sie nach dem „vulgus in populo“ in den eigenen „Unterwelten der Kultur" (Maase und Warneken 2003) suchte. Vom jeweiligen Wissen, Interesse und den Erfahrungen des Sammlers oder der sammelnden Institution hing also ab, auf Grundlage welcher „Sammlungsstrategie“ (wie man heute sagen würde) die Dinge ausgewählt wurden. Die Zeugnisse davon sind die Magazine und Inventarlisten der musealen Institutionen. Die Ausweitung der räumlichen, sozialen und zeitlichen Horizonte mit der Industrialisierung im 19. Jahrhundert führte zur Auflösung der bäuerlichen Lebenswelten. Diese wurden, zumindest in der westlichen Welt, wo sich der soziokulturelle Wandel besonders deutlich zeigte, in die regionalen Freilichtmuseen herübergerettet. Die Fachgeschichte der Volkskunde deutet Technik zunächst als Hintergrundfolie für zahlreiche Veränderungen in der Lebenswelt der Moderne.

\section{Die erfahrungsgeschichtliche Dimension der Technik}

Im Fach Volkskunde traten in den 1960er Jahren erstmals Studien auf, die wahlweise die „technische Welt" oder das „Eindringen der Technik in die Lebenswelt“ untersuchen (Bausinger 1961, Jacobeit 1965). Die Rolle der Technik für unsere Erfahrungen und in unserer Lebenswelt ist zum Thema eingehender kulturwissenschaftlicher Beschäftigung geworden - in einer „technischen Welt“ wird Technik bemerkt und unbemerkt in immer größerem Maße be- und genutzt (Bausinger 1961, vgl. auch 1981, Hengartner 1998, Scharfe 1993a, Schröder 2007). Technik bildet die Hintergrundfolie für die Veränderungen der Lebenswelt. So bringt die Massenmotorisierung zum Beispiel neue Praktiken der Pflege sozialer Kontakte hervor (beispielsweise Besuche über weite Entfernungen, Automobilvereine) und verändert generell räumliche, soziale und zeitliche Horizonte (Bausinger 1961).

\footnotetext{
3 Vgl. zum Begriff „Volk“ einführend Bauman und Briggs (2003:163-196), zum fachgeschichtlich spezifischen Gebrauch in der Volkskunde auch Bausinger (1961) und Hartmann (2001).
} 
Seit den 1970er Jahren haben sich die volkskundlichen Kulturwissenschaften auf das gemeinsame Interesse verständigt, den selbstverständlichen Alltag und seine geschichtliche Gewordenheit zu erforschen. Kultur, verstanden als "the whole way of life" (Raymond Williams) verwirklicht sich in alltäglicher Praxis, die auf jeweils vorhandenen Wissensbeständen beruht, und ist entsprechend ständig in Veränderung. Eine kulturwissenschaftlich informierte Technikforschung geht von diesen Prämissen aus und fokussiert daher besonders auf den sozio-kulturellen Wandel. Dabei fragt sie insbesondere danach, wie und auf welchen Wegen Technisches immer mehr in unseren Alltag eingedrungen ist (vgl. Hengartner und Rolshoven 1998). Die Integration von Technik in den Alltag verläuft keineswegs immer geradlinig. Am Beispiel des Telefonierens (Hengartner 1998, 2002) soll verdeutlicht werden, wie alltägliche unhinterfragte Handlungsweisen von Technischem durchdrungen sind und welche Fragestellungen eine kulturwissenschaftliche Technikforschung zum Zusammenhang zwischen Technik und Kultur hat.

Die Telefonie, die heute als selbstverständliche Kulturtechnik gilt, musste, nachdem es die ersten Telefone gab, zunächst erlernt werden, wobei die Verfügbarkeit von Netzen und Telefonapparaten eine wichtige Rolle für das Kommunikationsverhalten spielte. In der „Frühzeit“ der Telefonie - vor 1920 - gab es vor allem auf dem Land nur wenige Apparate und diese vorzugsweise in Geschäften und anderen öffentlichen Bereichen, sodass Telefonate vor allem der geschäftlichen Kurzkommunikation vorbehalten blieben. Die Unsicherheiten im ersten Umgang mit der neuen Technik konnten durch Bebilderungen und Hinweise („Fasse dich kurz!" $)$ sowie durch Telefonunterricht in den Schulen ausgeräumt werden. Mit der weiteren Verbreitung der Telefone in die Privathaushalte änderte sich auch das Telefonverhalten: private Gespräche dienten nun der Entspannung und der Pflege von sozialen Kontakten. ${ }^{4}$ Heute taucht der Telefonunterricht nicht mehr in den Stundenplänen auf - das Telefon ist selbstverständlich geworden und wird in familiären Sozialisationszusammenhängen erlernt. Dass diese ausführliche Art der Kommunikation wieder in öffentlichen Kontexten anzutreffen ist - in Form der Mobiltelefonie - war zwar von den Erfindern des „Handys“ nicht intendiert, spricht aber für das große „Gewohntsein“ (Hengartner 2004) an diese Kulturtechnik. Dass sich die Erfindung des Telefons im Alltag durchsetzte, hing von verschiedenen, mit der erscheinenden Moderne verbundenen, Faktoren ab: ein Kontext war die gestiegene räumliche Mobilität, in dem das private und geschäftliche „fernmündliche“ Sprechen offenbar gegenüber der Telegrafie oder dem weiterhin existierenden Brief Vorteile bot.

Der hier skizzierte erfahrungsgeschichtliche Ansatz kulturwissenschaftlicher Technikforschung betont, dass bestehende Praxen wichtig für den Erfolg oder

\footnotetext{
${ }^{4}$ Erwähnt sei ein weiteres Beispiel für die Einfügung der Kulturtechnik des Telefonierens in bestehende kulturelle und soziale Formen, zum Beispiel Gender-Muster; so wird das angeblich stundenlange Telefonieren weiblicher Teilnehmer nicht als „Quasseln“ sondern als Pflege von Familienkontakten deutbar.
} 
Misserfolg neuer Technik sind. Technik vereinfacht Handlungen oder verstärkt deren Effizienz, sie erschließt aber auch neue Handlungspotenziale, sodass völlig unintendierte Nutzungen entstehen können (Beck 1997, Latour 1996). Technik ist also nicht der Auslöser sozio-kulturellen Wandels (wie in der Überbewertung durch die „klassische“ Technikgeschichte mit ihrem genius inventor), sondern ein Bestandteil desselben. Soziales und Technik sind ineinander verwoben, die verschiedenen Ebenen des Handelns, des habitualisierten Umgangs mit Technik, die technischen Möglichkeiten und Nutzungen sowie weitere Kontexte müssen unterschieden und beschrieben werden, um die Komplexität des sozio-kulturellen Wandels erfassen zu können.

\section{Die Praxisdimension: Umgang mit Technik}

Mit dem Begriff der Technik als „Querschnittsdimension“ verweist der Kulturwissenschaftler Klaus Schönberger weiterhin darauf, dass neben den historischen Technisierungsprozessen auch weitere Ebenen, wie Umgangspraktiken mit Technik und ihre Materialität untersucht werden müssen. Unterschiedliche Akteure, wie Regierungsvertreter/innen, NGO-Beobachter/innen und andere, werden in unterschiedlichen Kontexten - dem, was in den Kulturwissenschaften „das Feld“ genannt wird - aktiv. Dabei bestehen nicht nur performative Unterschiede zwischen einer Konferenz der World Intellectual Property Organisation (WIPO) in Genf und einem Totenritual der Toraja auf Sulawesi, sondern auch Unterschiede im jeweiligen technischen Setting. In beiden Fällen ist auf höchst unterschiedliche Weise digitale Technik vorhanden, zum Beispiel als (Handy-)Filmtechnik. Zu den Teilprojekten unserer Forschergruppe, die die Cultural Property-Verhandlungen bei der WIPO beobachten, ist anzumerken, dass Kommunikations- und Transporttechnik überhaupt erst internationale Verhandlungen ermöglicht. Flugzeuge und andere Transportmittel ermöglichen die persönliche Anwesenheit über große räumliche Distanzen hinweg. Gleichzeitig sind die Mobilitätskosten in den letzten Jahrzehnten sehr gesunken, sodass ein größerer Austausch und engere persönliche Kontakte möglich sind. Auch Telefone, Faxtechnik und die Kommunikationstechnik insgesamt ermöglichen es den Verhandlungspartnern, in physischer Abwesenheit in Gespräche zu treten und erlauben informelle Kommunikationen. Während hier ein größerer Rahmen geschaffen ist, innerhalb dessen es zu Verständigungen, Eklats, und anderen Ereignissen unter den beteiligten Akteuren kommen kann, wird die Technik auch im Kleinen in den Verhandlungen aktiv: in Form des USBSticks. Bei der WIPO sammeln die Protokollierenden nach jeder Rede vor der Versammlung mit einem USB-Speichermedium die Manuskripte und Statements ein, die anschließend in die Tagungsdokumentation einfließen. Diese Überfülle an Dokumentation und das Abgleichen von Informationen vereinfacht die Datenverarbeitung und verbessert die Repräsentation kleinerer Länder und von NGOs, die ihre Positionen besser abstimmen, archivieren und damit gegenüber größeren Län- 
dern vertreten können. Als nicht vorhersehbare Folge werden aber auch Entscheidungsprozesse verlangsamt oder behindert, weil insbesondere durch die „virtuellen Archive" die Verhandelnden dazu diszipliniert werden, nur noch zuvor abgestimmte Verhandlungspositionen preiszugeben. Die Umgangsweisen mit digitaler Technik - zum Beispiel Mikrofonen, USB-Sticks und Laptops - ist als unhinterfragte Praxis in den Alltag der WIPO eingegangen. Weder Flugzeuge noch USBSticks sind aus dem Alltag wegzudenken, weil sie Handlungen „technisch hinterlegen“ oder überhaupt erst ermöglichen (vgl. Hengartner 2004, Schönberger 2007).

Die neu entstehenden Umgangspraktiken greifen auf vorhandene Wissensbestände zurück. Auch in der neuen Technik selbst befindet sich immer schon Vorgängertechnik, die den Alltag bereits durchdrungen hat und deren Funktionsweise (und damit bestehende Praktiken) das Zurückgreifen auf bestehendes Wissen erst ermöglicht. Diese Eigenschaft der Technik fasst Martin Scharfe mit dem Begriff der „Penetranz“ (1993a). So steckt in einem USB-Stick Speichertechnik, die zuvor in Computerfestplatten und später in mobilen Festplatten steckte und immer weiterentwickelt wurde. Die bisherigen Umgangsweisen mit der Vorgängertechnik durchdringen nun das weiterentwickelte Ding: zum Beispiel Reproduktionstechniken, die mit analogen Daten- und Tonträgern erlernt wurden und nun im Verbund mit digitalen Mitteln sich vervielfachende Kopiermöglichkeiten und geschwindigkeiten schaffen. Was mit dem Telefon und dem USB-Stick angedeutet wurde, erfährt mit dem „Internet“ eine neue Dimension: die problemlose Verbreitung und Speicherung von Daten. Die Anwendungen im Umfeld des Netzes unterstützen mittlerweile viele verschiedene Kommunikationen, die an tradierte $\mathrm{Mu}-$ ster anknüpfen, zum Beispiel die briefähnliche Kommunikation (E-Mail), die (mobil-)telefonische Unterhaltung (VoIP, Skype), aber auch Einweg-Kommunikationen wie Zeitung und Fernsehen. Weitere bisher unbekannte und nun extrem erfolgreiche Kommunikationsformen, wie das Bloggen, kommen hinzu. Eng mit dem Internet verbunden ist die Digitalisierung anderer Alltagsbereiche, zum Beispiel der privaten und geschäftlichen Kommunikation, neue Praktiken wie das OnlineEinkaufen, das „vernetzte“ wissenschaftliche Arbeiten (multilokale Forschergruppen), aber auch das Bereitstellen von Inhalten, was insbesondere klassischen Medien wie Zeitungen schwere Probleme bereitet. Gleichzeitig birgt die dezentrale und ungebundene Zirkulation der Daten auf vielen Servern neue Möglichkeiten der Archivierung und der Sicherung von Archivgut (Cohen 2001), bringt aber auch Veränderungen für den Zugang zu Archivgut mit sich, was einige Sammlungen in Bedrängnis bringt (Seeger 2001). 


\section{Die materielle Dimension der Technik oder: Der Datensatz im Zeitalter seiner digitalen Reproduzierbarkeit ${ }^{5}$}

Technik ist insbesondere im Zusammenhang mit nichtdinglichen kulturellen Phänomenen, wie der Performanz von Traditionen oder Klängen und Tönen, die Voraussetzung für ihre Verdinglichung. Mit der Digitalisierung von Daten sind die Daten gleichzeitig wieder von ihrer materiellen Gebundenheit gelöst - wobei materiell hier im doppelten Sinne als auf das Material und auf den Wert bezogen verstanden werden kann. Für digitale Daten ergeben sich völlig neue Möglichkeiten und Modi der Vervielfältigung: digitale Daten sind, einmal in der Online-Welt, fast kostenlos beliebig oft, an vielen Plätzen der Welt, ohne Datenverlust erreichbar, abrufbar, kopierbar und werden damit zum Verhandlungsgegenstand (UNESCO, WIPO). Ein Beispiel für die Umgehung von Schutzrechten an geistigem Eigentum ist das verlustfreie Kopieren von Musik über Online-Tauschplattformen („Napster"). Diese neue Praktik führte zu international beachteten Gerichtsprozessen, um die bestehenden Intellectual Property-Regeln durchzusetzen. Auch die Verbreitung von Kulturgütern hat sich durch ihre digitale Verfügbarkeit (zum Beispiel Bild-, Ton- und Videoportale) verändert, weshalb die Trägergruppen von bestimmten kulturellen Gütern über internationale Institutionen versuchen, den Zugang der Güter rechtlich zu regulieren oder exklusive Rechte an ihnen eingeräumt zu bekommen.

Die dingliche Gebundenheit der Information an den Datenträger - Dinge, Papiere, Tonträger - ist erloschen, sobald die “contents” hochgeladen und ,,im Internet“ sind. Die „technische Reproduzierbarkeit", deren Analyse Walter Benjamin in seinem kunstphilosophischen Aufsatz für Kunstwerke vornahm (1975[1936]), löst das Reproduzierte aus dem Bereich der Tradition heraus. Anstelle des einzelnen Kunstwerks tritt durch die Reproduktionstechnik ein massenweises Vorkommen eines Werks anstelle des einmaligen Vorkommens. Das einzelne Werk, das vormals ganz in die Tradition oder das Ritual eingebunden war, findet sich nun als Kopie in mannigfaltigen Kontexten wieder. Benjamin entwickelt seine Gedanken zur Reproduktionstechnik vor allem am Beispiel der Fotografie- beziehungsweise Filmtechnik. Im Zusammenhang mit der so genannten Digitalisierung können sie neu gelesen werden. Bei einer Kopie, und sei sie auch noch so hochwertig, ist die Geschichte des Kunstwerks (Einbindung in das Ritual, physische Veränderungen, Besitzerwechsel usw.), die seine „Aura“ ausmacht, entfallen. Dasselbe gilt umso mehr für den digitalen Datensatz, der kein „Original“ kennt und immer Kopie und Original zugleich ist. Für die Erforschung der Entstehung von Cultural Property lohnt es sich, die Folgen der Loslösung des Kunstwerks - oder einer jeglichen cultural expression - vom jeweiligen Traditionszusammenhang durch die digitale Reproduzierbarkeit genauer zu betrachten. Die Materialität digitaler Daten ist,

\footnotetext{
5 Überschrift geliehen von Walter Benjamin: „Das Kunstwerk im Zeitalter seiner technischen Reproduzierbarkeit“"(1975[1936]).
} 
unabhängig, ob sie auf einem Rechner oder einem anderen Datenträger gespeichert sind, bei Kopie und Original identisch. Damit stellt sich nicht mehr die Frage des physischen Aufbewahrungsortes des Originals, sondern nur noch die Frage des Zugangs mit Hilfe von Internetverbindungen und Programmen, die in der Lage sind, den digitalen Datensatz zu öffnen. Benjamin kannte trotz Photograph und Phonograph noch die Gewissheit des „Hier und Jetzt des Originals“. Dieses werde mit seiner massenweisen Reproduktion zwar entwertet, der Bestand des Kunstwerks jedoch und seine Echtheit blieben unangetastet. Bei der Entstehung von digitalen Objekten tritt eine grundlegende Veränderung ein: digitale Objekte, als Ansammlungen von „Nullen und Einsen“, haben keine „Objektgeschichte“, mit denen sie sich selbst authentisieren können. Authentizität muss aber, wenn Quellen wissenschaftlich geeignet sein sollen, gewährleistet sein. Archive verfügen oftmals über die Originalquellen ("stable, physical media", Levy 2000:30, passim) von Digitalisaten. Hier kann die Authentizität des Digitalisats über Metadaten des Originalobjekts hergestellt werden. „Originale“ Digitalisate verfügen jedoch über keine Objektgeschichte. In der archivarischen Diskussion (zum Beispiel CLIR 2000) wurde erkannt, dass sich ihre Authentizität nur über "social interaction" stabilisieren lässt (Levy 2000). Ursprünglich digitale Datensätze können durch die Anreicherung mit Metadaten oder Verwendung digitaler Signaturen, also soziale Kontrollmechanismen, authentisiert werden: "[S]ocial mechanisms of control promise to be the fundamental basis for the establishment of digital authenticity" (Hirtle 2000:20). In der analogen Welt zeichnen die Metadaten eines Objekts (zum Beispiel auf Karteikarten) seine Objektgeschichte auf. In der digitalen Welt werden sie selbst zur Geschichte des digitalen Datensatzes. Authentizität wird nicht mehr durch die aufgezeichnete dingliche Überlieferungsgeschichte, sondern durch soziale Mechanismen hergestellt. Archive überliefern nicht nur Objekte, sondern stellen digitale Objekte bereit und bürgen als vertrauenswürdige Institutionen für ihre Authentizität, respektive die Richtigkeit der Metadaten.

Zurzeit werden in vielen Archivprojekten Digitalisate von analogen Objekten hergestellt, wobei auch Archivalien betroffen sind, die nie publiziert wurden oder dafür vorgesehen waren. ${ }^{6}$ Im Zusammenhang mit der Digialisierung von "traditional cultural expressions", spielt weiterhin, wie auch Benjamin wusste, eine Rolle, dass Traditionen lebendig sind und sich im Gegensatz zu einem archivierten Objekt verändern (vgl. Benjamin 2006:19-20). Genau wie beim Kunstwerk ist auch hier die reproduzierte traditional cultural expression aus ihrem alltäglichen Zusammenhang herausgetreten und fixiert. Zum Beispiel kann eine Tonaufnahme aus dem Zusammenhang entstanden sein, traditionelles Liedgut für eine wissenschaftliche Hausarbeit aufzuzeichnen. Werden die Aufnahmen in einem Schallarchiv gelagert und Jahrzehnte später gefunden, so können sie zu neue Bedeutungen erlangen, zum Beispiel für die Nachfahren der aufgezeichneten Sänger (Danielson

${ }^{6}$ Institutionell und inhaltlich höchst unterschiedliche Beispiele sind das Indiana University Digital Library Program, Europeana oder die Digitalisierungsprojekte in den großen Phonogrammarchiven wie Wien oder Berlin, die unterschiedlichste Inhalte weltweit öffentlich zugänglich machen. 
2001). Aber auch im gesellschaftlichen Kontext können sich „öffentlich-westliche“ Archive, die bisher innerhalb klarer rechtlicher Grenzen operierten (zum Beispiel definiert durch Urheberrechte, Persönlichkeitsrechte und andere) plötzlich mit Rückgabeforderungen konfrontiert sehen, die eher ethischer als rechtlicher Art sind (vgl. Seeger 2001). Hier entstehen neue Herausforderungen für die Archive beziehungsweise andere Traditions-Träger der online recherchierbaren Digital„Quellen“: Materialien, die vielleicht nur zur Aufbewahrung oder wissenschaftlichen Erforschung in einer Institution abgegeben worden waren, können, falls sie schon digitalisiert und über das Internet der Öffentlichkeit zugänglich gemacht worden sind, nicht mehr zurückgerufen werden.

\section{Technik als Querschnittsdimension der Entstehung von Cultural Property und Kulturerbe}

Die Ungebundenheit der digitalen Objekte und die massenhafte Entstehung privater und öffentlicher digitaler Archive sind Kennzeichen der Digitalisierung. Erst die Digitalisierung hat das "enabling potential" (Schönberger 2006), dass Nutzer/innen Daten in großen Mengen kopieren, tauschen und sammeln können. Die Musikindustrie hat besonders mit einer unintendierten Folge der Digitalisierung zu tun: die unkontrollierte Ausbreitung von vormals nur auf Industrietonträgern zugänglichen Daten mit Hilfe von Festplatten in der Hand Musikliebhaber/innen schafft für die Musikindustrie große Dynamiken, auf die sie bislang keine Antwort gefunden haben. Durch die digitalen Speichermöglichkeiten erweitern sich ebenso die Möglichkeiten der Fixierung von kulturellen Ausdrucksformen (vgl. Beitrag Engelbrecht in diesem Band). Beim Filmen auf Zelluloid konnten Forscher/in und Forschungsteilnehmer/innen einfache Verträge schließen (meist mündlich, vgl. ebd.), weil klar war, dass die Film- und Tonrollen irgendwo in Europa in einem Keller lagern und von Zeit zu Zeit vorgeführt würden. Digitale Daten schaffen andere Verhandlungsgegenstände: plötzlich können die eigenen Tänze, Ideen, Lebensweisen weltweit und unkontrolliert zirkulieren und in völlig neue Zusammenhänge eingebaut und verwertet werden. Die massenhafte Vervielfältigung kann, ökonomisch betrachtet, einen Einfluss auf den Wert des Dargestellten haben. Das Beispiel des kambodschanischen Apsara-Tanzes (vgl. Beitrag Eggert in diesem Band) sei hier genannt: Innerhalb kürzester Zeit ist hier ein vormals nur dem Königshaus vorbehaltener Tanz als UNESCO "intangible heritage" zertifiziert worden und dadurch zu einer kulturellen Chiffre Kambodschas geworden. Damit entstehen neue Märkte im Tourismus-Sektor, wie Tanzschulen, Tanzgruppen, und Reiseandenken, die sich auf Apsara beziehen. Die massenhafte, auch multimediale, Verbreitung von kulturellen Gütern ist einer der Gründe einerseits für die Versuche, über Cultural Property-Regimes Ansprüche durchzusetzen oder andererseits über Kulturerbe-Politiken zumindest in zertifizierte Bahnen zu lenken. 
Bisher ist besonders auf die durch „die Technik“ hervorgebrachten Dynamiken eingegangen worden, was freilich eine verkürzte Fragestellung ist. Dies ist dem Anspruch geschuldet, in einem ersten Schritt zu beschreiben wie die kulturwissenschaftliche Technikforschung für die Untersuchung von Cultural Property und Cultural Heritage Forschungsperspektiven - Erfahrungsgeschichte, Techniknutzungen oder Materialität - eröffnen kann. Die zunehmende Einschreibung von Technik in die verschiedenen Bereiche und Ebenen der Lebenswelt wirft die Frage auf, wie und warum sich Technik überhaupt durchsetzt (und andere nicht). Betrachtet man einige technische „Leitfossile“ (Martin Scharfe), zum Beispiel Flugzeug, Auto, Telefon, Computer oder das Internet, so fällt auf, dass diese dazu führten, dass bestimmte Aufgaben mit dem Einsatz der gleichen Mittel effektiver zu bewältigen waren: schnellere und sicherere Fortbewegung, direkte mündliche Kommunikation, Verarbeitung immer komplexerer Daten mit weniger Aufwand sind einige Schlagwörter. Die technische Moderne und die bei der Entstehung von Cultural Property und Cultural Heritage beteiligten technischen Entwicklungen sind in kapitalistischen Wirtschaftsformen entstanden. Kulturwissenschaftliche Technikforschung muss hier auch nach den Akteuren des technischen Wandels fragen. Der Grund für die Durchsetzung einer Technik sind die alltäglichen wirtschaftlichen Interessen der Technikanwender, die mobiler sein wollen, die etwas zu transportieren haben, besser verwalten wollen, oder Wissen zugänglich machen wollen. Hier wird die „soziale Konstruktion der Technik“ (zum Beispiel Bijker et al. 1987) sichtbar, in der sich die Interessen der system builders ausdrücken. Die Nutzer/innen können dabei aber mitbestimmen, wie in Deutschland die Atom-Ein- und Ausstiege, in China die Internet-Zensur oder in vielen Bibliotheken die Handy-Verbote zeigen: Bei aller Veränderung von traditionellen Lebenswelten durch die Technik - darüber, welche Technik es geben soll und wie sie eingesetzt werden soll, entscheiden menschliche Akteure selbst.

\section{Technik, Archivierung und die Wiederanwesenheit der Kultur}

Die Veränderungen, die zu den Rettungsaktivitäten der Volkskultur während der Moderne geführt haben, werden heute unter dem Schlagwort der „Globalisierung“ zusammengefasst. Die Globalisierung schafft weltweit einen Veränderungsdruck für regionale Kulturen: Abwanderung in die Städte, neue Berufe oder Migrationsbewegungen verändern die Rahmenbedingungen für die Reproduktion von Kultur, weil überlieferte Traditionszusammenhänge ,verschwinden“. Die gegenwärtige Deutung der Globalisierung als Metaprozess, der regionale Kulturen bedrängt und nivelliert führt erneut zu umfassenden Inventarisierungsbemühungen. Im internationalen Kontext nahmen und nehmen die westlich-hochkulturell konzipierten UNESCO World Heritage und Property-Listen den Rettungsgedanken mit unterschiedlichen Schwerpunkten auf. Die Sammlung und Auflistung kultureller Phä- 
nomene hat bisher zu zahlreichen Kulturerbe-Listen (UNESCO World Heritage, Intangible Heritage, Memory of the World) geführt und wird aktuell weitergehend verhandelt (Cultural-Property-Regimes, WIPO).

Die kulturpolitische Praxis der Inwertsetzung von kulturellen Gütern (durch Hervorheben, Zertifizieren, Inventarisieren, Nutzen) ist nur eine Seite von Kulturerbe und Cultural Property. In den Ansprüchen an kulturelle Güter zeigt sich auch die globale Diffusion und Professionalisierung ethnographischer Arbeit, die bei den Prozessen der Konstruktion von Kulturerbe zur Anwendung kommt (und die das Thema einer detaillierten Untersuchung über die Personalunion von Forscher/innen, Berater/innen und den Austausch von spezifischem ethnographischem Wissen zwischen Forschung und UNESCO wäre). So wurden in der Volkskunde unter den Stichworten Folklorismus und Historismus in den 1980er Jahren ähnliche Phänomene diskutiert, die nun unter den Begriffen „Cultural Property“ beziehungsweise „Kulturerbe“ mit anderen Schwerpunkten in den Blick genommen werden. Nahm die Debatte damals vor allem Fragen der Erlebnis- und Kompensationsfunktion von Traditionen im regionalen Kontext auf, so werden die als „Kulturerbe“ wiederkehrenden Traditionen gegenwärtig als globales Phänomen und ihre Nutzung als Ressource untersucht. Mit der Verschiebung und Erweiterung ethnographischen Wissens über Kulturerbe und Cultural Property ändern sich auch die Schwerpunkte ihrer Regulierung: die 1954 im Angesicht der Erfahrungen des Zweiten Weltkriegs verabschiedete Haager Konvention sollte noch das bewegliche und unbewegliche "Property" der Nationalstaaten schützen - Gebäude, Kunstwerke, archäologische Stätten. Als knapp 20 Jahre später (1972) die erste Welterbekonvention in Kraft trat, trat zum (kunst)historisch bedeutsamen Kulturerbe auch das Naturerbe hinzu. Seit 2003 schützen die Unterzeichnerstaaten des „Übereinkommens zur Bewahrung des immateriellen Kulturerbes“ ergänzend die „Praktiken, Darbietungen, Ausdrucksformen, Kenntnisse und Fähigkeiten [...] sowie die damit verbundenen Instrumente, Objekte, Artefakte und Kulturräume [...], die Gemeinschaften, Gruppen und gegebenenfalls Individuen als Bestandteil ihres Kulturerbes ansehen" (UNESCO 2003). Die Auflistung und Zertifizierung von Kulturgütern verlangt also, wie die Begriffe anzeigen, eine Menge ethnographisches Wissen, um das Herausheben eines kulturellen Gutes angemessen zu begründen. Dabei kommt zunehmend Technik zum Einsatz, denn im Gegensatz zu Dingen müssen nichtdingliche Praktiken, Kenntnisse und Fähigkeiten medial aufgezeichnet werden, um sie mit einem Prädikat versehen zu können. Die Erweiterung des Property- beziehungsweise Heritage-Begriffs und damit des zu Schützenden geschieht also einerseits durch Technik: sie ist als Speicher für die immaterielle Kultur in die Entstehung und Verbreitung des Kulturguts involviert. Andererseits erweitert sich ständig das ethnographische Wissen über Kultur, das auch in die Kulturpolitik zurückwirkt, dessen Verbreitungswege und Wirkungsweisen jedoch erst in Ansätzen untersucht sind (vgl. Bendix und Welz 2002).

Erst durch die Aufzeichnungs- und Speichertechnik sind nichtdingliche kulturelle Ausdrucksformen auflistbar geworden. Eine Liste ist "a 'purposeful collecti- 
on' pretending indifference but calling for action", schreibt der Stadtplaner und Kulturökonom Mark J. Schuster (2002:04). Dabei bezieht er sich insbesondere auf die Zertifizierungsfunktion: eine Liste sagt nicht nur, was zu schützen ist (nämlich das Aufgeführte), sondern auch, dass etwas zu schützen ist. Eine weitere Eigenschaft von Listen ist, dass sie zur Vorbereitung von "policy actions" genutzt werden: "[Fe]deral listing may trigger a fuller range of policy actions that are embedded in state or local law." (Schuster 2002:08). Auch im internationalen Recht, wie die UNESCO-Konventionen und WIPO-Diskussionen zeigen, spielen Auflistungen eine wichtige Rolle für die Vorbereitungen von Schutz- oder Regulierungsmaßnahmen.

Die Auflistung von kulturellen Phänomenen wird erst dadurch möglich, dass die in der Liste aufgeführten Positionen sich direkt auf Inhalte beziehen. Diese Inhalte referenzieren im Falle des nichtdinglichen Kulturerbes, aber auch für immaterielle traditional cultural expressions (digitale) Archivalien. Die neuen Umgangspraktiken mit digitalen Daten und die Entstehung von online aufgelisteten Kulturinventaren haben zu einer Neubestimmung und Umdeutung des Archivbegriffs geführt, der durch die massenweise Archivierung digitaler Dokumente mitausgelöst wurde. In den Geistes- und Kulturwissenschaften werden Archive neu verhandelt: wurde das Archiv bis vor zwei Dekaden noch in den historischen „Hilfswissenschaften" als Ort gedacht, in dem (originales) Archivgut zuhanden ist, so ist der Archivbegriff nun ein erweiterter. Daten, die einmal von der analogen in die digitale Welt getreten sind, können über den gesamten Globus archiviert werden. Jedwede Sammlung kann am Anfang von Forschungen stehen und eine mögliche Grundlage für ein Archiv sein (vgl. te Heesen und Spary 2002). Damit ist das Archiv in der Foucault-Rezeption „das Gesetz der Gesamtheit dessen, das überhaupt gesagt werden kann“ (Ernst 2002:20) oder „das allgemeine System der Formation und der Transformation der Aussagen" (18).

Vor dem Hintergrund von online verfügbaren Texten in digitalen Archiven erscheint ethnographische Forschung zunehmend als „Kommentar“ - ein Genre, das auf die Kopräsenz eines anderen Texts verweist -, denn als Erzählung über ein abwesendes Objekt (vgl. Fabian 2008). Die „vormodernen“ Lebenswelten sind Ende des 19., Anfang des 20. Jahrhunderts in den Museen verschwunden. Viele der heute aufgelisteten Kulturgüter konnten durch Herausgreifen, technische Archivierung, Auflistung, Dokumentation konserviert werden, andere gegenwärtige Verhandlungsgüter sollen für ihre Trägergruppen bewahrt werden und ihnen zugute kommen. Cultural Property und Kulturerbe wären in dieser Lesart Kommentare zu kulturellen Phänomenen, die zunehmend aus ihrer Abwesenheit geholt werden. 




\title{
Der zunehmende Geltungsbereich von Cultural Properties und ihrer Politik ${ }^{1}$
}

\author{
Rosemary J. Coombe
}

\section{Einleitung: Der rechtliche, ökonomische und politische Kontext}

Die potenziellen Themen für eine Untersuchung der juristischen und sozialwissenschaftlichen Literatur zu Cultural Property haben sich im letzten Jahrzehnt exponentiell vervielfacht. Im internationalen Recht war es früher möglich, Cultural Property und kulturelles Erbe als zwei getrennte Kategorien zu betrachten, aber schon damals beklagten Kommentatoren die Tatsache, dass die entsprechenden Begriffe in den verschiedenen Sprachen selten Übersetzungen der gleichen Konzepte waren. Biens culturels, beni culturali, bienes culturales, Kulturgut und bens culturais zum Beispiel haben nicht die gleichen juristischen Bedeutungen (Frigo 2004:370). Solche Interpretationsschwierigkeiten erscheinen nunmehr provinziell. Auf jeden Fall versprechen diese Schwierigkeiten nur größer zu werden, je mehr sich diese Kategorien ausweiten, ihre Unterscheidungen zusammenbrechen und ihre Thematik und Sachgebiete stark zunehmen.

Das Konzept Cultural Property ist nicht länger ein esoterisches Rechtsgebiet, das sich dem Schutz von Altertümern und deren genauer Herkunft verschrieben hat,

\footnotetext{
1 Translated and reprinted, with permission, from the Annual Review of Law and Social Science, Volume 5 (C) 2009 by Annual Reviews — www.annualreviews.org. Übersetzung aus dem Englischen von Julia Heinecke.
} 
sondern bezieht sich heute auf immaterielle und materielle Güter - von Folklore ${ }^{2}$ über Nahrungsmittel und Lebensarten bis zu den Landschaftsräumen, aus denen sie stammen. Vom Saatgut bis zur Meereslandschaft - die Welt der Dinge mit kultureller Bedeutung und die Streitigkeiten um die Eigentumsrechte, die ihnen zugeschrieben werden und die ihrer Bedeutung entsprechen, haben an Umfang und Komplexität dramatisch zugenommen.

Die Ursachen und Konsequenzen der Zunahme an Cultural Property und an dem noch größeren Bereich der Ansprüche an kulturellen Rechten zu verstehen, ist ein natürliches Untersuchungsgebiet für Rechts- und Sozialwissenschaftler und das Feld für rechtssoziologische Studien. Jedoch ist wohl nur ein sehr kleiner Teil der verfügbaren Lehre so interdisziplinär ausgerichtet, wie es die Politik dieses dynamischen Feldes idealerweise erfordert. Wenige Wissenschaftler verstehen die internationalen rechtlichen Rahmenbedingungen und die transnationalen Politikinitiativen vollständig, die Regierungen, Nichtregierungsorganisationen (NGOs), Entwicklungsträger, multilaterale Institutionen, indigene Völker und Gemeinschaften in unterschiedlichem Maße antreiben, um "culture as a resource" (Yúdice 2003) zu behandeln. Etwas mehr hat sich getan, um diese Bewegung mit neuen Mustern der Kapitalbildung in einer globalen politischen Wirtschaft, in der Informationskapital (Castells 1996-1998) an neuer Bedeutung gewonnen hat (Verdery und Humphrey 2004, Watts 2006), in Beziehung zu setzen. Wissenschaftler fangen jedoch gerade erst an, die empirischen Ausprägungen des Informationskapitalismus beim Aufkommen von Kultur als Ressource zu berücksichtigen (Harvey 2001, Parry 2004, Whatmore 2002).

Kultur als Ressource betrachtet umfasst eine größere Bandbreite an Werten als Kultur, bei der, als reiner Vermögenswert aufgefasst, dazu geneigt wird, nur die ökonomische Seite zu betonen. Diese Werte beinhalten sozialen Zusammenhalt, Autonomie der Gemeinschaft, politische Anerkennung und Sorge über unangemessene Formen kultureller Aneignung und Falschdarstellung sowie den Verlust von Sprachen und lokalem Wissen. Die zuletzt genannten Befürchtungen stehen in engem Zusammenhang mit der Verbreitung neuer Kommunikationstechnologien, durch die es möglich geworden ist, kulturelle Formen in einer nie zuvor gekannten Geschwindigkeit zu reproduzieren und zu veröffentlichen (Burri-Nenova 2008). Hat die Digitalisierung den Prozess der sozialen Dekontextualisierung beschleunigt, so hat sie gleichzeitig aber auch die Wahrnehmung über die Ausbeutung von Ressourcen kulturellen Erbes geschärft und das politische Bewusstsein über die hieraus entstehenden Verletzungen erhöht (Coleman und Coombe 2009). Gleichzeitig wurden neue Initiativen für eine politisch sinnvolle Handhabung und ge-

\footnotetext{
2 A. d. Ü.: Der Begriff "folklore" wird in dieser Übersetzung beibehalten, da die Autorin ihn gemäß der wertfreien Konnotationen, um die man sich in internationalen juristischen Debatten bemüht, nutzt; er sollte nicht mit der gängigen pejorativen Deutung, die im deutschen Sprachgebrauch üblich geworden ist, gelesen werden.
} 
meinschaftliche Nutzung von Ressourcen kulturellen Erbes auf den Weg gebracht (Christen 2008, 2009, Kansa et al. 2005).

Weitere Forschungen sind nötig, um die Geltendmachung von Cultural Property mit dem politischen Klima, in dem indigene Völker sich beispiellose, neue Rechte gesichert haben, zu verbinden (Filbo und de Souza 2007, Gow 2008, Hirtz 2003, Sylvain 2002, 2005), und um die Revitalisierung indigener Rechte und Identitäten ebenso mit dem Neoliberalismus (Clark 2005, Coffey 2003, Hale 2002, Hristov 2005, Jung 2003, Perreault 2005, Speed 2008) in Beziehung zu setzen wie mit den auf Rechten basierenden Praktiken, die zunehmend angewandt werden, um sich neoliberaler Entwicklungsprogramme zu erwehren (Goodale 2007). Kulturelle Ansprüche sind ein zentraler Punkt im gemeinsamen Kampf vieler Randgruppen, für die Kultur ein Konzept ist, das reflexartig verwendet wird, um sich mit größeren staatlichen oder Nichtregierungsinstitutionen für die Behauptung von Identität, mit einer stärkeren Einbindung ins politische Leben, der Verteidigung der lokalen Autonomie und neuen Formen des Engagements in globalen Märkten (genauso wie Widerstand dagegen) zu befassen. Die wissenschaftliche Anerkennung dieser neuen Politik von Cultural Property und kultureller Rechte hat das akademische Interesse an den Bedingungen kulturellen Bewusstseins und den Beziehungen von Objektifizierung, Reifizierung, Authentizität und Dekontextualisierung erneuert (Clifford 2004, Handler 2003, Harrison 2000, Kaneff und King 2004, Kirsch 2005).

Kulturelle Rechte im internationalen Recht umfassen Rechte an geistigem Eigentum (oder, allgemeiner gesprochen, Rechte an moralischen und materiellen Interessen an Werken, für die es einen Urheber gibt), Rechte von Minderheiten, kulturelles Erbe zu pflegen und zu entwickeln, Rechte an der Teilnahme am kulturellen Leben, Rechte an der Profitierung von künstlerischen und wissenschaftlichen Leistungen sowie Rechte an internationaler kultureller Zusammenarbeit (Helfer 2007, Macmillan 2008, Symonides 1998, Yu 2007). Diese werden noch erweitert durch die Bestimmungen zum kulturellen Erbe in der UN-Erklärung über die Rechte der indigenen Völker von 2007, die selbst als Entwurf schon ein wichtiger Teil des Völkergewohnheitsrechtes war, um andere Rechte zu interpretieren (Coombe et al. 2007). Regionale Menschenrechtsinstrumente machen auch die kulturellen Rechte von Kollektivitäten geltend (Jovanovic 2005). UNESCOKonventionen jüngeren Datums haben einen neuen Schwerpunkt auf das immaterielle kulturelle Erbe und auf die kulturelle Vielfalt gelegt, was zu einer stärkeren Überprüfung von Kulturgütern von staatlicher Seite und einer größeren Reifizierung kultureller Traditionen geführt hat (Albro 2005a, 2005b, 2007). Egal, ob das Ziel die Entwicklung des ländlichen Raumes, Umweltverträglichkeit oder eine auf Rechten basierende Entwicklung ist: Eine Betonung auf der Aufrechterhaltung (und in einigen Fällen der Profitierung) von kulturellen Unterschieden hat auf der internationalen Bühne neue Bedeutung erlangt (Coombe 2005a, Ensor 2005, Radcliffe und Laurie 2006a, 2006b). Sicherlich schließen nicht alle Bemühungen um kulturelle Rechte auch Ansprüche auf Cultural Property ein. Doch in 
dem Maße, in dem man bei der Geltendmachung kultureller Rechte dazu neigt, eine neue, besitzanzeigende rhetorische Form anzunehmen, und die neoliberale ideologische Dominanz von Regierungsseite sowie institutionelle Reformprogramme eher marktbasierte Lösungen betonen, wird Cultural Property immer im politischen Blickfeld dieser Diskurse, Praktiken und Kontroversen sein.

Neue Formen, zum Beispiel von kultureller oder „Ethnoentwicklung“, die Ökotourismus und die Kultivierung von kulturell typischen Exportgütern beinhalten können, sind als Mittel eingesetzt worden, um die Wiederbelebung der ländlichen Wirtschaft, den sozialen Zusammenhalt, menschliche Sicherheit und politische Autonomie umzusetzen (Andolino et al. 2005, Aylwin und Coombe 2010, Laurie et al. 2005, Perreault 2003a, 2003b, Radcliffe 2006b, Rhoades 2006). Dies ist ein typischer Bereich neoliberaler Gouvernementalität, der sowohl multilaterale Institutionen als auch NGOs umfasst, die bestrebt sind, lokale Gemeinschaften zu stärken, Traditionen als Quellen des sozialen Kapitals anzuerkennen (Bebbington 2004b, Dervyttere 2004, Perreault 2003c) und anderweitig Menschen zu ermutigen, sich eine besitzanzeigende und unternehmerische Haltung hinsichtlich ihrer eigenen Kultur und der sozialen Reproduktionsbeziehungen, die sie traditionell getragen haben, anzueignen (Elyachar 2005, Greene 2004, Lowrey 2008). Diese Darstellungen haben ihren Ursprung in diversen internationalen Rechtsinstrumenten und deren Interpretation sowie in der institutionellen Politik (Kingsbury 1999), die auf diese reagiert, und im Diskurs über Menschen- und indigene Rechte, welcher die Reaktionen auf diese Politik auf lokaler, NGO- und transnationaler Ebene prägt. Letztere bieten normative Ressourcen für eine alternative Artikulierung von Kultur als Quelle einer Moralökonomie, einer sozialen Bedeutung und einer würdevollen Existenz (Edelman 2005, Gow 2008, Perreault 2005b, Saugestad 2001, StewartHarawira 2005).

Die Umsetzung mehrerer internationaler Vereinbarungen und neuer Programme rechtlicher Verhandlungen zeigen in der letzten Zeit eine Beschleunigung der globalen Politikgestaltung in Bezug auf Kultur. Das Übereinkommen über handelsbezogene Aspekte der Rechte des geistigen Eigentums (TRIPS, TradeRelated Aspects of Intellectual Property Agreement), die Biodiversitäts-Konvention (CBD, Convention on Biological Diversity)/Arbeitsgruppe für Artikel 8(j), die Vertragsentwurfverhandlungen des Intergovernmental Committee on Intellectual Property and Traditional Knowledge, Traditional Cultural Expression and Genetic Resources der Weltorganisation für Geistiges Eigentum (WIPO, World Intellectual Property Organization) zum Schutz des traditionellen Wissens und traditioneller kultureller Ausdrucksformen sowie Vorschläge von Staaten für ein internationales Rechtsinstrument zur Bindung von Mitgliedsstaaten, das Programm "Indigenous Knowledge for Development" der Weltbank und die Passage der UNESCO-Verträge zum Schutz und zur Förderung der Vielfalt kultureller Ausdrucksformen sowie zum Schutz des immateriellen Kulturerbes (Kulturkonvention, UNESCO Treaties on the Protection and Promotion of the Diversity of Cultural Expressions and the Protection of Intangible Cultural Heritage): Sie alle 
gestalten wohl soziale Beziehungen vor Ort um, während Orte gleichzeitig in transnationalen Aktivitätsnetzwerken verknüpft werden.

Auch wenn die Rolle der NGOs und multilateralen Institutionen in der internationalen Politikgestaltung und die politische Bedeutung von Indigenität als globale Menschenrechtsbewegung in den letzten zehn Jahren auf erhöhtes akademisches Interesse stieß, gibt es dennoch bis jetzt kaum akademische Anerkennung für die bedeutende Rolle dieser Institutionen in der Praxis, durch die Eigentumsbeziehungen zu Kultur evoziert werden. Sie bringen neue Vorstellungen von Tradition und Moderne in Bezug auf lokale Praktiken ein (Bebbington 2004a), wobei das lokale Verständnis von den Beziehungen zwischen Natur und Kultur überarbeitet wird. Hierbei wird die Bedeutung der sozialen Verbundenheit zum Ort betont und die Menschen vor Ort ermutigt, ihre territorialen Beziehungen in Hinsicht auf Kultur und Eigentum auszudrücken (Escobar 2001, 2003, 2008). NGOs im Umwelt- und ethnologischen Bereich spielen eine wichtige Rolle bei den Prozessen, in denen Menschen sich selbst als indigen verstehen lernen, als, in den Worten der CBD, "local communities embodying traditional lifestyles" oder als Träger traditionellen Wissens über die Umwelt (Li 2000a, 2000b, Tsing 1999). NGOs können neue Formen der Gouvernementalität unter neoliberalen Regierungen ausüben (Bebbington 2005, Bryant 2002a, 2002b), zum Beispiel jene, die versuchen, die biologische und kulturelle Vielfalt zu schützen, traditionelles Wissen und traditionelle kulturelle Ausdrucksformen zu lokalisieren, immaterielles Kulturerbe zu inventarisieren und typische Kulturgüter zu vermarkten (Coombe 2010a, 2010b). Hierbei handelt es sich um einen Bereich, der wenig rechtssoziologische Aufmerksamkeit erfährt.

Es ist unmöglich, die wissenschaftliche Literatur in all diesen Bereichen zu überprüfen, die für Cultural Property relevant ist. Stattdessen werde ich den Schwerpunkt auf Bereiche legen, auf die sich die Forschung besonders stark konzentriert hat. Hierbei argumentiere ich, dass die starke Zunahme an Ansprüchen auf Cultural Property als Indikator und Anstoß für Veränderungen in politischen Beziehungen eine wichtigere Rolle spielen könnte als als Bereich, der einer inländischen oder internationalen Eigentumsrechtsreform bedarf, auch wenn solche Reformen offenbar in unterschiedlichem Maße in verschiedenen Rechtsprechungen kurz vor der Durchführung stehen. Produktion, Austausch und Konsum von Cultural Property beinhalten die Konstruktion, Anerkennung und Akzeptanz von sozialen Gruppen und Gruppenidentiäten in weltweiten öffentlichen Sphären genauso, wie es die Kontrolle über die Objekte per se betrifft. Sich verändernde Praktiken, Verhaltensweisen, Einstellungen und Vorschriften in Bezug auf kulturelles Erbe signalisieren und spiegeln gleichzeitig Wandlungen in sozialen Beziehungen wider, die größere Muster später Moderne und Dekolonialisierung erkennen lassen. 


\section{Umdenken beim kulturellen Erbe}

Laut des Geografen David Harvey (2001) hat eine Beschäftigung mit der Vergangenheit und mit der richtigen Behandlung von materiellen Objekten dieser Vergangenheit eine lange Geschichte, die ein eher allgemeines menschliches Interesse an individuellen und Gruppenidentitäten widerspiegelt. Auch wenn die Verwendung materieller Kultur zur Stärkung nationaler Ideologien wohlbekannt ist, argumentiert Harvey, dass eine Betonung dieses modernen Phänomens über Gebühr eine Beschäftigung mit viel zentraleren Fragen zur Verwendung des Erbes bei der Erzeugung von Identitäten und der Legitimation von Macht ausschließen kann (Harvey 2001:320-33). Gleichwohl dominiert dieser spezielle Diskurs über Erbe, der sich im Europa des 19. Jahrhunderts entwickelt hat, weiterhin Theorie und Praxis auf der ganzen Welt, indem seine Werte als universell dargestellt werden. Seine Ursprünge sind verbunden mit der Entwicklung des Nationalismus im 19. Jahrhundert und der liberalen Moderne, und auch wenn konkurrierende Diskurse auftauchen, "the dominant discourse is intrinsically embedded with a sense of the pastoral care of the material past" (Smith 2006:17). Eine Beschäftigung mit dem kulturellen Erbe entstand aus der Besorgnis moderner Staaten über die nationale soziale Kohäsion und Identität und der Notwendigkeit, der Bevölkerung nationale Gefühle und staatsbürgerliche Verantwortung einzuimpfen.

Eine Beschäftigung mit Monumenten als Zeugen der Geschichte und als Kunstwerke, die sich in der französischen Vorstellung von patrimoine und der von der Romantik abgeleiteten englischen Konservationsbewegung widerspiegelt, bürgerte sich im 20. Jahrhundert international ein. Kritische Wissenschaftler zeigen, wie so die Macht der nationalen Eliten gestärkt, die Gestalt ausgedünnter Expertengremien aufrechterhalten, soziale Erfahrungsunterschiede geleugnet und nicht-nationale Gemeinschaftsidentitäten ignoriert und verschleiert werden, während die Öffentlichkeit zu passiven und unkritischen Konsumenten statt zu aktiven Schöpfern und Interpreten des Erbes gemacht werden.

In den letzten zwanzig Jahren haben Erbwissenschaftler ihre Aufmerksamkeit weg von konkreten Stätten, Objekten und Lokalitäten auf die durchdringende Nichtgreifbarkeit und Unvorhersehbarkeit von Erbe gelegt (Munjeri 2004). Das, was aus Dingen, Monumenten und Orten ein „Erbe“ macht, sind nicht inhärente kulturelle Werte oder eine ureigene Bedeutsamkeit, sondern "the present-day cultural processes and activities that are undertaken at and around them" (Smith 2006:3), durch die ihnen ein Wert und eine Bedeutung beigemessen werden. Solche Prozesse, wie die Verwaltung, die Erhaltung und Führung von Orten, Stätten und Objekten, sind daher grundlegend für ihre kulturelle Bewertung. Kurz, "heritage is a multi-layered performance - be this a performance of visiting, managing, interpretation or conservation - that embodies acts of remembrance and commemoration while negotiating and constructing a sense of place, belonging and understanding in the present" (ebd.). Der kulturelle Prozess der Identitätsbildung, der grundlegend und wesentlich für das Erbe ist, ist jedoch durch eine ideologische 
Betonung auf Dinge oder Objekte und deren Ursprung verschleiert worden - verknüpft mit und definiert durch Konzepte der Monumentalität und Ästhetik (4). Dieser ,autorisierte Erbdiskurs“ dient der Auslöschung subalterner und populärer Praktiken, durch die angenommene Werte infrage gestellt, die Bedeutungen aus der Vergangenheit verhandelt und überarbeitet sowie Gemeinschafts- und Gruppenidentitäten sozial projiziert, wahrgenommen und angezweifelt werden. Ein neues Verständnis von Erbe hat sich sowohl aus einer Gegenbewegung zur Professionalisierung des Kulturerbemanagementbereichs als auch aus den Kampfansagen der Minderheiten und indigenen Völker an die monologen Erzählungen über nationale Geschichte und Identität, die sich negativ auf ihre Darstellung und ihr Selbstverständnis auswirken, entwickelt.

Ein praktisches Beispiel, durch das sich archäologische und Erbstudien mit Identitätspolitik zu befassen begannen, beinhaltet kulturelles Ressourcenmanagement (CRM, Cultural Resource Management), die Politikstrategie und Vorgehensweisen, um Kulturerbe-Objekte, -Stätten, -Orte und -Monumente zu schützen, zu erhalten und/oder zu konservieren. Gleichzeitig werden durch diesen Prozess die archäologischen Datenbanken erhalten und gepflegt (1). Jene Gegenstände, die von Archäologen als von universellem kulturellen Wert gehandhabt werden (aber oft vom Staat als nationales Erbe beansprucht werden), sind für die Identität anderer oft äußerst wichtig, wie die Ausuferung von Konflikten zwischen Archäologen und indigenen Völkern auf dem amerikanischen Kontinent und in Australien deutlich zeigt. Durch CRM, so die Argumentation der Erbwissenschaftlerin Laurajane Smith (2-3), wird archäologisches Fachwissen und Kompetenz von öffentlichen Entscheidungsträgern aufgeboten, um die Führung oder Regulierung erlaubter Ausdrucksformen sozialer oder kultureller Identität zu unterstützen:

The way in which any heritage item, site or place is managed, interpreted and understood has a direct impact on how those people who associated with, or who associate themselves with, that heritage, are themselves understood and perceived. The past, and the material culture that symbolizes that past, plays an important part in creating, recreating and underpinning a sense of identity. [...] Various groups or organizations and interests may use the past to give historical and cultural legitimacy to a range of claims about themselves and their experiences in the present. (2)

Als eine Form der Fachkompetenz und als eine geistige Disziplin, die in westlichen Gesellschaften in Debatten über die Vergangenheit bevorzugt wird, ist Archäologie eine Form des Wissens, die als Regierungstechnologie fungiert. Das Wissen der Archäologie, ihre Techniken und Verfahren werden bei der Bevölkerungsregulierung und der Steuerung sozialer Probleme, die mit den Behauptungen über die Bedeutung der Vergangenheit und ihrem Erbe zusammenspielen, aufgeboten. Diese werden von Regierungen und Entscheidungsträgern, die durch CRM konkurrierende Forderungen und Behauptungen in Bezug auf die Vergangenheit von verschiedenen Interessenseiten klären und schlichten, benutzt. Darüber hinaus 
wird archäologisches Fachwissen herangezogen, um die Interessen und Bevölkerungen zu definieren, die mit sozialen Problemen, die sich mit einem besonderen Verständnis der Vergangenheit überschneiden, verbunden werden. Daher spielt diese Disziplin bei der Legitimierung oder Delegitimierung von Interessen eine Rolle, vor allem in postkolonialen Zusammenhängen, in denen Menschen versuchen, Ansprüche an Land, Souveränität und nationaler Identität durchzusetzen; "archaeological knowledge, and the discourse that frames that knowledge, can and does have a direct impact on people's sense of cultural identity, and thus becomes a legitimate target and point of contention for a range of interests" (3).

Die Thematik des kulturellen Erbes, so erkennen Wissenschaftler nun weithin an, ist nicht eine Ansammlung von materiellen Gegenständen der Vergangenheit Stätten, Orte und Objekte - mit inhärenten historischen Werten, die ordentlich besessen, kontrolliert und betrieben werden können. Vielmehr handelt es sich um einen Satz an Werten und Bedeutungen, die in einem größeren Bereich sozialer Praktiken angefochten und verhandelt werden. Solche Dinge haben einen Wert nicht nur wegen ihrer inhärenten Bedeutung, sondern aufgrund ihrer Rolle in der Überlieferung von Identitäten und Werten (Smith 2006). Eine wachsende Bewegung identifiziert und rechtfertigt die Wünsche, zum Beispiel lokale Gemeinschaften vollständig in das Management des Erbes einzubinden (Buggey und Mitchell 2008), und die archäologische Praxis hat, wie ich weiter unten diskutieren werde, sich langsam dahingehend entwickelt, indigene Kritik in Theorie und Forschung mit einzubeziehen (Meskell 2002, Nicholas und Bannister 2004).

Die Wertschätzung kulturellen Erbes ist sicherlich ein wiederbelebtes Betätigungsfeld der Kulturerbepolitik. Kulturelles Erbe wird nun von kritischen Fachleuten im Feld als "culture and landscape cared for by communities" verstanden, die in die Zukunft weitergegeben werden müssen, um dem Bedürfnis der Menschen nach Identität und Zugehörigkeit Rechnung zu tragen, gleichzeitig aber auch, um als Grundlage eines neuen Wirtschaftszweiges zu dienen (Loulanski 2006:209). Das Erbe überbrückt die Gräben zwischen Kultur und Wirtschaft (und zunehmend auch der Umwelt). Konservierung ist nicht mehr primär auf die Erhaltung von Monumenten ausgerichtet, sondern orientiert sich an zukünftigen Nutzungsformen für gesellschaftliche Zwecke. Sie bezieht charakteristische Lebensstile in einzigartigen Umgebungen ein und umfasst nicht mehr ausschließlich Ausstellungen, Archive oder touristische Stätten; vielmehr hat es eine Schwerpunktverlagerung weg von der Bestandswahrung zu nachhaltigen Benutzung gegeben (211). Staatlich geleitete Projekte zur Lagerung von Objekten in Institutionen werden zunehmend zugunsten von gemeinschaftsbasierter Forschung und Entwicklung mit einem Fokus auf die Verbesserung der Lebensumstände vor Ort und der Existenzgrundlagen zurückgewiesen (ebd.). Durch die Kombination von natürlicher und kultureller Umgebung wird der Schutz des Kulturerbes nun mit nachhaltiger Entwicklung verknüpft, und die Kulturerbepolitik orientiert sich jetzt an der Erhaltung der Einheit von materieller (Objekte) und immaterieller (gelebte Erfahrung und Praxis) Kultur. 
Nach Untersuchungen der Kulturwissenschaftlerin Lisa Breglia (2006) hat die neoliberale Politik die Kontroversen über die wahren Hüter des kulturellen Erbes vermehrt. Die Zerschlagung von Unternehmen in Staatsbesitz und die Dezentralisierung der Kontrolle über kulturelle Institutionen haben zeitgleich zu neuen Formen der Kommodifizierung und zu neuen Formen der Identitätspolitik geführt. Stätten und Objekte kulturellen Erbes sind zunehmend den Kräften des Marktes ausgeliefert, wo verlangt wird, dass sie ihre Erhaltung sprichwörtlich selbst finanzieren; ihre Eingliederung in neue Formen von Touristikunternehmen provoziert heftige Reaktionen bei jenen, die diese als ihr eigenes kulturelles Erbe betrachten. Breglia beschäftigt sich vor allem mit Baudenkmälern, aber eine ähnliche Politik ist auch in Bezug auf Besitztümer von weltweiter kultureller Bedeutung erkennbar.

Obwohl Cultural Property oft entweder als allgemeines Erbe der Menschheit oder als unveräußerliches Eigentum einer Nation wahrgenommen wird, sind die Stellen, die mit seinem Schutz, seiner Erhaltung, Förderung und Entwicklung befasst sind, tatsächlich deutlich vielfältiger. So haben zum Beispiel vielerorts auf der Welt bestimmte Familien seit mehreren Generationen die Rolle der Betreuer archäologischer und religiöser Stätten inne. Auch andere Institutionen, die die Erhaltung der Stätten, Ausgrabungen und Forschungen unterstützt haben, mögen eigene Interessen verfolgen. Soweit sich diese Stätten auf angestammtem Gebiet befinden, können nachkommende Gruppen spezielle Ansprüche haben. Zweifellos ist diese Interessenvielfalt nicht neu. Zahlreiche Parteien mit konkurrierenden und sich manchmal widersprechenden Eigentumsbeziehungen zum Kulturerbe und seiner ordnungsgemäßen Behandlung kommen jedoch vielleicht deutlicher zum Vorschein, sobald der Staat entweder seine Schutzbehörden zurückzieht, seine Autorität delegiert oder aber die Entwicklung dieser Ressourcen für den finanziellen Profit aggressiver vorantreibt. In aktuellen theoretischen Arbeiten über Erbe wurde darauf reagiert, indem man von der Untersuchung des Erbes als materielle Kultur wegkam und es stattdessen als politische Praxis sozialer Beziehungen versteht (Breglia 2006:14).

Dem internationalen Rechtsgebiet des immateriellen Kulturerbes wurde kürzlich ein Schwung an politischer Energie zugeführt, die 2007 in der Konvention zur Sicherung des immateriellen Kulturerbes (Convention for the Safeguarding of Intangible Cultural Heritage) resultierte. In dieser Konvention wird der Schwerpunkt von der Anerkennung als nationale Meisterwerke auf die Erhaltung von gelebtem Erbe an der Grenze zwischen Natur und Kultur verlagert, wie sie durch die aktive Teilhabe der Gemeinschaften den Menschenrechten und den Prinzipien der nachhaltigen Entwicklung entsprechend gepflegt wird (Aikawa-Faure 2009). Ein weiteres Betätigungsfeld der Kulturpolitik verheißt durch die Erwähnungen der kulturellen Bedeutung und das Zelebrieren des kulturellen Unterschiedes, wie es die rechtliche und behördliche Umsetzung dieser Konvention anzutreiben verspricht, belebt zu werden (Bendix 2009a). 


\section{Die Politik des indigenen Kulturerbes}

Die vielleicht bemerkenswerteste Wandlung sozialer Praktiken rund um die Kulturerbepolitik beinhaltet die Rechte auf materielle Artefakte und geistiges Eigentum, die mit Stätten von kultureller Bedeutung für indigene Völker verbunden werden. Heftige Debatten darüber, wer die Vergangenheit kontrolliert, wer den Zugang zu Stätten, Material und Informationen regelt und wessen Interpretationen der Vergangenheit gelten sollten, haben die archäologische, kulturanthropologische und museale Forschung charakterisiert, wobei es insbesondere um Objekte von Bedeutung für Aborigines in Australien, First Nations in Kanada und Native Americans geht. Frühe Diskussionen legten den Schwerpunkt auf die Repatriierung, wobei Vorwürfe zur kulturellen Aneignung und zur Ordnungsmäßigkeit des Besitzes kultureller Güter umgekehrt und alte Ansprüche in Bezug auf künstlerische Artefakte wiederholt wurden (Glass 2004). Reaktionen auf die Vorwürfe von Aneignungen sind in ihrer Natur jedoch fortwährend weniger exklusiv, sondern zunehmend inklusiv geworden.

Archäologen behandelten ihr Material herkömmlicherweise als empirische Aufzeichnungen einer universell definierten kulturellen Vergangenheit, die das wissenschaftliche Verständnis eines allgemeinen Kulturerbes bereichert haben. Keiner lebenden Gruppe wurde zuerkannt, irgendein berechtigtes Recht zu haben, den Forschungsauftrag der wissenschaftlichen Experten zu begrenzen (Nicholas und Wylie 2009:15). Solche Überzeugungen wurden einer verstärkten Überprüfung unterzogen. Nunmehr ist bestätigt, dass sehr wenige Archäologen ihre wissenschaftliche Arbeit gänzlich unvoreingenommen betreiben, und die Nachfahren der Gemeinschaften haben diese Bedingungen infrage gestellt. Archäologen sind zunehmend einer größeren Gruppe von Anspruchsberechtigten gegenüber rechenschaftspflichtig, die die Privilegierung ihrer angeblich gänzlich wissenschaftlichen Interessen nicht akzeptieren. Die Passage im Native American Grave Protection and Repatriation Act (NAGPRA) in den USA von 1990 war nur eine von vielen Würdigungen der Rechte der nachkommenden Gemeinschaften, die Fachleute nunmehr anerkennen. Forderungen nach der Rückgabe von Artefakten, historischen Fotografien und ethnografischen Informationen sind allgemein üblich geworden. Ethische Fragen nach der Rechenschaftspflicht und professionellen Verantwortung gehen jetzt über Fragen der Verwaltung der archäologischen Aufzeichnungen hinaus und umfassen Verantwortlichkeiten für das Wohl und die Ermächtigung dieser nachkommenden Gemeinschaften, deren Cultural Property (nicht nur Objekte von kultureller Bedeutung für sie, sondern auch solche Besitztümer, die für ihre Kultur als repräsentativ gelten) in die archäologische Forschung einbezogen sind.

Wenngleich einige Gemeinschaften einschließlich einiger Nationalstaaten exklusive Eigentumsmodelle eingeführt haben - an ihrem Kulturerbe interessierten Forschern den Zugang zu verwehren, alle entstehenden Rechte am geistigen Eigentum aus der Forschung zu beanspruchen und/oder auf eine Entschädigung 
und Nutzungsgebühren zu bestehen -, so sind doch auch innovativere Gewinnbeteiligungsmodelle entstanden. Einige professionelle Archäologen und Kulturanthropologen nehmen immer noch ein Besitzrecht an ihren Entdeckungen für sich in Anspruch und ziehen nicht die Notwendigkeit in Betracht, die Interessen der nachkommenden Gemeinschaften zu berücksichtigen, andere jedoch sind sensibler geworden für die Dynamik der Kolonialmächte, durch die kulturelle Aufzeichnungen einiger Völker historisch bedingt zu den wissenschaftlichen Leistungen anderer wurden. Indigene Völker können eine erheblich andere Beziehung zu dem haben, was wir Geschichte nennen - antike Artefakte, menschliche Überreste und kulturell bedeutsame Plätze können in ihren einzigartigen Moralökonomien eine besondere Währung und/oder spirituelle Eigenschaften bewahren. Dies respektvoll anzuerkennen, hat neue Prozesse der Beratung, Reziprozität und gemeinschaftlicher Praxis mit sich gebracht:

Some of the most creative of these initiatives are predicated on a commitment to involve Indigenous peoples directly in the process of archaeology, a process that often significantly reframes and enriches archaeological practice. Descendant Indigenous communities often raise questions that archaeologists had never addressed, and their traditional knowledge is vital for understanding the material traces of antecedent land-use patterns, resourceharvesting practices, and a range of other more social aspects of past lifeways (Nicholas und Wylie 2009:18).

Die Übernahme von Idealen der gemeinschaftlichen Praxis seitens der Archäologie führte zu einer Erweiterung des akademischen Diskurses und einer disziplinären Praktik, die nicht nur ethisch verantwortungsbewusster ist, sondern auch theoretisch auf festerem Grund steht (18-19). Indigene Gemeinschaften können jetzt die Leitung von Forschungsprojekten, die ihr Gebiet, ihre materielle Geschichte oder ihr kulturelles Erbe einbeziehen, übernehmen, ausführliche Beratungsvorschriften entwickeln, Publikationsformen, die gesellschaftlichen Schaden auslösen könnten, begrenzen und/oder den Zugang zu Kunsthandwerk einschränken und von Richtlinien Gebrauch machen, die die Ziele der Gemeinschaft unterstützen sollen (Nicholas 2008). Die kreative Anwendung der Gesetze zum geistigen Eigentum haben es einigen indigenen Völkern ermöglicht, die unangemessene Nutzung des Kulturerbes einzuschränken. Potenzielle Gesetze versprechen weitere Eigentumsformen für Schutz und Entschädigungen, wie jene Vorschläge, die indigenes Kulturerbe, traditionelles Wissen und traditionelle kulturelle Ausdrucksformen schützen sollen (Coombe 2008). Dort, wo Forscher und die Menschen vor Ort es schaffen, wohlwollende Beziehungen aufzubauen, lassen sich ihre gemeinsamen Interessen kombinieren, um den gegenwärtigen Bedarf an Arbeitsplätzen in der Gemeinschaft, an Ressourcenmanagement, Spracherhaltung, Bildung und nachhaltigen Formen der lokalen Entwicklung anzugehen oder um Gebietsansprüche zu unterstützen. $\mathrm{Ob}$ wir diese als Ergebnis der Anerkennung von indigenem Cultural Property betrachten oder einfach als eine kreative Form der Vermeidung von Eigentumslösungen oder 
nicht - es bestehen kaum Zweifel daran, dass solche Aktivitäten mit Gewinn auf beiden Seiten eine erhöhte ethische Sensibilität im Umgang mit kulturellen Rechte erkennen lassen.

Diese dynamischen neuen Formen der sozialen Beziehung charakterisieren darüber hinaus auch andere Gebiete der Auseinandersetzung, die im eher konventionellen Sinne als Fragen um Cultural Property betrachtet werden, vor allem im musealen Kontext. Es ist anerkannt, dass heutige Ursprungsgemeinschaften Interesse an Eigentum mit kultureller Bedeutung haben, das weder von internationalistischen Bekenntnissen zur Erhaltung des Welterbes noch von Staatsinteressen bezüglich der Kontrolle des nationalen Kulturerbes noch von Bekenntnissen zu den Kräften des Marktes für die Distribution richtig umfasst wird. Im gesamten Pazifischen Raum, zum Beispiel, haben Museen eine bedeutende Rolle in den Verhandlungen über die konkurrierenden Interessen an Cultural Property gespielt, um sicherzustellen, dass das öffentlichen Interesse weder ignoriert noch die Ursprungsgemeinschaften verprellt werden. Beispielsweise wurde Te Papa Tongarewa, das Museum of New Zealand, komplett umgebaut und neu organisiert, um die bikulturelle Natur des Staates und die Gleichberechtigung seiner Gründergesellschaften anzuerkennen. Maori taonga oder kulturelle Schätze werden durch die Einrichtung von Pflegschaften im Museum gehalten. Dies kann den Verzicht auf Objekte beinhalten oder bedeuten, sie auf kulturell sensible Art und Weise auszustellen, vor allem aber werden Vertreter und Führungspersönlichkeiten der Maori in den Entscheidungsprozess einbezogen. So erklärt die Anthropologin Heidi Geismar:

Rather than a condition of ownership, this notion of guardianship develops relationships of consultation and collaboration. The acknowledgment that property is a relationship rather than an object (so evident to property theorists, yet so obfuscated [in Cultural Property debates]...) suggests an alternative view of Cultural Property, which acknowledges the political and social relations that objects are enmeshed within as vital to their identities... Ownership does not only imply the right to freely do what one wants to with an object; it is far expanded beyond this commodity logic and also implies a state of responsibility. The two are not mutually incompatible. The notion of property (and Cultural Property) implies entitlement, use, placement, and circulation as well as commoditization (2008:115).

Das Konzept der Museumspflegschaft hat sich in der indigenen Welt und innerhalb von Diasporagemeinden verbreitet. Auch wenn die Rückführung von Objekten eine Handlungsoption ist, kann die Anerkennung von Pflegschaften die Bewahrung von Cultural Property in den öffentlichen Museen tatsächlich ermöglichen und ihren Gebrauch und ihre Ausstellung fördern. Die Teilhabe von Künstlern, Forschern und Ältesten aus den Ursprungsgemeinschaften an der Museumsarbeit kann eine produktive Quelle für neue Ideen sein, wobei der Schwerpunkt von festen Objekten, die Individuen, Gruppen oder Institutionen gehören, verlagert wird "to a more relational understanding of the dynamic links between people and 
things" (Geismar 2008:116). In der Tat ist die Anerkennung der Besonderheit indigener kuratorischer Praxis in Übereinstimmung mit dem Verständnis, dass Museen eine aktive Rolle bei der Erhaltung des immateriellen Kulturerbes spielen und dafür neue Partnerschaften mit Gemeinschaften benötigen, entstanden. Diese Anstrengungen verlagern "museological thinking and practice from a focus on objects and material culture to a focus on people and the sociocultural practices, processes, and interactions associated with their cultural expressions" (Kreps 2009:194).

In vielen Fällen, die indigene Völker betreffen, haben die neuen musealen Prinzipien bezüglich Zugang, Verwendung und Interpretation Gemeinschaften wieder mit ihrem kulturellen Erbe verbunden und die Anerkennung der Rolle der Vergangenheit in der Gegenwart gestärkt und so den Stolz auf die eigene Kultur wiederbelebt. Solche Prinzipien gründen auf Beziehungen von Respekt und erkennen an, dass westliche Auffassungen von Privateigentum der Beziehung zwischen kulturellen Besitztümern und der Identität in indigenen Gemeinschaften nicht unbedingt gerecht werden (Bell et al. 2008). So meint der Kulturanthropologe Brian Noble (2008:465), dass "owning as property" (Besitz als Eigentum) die Exklusivität in Hinsicht auf Besitztum und Übertragbarkeit zum Zwecke des Austausches und der Vermögensmaximierung betont, während "owning as belonging" (Besitz als Zugehörigkeit) den Schwerpunkt auf Transaktionen legt, die die Beziehungen von Respekt und Verantwortung innerhalb und zwischen den Völkern stärken. Zum Beispiel können eine starke Verbundenheit mit und Verpflichtungen gegenüber Objekten von bedeutendem kulturellen Wert für indigene Gemeinschaften einhergehen mit bestimmten Formen von Unübertragbarkeit:

[T] ransfer and other forms of exchange of Cultural Property tend to strengthen, deepen and extend social and emotional connections among people, their histories, their material productions, their knowledge, their lands, their kin groups, and the Creator, rather than effect a separation, as would be expected of the predominantly Western understanding of property as a commodity... [T] o reduce this connection to a simple relation between property and identity is to be too narrow. Modes of exchange, and relationships and obligations created through exchange, are also crucial to social and political formation (474).

Ein Weg, um andere Praktiken von Besitztum neben der westlichen Gesetzmäßigkeit anzuerkennen, ist, eine Form des gegenseitigen Respekts und der Anerkennung auszuüben, dem wohl die Mehrheit sowohl der Eigentums- als auch der Kulturtheoretiker weiterhin ausweichen. Letztendlich heißt es anzuerkennen, dass Cultural Property nur eine Dimension der kulturellen Rechte ist - eine Kategorie der Menschenrechte, die verstärkt den Schwerpunkt auf moralische Rechte, kollektive kulturelle Identität, kulturelle Integrität, kulturelle Zusammenarbeit, transkulturelle Kommunikation und interkulturellen Austausch legt. 


\section{$4 \quad$ Kultur und Entwicklung}

"[C]ulture has recently acquired a new visibility and salience in development thinking and practice" (Radcliffe 2006b:1). Seit den späten 1990er-Jahren ist Kultur ein Kernstück der Entwicklungspraxis gewesen; diese signalisiert Bedenken ob der Aufrechterhaltung der kulturellen Vielfalt, der Respektierung der lokalen Wertesysteme, die den sozialen Zusammenhalt sicherstellen, und der Beendigung der Diskriminierung sozialer Randgruppen (1-8). Egal, ob das Ziel die ländliche Entwicklung oder ökologische Nachhaltigkeit ist: Eine Betonung auf die Pflege und in manchen Fällen auf die Profitierung von kulturellen Unterschieden hat eine neue Bedeutung erlangt (Clarke 2001, Coombe 2005a, Coombe et al. 2007, Radcliffe und Laurie 2006a, 2006b).

Wir sind Zeugen einer wachsenden Besitzgier in Bezug auf kulturelle Formen geworden, genau zu der Zeit, in der Kultur neu bewertet wird, nicht nur von indigenen Völkern (Brown 2003, 2005), sondern auch von Gemeinden, Regionen und nationalen Regierungen. Die zuletzt genannten Interessenvertreter betrachten kulturelle Ausdrucksformen, kulturelle Unterschiede und kulturelle Vielfalt als Quellen von Sinn und Wert, die den gesellschaftlichen Zusammenhalt fördern, LandStadt-Migration verhindern, neue Möglichkeiten für den Lebensunterhalt bieten und die natürlich das Potenzial haben, neue Einkommensquellen zu erschließen. Geistiges Eigentum ist für diese Initiativen zentral und neue Formen von Suigeneris-Rechten werden in zahlreichen Foren diskutiert, wo Traditionen und die Erhaltung von Kultur neue Dringlichkeit bekommen haben. Diese Überlegungen beziehen eine Reihe von Akteuren ein, einschließlich indigener Völker mit neuer Stimme, religiöser Diasporagemeinden, Bauern, Heiler, Kunsthandwerker sowie eines wachsenden Aufgebots an NGOs.

Die kulturelle Wende im Entwicklungsbereich hat im Zusammenhang mit der neoliberalen Politikgestaltung und den Widerständen dagegen stattgefunden. Kultur wird als Wert aufgefasst, der einem Ort, einer Gruppe, einer Institution, einer Ressourcenmanagementstrategie oder einer materiellen Produktionsstätte zugeschrieben werden kann (Radcliffe 2006c:229-31). Wenn Kultur zunehmend als neue Grundlage für Kapitalbildung gesehen wird, kann sie ebenso gut bei strategischen Interventionen in der Bildung von gegenseitigem Respekt, Anerkennung und Würde eingesetzt werden. In anderen Worten: Eine Anerkennung von Cultural Property kann ein Bewusstsein für die Notwendigkeit von kulturellen Rechten schaffen. Wie die Geografin Sarah Radcliffe (2006c) ausführt, wird Kultur durch die Vorliebe der Entwicklungsinstitutionen, von Kultur als ein Produkt zu sprechen, als ein Satz an materiellen Objekten und charakteristischen Verhaltensweisen behandelt, wodurch die Suche nach kulturell typischen Produkten und Dienstleistungen für die globalen Märkte befördert wird. Ersatzweise wird bei der Behandlung von Kultur als Institution der Entwicklungsschwerpunkt auf charakteristische Formen der Organisation, Regulierung und Führung gelegt (235-236). Deutlich seltener jedoch erkennen Entwicklungsbestrebungen kulturelle Traditionen als 
Quellen der Innovation und politischen Hoffnung an, in denen Menschen versuchen, eine bestimmte Wahrnehmung, wer sie sind und was für eine wirtschaftliche und politische Zukunft sie sich wünschen, auszudrücken und zu formen (Appadurai 2004, Bebbington 2005).

Zur Illustration: Ursprungsbezeichnungen und geografische Hinweise - geografische Namen, die die Herkunft eines Gutes kennzeichnen, wo "the quality and characteristics exhibited by the product are essentially attributable to the geographical environment, including natural and human factors" (Höpperger 2007:3) - sind angewandte Formen des Schutzes geistigen Eigentums, um Produktionsbedingungen vor Ort aufrechtzuerhalten und um traditionelle Methoden und Tätigkeiten anzuerkennen und wertzuschätzen. Historisch gesehen dienten sie dazu, die ländlichen Traditionen der europäischen Eliten zu schützen; in einigen Bereichen kennzeichnen sie nunmehr die tatsächliche Existenz von lokalen kulturellen Unterschieden (Fillippucci 2004). Zunehmend werden sie als Mittel zur Förderung der Entwicklung anderer betrachtet, deren Traditionen dadurch einen neuen Wert erhalten können. Diese marktbasierten Instrumente können missbraucht werden, besonders von Staaten, die eher bestrebt sind, neue Quellen für den Exporterlös aufzumachen, als die Traditionen von Gemeinschaften zu fördern (Chan 2008). In dem Maße, in dem solche Kennzeichnungen lokale Kultur reifizieren, entsteht das Risiko, dass sie lokale Praktiken festlegen oder einfrieren, statt dafür zu sorgen, dass diese fortwährend neu erzeugt werden können. Darüber hinaus sind sie in der Verwaltung kostspielig, technisches Know-how ebenso wie größere Investitionen ins Marketing sind nötig, um Gewinne zu erzielen. Größere öffentliche Investitionen und/oder internationale Unterstützung oder von NGOs werden notwendig sein, um die mächtigsten privaten Akteure einer Gemeinschaft davon abzuhalten, diese Gelegenheiten zur Monopolbildung zu nutzen. Diese Herausforderungen sind jedoch nicht unüberwindbar, und viele Staaten, NGOs und Entwicklungsbehörden haben sich diese Instrumente zu eigen gemacht, um Potenzial sowohl für eine ökologische Nachhaltigkeit (Larson 2007) als auch für eine ökonomische Entwicklung zu halten, indem Märkte für kulturell charakteristische Güter geschaffen werden (Bramley und Kirsten 2007, Aylwin und Coombe 2010, Coombe et al. 2007).

Minderheiten und indigene Gemeinschaften haben auch affirmative Rechte an geistigem Eigentum geltend gemacht und darauf bestanden, dass ihre spezifischen Traditionen wichtige Quellen von symbolischem Wert seien. Sie versuchen, Kapital zu schlagen aus der symbolischen Ressource, die Authentizität in einem globalen Markt besitzt, in dem einige Konsumenten das Heterogene in einem homogenen Feld wertschätzen und nach dem Unterschied im Meer der Gleichheit suchen (z. B. Maori-Markenzeichen in Neuseeland und Gütezeichen der First Nations in Kanada). Ermutigt werden sie durch internationale Institutionen wie die UNES$\mathrm{CO}$, die die Komplementarität von kulturellen und ökonomischen Entwicklungsaspekten betonen und den interkulturellen Austausch als politisches und soziales Gut fördern. Wie auch immer - Kennzeichnungen, die die Herkunftsbedingungen 
anzeigen (das schließt auch Kollektivmarken und Gütezeichen ein) haben eine neue Popularität erreicht als Mittel, um die kulturell charakteristischen Produktionsformen und auf Traditionen gründenden Güter zu schützen und darzustellen und gleichzeitig nachhaltige Entwicklungsziele zu erreichen (Aylwin und Coombe 2010). Durch die Möglichkeit ihres kollektiven Besitzes und Managements werden sie zu besonders attraktiven Mitteln, um traditionelle Produktionszusammenhänge und soziale Reproduktionszusammenhänge zu erhalten anstatt Ungleichheiten vor Ort zu verschärfen. Der öffentliche Charakter der Rechte, die sich aus ihrem Gebrauch ergeben, weckt Hoffnungen auf die Erhaltung von örtlich begrenzten Produktionsstrategien, die auf historischen Erinnerungen aufbauen und gleichzeitig den lokalen kulturellen Stolz entwickeln.

Diese Mittel für den Schutz und die Darstellung von Cultural Property kann für eine ganze Reihe von sozialen Akteuren attraktiv sein, gerade weil sie Entwicklungen, die sich an der Behandlung von Kultur als ein Produkt orientieren, mit der Anerkennung von Kultur als Institution kombinieren, gleichzeitig sind sie vielversprechend für die Gemeinschaften, die sowohl nach Anerkennung ihrer kulturellen Rechte als auch nach Verbesserungen ihrer Lebensbedingungen streben. Kulturelle Eigenschaften werden für verschiedene Zwecke eingesetzt. Laut Rechtswissenschaftler Madhavi Sunder (2007:106) hat man in der Geschichte indigene Völker und sogenannte traditionelle Gemeinschaften als Mitwirkende am oder Schützer des öffentlichen Bereichs verstanden. Ihren traditionellen Beitrag als Innovation anzuerkennen wurde entweder als Widerspruch in sich zurückgewiesen oder als eine Form des neoliberalen falschen Bewusstseins dämonisiert, das Rechte am geistigen Eigentum in Form von Pflegschaften ausdehnt, die über den geeigneten Bereich von Kultivierung hinausgehen. Dennoch ist die kreative Verwendung geografischer Hinweise ein Beispiel, durch das Kultur und Kommerz eine Verbindung eingehen und Tradition durch ihre Kommerzialisierung potenziell erhalten wird: "[T]hird-world artisans recognize that '[e]xcept in a museum setting, no traditional craft skill can be sustained unless it has a viable market"' (Sunder 2007:111). Sunder sieht dies in Übereinstimmung mit dem Ansatz menschlicher Fähigkeit zur Entwicklung, der Entwicklung als jedwedes Handeln versteht, das die menschlichen Fähigkeiten erweitert, durch die Menschen zentrale Freiheiten erreichen, einschließlich der Freiheit, am Markt teilzunehmen und dafür bezahlt zu werden (121). Die Anerkennung indigener und/oder traditioneller Völker als Urheber und Innovatoren verbessert ihren Zugang zu lebenswichtigen Gütern, fördert Entwicklungsziele und verbessert interkulturelle Beziehungen (ebd.).

Man mag wohl argumentieren, dass das enorme Interesse an traditionellem Wissen und seiner Erhaltung in Kreisen der internationalen Politik mehr mit der Identifizierung und dem Anzapfen von Reservoiren an Einblicken, Techniken und systemischem Wissen, die vielversprechend für zukünftige Entwicklungen in Wissenschaft und Technologie sind, zu tun hat als mit der Erhaltung der Existenzgrundlagen der Menschen vor Ort, der Linderung ihrer Armut oder der Förderung ihrer politischen Autonomie. Gleichwohl bietet der Diskurs in dem Maße, in dem 
er den Boden für eine Anerkennung und Aufwertung kultureller Unterschiede bereitet, auch ein Mittel, um Verknüpfungen mit anderen Menschenrechten in Bezug auf kulturelle Unterschiede herzustellen, und somit einen versteckten Grund, um weitere politische Forderungen voranzutreiben.

Weltweite Versuche, traditionelles Wissen zu respektieren, zu erhalten und wertzuschätzen, entpolitisieren wohl Positionen zu Verarmung, indem ein akzeptablerer Mantel der Kultur über die Bedingungen der sozialen Marginalisierung geworfen wird. Aber um die Bedeutung kultureller Vielfalt durch den Erhalt der biologischen Vielfalt anzukennen, so meine Argumentation, sollte kulturelle Vielfalt nicht in abstrakter, reifizierter oder museologischer Hinsicht anerkannt werden, sondern als entstehendes internationales Menschenrecht, das die Wechselbeziehung zwischen den Rechten auf Nahrung, Souveränität, territorialer Sicherheit und gemeinsamem Erbe bekräftigt (Coombe 2005b). Zumindest ein Teil der ideologischen Rolle der Kultur in diesen neuen Forderungen bezieht sich auf einen grundlegenden Weg hin zu Umwandlungen in der Kapitalbildung, was den Druck zunehmend vergrößert, Informationen nutzbar zu machen, damit diese gesammelt und in Werke des geistigen Eigentums umgesetzt werden können.

Der Antrieb, lokales Wissen und Praktiken der Menschen als innovative Werke - Formen des immateriellen oder geistigen Eigentums -, die ganzheitlich auf eine indigene Identität oder einen traditionellen Lebensstil bezogen sind, zu repräsentieren, entwickelt sich aus dieser politischen Ökonomie. In genau diesen Kontext muss man Versuche der Kulturalisierung oder Indigenisierung von Wissen einordnen, damit dieses nicht mehr nur eine einfache Information ist, sondern stattdessen zu der wertvolleren Form eines Werkes wird (Coombe 2003). Nur dann können Ansprüche auf den Besitz, die Kontrolle, Erhaltung und Pflege von Wissen gestellt werden, nur dann werden die Menschen respektiert. Im Verlauf ihrer Interpretation in den letzten zehn Jahren ist die Konvention zur Biologischen Vielfalt (CBD) zum Fokus der Energie vieler Dritte-Welt-Regierungen, indigener Völker und Nichtregierungs- oder zivilgesellschaftlicher Organisationen geworden, weil sie der einzige bedeutende internationale und rechtlich bindende Vertrag zu sein scheint, der das Potenzial hat, den neoliberalen Imperativen der TRIPsVereinbarung entgegenzutreten (McAfee 1999). Da indigene Völker aktivere und gewieftere Teilnehmer im Kreise dieser Politikgestaltung geworden sind und sie geschärfte Fachkompetenz aus anderen Veranstaltungen der Vereinten Nationen eingebracht haben, haben sie Fragestellungen zu kultureller Integrität, demokratischer Entscheidungsfindung, Verantwortlichkeit und Selbstbestimmung direkt auf den Verhandlungstisch gelegt. Ihre Möglichkeiten, dies zu tun, werden durch die rhetorischen Druckmittel, die internationale Menschenrechtsnormen bieten, und den zentralen, wenn auch mehrdeutigen Platz, den die Kultur darin einnimmt, in hohem Maße unterstützt.

Viele indigene Völker (und viele, von denen man annimmt, sie pflegten einen traditionellen Lebensstil) leben in oder unterliegen der Gerichtsbarkeit von Staaten, mit denen sie ein langes historisches Verhältnis voll Misstrauen, Betrug und Ge- 
walt verbindet. Statt Delegierten des Staates bei der CBD, die ihre Interessen vertreten sollen, zu trauen, haben sie die Programme, Foren, Förderungs- und PRMöglichkeiten der CBD genutzt, um die Legitimierung und Unterstützung der Declaration on the Rights of Indigenous Peoples (Erklärung über die Rechte der indigenen Völker) voranzubringen, deren Entwurf fast gleichzeitig mit den Debatten über die Umsetzung der CBD verhandelt wurde. Die Verhandlungen über den Entwurf, der $2007 \mathrm{zu}$ einer Erklärung führte, die inzwischen fast alle Staaten unterzeichnet haben, haben ein bestimmtes Vokabular zu Darstellungen und Ansprüchen geschaffen, die ständig in so vielen rechtlichen Zusammenhängen wiederholt wurden, dass sie schlussendlich als eine Form des Völkergewohnheitsrechts betrachtet werden können.

Gemäß internationaler Gesetze können nur Völker Selbstbestimmung fordern und alle Völker haben Kultur. Die Rechte der indigenen Völker auf ihr Land, ihre Gebiete und Ressourcen werden als Ableitung von ihrer Kultur und ihren spirituellen Traditionen anerkannt. Völker haben das Recht, ihre kulturelle Entwicklung fortzuführen sowie ihre kulturellen Traditionen wiederzubeleben und zu schützen. Weiterhin ist anerkannt, dass indigene Völker das Recht haben, ihr geistiges Eigentum und Cultural Property zu kontrollieren. Dies schließt Rechte auf bestimmte Maßnahmen zur Kontrolle, Entwicklung und Schutz ihrer Wissenschaften, Technologien und kulturellen Manifestierungen (inklusive des Wissens über lokale genetische Ressourcen) ein. Gesetzliche Richtlinien zum Schutz des indigenen Erbes definieren dieses so, dass es von Generation zu Generation tradiertes Wissen einschließt, das zu einem bestimmten Volk oder seinem Gebiet gehört. Der Schwerpunkt wird hierbei auf den dynamischen und innovativen Charakter traditionellen Wissens gelegt. Darüber hinaus hat die Schaffung der rechtlichen und politischen Kategorie von traditionellem Wissen wiederum die politischen Bedingungen geschaffen, durch die traditionelle kulturelle Ausdrucksformen mit allen Vor- und Nachteilen verstanden werden als Cultural Property, das verwaltet werden muss.

\section{Traditionelle kulturelle Ausdrucksformen (,Traditional Cultural Expressions")}

Viele der Beispiele in diesem Beitrag setzen voraus, dass ein gewisses Niveau bzw. eine gewisse Art von Schutz in Bezug auf traditionelle Kulturgüter geltend gemacht werden kann oder muss. Der Schutz kultureller Ausdrucksformen vor illegaler Aneignung, falscher Darstellung und unerlaubter Kommerzialisierung ist in westlichen Gesetzen jedoch ein Bereich, der in geistiges Eigentum, kulturelle Menschenrechte, gewohnheitsrechtliche Delikthaftung und, seit Kurzem, in indigene Rechtsansprüche fragmentiert ist (Girsberger 2008, Graber 2008, Macmillan 2008). Trotz jahrelanger internationaler Verhandlungen und transnationaler Fürsprache wurde keine Einigung über die Ratsamkeit entweder einer globalen Ordnung oder der Anwendung des Gewohnheitsrechts als praktikables Schutzinstrument erzielt 
(Wendland 2008). Der Schutz selbst ist ein Konzept mit vielfachen und sich widersprechenden Bedeutungen, die von der Ermöglichung der Kommerzialisierung bis zur Verhinderung derselben reichen, je nach Inhalt und seiner gesellschaftlichen Bedeutung. Die digitale Kommunikation hat die Bedenken in diesem Bereich verstärkt und die Risiken der widerrechtlichen Aneignung und Dekontextualisierung erhöht, während sie gleichzeitig Gemeinschaften neue Möglichkeiten bietet, von der Förderung neuer Nutzungen traditioneller Kulturausdrucksformen, die eine nachhaltige Entwicklung voranbringen, zu profitieren (Antons 2008a, 2008b, Burri-Nenova 2008, Sahlfeld 2008).

Das wachsende Interesse am Schutz traditioneller kultureller Ausdrucksformen deutet jedoch zumindest genauso auf Staatsinteressen an der Lokalisierung und Förderung neuer Investitionen, kultureller Exportprodukte und touristischer Möglichkeiten hin, wie es ein Zeichen der Sorge ist über die Lebensbedingungen und das Wohl derjenigen indigenen Völker und Minderheiten, die sehr wahrscheinlich über bestimmte kulturelle Ressourcen verfügen. Moderne Staaten haben eine lange Geschichte hinsichtlich der Vereinnahmung kultureller Traditionen von Minderheiten in ein verstaatlichtes Kulturerbe. In der Tat hat das Konzept von Tradition selbst seinen Ursprung in der Moderne und der Konstituierung eines unzivilisierten, vormodernen oder nicht westlichen Anderen, das der Erlösung durch Zivilisierungsprozesse bedarf.

In einer exzellenten Studie über die Geschichte dieser Kategorie zeigt die Historikerin Monika Dommann (2008), wie "folklore" definiert wurde als "knowledge of the people" (Wissen der Menschen), das von der Moderne unberührt war. Sie wurde auch als Beweis einer menschlichen Vergangenheit betrachtet, die unweigerlich mit dem aufkommenden Fortschritt verschwinden würde, wenn die moderne nationale Wissenschaft sie nicht für die Nachwelt rettete. Als zentralen Punkt bei den nationenbildenden Projekten und den Kolonialherrschaften des Europa im 19. Jahrhundert beinhaltete die Konstruktion typischer Kulturtraditionen in der Kreierung nationaler und kolonial erdachter Gemeinschaften häufig die Reifizierung charakteristischer Bräuche ländlicher Völker und/oder Stammesgruppen. Nationale Archive wurden geschaffen, um das Kulturmaterial zu beherbergen. Das Eigentum an diesen materiellen Objekten hielt normalerweise der Staat, der kulturelle Inhalt aber sollte als öffentlicher Bereich gelten, wodurch sich der Wert solchen Materials leicht ausbeuten und beschlagnahmen ließ. Zum Beispiel wurden bei traditioneller Musik jedwede Eigentumsrechte nur bei Originalaufnahmen und in neuen Arrangements, die auf vorherige Kompositionen basierten, gehalten.

Diese Fragmentierung von Rechtsansprüchen, ermöglicht durch die historischen Bedingungen, unter denen dieses Kulturmaterial bewertet, gesammelt und ausgebeutet wurde, hat neue Kontroversen über Cultural Property hervorgerufen (Coleman und Coombe 2009). Postkoloniale Staaten haben lange die Universalität der Gesetze des 19. Jahrhunderts angefochten, die die Enteignung ihres Kulturerbes möglich machte, als eine Weiterführung von Verletzungen ausgelöst durch den Kolonialismus, die sie von einer vollen gesellschaftlichen Entwicklung ausschloss. 
Das System „ein Staat, eine Stimme“ in der WIPO ermöglicht sogenannten Entwicklungsländern, das Thema auf dem globalen Verhandlungstisch zu halten. Einige Staaten zum Beispiel haben Folklore ins Urheberrecht eingegliedert und damit einen lebendigen nationalen öffentlichen Bereich geschaffen, unter dem neue Formen der Kreativität und kulturellen Wiederbelebung aufblühen (Goodman 2002, 2005). Die UNESCO hat sich dieser Fragestellung 1989 angenommen und Folklore in das „universelle Erbe der Menschheit“ aufgenommen, was neue Kritik hervorrief, als sowohl indigene Völker als auch sogenannte traditionelle Gemeinschaften als potenzielle Interessenvertreter auftauchten. Die WIPO hat wieder die Führung bei den internationalen Politikverhandlungen, die dieses neue Gebiet von Rechteinhabern anerkennen, übernommen. Sie hat auch den Begriff „traditionelle kulturelle Ausdrucksformen" (traditional cultural expressions) hinzugefügt, um den Begriff "folklore" für diejenigen zu ersetzen, die letzteren als anachronistische Referenz an ein eingefrorenes kulturelles Archiv betrachten, was für sie stattdessen ein Bereich mit dynamischen Ressourcen für weiterführende Innovationen ist.

Fragestellungen zur Gesetzgebung und Selbstbestimmung versprechen eine weitere Komplizierung dieses Gebiets der entstehenden Rechte und Verantwortlichkeiten, vor allem in Anbetracht der vielfachen Bedeutungen, die das Konzept des Gewohnheitsrechts begleiten, das so oft als Mittel zur Anerkennung traditioneller Systeme von Kulturmanagement sowie zur Förderung der politischen Selbstbestimmung indigener und Minderheitenvölker vorgebracht wird. Der sogenannte Schutz traditioneller kultureller Ausdrucksformen (etwa der Schutz traditionellen Wissens) ist wohl die fragwürdige politische Arbeit zentralisierter moderner juristischer Systeme, die versuchen, die kulturellen Systeme peripherer Gesellschaften einzugliedern, und dabei dazu neigen, dies mit einer seltsamen Art von Scheuklappendenken zu tun (Teubner und Fischer-Lescano 2008).

Aggressive weltweite Expansionen des westlichen Systems des geistigen Eigentums, die von neuen Strategien der Kapitalbildung und nationalen Politikzielen zur Erhaltung kultureller und biologischer Vielfalt angetrieben werden, resultieren häufig in dienlichen Ansätzen zu traditionellen kulturellen Ausdrucksformen, die im Konflikt stehen mit den Bedürfnissen, Werten und Rationalitäten lokaler Gemeinschaften. Kulturanthropologische Studien über nationale Anstrengungen zum Schutz traditioneller kultureller Ressourcen illustrieren ironischerweise die Verletzbarkeit von gesellschaftlichen Minderheiten beim staatlichen Einsatz kolonialer Gewohnheitsrechtsordnungen - wobei sie die Besonderheit der sozialen Prozesse, durch die Wissen und kulturelle Ausdrucksformen generiert werden, bei der Nutzbarmachung von Tradition für moderne Märkte ignorieren (z. B. Aragon 2008, Aragon und Leach 2008, Balliger 2007, Green 2007, Scher 2002).

Als Konsequenz müssen diejenigen, die versuchen, die Vitalität spezieller lokaler und regionaler Wissensformen und kultureller Ausdrucksformen aufrechtzuerhalten, sich selbst verteidigen gegen die Übergriffe der modernen globalen Wissenschaft, einer verallgemeinernden Ästhetik, neue Arten der Kapitalbildung und nationale Eliten, die nach neuen Formen von ausbeutbaren Ressourcen gieren. Um 
dies zu tun, scheint es, dass sie oft ihre eigenen spezifischen Bestrebungen durch die Rhetorik der Menschenrechte auf Kultur artikulieren, ein Begriff, der zunehmend belebt wird durch Gemeinschaftsinvestitionen in die Pflege und Unterstützung von Identitäten, gesellschaftlichen Systemen, Existenzgrundlagen und alternativen Wertesystemen. So überlegt Fiona Macmillan:

$[\mathrm{P}]$ erhaps, however, there is still enough vitality in the more specific concept of cultural rights to offer a political and legal counterbalance to the power of the WTO system. The UNESCO Conventions concluded this century might be thought to demonstrate this proposition. Nevertheless, the question of how we make cultural rights strong enough and specific enough to confer proper legal protection remains (2008:62).

\section{Schlußbetrachtung}

Viele Wissenschaftler bleiben skeptisch bezüglich des Werts und der Konsequenzen einer Verbindung zwischen der anthropologischen Vorstellung von Kultur und dem juristischen Konzept von Eigentum, vor allem insofern, als kritische Theoretiker jetzt Kultur in symbolischen Prozessen verorten, die fortwährend in gesellschaftlichen Praktiken, die sich mit Machtbeziehungen regelmäßig überschneiden, neu geschaffen werden. Ein solches Verständnis geht eher unbehaglich einher mit einer Vision von Kultur "as a bounded entity, the properties of which can be inventoried"” (Handler 2003:356). In dem Maße, in dem Initiativen zur Erhaltung von Erbe und Cultural Property dazu neigen, einen objektivierenden Ansatz anzunehmen, können sie die symbolischen Prozesse, die sie schützen möchten, grundlegend verändern, indem sie sich zu eng auf Objekte, Stätten und Traditionen konzentrieren, zum Nachteil der semiotischen Dimensionen von Kultur (361-363).

Um die Fragestellung zu Cultural Property anzugehen, muss man notwendigerweise die Postulierung und Positionierung von sozialen Identitäten ansprechen. Kollektive Identitäten sind niemals objektiv gegeben, und Gruppen haben keine objektiv begrenzte Existenz: "Power is fundamentally engaged within claims of cultural appropriation and claims to 'culture' - both in attempts to address historical imbalances, such as past histories of dispossession and colonisation and also in the renegotiation of contemporary positions within societies" (Anderson 2009:192). Die Rhetorik des kulturellen Besitzes mag zu absurden Forderungen führen (Comaroff und Comaroff 2009), vor allem wenn gegenwärtige soziale Kategorien für die Geltendmachung von Besitz in Bezug auf historische Objekte eingesetzt werden, die schon viel älter sind als jene Identitäten, die sie jetzt beanspruchen (Appiah 2006). Auch jetzt noch verstehen wir das Konzept von Eigentum grundlegend falsch, wenn wir uns primär auf das westliche Modell des exklusiven individuellen oder gemeinsamen Besitzes konzentrieren, wie es so viele Kritiker des kulturellen und geistigen Eigentums implizit tun. Wie die Rechtswissen- 
schaftler Carpenter et al. (2009) darstellen, verbinden die Kritiker von Cultural Property es fälschlicherweise mit einem engen und fundamentalistischen Paradigma von Eigentum, das Entfremdung, Exklusivität und Kommodifizierung betont. Es würde jedoch klug erscheinen, die Fetischisierung einer besonderen Eigentumsvorstellung schlicht zu vermeiden, um gewissen Fetischisierungen von Kultur entgegenzutreten. Eigentum spielt viele Rollen in Gesellschaften, es manifestiert sich selbst in Ideologien, unterschiedlichen rechtlichen Systemen, sozialen Beziehungen, sozialen Praktiken und in der Beziehung zwischen all diesen (von BendaBeckmann et al. 2006). Genau dieses Thema des Cultural Property fordert größere kritische Reflexivität hinsichtlich der unterschiedlichen Formen von Eigentum ebenso wie eine verbesserte Überprüfung westlicher Vorurteile in Bezug auf Eigentum.

Die erhellende Überprüfung der interdisziplinären wissenschaftlichen Literatur bezüglich des hier dargestellten Cultural Property gibt zu erkennen, dass Besitz- und Eigentumsansprüche auf der Grundlage der kulturellen Bindung zu Dingen - materiellen und immateriellen, greifbaren und nicht greifbaren - unter den Bedingungen des Neoliberalismus, des Informationskapitalismus und der Einrichtung neuer Menschenrechtsordnungen stark zunehmen. Neue Themen, Institutionen, Gesetze und Felder transnationaler Politik tauchen gleichzeitig auf. Dennoch hinken die Versuche, neue Ordnungen von staatsbasierten Eigentumsrechten zu konstruieren, weit hinter den traditionellen Bräuchen, gegenwärtigen Sitten und vor allem hinter den neuen Praktiken, Vorschriften, Ethikformen und Beziehungen gegenseitigen Respekts und Anerkennung, die durch die Ansprüche auf Cultural Property ausgelöst wurden, hinterher. In den letzten zwanzig Jahren sind wir nun Zeugen eines neuen und vitalen Bereichs der kulturellen Rechtsnormen und -praktiken geworden, die sich im Schatten von Cultural Property entwickeln, um noch durch die formalen Systeme der westlichen Gesetzgebung für rechtsgültig erklärt zu werden. Dieses neue Feld der verhandelten Anstandsformen hält wohl ein genauso großes, wenn nicht noch größeres Versprechen auf plurikulturelle Ethikformen und interkulturelle Zukunftsaussichten bereit, als es das durch das Gesetz legitimierte Cultural Property leisten kann. In gegenseitiger Beziehung stehende Vorstellungen von Eigentum und Kultur sind weltweit auf unterschiedlichste Art und Weise aktiv, die größere kritische Aufmerksamkeit von Sozialrechtswissenschaftlern erfordern. 




\section{Teil 5}

Ausblick 



\title{
On Cultural Property and Its Protection: A Law and Economics Comment
}

\author{
Ejan Mackaay
}

\section{Introduction}

The term cultural property seems to have come into vogue after the Second World War as part of efforts to prevent the recurrence of the massive war-time destruction of objects of cultural significance to various groups and, in some cases, to all of humanity. The 1954 Convention for the Protection of Cultural Property in the Event of Armed Conflict (UNESCO 1954) symbolises those efforts.

Destruction is not the only doomsday scenario for cultural property. Removal of objects from their owners or region of origin is another concern. This, too, had occurred during the Second World War with the nazis' looting treasures of all kinds from occupied territories, not to mention the massive confiscation of the property of their Jewish victims everywhere. But the concern was older, as Merryman (1985) for one shows in the story of the Elgin marbles, brought from Greece to England during the $19^{\text {th }}$ century. This concern has found expression in a 1970 UNESCO treaty (UNESCO 1970) and in a 1995 Unidroit Convention (UNIDROIT 1995) seeking to halt international traffic in cultural property.

These concerns have been extended to prevent culturally significant objects or intangibles from falling into ruins, oblivion or insignificance by neglect or by commercial exploitation. Further objects of concern are traditional knowledge held by tribes and, within their environment, plants with medicinal or pharmaceutical significance for industries in the developed world; the concern here is not merely their possible disappearance but also their exploitation without due, in particular 
financial, recognition for current holders of that knowledge. The concern to protect traditional knowledge finds expression inter alia in the 2003 Paris Convention for the Safeguarding of the Intangible Cultural Heritage (UNESCO 2003) and in the 2005 Paris Convention on the Protection and Promotion of the Diversity of Cultural Expressions (UNESCO 2005a).

From here it is but one step to the question of the extinction of animal and plant species and the disappearance of rain forests as a result of encroachment by those seeking economic development. This concern - to maintain biodiversity - is generally considered to lie outside the perimeter of cultural property protection. ${ }^{1}$

\section{What is Cultural Property}

This brief overview of treaties dealing with the protection of one or other form of cultural property makes obvious that the term is used to cover a wide variety of matters. What precisely should be included amongst the objects we seek to preserve because of their cultural significance, collectively designated as "cultural property" or "(cultural) heritage"? Surely not everything belonging to the lifestyles of earlier times would qualify, as that would simply stifle development. Cultural property appears to be reserved for objects, tangible as well as intangible, that are significant expressions of the culture or collective identity of humanity as a whole or of specific groups and that are considered to be particularly worthy of preservation because of artistic, archaeological, ethnological or historical interest (Merryman 1986). Examples would be collections of fauna, flora, minerals or anatomy; historical buildings or landscapes, religious or other; antiquities; significant art of all kinds; traditional knowledge; folklore, customs and rites (see UNESCO 1970, art. 1). The French language used in Quebec might be seen here as cultural property to be preserved.

Cultural property need not necessarily take traditional forms, although much that is currently recognised as such does. In his interesting paper elsewhere in this volume, Johannes Müske stresses that new technologies can be used to capture the expressions of cultural property, such as sounds and tones, or the funeral rituals on Bali. But that is not the end of the story, since if culture is taken as "the whole way of life", much of current culture is expressed in ways conditioned by newly available technologies and hence evolving at the pace at which these technologies appear. This is obvious for communication technologies, from the telephone, through television, to mobile phones, Skype and web 2.0, as much as for transportation technologies which give us a mobility inconceivable until now, as well as the identity, social and artistic practices that come with them. So technology does not necessarily erase culture, but may also give expression to new forms of it. Science, for one, will never be the same since the internet. Art may not be either, if it is

\footnotetext{
${ }^{1}$ For an overview of that subject, see Biller 2010.
} 
separated from the physical supports with which it had become associated in the older culture. What of all this wealth of new cultural expression is significant and how it should be preserved and made accessible in digital form are unresolved questions lying ahead of us and requiring our attention. Authenticity may take on an altogether different meaning in this context (Bendix 1997).

\section{What is Special About Cultural Property}

The use of the term "cultural property" suggests that there is something special about the class of phenomena so designated that requires our attention and perhaps specific measures for their protection or preservation. Special protection should not be granted lightly, since elites and other special interest groups in society have been known to clamour for special protection as a cover for preserving their privileges; the effect of such protection has invariably been to slow down innovation to the detriment of society at large and of the less well-off in particular.

The argument that special measures are necessary because the commodities or services are essential to our well-being is not in itself convincing either. Many essential commodities and services are available through ordinary market processes with the associated property rights and other legal infrastructure, without most of us giving it a second thought. This is obvious for food and other current consumption objects. Closer to cultural property, the preservation of antiques seems to work well enough through private markets. For individually produced or very rare artefacts like paintings, public initiative such as museums may have to complement market processes.

So what is special about cultural property? In their paper elsewhere in this volume, Bicskei, Bizer and Gubaydulina point to its being associated with personal identity and dignity of members of a group. In a recent book by Akerlof and Kranton (2010) argue that identity makes a useful addition to the economist's toolkit, as it explains how persons assume roles, accept norms and make decisions. Their view is that it allows one to account for some decisions that in the traditional full rationality model used by economists would appear anomalous, such as why immigrant children adopt the accent of their peers rather that of their parents or how Codes of honour operate effectively in military and other circles (Akerlof und Kranton 2010:11). In their contribution to the volume, Hauser-Schäublin and Klenke explicitly link identity and cultural heritage. For our purposes, the argument would be that imperilling cultural property that supports persons' identity or dignity would dissolve (some of) the cement of society.

Why can the provision of this kind of cultural property not be left to ordinary market processes? Economists would look for an answer in the public good characteristics of the phenomenon in question. It is perhaps helpful to look for inspiration at a recent contribution to that literature dealing with biodiversity (Biller 2010:174). The central reasons for biodiversity requiring our attention consist 
mainly of three factors: (1) a potential large "scale" effect; (2) irreversibility; (3) uncertainty, to which one might add that (4) few ecosystems are undisturbed by human activity.

It is worth pondering to what extent these factors are transposable to the cultural diversity context; "human activity" might be taken here as the corrosive effect of contact with cultures based on a developed economy, mass production and standardised language. Irreversibility and uncertainty appear to be present for some forms of cultural property as well. Is protection of cultural property somewhat like erosion control in ecosystems? That would raise a collective action problem: the result will only come about with everyone or nearly everyone contributing. The temptation to free ride may be too strong for some and the enterprise may come to naught unless public authority steps in.

\section{Who Decides What to Designate as Cultural Property}

Since not all cultural expression is at once significant and in (apparent) need of protection, the question arises of who should decide what qualifies. In open economies, one would be tempted to reach for the background rule, which is to leave that decision with the owner of the object or the persons directly affected. The drawback of the first solution is obvious in the title of a book by Sax (1999): should one let Rembrandts be used as dartboards or ritually burnt during a funeral ceremony for a very wealthy deceased? If the decision is left to a group, one faces the question of who controls the decision-making procedure within it. How are conflicting views reconciled or conflicts resolved (for instance as regards the filming of traditional funeral rites by western film makers, as highlighted in Engelbrecht's contribution elsewhere in this volume)? The preferences of the chief or of an elite might be imposed on the rest of the group, usually to the detriment of innovation by the unorthodox. When the decision is left with the authorities at the level of the nation state, how is one to ensure that what matters for persons' identity at the local level gets its due in a playing field comprising many millions of persons? In their conclusion, Hauser-Schäublin and Klenke express misgivings about how well local interests are going to be represented in the negotiations amongst nations states at the UNESCO level. The risk of infighting amongst interest groups is obvious.

The relevance of these considerations comes out when one considers what happens in the case of a mistaken decision. A decision to protect something as cultural property comes with restrictions on what can be done with it or to it. On experience, the locals may learn that the costs outweigh the benefits. Who should have a say in the decision to reverse the decision? Perhaps here too one should consider the principle of subsidiarity: leave the decision at the lowest possible level at which the interests of all persons affected will weigh in in the decision. Pesel- 
mann and Socha elsewhere in this volume examine the problem and how it was resolved in the case of the Elbe Valley.

The subsidiarity principle would be of little guidance where the object in question is designated as part of the common heritage of all of mankind. Merryman, in a path-breaking paper in 1986, appears to have in mind this kind of object in highlighting the "internationalist" view of cultural property: look at cultural property as "components of a common human culture, whatever their places of origin or present location, independent of property rights or national jurisdiction" (Merryman 1986:831). He adds: "Another way of thinking about cultural property is as part of a national cultural heritage. This gives nations a special interest, implies the attribution of national character to objects, independently of their location or ownership, and legitimizes national export controls and demands for the "repatriation" of cultural property. As a corollary of this way of thinking, the world divides itself into source nations and market nations" (832).

It appears, however, that the opposite or "nationalist" view of cultural property has carried the day: what is cultural property is decided by or within nation states. Where cultural property is considered of significance for all of humanity, even national authorities would have their hands tied. Yet how this works out in practice will very much depend on the priorities of those who provide the funds for preservation.

\section{How to Protect Cultural Property}

Considering the extraordinary variety of phenomena designated as "cultural property", it is hardly surprising to find a bewildering variety of institutions used or advocated for their preservation: regulation of use, trade, import and export; certification and licensing; intellectual property rights; subsidies or tax advantages; nationalisation. Most of these reflect the view that ordinary market processes with the associated property rights and legal infrastructure won't do the job because of the public good character of cultural property objects or significant negative externalities associated with their loss.

The large scale of the loss of cultural property, its sometimes unique character and the irreversibility of its loss would seem to militate for extraordinary measures of preservation. Yet the level of precautions against accidents is never boundless; it should be adjusted to, but limited by, the loss they are designed to prevent - a principle known in law-and-economics as the Hand test (Mackaay und Rousseau 2008:335). Given the uncertainty regarding the speed at which loss of cultural property will occur and the evaluation of the resulting loss, it may be difficult to determine the appropriate level of precaution with precision. Some will be tempted to err of the side of caution and invoke the - rather indeterminate - precautionary principle. 
Beyond these general considerations, can we develop some general guidelines? A good place to start may be Biller's policy recommendations in the paper on biodiversity: (1) Eliminate perverse incentives; (2) Privatise protection where feasible and involve local communities; (3) Combine non-excludable attributes with excludable ones and take advantage of markets where these can deliver such tied goods; (4) Ensure the provision of cultural property related public goods (Biller 2010:170-172).

Perverse incentives are present when some persons can make money from activities that create losses to others (externalities) or can draw public money for activities without perceptible benefit to the public at large (rent-seeking). One may be tempted to think that trade in cultural objects, removing them from their region of origin, would qualify as an externality and that the appropriate answer would be to prohibit it. But this would increase the value of such objects, create a black market and draw in shady operators who can handle the risk of operating in such a market (Batour 1982). Surprisingly, we have learnt in trying to halt the decline of the rhinoceros and elephant populations in Africa, that is it better to allow some trade of the protected objects but in controlled fashion and to provide for access to interested persons to see them locally, giving locals an interest in providing the access (recommendation 2). It is not obvious whether this argument affects the question of whether cultural artefacts removed from their region of origin should be returned there - the Elgin marbles question mentioned above.

For intangible cultural property such as dance forms, discussed in Eggert's paper elsewhere in the collection, the broad guidelines suggest that experimentation of new forms should not be stopped (encouraging local entrepreneurship), though official certification might be reserved for the authentic forms. Some of the revenue generated by the marketing of new forms might be recycled into funds for research on the classical forms and training of young performers. This would seem to be a more promising tack than prohibiting any but the authentic forms from being expressed, as Lankau discusses in his paper in this volume.

Should one attempt to stop outsiders from drawing inspiration from these forms for developing further derivative art? This may look like a touchy subject, but consider Gauguin's paintings or van Gogh drawing inspiration from Hiroshige (Geller 1998), which we seem to find unobjectionable? Fencing off intellectual creations from imitators and followers is done in the intellectual property world through intellectual property rights. But all of these have built-in limitations to restrict their effect to what is presumed to be minimally necessary to encourage those who make such creations, as Zimbehl explains in his contribution to the volume. Beyond it, information circulates freely and much of the economic development as we know it is based on innovations building onto earlier innovations. So far as we now understand it, this is not a constraint arbitrarily invented by the developed world to protect its privileged position, but fundamental to lifting oneself out of poverty through the advancement and application of knowledge. Whether there are other but equally promising ways of lifting the poorest nations 
out of poverty is an open question. Experimentation with other forms has not, so far, convincingly come up with any and has turned out in many cases to be extremely painful in terms of suffering of ordinary humans who were (unwilling) participants in the experiment.

Granting rights to communities without a clear justification of their incentive effects on creation requires an entirely different logic, as Hilty (2009) has shown in a recent paper. Should such sui generis rights be granted as incentives to preserve traditional knowledge? To pursue the idea, those rights would have to be perpetual; there would be a problem of who decides on what to do with them: within the community holding the rights, there could be opposition between traditionalists seeking to keep knowledge local and innovators tempted to give access against payment or to become entrepreneurs exploiting the knowledge themselves. If the latter are allowed to pursue their preferred option, would the knowledge then - in due course - become part of the stock of accessible accumulated human knowledge? Moreover, effective exploitation of knowledge in the medicinal and pharmaceutical domains may require operation at a very substantial scale, given the size of the risks of harm to humans involved; this may act as an entry barrier, but how serious it is is unclear, considering the phenomenal growth of big pharma in India and Brazil. As Kilian Bizer and Gerald Spindler plan to demonstrate in their follow-up work: for a project requiring the consent of various rights holders, having too many rights, each with an effective veto right, may lead to a tragedy of the anticommons, effectively stifling development. ${ }^{2}$ In some cases, such as the tradecraft of goldsmiths' being patented discussed in the Hauser-Schäublin and Klenke paper, the wiser course might be to leave this knowledge unpatentable, in the public domain, forcing people to make their money on the artefacts they can produce with it.

A brief observation should be added on the role of the UNESCO recognition of world heritage status to particular objects or monuments. It illustrates the difficulty highlighted above of who has a voice in the decision to recognise something as cultural property. The recognition itself acts economically like a - rather exclusive - standard or a collective trade-mark somewhat along the lines of geographical indications. ${ }^{3}$ A trade-mark conveys to consumers information that would otherwise much costlier or even impossible to glean, simplifying and accelerating their decision as to whether to acquire or go and visit the object in question. The trademark itself does not stop anyone from offering similar ware but with a different label, perhaps quite successfully as French wine growers, insisting on their appellations controlees, discovered to their dismay in competition with wines from Australia, South Africa and Chile: consumers were not willing to pay premium price for those labels. So whilst there appears to be much "horse trading" around UNESCO

\footnotetext{
2 Heller, who first articulated the concept in a paper in 1988, summarises the literature in a recent book (Heller 2008).

${ }^{3}$ A good summary of which may be found in Hughes 2007.
} 
recognition of world heritage status, the result is probably less distorting of ordinary market processes than one might fear.

\section{Conclusion}

Cultural property sounds like a powerful battle cry to spur people into action to protect common heritage. Terms matter, as Groth stresses in his paper in this collection. Stirring images can, however, be misleading. People may believe that extending copyright will protect poor authors, rather than the fat cats, or that a trade embargo is the best way of preserving cultural artefacts, whereas it is never fully effective and generally conjures up a black market with the attendant corruption of officials who supervise the embargo. Good intentions are no excuse for bad results.

In this brief comment, I have raised doubts as to the soundness of cultural property as an analytical concept. Many of the concerns that lie behind it appear to mirror the North-South or developing-developed nations debate. A good part of significant cultural property or world heritage is situated in developing countries, whereas the greatest concern to preserve it may be present in developed countries. One may be concerned that economic development lifting the poorest nations out of their poverty may be bought with a significant loss of cultural property, and want to do something about it. Market processes are not naturally protective of cultural property unless it is backed by willingness to pay.

The difficulty is that the institutions that spring to mind to protect cultural property may have significant costs in terms of development and access to knowledge. The challenge is to understand the function of existing market and nonmarket institutions and to tailor adjustments to them so as achieve significant protection of cultural protection at acceptable cost. 




\section{Autoren}

Regina Bendix ist seit 2001 Professorin für Kulturanthropologie/Europäische Ethnologie an der Georg-August-Universität Göttingen. Aus ihren wissenshistorischen Arbeiten (In Search of Authenticity, Madison 1997) sowie ethnographischer Arbeit im Bereich von Tourismus und Kultur erwuchs auch das Interesse zu Fragen nach Kulturerbe und Kultureigentum innerhalb der größeren Matrix von Kultur im Spannungsfeld von Wirtschaft und Politik. Sie ist Sprecherin der DFG-Forschergruppe 772.

Marianna Bicskei ist wissenschaftliche Mitarbeiterin am Lehrstuhl für Wirtschaftspolitik und Mittelstandsforschung an der Georg-August-Universität Göttingen. Sie ist Mitglied in der interdisziplinären DFG-Forschergruppe zu Cultural Property der Georg-August-Universität Göttingen. In dem Teilprojekt „Recht und Ökonomik von Cultural Property: Eine institutionenökonomische Analyse der Regelbildung“ fokussiert sie sich auf die ökonomischen Aspekten der Schutzwürdigkeit kultureller Güter.

Kilian Bizer ist seit 2004 Professor für Wirtschaftspolitik und Mittelstandsforschung und Direktor des Volkswirtschaftlichen Instituts für Mittelstand und Handwerk an der GeorgAugust-Universität Göttingen. Kulturelle Eigentumsrechte sind nicht nur politökonomische Verhandlungsergebnisse auf nationaler und internationaler Ebene, sondern können sowohl Anreiz als auch Hemmnis für wirtschaftliche Entwicklung insbesondere von kleinen und mittleren Unternehmen sein. Aus diesem Grund ist es von hohem ökonomischen Interesse, die Aushandlungsprozesse der kulturellen Verfügungsrechte und die von ihnen ausgehenden Wirkungen zu untersuchen.

Rosemary Coombe ist Tier One Canada Research Chair der Law, Communication and Cultural Studies an der York University in Toronto. Die Rechtswissenschaftlerin und Kulturanthropologin ist international ausgewiesene Expertin auf dem Bereich der kulturellen, politischen und sozialen Implikationen von Gesetzgebung um geistiges Eigentum. Sie ist Autorin einer der Hauptstudien zum Thema geistiges Eigentum und Kulturpolitik (The Cultural Life of Intellectual Properties, Durham 1998).

Aditya Eggert studierte Internationales Informationsmanagement mit Schwerpunkt Interkulturelle Kommunikation an den Universitäten Hildesheim, Göttingen und Antananarivo, Madagaskar. Während des Stiftungskollegs für internationale Aufgaben der Robert Bosch Stiftung und der Studienstiftung des deutschen Volkes im Jahr 2007/2008 spezialisierte sie sich auf kulturelles Erbe und Tourismus. Seit 2009 ist sie wissenschaftliche Mitarbeiterin am Institut für Ethnologie der Georg-August-Universität Göttingen und promoviert als Mitglied der DFG Forschergruppe zu Cultural Property über die Konstituierung von immateriellem Kulturerbe in Kambodscha. 
Beate Engelbrecht ist seit 1985 Referentin für Ethnologie am IWF Wissen und Medien (Institut für den Wissenschaftlichen Film) und seit 1986 Lehrbeauftragte für Visuelle Anthropologie an der Universität Göttingen. Sie arbeitet als ethnographische Filmemacherin über Handwerk und Feste in Mexiko, Afrika und Indonesien. Fragen der Theorie und Methoden des ethnographischen Films wie auch des Urheberrechts, der Archivierung und digitalen Nutzung audiovisueller Medien sind ihre Arbeitsschwerpunkte.

Stefan Groth studierte Soziologie, Kulturanthropologie, Wirtschafts- und Sozialpsychologie und Public Relations in Göttingen und Udine. Seit 2008 ist er wissenschaftlicher Mitarbeiter und Promovend in der DFG-Forschergruppe zu Cultural Property am Institut für Kulturanthropologie/Europäische Ethnologie der Georg-August-Universität Göttingen und arbeitet zu internationalen Verhandlungen um traditionelles Wissen.

Zulia Gubaydullina ist seit Juli 2009 akademische Rätin an der Professur für Wirtschaftspolitik und Mittelstandsforschung an der Universität Göttingen. Sie studierte Wirtschaftswissenschaften an der Staatlichen Universtität Kasan (Russland) und promovierte zum Dr. rer. pol. an der Technischen Universität Darmstadt. Seit 2008 ist sie Mitglied der DFGForschergruppe zu Cultural Property.

Brigitta Hauser-Schäublin ist Professorin für Ethnologie an der Universität Göttingen. Auf ihren Feldforschungen in Papua-Neuguinea (zwischen 1972 und 1985) und auf Bali, Indonesien (seit 1988), hat sie sich auch mit dem Handeln von lokalen und regionalen Akteuren am Schnittpunkt zwischen Kultur, Kunst, Politik und internationalem Tourismus befasst. Fragen des kulturellem Eigentums wurden auch im Rahmen internationaler Ausstellungen der Göttinger James Cook Sammlung in Hawai'i, Canberra und Bonn (zwischen 2006 und 2010) virulent und haben den Blick für entsprechende Forschungsfragen geschärft.

Karin Klenke studierte Ethnologie und Soziologie an den Universitäten Göttingen und Kopenhagen und war anschließend Stipendiatin im DFG-geförderten Graduiertenkolleg Identität und Differenz an der Universität Trier. In ihrer Dissertation zum Thema Körper, Schönheit und Geschlecht in Tana Karo, Nord-Sumatra/Indonesien analysierte sie die zunehmende Bedeutung von Schönheitspraxen im Kontext gesellschaftlicher Transformationsprozesse. Seit 2008 ist sie wissenschaftliche Mitarbeiterin in der Forschergruppe Cultural Property. Ihr Projekt zum Thema des Nominierungsprozesses von Tana Toraja als UNESCO-Weltkulturerbe fokussiert die Transformationen und Multiplikationen der Bedeutungen und Werte der nominierten Orte zwischen lokaler und transnationaler Ebene.

Matthias Lankau studierte internationale Betriebswirtschaftslehre in Nürnberg und Sydney, sowie internationale Volkswirtschaftslehre in Göttingen. Seit 2008 ist er wissenschaftlicher Mitarbeiter und Promovend in der DFG-Forschergruppe zu Cultural Property an der Wirtschaftswissenschaftlichen Fakultät der Georg-August-Universität Göttingen und fokussiert sich auf internationalen Verhandlungen um traditionelles Wissen. 
Ejan Mackaay war von 1972 bis zu seiner Pensionierung Ende 2008 Professor an der Juristischen Fakultät der Universität von Montreal. Von 1999 bis 2003 leitete der das Forschungszentrum für öffentliches Recht und von 2005 bis 2008 das Centre for the Law of Business and International Trade. In der letzten Phase seiner Karriere konzentrierte sich sein Forschungsinteresse auf Intellectual Property und auf die wirtschaftswissenschaftliche Analyse des Rechts, worüber er 2008 zusammen mit Stéphane Rousseau die erste französischsprachige Abhandlung veröffentlichte.

Sven Mißling ist wissenschaftlicher Angestellter am Lehrstuhl Prof. Dr. P.-T. Stoll, Institut für Völkerrecht und Europarecht der Georg-August-Universiät Göttingen und Mitglied der DFG-Forschergruppe zu Cultural Property. Die Beschäftigung mit Aspekten des nationalen, europäischen und internationalen Kulturrechts, insbesondere mit der UNESCO, stellt unter anderem einen Schwerpunkt seiner aktuellen Forschungsarbeit dar. Seine Dissertation befasst sich mit der „Kunst in Art. 5 Abs. 3 Satz 1 GG - Grundrechtliche(r) Struktur und gesellschaftliche(r) Funktion".

Johannes Müske ist wissenschaftlicher Mitarbeiter und Promotionsstudent am Institut für Volkskunde/Kulturanthropologie der Universität Hamburg. Er studierte Volkskunde, Rechtswissenschaft, Betriebswirtschaftslehre und Museumsmanagement an den Universitäten Hamburg und Sevilla. Er ist Mitglied im Forschungskolleg Kulturwissenschaftliche Technikforschung und der interdisziplinären DFG-Forschergruppe zum Thema Cultural Property, wo er den Zusammenhang zwischen Speichertechnik und der Entstehung eines „Kulturguts“ Klang untersucht.

Arnika Peselmann ist seit ihrem Magisterabschluss 2008 im Fach Kulturanthropologie/Europäische Ethnologie an der Georg-August-Universität Göttingen als Koordinatorin der interdisziplinären DFG-Forschergruppe zu Cultural Property tätig. In ihrem empirisch angelegten Dissertationsvorhaben untersucht sie die ideellen und ökonomischen Inwertsetzungsprozesse von kulturellen Elementen im Kontext von UNESCO-Welterbenominierungen.

Philipp Socha ist Doktorand und wissenschaftliche Hilfskraft bei Prof. Dr. P.-T. Stoll, Institut für Völkerrecht und Europarecht der Georg-August-Universiät Göttingen und Mitglied der DFG-Forschergruppe zu Cultural Property. Nach seinem Studium in Göttingen und Toronto in den Bereichen des internationalen Rechts geistigen Eigentums und dem Kulturvölkerrecht, beschäftigt er sich in seiner Promotion mit Gruppenrechten und Kollektiven im Völkerrecht

Philipp Zimbehl ist seit seinem Diplomabschluss in Rechtswissenschaften im Juli 2009 am Lehrstuhl für Bürgerliches Recht, Handels- und Wirtschaftsrecht, Rechtsvergleichung, Multimedia- und Telekommunikationsrecht von Prof. Dr. Gerald Spindler mit der Arbeit in der interdisziplinären DFG-Forschergruppe zu Cultural Property im Teilprojekt „Recht und Ökonomik von Cultural Property: Eine institutionenökonomische Analyse der Regelbildung“ betraut. Sein Dissertationsvorhaben beschäftigt sich mit der Bewertung von Ansätzen zum Schutz von Folklore aus rechtsökonomischer Sicht. 



\section{Literaturverzeichnis}

Adams, Kathleen M.

1984 Come to Tanah Toraja, "Land of the Heavenly Kings": Travel Agents as

Brokers in Ethnicity. Annuals of Tourism Research 11(3):469-485.

1997 Constructing and Contesting Chiefly Authority in Contemporary Tana

Toraja, Indonesia. In Chiefs Today. Geoffrey M. White und Lamont

Lindstrom, Hrsg. S. 264-275. Stanford: Stanford University Press.

1998 More than an Ethnic Marker: Toraja Art as Identity Negotiator. American

Ethnologist 25(3):327-351.

2006 Art as politics: Re-crafting Identities, Tourism, and Power in Tana Toraja, Indonesia. Honolulu: University of Hawai'i Press.

2009 Courting and Consorting with the Global: The Local Politics of an emerging World Heritage Site in Sulawesi, Indonesia." In Victor T. King, Micheal Parnwell und Michael Hitchcock, Hrsg. Heritage Tourism in Southeast Asia. Honolulu: Nias Press and University of Hawai'i Press.

Aikawa-Faure, Noriko

2009 From the Proclamation of Masterpieces to the Convention for the

Safeguarding of Intangible Heritage. In Intangible Heritage. Laurajane Smith and Natsuko Akagawa, Hrsg. S.13-45. London/New York : Routledge.

Akerlof, George A., und Rachel E. Kranton

2000 Economics and Identity. The Quarterly Journal of Economics 115(3):715753.

Akerlof, George A. und Rachel E. Kranton

2010 Identity Economics: How Our Identities Shape Our Work, Wages, and

Well-Being. Princeton: Princeton University Press.

Albrecht, Helmut, Jane Gradtke und Jens Kugler

2007 UNESCO-Welterbe-Projekt Montanregion Erzgebirge. Realisierungstudie

2007. Freiberg: Förderverein Montanregion Erzgebirge e.V.

Albro, Robert

2005a The Challenges of Asserting, Promoting, and Performing Cultural Heritage. Theory of Cultural Heritage 1:1-8.

2005b Managing Culture at Diversity's Expense: Thoughts on UNESCO's newest Cultural Policy. The Journal of Arts Management, Law and Society 35(3):247-254. 
2007 The Terms of Participation in Recent UNESCO Cultural Policy Making. In Safeguarding Intangible Cultural Heritage: Challenges and Approaches. Janet Blake, Hrsg. S. 109-128. Powys: Builth Wells.

Anderson, Benedict

1983 Imagined Communities: Reflections on the Origin and Spread of Nationalism. London: Verso.

Anderson, Jane E.

2009 Law, Knowledge and Culture: the Production of Indigenous Knowledge in Intellectual Property Law. Cheltenham: Edward Elgar.

Andolino, Robert, Sarah Radcliffe, und Nina Laurie

2005 Development and Culture: Transnational Identity Making in Bolivia.

Political Geography 24(6):678-702.

Anico, Marta, und Elsa Peralta

2009 Introduction. In Heritage and Identity. Engagement and Demission in the Contemporary World. Marta Anico und Elsa Peralta Hrsg. S. 1-11. London: Routledge.

Antons, Christoph

2008a Traditional Cultural Expressions and their Significance for Development in a Digital Environment: Examples from Australia and Southeast Asia. In Intellectual Property and Traditional Cultural Expressions in a Digital Environment. Christoph Beat Graber und Mira Burri-Nenova, Hrsg. S. 287302. Cheltenham: Edward Elgar.

2008b Traditional Knowledge, Traditional Cultural Expressions and Intellectual Property Law in the Asia-Pacific Region. The Hague: Kluwer Law International.

2009 What Is "Traditional Cultural Expression"? International Definitions and Their Application in Developing Asia. WIPO Journal 1:111-123.

Appadurai, Arjun

1986 Introduction: Commodities and the Politics of Value. In The Social Life of Things: Commodities in Cultural Perspective. Arjun Appadurai, Hrsg. S. 3-63. Cambridge: Cambridge University Press.

1990 Disjuncture and Difference in the Global Cultural Economy. In Global Culture. Mike Featherstone, Hrsg. S. 295-310. London: Sage.

1996 Modernity at Large: Cultural Dimensions of Globalization. Minneapolis: University of Minnesota Press.

2004 The Capacity to Aspire: Culture and the Terms of Recognition. In Culture and Public Action: A Cross-Disciplinary Dialogue on Developement Policy. Vijayendra Rao und Michael Walton, Hrsg. S. 59-84. Stanford: Stanford University Press. 
Appiah, Kwame Anthony

2006 Cosmopolitanism: Ethics in a World of Strangers. New York: Norton.

Aragon, Lorraine V.

2008 The Local Commons as a Missing Middle in Debates over Indigenous

Knowledge and Intellectual Property Law. Vortrag zum Annual Meeting der

American Anthropological Association, San Francisco.

Aragon, Lorraine V. und James Leach

2008 Arts and Owners: Intellectual Property Law and the Politics of Scale in

Indonesian Arts. American Ethnologist 35(4):607-631.

Asch, Timothy

1988 Collaboration in Ethnographic Filmmaking: A Personal View. In

Anthropological Filmmaking. Jack R. Rollwagen, Hrsg. S. 1-29. Chur:

Harwood.

Assmann, Aleida

2009 Erinnerungsräume: Formen und Wandlungen des kulturellen

Gedächtnisses. München: Beck.

Attar, Mohsen al, Nicole Aylwin und Rosemary J. Coombe

2009 Indigenous Cultural Heritage Rights in International Human Rights Law.

In Protection of First Nations Cultural Heritage: Laws, Policy, and Reform.

Catherine E. Bell und Robert K. Paterson, Hrsg. S. 311-342. Vancouver: UBC Press.

Audi, Alan

2007 A Semiotics of the Cultural Property Argument. International Journal of

Cultural Property 14(2):131-156.

Auerbach, Konrad

2000 Museumsführer Erzgebirgisches Spielzeugmuseum Seiffen. Seiffen:

Erzgebirgisches Spielzeugmuseum Seiffen.

Australian Film Commission

2003 Issues Paper: Towards a Protocol for Filmmakers Working with

Indigenous Content and Indigenous Communities. http://www.wipo.int/tk/

en/folklore/creative_heritage/policy/link0008.html (Zugriff am 07.03.2010).

Aylwin, Nicole und Rosemary J. Coombe

2010 Marks Indicating Conditions of Origin in Rights-Based and Sustainable

Development. In Human Rights, Development and Restorative Justice: An

Osgoode Reader. Peer Zumbansen und Ruth Buchanan, Hrsg. Oxford: Hart.

BPSTT (Badan Pusat Statistik Kabupaten Tana Toraja)

2007 Tana Toraja dalam angka: Tana Toraja in Figures 2006. Rantepao:

Kabupaten Tana Toraja. 
Ballhaus, Edmund und Beate Engelbrecht, Hrsg.

1995 Der ethnographische Film. Einführung in Methoden und Praxis. Berlin:

Diedrich Reimer.

Balliger, Robin

2007 The Politics of Cultural Value and the Value of Cultural Politics:

International Intellectual Property Legislation in Trinidad. In Trinidad Carnival:

The Cultural Politics of a Transnational Festival. Garth L. Green und Philip W.

Scher, Hrsg. S. 198-214. Bloomington: Indiana University Press.

Bappert, Walter

1962 Wege zum Urheberrecht: Die geschichtliche Entwicklung des

Urheberrechtsgedankens. Frankfurt am Main: Klostermann.

Barthes, Roland

1972[1957] Mythologies. Jonathan Cape, Übersetzer. New York: Noonday.

Bator, Paul M.

1982 An Essay on the International Trade in Art. Stanford Law Review

(34):275-384.

Bauman, Richard und Charles L. Briggs

2003 Voices of Modernity: Language Ideologies and the Politics of Inequality.

Cambridge: Cambridge University Press.

Bausinger, Hermann

1961 Volkskultur in der technischen Welt. Stuttgart: Kohlhammer.

1966 Zur Kritik der Folklorismuskritik. In Populus Revisus: Beiträge zur

Erforschung der Gegenwart. Hermann Bausinger, Hrsg. S.61-75. Tübingen:

Tübinger Vereinigung für Volkskunde.

1981 Technik im Alltag: Etappen der Aneignung. Zeitschrift für Volkskunde 77(2): 227-242.

1988 Da Capo: Folklorismus. In Sichtweisen der Volkskunde: Zur Geschichte und Forschungspraxis einer Disziplin. Albrecht Lehmann und Andreas Kuntz, Hrsg. S. 321-329. Berlin: Reimer.

Bausinger, Hermann, Utz Jeggle, Gottfried Korff und Martin Scharfe, Hrsg. 1999 Grundzüge der Volkskunde. Darmstadt: Wissenschaftliche

Buchgesellschaft.

Bebbington, Anthony

2004a NGOs and Uneven Development: Geographies of Development

Intervention. Progress in Human Geography 28(6):725-745.

2004b Social Capital and Development Studies 1: Critique, Debate, Progress?

Progress in Development Studies 4:343-349.

2005 Culture and Public Action: A Cross-Disciplinary Dialogue on

Development Policy. American Anthropologist 107(2):305-306. 
Beck, Stefan

1997 Umgang mit Technik: Kulturelle Praxen und kulturwissenschaftliche

Forschungskonzepte. Berlin: Akademie-Verlag.

Beckert, Jens

2005 The Moral Embeddedness of Markets. MPIfG Discussion Paper 05(6):1-

22.

2007 Die soziale Ordnung von Märkten. MPIfG Discussion Paper 07(6):1-26.

Behnam, Awni

1998 The Group System. In Multilateral Diplomacy: The United Nations System at Geneva. A Working Guide. Marcel A. Boisard und Evgeny M.

Chossudovsky, Hrsg. S. 193-204. The Hague: Kluwer Law International.

Bell, Catherine Edith

2008 First Nations Cultural Heritage: a Selected Survey of Issues and Initiatives.

In First Nations Cultural Heritage and Law: Case Studies, Voices, and

Perspectives. Catherine Edith Bell und Val Napoleon, Hrsg. S. 367-414.

Vancouver: UBC Press.

Bell, Catherine Edith und Robert K. Paterson

2008 Protection of First Nations Cultural Heritage: Laws, Policy, and Reform. Vancouver: UBC Press.

Benda-Beckmann, Franz von, Keebet von Benda-Beckmann und Melanie Wiber 2006a Introduction: The Properties of Property. In Changing Properties of Property. Franz von Benda-Beckmann, Keebet von Benda-Beckmann, und Melanie Wiber, Hrsg. S.1-40. New York: Berghahn.

Benda-Beckmann, Franz von, Keebet von Benda-Beckmann und Melanie Wiber, Hrsg.

2006b Changing Properties of Property. New York: Berghahn

Bendix, Regina

1997 In Search of Authenticity: The Formation of Folklore Studies. Madison:

University of Wisconsin Press.

2000 Heredity, Hybridity and Heritage from one Fin-de-Siecle to the Next. In

Folklore, Heritage Politics and Ethnic Diversity: A Festschrift for Barbro

Klein. Pertti J. Anttonen und Babro Klein, Hrsg. S. 37-54. Botkyrka:

Multicultural Center.

2007 Kulturelles Erbe zwischen Wirtschaft und Politik: Ein Ausblick. In

Prädikat „Heritage“. Wertschöpfungen aus kulturellen Ressourcen. Dorothee

Hemme, Markus Tauschek und Regina Bendix, Hrsg. S. 337-356. Berlin: LIT.

2009a Heritage between Economy and Politics: An Assessment from the

Perspective of Cultural Anthropology. In Intangible Heritage. Laurajane Smith und Noriko Akagawa, Hrsg. S. 253-69. London: Routledge.

2009b Possession, Onwership and Responsibility. Traditiones 38(2):181-199. 
Bendix, Regina und Valdimar T. Hafstein

2009 Culture and Property. An Introduction. Ethnologia Europaea 39(2):5-10.

Bendix, Regina und Gisela Welz, Hrsg.

2002 Kulturwissenschaft und Öffentlichkeit. Amerikanische und deutschsprachige Volkskunde im Dialog. Frankfurt am Main: Institut für Kulturanthropologie und Europäische Ethnologie.

Benjamin, Walter 2006[1936] Das Kunstwerk im Zeitalter seiner technischen Reproduzierbarkeit. Frankfurt am Main: Suhrkamp.

Benkler, Yochai

1999 Free as the Air to Common Use: First Amendment Constraints on Enclosure of the Public Domain. New York University Law Review 74(2):354-446.

Berger, Karl C., Hrsg.

2009 Erb.gut? Kulturelles Erbe in Wissenschaft und Gesellschaft. Wien: Selbstverlag des Vereins für Volkskunde.

Berndt, Joachim 1998 Internationaler Kulturgüterschutz. Abwanderungsschutz, Regelungen im innerstaatlichen Recht, im Europa- und Völkerrecht. Köln: Heymann.

Berryman, Cathryn A.

1993 Toward More Universal Protection of Intangible Cultural Property. Journal of Intellectual Property Law 1(2):293-333.

Beus, Jos de

1996 The Value of National Identity. In The Value of Culture: On the

Relationshiph between Economics and Arts. Arjo Klamer, Hrsg. S. 166-186. Amsterdam: Amsterdam University Press.

Bhatia, Vijay K., Jan Engberg und Maurizio Gotti, Hrsg. 2005 Vagueness in Normative Texts. Bern: Peter Lang.

Bhatia, Vijay K., Christopher N. Candlin und Paola Evangelisti Allori, Hrsg. 2008 Language, Culture and the Law: The Formulation of Legal Concepts across Systems and Cultures. Bern: Peter Lang.

Bijker, Wiebe E.

1995 Of Bicycles, Bakelites, and Bulbs: Toward a Theory of Sociotechnical Change. Cambridge: MIT Press.

Bijker, Wiebe E., Thomas P. Hughes und Trevor J. Pinch, Hrsg. 1987 The Social Construction of Technological Systems: New Directions in the Sociology and History of Technology. Cambridge: MIT Press. 
Biller, Dan

2010 The Economics of Biodiversity Loss. In Solutions for the World's Biggest Problems: Costs and Benefits. Bjørn Lomborg, Hrsg. S. 162-177. Cambridge: Cambridge University Press.

Binder, Beate, Wolfgang Kaschuba und Peter Niedermüller, Hrsg. 2001 Inszenierung des Nationalen: Geschichte, Kultur und die Politik der Identitäten am Ende des 20. Jahrhunderts. Köln: Böhlau.

Bizot, Francois

1973 Histoire du Reamker. Récit recueilli et présente. Phnom Penh: Cambodian Institute for Cooperation and Peace.

Blake, Janet

2008 Safeguarding Intangible Heritage under UNESCO's 2003 Convention. In

Il patrimonio immateriale secondo l'UNESCO: analisi e prospettive. Chiara Bortolotto, Hrsg. S.49-67. Rom: Ist. Poligrafico dello Stato.

2009 UNESCO's 2003 Convention on Intangible Cultural Heritage. The Implications of Community Involvement in "Safeguarding". In Intangible Heritage. Laurajane Smith und Natsuko Akagawa, Hrsg. S.45-74. London: Routledge.

Bois, George

1906 Le Sculpteur Rodin et les Danseuses Cambodgiennes. L'Illustration 3309:64-64.

Bortolotto, Chiara, Hrsg.

2008 Il patrimonio immateriale secondo l'UNESCO: analisi e prospettive. Rom: Ist. Poligrafico dello Stato

Boyle, James

2008 The Public Domain: Enclosing the Commons of the Mind. New Haven: Yale University Press.

Braithwaite, John, und Peter Drahos

2000 Global Business Regulation. Cambridge: Cambridge University Press.

Bramley, Cerkia und Johann F. Kirsten

2007 Exploring the Economic Rationale for Protecting Geographical Indicators in Agriculture. Agrekon 46(1):69-93.

Brams, Steven J.

1975 Game Theory and Politics. London: Free Press.

Bräuchler, Birgit

2007 Ein Comeback der Tradition? Die Revitalisierung von Adat in Ostindonesien. Zeitschrift für Ethnologie 132(1):37-57. 
Breglia, Lisa

2006 Monumental Ambivalence: The Politics of Heritage. Austin: University of Texas Press.

Briggs, Charles L.

1993 Linguistic Ideologies and the Naturalization of Power in Warao Discourse.

Pragmatics 2(3):387-404.

Brown, Michael F.

1998 Can Culture Be Copyrighted? Current Anthropology 39(2):193-222.

2003 Who Owns Native Culture? Cambridge: Harvard University Press.

2004 Heritage as Property. In Property in Question. Value Transformation in the Global Economy. Katherine Verdery und Caroline Humphrey, Hrsg. S. 49-68. Oxford: Berg.

2005 Heritage Trouble: Recent Work on the Protection of Intangible Cultural Property. Journal of Cultural Property 12(01):40-61.

Brunet, Jacques

1969 Nang Sbek. Théatre d'ombres dansé du Cambodge. Berlin: Institut

International d'Etudes Comparatives de la Musique. Berlin.

Bryant, Raymond L.

2002a False Prophets? Mutant NGOs and Philippine Environmentalism. Society

\& Natural Resources 15(7):629-639.

2002b Non-governmental Organizations and Governmentality: "Consuming"

Biodiversity and Indigenous People in the Philippines. Political Studies 50(2):268-292.

Buddhist Institute

1937 Reamker. Volume 1-80. Phnom Penh: Buddhist Institute.

Buggey, Susan und Nora Mitchell

2008 Cultural Landscapes: Venues for Community-based Conservation. In

Cultural Landscapes: Balancing Nature and Heritage in Preservation Practice.

Richard W. Longstreth, Hrsg. S. 164-179. Minneapolis: University of Minnesota Press.

Bumbaru, Dinu

2006 Overview of the Development of Trends in Heritage Management since the 1964 Venice Charter. In Proceedings. International Conference on the Safeguarding of Tangible and Intangible Cultural Heritage: Towards an Integrated Approach. UNESCO/Agency for Cultural Affairs (Japan), Hrsg. S.42-74. Paris: UNESCO.

Bundesregierung der Bundesrepublik Deutschland 2007 Gutachten der Bundesregierung zur Bindungswirkung des UNESCOÜbereinkommens zum Schutz des Natur- und Kulturerbes der Welt. Berlin. 
Burri-Nenova, Mira

2008 The Long Tail of the Rainbow Serpent: New Technologies and the

Protection and Promotion of Traditional Cultural Expressions. In Intellectual

Property and Traditional Cultural Expressions in a Digital Environment.

Christoph Beat Graber und Mira Burri-Nenova, Hrsg. S. 205-236.

Cheltenham: Edward Elgar.

Busse, Mark

2008 Museums and the Things in Them Should Be Alive. International Journal of Cultural Property 15(2):189-200.

Cadena, Marisol de la und Orin Starn

2007 Indigenous Experience Today. Oxford: Berg.

Cambodian Living Arts (CLA)

2007 Masters Teaching. http://www.cambodianlivingarts.org/projects/teaching (Zugriff am 08.04.2010).

Carneiro da Cunha, Manuela

2009 "Culture" and Culture: Traditional Knowledge and Intellectual Rights.

Chicago: Prickly Paradigm Press.

Carpenter, Kristen A., Sonia K. Katyal und Angela R. Riley

2009 In Defense Of Property. The Yale Law Journal 118:1022-1125.

Carstens, Janet, und Stephan Hugh-Jones, Hrsg.

1995 About the House. Lévi-Strauss and Beyond. Cambridge: Cambridge

University Press.

Castells, Manuel

1996-1998 The Information Age: Economy, Society and Culture, Volume 1-3.

Cambridge: Blackwell.

CBC News

2000 "Strike Fear in the Heart of the White Man": Mugabe.

http://www.cbc.ca/world/story/2000/12/14/mugabe001214.html (Zugriff am 13.09.2009).

Chan, Anita Say

2008 E-Governance for Artisans: Intellectual Property, Networked Culture, and the Promiscuity of Freedom in Peru. Vortrag zum Annual Meeting der American Anthropolgist Association, San Francisco.

Cheng, Sao-Wen

2006 Cultural Goods Creation, Cultural Capital Formation, Provision of

Cultural Services and Cultural Atmosphere Accumulation. Journal of Cultural Economics 30:263-286. 
Chibnik, Michael

2003 Crafting Tradition: The Making and Marketing of Oaxacan Wood

Carvings. Austin: University of Texas Press.

Chrispeels, Erik

1998 Procedures of Multilateral Conference Diplomacy. In Multilateral

Diplomacy: The United Nations System at Geneva. A Working Guide. Marcel

A. Boisard und Evgeny M. Chossudovsky, Hrsg. S. 119-136. The Hague:

Kluwer Law International.

Christen, Kimberly A.

2005 Gone Digital: Aboriginal Remix and the Cultural Commons. International Journal of Cultural Property 12(3):315-345.

2008 Aboriginal Business: Alliances in a Remote Australian Town. Santa Fe:

School for Advanced Research Press.

Clark, A. Kim

2005 Ecuadorian Indians, the Nation, and Class in Historical Perspective:

Rethinking a "New Social Movement”. Anthropologica 47:53-65.

Clarke, Gerard

2001 From Ethnocide to Ethnodevelopment? Ethnic Minorities and Indigenous

Peoples in Southeast Asia. Third World Quarterly 22(3):413-436.

Clarke, Kamari Maxine

2004 Mapping Yorùbá Networks: Power and Agency in the Making of

Transnational Communities. Durham: Duke University Press.

Clifford, James

2004 Looking Several Ways: Anthropology and Native Heritage in Alaska.

Current Anthropology 45(1):5-23.

Clift, Charles

2007 Is Intellectual Property Protection a Good Idea? In Local Science vs.

Global Science. Approaches to Indigenous Knowledge in International

Development. Paul Sillitoe, Hrsg. S. 191-208. New York: Berghahn.

Coffey, Mary K.

2003 From Nation to Community: Museums and the Reconfiguration of

Mexican Society under Neoliberalism. In Foucault, Cultural Studies, and

Governmentality. Jack Z. Bratich, Jeremy Packer und Cameron McCarthy,

Hrsg. S. 207-243. Albany: State University of New York Press.

Cohen, Elizabeth

2001 Preservation of Audio. In Folk Heritage Collections in Crisis. Council on

Library and Information Resources Reports, Hrsg. S. 20-27. Washington:

CLIR. 
Coleman, Elizabeth Burns, Rosemary J. Coombe und Fiona MacArailt 2009 A Broken Record: Subjecting Music to Cultural Rights. In Ethics of

Cultural Appropriation. James O. Young und Conrad G. Brunk, Hrsg. S. 173210. Oxford: Wiley-Blackwell.

Comaroff, John L. und Jean Comaroff 2009 Ethnicity, Inc. Chicago: University of Chicago Press.

Copinger, Walter Arthur, Edmund Purcell Skone James und Kevin M. Garnett 2005 Copinger and Skone James on Copyright Vol. 2. London: Sweet \& Maxwell.

Coombe, Rosemary J.

1991 Objects of Property and Subjects of Politics: Intellectual Property Law and Democratic Dialogue. Texas Law Review 69:1853-1880.

1997 Cultural Appropriations. London: Routledge.

1998 The Cultural Life of Intellectual Property. Authorship, Appropriation, and the Law. Durham: Duke University Press.

2003a Fear, Hope, and Longing for the Future of Authorship and a Revitalized

Public Domain in Global Regimes of Intellectual Property. De Paul Law

Review. 52:1171-1192.

2003b Works in Progress: Indigenous Knowledge, Biological Diversity and

Intellectual Property in a Neoliberal Era. In Globalization under Construction : Governmentality, Law, and Identity. Richard Warren Perry und Bill Maurer, Hrsg. S. 273-314. Minneapolis: University of Minnesota Press.

2005a Legal Claims to Culture in and Against the Market: Neoliberalism and the Global Proliferation of Meaningful Difference. Law, Culture and the Humanities 1(1):32-55.

2005b Protecting Traditional Environmental Knowledge and New Social

Movements in the Americas: Intellectual Property, Human Right or Claims to an Alternative Form of Sustainable Development? Florida Journal of International Law 17:115-136.

2007 The Work of Rights at Governmentality's Limits. Anthropologica 49(2):284-299.

2008 First Nations' Intangible Cultural Heritage Concerns: Prospects for

Protection of Traditional Knowledge and Traditional Cultural Expressions in International Law. In Protection of First Nations Cultural Heritage: Laws, Policy, and Reform. Catherine E. Bell und Robert K. Paterson, Hrsg. S. 247277. Vancouver: UBC Press.

2010a Cultural Agencies: The "Construction" of Community Subjects and Their Traditions. In The Making and Unmaking of Intellectual Property. Mario Biagioli, Peter Jaszi und Martha Woodmansee, Hrsg. Chicago: University of Chicago Press. 
2010b Owning Culture: Locating Communities and Their Properties. In Ownership and Appropriation. Mark Busse und Veronica Strang, Hrsg. London: Berg.

Coombe, Rosemary J., Steven Schnoor und Mohsen Ahmed

2007 Bearing Cultural Distinction: Informational Capital and New Expectations for Intellectual Property. University of California Davis Law Review 40(3):891-917.

Cornu, Marie

2004 La Protection du Patrimoine Culturel Immatériel. In Intérêt Culturel et Mondialisation, Vol. 2: Les Aspects Internationaux. Nébila Mezghani und Marie Cornu, Hrsg., S.197-218. Paris: L'Harmattan.

Council on Library and Information Resources (CLIR), Hrsg. 2000 Authenticity in a Digital Environment. Washington: CLIR.

Crawford, Peter I. und David Turton, Hrsg. 1992 Film as Ethnography. Manchester: Manchester University Press.

Cross, John G.

1977 Negotiation as a Learning Process. In The Negotiation Process. Theories and Applications. I. William Zartman, Hrsg. S. 29-54. Beverly Hills: Sage.

Crystal, Eric

1970 Toraja Town. University of California, Berkeley: Ph.D. Dissertation.

1974 Cooking-Pot politics: A Toraja Village Study. Indonesia 18:119-152.

Danielson, Virginia

2001 Stating the Obvious: Lessons Learned Attempting Access to Archival Audio Collections. In Folk Heritage Collections in Crisis. Council on Library and Information Resources, Hrsg. S. 4-13. Washington: CLIR.

Davidson, Jamie S. und David Henlev, Hrsg.

2007 The Revival of Tradition in Indonesian Politics: The Deployment of Adat from Colonialism to Indigenism. London: Routledge.

Dervyttere, Anne

2004 Indigenous Peoples, Development with Identity and the Inter-American Development Bank: Challenges and Opportunities. In Lessons of Indigenous Development in Latin America: The Proceedings of a World Bank Workshop on Indigenous Peoples Development. Shelton H. Davis, Jorge Enrique Uquillas und Melanie A. Eltz, Hrsg. S. 23-30. Washington: World Bank Environ. Soc. Sustain. Dev. Dep.

Deutsche UNESCO-Komission

2006 Übereinkommen über Schutz und Förderung der Vielfalt kultureller Ausdrucksformen. Magna Charta der Internationalen Kulturpolitik. Bonn: Deutsche UNESCO-Kommission. http://www.unesco.de/kkvbroschuere.html?\&L=0 (Zugriff am 28.01.09). 
Dijk, Teun A. van

1998 Ideology: A Multidisciplinary Approach. London: Sage.

Dommann, Monika

2008 Lost in Tradition? Reconsidering the History of Folklore and its Legal

Protection since 1800. In Intellectual Property and Traditional Cultural

Expressions in a Digital Environment. Christoph Beat Graber und Mira Burri-

Nenova, Hrsg. S. 3-16. Cheltenham: Edward Elgar.

Donzelli, Aurora

2007 Copyright and Authorship: Ritual Speech and the New Market of Words in Toraja. In Learning Religion: Anthropological Approaches. David Berliner und Ramón Sarró, Hrsg. S. 141-159. Oxford: Berghahn.

Dow, James R. und Lixfeld, Hannsjost, Hrsg.

1986 German Volkskunde: a Decade of Theoretical Confrontation, Debate, and Reorientation (1967-1977). Bloomington: Indiana University Press.

Druckman, Daniel

2001 Turning Points in International Negotiation. Journal of Conflict

Resolution 45:519-544.

Dutfield, Graham und Uma Suthersanen

2008 Global Intellectual Property Law. Cheltenham: Edward Elgar.

Edelmann, Marc

2005 Bringing the Moral Economy back in... to the Study of 21st-Century

Transnational Peasant Movements. American Anthropologist 107(3):331-345.

Elyachar, Julia

2005 Markets of Dispossession: NGOs, Economic Development, and the State in Cairo. Durham: Duke Univ. Press.

Engels, Friedrich

1884 Der Ursprung der Familie, des Privateigentums und des Staats. Hottingen-

Zürich: Verlag der Schweizerischen Volksbuchhandlung.

Ensor, Jonathan

2005 Linking Rights and Culture. In Reinventing Development? Translating

Rights-based Approaches from Theory into Practice. Paul Gready und

Jonathan Ensor, Hrsg. S. 254-277. London: Zed Books.

Ernst, Wolfgang

2002 Das Rumoren der Archive: Ordnung aus Unordnung. Berlin: Merve.

Escobar, Arturo

2001 Culture Sits in Places: Reflections on Globalism and Subaltern Strategies

of Localization. Political Geography 20(2):139-174.

2003 Place, Nature, and Culture in Discourses of Globalization. In Localizing

Knowledge in a Globalizing World: Recasting the Area Studies Debate. Ali 
Mirsepassi, Amrita Basu und Frederick Sturton Weaver, Hrsg. S. 37-59. Syracuse: Syracuse University Press.

2008 Territories of Difference: Place, Movements, Life, Redes. Durham: Duke University Press.

Evans-Pritchard, Deirdre

1987 The Portal Case: Authenticity, Tourism, Traditions, and the Law. Journal of American Folklore 100(397):287-296.

Fabian, Johannes

1983 Time and the Other: How Anthropology Makes Its Object. New York:

Columbia University Press.

2008 Ethnography as Commentary: Writing from the virtual Archive. Durham:

Duke Univ. Press.

Farley, Christine Haight

1997 Protecting Folklore: Is Intellectual Property the Answer? Connecticut Law Review 30(1):1-58.

Fechner, Frank, Hrsg.

1996 Prinzipien des Kulturgüterschutzes. Ansätze im deutschen, europäischen und internationalen Recht. Berlin: Duncker \& Humblot.

Filbo, C. F. und M. de Souza

2007 Multiculturalism and Collective Rights (in Latin America). In Another Knowledge is Possible: Beyond Northern Epistemologies. Boaventura de Sousa Santos, Hrsg. S. 74-114. London: Verso.

Filippucci, Paola

2004 A French Place without a Cheese: Problems with Heritage and Identity in Northeastern France. Focaal 44:72-86.

Forsyth, Miranda

2003 Intellectual Property Laws in the South Pacific: Friend or Foe? Journal of South Pacific Law 7(1). http://www.paclii.org/journals/fjSPL/vol07no1/

8.shtml (Zugriff am 05.05.2010).

Francioni, Francesco

2008 The 1972 World Heritage Convention: An Introduction. In The 1972

World Heritage Convention: A Commentary. Francesco Francioni und

Federico Lenzerini, Hrsg. S. 3-9. Oxford: Oxford University Press.

Francois, Etienne und Hagen Schulze, Hrsg.

2002 Deutsche Erinnerungsorte. München: Beck.

Francois, Patrick und Tanguy van Ypersele

2002 On the Protection of Cultural Goods. Journal of International Economics 56(2):359-369. 
Frey, Bruno S.

2008 What Values should Count in the Arts? The Tension between Economic

Effects and Cultural Value. In Beyond Price: Value in Culture, Economics, and the Arts. Michael Hutter und David Throsby, Hrsg. S. 261-269. New York: Cambridge University Press.

Frey, Bruno S. und Stephan Meier

2006 The Economics of Museums. In Handbook of the Economics of Art and Culture. Victor A. Ginsburgh und David Throsby, Hrsg. S. 1017-1047.

Amsterdam: Elsevier.

Friedreich, Sönke

2005 Das heilige Land. Frömmigkeit und der Diskurs um regionale Identität im

Erzgebirge. In Ort. Arbeit. Körper. Ethnografie Europäischer Modernen.

Beate Binder, Silke Göttsch, Wolfgang Kaschuba und Konrad Vanja, Hrsg. S. 225-232. Berlin: Waxmann.

2009 Wie man ein Kulturerbe auschlägt. Städtische Selbstbilder und urbane

Pfadabhängigkeiten im Streit um das UNESCO-Weltkulturerbe Dresdner

Elbtal. In Erb.gut? Kulturelles Erbe in Wissenschaft und Gesellschaft. Karl C.

Berger, Margot Schindler und Ingo Schneider, Hrsg. S.171-180. Wien:

Selbstverlag des Vereins für Volkskunde.

Frigo, Manlio

2004 Cultural Property vs. Cultural Heritage: A "Battle of Concepts" in

International Law? International Review of the Red Cross 86(854):367-378.

Fukutomi, Tomoko

1999 Cambodian Shadow Theatre, Sbek Thom, Reamker. Asian Literature (Asia

Bungaku) 5. Tokio: Asia Bunkasha.

2001 Cambodian Shadow Theatre, Sbek Thom, Reamker. Asian Literature (Asia

Bungaku) 6. Tokio: Asia Bunkasha.

Gad, Mohamed Omar

2006 Representational Fairness in WTO Rule Making: Negotiating,

Implementing and Disputing the TRIPS Pharmaceutical-Related Provisions.

London: British Institute of International and Comparative Law.

Gal, Susan und Judith T. Irvine

2000 Language Ideology and Linguistic Differentiation. In Regimes of

Language: Ideologies, Polities and Identities. Paul V. Kroskrity, Hrsg. S. 35-84.

Santa Fe: School of American Research Press.

Gal, Susan

2005 Language Ideologies Compared. Journal of Linguistic Anthropology 15(1):23-37. 
Garsten, Christina und Anna Hasselström

2003 Risky Business: Discourses of Risk and (Ir)Responsibility in Globalizing

Markets. Ethnos 68(2):249-270.

Geismar, Haidy

2008 Cultural Property, Museums, and the Pacific: Reframing the Debates.

International Journal of Cultural Property 15(02):109-122.

Geller, Paul Edward

1998 Hiroshige vs. Van Gogh: Resolving the Dilemma of Copyright Scope in

Remedying Infringement. Journal of the Copyright Society of the USA 46:3970.

Genius-Devime, Barbara

1996 Bedeutung und Grenzen des Erbes der Menschheit im völkerrechtlichen

Kulturgüterschutz. Baden-Baden: Nomos.

Ghafele, Roya

2005 Unlocking the Hidden Potential of Traditional Knowledge: Building up a

Phytopharmaka Market. Präsentation zum "WIPO National Seminar on

Omani Traditional Values in a Globalized World: The Intellectual Property

Challenge", Muscat, Oman. http://www.wipo.int/meetings/en/details.jsp? meeting_id=7445 (Zugriff am 15.02.2010).

Girsberger, Martin A.

2008 Legal Protection of Traditional Cultural Expressions: A Policy Perspective.

In Intellectual Property and Traditional Cultural Expressions in a Digital

Environment. Christoph Beat Graber und Mira Burri-Nenova, Hrsg. S. 123-

149. Cheltenham: Edward Elgar.

Glass, Aaron

2004 Return to Sender: On the Politics of Cultural Property and the Proper Address of Art. Journal of Material Culture 9(2):115-139.

Goldstein, Paul

1994 Copyright's Highway. From Gutenberg to the Celestial Jukebox. New

York: Hill and Wang.

2001 International Copyright: Principles, Law, and Practice. Oxford: Oxford

University Press.

Goodale, Mark

2007 Locating Rights, Envisioning Law between the Global and the Local. In

The Practice of Human Rights: Tracking Law between the Global and the

Local. Mark Goodale und Sally Engle Merry, Hrsg. S. 1-38. Cambridge:

Cambridge University Press.

Goodman, Jane E.

2002 Stealing our Heritage? Women's Folksongs, Copyright Law, and the Public

Domain in Algeria. Africa Today 49:85-97. 
2005 Berber Culture on the World Stage: From Village to Video. Bloomington : Indiana University Press.

Gow, David D.

2008 Countering Development: Indigenous Modernity and the Moral Imagination. Durham: Duke University Press.

Graber, Christoph Beat

2008 Using Human Rights to Tackle Fragmentation in the Field of Traditional Cultural Expressions: An Institutional Approach. In Intellectual Property and Traditional Cultural Expressions in a Digital Environment. Christoph Beat Graber und Mira Burri-Nenova, Hrsg. S. 96-122. Cheltenham: Edward Elgar.

Graber, Christoph Beat und Mira Burri-Nenova, Hrsg.

2008 Intellectual Property and Traditional Cultural Expressions in a Digital Environment. Cheltenham: Edward Elgar.

Graburn, Nelson

1976 Ethnic and Tourist Arts: Cultural Expressions from the Fourth World. Berkeley: University of California Press.

Green, Garth L.

2007 "Come to Life": Authenticity, Value, and the Carnival as Cultural

Commodity in Trinidad and Tobago. Identities: Global Studies in Culture and Power 14(1):203-224.

Greene, Shane

2004 Indigenous People Incorporated? Culture as Politics, Culture as Property in Pharmaceutical Bioprospecting. Current Anthropology 45(2):211-237.

Grenet, Sylvie

2008 The Ethnology Department of the French Ministry of Culture and the Issues of Intangible Cultural Heritage in France. In Il Patrimonio Immateriale secondo l'UNESCO: Analisi e Prospettive. Chiara Bortolotto, Hrsg. S. 85-95. Rom: Ist. Poligrafico dello Stato.

Groschwitz, Helmut

2009 Welterbe als Marke. Kulturelles Erbe und die Produktion kultureller Labels am Beispiel der Welterbestätten Regensburger Altstadt mit Stadtamhof. In Erb.gut? Kulturelles Erbe in Wissenschaft und Gesellschaft. Karl C. Berger, Margot Schindler und Ingo Schneider, Hrsg. S. 215-224. Wien: Selbstverlag des Vereins für Volkskunde.

Groslier, George

1913 Artiste peintre. Danseuses cambodgiennes anciennes et modernes. Paris: Augustin Challamel.

1929 Le Theatre et la danse au Cambodge. Journal Asiatique 214:125-143. 
Groth, Stefan

2009 Tradition und Wert: Die Weltorganisation für Geistiges Eigentum,

Cultural Property und die Vorzeitigkeit von Tradition. Kulturen 2:33-39.

Gruber, Lloyd

2000 Ruling the World: Power Politics and the Rise of Supranational

Institutions. Princeton: Princeton University Press.

Gugerli, David

2007 Die Welt als Datenbank: Zur Relation von Softwareentwicklung, Abfragetechnik und Deutungsautonomie. In Nach Feierabend: Zürcher Jahrbuch für Wissensgeschichte 3. David Gugerli, Michael Hagner, Philipp Sarasin und Jakob Tanner, Hrsg. S.11-36. Zürich: Diaphanes.

Gygi, Beat

1990 Internationale Organisationen aus der Sicht der neuen politischen

Ökonomie. Heidelberg: Physica.

Hafstein, Valdimar

2004 The Politics Of Origins: Collective Creation Revisited. Journal of American Folklore 117(465):300-315.

2007 Claiming Culture: Intangible Heritage Inc., FolkloreC, Traditional

Knowledge ${ }^{\mathrm{TM}}$. In Prädikat Heritage. Wertschöpfung aus kulturellen

Ressourcen. Dorothee Hemme, Markus Tauschek und Regina Bendix, Hrsg. S.

75-100. Münster: LIT.

2009 Intangible Heritage as a List: From Masterpieces to Representation. In

Intangible Heritage. Laurajane Smith und Natsuko Akagawa, Hrsg. S. 93-112.

London: Routledge.

Hale, Charles R.

2002 Does Multiculturalism Menace? Governance, Cultural Rights and the

Politics of Identity in Guatemala. Journal of Latin American Studies 34(3):485524.

Handler, Richard

2003 Cultural Property and Culture Theory. Journal of Social Archaeology 3(3):353-365.

Hann, Chris M.

1998 Introduction: The Embededness of Property. In Property Relations.

Renewing the Anthropological Tradition. Chris M. Hann, Hrsg. S. 1-47.

Cambridge: Cambridge University Press.

Hannerz, Ulf

1996 Transnational Connections. Culture, People, Places. London: Routledge.

Hardin, Garret

1968 The Tragedy of the Commons. Science 162:1243-1248. 
Harrison, Simon

1999 Identity as a Scarce Resource. Social Anthropology 7(3):239-251.

2000 From Prestige Goods to Legacies: Property and the Objectification of

Culture in Melanesia. Comparative Studies in Society and History 42(3):662679.

Hartmann, Andreas

2001 Die Anfänge der Volkskunde. In Grundriß der Volkskunde: Einführung in die Forschungsfelder der europäischen Ethnologie. Rolf Wilhelm Brednich, Hrsg. S. 9-30. Berlin: Reimer.

Harvey, David C.

2001 Heritage Pasts and Heritage Presents: Temporality, Meaning, and the

Scope of Heritage Studies. International Journal of Heritage Studies 7:319-38.

Hayden, Cori

2003 When Nature Goes Public: The Making and Unmaking of Bioprospecting in Mexico. Princeton: Princeton University Press.

Hegel, Georg Wilhelm Friedrich

2006[1807] Phänomenologie des Geistes. Hamburg: Meiner.

Helfer, Laurence R.

2007 Toward a Human Rights Framework for Intellectual Property. University of California Davis Law Review 40:971-1020.

Heller, Michael A.,

2008 The Gridlock Economy: How Too Much Ownership Wrecks Markets,

Stops Innovation, and Costs Lives. New York: Basic Books.

Hemme, Dorothee

2009 Märchenstraßen - Lebenswelten. Zur kulturellen Konstruktion einer touristischen Themenstraße. Münster: LIT.

Hemme, Dorothee, Markus Tauschek und Regina Bendix, Hrsg.

2007a Prädikat „Heritage“. Wertschöpfung aus kulturellen Ressourcen. Münster: LIT.

Hemme, Dorothee, Markus Tauschek und Regina Bendix

2007b Vorwort. In Prädikat „Heritage“: Wertschöpfungen aus kulturellen

Ressourcen. Dorothee Hemme, Markus Tauschek und Regina Bendix, Hrsg. S.

7-17. Münster: LIT.

Hengartner, Thomas

2002 Das Telefon wird alltäglich. Zu einer Alltags- und Erfahrungsgeschichte

des Telefons. In Telemagie: 150 Jahre Telekommunikation in der Schweiz.

Kurt Stadelmann und Thomas Hengartner, Hrsg. S. 66-153. Zürich: Chronos. 2004 Zur „Kultürlichkeit“ von Technik: Ansätze kulturwissenschaftlicher

Technikforschung. In Technikforschung: zwischen Reflexion und

Dokumentation. Schweizerische Akademie der Geistes- und 
Sozialwissenschaften, Hrsg. S. 39-57. Bern: Schweizerische Akademie der Geistes- und Sozialwissenschaften.

2009 Von Schreib-, Spech- und Denkmaschinen. Zum Verhältnis von Mensch, Kultur und Technik. In Geisteswissenschaften in der Offensive: Hamburger Standortbestimmungen. Jörg Dierken, Hrsg. S. 255-275. Hamburg:

Europäische Verlagsanstalt.

Hengartner, Thomas und Johanna Rolshoven, Hrsg.

1998 Technik - Kultur: Formen der Veralltäglichung von Technik -

Technisches als Alltag. Zürich: Chronos.

Herz, Richard

1993 Legal Protection for Indigenous Cultures: Sacred Sites and Communal

Rights. Virginia Law Review 79(3):691-716.

Heywood, Denise

2008 Cambodian Dance. Celebration of the Gods. Bangkok: River Books.

Hilf, Meinhard

1973 Die Auslegung mehrsprachiger Verträge. Berlin: Springer.

Hill, Jane $\mathrm{H}$.

2008 The Everyday Language of White Racism. Malden: Wiley-Blackwell.

Hilty, Reto M.

2009 Rationales for the Legal Protection of Intangible Goods and Cultural

Heritage. Max Planck Institute for Intellectual Property, Competition \& Tax

Law Research Paper Series 9-10:1-25. http://ssrn.com/abstract=1470602 (Zugriff am 05.06.2010).

Hirschman, Albert

1945 National Power and the Structure of Foreign Trade. Berkeley: University of California Press.

Hirtle, Peter B.

2000 Archival Authenticity in a Digital Age. In Authenticity in a Digital

Environment. Council on Library and Information Resources, Hrsg. S. 8-23.

Washington: CLIR.

Hirtz, Frank

2003 It Takes Modern Means to be Traditional: On Recognizing Indigenous

Cultural Communities in the Philippines. Development \& Change 34(5):887917.

Hristov, Jasmin

2005 Indigenous Struggles for Land and Culture in Cauca, Columbia. Journal of Peasant Studies 32:88-117. 
Höpperger, Marcus

2007 Geographical Indications in the International Arena: The Current

Situation. http://www.wipo.int/edocs/mdocs/geoind/en/wipo_geo_bei_07/

wipo_geo_bei_07_www_81753.ppt (Zugriff am 06.05.2010).

Holznagel, Anke

1999 Eigentum und Erbschaft bei den Minangkabau in West Sumatra. Vortrag an der Universität Hannover. http://www.ganzrecht.de/index.php?id= 4,55,1,0,1,0 (Zugriff am 13.02.2010).

Honneth, Axel

1995 The Struggle for Recognition: The Moral Grammar of Social Conflicts.

Cambridge: MIT Press.

Hönes, Ernst-Rainer

2009 Internationaler Denkmal-, Kulturgüter- und Welterbeschutz.

Schriftenreihe des Deutschen Nationalkomitees für Denkmalschutz 74. Bonn:

Deutsches Nationalkomitee für Denkmalschutz.

Huber, Birgit

2005 Open Source - Software und „kulturelles Erbe“ indigener Bevölkerungen zwischen Markt und alternativer Rationalität. Von der Anthropologie des Rechts zu einer Antrhopologie als Basis des Rechts. In Recht und Religion im Alltagsleben: Perspektiven der Kulturforschung. Manfred Seifert und Winfried Helm, Hrsg. S. 41-59. Passau: Klinger.

Hug, Simon

2008 Multilateral Bargaining at the 2004 IGC and Referendums: An Empirical Assessment. Paper zur Konferenz "Direct Democracy in and around Europe: Integration, Innovation, Illusion and Ideology." Aarau, 02-04.10.2008.

Hughes, Justin

2007 Champagne, Feta, and Bourbon: The Spirited Debate About Geographical Indications. Hastings Law Journal 58:299-386.

Husmann, Rolf und Ingrid Wellinger

1992 A Bibliography of Ethnographic Film. Münster: LIT.

Hutton, Chris

2009 Language, Meaning and the Law. Edingburgh: Edingburgh University Press.

ICOMOS

2007 The Role of ICOMOS in the World Heritage Convention. http://www. international.icomos.org/world_heritage/icomoswh_eng.htm (Zugriff am 12.05.2010). 
Idrus, Nurul Ilmi

2007 Property, Inheritance and Authority: A Case Study of Land Resource

Management in Sangalla', Tana Toraja. http://sulawesi.cseas.kyoto-

u.ac.jp/final_reports2007/article/27-nurul.pdf (Zugriff am 25.10.2009).

Indonesian Media Law and Policy Centre et al.

2005 Lokakarya: The Impact of Intellectual Property Laws on Indonesian

Traditional Arts. http://www.wcl.american.edu/pijip/documents/ngos

07292005.doc (Zugriff am 06.09.2009)

IUCN (International Union for Conservation of Nature)

2001 IUCN Evaluation of World Heritage Nominations: Guidelines for

Reviewers of Cultural Landscapes - The Assessment of Natural Values in

Cultural Landscapes. http://cmsdata.iucn.org/downloads/guidelines_for_

reviewers_of_cls.pdf (Zugriff am 05.05.2010).

Isomura, Hisanori

2004 Le Japon et le Patrimoine Immatériel. In Le Patrimoine Culturel

Immatériel. Les Enjeux, les Problématiques, les Pratiques. Jean Duvignaud,

Chérif Khaznadar, Akinwumi Isola und Françoise Gründ, Hrsg. S. 41-48.

Paris: Babel.

Jackenkroll, Martina

2008 I-Tüpfelchen UNESCO-Welterbe? Lokale Deutungsmuster der

„Heritageifizierung“ des Dresdner Elbtals. Volkskunde in Sachsen 20:171-187.

Jacobeit, Wolfgang

1965 Bäuerliche Arbeit und Wirtschaft: Ein Beitrag zur Wissenschaftsgeschichte der deutschen Volkskunde. Berlin: Akademie-Verlag.

Jacobs, Marc

2007 Das Konventionsprojekt der UNESCO zum immateriellen Kulturerbe.

Von dem „deus ex machina“ und einem „Meisterwerk der Kompromisse“ und seiner politischen Umsetzung. UNESCO heute 07(1):9-15.

Janeba, Eckhard

2004 International Trade and Cultural Identity. In NBER Working Paper Series.

Cambridge: National Bureau of Economic Research.

Janke, Terry

2003 Minding Culture: Case Studies on Intellectual Property and Traditional

Cultural Expressions. Genf: World Intellectual Property Organization.

Jansen, Bernhard

1999 Die Verwendung der EU Amtssprache in Internationalen Abkommen.

EuZW. Europäische Zeitung für Wirtschaftsrecht 1:1-32. 
Jaszi, Peter

2009 Kebudayaan Tradisional: suatu langkah maju untuk perlindungan

Indonesia. Lembaga Studi Pers dan Pembangunan: Ford Foundation Jakarta, American University.

Jenkins, Richard

2004 Social Identity. New York: Routledge.

Jenzen, Igor A.

2006 Spiel mit den Grenzen. Die Neukonzeption des Museums für Sächsische Volkskunst. In Grenzen und Differenzen. Zur Macht sozialer und kultureller Grenzziehung. Thomas Hengartner und Johannes Moser, Hrsg. S. 579-586.

Leipzig: Leipziger Universitätsverlag.

Johnson, H. D. N.

1962 International Court of Justice Judgements of May 26, 1961 and June 15, 1962: The Case concerning the Temple of Preah Vihear. International and

Comparative Law Quarterly 11(4):1183-1204.

Jovanovic, Miodrag A.

2005 Recognizing Minority Identities through Collective Rights. Human Rights Quarterly 27:625-51.

Jung, Courtney

2003 The Politics of Indigenous Identity: Neoliberalism, Cultural Rights, and the Mexican Zapatistas. Social Research 70(2):433-462.

Kamil, Sulaeman

2000 Protection of Traditional Knowledge in Indonesia: Review. Paper zum

"UNCTAD Expert Meeting on Systems and National Experiences for

Protecting Traditional Knowledge, Innovations and Practices", Genf, 30.10.-

01.11.2000.

Kamusella, Tomasz

2009 The Politics of Language and Nationalism in Central Europe. Houndmills:

Palgrave Macmillan.

Kaneff, Deema, und Alexander D. King

2004 Introduction: Owning Culture. Focaal 44:3-19.

Kansa, Eric C., Jason Schultz und Ahrash N. Bissell

2005 Protecting Traditional Knowledge and Expanding Access to Scientific

Data: Juxtaposing Intellectual Property Agendas via a Model. International

Journal of Cultural Property 12(3):285-314.

Kaschuba, Wolfgang

2006 Einführung in die europäische Ethnologie. München: Beck. 
Kasten, Erich

2004 Ways of Owning and Sharing Cultural Property. In Properties of Culture Culture as Property. Pathways to the Reform in Post-Soviet Siberia. Erich Kasten, Hrsg. S. 9-34 Berlin: Reimer.

Kaufmann, Johan

1998 Some Practical Aspects of United Nations Decision-Making, Tactics and Interaction between Delegates. In Multilateral Diplomacy: The United Nations System at Geneva. A Working Guide. Marcel A. Boisard und Evgeny M. Chossudovsky, Hrsg. S. 231-246. The Hague: Kluwer Law International.

Kearney, Michael

1995 The Local and the Global: The Anthropology of Globalization and

Transnationalism. Annual Review of Anthropology 24:547-565.

Kingsbury, Benedict W.

1999 Operating Policies of International Institutions as Part of the Law Making

Process: The World Bank and Indigenous Peoples. In The Reality of

International Law: Essays in Honour of Ian Brownlie. Guy S. Goodwin-Gill,

Stefan Talmon und Ian Brownlie, Hrsg. S. 323-47. Oxford: Clarendon.

Kirsch, Stuart

2005 Property Limits: Debates on the Body, Nature and Culture. In Translations and Creations: Property Debates and the Stimulus of Melanesia. Marilyn Strathern und Eric Hirsch, Hrsg. S. 21-39. Oxford: Berghahn.

Kirshenblatt-Gimblett, Barbara

2004 Intangible Heritage as a Metacultural Production. Museum International 56 (1-2):52-65.

2006 World Heritage and Cultural Economics. In Museum Frictions. Public Cultures/Global Transformations. Ivan Karp, Corinne A. Kratz, Lznn Szwaja und Tomas Ybarra-Frausto, Hrsg. S. 161-203. Durham: Duke University Press.

Kis-Jovak, Jowa Imre, Hetty Nooy-Palm und Reimar Schefold, Hrsg.

1988 Banua Toraja: Changing Patterns in Architecture and Symbolism Among the Sa'dan Toraja, Sulawesi, Indonesia. Amsterdam: Royal Tropical Institute.

Klamer, Arjo und Peter-Wim Zuidhof

1999 The Values of Cultural Heritage: Merging Economic and Cultural Appraisals. In Economics and Heritage Conservation. Randy Mason, Hrsg. S. 23-62. Los Angeles: Getty Conservation Institute.

Kollewe, Carolin

2007 Wie „böse Steine“ zu „wertvollen Stücken“ werden: Produktion kulturellen Erbes und Konstruktion lokaler Identität während des Aufbaus eines Gemeindemuseums in Südmexiko. In Prädikat „Heritage“: 
Wertschöpfungen aus kulturellen Ressourcen. Dorothee Hemme, Markus

Tauschek und Regina Bendix, Hrsg. S.253-277. Berlin: LIT.

Kolliopoulos, Alexandros

2005 La Convention de l'UNESCO sur la diversité des expressions culturelles.

AFDI 51:487-511.

Koppel, Oliver

2008 Patente: Unverzichtbarer Schutz geistigen Eigentums. Wirtschaftsdienst 88(12):775-780.

Korff, Gottfried

1992 Volkskunst als ideologisches Konstrukt? Fragen und Beobachtung zum

politischen Einsatz der „Volkskunst“ im 20. Jahrhundert. Jahrbuch für Volkskunde 15:23-50.

Kreps, Christina

2009 Indigenous Curation, Museums, and Intangible Cultural Heritage. In

Intangible Heritage. Laurajane Smith und Natsuko Akagawa, Hrsg. S. 193-208.

London: Routledge.

Kroskrity, Paul V., Hrsg.

2000 Regimes of Language: Ideologies, Polities and Identities. Santa Fe: School of American Research Press.

Krüss, James

1962 Timm Thaler oder das verkaufte Lachen. Hamburg: Oetinger.

Kurniawan, Joeni Arianto

2008 Hukum Adat dan Problematika Hukum. Yuridika 23(1):1-21.

Kusumadara, Afifah

2008 Problems of Enforcing Intellectual Property Laws in Indonesia. Paper zur

Konferenz "The Law of International Business Transactions: A Global

Prespective", Hamburg, 10-12.04.2008.

Landes, William M. und Richard A. Posner

1989 An Economic Analysis of Copyright Law. Journal of Legal Studies 18(2):325-363.

2003 The Economic Structure of Intellectual Property Law. Cambridge:

Belknap Press.

2004 The Political Economy of Intellectual Property Law. Washington: AEI-

Brookings Joint Center for Regulatory Studies.

Larson, Jorge

2007 Relevance of Geographical Indications and Designations of Origin for the

Sustainable Use of Genetic Resources. http://www.underutilized-

species.org/Documents/PUBLICATIONS/gi_larson_lr.pdf (Zugriff am

05.05.2010). 
Latour, Bruno

1996 Der Berliner Schlüssel. Erkundungen eines Liebhabers der

Wissenschaften. Berlin: Akademie-Verlag.

1997 Nous n'Avons Jamais Été Modernes. Paris: La Découverte.

1999 Aramis, or the Love of Technology. Cambridge: Harvard University Press.

Lau, Kimberly J.

2000 New Age Capitalism: Making Money East of Eden. Philadelphia:

University of Pennsylvania Press.

Laurie, Nina, Robert Andolina und Sarah Radcliffe

2005 Ethnodevelopment: Social Movements, Creating Experts and

Professionalising Indigenous Knowledge in Ecuador. Antipode 37(3):470-496.

Lazear, Edward P.

1999 Culture and Language. Journal of Political Economy 107(S6):95-126.

Lee, Richard Borshay

2006 Twenty-First Century Indigenism. Anthropological Theory 6(4):455-479.

Lehmann, Albrecht und Andreas Kuntz Hrsg.

1988 Sichtweisen der Volkskunde: Zur Geschichte und Forschungspraxis einer

Disziplin. Berlin: Reimer.

Lerm, Susanne

2005 Pressemitteilung Fraktion Bündnis 90/Die Grünen: „Was ist der

UNESCO-Titel wert?“, 09.11.2005. http://www.welterbe-

erhalten.de/uploads/unesco/wert_welterbe.pdf (Zugriff am 05.05.2010).

Levy, David M.

2000 Where's Waldo? Reflections on Copies and Authenticity in a Digital

Environment. In Authenticity in a Digital Environment. Council on Library

and Information Resources, Hrsg. S. 24-31. Washington: CLIR.

Lewinski, Silke von

2003 Final Considerations. In Indigenous Heritage and Intellectual Property.

Silke von Lewinski, Hrsg. S.507-528. The Hague: Kluwer Law International.

2004 Protectiong Cultural Expressions: The Perspective of Law. In Properties of

Culture - Culture as Property: Pathways to the Reform in Post-Soviet Siberia.

Erich Kasten, Hrsg. S. 111-127. Berlin: Reimer.

Li, Tania Murray

2000a Articulating Indigenous Identity in Indonesia: Resource Politics and the

Tribal Slot. Comparative Studies in Society and History 42(1):149-179.

2000b Locating Indigenous Environmental Knowledge in Indonesia. In

Indigenous Environmental Knowledge and Its Transformations: Critical

Anthropological Perspectives. Roy Ellen, Peter Parkes und Alan Bicker, Hrsg.

S. 121-149. Amsterdam: Harwood. 
2007 Adat in Central Sulawesi: Contemporary Deployments. In The Revival of Tradition in Indonesian Politics: The Deployment of Adat from Colonialism to Indigenism. Jamie S. Davidson und David Henley, Hrsg. S. 337-370. New York: Routledge.

Liebig, Klaus

2008 Geistige Eigentumsrechte aus der Perspektive der Entwicklungsländer: Ein zwiespältiges Instrument. Wirtschaftsdienst 88(12):780-783.

Loulanski, Tolina

2006 Revising the Concept for Cultural Heritage: The Argument for a

Functional Approach. International Journal of Cultural Property 13(2):207233.

Lowrey, Kathleen

2008 Incommensurability and New Economic Strategies among Indigenous and Traditional Peoples. Journal of Political Ecology 15:61-74.

Lucas-Schloetter, Agnes

2008 Folklore. In Indigenous Heritage and Intellectual Property: Genetic

Resources, Traditional Knowledge and Folklore. Silke von Lewinski, Hrsg. S.

259-370. The Hague: Kluwer Law International.

Luttermann, Claus

1999 Rechtssprachenvergleich in der Europäischen Union: Ein Lehrbuchfall:

EuZW: Europäische Zeitung für Wirtschaftsrecht 13:401-416.

Maase, Kaspar

1998 Nahwelt zwischen „Heimat“ und „Kulisse“. Anmerkungen zur

volkskundlich-kulturwissenschaftlichen Regionalitätsforschung. Zeitschrift für Volkskunde 94:53-70.

Maase, Kaspar und Bernd Jürgen Warneken

2003 Der Widerstand des Wirklichen und die Spiele sozialer Willkür. Zum wissenschaftlichen Umgang mit den Unterwelten der Kultur. In Unterwelten der Kultur: Themen und Theorien der volkskundlichen Kulturwissenschaft. Kaspar Maase und Bernd Jürgen Warneken, Hrsg. S. 7-24. Köln: Böhlau.

MacDougall, David

1998 Transcultural Cinema. Lucien Taylor, Hrsg. Princeton: Princeton University Press.

Mackaay, Ejan

2007 The Economics of Intellectual Property Rights in Civil Law Systems. In Economic Analysis of Law: A European Perspective. Aristides N. Hatzis, Hrsg. Cheltenham: Edward Elgar.

Mackaay, Ejan und Stéphane Rousseau

2008 Analyse Économique du Droit. Paris: Dalloz-Sire. 
Macmillan, Fiona

2008 Human Rights, Cultural Property, and Intellectual Property: Three

Concepts in Search of a Relationship. In Intellectual Property and Traditional

Cultural Expressions in a Digital Environment. Chrisoph Beat Graber und

Mira Burri-Nenova, Hrsg. S. 50-63. Cheltenham: Edward Elgar.

Marcus, George E.

1995 Ethnography in/of the World System: The Emergence of Multi-Sited

Ethnography. Annual Review of Anthropology 24:95-117.

Marcus, George E. und Fred R. Myers

1995 The Traffic in Culture: Refiguring Art and Anthropology. Berkeley:

University of California Press.

Martínez Novo, Carmen

2005 Who Defines Indigenous? Identities, Development, Intellectuals, and the

State in Northern Mexico. New Brunswick: Rutgers University Press.

Mariotti, Luciana

2008 Prospettive Italiane della Convenzione per la Salvaguardia del Patrimonio

Culturale Immateriale. Ipotesi di Analisi tra Antropologia e Giuridiche. In Il

Patrimonio Immateriale Secondo l'UNESCO: Analisi e Prospettive. Chiara

Bortolotto, Hrsg. S.67-85. Rom: Ist. Poligrafico dello Stato.

Marrie, Henrietta

2009 The UNESCO Convention for the Safeguarding of the Intangible Cultural Heritage and the Protection and Maintenance of the Intangible Cultural Heritage of Indigenous People. In Intangible Heritage. Laurajane Smith und Natsuko Akagawa, Hrsg. S. 169-193. London: Routledge.

Mas-Colell, Andreu 1999 Should Cultural Goods be Treated Differently? Journal of Cultural Economics 23:87-93.

McAfee, Kathleen 1999 Selling Nature to Save it? Biodiversity and Green Developmentalism.

Environment and Planning D: Society and Space 17(2):133-154.

McKelvey, Richard D. und Peter C. Ordeshook

1984 An Experimental Study of the Effects of the Procedural Rules on

Committee Behavior. The Journal of Politics 46:182-205.

McLeod, Kembrew

2001 Owning Culture: Authorship, Ownership, and Intellectual Property Law.

New York: Peter Lang.

Merki, Christoph Maria

2002 Der holprige Siegeszug des Automobils 1895-1930: Zur Motorisierung des

Strassenverkehrs in Frankreich, Deutschland und der Schweiz. Wien: Böhlau. 
Merryman, John Henry,

1985 Thinking about the Elgin Marbles. Michigan Law Review 83:1881-1923.

1986 Two Ways of Thinking about Cultural Property. American Journal of International Law 80:831-853.

Meskell, Lynn

2002 The Intersections of Identity and Politics in Archaeology. Annual Review of Anthropology 31:279-301.

Miura, Keiko

1994 Information on Cambodian Shadow Puppets. Unveröffentlichtes Arbeitspapier.

2004 Contested Heritage. People of Angkor. Ph.D. Thesis. London: Unversity of London, SOAS.

2005 Conservation of a "Living Heritage Site". A Contradiction in Terms? Conservation and Management of Archaeological Sites 7:3-18.

2009 From Property to Heritage: Different Notions, Rules of Ownership and Practices of New and Old Actors in Angkor World Heritage Site. Beitrag zur Klausurtagung 2009 der DFG-Forschergruppe Cultural Property, Göttingen.

Mißling, Sven und Maleen Watermann

2009 Die doppelte Verantwortung der UNESCO. Zur zwiespältigen Ernennung des Tempels von Preah Vihear zum Weltkulturerbe. Vereinte Nationen 9(6):249-256.

Mißling, Sven

n.d. Die Kunst in Art. 5 Abs. 3 Satz 1 GG: Grundrechtliche Struktur und Funktion in der Gesellschaft. Ph.D. Dissertation, Göttingen: Institut für Völkerrecht und Europarecht, Georg August Universität Göttingen.

Ministry of Culture and Fine Arts (MoCFA)

2004a Sbek Thom. Khmer Shadow Theatre of Cambodia. Document for a National Candidature for the Proclamation of Masterpieces of the Oral and Intangible Heritage of Humanity. Phnom Penh: Ministry of Culture and Fine Arts/UNESCO.

2004b Proclamation of Masterpieces of the Oral and Intangible Heritage of Humanity. Document Submitted for Candidature of Sbek Thom Khmer Shadow Theatre of Cambodia. Phnom Penh: Ministry of Culture and Fine Arts/UNESCO.

Morgan, Lewis Henry

1878 Ancient Society or Researches in the Lines of Human Progress from

Savagery through Barbarism to Civilization. New York: Holt.

Moser, Hans

1962 Vom Folklorismus in unserer Zeit. Zeitschrift für Volkskunde 58:177-209. 
Münch, Ingo von

2002 Sprechen und Schweigen im Recht. NJW: Neue Juristische Wochenschrift 28:1995-2001.

Müller, Michael

2010 Kirche rückt Welterbe-Bewerbung in den Fokus. Annaberger Zeitung. 19.01.2010. http://www.freiepresse.de/nachrichten/regionales/erzgebirge/ annaberg/ 1663926.php (Zugriff am 05.05.2010).

Munjeri, Dawson

2004 Tangible and Intangible Heritage: From Difference to Convergence. Museum International 56(1-2):12-20.

Musitelli, Jean

2002 World Heritage - Between Universalism and Globalization. International Journal of Cultural Property IJCP 11(2):323-336.

2005 L'invention de la Diversité Culturelle. AFDI 51:512-523.

Myers, Fred R.

2001 The Empire of Things: Regimes of Value and Material Culture. Santa Fe: School of American Research Press.

2002 Painting Culture: The Making of an Aboriginal High Art. Durham: Duke University Press.

2004 Ontologies of the Image and Economies of Exchange. American

Ethnologist 31(1):5-20.

Nafziger, James A. R. und Tullio Scovazzi, Hrsg.

2008 The Cultural Heritage of Mankind. Leiden: Nijhoff.

Neth, Baromey

2009 Angkor as World Heritage Site and the Development of Tourism. A Study on Indestination Revenue in the Accomodation Sector in Siem Reap Angkor Region. Beitrag zur Klausurtagung 2009 der DFG-Forschergruppe Cultural Property, Göttingen.

Neuwirth, Rostam J.

2006 United in Divergency: A Commentary on the UNESCO Convention on the Protection and Promotion of the Diversity of Cultural Expressions. ZaöRV 66:819-862.

New, William

2009 WIPO Ponders New Negotiating Style; Ddg Positions Opening; Patent Filings Falter. http:/ /www.ip-watch.org/weblog/index.php?p=1412 (Zugriff am 15.04.2010).

Nicholas, Geroge P.

2005 The Persistence of Memory; the Politics of Desire: Archaeological Impacts on Aboriginal Peoples and their Response. In Indigenous Archaeologies: 
Decolonizing Theory and Practice. Claire Smith und Hans Martin Wobst, Hrsg. S. 81-103. London: Routledge.

2008 Policies and Protocols for Archaeological Sites and Associated Cultural and Intellectual Property. In Protection of First Nations Cultural Heritage:

Laws, Policy, and Reform. Catherine Edith Bell und Robert K. Paterson, Hrsg. S. 203-22. Vancouver: UBC Press.

Nicholas, George P. und Alison Wylie 2009 Archaeological Finds: Legacies of Appropriation, Modes of Response. In Ethics of Cultural Appropriation. James O. Young, und Conrad G. Brunk, Hrsg. S.11-51. Oxford: Wiley-Blackwell.

Nicholas, George P., und Kelly P. Bannister 2004 Copyrighting the Past? Emerging Intellectual Property Rights Issues in Archaeology. Current Anthropology 45(3):327-350.

Niezen, Ronald

2003 The Origins of Indigenism: Human Rights and the Politics of Identity.

Berkeley: University of California Press.

Noble, Brian.

2008 Owning as Belonging, Owning as Property: the Crisis of Power and

Respect in First Nations Heritage Transactions with Canada. In First Nations

Cultural Heritage and Law: Case Studies, Voices, and Perspectives. Catherine

Edith Bell und Val Napoleon, Hrsg. S. 465-488. Vancouver: UBC Press.

Nolte, Georg

2008 Kulturelle Vielfalt als Herausforderung für das Völkerrecht. Berichte der Gesellschaft für Völkerrecht 43:1-33.

Nora, Pierre

1984 Les Lieux de Mémoire. Paris: Gallimard.

1998 Zwischen Geschichte und Gedächtnis. Frankfurt am Main: Fischer.

Nora, Pierre und Étienne François

2005 Erinnerungsorte Frankreichs. München: Beck.

Nordmann, Matthias

2001 Rechtsschutz von Folkloreformen. Baden-Baden: Nomos.

Nooy-Palm, Hetty

1979 The Sa'dan-Toraja: a Study of their Social Life and Religion 1:

Organization, Symbols and Beliefs. Verhandelingen van het Koninklijk

Instituut voor Taal-, Land- en Volkenkunde 87. The Hague: Nijhoff.

1986 The Sa'dan-Toraja: A Study of Their Social Life and Religion 2: Rituals of the East and West. Verhandelingen van het Koninklijk Instituut voor Taal-, Land- en Volkenkunde, 118. Dordrecht: Foris. 
2001 The Ancestral House of the Sa'dan Torarja, Sulawesi, Indonesia. In Architectural Anthropology. Mari-Jose Amerlinck, Hrsg. S.145-170. Westport: Bergin \& Garvey.

Noyes, Dorothy

2006 The Judgement of Solomon: Global Protections for Tradition and the Problem of Community Ownership. Cultural Analysis 5:27-56.

Odendahl, Kerstin

2005a Die Bewahrung des immateriellen Erbes als neues Thema des

Völkerrechts, SZIER/RSDIE 15(3):445-459.

2005b Kulturgüterschutz. Entwicklung, Struktur und Dogmatik eines ebenenübergreifenden Normensystems. Tübingen: Mohr Siebeck.

O'Keefe, Patrick J. und Lyndel V. Prott 1992 "Cultural Heritage" or "Cultural Property"? International Journal of Cultural Property 1(2):307-320.

Ole Kaunga, Johnson 2006 Experiences from Kenya. Background Information Prepared for the WIPO Panel on Indigenous and Local Communities' Concerns and Experiences in Promoting, Sustaining and Safeguarding their Traditional Knowledge, Traditional Cultural Expressions and Genetic Resources, Geneva. http://www.kipo.ke.wipo.net/export/sites/www/tk/en/ngoparticipation/ind _loc_com/pdf/ole-kaunga.pdf (Zugriff am 13.09.2009).

Ong, Aihwa und Stephen J. Collier, Hrsg.

2004 Global Assemblages: Technology, Politics and Ethics as Anthropological Problems. Malden: Blackwell.

Opielka, Michael

2006 Gemeinschaft in Gesellschaft. Wiesbaden: VS Verlag für

Sozialwissenschaften.

OVG Bautzen

2007 Beschluss vom 09.03.2007 - 4 BS 216/06. Landes- und

Kommunalverwaltung 11:520.

Parry, Bronwyn

2004 Trading the Genome: Investigating the Commodification of Bioinformation. New York: Columbia University Press.

Pech, Tum Krevel

1995 Sbek Thom: Khmer Shadow Theatre. T. P. Sos Kem, Übersetzer. N.d.: Southeast Asia Program, Cornell University, UNESCO, JSRC.

Pelliot, Paul

1902 Mémoires sur les coutumes du Cambodge, par Tcheou Ta Kouan. Bulletin de l'École Française d'Extrême-Orient 2:123-177. 
Perreault, Thomas

2003a A People with our own Identity: Toward a Cultural Politics of

Development in Ecuadorian Amazonia. Environment and Plannning D:

Society and Space 21(5):583-606.

2003b Changing Places: Transnational Networks, Ethnic Politics, and

Community Development in the Ecuadorian Amazon. Political Geography

22(1):61-88.

2003c Social Capital, Development, and Indigenous Politics in Ecuadorian

Amazonia. Geographical Review 93(3):328-349.

2005 Geographies of Neoliberalism in Latin America. Environment and

Planning A 37(2):191-201.

Pfaffenberger, Bryan

1992a Social Anthropology of Technology. Annual Review of Anthropology

21:491-516.

1992b Technological Dramas. Science, Technology \& Human Values 17(3):282_

312.

Philips, Susan A.

2000 Constructing a Tongan Nation-State through Language Ideology in the

Courtroom. In Regimes of Language: Ideologies, Polities and Identities. Paul

V. Kroskrity, Hrsg. S. 229-258. Santa Fe: School of Amercian Research Press.

Pilcher, Jeffrey M.

1998 Que Vivan los tamales!: Food and the Making of Mexican Identity.

Albuquerque: University of New Mexico Press.

Postma, Metje und Peter Ian Crawford, Hrsg.

2006 Reflecting Visual Ethnography. Using the Camera in Anthropological

Research. Hoejberg: Intervention Press.

Povinelli, Elizabeth A.

2002 The Cunning of Recognition: Indigenous Alterities and the Making of

Australian Multiculturalism. Durham: Duke University Press.

Pratt, Mary Louise

2007 Afterword: Indigeneity Today. In Indigenous Experience Today. Marisol

de la Cadena und Orin Starn, Hrsg. S. 397-404. Oxford: Berg.

Putnam, Robert D.

1988 Diplomacy and Domestic Politics: The Logic of Two-Level Games.

International Organization 42(3):427-460.

Radcliffe, Sarah A.

2006a Conclusions: the Future of Culture and Development. In Culture and

Development in a Globalizing World: Geographies, Actors, and Paradigms.

Sarah A. Radcliffe, Hrsg. S. 228-237. London: Routledge. 
2006b Culture and Development in a Globalizing World: Geographies, Actors, and Paradigms. London: Routledge.

2006c Culture in Development Thinking: Geographies, Actors, and Paradigms. In Culture and Development in a Globalizing World : Geographies, Actors, and Paradigms. Sarah A. Radcliffe, Hrsg. S. 1-29. London: Routledge.

Radcliffe, Sarah A. und Nina Laurie

2006a Culture and Development: Taking Culture Seriously in Development for Andean Indigenous People. Environment and Plannning D: Society and Space 24(2):1-18.

2006b Indigenous Groups, Culturally Appropriate Development and the Sociospatial Fix of Andean Development. In Culture and Development in a Globalizing World: Geographies, Actors, and Paradigms. Sarah A. Radcliffe, Hrsg. S. 83-106. London: Routledge.

Ramsauer, Thomas

2005 Geistiges Eigentum und kulturelle Identität: eine Untersuchung zum immaterialgüterrechtlichen Schutz autochthoner Schöpfungen. München: Beck.

Rehbinder, Manfred und Heinrich Hubmann

2010 Urheberrecht: Ein Studienbuch. München: Beck.

Rehfeld, Nina

2009 Tod in der Heiler-Welt. Financial Times Deutschland, 22.10.2009.

http://www.ftd.de/lifestyle/outofoffice/:out-of-office-tod-in-der-heilerwelt/50026637.html (Zugriff am 05.05.2010).

Renninger, John P.

1989 The Failure to Launch Global Negotiations at the $11^{\text {th }}$ Special Session of the General Assembly. In Effective Negotiation: Case Studies in Conference Diplomacy. Johan Kaufmann, Hrsg. S. 231-254. Dordrecht: Nijhoff.

Renzikowski, Joachim

2002 Toleranz und die Grenzen des Strafrechts. In Gedächtnisschrift für Dieter Meurer. Eva Graul und Gerhard Wolf, Hrsg. S. 179-189. Berlin: De Gruyter Recht.

Rhoades, Robert E., Hrsg.

2006 Development with Identity: Community, Culture and Sustainability in the Andes. Cambridge: CABI Publishing.

Ridler, Neil B.

1986 Cultural Identity and Public Policy: An Economic Analysis. Journal of Cultural Economics 10(2):45-56. 
Riley, Angela R.

2000 Recovering Collectivity: Group Rights to Intellectual Property in Indigenous Communities. Cardozo Arts \& Entertainment Law Journal 18:175225.

2010 Sucking the Quileute Dry. The New York Times, 07.02.2010. http:// www.nytimes.com/2010/02/08/opinion/08riley.html (Zugriff am 05.05.2010).

Roussin, Lucille A.

2003 Cultural Heritage and Identity. Cardozo Journal of International and Comparative Law 11:707-710.

Ruby, Jay

1991 Speaking For, Speaking About, Speaking With, or Speaking Alongside: An Anthropological and Documentary Dilemma. Visual Anthropology Review 7(2):50-67.

2000 Picturing Culture. Explorations of Film \& Anthropology. Chicago:

University of Chicago Press.

Ruiz Fabri, Hélèn

2007 Jeux dans la Fragmentation: la Convention sur la Promotion et la

Protection de la Diversité des Expressions Culturelles. RGDIP 111:43-88.

Sahlfeld, Miriam

2008 Commercializing Cultural Heritage? Criteria for a Balanced

Instrumentalization of Traditional Cultural Expressions for Development in a

Globalized Digital Environment. In Intellectual Property and Traditional

Cultural Expressions in a Digital Environment. Christoph Beat Graber und

Mira Burri-Nenova, Hrsg. S. 256-86. Cheltenham: Edward Elgar.

Sardjono, Agus

2007 The Development of Indonesian Intellectual Property Laws in the Legal

Reform Era: Between Need and Reality. In Reforming Laws and Institutions in Indonesia: An Assessment. Naoyuki Sakumoto und Hikmahanto Juwana, Hrsg. S. 145-186. Chiba: Institute of Developing Economies, Japan External Trade Organization.

Satish, N. G.

2003 Rediscovering Traditional Knowledge: A Case Study of Neem.

International Journal of Information Technology and Management 2(3):184 196.

Saugestad, Sidsel

2001 The Inconvenient Indigenous: Remote Area Development in Botswana,

Donor Assistance and the First People of the Kalahari. Uppsala: Nordic Africa Institute. 
Saveros, Pou

1977 Ramakerti (XVIe-XVIIe Siècles). Volume 1-2. Paris: Ecole Française d'Extrême-Orient.

Sax, Joseph L,

1999 Playing Darts with a Rembrandt: Public and Private Rights in Cultural

Treasures. Ann Arbor: University of Michigan Press.

Scarduelli, Pietro

2005 Dynamics of Cultural Change among the Toraja of Sulawesi: The

Commoditization of Tradition. Anthropos 100(2):389-400.

Scafidi, Susan

2005 Who Owns Culture? Appropriation and Authenticity in American Law.

News Brunswick: Rutgers University Press.

Schack, Haimo

2007 Urheber- und Urhebervertragsrecht. Tübingen: Mohr Siebeck.

Schäfer, Stefan

2009 Die Zukunft des UNESCO-Welterbesystems: Reformansätze für das 21. Jahrhundert. Vereinte Nationen 7(6): 243-256

Scharfe, Martin

1993a Technik und Volkskultur. In Kultur und Technik: Zu ihrer Theorie und

Praxis in der modernen Lebenswelt. Wolfgang König und Marlene Landsch, Hrsg. S. 43-69. Frankfurt am Main: Peter Lang.

1993b Utopie und Physik: Zum Lebensstil der Moderne. In Der industrialisierte

Mensch. Michael Dauskardt und Helge Gerndt, Hrsg. S. 73-90. Münster: Ardey.

2009 Kulturelle Materialität. In Erb.gut? Kulturelles Erbe in Wissenschaft und Gesellschaft. Karl C. Berger, Hrsg. S. 15-33. Wien: Selbstverlag des Vereins für Volkskunde.

Scher, Philip W.

2002 Copyright Heritage: Preservation, Carnival and the State in Trinidad. Anthropological Quarterly 75(3):453-484.

Schieffelin, Bambi B., Kathryn A. Woolard und Paul V. Kroskrity 1998 Language Ideologies: Practice and Theory. Oxford: Oxford University Press.

Schlinkert, Jana C.

2007 Lebendige folkloristische Ausdrucksweisen traditioneller Gemeinschaften: rechtliche Behandlungsmöglichkeiten auf internationaler Ebene. Berlin: BWV.

Schneider, Franka

2009a Städtische Arenen volkskundlicher Wissensarbeit. Die internationale Volkskunstausstellung 1909 im Berliner Warenhaus Wertheim. In Horizonte 
ethnographischen Wissens. Eine Bestandsaufnahme. Ina Dietzsch, Wolfgang Kaschuba und Leonore Scholze-Irrlitz, Hrsg. S. 54-86. Wien: Böhlau.

Schneider, Irene

2009b Civil Society and Legislation: Development of the Human Rights

Situation in Iran 2008. In Islam and Human Rights. Hatem Elliesie, Hrsg. S. 339-366. Frankfurt am Main: Peter Lang.

Schönberger, Klaus

2006 Using ICT and Socio-Cultural Change: Persistent and Recombinant

Practices in Using Weblogs. In Cultural Attitudes towards Technology and

Communication. Fay Sudweeks, Fay Hrachovec und Charles Ess, Hrsg. S.

642-658. Sidney: Murdoch.

2007 Technik als Querschnittsdimension: Kulturwissenschaftliche

Technikforschung am Beispiel von Weblog-Nutzung in Frankreich und

Deutschland. Zeitschrift für Volkskunde 103(2):197-221.

Schorlemer, Sabine von

1992 Internationaler Kulturgüterschutz. Ansätze zur Prävention im Frieden sowie im bewaffneten Konflikt. Berlin: Duncker \& Humblot.

2005 Kulturpolitik im Völkerrecht verankert. Das neue UNESCO-

Übereinkommen zum Schutz der kulturellen Vielfalt. Vereinte Nationen 05(6):217-224.

2006 Die Dresdner Brücken-Posse. Blätter für deutsche und internationale Politik 11(51):1312-1315.

Schramm, Manuel

2003 Konsum und regionale Identität in Sachsen 1880-2000. Die

Regionalisierung von Konsumgütern im Spannungsfeld von Nationalisierung und Globalisierung. Stuttgart: Franz Steiner.

Schröder, Hans Joachim

2007 Technik als biographische Erfahrung 1930-2000: Dokumentation und

Analyse lebensgeschichtlicher Interviews. Zürich: Chronos.

Schürch, Franziska

2008 Landschaft, Senn und Kuh: Die Entdeckung der Appenzeller Volkskunst.

Basel: Waxmann.

Schuster, Mark J.

2002 Making a List and Checking it Twice: The List as a Tool of Historic

Preservation. Harris School of Public Policy Studies Working Papers 0303.

http://harrisschool.uchicago.edu/about/publications/working-

papers/pdf/wp_03_03.pdf (Zugriff am 07.05.2010). 
Scovazzi, Tullio

2008 La notion du patrimoine culturel de l'humanité dans les instruments internationaux. In The Cultural Heritage of Mankind. James A. R. Nafziger und Tullio Scovazzi, Hrsg. S. 3-142. Leiden: Nijhoff.

Seeger, Anthony

2001 Intellectual Property and Audiovisual Archives and Collections. In Folk Heritage Collections in Crisis. Council on Library and Information Resources, Hrsg. S. 32-46. Washington: Council on Library and Information Resources. 2009 Lessons Learned from the ICTM (NGO) Evaluation of Nominations for the UNESCO Masterpiece of the Oral and Intangible Heritage of Humanity 2001-2005. In Intangible Heritage. Laurajane Smith und Natsuko Akagawa, Hrsg. S. 112-129. London: Routledge.

Seleti, Yonah

2009 Promoting Access to Traditional African Medicine, Managing the IPR: The Case of South Africa. Präsentation zur "Conference on Intellectual Property and Public Policy Issues." http://www.wipo.int/export/sites/www/meetings/ en/2009/ip_gc_ge/presentations/seleti.pdf (Zugriff am 13.09.2009).

Sherwood-Edwards, Mark

1994 The Redundancy of Originality. International Review of Industrial Property and Copyright Law. 25(5):658-689.

Siegrist, Hannes und David Sugarmann

1999 Eigentum im internationalen Vergleich (18.-20. Jahrhundert). Göttingen:

Vandenhoeck \& Ruprecht.

Silverstein, Michael

1976 Shifters, Linguistic Categories, and Cultural Description. In Meaning in Anthropology. Keith H. Basso und Henry A. Selby, Hrsg. S. 11-55. Santa Fe: School of American Research Press.

1979 Language Structure and Linguistic Ideology. In The Elements: A

Parasession on Linguistic Units and Levels. Paul R. Cline, William F. Hanks und Carol L. Hofbauer, Hrsg. S. 193-247. Chicago: Chicago Linguistic Society. 2003 Translation, Transduction, Transformation: Skating "Glossando" on Thin Semiotic Ice. In Translating Cultures: Perspectives on Translation and Anthropology. Paula G. Rubel und Abraham Rosman, Hrsg. S. 75-105.

Oxford: Berg.

2004 "Cultural" Concepts and the Language-Culture Nexus. Current Anthropology 45(5):621-652.

Simmel, Georg

2009 Philosophie des Geldes. Frankfurt am Main: Suhrkamp.

Singh, L.P.

1962 The Thai-Cambodian Temple Dispute. Asian Survey 2(8):23-26. 
Sjöstedt, Gunnar, Bertram I. Spector und I. William Zartman

1994 The Dynamics of Regime-Building Negotiations. In Negotiating

International Regimes. Lessons Learned from the United Nations Conference on Environment and Development (UNCED). Bertram I. Spector, Gunnar

Sjöstedt und I. William Zartman, Hrsg. S. 3-20. London: Graham \& Trotham.

Smith, Laurajane

2004 Archaeological Theory and the Politics of Cultural Heritage. London:

Routledge.

2006 Uses of Heritage. Abingdon: Routledge,

Smith, Laurajane und Natsuko Akagawa

2009 Intangible Heritage. London: Routledge.

Snidal, Duncan

1985 The Game Theory of International Politics. World Politics 38(1):25-57.

Sola, Angélica

2008 Quelques réflexions à propos de la Convention pour la sauvegarde du

patrimoine culturel immatériel. In The Cultural Heritage of Mankind. James A.

R. Nafziger und Tullio Scovazzi, Hrsg. S. 487-527. Leiden: Nijhoff.

Secretatiat of the Pacific Community

2002a Guidelines for Developing National Legislation for the Protection of

Traditional Knowledge and Expressions of Culture Based on the Pacific Model

Law 2002. http://www.wipo.int/export/sites/www/tk/en/laws/pdf/

spc_guidelines.pdf (Zugriff am 25.05.2010).

2002b Regional Framework for the Protection of Traditional Knowledge and

Expressions of Culture. http://www.wipo.int/export/sites/www/tk/en/laws/

pdf/spc_framework.pdf (Zugriff am 25.05.2010).

Speed, Shannon

2008 Rights in Rebellion: Indigenous Struggle and Human Rights in Chiapas.

Palo Alto: Stanford University Press.

Srinivas, Burra

2008 The UNESCO Convention for the Safeguarding of the Intangible

Heritage. In The Cultural Heritage of Mankind. James A.R. Nafziger und Tullio

Scovazzi, Hrsg. S. 529-556. Leiden: Nijhoff.

Stadelmann, Kurt und Thomas Hengartner, Hrsg.

2002 Telemagie: 150 Jahre Telekommunikation in der Schweiz. Zürich:

Chronos.

Stewart-Harawira, Makere

2005 The New Imperial Order: Indigenous Responses to Globalization.

London: Zed Books. 
Stoll, Peter-Tobias und Anja von Hahn

2008 Indigenous Peoples, Indigenous Knowledge and Indigenous Resources in International Law. In Indigenous Heritage and Intellectual Property. Silke von Lewinski, Hrsg. S. 7-52. Alphen: Kluwer Law International.

Sunder, Madhavi

2007 The Invention of Traditional Knowledge. Law \& Contemporary Problems 70:97-124.

Sykes, Alan O.

2004 The Economics of Public International Law. University of Chicago Law \& Economics Olin Working Papers 216:1-81.

Sylvain, Renée

2002 Land, Water, and Truth: San Identity and Global Indigenism. American Anthropologist 104(4):1074-1085.

2005 Disorderly Development: Globalization and the Idea of "Culture" in the Kalahari. American Ethnologist 32(3):354-370.

Symonides, Janusz

1998 The Implementation of Cultural Rights by the International Community.

Gazette 60(1):7-25.

Szasz, Paul

1989 Reforming the Multilateral Treaty-Making Process: Opportunity Missed. In International Law at a Time of Perplexity. Essays in Honour of Shabtai

Rosenne. Yoram Dinstein, Hrsg. S. 909-939. Leiden: Martinus Nijhoff. 1992 International Norm-Making. In Environmental Change and International Law. Edith Brown Weiss, Hrsg. S. 41-80. Tokyo: United Nations University Press.

Tauschek, Markus

2009 Cultural Property as Strategy: The Carnival of Binche, the Creation of Cultural Heritage and Cultural Property. Ethnologia Europaea 39(2):67-80.

Te Heesen, Anke und Emma C. Spary, Hrsg.

2001 Sammeln als Wissen: Das Sammeln und seine wissenschaftsgeschichtliche Bedeutung. Göttingen: Wallstein-Verlag.

Teubner, Gunter und Andreas Fischer-Lescano

2008 Cannibalizing Epistemes: Will Modern Law Protect Traditional Cultural

Expressions? In Intellectual Property and Traditional Cultural Expressions in a Digital Environment. Christoph Beat Graber und Mira Burri-Nenova, Hrsg. S. 17-48. Cheltenham: Edward Elgar.

The Economist

2010 The "Indigenisation" of Zimbabwe: Foreigners and Local Whites Out. http://www.economist.com/world/middle-east/displaystory.cfm?story_id $=15955482$ (Zugriff am 10.05.2010). 
Thierry, Solange

1963 Les danses sacrées au Cambodge. In Sources Orientales VI. N.d. S. 343-

373. Paris: Editions du Seuil.

Thiounn, Samdech Chaufea

1956[1930] Danses cambodgiennes. Phnom Penh: Buddhist Institute.

Throsby, David

2003 Determining the Value of Cultural Goods: How Much (or How Little)

Does Contingent Valuation Tell Us? Journal of Cultural Economics 27:275285.

Toivanen, Reetta

2007 Linguistic Diversity and the Paradox of Rights Discourse. In The

Language Question in Europe and Diverse Societies: Political, Legal and Social

Perspectives. Dario Castiglione und Chris Longman, Hrsg. S. 101-121.

Oxford: Hart.

Tollison, Robert D. und Thomas D. Willet

1979 An Economic Theory of Mutually Advantageous Issue Linkages in

International Negotiations. International Organization 33(4):425-449.

Torsen, Molly A.

2008 Intellectual Property and Traditional Cultural Expressions: A Synopsis of

Current Issues. Intercultural Human Rights Law Review 3:199-214.

Tschofen, Bernhard

2007 Antreten, ablehnen, verwalten? Was der Heritage Boom den

Kulturwissenschaften aufträgt. In Prädikat Heritage. Wertschöpfung aus

kulturellen Ressourcen. Dorothee Hemme, Markus Tauschek und Regina

Bendix, Hrsg. S. 19-32. Münster: LIT.

Triebel, Volker und Stephan Balthasar

2004 Auslegung Englischer Vertragstexte unter Deutschen Vertragsstatut:

Fallstricke des Art. 32 I Nr. 1 EGBGB. NJW. Neue Juristische Wochenschrift 31:2189-2196.

Trouillot, Michel-Rolph

2003 Global Transformations: Anthropology and the Modern World.

Houndmills: Palgrave Macmillan.

Trumbore, Peter F.

1998 Public Opinion as a Domestic Constraint in International Negotiations:

Two-Level Games in the Anglo-Irish Peace Process. International Studies Quarterly 42(3):545-565.

Tsing, Anna L.

1999 Becoming a Tribal Elder and other Development Fantasies. In

Transforming the Indonesian Uplands: Marginality, Power and Production.

Tania Murray Li, Hrsg. S. 159-202. Amsterdam: Harwood. 
2005 Friction: An Ethnography of Global Connection. Princeton: Princeton University Press.

Turnbridge, John E.und Gregory John Ashworth

1996 Dissonant Heritage: The Management of the Past as a Resource in

Conflict. Chichester: Wiley.

Tylor, Edward Burnett

1871 Primitive Culture: Researches into the Development of Mythology,

Philosophy, Religion, Language, Art and Custom. London: Murray.

Ueki, Yukinobu

2006 Unique Features of Japan's Intangible Cultural Heritage and Its Protection.

A Case Study from Japan. In Proceedings. International Conference on the

Safeguarding of Tangible and Intangible Cultural Heritage: Towards an

Integrated Approach. S.114-130. Paris: UNESCO/Agency for Cultural Affairs (Japan).

UN

1980 Review on Multilateral Treaty-Making Process. United Nations.

2000 Human Rights of Indigenous Peoples, Commission on Human Rights.

Report of the Seminar on the Draft Principles and Guidelines for the

Protection of the Heritage of Indigenous People. United Nations. Vol.

E/CN.4/Sub.2/2000/26.

2007 Declaration on the Rights of Indigenous Peoples United Nations.

UNESCO

1954 Haager Protokoll über den Schutz von Kulturgut bei bewaffneten

Konflikten (Convention for the Protection of Cultural Property in the Event of Armed Conflict). Den Haag: UNESCO.

1970 Übereinkommen über Massnahmen zum Verbot und zur Verhütung der rechtswidrigen Einfuhr, Ausfuhr und Übereignung von Kulturgut (Convention on the Means of Prohibiting and Preventing the Illicit Import, Export and Transfer of Ownership of Cultural Property). Paris: UNESCO.

1972 Übereinkommen zum Schutz des Kultur- und Naturgutes der Welt

(Convention Concerning the Protection of the World Cultural and Natural Heritage). Paris: UNESCO.

1982 Mexico City Declaration on Cultural Policies. Mexiko Stadt: UNESCO.

2001 Universal Declaration on Cultural Diversity. Paris: UNESCO.

2003 Übereinkommen zur Bewahrung des immateriellen Kulturerbes

(Convention for the Safeguarding of the Intangible Cultural Heritage). Paris:

UNESCO.

2005a Konvention zum Schutz und zur Förderung der Vielfalt kultureller Ausdrucksformen (Convention on the Protection and Promotion of the Diversity of Cultural Expressions). Paris: UNESCO. 
2005b Operational Guidelines for the Implementation of the World Heritage Convention. Paris: UNESCO.

2009 The Representative List of the Intangible Cultural Heritage of Humanity: Indonesian Batik. http://www.unesco.org/culture/ich/index.php?RL $=00170$ (Zugriff am 28.01.2010).

UNESCO und MoCFA

2004 Inventory of Intangible Cultural Heritage of Cambodia. Phnom Penh: JSRC.

UNIDROIT

1995 Convention on Stolen or Illegally Exported Cultural Objects.

http://www.unidroit.org/english/conventions/1995culturalproperty/1995cult uralproperty-e.htm (Zugriff am 05.05.2010).

Utomo, Tomi Suryo

2009 Kepemilikan dan benefit sharing terhadap komersialiasi sumber days genetika, pengetahuan tradisional dan folklor (GRTKF) di dalam sistem hukum Indonesia. Fokus 6(3):14-29.

Vaidhyanathan, Siva

2001 Copyrights and Copywrongs: The Rise of Intellectual Property and how it Threatens Creativity. New York: New York University Press.

Verdery, Katherine und Caroline Humphrey

2004 Property in Question: Value Transformation in the Global Economy. Oxford: Berg.

Visiting Arts

2001 Cambodia Arts Directory: Visiting Arts South East Asia Regional Arts Profile. London: Visiting Arts.

Volkman, Toby Alice

1985 Feasts of Honor: Ritual and Change in the Toraja Highlands. Urbana: University of Illinois Press.

Wagner, Markus

2008 The Temple of Preah Vihear Case. In Max Planck Encyclopedia of Public International Law (EPIL). Rüdiger Wolfrum, Hrsg. http://www.mpepil.com/ subscriber_article?id=/epil/entries/law-9780199231690-e216 (Zugriff am 10.03.2010).

Wandtke, Artur-Axel et al.

2009 Praxiskommentar zum Urheberrecht. München: Beck.

2010 Fallsammlung zum Urheber- und Medienrecht für Studium,

Fachanwaltsausbildung und Praxis. München: Beck.

Wandtke, Artur-Axel und Claire Dietz

2009 Urheberrecht. Berlin: De Gruyter Recht. 
Watanabe, Akiyoshi

2006 The Japanese System for the Safeguarding of Cultural Heritage. In

Proceedings. International Conference on the Safeguarding of Tangible and

Intangible Cultural Heritage: Towards an Integrated Approach, S. 74-98. Paris:

UNESCO/Agency for Cultural Affairs (Japan).

Waterson, Roxana

1990 The Living House: An Anthropology of Architecture in South-East Asia.

Oxford: Oxford University Press.

2003 The Immortality of the House in Tana Toraja. In The House in Southeast

Asia. Stephen Sparks und Signe Howell, Hrsg. S. 34-52. London: Routledge.

2009 Paths and Rivers. Sa'dan Toraja Society in Transformation. Leiden:

KITLV Press.

Watts, Michael

2006 Culture, Development and Global Neo-Liberalism. In Culture and

Development in a Globalizing World: Geographies, Actors, and Paradigms.

Sarah A. Radcliffe, Hrsg. S. 30-57. London: Routledge.

Weigelt, Frank- André

2008 Cultural Property und Cultural Heritage. Eine vergleichend ethnologische

Analyse internationaler UNESCO-Konzeptionen. Saarbrücken: VDM.

Weismantel, Mary

2006 Ayllu: Real and Imagined Communities in the Andes. In The Seductions of

Community: Emancipations, Oppressions, Quandaries. Gerald W. Creed,

Hrsg. S. 77-100. Santa Fe: School of American Research Press.

Welker, Marina A.

2009 "Corporate Security Begins in the Community": Mining, the Corporate

Social Responsibility Industry, and Environmental Advocacy in Indonesia.

Cultural Anthropology 24(1):142-179.

Welz, Gisela

1998 Moving Targets. Feldforschung unter Mobilitätsdruck. Zeitschrift für Volkskunde 2:177-194.

2004 Multiple Modernities: The Transnationalisation of Cultures. Research

Group Transnationalism Working Paper 5:1-20.

Wendland, Wend

2002a Intellectual Property, Traditional Knowledge and Folklore: WIPO’s

Exploratory Program, Part I. International Review of Industrial Property and

Copyright Law 33(4):485-504.

2002b Intellectual Property, Traditional Knowledge and Folklore: WIPO’s

Exploratory Program, Part II. International Review of Industrial Property and Copyright Law 33(5):606-621.

2008 "It's a small world (after all)": Some Reflections on Intellectual Property and Traditional Cultural Expressions. In Intellectual Property and Traditional 
Cultural Expressions in a Digital Environment. Christoph Beat Graber und Mira Burri-Nenova, Hrsg. S. 150-182. Cheltenham: Edward Elgar.

Whatmore, Sarah

2002 Hybrid Geographies: Natures, Cultures, Spaces. London: Sage.

Whitby-Last, Kathryn

2008 Cultural Landscapes. In The 1972 World Heritage Convention: A

Commentary. Francesco Francioni und Federico Lenzerini, Hrsg. S. 51-63.

Oxford: Oxford University Press.

Wiedemann, Andreas

2007 „Komm mit uns das Grenzland aufbauen!“: Ansiedlung und neue

Strukturen in den ehemaligen Sudetengebieten 1945-1952. Essen: Klartext. WIPO

1998 General Rules of Procedure. 399 (FE) Rev. 3.

2000 WIPO General Assembly: Matters Concerning Intellectual Property and Genetic Resources, Traditional Knowledge and Folklore. WO/GA/26/6. 2001a Intergovernmental Committee on Intellectual Property and Genetic

Resources, Traditional Knowledge and Folklore. Rules of Procedure.

WIPO/GRTKF/IC/1/2.

2001b Intergovernmental Committee on Intellectual Property and Genetic

Resources, Traditional Knowledge and Folklore. Report Adopted by the

Committee. WIPO/GRTKF/IC/2/16.

2003a Intergovernmental Committee on Intellectual Property and Genetic

Resources, Traditional Knowledge and Folklore. Consolidated Analysis of the

Legal Protection of Traditional Cultural Expressions/Expressions of Folklore. WIPO/GRTKF/IC/5/3.

2003b Intergovernmental Committee on Intellectual Property and Genetic

Resources, Traditional Knowledge and Folklore. The Protection of Traditional Cultural Expressions/Expressions of Folklore: Legal and Political Options. WIPO/GRTKF/IC/6/3.

2006a Booklet: Intellectual Property and Traditional Cultural

Expressions/Folklore. Geneva: World Intellectual Property Organization.

2006b Intergovermental Committee on Intellectual Property and Genetic

Resources, Traditional Knowledge and Folklore. The Protection of Traditional

Cultural Expressions/Expressions of Folklore: Revised Objections and

Principles. WIPO/GRTKF/IC/9/4.

2006c Intergovermental Committee on Intellectual Property and Genetic

Resources, Traditional Knowledge and Folklore. The Protection of Traditional

Cultural Expressions/Expressions of Folklore: Updated Draft Outline of

Policy Options and Legal Mechanisms. WIPO/GRTKF/IC/9/INF/4. 2007 Intergovernmental Committee on Intellectual Property and Genetic

Resources, Traditional Knowledge and Folklore. Bandung Declaration on the

Protection of Traditional Cultural Expressions, Traditional Knowledge, and 
Genetic Resources. Document submitted by the Delegation of Indonesia. WIPO/GRTKF/IC/11/12.

2008a Intergovernmental Committee on Intellectual Property and Genetic

Resources, Traditional Knowledge and Folklore. Second Provisional List of Participants. WIPO/GRTKF/IC/13/INF/1 Prov. 2.

2008b WIPO's New Director General Outlines Challenges and Priorities. http://www.wipo.int/about-wipo/en/dgo/dg_gurry_acceptance_speech _2008.html (Zugriff am 04.05.2010).

2010 WIPO Intellectual Property Handbook: Policy, Law and Use. http:// www.wipo.int/about-ip/en/iprm (Zugriff am 27.01.2010).

Wolf, Rainer

2008 Weltkulturvölkerrecht und nationalstaatliche Umsetzung. Natur und Recht 30:311-319.

Woolard, Kathryn A. und Bambi B. Schieffelin

1994 Language Ideology. Annual Review of Anthropology 23(1):55-82.

World Fair Trade Organization

2007 IFAT Standards for Fair Trade Organizations. http://www.wfto.com/ index.php?option $=$ com_content\&task $=$ view\&id $=39 \&$ Itemid $=125($ Zugriff am 25.05.2010).

Yu, Peter K.

2007 Reconceptualizing Intellectual Property Interests in a Human Rights

Framework. University of California Davis Law Review 40:1039-149.

Yúdice, George

2003 The Expediency of Culture: Uses of Culture in the Global Era. Durham:

Duke University Press.

Zimmermann, Harm-Peer

2009 Memory, Markt und Medien. Analyse des UNESCO-Programms

"Memory of the World" im Hinblick auf die Fragen der Kommerzialisierung und Popularisierung. In Zwischen Identität und Image. Die Popularität der Brüder Grimm in Hessen. Harm-Peer Zimmermann, Hrsg. S. 543-571. Marburg: Jonas. 
ann Eigentum an Kultur sinnvoll sein? Das Interesse, Cultural Property dem Markt zuzuführen oder dies zu verhindern und hierdurch kollektiven oder individuellen, ideologischen oder ökonomischen Gewinn zu schaffen, gestaltet sich unter den stark divergierenden Bedingungen, die Akteure in einer postkolonialen, spätmodernen Welt vorfinden. Die interdisziplinäre DFG-Forschergruppe zur Konstituierung von Cultural Property beleuchtet diese seit einigen Jahren in der Öffentlichkeit mit wachsender Brisanz verhandelte Frage. Die Forschergruppe fragt nach der Konstituierung von Cultural Property im Spannungsfeld von kulturellen, wirtschaftlichen, juristischen und hiermit auch gesellschaftspolitischen Diskursen. Dies bedingt auch die in dieser fokussierten Form neue Zusammenarbeit von Fachwissenschaftler/innen aus Kultur- und Sozialwissenschaften sowie Rechts-und Wirtschaftswissenschaften. Die Unterschiedlichkeit des disziplinären Zugriffs auf einen Forschungsbereich zeigt sich in den in diesem Band vermittelten ersten Ergebnissen aus der laufenden Forschung genauso deutlich wie die Notwendigkeit, disziplinäre Standpunkte in gemeinsamer Arbeit zusammenzuführen, um den Konstituierungsprozess von Cultural Property zu verstehen.

ISBN: 978-3-941875-61-6

ISSN: $2190-8672$ 